\title{
Copper-Catalyzed Photo-Induced Enantioselective Dual Carbofunctionalization of Alkenes
}

Yajing Zhanga, Youwen Sun, ${ }^{\mathrm{b}}$ Bin Chen, ${ }^{\mathrm{b}}$ Meichen Xu, ${ }^{\mathrm{b}}$ Chen li, ${ }^{\mathrm{b}}$ Dayong Zhang ${ }^{\star \mathrm{a}}$ and Guozhu Zhang*b

anstitute of Pharmaceutical Science, China Pharmaceutical University, Nanjing, 210009, China

'SState Key Laboratory of Organometallic Chemistry, Shanghai Institute of Organic Chemistry, Center for Excellence in Molecular Synthesis, University of Chinese Academy of Sciences, Chinese Academy of Sciences, 345 Lingling Road, Shanghai 200032, P. R. China 


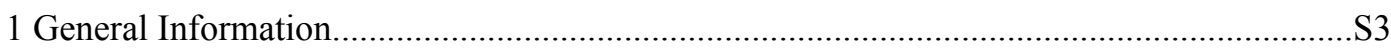

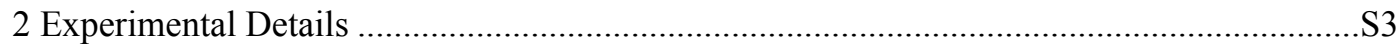

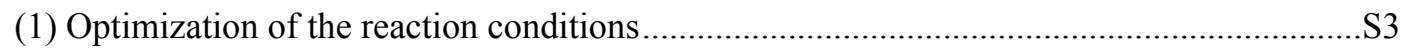

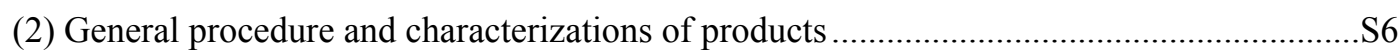

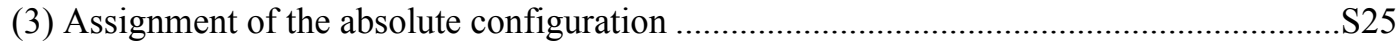

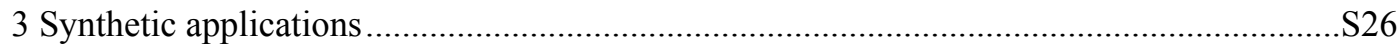

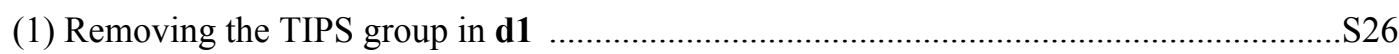

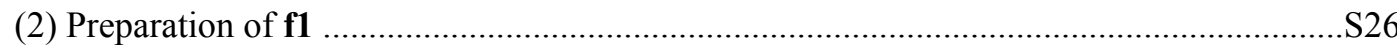

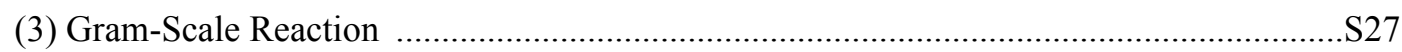

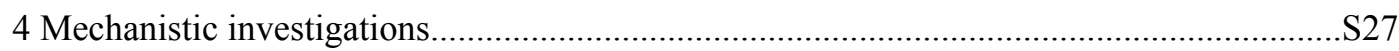

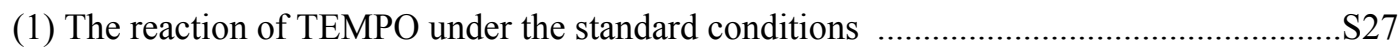

(2) Considerations of the reaction's active intermediate. ................................................... 228

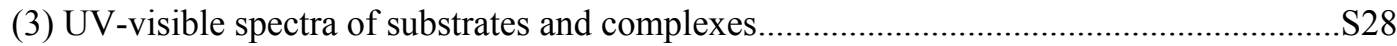

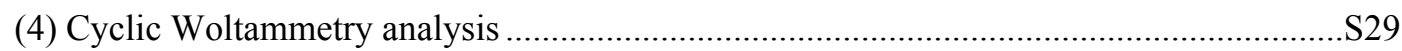

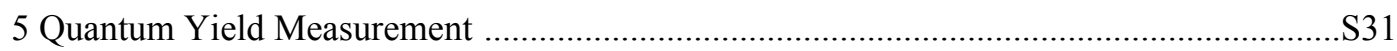

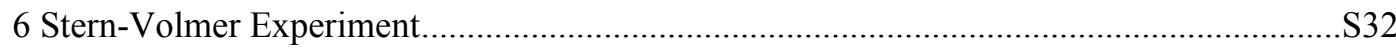

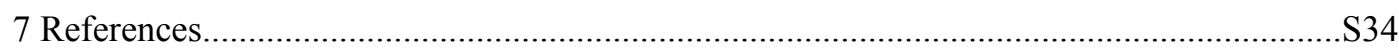

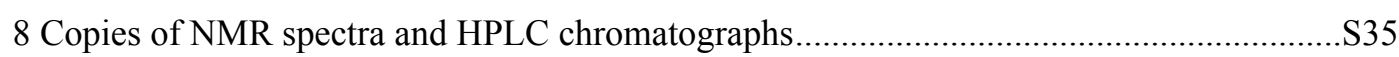




\section{General Information}

All reagents were purchased from commercial suppliers and all reactions were performed in glove box under a nitrogen atmosphere. NMR spectra were recorded on Bruker (400 MHz), Varian Inova (400 MHz) or Aglient (400 MHz) spectrometer. ${ }^{1} \mathrm{H}-\mathrm{NMR}$ spectra chemical shifts $(\delta)$ are given in ppm (parts per million) with TMS as internal standard and NMR solvent was CDCl3 (77.16 ppm for ${ }^{13} \mathrm{C}, 0 \mathrm{ppm}$ for ${ }^{1} \mathrm{H}$ ). The multiplicities are abbreviated as follows: s (singlet) $\mathrm{d}$ (doublet) $\mathrm{t}$ (triplet) $\mathrm{q}$ (quartet) and $\mathrm{m}$ (multiplet). High performance liquid chromatography (HPLC) was performed on Aglilent 1260, Ultimate 3000 RS Autosampler and Water e2695. Mass spectra were carried on EIHR (GC-TOF), GCMS-QP2020 spectrometer. All IR spectra were performed with Nicolet 380 and the significant wave numbers $\tilde{\mathrm{v}}$ are showed in $\mathrm{cm}^{-1}$ in brackets. Optical rotation was performed on Rudolph-Autopol I. UV/Vis absorption spectra were recorded on Varian Cary 100. Cyclic voltammetry were obtained from Shanghai Chen Hua CHI660. Fluorescence spectrum was obtained from HITACHI F-2700. Photolysis experiments were performed on FZ-A Photolysis Spectrometer. Materials: Unless stated otherwise, acetonitrile and the substrates were purchased from commercial sources without further purification. Blue LED lamps (40W, Kessil A160WE tuna blue) were used for reactions. (trifluoromethyl)benzene was internal standard for ${ }^{19} \mathrm{~F}$ NMR

\section{Experimental Details}

\section{(1) Optimization of the reaction conditions}

\section{Table 1. Ligand Screening}

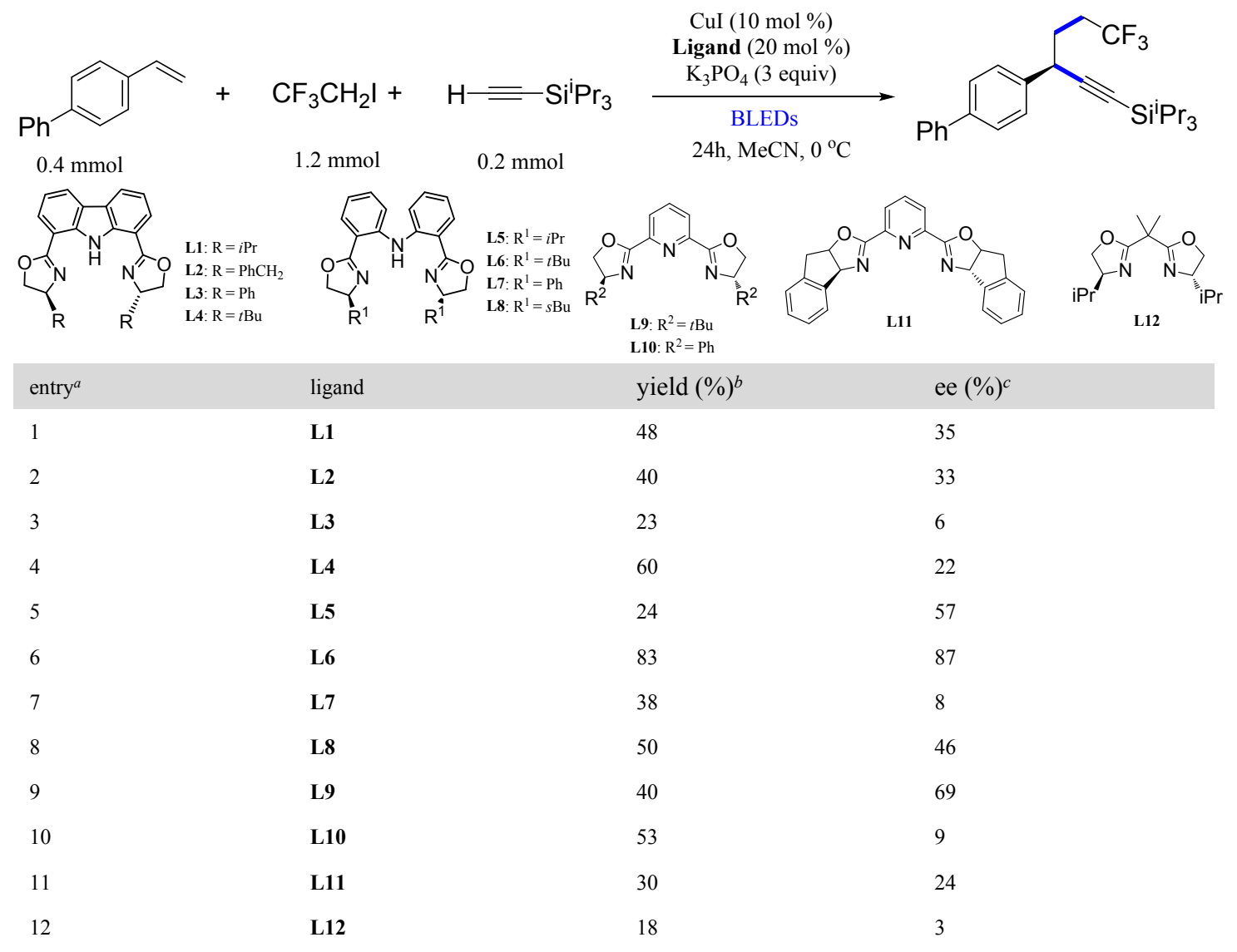


Talbe 2. Copper salt Screening

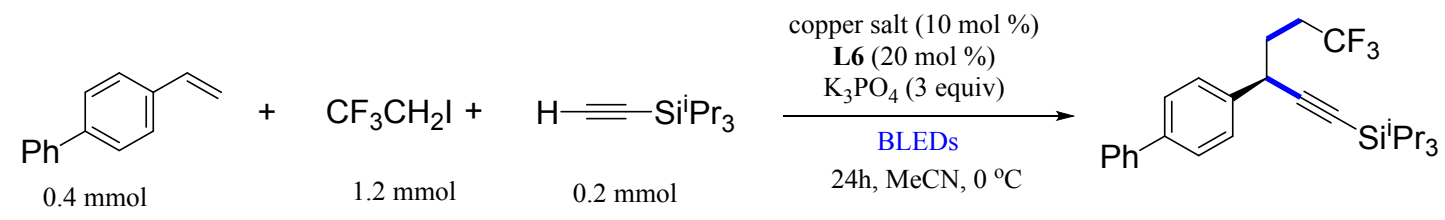

\begin{tabular}{llll}
\hline entry ${ }^{a}$ & copper salt & yield $(\%)^{b}$ & ee $(\%)^{c}$ \\
1 & $\mathrm{CuI}$ & 83 & 87 \\
2 & $\mathrm{CuBr}$ & 64 & 82 \\
3 & $\mathrm{CuCl}$ & 73 & 86 \\
4 & $\mathrm{Cu}(\mathrm{MeCN})_{4} \mathrm{PF}_{4}$ & 38 & 83 \\
5 & $\mathrm{CuCN}$ & 40 & 86 \\
6 & $\mathrm{Cu}(\mathrm{ClO})_{2} \cdot 6 \mathrm{H}_{2} \mathrm{O}$ & 82 & 84 \\
7 & $\mathrm{CuOAc}$ & 34 & 89 \\
8 & $\mathrm{Cu}(\mathrm{OTf})_{2}$ & 32 & \\
\\
5
\end{tabular}

\section{Table 3. Base Screening}

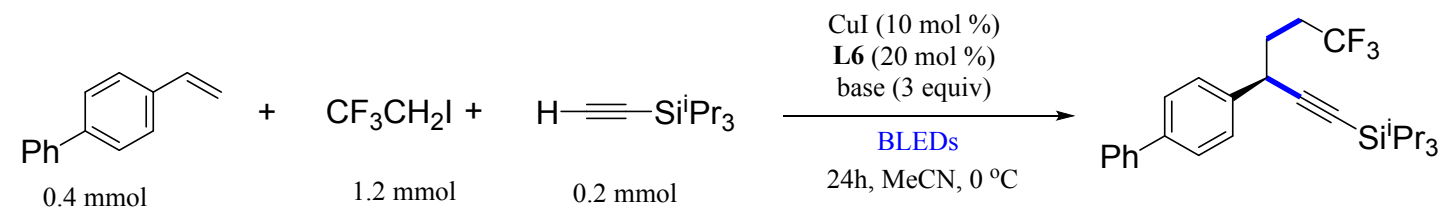

\begin{tabular}{|c|c|c|c|}
\hline entry $^{a}$ & base & yield $(\%)^{b}$ & ee $(\%)^{c}$ \\
\hline 1 & $\mathrm{~K}_{3} \mathrm{PO}_{4}$ & 83 & 87 \\
\hline 2 & $\mathrm{~K}_{2} \mathrm{CO}_{3}$ & 70 & 80 \\
\hline 3 & $\mathrm{Na}_{2} \mathrm{CO}_{3}$ & 15 & 20 \\
\hline 4 & $\mathrm{CS}_{2} \mathrm{CO}_{3}$ & 34 & 38 \\
\hline 5 & $\mathrm{Li}_{2} \mathrm{CO}_{3}$ & 10 & ND \\
\hline 6 & $\mathrm{~K}_{2} \mathrm{HPO}_{4}$ & 40 & 34 \\
\hline 7 & $\mathrm{Et}_{3} \mathrm{~N}$ & NR & ND \\
\hline 8 & $\mathrm{LiOBu}$ & 55 & 67 \\
\hline $9^{d}$ & $\mathrm{KOBu}$ & 40 & 92 \\
\hline
\end{tabular}

${ }^{a}$ The reactions were carried out at room temperature.

${ }^{b}$ Yields determined by ${ }^{1}$ HNMR analysis.

${ }^{c}$ Determined by chiral HPLC analysis.

${ }^{d}$ The reactions were carried out at $0{ }^{\circ} \mathrm{C}$.

NR: no reaction

ND : not determined

Table 4. Solvent Screening 


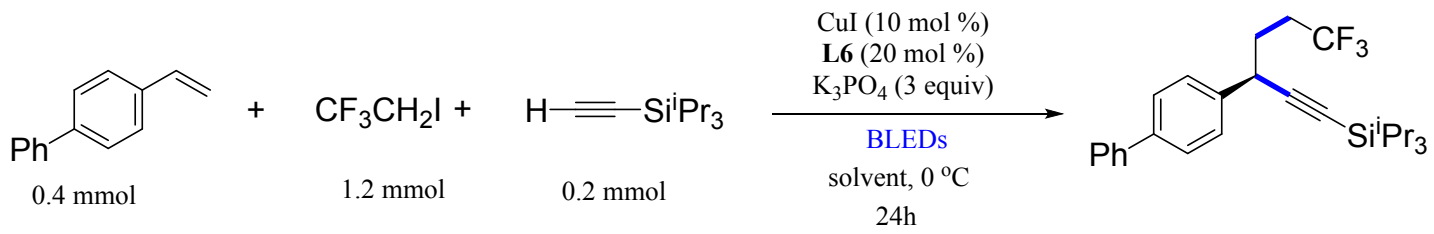

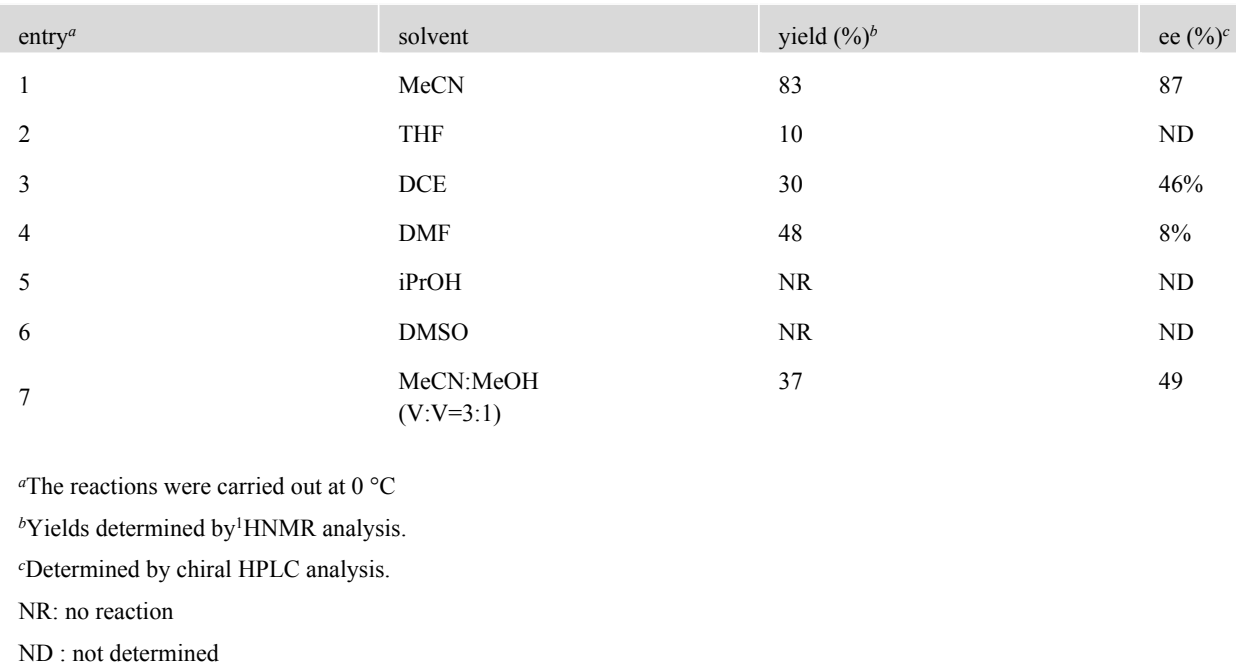

\section{Combining above studies}

We first studied the reaction between triisopropylsilylacetylene, 4-vinyl biphenyl, and trifluoroethyl iodide catalyzed by copper under photo-irradiation. After extensive optimization of the reaction parameters, the photoinduced copper-catalyzed three-component coupling reactions proceeded smoothly providing the product in excellent ee. The most critical one is the use of an unexpected $t$ Bu-BOPA (bisoxazoline diphenylamine) as the ligand (Table 1 , entry 1$)$.

Initially, $i$ Pr-Cbzbox was chosen as the chiral ligand, and the product was obtained in $48 \%$ yield with $35 \%$ ee (Table 1, entry 2). Among various Cbzbox ligands screened, $i$ Pr-Cbzbox gave a higher ee (Table 1, Entries 3-5). To our surprise, when $i \operatorname{Pr}-B O P A$ was tested, a significant improvement in the enantioselectivity was observed with no obvious change in yield (Table 1, Entry 6 ). ${ }^{16}$ Further screening of the ligand led to $t$ Bu-BOPA as the optimal one with $83 \%$ yield and $87 \%$ ee (Table 1, Entry 7). Lowering the temperature to $0{ }^{\circ} \mathrm{C}$ further improved the enantioselectivity to $96 \%$ with slightly decreased yield. Privileged chiral ligands such as Pybox and Box were less effective for this reaction with lower enantioselectivity and yield obtained (Table 1, Entries 10-13). Control experiments suggested the essential role of copper and light since no desired product was observed in the absence of either of these two factors (Table 1; Entries 14, 15). Interestingly, the reactions without ligands still provided the product, albeit in low yield. This result suggested copper-acetylide presumably served as the photocatalyst but with low efficiency under current reaction conditions (Table 1, Entry 16).

Various deviations from the standard reaction conditions was illustrated (See the Supporting Information for details). $\mathrm{CuI}$ produced a slightly better yield among the copper salts tested. Solvent screening revealed that acetonitrile gave the best result. In addition, $\mathrm{K}_{3} \mathrm{PO}_{4}$ outperformed other bases. To maintain a better balance of cost and reaction efficiency, $0.6 \mathrm{mmol}$ of trifluoroethyl iodide (c1) was used for further studies (Table 1, Entries 17, 18). Lowering the catalyst loading influenced the product yield and ee (Table 1, Entry 19).

Table 5. Optimization of the Reaction Conditions 


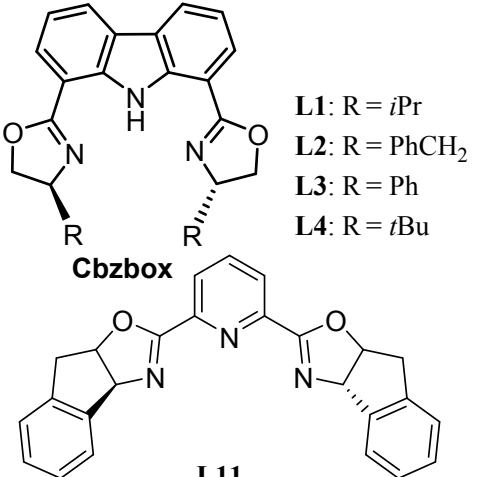

L11<smiles>C=Cc1ccc(-c2ccccc2)cc1</smiles>

a1

2 equiv

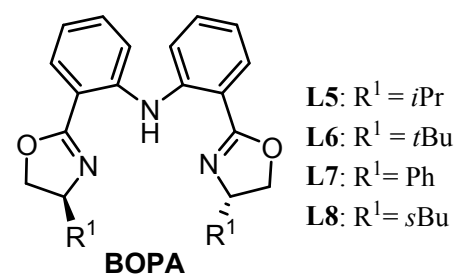<smiles>CC(C)OC(=O)[C@@H](C)C(C)C</smiles>

L12

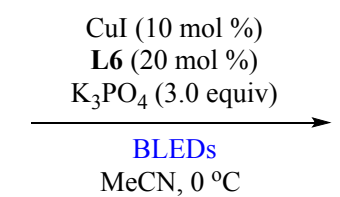

$24 \mathrm{~h}$<smiles>[R]C1COC(c2cccc(C3=NC([R])CO3)n2)=N1</smiles>

L9: $\mathrm{R}^{2}=t \mathrm{Bu}$

L10: $R^{2}=P h$

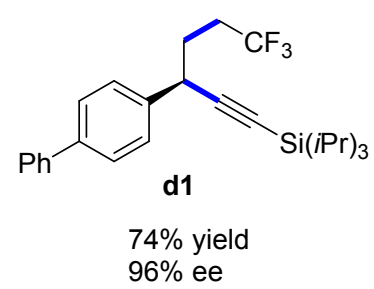

\begin{tabular}{|c|c|c|c|}
\hline entry $^{a}$ & base & yield $(\%)^{b}$ & ee $(\%)^{c}$ \\
\hline 1 & None & $74^{d}$ & 96 \\
\hline $2^{e}$ & L1 & 48 & 35 \\
\hline $3^{e}$ & L2 & 40 & 33 \\
\hline $4^{e}$ & L3 & 23 & 6 \\
\hline $5^{e}$ & L4 & 60 & 22 \\
\hline $6^{e}$ & L5 & 24 & 57 \\
\hline $7^{e}$ & L6 & 83 & 87 \\
\hline $8^{e}$ & L7 & 38 & 8 \\
\hline $9^{e}$ & L8 & 50 & 46 \\
\hline $10^{e}$ & L9 & 40 & 69 \\
\hline $11^{e}$ & L10 & 53 & 9 \\
\hline $12^{e}$ & L11 & 30 & 24 \\
\hline $13^{e}$ & L12 & 18 & 3 \\
\hline $14^{e}$ & No copper & - & ND \\
\hline $15^{e}$ & No light & - & ND \\
\hline $16^{e}$ & Without ligand & trace & ND \\
\hline $17^{e}$ & $\mathbf{c 1}(0.3 \mathrm{mmol})$ & 21 & 96 \\
\hline $18^{e}$ & c1 (1 mmol) & 88 & 96 \\
\hline $19^{e}$ & 10 mol\% $\% \mathbf{~} 6$ & 74 & 82 \\
\hline
\end{tabular}

${ }^{a}$ The reactions were carried out using 4-vinyl biphenyl (0.4 mmol), $\mathrm{CF}_{3} \mathrm{CH}_{2} \mathrm{I}(1.2 \mathrm{mmol})$, triisopropylsilylacetylene (0.2 mmol), $\mathrm{Cu}(\mathrm{I})(10 \mathrm{~mol} \%)$, and ligand $(20 \mathrm{~mol} \%)$ at $0{ }^{\circ} \mathrm{C}$, unless noted otherwise. ${ }^{b}$ Yields determined by ${ }^{1} \mathrm{H}$ NMR analysis using diethyl phthalate as an internal standard. ${ }^{c}$ Determined by chiral HPLC analysis, and the absolute configuration was assigned by comparison with reported example (see the supporting information for further details). ${ }^{d}$ Isolated yield. ${ }^{e}$ The reactions were carried out at room temperature. N.D. $=$ not determined.

\section{(2) Genernal procedure and characterizations of products}

Under nitrogen atmosphere, $\mathbf{L 6}(0.02 \mathrm{mmol}, 20 \mathrm{~mol} \%)$, $\mathrm{CuI}(0.01 \mathrm{mmol}, 10 \mathrm{~mol} \%), \mathrm{K}_{3} \mathrm{PO}_{4}(0.3$ mmol, 3.0 equiv), alkyne ( $0.2 \mathrm{mmol}, 1$ equiv), olefins ( $0.4 \mathrm{mmol}, 2.0$ equiv) and alkyl/aryl iodides (1.2 mmol, 6.0 equiv) were added in a dried reaction vessel with $2 \mathrm{~mL} \mathrm{MeCN}$, and the reaction 
mixture was stirred at $0{ }^{\circ} \mathrm{C}$ under Blue LED for $24 \mathrm{~h}$. After completion, the reaction was quenched and the suspension was filtered through a pad of silica gel, rinsed with $\mathrm{CH}_{2} \mathrm{Cl}_{2}$. The eluent was evaporated in vacuo and the residual oil was purified by column chromatography (petroleum ether and ethyl acetate).

(R)-(3-([1,1'-biphenyl]-4-yl)-6,6,6-trifluorohex-1-yn-1-yl)triisopropylsilane (d1)<smiles>FC(F)(F)CCC(C#C[AsH2-])c1ccc(-c2ccccc2)cc1</smiles>

Prepared following the general procedure showed above using 1-ethenyl-4-phenylbenzene (72.1 mg, $0.4 \mathrm{mmol}, 2.0$ equiv), triisopropylsilylacetylene ( $36.4 \mathrm{mg}, 0.2 \mathrm{mmol}, 1.0$ equiv) and 1, 1, 1-trifluoro2-iodoethane ( $251.9 \mathrm{mg}, 1.2 \mathrm{mmol}, 6.0$ equiv). The desired product was purified by column chromatography (petroleum ether) as colorless oil (65.7 mg, $74 \%$ ).

${ }^{1} \mathrm{H}$ NMR (400 MHz, $\left.\mathrm{CDCl}_{3}\right): \delta$ 7.60-7.56 (m, 4H), 7.46-7.42 (m, 4H), 7.36-7.32 (m, $\left.1 \mathrm{H}\right), 3.87(\mathrm{dd}$, $J=8.4,5.2 \mathrm{~Hz}, 1 \mathrm{H}), 2.36-2.24(\mathrm{~m}, 2 \mathrm{H}), 2.13-1.93(\mathrm{~m}, 2 \mathrm{H}), 1.15-1.10(\mathrm{~m}, 21 \mathrm{H})$.

${ }^{13} \mathrm{C}$ NMR $\left(101 \mathrm{MHz}, \mathrm{CDCl}_{3}\right): \delta 140.8,140.2,139.5,128.9,127.9,127.52,127.46,127.3(\mathrm{q}, J=$ $277.2 \mathrm{~Hz}), 127.2,107.8,85.4,37.5,31.6(\mathrm{q}, J=29.1 \mathrm{~Hz}), 31.0(\mathrm{q}, J=2.6 \mathrm{~Hz}), 18.8(\mathrm{~d}, J=0.5 \mathrm{~Hz})$, 11.4 .

${ }^{19} \mathrm{~F}$ NMR $\left(376 \mathrm{MHz}, \mathrm{CDCl}_{3}\right): \delta-65.03$ (t, $\left.J=10.7 \mathrm{~Hz}, 3 \mathrm{~F}\right)$. IR (neat) $\mathrm{cm}^{-1} \tilde{\mathrm{v}}: 3060,3030,2942$, 2892, 2866, 2170, 1486, 1459, 1385, 1256, 1219, 1136, 1061, 1005, 882, 862, 837, 763, 695, 664, 611, 580. HRMS (EI+ $70 \mathrm{eV}): \mathrm{C}_{27} \mathrm{H}_{35} \mathrm{~F}_{3} \mathrm{Si}[\mathrm{M}]^{+}$: calcd: 444.2460, found: 444.2455 .

$\left[\mathrm{a}_{\mathrm{D}}{ }^{24}=9.9\left(\mathrm{c}=0.28, \mathrm{CH}_{2} \mathrm{Cl}_{2}\right) \mathrm{SFC}\left(\mathrm{OJ}-3,0.3 * 1.5 \mathrm{~cm}, 5 \mu \mathrm{m}\right.\right.$, column T: $28^{\circ} \mathrm{C}$, background press: $2000 \mathrm{psi}, \mathrm{CO}_{2} / \mathrm{MeOH}=98 / 2$, flow $1.0 \mathrm{~mL} / \mathrm{min}$, detection at $254 \mathrm{~nm}$ ) retention time $=3.4 \mathrm{~min}$ (minor) and $4.7 \mathrm{~min}$ (major), ee $=96 \%$.

\section{(R)-triisopropyl(6,6,6-trifluoro-3-phenylhex-1-yn-1-yl)silane (d2)}

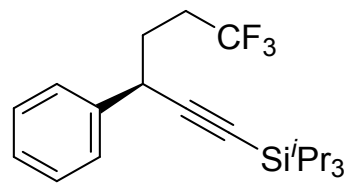

Prepared following the general procedure showed above using styrene $(41.6 \mathrm{mg}, 0.4 \mathrm{mmol}, 2.0$ equiv), triisopropylsilylacetylene (36.4 mg, $0.2 \mathrm{mmol}, 1.0$ equiv) and 1, 1, 1-trifluoro-2-iodoethane ( $251.9 \mathrm{mg}, 1.2 \mathrm{mmol}, 6.0$ equiv). The desired product was purified by column chromatography (petroleum ether) as colorless oil (52.2 $\mathrm{mg}, 71 \%$ ).

${ }^{1} \mathrm{H}$ NMR $\left(400 \mathrm{MHz}, \mathrm{CDCl}_{3}\right): \delta$ 7.39-7.32 (m, 4H), 7.27-7.24 (m, 1H), $3.82(\mathrm{dd}, 1 \mathrm{H}, J=8.0,5.2$ $\mathrm{Hz}), 2.32-2.20$ (m, 2H), 2.09-1.89 (m, 2H), 1.09 (s, 21H).

${ }^{13} \mathrm{C}$ NMR (101 MHz, $\mathrm{CDCl}_{3}$ ): $\delta 140.4,128.8,127.5,127.28$ (q, $\left.J=277.0 \mathrm{~Hz}\right), 127.26,107.9,85.2$, $37.8,31.6(\mathrm{q}, J=29.0 \mathrm{~Hz}), 31.0(\mathrm{q}, J=2.7 \mathrm{~Hz}), 18.8,11.4$.

${ }^{19} \mathrm{~F}$ NMR (376 MHz, $\mathrm{CDCl}_{3}$ ): $\delta-66.09$ (t, $J=10.7 \mathrm{~Hz}, 3 \mathrm{~F}$ ). IR (neat) $\mathrm{cm}^{-1} \tilde{\mathrm{v}}: 2944,2893,2866$, 2172, 1457, 1385, 1255, 1136, 1061, 1031, 1107, 882, 816, 663, 633,584, 566.

HRMS (EI+ $70 \mathrm{eV}): \mathrm{C}_{21} \mathrm{H}_{31} \mathrm{~F}_{3} \mathrm{Si}[\mathrm{M}]^{+}$: calcd: 368.2147 , found: 368.2154. [ a $]_{\mathrm{D}} 32=5.9$ (c $=0.60$, $\mathrm{CH}_{2} \mathrm{Cl}_{2}$ ). HPLC (OD-3, $0.46 * 25 \mathrm{~cm}, 5 \mu \mathrm{m}, 100 \%$ hexane, flow $0.5 \mathrm{~mL} / \mathrm{min}$, detection at $214 \mathrm{~nm}$, $16{ }^{\circ} \mathrm{C}$ ) retention time $=9.0 \mathrm{~min}$ (minor) and $9.6 \mathrm{~min}$ (major), ee $=97 \%$.

(R)-(3-(4-chlorophenyl)-6, 6, 6-trifluorohex-1-yn-1-yl) triisopropylsilane (d3) 
<smiles>Cc1cccc(C#CC(CCC(F)(F)F)c2ccccc2)c1</smiles>

Chemical Formula: $\mathrm{C}_{19} \mathrm{H}_{17} \mathrm{~F}_{3}$

Exact Mass: 302.1282

Prepared following the general procedure showed above using 4-chlorostyrene $(55.4 \mathrm{mg}, 0.4 \mathrm{mmol}$, 2.0 equiv), triisopropylsilylacetylene $(36.4 \mathrm{mg}, 0.2 \mathrm{mmol}, 1.0$ equiv) and 1, 1, 1-trifluoro-2iodoethane $(251.9 \mathrm{mg}, 1.2 \mathrm{mmol}, 6.0$ equiv). The desired product was purified by column chromatography (petroleum ether) as colorless oil (52.3 mg, $65 \%$ ).

${ }^{1} \mathrm{H}$ NMR $\left(400 \mathrm{MHz}, \mathrm{CDCl}_{3}\right): \delta 7.32(\mathrm{~s}, 4 \mathrm{H}), 3.80(\mathrm{dd}, J=8.8,5.2 \mathrm{~Hz}, 1 \mathrm{H}), 2.32-2.18(\mathrm{~m}, 2 \mathrm{H}), 2.06-$ $1.86(\mathrm{~m}, 2 \mathrm{H}), 1.09(\mathrm{~s}, 21 \mathrm{H})$

${ }^{13} \mathrm{C}$ NMR (101 MHz, $\left.\mathrm{CDCl}_{3}\right): \delta 139.0,133.1,129.0,128.8,127.2(\mathrm{q}, J=277.2 \mathrm{~Hz}), 107.3,85.8$, 37.2, 31.6 (q, $J=29.1 \mathrm{~Hz}), 30.9$ (q, $J=2.6 \mathrm{~Hz}), 18.8(\mathrm{~d}, J=0.4 \mathrm{~Hz}), 11.3$.

${ }^{19} \mathrm{~F}$ NMR (376 MHz, $\left.\mathrm{CDCl}_{3}\right): \delta-66.09(\mathrm{t}, J=10.7 \mathrm{~Hz}, 3 \mathrm{~F})$. IR (neat) $\mathrm{cm}^{-1} \tilde{\mathrm{v}}: 2943,2891,2866$, 2172, 1490, 1461, 1386, 1255, 1014, 1137, 882, 828, 675, 662, 624, 552, 505.

HRMS (EI+ $70 \mathrm{eV}): \mathrm{C}_{21} \mathrm{H}_{30} \mathrm{ClF}_{3} \mathrm{Si}[\mathrm{M}]^{+}:$calcd: 402.1757, found: 402.1755. [ a $]_{\mathrm{D}}{ }^{32}=7.9(\mathrm{c}=0.49$, $\mathrm{CH}_{2} \mathrm{Cl}_{2}$ ). HPLC (OD-3, $0.46 * 25 \mathrm{~cm}, 5 \mu \mathrm{m}, 100 \%$ hexane, flow $0.3 \mathrm{~mL} / \mathrm{min}$, detection at $214 \mathrm{~nm}$, $16{ }^{\circ} \mathrm{C}$ ) retention time $=8.9 \mathrm{~min}$ (major) and $9.4 \mathrm{~min}$ (minor), ee $=96 \%$.

(R)- (3-(4-bromophenyl)-6,6,6-trifluorohex-1-yn-1-yl)triisopropylsilane (d4)<smiles>FC(F)(F)CCC(C#C[SeH])c1ccc(Br)cc1</smiles>

Prepared following the general procedure showed above using 4-bromostyrene $(72.3 \mathrm{mg}, 0.4 \mathrm{mmol}$, 2.0 equiv), triisopropylsilylacetylene $(36.4 \mathrm{mg}, 0.2 \mathrm{mmol}, 1.0$ equiv) and 1, 1, 1-trifluoro-2iodoethane (251.9 mg, $1.2 \mathrm{mmol}, 6.0$ equiv). The desired product was purified by column chromatography (petroleum ether) as colorless oil (60.6 mg, $68 \%$ ).

${ }^{1} \mathrm{H}$ NMR (400 MHz, $\left.\mathrm{CDCl}_{3}\right): \delta$ 7.49-7.45 (m, 2H), $\delta 7.28-7.25(\mathrm{~m}, 2 \mathrm{H}), 3.78(\mathrm{dd}, J=8.8,5.6 \mathrm{~Hz}$, $1 \mathrm{H}), 2.31-2.19(\mathrm{~m}, 2 \mathrm{H}), 2.06-1.86(\mathrm{~m}, 2 \mathrm{H}), 1.08-1.03(\mathrm{~m}, 21 \mathrm{H})$.

${ }^{13} \mathrm{C}$ NMR $\left(101 \mathrm{MHz}, \mathrm{CDCl}_{3}\right): \delta 139.6,131.9,129.2,127.1(\mathrm{q}, J=277.2 \mathrm{~Hz}), 121.2,107.2,85.8$, $37.3,31.6(\mathrm{q}, J=29.1 \mathrm{~Hz}), 30.8(\mathrm{q}, J=2.6 \mathrm{~Hz}), 18.8(\mathrm{~d}, J=0.6 \mathrm{~Hz}), 11.3$.

${ }^{19} \mathrm{~F}$ NMR $\left(376 \mathrm{MHz}, \mathrm{CDCl}_{3}\right): \delta-66.08(\mathrm{t}, J=10.7 \mathrm{~Hz}, 3 \mathrm{~F})$. IR (neat) $\mathrm{cm}^{-1}$ ṽ: 2943, 2892, 2865, 2172, 1487, 1461, 1387, 1255, 1219, 1137, 1071, 1010, 882, 828, 675, 662, 635, 622, 551, 506.

HRMS (EI+ $70 \mathrm{eV}): \mathrm{C}_{21} \mathrm{H}_{30} \mathrm{BrF}_{3} \mathrm{Si}[\mathrm{M}]^{+}:$calcd:446.1252, found: 446.1256. [ $\left.{ }^{\mathrm{a}}\right]_{\mathrm{D}}{ }^{21}=11.5(\mathrm{c}=0.71$ $\mathrm{CH}_{2} \mathrm{Cl}_{2}$ ). HPLC (PC-2, 0.46*25 cm, $5 \mu \mathrm{m}, 100 \%$ hexane, flow $0.3 \mathrm{~mL} / \mathrm{min}$, detection at $214 \mathrm{~nm}$, $16{ }^{\circ} \mathrm{C}$ ) retention time $=15.7 \mathrm{~min}$ (minor) and $16.5 \mathrm{~min}$ (major), ee $=94 \%$

(R)-triisopropyl(6, 6, 6-trifluoro-3-(p-tolyl)hex-1-yn-1-yl)silane (d5)<smiles>Cc1ccc(C(C#C[Se-])CCC(F)(F)F)cc1</smiles>

Prepared following the general procedure showed above using 1-methyl-4-vinylbenzene (47.3 mg, 
$0.4 \mathrm{mmol}, 2.0$ equiv), triisopropylsilylacetylene ( $36.4 \mathrm{mg}, 0.2 \mathrm{mmol}, 1.0$ equiv) and 1, 1, 1-trifluoro2-iodoethane ( $251.9 \mathrm{mg}, 1.2 \mathrm{mmol}, 6.0$ equiv). The desired product was purified by column chromatography (petroleum ether) as colorless oil (53.5 mg, $70 \%$ ).

${ }^{1} \mathrm{H}$ NMR $\left(400 \mathrm{MHz}, \mathrm{CDCl}_{3}\right): \delta 7.27(\mathrm{~d}, J=7.2 \mathrm{~Hz}, 2 \mathrm{H}), \delta 7.15(\mathrm{~d}, J=8.0 \mathrm{~Hz}, 2 \mathrm{H}), 3.79(\mathrm{dd}, J=$ 7.6, 6.0Hz, 2H 1H), 2.34 (s, 3H), 2.30-2.18 (m, 2H), 2.06-1.87 (m, 2H), 1.09-1.03 (m, 21H).

${ }^{13} \mathrm{C}$ NMR $\left(101 \mathrm{MHz}, \mathrm{CDCl}_{3}\right): \delta 137.4,136.9,129.4,127.4,127.3(\mathrm{q}, J=277.1 \mathrm{~Hz}), 108.2,84.9$, 37.4, 34.5, 31.6 (q, $J=29.0 \mathrm{~Hz}), 31.0$ (q, $J=2.5 \mathrm{~Hz}), 21.2,18.8,11.4$.

${ }^{19} \mathrm{~F}$ NMR $\left(376 \mathrm{MHz}, \mathrm{CDCl}_{3}\right): \delta-66.08$ (t, $\left.J=10.7 \mathrm{~Hz}, 3 \mathrm{~F}\right)$. IR (neat) $\mathrm{cm}^{-1} \tilde{\mathrm{v}}: 2943,2892,2865$, 2171, 1458, 1385, 1256, 1136, 1007, 882, 816, 663, 566.

HRMS (EI+ $\left.{ }^{+}, 70 \mathrm{eV}\right): \mathrm{C}_{22} \mathrm{H}_{33} \mathrm{~F}_{3} \mathrm{Si}[\mathrm{M}]^{+}$: calcd: 382.2304, found: 382.2307. [ a $]_{\mathrm{D}}^{25}=7.2$ (c = 0.10, $\mathrm{CH}_{2} \mathrm{Cl}_{2}$ ). HPLC (PA-2, 0.46*25 cm, $5 \mu \mathrm{m}, \mathrm{MeCN} / \mathrm{H}_{2} \mathrm{O}=60 / 40$ flow $0.7 \mathrm{~mL} / \mathrm{min}$, detection at 214 $\mathrm{nm}, 25^{\circ} \mathrm{C}$ ) retention time $=30.8 \mathrm{~min}$ (minor) and $34.8 \mathrm{~min}$ (major), ee $=96 \%$.

(R)-(3-(4-(tert-butyl)phenyl)-6,6,6-trifluorohex-1-yn-1-yl)triisopropylsilane (d6)<smiles>CC(C)(C)c1ccc(C(C#C[Se-])CCC(F)(F)F)cc1</smiles>

Prepared following the general procedure showed above using 4-butylstyrene $(64.1 \mathrm{mg}, 0.4 \mathrm{mmol}$, 2.0 equiv), triisopropylsilylacetylene ( $36.4 \mathrm{mg}, 0.2 \mathrm{mmol}, 1.0$ equiv) and 1, 1, 1-trifluoro-2iodoethane ( $251.9 \mathrm{mg}, 1.2 \mathrm{mmol}, 6.0$ equiv). The desired product was purified by column chromatography (petroleum ether) as colorless oil (57.7 mg, $68 \%$ ).

${ }^{1} \mathrm{H} \mathrm{NMR}\left(400 \mathrm{MHz}, \mathrm{CDCl}_{3}\right.$ ): $\delta 7.33(\mathrm{dd}, J=19.2,7.6 \mathrm{~Hz}, 4 \mathrm{H}), 3.81(\mathrm{dd}, J=8.0,5.2 \mathrm{~Hz}, 1 \mathrm{H}), 2.32-$ $2.20(\mathrm{~m}, 2 \mathrm{H}), 2.08-1.89(\mathrm{~m}, 2 \mathrm{H}), 1.32(\mathrm{~s}, 9 \mathrm{H}), 1.09-1.04(\mathrm{~m}, 21 \mathrm{H})$.

${ }^{13} \mathrm{C}$ NMR $\left(101 \mathrm{MHz}, \mathrm{CDCl}_{3}\right): \delta 150.1,137.3,127.3(\mathrm{q}, J=277.6 \mathrm{~Hz}), 127.1,125.7,108.1,85.1$, 37.3, 34.6, 31.6 (q, $J=28.9 \mathrm{~Hz}), 31.5,31.0$ (q, $J=2.6 \mathrm{~Hz}), 18.8$ (d, $J=0.5 \mathrm{~Hz}), 11.4$.

${ }^{19} \mathrm{~F}$ NMR $\left(376 \mathrm{MHz}, \mathrm{CDCl}_{3}\right): \delta-66.06(\mathrm{t}, J=10.9 \mathrm{~Hz}, 3 \mathrm{~F})$. IR (neat) $\mathrm{cm}^{-1} \tilde{\mathrm{v}}: 2959,2866,2349$, 2322, 2173, 1462, 1386, 1258, 1138, 1103, 1017, 1007, 883, 832, 802, 670, 664, 585.

HRMS (EI+ $\left.{ }^{+}, 70 \mathrm{eV}\right): \mathrm{C}_{25} \mathrm{H}_{39} \mathrm{~F}_{3} \mathrm{Si}[\mathrm{M}]^{+}$: calcd: 424.2773 , found: 424.2777. [ $\left.{ }^{a}\right]_{\mathrm{D}}{ }^{32}=4.5$ (c $=0.40$, $\mathrm{CH}_{2} \mathrm{Cl}_{2}$ ). HPLC (PC-2, $0.46 * 25 \mathrm{~cm}, 5 \mu \mathrm{m}, 100 \%$ hexane, flow $0.3 \mathrm{~mL} / \mathrm{min}$, detection at $214 \mathrm{~nm}, 16^{\circ} \mathrm{C}$ ) retention time $=11.6 \mathrm{~min}$ (minor) and $12.3 \mathrm{~min}$ (major), ee $=94 \%$.

(R)-triisopropyl(6,6,6-trifluoro-3-(4-methoxyphenyl)hex-1-yn-1-yl)silane (d7)<smiles>COc1ccc(C(C#C[SiH2])CCC(F)(F)F)cc1</smiles>

Prepared following the general procedure showed above using 4-methoxystyrene $(68.8 \mathrm{mg}, 0.4$ mmol, 2.0 equiv), triisopropylsilylacetylene ( $36.4 \mathrm{mg}, 0.2 \mathrm{mmol}, 1.0$ equiv) and 1, 1, 1-trifluoro-2iodoethane $(251.9 \mathrm{mg}, 1.2 \mathrm{mmol}, 6.0$ equiv). The desired product was purified by column chromatography (petroleum ether: ethyl acetate $=100: 1)$ as colorless oil $(50 \mathrm{mg}, 63 \%)$

${ }^{1} \mathrm{H}$ NMR (400 MHz, CDCl $): \delta 7.29(\mathrm{~d}, J=8.4 \mathrm{~Hz}, 1 \mathrm{H}), 6.87(\mathrm{~d}, J=8.8 \mathrm{~Hz}, 1 \mathrm{H}), 3.78$ (dd, $J=8.4$, $2.8 \mathrm{~Hz}, 1 \mathrm{H}), 2.30-2.18(\mathrm{~m}, 2 \mathrm{H}), 2.05-1.86(\mathrm{~m}, 2 \mathrm{H}), 1.09-1.06(\mathrm{~m}, 21 \mathrm{H})$.

${ }^{13} \mathrm{C}$ NMR (101 MHz, $\left.\mathrm{CDCl}_{3}\right): \delta 158.8,132.5,128.5,114.1,127.3(\mathrm{q}, J=277.2 \mathrm{~Hz}), 108.3,84.9$, $55.4,37.0,31.5(\mathrm{q}, J=29.0 \mathrm{~Hz}), 31.1(\mathrm{q}, J=2.4 \mathrm{~Hz}), 18.8(\mathrm{~d}, J=0.5 \mathrm{~Hz}), 11.4$.

${ }^{19} \mathrm{~F}$ NMR $\left(376 \mathrm{MHz}, \mathrm{CDCl}_{3}\right): \delta-66.07$ (t, $\left.J=10.9 \mathrm{~Hz}, 3 \mathrm{~F}\right) . \mathrm{IR}$ (neat) $\mathrm{cm}^{-1} \tilde{\mathrm{v}}: 2943,2865,2171$, 


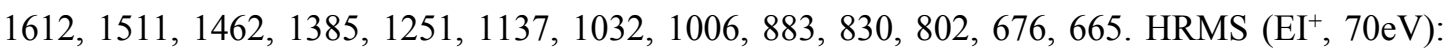
$\mathrm{C}_{22} \mathrm{H}_{33} \mathrm{~F}_{3} \mathrm{OSi}[\mathrm{M}]^{+}$: calcd: 398.2253 , found: 398.2259. [ a $]_{\mathrm{D}}{ }^{29}=6.4\left(\mathrm{c}=0.40, \mathrm{CHCl}_{3}\right)$ HPLC (AD$\mathrm{H}, 0.46 * 25 \mathrm{~cm}, 5 \mu \mathrm{m}, 100 \%$ hexane , flow $0.6 \mathrm{~mL} / \mathrm{min}$, detection at $210 \mathrm{~nm}, 40{ }^{\circ} \mathrm{C}$ ) retention time $=6.5 \mathrm{~min}$ (minor) and $6.8 \mathrm{~min}$ (major), ee $=93 \%$

(R)-triisopropyl(6,6,6-trifluoro-3-(4-(trifluoromethyl)phenyl)hex-1-yn-1-yl)silane (d8)<smiles>FC(F)(F)CCC(C#C[Se-])c1ccc(C(F)(F)F)cc1</smiles>

Prepared following the general procedure showed above using 4-(trifluoromethyl) styrene (68.8 $\mathrm{mg}$, $0.4 \mathrm{mmol}, 2.0$ equiv), triisopropylsilylacetylene ( $36.4 \mathrm{mg}, 0.2 \mathrm{mmol}, 1.0$ equiv) and 1, 1, 1-trifluoro2-iodoethane (251.9 mg, $1.2 \mathrm{mmol}, 6.0$ equiv). The desired product was purified by column chromatography (petroleum ether: ethyl acetate $=100: 1$ ) as colorless oil (41.8 $\mathrm{mg}, 48 \%$ ).

${ }^{1} \mathrm{H}$ NMR $\left(400 \mathrm{MHz}, \mathrm{CDCl}_{3}\right): \delta 7.56(\mathrm{dd}, J=39.6,8.0 \mathrm{~Hz}, 4 \mathrm{H}), 3.89(\mathrm{dd}, J=8.4,5.6 \mathrm{~Hz}, 1 \mathrm{H}), 2.32-$ $2.22(\mathrm{~m}, 2 \mathrm{H}), 2.10-1.89(\mathrm{~m}, 2 \mathrm{H}), 1.09-1.07(\mathrm{~m}, 21 \mathrm{H})$

${ }^{13} \mathrm{C}$ NMR (101 MHz, $\left.\mathrm{CDCl}_{3}\right): \delta 144.5,129.7$ (q, $\left.J=32.6 \mathrm{~Hz}\right), 127.1(\mathrm{q}, J=277.2 \mathrm{~Hz}), 125.8(\mathrm{q}, J$ $=3.8 \mathrm{~Hz}), 124.2(\mathrm{q}, J=273.0 \mathrm{~Hz}), 106.7,86.3,37.7,31.6(\mathrm{q}, J=29.2 \mathrm{~Hz}), 30.9(\mathrm{q}, J=2.5 \mathrm{~Hz})$, $18.7(\mathrm{~d}, J=0.5 \mathrm{~Hz}), 11.3$.

${ }^{19} \mathrm{~F}$ NMR (376 MHz, $\mathrm{CDCl}_{3}$ ): $\delta-62.49$ (s, 3F), -66.11 (t, $\left.J=10.7 \mathrm{~Hz}, 3 \mathrm{~F}\right)$. IR (neat) $\mathrm{cm}^{-1} \tilde{\mathrm{v}}: 2946$, 2867, 2175, 1326, 1259, 1131, 801,678. IR (neat) $\mathrm{cm}^{-1} \mathrm{v}: 2946,2867,1462,1326,1259,1131,1069$, 801, 677, 627. HRMS (EI+ $70 \mathrm{eV}): \mathrm{C}_{22} \mathrm{H}_{30} \mathrm{~F}_{6} \mathrm{Si}[\mathrm{M}]^{+}$: calcd: 436.2021, found: 436.2016. [ a $]_{\mathrm{D}}{ }^{32}=$ $6.8\left(\mathrm{c}=0.60, \mathrm{CHCl}_{3}\right)$. HPLC $(\mathrm{PC}-2,0.46 * 25 \mathrm{~cm}, 5 \mu \mathrm{m}, 100 \%$ hexane, flow $0.5 \mathrm{~mL} / \mathrm{min}$, detection at $214 \mathrm{~nm}, 16^{\circ} \mathrm{C}$ ) retention time $=8.3 \mathrm{~min}$ (major) and $8.8 \min$ (minor), ee $=67 \%$.

(R)-(3-(3-bromophenyl)-6,6,6-trifluorohex-1-yn-1-yl)triisopropylsilane (d9)<smiles>FC(F)(F)CCCCCC[As]C#CC(CCC(F)(F)F)c1cccc(Br)c1</smiles>

Prepared following the general procedure showed above using 1-bromo-3-ethenylbenzene (72.3 mg, $0.4 \mathrm{mmol}, 2.0$ equiv), triisopropylsilylacetylene $(36.4 \mathrm{mg}, 0.2 \mathrm{mmol}, 1.0$ equiv) and 1, 1, 1-trifluoro2-iodoethane ( $251.9 \mathrm{mg}, 1.2 \mathrm{mmol}, 6.0$ equiv). The desired product was purified by column chromatography (petroleum ether) as colorless oil $(63.3 \mathrm{mg}, 71 \%$,).

${ }^{1} \mathrm{H}$ NMR $\left(400 \mathrm{MHz}, \mathrm{CDCl}_{3}\right): \delta$ 7.59-7.58 (m, 1H), 7.41-7.30 (m, 2H), 7.23-7.20 (m, 1H), $3.79(\mathrm{dd}$, $J=8.8,5.2 \mathrm{~Hz}, 1 \mathrm{H}), 2.32-2.20(\mathrm{~m}, 2 \mathrm{H}), 2.08-1.87(\mathrm{~m}, 2 \mathrm{H}), 1.09-1.04(\mathrm{~m}, 21 \mathrm{H})$.

${ }^{13} \mathrm{C}$ NMR (101 MHz, $\mathrm{CDCl}_{3}$ ): $\delta 142.8,130.8,130.4,130.3,127.1$ (q, $\left.J=277.4 \mathrm{~Hz}\right), 126.1,122.8$, 106.9, 86.2, 37.5, 31.6 (q, $J=29.1 \mathrm{~Hz}), 30.8$ (q, $J=2.7 \mathrm{~Hz}), 18.8,11.3$.

${ }^{19} \mathrm{~F}$ NMR (376 MHz, CDCl $)$ ): $\delta-66.06$ (t, $\left.J=10.7 \mathrm{~Hz}, 3 \mathrm{~F}\right)$. IR (neat) $\mathrm{cm}^{-1} \tilde{\mathrm{v}}: 2943,2892,2865,2172$, 1463, 1386, 1255, 1137, 1031, 782, 720, 695, 676, 663, 614. HRMS (EI, $70 \mathrm{eV}): \mathrm{C}_{21} \mathrm{H}_{30} \mathrm{BrF}_{3} \mathrm{Si}$ $[\mathrm{M}]^{+}$: calcd: 446.1252, found: 446.1245. [ a $]_{\mathrm{D}}^{32}=19.3\left(\mathrm{c}=0.69, \mathrm{CH}_{2} \mathrm{Cl}_{2}\right)$.

HPLC (OD-H, 0.46*25 cm, $5 \mu \mathrm{m}$, hexane / isopropanol $=99 / 1$, flow $0.5 \mathrm{~mL} / \mathrm{min}$, detection at $210 \mathrm{~nm}, 25^{\circ} \mathrm{C}$ ) retention time $=6.5 \mathrm{~min}$ (minor ) and $6.9 \min$ (major), ee $=96 \%$.

(R)-(3-(2-chlorophenyl)-6,6,6-trifluorohex-1-yn-1-yl)triisopropylsilane (d10) 
<smiles>FC(F)(F)CCC(C#C[AsH2-])c1ccccc1Cl</smiles>

Prepared following the general procedure showed above using 1-chloro-2-ethenylbenzene $(55.4 \mathrm{mg}$, $0.4 \mathrm{mmol}, 2.0$ equiv), triisopropylsilylacetylene ( $36.4 \mathrm{mg}, 0.2 \mathrm{mmol}, 1.0$ equiv) and 1, 1, 1-trifluoro2-iodoethane ( $251.9 \mathrm{mg}, 1.2 \mathrm{mmol}, 6.0$ equiv). The desired product was purified by column chromatography (petroleum ether) as colorless oil (33.8 mg, $42 \%$,).

${ }^{1} \mathrm{H}$ NMR $\left(400 \mathrm{MHz}, \mathrm{CDCl}_{3}\right): \delta 7.69(\mathrm{dd}, J=7.6,1.6 \mathrm{~Hz}, 1 \mathrm{H}), 7.35(\mathrm{dd}, J=7.6,1.2 \mathrm{~Hz}, 1 \mathrm{H}), 7.21$ (td, $J=7.6,1.2 \mathrm{~Hz}, 1 \mathrm{H}), 7.21(\mathrm{td}, J=7.6,1.6 \mathrm{~Hz}, 1 \mathrm{H}), 4.28(\mathrm{dd}, J=8.8,4.4 \mathrm{~Hz} 1 \mathrm{H}), 2.39-2.27(\mathrm{~m}$, 2H), 2.13-2.04 (m, 21H), 1.90-1.81(m, 21H), 1.10-1.07 (m, 21H).

${ }^{13} \mathrm{C}$ NMR (101 MHz, $\left.\mathrm{CDCl}_{3}\right): \delta 138.0,132.9,129.8,129.4,128.7,127.3,127.2(\mathrm{q}, J=277.1 \mathrm{~Hz})$, 107.2, 85.6, 35.0, 31.8 (q, $J=29.1 \mathrm{~Hz}), 29.0$ (q, $J=2.7 \mathrm{~Hz}), 18.8(\mathrm{~d}, J=1.1 \mathrm{~Hz}), 11.4$.

${ }^{19} \mathrm{~F}$ NMR (376 MHz, $\left.\mathrm{CDCl}_{3}\right): \delta-66.14$ (t, $\left.J=10.7 \mathrm{~Hz}, 3 \mathrm{~F}\right)$. IR (neat) $\mathrm{cm}^{-1} \tilde{\mathrm{v}}$ : 2943, 2893, 2866, 2172, 1466, 1386, 1333, 1255, 1220, 1139, 1107, 1064, 1031, 1006, 882, 753, 663, 612, 576, 457. HRMS (EI ${ }^{+}, 70 \mathrm{eV}$ ): $\mathrm{C}_{21} \mathrm{H}_{30} \mathrm{ClF}_{3} \mathrm{Si}[\mathrm{M}]^{+}:$calcd: 402.1757, found: 402.1760.[ a $]_{\mathrm{D}}^{25}=10.3$ (c=0.19, $\mathrm{CH}_{2} \mathrm{Cl}_{2}$ ). HPLC (PA-2, 0.46*25 cm, $5 \mu \mathrm{m}, \mathrm{MeCN} / \mathrm{H}_{2} \mathrm{O}=60 / 40$, flow $0.7 \mathrm{~mL} / \mathrm{min}$, detection at $214 \mathrm{~nm}, 25^{\circ} \mathrm{C}$ ) retention time $=34.8 \mathrm{~min}$ (minor) and $44.3 \mathrm{~min}$ (major), ee $=95 \%$

(R)-triisopropyl (6,6,6-trifluoro-3-(2-fluorophenyl) hex-1-yn-1-yl)silane (d11)<smiles>Fc1ccccc1C(C#C[SeH]C(F)(F)F)CCC(F)(F)F</smiles>

Prepared following the general procedure showed above using 2-fluorostyrene ( $48.8 \mathrm{mg}, 0.4 \mathrm{mmol}$, 2.0 equiv), triisopropylsilylacetylene $(36.4 \mathrm{mg}, 0.2 \mathrm{mmol}, 1.0$ equiv) and 1, 1, 1-trifluoro-2iodoethane ( $251.9 \mathrm{mg}, 1.2 \mathrm{mmol}, 6.0$ equiv). The desired product was purified by column chromatography (petroleum ether) as colorless oil (36.3 mg, $47 \%$ ).

${ }^{1} \mathrm{H}$ NMR $\left(400 \mathrm{MHz}, \mathrm{CDCl}_{3}\right): \delta 7.60(\mathrm{td}, J=7.6,1.6 \mathrm{~Hz}, 1 \mathrm{H}), 7.28-7.23(\mathrm{~m}, 1 \mathrm{H}), 7.15(\mathrm{td}, J=7.6$, $0.8 \mathrm{~Hz}, 1 \mathrm{H}), 7.06-7.01(\mathrm{~m}, 1 \mathrm{H}), 4.16(\mathrm{dd}, J=8.8,5.2 \mathrm{~Hz} 1 \mathrm{H}), 2.34-2.22(\mathrm{~m}, 2 \mathrm{H}), 2.10-1.88(\mathrm{~m}$, $2 \mathrm{H}), 1.14-1.09(\mathrm{~m}, 21 \mathrm{H})$.

${ }^{13} \mathrm{C}$ NMR (101 MHz, $\left.\mathrm{CDCl}_{3}\right): \delta$ 142.8, 130.8, 130.4, 130.3, 127.1 (q, $\left.J=277.2 \mathrm{~Hz}\right), 126.1,122.8$, 106.9, 86.2, 37.4, 31.6 (q, $J=29.1 \mathrm{~Hz}), 30.8$ (q, $J=2.6 \mathrm{~Hz}), 18.8(\mathrm{~d}, J=0.3 \mathrm{~Hz}), 11.3$.

${ }^{19} \mathrm{~F}$ NMR (376 MHz, $\left.\mathrm{CDCl}_{3}\right): \delta-66.17(\mathrm{t}, J=10.7 \mathrm{~Hz}, 3 \mathrm{~F}),-119.41--119.45(\mathrm{~m}, 1 \mathrm{~F})$. IR (neat) $\mathrm{cm}^{-1}$ ṽ: $2943,2892,2865,2172,1462,1386,1255,1139,1032,1007,882,782, .720,696,677$. HRMS ( $\left.\mathrm{EI}^{+}, 70 \mathrm{eV}\right): \mathrm{C}_{21} \mathrm{H}_{30} \mathrm{~F} \mathrm{~S}_{4} \mathrm{Si}[\mathrm{M}]^{+}$: calcd: 386.2053, found: 386.2057. [ $\left.{ }^{a}\right]_{\mathrm{D}}{ }^{25}=2.9$ (c $=0.10, \mathrm{CH}_{2} \mathrm{Cl}_{2}$ ). HPLC (PA-2, 0.46*25 cm, $5 \mu \mathrm{m}, \mathrm{MeCN} / \mathrm{H}_{2} \mathrm{O}=60 / 40$, flow $0.7 \mathrm{~mL} / \mathrm{min}$, detection at $214 \mathrm{~nm}$, $25{ }^{\circ} \mathrm{C}$ ) retention time $=24.9 \mathrm{~min}$ (minor) and $28.4 \mathrm{~min}$ (major), ee $=92 \%$

(R)-triisopropyl(6,6,6-trifluoro-3-(naphthalen-2-yl)hex-1-yn-1-yl)silane (d12)<smiles>FC(F)(F)CCCCC[Se]C#CC(CCC(F)(F)F)c1ccc2ccccc2c1</smiles>

Prepared following the general procedure showed above using 1-vinylnaphthalene (61.7 $\mathrm{mg}, 0.4$ mmol, 2.0 equiv), triisopropylsilylacetylene (36.4 mg, $0.2 \mathrm{mmol}, 1.0$ equiv) and 1, 1, 1-trifluoro-2- 
iodoethane ( $251.9 \mathrm{mg}, 1.2 \mathrm{mmol}, 6.0$ equiv). The desired product was purified by column chromatography (petroleum ether: ethyl acetate $=50: 1)$ as colorless oil $(60.2 \mathrm{mg}, 72 \%)$.

${ }^{1} \mathrm{H}$ NMR (400 MHz, $\left.\mathrm{CDCl}_{3}\right): \delta 7.89(\mathrm{~s}, 1 \mathrm{H}), 7.84-7.79(\mathrm{~m}, 3 \mathrm{H}), 7.51-7.45(\mathrm{~m}, 3 \mathrm{H}), 3.99(\mathrm{dd}, J=$ 7.6, 5.6 Hz, 1H), 2.37-2.22 (m, 2H), 2.18-1.99 (m, 2H), 1.12-1.09 (m, 21H).

${ }^{13} \mathrm{C}$ NMR $\left(101 \mathrm{MHz}, \mathrm{CDCl}_{3}\right): \delta 137.7,133.5,132.7,128.6,128.0,127.8,127.3$ (q, $\left.J=277.2 \mathrm{~Hz}\right)$, 126.4, 126.3, 126.0, 125.6, 107.9, 85.6. 37.9, 31.6 (q, $J=29.0 \mathrm{~Hz}$ ), 30.7 (q, $J=2.6 \mathrm{~Hz}), 18.8,11.4$. ${ }^{19} \mathrm{~F}$ NMR $\left(376 \mathrm{MHz}, \mathrm{CDCl}_{3}\right): \delta-66.04(\mathrm{t}, J=10.7 \mathrm{~Hz}, 3 \mathrm{~F})$. IR (neat) $\mathrm{cm}^{-1} \tilde{\mathrm{v}}: 3089,3056,2942$, 2865, 2170, 1461, 1385, 1254, 1136, 1105, 1007, 882, 856, 816, 749, 665, 646, 620, 476.

HRMS (EI+ $\left.{ }^{+}, 70 \mathrm{eV}\right): \mathrm{C}_{25} \mathrm{H}_{33} \mathrm{~F}_{3} \mathrm{Si}[\mathrm{M}]^{+}$: calcd: 418.2304, found: 418.2300. [ a $]_{\mathrm{D}}^{32}=2.8(\mathrm{c}=0.34$, $\mathrm{CH}_{2} \mathrm{Cl}_{2}$ ). HPLC (OD-3, $0.46 * 25 \mathrm{~cm}, 5 \mu \mathrm{m}, 100 \%$ hexane, flow $0.5 \mathrm{~mL} / \mathrm{min}$, detection at $214 \mathrm{~nm}, 16{ }^{\circ} \mathrm{C}$ ) retention time $=18.3 \min$ (major ) and $19.2 \min$ (minor), ee $=96 \%$

(R)-triisopropyl(6,6,6-trifluoro-3-(thiophen-2-yl)hex-1-yn-1-yl)silane (d13)

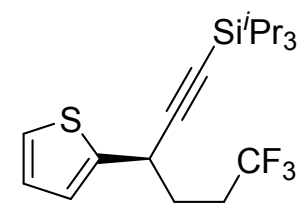

Prepared following the general procedure showed above using 2-ethenylthiophene (44.1 mg, 0.4 mmol, 2.0 equiv), triisopropylsilylacetylene ( $36.4 \mathrm{mg}, 0.2 \mathrm{mmol}, 1.0$ equiv) and 1, 1, 1-trifluoro-2iodoethane (251.9 mg, $1.2 \mathrm{mmol}, 6.0$ equiv). The desired product was purified by column chromatography (petroleum ether: ethyl acetate $=50: 1$ ) as colorless oil (51.6 mg, $69 \%$ ).

${ }^{1} \mathrm{H}$ NMR (400 MHz, CDCl $): \delta 7.20(\mathrm{dd}, J=5.2,1.2 \mathrm{~Hz}, 1 \mathrm{H}), 7.00-6.94(\mathrm{~m}, 2 \mathrm{H}), 6.95$ (dd, $J=5.2$, $3.6 \mathrm{~Hz}, 1 \mathrm{H}), 4.11(\mathrm{dd}, J=8.0,5.2 \mathrm{~Hz}, 1 \mathrm{H}), 2.37-1.98(\mathrm{~m}, 4 \mathrm{H}), 1.099-1.095(\mathrm{~m}, 21 \mathrm{H})$.

${ }^{13} \mathrm{C}$ NMR (101 MHz, $\left.\mathrm{CDCl}_{3}\right): \delta 143.9,126.9,124.9,124.5,127.2(\mathrm{q}, J=277.1 \mathrm{~Hz}), 106.9,85.2$. 33.0, $31.2(\mathrm{q}, J=29.2 \mathrm{~Hz}), 30.0(\mathrm{q}, J=2.6 \mathrm{~Hz}), 18.7,11.4$.

${ }^{19} \mathrm{~F}$ NMR (376 MHz, $\left.\mathrm{CDCl}_{3}\right): \delta-66.06(\mathrm{t}, J=10.7 \mathrm{~Hz}, 3 \mathrm{~F})$. IR (neat) $\mathrm{cm}^{-1} \tilde{\mathrm{v}}: 2943,2892,2866$,

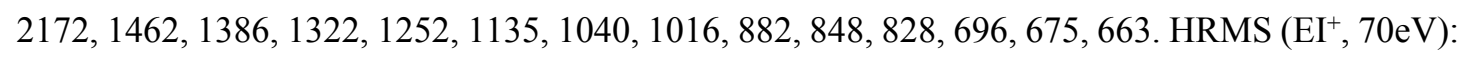
$\mathrm{C}_{25} \mathrm{H}_{33} \mathrm{~F}_{3} \mathrm{Si}[\mathrm{M}]^{+}$: calcd: 374.1711, found: 374.1706. [ $\left.{ }^{\alpha}\right]_{\mathrm{D}}{ }^{25}=10.1\left(\mathrm{c}=0.49, \mathrm{CH}_{2} \mathrm{Cl}_{2}\right)$.

HPLC (AD-H, $0.46 * 25 \mathrm{~cm}, 5 \mu \mathrm{m}, 100 \%$ hexane, flow $0.3 \mathrm{~mL} / \mathrm{min}$, detection at $250 \mathrm{~nm}, 25^{\circ} \mathrm{C}$ ) retention time $=11.9 \mathrm{~min}$ (minor ) and $12.5 \mathrm{~min}$ (major), ee $=94 \%$

(R)-(3-([1, 1'-biphenyl]-4-yl)-6,6,6-trifluorohex-1-yn-1-yl)trimethylsilane (d14)<smiles>CC#CC(CCC(F)(F)F)c1ccc(-c2ccccc2)cc1</smiles>

Prepared following the general procedure showed above using 1-ethenyl-4-phenylbenzene (72.1 mg, $0.4 \mathrm{mmol}, 2.0$ equiv), trimethylsilylacetylene (19.6 mg, $0.2 \mathrm{mmol}, 1.0$ equiv) and 1, 1, 1-trifluoro2-iodoethane (251.9 mg, $1.2 \mathrm{mmol}, 6.0$ equiv). The desired product was purified by column chromatography (petroleum ether: ethyl acetate $=100: 1$ ) as colorless oil $(46.8 \mathrm{mg}, 65 \%$ ).

${ }^{1} \mathrm{H}$ NMR (400 MHz, CDCl $): \delta$ 7.58-7.55 (m, 4H), 7.44-7.40 (m, 4H), 7.35-7.31 (m, 1H), $3.79(\mathrm{dd}$, $J=7.6,6.4 \mathrm{~Hz}, 1 \mathrm{H}), 2.30-2.18(\mathrm{~m}, 2 \mathrm{H}), 2.09-1.92(\mathrm{~m}, 2 \mathrm{H}), 0.21(\mathrm{~s}, 9 \mathrm{H})$.

${ }^{13} \mathrm{C}$ NMR $\left(101 \mathrm{MHz}, \mathrm{CDCl}_{3}\right): \delta 140.8,140.34,139.31,128.9,127.9,127.6,127.5,127.3$ (q, $J=$ $277.2 \mathrm{~Hz}), 127.2,106.2,89.2,37.4,31.6(\mathrm{q}, J=29.0 \mathrm{~Hz}), 30.6$ (q, $J=2.6 \mathrm{~Hz}), 0.2$.

${ }^{19} \mathrm{~F}$ NMR $\left(376 \mathrm{MHz}, \mathrm{CDCl}_{3}\right): \delta-65.91(\mathrm{t}, J=10.9 \mathrm{~Hz}, 3 \mathrm{~F})$. IR (neat) $\mathrm{cm}^{-1} \tilde{\mathrm{v}}: 3030,2959,2899$, $2173,1487,1386,1251,1219,1135,1006,838,761,696,654,577$. HRMS (EI $\left.{ }^{+}, 70 \mathrm{eV}\right): \mathrm{C}_{22} \mathrm{H}_{23} \mathrm{~F}_{3} \mathrm{Si}$ 
$[\mathrm{M}]^{+}$: calcd: 360.1521 , found: 360.1523 . [ a $]_{\mathrm{D}}{ }^{21}=5.8\left(\mathrm{c}=0.14, \mathrm{CH}_{2} \mathrm{Cl}_{2}\right)$. SFC $(\mathrm{OJ}-3,0.3 * 1.5 \mathrm{~cm}$, $5 \mu \mathrm{m}$, column T: $28{ }^{\circ} \mathrm{C}$, background press: $2000 \mathrm{psi}, \mathrm{CO}_{2} / \mathrm{MeOH}=99.5 / 0.5$, flow $1.0 \mathrm{~mL} / \mathrm{min}$, detection at $254 \mathrm{~nm}$ ) retention time $=8.8 \mathrm{~min}$ (minor) and $9.7 \min$ (major), ee $=87 \%$.

(R)-(3-([1, 1'-biphenyl]-4-yl)-6,6,6-trifluorohex-1-yn-1-yl)triethylsilane (d15)<smiles>CCCC#CC(CCC(F)(F)F)c1ccc(-c2ccccc2)cc1</smiles>

Prepared following the general procedure showed above using 1-ethenyl-4-phenylbenzene ( $72.1 \mathrm{mg}$, $0.4 \mathrm{mmol}, 2.0$ equiv), triethylsilylacetylene ( $28.0 \mathrm{mg}, 0.2 \mathrm{mmol}, 1.0$ equiv) and 1, 1, 1-trifluoro-2iodoethane ( $251.9 \mathrm{mg}, 1.2 \mathrm{mmol}, 6.0$ equiv). The desired product was purified by column chromatography (petroleum ether: ethyl acetate $=100: 1)$ as colorless oil $(49.0 \mathrm{mg}, 61 \%)$.

${ }^{1} \mathrm{H}$ NMR $\left(400 \mathrm{MHz}, \mathrm{CDCl}_{3}\right): \delta$ 7.60-7.56 (m, 4H), 7.46-7.43 (m, 4H), 7.36-7.33 (m, 1H), $3.84(\mathrm{dd}$, $J=8.0,6.0 \mathrm{~Hz}, 1 \mathrm{H}), 2.34-2.22(\mathrm{~m}, 2 \mathrm{H}), 2.11-1.93(\mathrm{~m}, 2 \mathrm{H}), 1.02(\mathrm{t}, J=7.8 \mathrm{~Hz}, 9 \mathrm{H}), 0.64(\mathrm{q}, J=8.0$ $\mathrm{Hz}, 6 \mathrm{H})$.

${ }^{13} \mathrm{C}$ NMR (101 MHz, $\left.\mathrm{CDCl}_{3}\right): \delta 140.8,140.3,139.4,128.9,127.9,127.55,127.46,127.3(\mathrm{q}, J=$ $277.2 \mathrm{~Hz}), 127.21,107.3,86.6,37.4,31.6(\mathrm{q}, J=29.0 \mathrm{~Hz}), 30.8$ (q, $J=2.5 \mathrm{~Hz}), 7.7,4.6$.

${ }^{19} \mathrm{~F}$ NMR $\left(376 \mathrm{MHz}, \mathrm{CDCl}_{3}\right): \delta-65.99(\mathrm{t}, J=10.7 \mathrm{~Hz}, 3 \mathrm{~F})$. IR (neat) $\mathrm{cm}^{-1}$ ṽ: $3030,2943,2864$, 2171, 1487, 1461, 1385, 1255, 1218, 1135, 1006, 882, 837, 763, 864, 696, 664, 579. HRMS (EI+, 70eV): $\mathrm{C}_{24} \mathrm{H}_{29} \mathrm{~F}_{3} \mathrm{Si}[\mathrm{M}]^{+}$: calcd: 402.1991, found: 402.1998. [ a $]_{\mathrm{D}}^{23}=7.8\left(\mathrm{c}=0.54, \mathrm{CH}_{2} \mathrm{Cl}_{2}\right)$. SFC (OJ-2, $0.3 * 1.5 \mathrm{~cm}, 5 \mu \mathrm{m}$, column T: $28{ }^{\circ} \mathrm{C}$, background press: $2000 \mathrm{psi}, \mathrm{CO}_{2} / \mathrm{MeOH}=$ $98 / 2$, flow $1.0 \mathrm{~mL} / \mathrm{min}$, detection at $254 \mathrm{~nm}$ ) retention time $=4.6 \mathrm{~min}$ (minor) and $6.1 \mathrm{~min}$ (major), ee $=88 \%$.

(R)-4-(1,1,1-trifluorodec-5-yn-4-yl)-1,1'-biphenyl (d16)<smiles>CCCCC#CC(CCC(F)(F)F)c1ccc(-c2ccccc2)cc1</smiles>

According to the general procedure, $\mathbf{d 1 6}$ was obtained from 1-ethenyl-4-phenylbenzene (72.1 mg, $0.4 \mathrm{mmol}, 2.0$ equiv), 1-hexyne (16.4 mg, $0.2 \mathrm{mmol}, 1.0$ equiv) and 1, 1, 1-trifluoro-2-iodoethane (251.9 mg, $1.2 \mathrm{mmol}, 6.0$ equiv). The desired product was isolated by column chromatography (petroleum ether: ethyl acetate $=100: 1$ ) as colorless oil (46.8mg, $68 \%$ ).

${ }^{1} \mathrm{H}$ NMR (400 MHz, $\left.\mathrm{CDCl}_{3}\right): \delta$ 7.59-7.55 (m, 4H), 7.46-7.41 (m, 4H), 7.36-7.32 (m, 1H), $3.75(\mathrm{td}$, $J=6.0,2.8 \mathrm{~Hz}, 1 \mathrm{H}$ ), $2.33-2.19$ (m, 4H), 2.07-1.90 (m, 2H), 1.57-1.42(m, 4H), 0.94 (t, $J=7.2 \mathrm{~Hz}$, $3 \mathrm{H})$.

${ }^{13} \mathrm{C}$ NMR (101 MHz, $\mathrm{CDCl}_{3}$ ): $\delta 140.9,140.4,140.1,128.9,127.9,127.5,127.4,127.4$ (q, $J=276.5$ $\mathrm{Hz}), 127.2,85.0,79.8,36.6,31.8$ (q, $J=28.9 \mathrm{~Hz}), 31.2,30.9$ (q, $J=2.2 \mathrm{~Hz}), 22.1,18.6,13.8$. ${ }^{19} \mathrm{~F}$ NMR (376 MHz, CDCl $): \delta-65.96$ (t, $\left.J=10.7 \mathrm{~Hz}, 3 \mathrm{~F}\right)$. IR (neat) $\mathrm{cm}^{-1} \tilde{\mathrm{v}}: 3030,2957,2932,2873$, $1486,1385,1255,1221,1130,1097,838,762,733,696,578$. HRMS $\left(\mathrm{EI}^{+}, 70 \mathrm{eV}\right): \mathrm{C}_{22} \mathrm{H}_{23} \mathrm{~F}_{3}[\mathrm{M}]^{+}$: calcd: 344.1752 , found: 344.1750 . [ a $]_{\mathrm{D}}{ }^{27}=3.00\left(\mathrm{c}=0.50, \mathrm{CH}_{2} \mathrm{Cl}_{2}\right)$. HPLC (OD-H, $0.46 * 25 \mathrm{~cm}, 5$ $\mu \mathrm{m}$, hexane $/$ isopropanol $=99.6 / 0.4$, flow $0.5 \mathrm{~mL} / \mathrm{min}$, detection at $210 \mathrm{~nm}, 40{ }^{\circ} \mathrm{C}$ ) retention time $=9.4 \mathrm{~min}$ (major) and $10.6 \mathrm{~min}$ (minor), ee $=88 \%$

(R)-4-(1, 1,1-trifluoro-9-phenylnon-5-yn-4-yl)-1,1'-biphenyl (d17) 
<smiles>FC(F)(F)CCC(C#CCCCc1ccccc1)c1ccc(-c2ccccc2)cc1</smiles>

Prepared following the general procedure showed above using 1-ethenyl-4-phenylbenzene ( $72.1 \mathrm{mg}$, $0.4 \mathrm{mmol}, 2.0$ equiv), (pent-4-yn-1-yl) benzene ( $28.8 \mathrm{mg}, 0.2 \mathrm{mmol}, 1.0$ equiv) and 1, 1, 1-trifluoro2-iodoethane ( $251.9 \mathrm{mg}, 1.2 \mathrm{mmol}, 6.0$ equiv). The desired product was purified by column chromatography (petroleum ether: ethyl acetate $=100: 1)$ as colorless oil $(49.5 \mathrm{mg}, 61 \%)$.

${ }^{1} \mathrm{H}$ NMR (400 MHz, $\left.\mathrm{CDCl}_{3}\right): \delta$ 7.59-7.55 (m, 4H), 7.44-7.41 (m, 4H), 7.35-7.26 (m, 3H), 7.20-7.18 (m, 3H), 3.77-3.75 (m, 1H), 2.74 (t, $J=7.6 \mathrm{~Hz} 2 \mathrm{H}), 2.29-2.22$ (m, 4H), 2.09-1.95 (m, 2H), 1.91$1.83(\mathrm{~m}, 2 \mathrm{H})$.

${ }^{13} \mathrm{C}$ NMR (101 MHz, $\left.\mathrm{CDCl}_{3}\right): \delta 141.7,140.9,140.3,140.2,128.9,128.7,128.5,127.9$ 127.5, 127.4, 127.3 (q, $J=277.2 \mathrm{~Hz}), 127.2,126.1,84.5,80.5,36.6,35.1 .31 .8$ (q, $J=28.9 \mathrm{~Hz}), 30.8$ (q, $J=2.4$ $\mathrm{Hz}), 30.8,18.4$.

${ }^{19} \mathrm{~F}$ NMR $\left(376 \mathrm{MHz}, \mathrm{CDCl}_{3}\right): \delta-65.85(\mathrm{t}, J=10.9 \mathrm{~Hz}, 3 \mathrm{~F})$. IR (neat) $\mathrm{cm}^{-1} \tilde{\mathrm{v}}: 3061,3028,2939$, 2859, 1487, 1454, 1386, 1256, 1220, 1129, 1100, 839, 798, 764, 743, 697, 579. HRMS (EI $\left.{ }^{+}, 70 \mathrm{eV}\right)$ : $\mathrm{C}_{27} \mathrm{H}_{25} \mathrm{~F}_{3}[\mathrm{M}]^{+}$: calcd: 406.1908, found: 406.1906. [ $\left.{ }^{a}\right]_{\mathrm{D}}{ }^{24}=3.8\left(\mathrm{c}=0.20, \mathrm{CH}_{2} \mathrm{Cl}_{2}\right)$. HPLC (OD-H, $0.46 * 25 \mathrm{~cm}, 5 \mu \mathrm{m}$, hexane $/$ isopropanol $=99 / 1$, flow $0.8 \mathrm{~mL} / \mathrm{min}$, detection at $210 \mathrm{~nm}, 40{ }^{\circ} \mathrm{C}$ ) retention time $=6.3 \mathrm{~min}$ (minor ) and $8.3 \mathrm{~min}$ (major), ee $=90 \%$

(R)-4-(1,1,1-trifluoro-7,7-dimethyloct-5-yn-4-yl)-1, 1'-biphenyl (d18)<smiles>CC(C)(C)C#CC(CCC(F)(F)F)c1ccc(-c2ccccc2)cc1</smiles>

Prepared following the general procedure showed above using 1-ethenyl-4-phenylbenzene ( $72.1 \mathrm{mg}$, $0.4 \mathrm{mmol}, 1.0$ equiv), 3, 3-dimethyl-butyne ( $16.4 \mathrm{mg}, 0.2 \mathrm{mmol}, 1.0$ equiv) and 1, 1, 1-trifluoro-2iodoethane $(251.9 \mathrm{mg}, 1.2 \mathrm{mmol}, 6.0$ equiv). The desired product was purified by column chromatography (petroleum ether: ethyl acetate $=100: 1)$ as white solid (41.9 mg, $61 \%$ ).

${ }^{1} \mathrm{H}$ NMR (400 MHz, $\left.\mathrm{CDCl}_{3}\right): \delta$ 7.59-7.55 (m, 4H), 7.46-7.41 (m, 4H), 7.36-7.32 (m, 1H), $3.75(\mathrm{dd}$, $J=7.6,6.0 \mathrm{~Hz} 1 \mathrm{H}), 2.30-2.18(\mathrm{~m}, 2 \mathrm{H}), 2.06-1.86(\mathrm{~m}, 2 \mathrm{H}), 1.27(\mathrm{~s}, 9 \mathrm{H})$

${ }^{13} \mathrm{C}$ NMR (101 MHz, $\left.\mathrm{CDCl}_{3}\right): \delta 163.3,160.8,136.24,136.20,129.0,128.9,127.2(\mathrm{q}, J=277.2 \mathrm{~Hz})$, 115.7, 115.5, 107.7, 85.6, 37.1, 31.6 (q, $J=29.1 \mathrm{~Hz}), 31.1(\mathrm{q}, J=1.7 \mathrm{~Hz}), 18.8(\mathrm{~d}, J=0.6 \mathrm{~Hz}), 11.4$. ${ }^{19} \mathrm{~F}$ NMR $\left(376 \mathrm{MHz}, \mathrm{CDCl}_{3}\right): \delta-65.99(\mathrm{t}, J=10.9 \mathrm{~Hz}, 3 \mathrm{~F}) . \mathrm{IR}$ (neat) $\mathrm{cm}^{-1}$ ṽ: $3030,2961,2901$, 2872, 2173, 1487, 1385, 1250, 1219, 1138, 1005, 837, 762, 693, 653, 578. HRMS ( $\mathrm{EI}^{+}, 70 \mathrm{eV}$ ): $\mathrm{C}_{22} \mathrm{H}_{23} \mathrm{~F}_{3}$ calcd: 344.1752, found: 344.1743 [ $\left.{ }^{a}\right]_{\mathrm{D}}{ }^{26}=4.8\left(\mathrm{c}=0.12, \mathrm{CH}_{2} \mathrm{Cl}_{2}\right)$. HPLC (OD-H, $0.46 * 25$ $\mathrm{cm}, 5 \mu \mathrm{m}$, hexane $/$ isopropanol $=99.6 / 0.4$, flow $0.5 \mathrm{~mL} / \mathrm{min}$, detection at $210 \mathrm{~nm}, 40{ }^{\circ} \mathrm{C}$ ) retention time $=7.8 \mathrm{~min}$ (major) and $8.9 \mathrm{~min}$ (minor), ee $=95 \%$.

(R)-4-(1,1,1-trifluoro-7-methyloct-5-yn-4-yl)-1, 1'-biphenyl (d19)<smiles>CC(C)C#CC(CCC(F)(F)F)c1ccc(-c2ccccc2)cc1</smiles>

Prepared following the general procedure showed above using 1-ethenyl-4-phenylbenzene (72.1 mg, 
$0.4 \mathrm{mmol}, 2.0$ equiv), 3-methyl-butyne (13.6 mg, $0.2 \mathrm{mmol}, 1.0$ equiv) and 1, 1, 1-trifluoro-2iodoethane $(251.9 \mathrm{mg}, 1.2 \mathrm{mmol}, 6.0$ equiv). The desired product was purified by column chromatography (petroleum ether: ethyl acetate $=100: 1)$ as colorless oil $(41.6 \mathrm{mg}, 63 \%)$.

${ }^{1} \mathrm{H}$ NMR $\left(400 \mathrm{MHz}, \mathrm{CDCl}_{3}\right): \delta$ 7.59-7.55 (m, 4H), 7.45-7.41 (m, 4H), 7.36-7.32 (m, 1H), 3.77-3.73 (m, 1H), 2.69-2.58 (m, 1H), 2.33-2.16 (m, 2H), 2.07-1.88 (m, 2H), $1.21(\mathrm{~d}, J=6.8 \mathrm{~Hz}, 6 \mathrm{H})$.

${ }^{13} \mathrm{C}$ NMR $\left(101 \mathrm{MHz}, \mathrm{CDCl}_{3}\right): \delta 140.9,140.4,140.1,128.9,127.9,127.5,127.40,127.37$ (q, $J=$ 277.2 Hz), 127.2, 90.8, 79.0, 36.4, 31.7 (q, J = 28.8 Hz), 31.0(q, $J=2.6 \mathrm{~Hz}), 23.5$ (d, $J=1.3 \mathrm{~Hz}$ ), 20.8 .

${ }^{19} \mathrm{~F}$ NMR (376 MHz, CDCl $): \delta-65.97(\mathrm{t}, J=10.9 \mathrm{~Hz}, 3 \mathrm{~F})$. IR (neat) $\mathrm{cm}^{-1} \tilde{\mathrm{v}}$ : 2963, 2922, 2877, $2852,1487,1448,1260,1092,1021,863,798,695,502$. HRMS ( $\left.\mathrm{EI}^{+}, 70 \mathrm{eV}\right): \mathrm{C}_{21} \mathrm{H}_{21} \mathrm{~F}_{3}[\mathrm{M}]^{+}:$calcd: 330.1595, found: 330.1587. [ $\mathrm{a}]_{\mathrm{D}}{ }^{26}=1.7\left(\mathrm{c}=0.45, \mathrm{CH}_{2} \mathrm{Cl}_{2}\right.$ ). HPLC (OD-H, $0.46 * 25 \mathrm{~cm}, 5 \mu \mathrm{m}$, hexane $/$ isopropanol $=99.6 / 0.4$, flow $0.5 \mathrm{~mL} / \mathrm{min}, \quad\left(\right.$ detection at $\left.210 \mathrm{~nm}, 40{ }^{\circ} \mathrm{C}\right)$ retention time $=$ $8.6 \mathrm{~min}$ (major) and $9.8 \mathrm{~min}$ (minor), ee $=93 \%$.

(R)-4-(1,1,1-trifluoro-8-methylnon-5-yn-4-yl)-1,1'-biphenyl (d20)<smiles>CC(C)CC#CC(CCC(F)(F)F)c1ccc(-c2ccccc2)cc1</smiles>

Prepared following the general procedure showed above using 1-ethenyl-4-phenylbenzene ( $72.1 \mathrm{mg}$, $0.4 \mathrm{mmol}, 2.0$ equiv), 4-methyl-pentyne ( $16.4 \mathrm{mg}, 0.2 \mathrm{mmol}, 1.0$ equiv) and 1, 1, 1-trifluoro-2iodoethane $(251.9 \mathrm{mg}, 1.2 \mathrm{mmol}, 6.0$ equiv). The desired product was purified by column chromatography (petroleum ether: ethyl acetate $=100: 1)$ as colorless oil $(45.4 \mathrm{mg}, 66 \%$ ).

${ }^{1} \mathrm{H}$ NMR (400 MHz, $\left.\mathrm{CDCl}_{3}\right): \delta$ 7.59-7.55 (m, 4H), 7.45-7.41 (m, 4H), 7.35-7.31 (m, 1H), 3.77 (td, $J=6.0,2.8 \mathrm{~Hz}, 1 \mathrm{H}), 2.31-2.20$ (m, 2H), 2.15 (dd, $J=6.4,2.4 \mathrm{~Hz}, 2 \mathrm{H}), 2.08-1.89$ (m, 2H), 1.88-1.79 (m, 1H), $1.01(\mathrm{~d}, J=6.4 \mathrm{~Hz}, 6 \mathrm{H})$.

${ }^{13} \mathrm{C}$ NMR $\left(101 \mathrm{MHz}, \mathrm{CDCl}_{3}\right): \delta 140.9,140.4,140.1,128.9,127.9,127.5,127.41,127.37$ (q, $J=$ $277.1 \mathrm{~Hz}), 127.2,83.9,80.8,36.6,31.8$ (q, $J=28.8 \mathrm{~Hz}), 31.0(\mathrm{q}, J=2.5 \mathrm{~Hz}), 28.4,28.1,22.2$.

${ }^{19} \mathrm{~F}$ NMR (376 MHz, CDCl $): \delta-65.93(\mathrm{t}, J=10.7 \mathrm{~Hz}, 3 \mathrm{~F}) \mathrm{HRMS}\left(\mathrm{EI}^{+}, 70 \mathrm{eV}\right): \mathrm{C}_{22} \mathrm{H}_{23} \mathrm{~F}_{3}[\mathrm{M}]^{+}$: calcd: 344.1752 , found: 344.1749 . [ $\left.{ }^{\alpha}\right]_{D}{ }^{24}=5.9\left(\mathrm{c}=0.14, \mathrm{CH}_{2} \mathrm{Cl}_{2}\right.$ ). IR (neat) $\mathrm{cm}^{-1} \tilde{\mathrm{v}}: 3057,2958$, 2928, 2871, 2834, 1486, 1455, 1385, 1255, 1219, 1129, 1100, 1009, 839, 769, 696, 577, 519. HPLC (OD-H, $0.46 * 25 \mathrm{~cm}, 5 \mu \mathrm{m}$, hexane $/$ isopropanol $=99.6 / 0.4$, flow $0.5 \mathrm{~mL} / \mathrm{min}$, detection at $210 \mathrm{~nm}, 40{ }^{\circ} \mathrm{C}$ ) retention time $=5.7 \mathrm{~min}$ (major) and $6.5 \mathrm{~min}$ (minor), ee $=89 \%$

(R)-4-(1-cyclopropyl-6,6,6-trifluorohex-1-yn-3-yl)-1, 1'-biphenyl (d21)<smiles>FC(F)(F)CCC(C#CC1CC1)c1ccc(-c2ccccc2)cc1</smiles>

Prepared following the general procedure showed above using 1-ethenyl-4-phenylbenzene ( $72.1 \mathrm{mg}$, $0.4 \mathrm{mmol}, 2.0$ equiv), cyclopropyl acetylene ( $13.2 \mathrm{mg}, 0.2 \mathrm{mmol}, 1.0$ equiv) and 1, 1, 1-trifluoro-2iodoethane (251.9 mg, $1.2 \mathrm{mmol}, 6.0$ equiv). The desired product was purified by column chromatography (petroleum ether: ethyl acetate $=100: 1)$ as colorless oil $(40.6 \mathrm{mg}, 62 \%)$.

${ }^{1} \mathrm{H}$ NMR (400 MHz, $\left.\mathrm{CDCl}_{3}\right)$ : $\delta$ 7.58-7.54 (m, 4H), 7.45-7.39 (m, 4H), 7.36-7.32 (m, 1H), 3.73-3.70 (m, 1H), 2.30-2.15 (m, 2H), 2.05-1.88(m, 2H), 1.33-1.26 (m, 1H), 0.80-0.66(m, 4H).

${ }^{13} \mathrm{C}$ NMR $\left(101 \mathrm{MHz}, \mathrm{CDCl}_{3}\right): \delta 140.9,140.3,140.1,128.9,127.8,127.5,127.4,127.2,127.3$ (q, $J=$ 
277.2 Hz), 87.9, 75.1, 36.5, 31.7 (q, $J=28.9 \mathrm{~Hz}), 30.8$ (q, $J=2.5 \mathrm{~Hz}), 8.4(\mathrm{~d}, J=5.6 \mathrm{~Hz}), 0.3$.

${ }^{19} \mathrm{~F}$ NMR (376 MHz, $\mathrm{CDCl}_{3}$ ): $\delta-66.96$ (t, $J=10.9 \mathrm{~Hz}, 3 \mathrm{~F}$ ). IR (neat) $\mathrm{cm}^{-1} \tilde{\mathrm{v}}: 3057,3028,2948,2872$, 2240, 1486, 1455, 1385, 1255, 1219, 1132, 1102, 1007, 987, 962, 861, 811, 736, 696, 577, 503. HRMS ( $\mathrm{EI}^{+}, 70 \mathrm{eV}$ ): $\mathrm{C}_{21} \mathrm{H}_{19} \mathrm{~F}_{3}[\mathrm{M}]^{+}$: calcd: 328.1439, found: 328.1431. [ a $]_{\mathrm{D}}{ }^{26}=4.9$ (c $=0.96$, $\mathrm{CH}_{2} \mathrm{Cl}_{2}$ ). HPLC (OD-H, $0.46 * 25 \mathrm{~cm}, 5 \mu \mathrm{m}$, hexane / isopropanol $=99.6 / 0.4$, flow $0.5 \mathrm{~mL} / \mathrm{min}$, (detection at $210 \mathrm{~nm}, 40{ }^{\circ} \mathrm{C}$ ) retention time $=8.9 \mathrm{~min}$ (major) and $10.7 \mathrm{~min}$ (minor), ee $=88 \%$.

(R)-3-(6,6,6-trifluoro-3-phenylhex-1-yn-1-yl) pyridine (d22)<smiles>FC(F)(F)CCC(C#Cc1cccnc1)c1ccccc1</smiles>

Prepared following the general procedure showed above using styrene (41.6 mg, $0.4 \mathrm{mmol}, 2.0$ equiv), 3-ethynylpyridine (20.6 mg, $0.2 \mathrm{mmol}, 1.0$ equiv) and 1, 1, 1-trifluoro-2-iodoethane (251.9 $\mathrm{mg}, 1.2 \mathrm{mmol}, 6.0$ equiv). The desired product was purified by column chromatography (petroleum ether: ethyl acetate $=40: 1)$ as colorless oil (30.0 $\mathrm{mg}, 52 \%)$.

${ }^{1} \mathrm{H}$ NMR $\left(400 \mathrm{MHz}, \mathrm{CDCl}_{3}\right): \delta 8.69-8.68(\mathrm{~m}, 1 \mathrm{H}), 8.53(\mathrm{dd}, J=4.8,1.6 \mathrm{~Hz}, 1 \mathrm{H}), 7.73(\mathrm{dt}, J=8.0$, $2.0 \mathrm{~Hz}, 1 \mathrm{H}), 7.43-7.23(\mathrm{~m}, 6 \mathrm{H}), 3.98(\mathrm{t}, J=7.0 \mathrm{~Hz}, 1 \mathrm{H}), 2.34-2.22(\mathrm{~m}, 2 \mathrm{H}), 2.18-2.06(\mathrm{~m}, 2 \mathrm{H})$.

${ }^{13} \mathrm{C}$ NMR (101 MHz, $\left.\mathrm{CDCl}_{3}\right): \delta 152.5,148.7,140.0,138.7,129.0,127.6,127.5,127.1$ (q, $J=277.5$ $\mathrm{Hz}), 123.1,120.4,93.2,81.2,37.4,31.8(\mathrm{q}, J=29.1 \mathrm{~Hz}), 31.5(\mathrm{q}, J=2.7 \mathrm{~Hz})$.

${ }^{19} \mathrm{~F}$ NMR (376 MHz, $\left.\mathrm{CDCl}_{3}\right): \delta-65.96(\mathrm{t}, J=10.7 \mathrm{~Hz}, 3 \mathrm{~F})$. IR (neat) $\mathrm{cm}^{-1} \tilde{\mathrm{v}}: 3030,2923,2230$, 1454, 1407, 1388, 1220, 1136, 1108, 1005, 978, 805, 701, 573. HRMS (EI+ $70 \mathrm{eV}): \mathrm{C}_{17} \mathrm{H}_{14} \mathrm{~F}_{3} \mathrm{~N}[\mathrm{M}]^{+}$ : calcd: 289.1078, found: 289.1075. [ a ] ${ }^{21}=-3.2\left(\mathrm{c}=0.50, \mathrm{CH}_{2} \mathrm{Cl}_{2}\right)$ HPLC (AD-H, $0.46 * 25 \mathrm{~cm}, 5$ $\mu \mathrm{m}$, hexane $/$ isopropanol $=99.5 / 0.5$, flow $0.5 \mathrm{~mL} / \mathrm{min}$, detection at $210 \mathrm{~nm}, 40{ }^{\circ} \mathrm{C}$ ) retention time $=17.9 \mathrm{~min}$ (minor ) and $23.6 \mathrm{~min}$ (major), ee $=82 \%$.

(R)-1-methyl-3-(6,6,6-trifluoro-3-phenylhex-1-yn-1-yl) benzene (d23)<smiles>Cc1cccc(C#CC(CCC(F)(F)F)c2ccccc2)c1</smiles>

Prepared following the general procedure showed above using styrene $(41.6 \mathrm{mg}, 0.4 \mathrm{mmol}, 2.0$ equiv), 3-ethynyltoluene (23.2 mg, $0.2 \mathrm{mmol}, 1.0$ equiv) and 1, 1, 1-trifluoro-2-iodoethane (251.9 $\mathrm{mg}, 1.2 \mathrm{mmol}, 6.0$ equiv).The desired product was purified by column chromatography (petroleum ether: ethyl acetate $=100: 1)$ as colorless oil $(36.8 \mathrm{mg}, 61 \%)$.

${ }^{1} \mathrm{H}$ NMR (400 MHz, CDCl $): \delta$ 7.44-7.34 (m, 4H), 7.30-7.18 (m, 4H), 7.13-7.11 (m, 1H), $3.95(\mathrm{dd}$, $J=8.0,5.6 \mathrm{~Hz}, 1 \mathrm{H}), 2.36-2.23(\mathrm{~m}, 5 \mathrm{H}), 2.17-2.00(\mathrm{~m}, 2 \mathrm{H})$.

${ }^{13} \mathrm{C}$ NMR (101 MHz, $\left.\mathrm{CDCl}_{3}\right): \delta 140.6,138.2,132.4,129.2,128.6,128.90,128.87,128.4,127.5$, $127.4,127.3$ (q, $J=277.2 \mathrm{~Hz}), 123.1,89.1,84.8,37.4,31.8$ (q, $J=28.9 \mathrm{~Hz}), 30.7$ (q, $J=2.6 \mathrm{~Hz})$, 21.3. ${ }^{19} \mathrm{~F}$ NMR $\left(376 \mathrm{MHz}, \mathrm{CDCl}_{3}\right): \delta-65.94(\mathrm{t}, J=10.7 \mathrm{~Hz}, 3 \mathrm{~F})$

IR (neat) $\mathrm{cm}^{-1} \tilde{\mathrm{v}}$ : 3063, 3030, 2923, 2853, 1602, 1490, 1454, 1387, 1257, 1108, 1008, 785, 696. HRMS (EI $\left.{ }^{+}, 70 \mathrm{eV}\right): \mathrm{C}_{19} \mathrm{H}_{17} \mathrm{~F}_{3}[\mathrm{M}]^{+}$: calcd: 302.1282, found: 302.1290. [ a $]_{\mathrm{D}}^{29}=-5.6$ (c = 0.94, $\mathrm{CHCl}_{3}$ ) HPLC (AD-H, $0.46 * 25 \mathrm{~cm}, 5 \mu \mathrm{m}, 100 \%$ hexane, flow $1 \mathrm{~mL} / \mathrm{min}$, detection at $210 \mathrm{~nm}$, $40{ }^{\circ} \mathrm{C}$ ) retention time $=5.3 \mathrm{~min}$ (minor) and $5.8 \mathrm{~min}$ (major), ee $=84 \%$ 
<smiles>FC(F)(F)CCC(C#Cc1cccc(Cl)c1)c1ccccc1</smiles>

Prepared following the general procedure showed above using styrene $(41.6 \mathrm{mg}, 0.4 \mathrm{mmol}, 2.0$ equiv), 4-chlorophenylacetylene (27.3 mg, $0.2 \mathrm{mmol}, 1.0$ equiv) and 1, 1, 1-trifluoro-2-iodoethane ( $251.9 \mathrm{mg}, 1.2 \mathrm{mmol}, 6.0$ equiv). The desired product was purified by column chromatography (petroleum ether: ethyl acetate $=100: 1$ ) as colorless oil $(41.8 \mathrm{mg}, 65 \%$ ).

${ }^{1} \mathrm{H}$ NMR (400 MHz, $\mathrm{CDCl}_{3}$ ): $\delta$ 7.48-7.45(m, 3H), 7.42-7.35 (m, 3H), 7.31-7.18 (m, 3H), 4.03 (dd, $J=8.4,5.6 \mathrm{~Hz}, 1 \mathrm{H}), 2.42-2.30(\mathrm{~m}, 2 \mathrm{H}), 2.20-2.03(\mathrm{~m}, 2 \mathrm{H})$.

${ }^{13} \mathrm{C}$ NMR (101 MHz, $\left.\mathrm{CDCl}_{3}\right): \delta .140 .2,136.2,133.4,129.4,129.3,128.9,127.6,127.5,127.3$ (q, $J$ $=277.2 \mathrm{~Hz}), 126.6,123.1,95.0,81.6,37.6,31.7(\mathrm{q}, J=29.0 \mathrm{~Hz}), 30.8(\mathrm{q}, J=2.6 \mathrm{~Hz})$.

${ }^{19} \mathrm{~F}$ NMR (376 MHz, $\left.\mathrm{CDCl}_{3}\right): \delta-65.95$ (t, $\left.J=10.5 \mathrm{~Hz}, 3 \mathrm{~F}\right)$.

IR (neat) $\mathrm{cm}^{-1} \tilde{\mathrm{v}}$ : 3064, 3030, 2926, 2854, 1493, 1473, 1387, 1256, 1120, 1134, 1108, 1004, 753, 700, 573. HRMS (EI+ $70 \mathrm{eV}$ ): $\mathrm{C}_{18} \mathrm{H}_{14} \mathrm{ClF}_{3}[\mathrm{M}]^{+}$: calcd: 322.0736, found: 322.0742. [ a $]_{\mathrm{D}}{ }^{29}=-3.0$ $\left(\mathrm{c}=0.33, \mathrm{CHCl}_{3}\right) \mathrm{HPLC}(\mathrm{AD}-\mathrm{H}, 0.46 * 25 \mathrm{~cm}, 5 \mu \mathrm{m}, 100 \%$ hexane, flow $1 \mathrm{~mL} / \mathrm{min}$, detection at 210 $\mathrm{nm}, 40{ }^{\circ} \mathrm{C}$ ) retention time $=5.9 \mathrm{~min}$ (minor ) and $6.7 \mathrm{~min}$ (major), ee $=69 \%$.

(R)-2-(6,6,6-trifluoro-3-phenylhex-1-yn-1-yl) thiophene (d25)<smiles>FC(F)(F)CCC(C#Cc1cccs1)c1ccccc1</smiles>

Prepared following the general procedure showed above using styrene (41.6 mg, $0.4 \mathrm{mmol}, 2.0$ equiv), 2-ethynylthiophene ( $21.6 \mathrm{mg}, 0.2 \mathrm{mmol}, 1.0$ equiv) and 1, 1, 1-trifluoro-2-iodoethane (251.9 $\mathrm{mg}, 1.2 \mathrm{mmol}, 6.0$ equiv). The desired product was purified by column chromatography (petroleum ether: ethyl acetate $=50: 1)$ as colorless oil $(37.6 \mathrm{mg}, 64 \%)$.

${ }^{1} \mathrm{H}$ NMR $\left(400 \mathrm{MHz}, \mathrm{CDCl}_{3}\right): \delta$ 7.41-7.35 (m, 4H), 7.31-7.26 (m, 1H), 7.25-7.22 (m, 1H), 7.20-7.19 (m, 1H), $3.97(\mathrm{t}, J=7.0 \mathrm{~Hz}, 1 \mathrm{H}), 2.33-2.19(\mathrm{~m}, 2 \mathrm{H}), 2.16-2.02(\mathrm{~m}, 2 \mathrm{H})$.

${ }^{13} \mathrm{C}$ NMR (101 MHz, $\left.\mathrm{CDCl}_{3}\right): \delta 140.2,131.9,129.0,127.5,127.1,126.9,123.2,127.2$ (q, $J=277.2$ $\mathrm{Hz}), 93.5,77.7,37.6,31.7$ (q, $J=29.0 \mathrm{~Hz}), 30.5$ (q, $J=2.6 \mathrm{~Hz}), 21.3 .{ }^{19} \mathrm{~F}$ NMR $\left(376 \mathrm{MHz}, \mathrm{CDCl}_{3}\right)$ : $\delta-65.93(\mathrm{t}, J=10.7 \mathrm{~Hz}, 3 \mathrm{~F})$

IR (neat) $\mathrm{cm}^{-1} \tilde{\mathrm{v}}$ : 3064, 3030, 2946, 2874, 1493, 1453, 1387, 1255, 1119, 1133, 1106, 999, 848, 758, 697, 571. HRMS (EI+ $70 \mathrm{eV}$ ): $\mathrm{C}_{16} \mathrm{H}_{13} \mathrm{~F}_{3} \mathrm{~S}[\mathrm{M}]^{+}$: calcd: 294.0690 found: 294.0688 [ a $]_{\mathrm{D}}^{29}=-5.6$ (c $\left.=0.94, \mathrm{CHCl}_{3}\right)$. HPLC $(\mathrm{AD}-\mathrm{H}, 0.46 * 25 \mathrm{~cm}, 5 \mu \mathrm{m}$, hexane $/$ isopropanol $=99.8 / 0.2$, flow $0.6 \mathrm{~mL} / \mathrm{min}$, detection at $210 \mathrm{~nm}, 40{ }^{\circ} \mathrm{C}$ ) retention time $=9.6 \mathrm{~min}$ (minor ) and $10.8 \mathrm{~min}$ (major), ee $=64 \%$.

(R)-4-(6,6,6-trifluoro-1-(4-methoxyphenyl) hex-1-yn-3-yl)-1,1'-biphenyl (d26)<smiles>COc1ccc(C#CC(CCC(F)(F)F)c2ccc(-c3ccccc3)cc2)cc1</smiles> 
Prepared following the general procedure showed above using 1-ethenyl-4-phenylbenzene (72.1 mg, $0.4 \mathrm{mmol}, 2.0$ equiv), 4-ethynylanisole (26.4 mg, $0.2 \mathrm{mmol}, 1.0$ equiv) and 1, 1, 1-trifluoro-2iodoethane (251.9 mg, $1.2 \mathrm{mmol}, 6.0$ equiv).The desired product was purified by column chromatography (petroleum ether: ethyl acetate $=40: 1)$ as colorless oil $(56.7 \mathrm{mg}, 72 \%)$.

${ }^{1} \mathrm{H}$ NMR (400 MHz, CDCl $)$ : $\delta$ 7.60-7.58 (m, 4H), 7.50-7.33 (m, 7H), 6.87-6.83 (m, 2H), 3.99 (dd, $J=8.0,5.6 \mathrm{~Hz}, 1 \mathrm{H}), 3.82(\mathrm{~s}, 3 \mathrm{H}), 2.39-2.26(\mathrm{~m}, 2 \mathrm{H}), 2.19-2.02(\mathrm{~m}, 2 \mathrm{H})$.

${ }^{13} \mathrm{C} \mathrm{NMR}\left(101 \mathrm{MHz}, \mathrm{CDCl}_{3}\right): \delta 159.6,140.8,140.4,139.8,133.2,128.9,128.0,127.6,127.5,127.3$ $(\mathrm{q}, J=277.4 \mathrm{~Hz}), 127.2,115.4,114.1,87.9,84.5,55.5,37.1,31.8(\mathrm{q}, J=28.9 \mathrm{~Hz}), 30.7$ (q, $J=2.3$ $\mathrm{Hz})$.

${ }^{19} \mathrm{~F}$ NMR $\left(376 \mathrm{MHz}, \mathrm{CDCl}_{3}\right): \delta-65.90(\mathrm{t}, J=10.7 \mathrm{~Hz}, 3 \mathrm{~F})$

IR (neat) $\mathrm{cm}^{-1} \tilde{\mathrm{v}}: 3030,2954,2838,1605,1509,1486,1386,1289,1247,1134,1103,1031,1005$, 832, 764, 736, 697, 579. HRMS (EI+ $70 \mathrm{eV}): \mathrm{C}_{25} \mathrm{H}_{21} \mathrm{~F}_{3} \mathrm{O}[\mathrm{M}]^{+}$: calcd: 394.1545, found: 394.1544. $\left[{ }^{a}\right]_{D}^{28}=-7.9\left(\mathrm{c}=0.40, \mathrm{CHCl}_{3}\right)$. HPLC (AD-H, 0.46*25 cm, $5 \mu \mathrm{m}$, hexane $/$ isopropanol = 90.0/10.0, flow $0.6 \mathrm{~mL} / \mathrm{min}$, detection at $210 \mathrm{~nm}, 40{ }^{\circ} \mathrm{C}$ ) retention time $=8.5 \mathrm{~min}$ (minor ) and $9.8 \mathrm{~min}$ (major), ee $=66 \%$.

(R)- (3-([1, 1'-biphenyl]-4-yl)-6, 6-difluorohex-1-yn-1-yl) triisopropylsilane (d27)

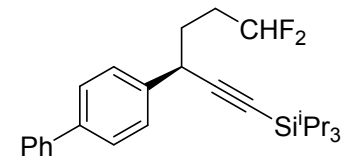

Prepared following the general procedure showed above using 1-ethenyl-4-phenylbenzene (72.1 mg, $0.4 \mathrm{mmol}, 2.0$ equiv), triisopropylsilylacetylene (36.4 mg, $0.2 \mathrm{mmol}, 1.0$ equiv) and 1, 1-difluoro2-iodoethane (230.3 $\mathrm{mg}, 1.2 \mathrm{mmol}, 6.0$ equiv). The desired product was purified by column chromatography (petroleum ether: ethyl acetate $=100: 1)$ as colorless oil $(53.6 \mathrm{mg}, 63 \%)$.

${ }^{1} \mathrm{H}$ NMR (400 MHz, $\left.\mathrm{CDCl}_{3}\right): \delta$ 7.60-7.55 (m, 4H), 7.46-7.41 (m, 4H), 7.36-3.32 (m, 1H), $5.84(\mathrm{tt}$, $J=56.8,4.4 \mathrm{~Hz} 1 \mathrm{H}), 3.84(\mathrm{dd}, J=8.4,5.2 \mathrm{~Hz} 1 \mathrm{H}), 2.12-1.84(\mathrm{~m}, 4 \mathrm{H}), 1.11-1.10(\mathrm{~m}, 21 \mathrm{H})$.

${ }^{13} \mathrm{C}$ NMR $\left(101 \mathrm{MHz}, \mathrm{CDCl}_{3}\right): \delta 140.9,140.1,128.9,128.0,127.43,127.40,127.2,117.1(\mathrm{t}, J=$ $240.0 \mathrm{~Hz}), 108.4,84.9,37.9,31.9(\mathrm{t}, J=21.3 \mathrm{~Hz}), 31.1(\mathrm{t}, J=5.1 \mathrm{~Hz}), 18.8,11.4$.

${ }^{19} \mathrm{~F}$ NMR (376 MHz, $\mathrm{CDCl}_{3}$ ): $\delta$-115.78- -116.07 (m, 2F). IR (neat) $\mathrm{cm}^{-1}$ ṽ: 2942, 2894, 2864, 2169 , 1487, 1462, 1403, 1260, 1122, 1051, 1033, 1009, 882, 840, 763, 736, 697, 675, 497.

HRMS (EI+ $70 \mathrm{eV}$ ): $\mathrm{C}_{27} \mathrm{H}_{36} \mathrm{~F}_{2} \mathrm{Si}[\mathrm{M}]^{+}$: calcd: 426.2554, found: 426.2552. [ a $]_{\mathrm{D}}{ }^{32}=13.4$ (c $=0.30$, $\mathrm{CH}_{2} \mathrm{Cl}_{2}$ ). HPLC (OD-H, $0.46 * 25 \mathrm{~cm}, 5 \mu \mathrm{m}$, hexane / isopropanol $=99 / 1$, flow $0.5 \mathrm{~mL} / \mathrm{min}$, detection at $210 \mathrm{~nm}, 40{ }^{\circ} \mathrm{C}$ ) retention time $=7.6 \mathrm{~min}$ (major) and $10.0 \mathrm{~min}$ (minor), ee $=94 \%$

(R)- (3-([1, 1'-biphenyl]-4-yl)-6-fluorohex-1-yn-1-yl) triisopropylsilane (d28)

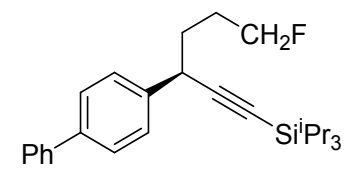

Prepared following the general procedure showed above using 1-ethenyl-4-phenylbenzene (72.1 mg, $0.4 \mathrm{mmol}, 2.0$ equiv), triisopropylsilylacetylene ( $36.4 \mathrm{mg}, 0.2 \mathrm{mmol}, 1.0$ equiv) and 1-fluoro-2iodoethane (208.7 mg, $1.2 \mathrm{mmol}, 6.0$ equiv). The desired product was purified by column chromatography (petroleum ether: ethyl acetate $=100: 1)$ as colorless oil $(58.0 \mathrm{mg}, 71 \%)$.

${ }^{1} \mathrm{H}$ NMR (400 MHz, $\left.\mathrm{CDCl}_{3}\right): \delta$ 7.60-7.55 (m, 4H), 7.47-7.41 (m, 4H), 7.35-7.32 (m, 1H), 4.58-4.38 $(\mathrm{m}, 2 \mathrm{H}), 3.82(\mathrm{dd}, J=8.0,4.4 \mathrm{~Hz} 1 \mathrm{H}), 2.04-1.82(\mathrm{~m}, 4 \mathrm{H}), 1.103-1.098(\mathrm{~m}, 21 \mathrm{H})$.

${ }^{13} \mathrm{C}$ NMR $\left(101 \mathrm{MHz}, \mathrm{CDCl}_{3}\right): \delta 141.0,140.7,139.8,128.9,128.0,127.3,127.2,109.2,84.2,83.9$ (d, $J=165.6 \mathrm{~Hz}), 38.2,34.7(\mathrm{~d}, J=4.5 \mathrm{~Hz}), 28.3(\mathrm{~d}, J=19.9 \mathrm{~Hz}), 18.8,11.4$. 
${ }^{19} \mathrm{~F}$ NMR (376 MHz, $\mathrm{CDCl}_{3}$ ): $\delta$-218.48- -218.89 (m, 1F). IR (neat) $\mathrm{cm}^{-1} \tilde{\mathrm{v}}: 2941,2893,2863,2168$, 1467, 1461, 1007, 996, 881, 838, 763, 696, 665, 496. HRMS (EI $\left.{ }^{(+)}, 70 \mathrm{eV}\right): \mathrm{C}_{27} \mathrm{H}_{37} \mathrm{FSi}[\mathrm{M}]^{+}:$calcd: 408.2649, found: 408.2641. [ a $]_{\mathrm{D}}^{32}=6.9\left(\mathrm{c}=0.14, \mathrm{CH}_{2} \mathrm{Cl}_{2}\right)$. HPLC (OD-H, 0.46*25 cm, $5 \mu \mathrm{m}$, hexan $/$ isopropanol $=99 / 1$, flow $0.5 \mathrm{~mL} / \mathrm{min}$, detection at $210 \mathrm{~nm}, 40{ }^{\circ} \mathrm{C}$ ) retention time $=7.3 \mathrm{~min}$ (major) and $8.3 \mathrm{~min}$ (minor), ee $=85 \%$

(R)- (3-([1,1'-biphenyl]-4-yl)-6, 6,7,7-tetrafluorohept-1-yn-1-yl) triisopropylsilane (d29)<smiles>FC(F)(F)CCC(C#C[SeH2])c1ccc(-c2ccccc2)cc1</smiles>

Prepared following the general procedure showed above using 1-ethenyl-4-phenylbenzene ( $72.1 \mathrm{mg}$, $0.4 \mathrm{mmol}, 2.0$ equiv), triisopropylsilylacetylene (36.4 mg, $0.2 \mathrm{mmol}, 1.0$ equiv) and 1, 1, 2, 2tetrafluoro-1-iodopropane ( $290.3 \mathrm{mg}, 1.2 \mathrm{mmol}, 6.0$ equiv). The desired product was purified by column chromatography (petroleum ether: ethyl acetate $=100: 1)$ as colorless oil $(79.2 \mathrm{mg}, 80 \%)$. ${ }^{1} \mathrm{H}$ NMR $\left(400 \mathrm{MHz}, \mathrm{CDCl}_{3}\right): \delta$ 7.60-7.57 (m, 4H), 7.48-7.41 (m, 4H), 7.36-7.32 (m, 1H), $5.69(\mathrm{tt}$, $J=54.0,2.4 \mathrm{~Hz}, 1 \mathrm{H}), 3.87(\mathrm{dd}, J=8.8,4.8 \mathrm{~Hz}, 1 \mathrm{H}), 2.32-1.93(\mathrm{~m}, 4 \mathrm{H}), 1.14-1.10$ (m, 21H).

${ }^{13} \mathrm{C}$ NMR $\left(101 \mathrm{MHz}, \mathrm{CDCl}_{3}\right): \delta 140.9,140.1,139.8,128.9,127.9,127.5,127.4,127.2,118.2(\mathrm{tt}, J$ $=246.9,29.5 \mathrm{~Hz}), 110.4(\mathrm{tt}, J=250.3,41.7 \mathrm{~Hz}), 108.0,85.3,37.9,29.6$ (t, $J=3.0 \mathrm{~Hz}), 27.6(\mathrm{t}, J=$ $22.5 \mathrm{~Hz}), 18.8(\mathrm{~d}, J=1.0 \mathrm{~Hz}), 11.4$.

${ }^{19} \mathrm{~F}$ NMR $\left(376 \mathrm{MHz}, \mathrm{CDCl}_{3}\right): \delta-115.71--115.93(\mathrm{~m}, 2 \mathrm{~F}),-135.21(\mathrm{dd}, J=54.1,3.4 \mathrm{~Hz}, 2 \mathrm{~F})$. IR (neat) $\mathrm{cm}^{-1} \tilde{\mathrm{v}}$ : 2968, 2943, 2892, 2866, 2169, 1487, 1463, 1380, 1230, 1160, 1104, 950, 882, 837, 816, 764, 697,493. HRMS (EI+ $\left.{ }^{+}, 70 \mathrm{eV}\right): \mathrm{C}_{28} \mathrm{H}_{36} \mathrm{~F}_{4} \mathrm{Si}[\mathrm{M}]^{+}$: calcd: 476.2522 , found: 476.2525. [ a $]_{\mathrm{D}}{ }^{32}$ $=5.6\left(\mathrm{c}=0.72, \mathrm{CH}_{2} \mathrm{Cl}_{2}\right)$. HPLC $(\mathrm{OD}-\mathrm{H}, 0.46 * 25 \mathrm{~cm}, 5 \mu \mathrm{m}$, hexane $/$ isopropanol $=99 / 1$, flow 0.5 $\mathrm{mL} / \mathrm{min}$, detection at $210 \mathrm{~nm}, 40{ }^{\circ} \mathrm{C}$ ) retention time $=7.2 \mathrm{~min}$ (major) and $10.4 \mathrm{~min}$ (minor), ee $=96 \%$.

(R)- (3-([1,1'-biphenyl]-4-yl)-5,5,6,6, 6-pentafluorohex-1-yn-1-yl) triisopropylsilane (d30)<smiles>FC(F)(F)CCC(C#C[Se]c1ccccc1)c1ccc(-c2ccccc2)cc1</smiles>

Prepared following the general procedure showed above using 1-ethenyl-4-phenylbenzene ( $72.1 \mathrm{mg}$, $0.4 \mathrm{mmol}, 2.0$ equiv), triisopropylsilylacetylene (36.4 mg, $0.2 \mathrm{mmol}, 1.0$ equiv) and iodopentafluoroethane $(295.1 \mathrm{mg}, 1.2 \mathrm{mmol}, 6.0$ equiv). The desired product was purified by column chromatography (petroleum ether: ethyl acetate $=100: 1)$ as colorless oil $(72.0 \mathrm{mg}, 75 \%$ ). ${ }^{1} \mathrm{H}$ NMR (400 MHz, CDCl $): \delta 7.60-7.57(\mathrm{~m}, 4 \mathrm{H}), 7.50-7.41(\mathrm{~m}, 4 \mathrm{H}), 7.36-7.32(\mathrm{~m}, 1 \mathrm{H}), 4.17(\mathrm{dd}$, $J=9.6,4.8 \mathrm{~Hz}, 1 \mathrm{H}), 2.68-2.36(\mathrm{~m}, 2 \mathrm{H}), 1.09-1.06(\mathrm{~m}, 21 \mathrm{H})$.

${ }^{13} \mathrm{C}$ NMR $\left(101 \mathrm{MHz}, \mathrm{CDCl}_{3}\right): \delta 140.7,140.6,139.3,129.0,127.9,127.7,127.6,123.7-111.7(\mathrm{~m}$, 2C), 106.9, $85.3(\mathrm{~d}, J=1.0 \mathrm{~Hz}), 39.8(\mathrm{t}, J=20.7 \mathrm{~Hz}), 31.4(\mathrm{t}, J=3.0 \mathrm{~Hz}), 18.7,11.4$.

${ }^{19} \mathrm{~F}$ NMR (376 MHz, $\mathrm{CDCl}_{3}$ ): $\delta-85.74(\mathrm{~s}, 3 \mathrm{~F}),-116.21--118.45(\mathrm{~m}, 2 \mathrm{~F})$. IR (neat) $\mathrm{cm}^{-1} \tilde{\mathrm{v}}: 3029$, 2939, 2892, 2863, 2168, 1486, 1463, 1010, 995, 883, 839, 763, 734, 674. HRMS (EI ${ }^{+}, 70 \mathrm{eV}$ ): $\mathrm{C}_{27} \mathrm{H}_{33} \mathrm{~F} 5 \mathrm{Si}[\mathrm{M}]^{+}$: calcd: 480.2272 , found: 480.2269. [ a $]_{\mathrm{D}}^{25}=11.9$ (c $=2.85, \mathrm{CH}_{2} \mathrm{Cl}_{2}$ ). HPLC (OD$\mathrm{H}, 0.46^{*} 25 \mathrm{~cm}, 5 \mu \mathrm{m}$, hexane / isopropanol $=99.7 / 0.3$, flow $0.5 \mathrm{~mL} / \mathrm{min}$, detection at $210 \mathrm{~nm}$, $40{ }^{\circ} \mathrm{C}$ ) retention time $=10.7 \mathrm{~min}$ (major) and $14.5 \mathrm{~min}$ (minor), ee $=98 \%$

(R)-(3-([1,1'-biphenyl]-4-yl)-5-fluoropent-1-yn-1-yl) triisopropylsilane (d31) 
<smiles>[18OH]C#CC(CCF)c1ccc(-c2ccccc2)cc1</smiles>

Prepared following the general procedure showed above using 1-ethenyl-4-phenylbenzene ( $72.1 \mathrm{mg}$, $0.4 \mathrm{mmol}, 2.0$ equiv), triisopropylsilylacetylene ( $36.4 \mathrm{mg}, 0.2 \mathrm{mmol}, 1.0$ equiv) and fluoro (iodo) methane (192.0 mg, $1.2 \mathrm{mmol}, 6.0$ equiv). The desired product was purified by column chromatography (petroleum ether: ethyl acetate $=100: 1)$ as colorless oil $(56.7 \mathrm{mg}, 72 \%)$.

${ }^{1} \mathrm{H}$ NMR $\left(400 \mathrm{MHz}, \mathrm{CDCl}_{3}\right): \delta$ 7.61-7.56 (m, 4H), 7.49-7.42 (m, 4H), 7.36-7.32 (m, 1H), 4.82-4.47 $(\mathrm{m}, 2 \mathrm{H}), 3.98(\mathrm{dd}, J=9.2,5.6 \mathrm{~Hz}, 1 \mathrm{H}), 2.26-2.00(\mathrm{~m}, 2 \mathrm{H}), 1.12-1.10(\mathrm{~m}, 21 \mathrm{H})$.

${ }^{13} \mathrm{C}$ NMR $\left(101 \mathrm{MHz}, \mathrm{CDCl}_{3}\right): \delta 140.9,140.2,140.0,128.9,128.0,127.5,127.4,127.2,108.5,84.5$, $81.9(\mathrm{~d}, J=165.5 \mathrm{~Hz}), 39.7$ (d, $J=19.8 \mathrm{~Hz}), 34.6(\mathrm{~d}, J=5.4 \mathrm{~Hz}), 18.8(\mathrm{~d}, J=0.7 \mathrm{~Hz}), 11.4$.

${ }^{19} \mathrm{~F}$ NMR (376 MHz, CDCl $)$ ): $\delta$-221.37- -221.75 (m, 1F). IR (neat) $\mathrm{cm}^{-1} \tilde{\mathrm{v}}: 3030,2942,2893,2864$, 2169, 1486, 1463, 1385, 1260, 1073, 1013, 996, 883, 834, 763, 734, 697, 667, 556.

HRMS (EI+ $\left.{ }^{+}, 70 \mathrm{eV}\right): \mathrm{C}_{26} \mathrm{H}_{35} \mathrm{FSi}[\mathrm{M}]^{+}$: calcd: 394.2492, found: 394.2486. [ a $]_{\mathrm{D}}{ }^{32}=7.9$ (c $=0.10$, $\mathrm{CH}_{2} \mathrm{Cl}_{2}$ ). HPLC (OD-H, $0.46 * 25 \mathrm{~cm}, 5 \mu \mathrm{m}$, hexane / isopropanol $=99.8 / 0.2$, flow $0.5 \mathrm{~mL} / \mathrm{min}$, detection at $210 \mathrm{~nm}, 40^{\circ} \mathrm{C}$ ) retention time $=19.3 \mathrm{~min}$ (major) and $22.3 \mathrm{~min}$ (minor), ee $=94 \%$.

(R)-(3-([1,1'-biphenyl] - 4 -yl) oct-1-yn-1-yl) triisopropylsilane (d32)

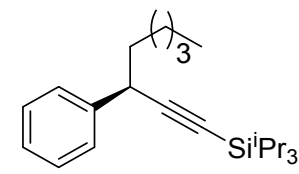

Prepared following the general procedure showed above using styrene $(41.7 \mathrm{mg}, 0.4 \mathrm{mmol}, 2.0$ equiv), triisopropylsilylacetylene ( $36.4 \mathrm{mg}, 0.2 \mathrm{mmol}, 1.0$ equiv) and 1-iodobutane (220.9 mg, 1.2 mmol, 6.0 equiv). The desired product was purified by column chromatography (petroleum ether) as colorless oil ( $34.0 \mathrm{mg}, 50 \%)$.

${ }^{1} \mathrm{H}$ NMR (400 MHz, CDCl $): \delta 7.39-7.38(\mathrm{~m}, 2 \mathrm{H}), 7.32-7.29(\mathrm{~m}, 2 \mathrm{H}), 7.23-7.19(\mathrm{~m}, 1 \mathrm{H}), 3.69(\mathrm{t}, J$ $=7.2 \mathrm{~Hz} 1 \mathrm{H}), 1.74-1.68(\mathrm{~m}, 2 \mathrm{H}), 1.52-1.43(\mathrm{~m}, 2 \mathrm{H}), 1.33-1.24(\mathrm{~m}, 4 \mathrm{H}), 1.093-1.085(\mathrm{~m}, 21 \mathrm{H})$.

${ }^{13} \mathrm{C}$ NMR $\left(101 \mathrm{MHz}, \mathrm{CDCl}_{3}\right): \delta 142.5,128.4,127.6,126.6,110.2,83.2,39.2,39.0,31.6,27.1,22.7$, $18.8(\mathrm{~d}, J=3.2 \mathrm{~Hz}), 14.2,11.5$. IR (neat) $\mathrm{cm}^{-1} \tilde{\mathrm{v}}: 2938,2892,2862,2169,1601,1493,1461,1071$, 1015, 994, 882, 755, 697, 664, 613.HRMS (EI $\left.{ }^{(+)}, 70 \mathrm{eV}\right): \mathrm{C}_{23} \mathrm{H}_{38} \mathrm{Si}[\mathrm{M}]^{+}:$calcd:342.2743, found: 342.2739. [ a $]_{\mathrm{D}}{ }^{28}=8.3\left(\mathrm{c}=0.16, \mathrm{CH}_{2} \mathrm{Cl}_{2}\right)$. HPLC (PC-3, 0.46*25 cm, $5 \mu \mathrm{m}, \mathrm{MeCN} / \mathrm{H}_{2} \mathrm{O}=$ $70.0 / 30.0$ flow $0.7 \mathrm{~mL} / \mathrm{min}$, detection at $214 \mathrm{~nm}, 25{ }^{\circ} \mathrm{C}$ ) retention time $=13.7 \mathrm{~min}$ (major) and $14.5 \mathrm{~min}$ (minor), ee $=95 \%$.

(R)-triisopropyl (5-methyl-3- phenylhex-1-yn-1-yl) silane (d33)

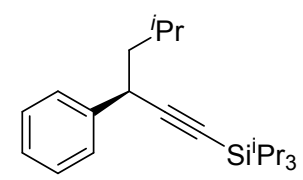

Prepared following the general procedure showed above using styrene $(41.7 \mathrm{mg}, 0.4 \mathrm{mmol}, 2.0$ equiv), triisopropylsilylacetylene (36.4 mg, $0.2 \mathrm{mmol}, 1.0$ equiv) and 2-iodopropane (204.0 mg, 1.2 mmol, 6.0 equiv). The desired product was purified by column chromatography (petroleum) as colorless oil (36.7 mg, $56 \%)$.

${ }^{1} \mathrm{H}$ NMR (400 MHz, $\left.\mathrm{CDCl}_{3}\right): \delta$ 7.39-7.36 (m, 2H), 7.32-7.28 (m, 2H), 7.23-7.19 (m, 1H), $3.72(\mathrm{dd}$, 
$J=10.0,5.6 \mathrm{~Hz} 1 \mathrm{H}), 1.95-1.85(\mathrm{~m}, 1 \mathrm{H}), 1.72-1.65(\mathrm{~m}, 1 \mathrm{H}), 1.50-1.43(\mathrm{~m}, 1 \mathrm{H}), 1.084-1.077(\mathrm{~m}$, $21 \mathrm{H}), 0.95(\mathrm{dd}, J=13.2,6.8 \mathrm{~Hz} 1 \mathrm{H})$

${ }^{13} \mathrm{C}$ NMR $\left(101 \mathrm{MHz}, \mathrm{CDCl}_{3}\right): \delta 142.9,128.5,127.5,126.6,110.2,83.1,48.7,37.2,26.3,23.3,21.8$, $18.8(\mathrm{~d}, J=0.9 \mathrm{~Hz}), 11.5$. IR (neat) $\mathrm{cm}^{-1} \tilde{\mathrm{v}}: 3029,2942,2865,2168,1493,1463,1384,1367,1260$, 1015, 995, 882, 803, 753, 698, 666. HRMS (EI $\left.{ }^{(+)}, 70 \mathrm{eV}\right): \mathrm{C}_{22} \mathrm{H}_{36} \mathrm{Si}[\mathrm{M}]^{+}$: calcd: 328.2586 , found: 328.2588. [ a $]_{\mathrm{D}}{ }^{28}=12.7\left(\mathrm{c}=0.31, \mathrm{CH}_{2} \mathrm{Cl}_{2}\right)$. HPLC (PC-3, 0.46*25 cm, $5 \mu \mathrm{m}, \mathrm{MeCN} / \mathrm{H}_{2} \mathrm{O}=$ $60.0 / 40.0$ flow $0.7 \mathrm{~mL} / \mathrm{min}$, detection at $214 \mathrm{~nm}, 25{ }^{\circ} \mathrm{C}$ ) retention time $=28.1 \mathrm{~min}$ (major) and $29.5 \mathrm{~min}$ (minor), ee $=90 \%$.

(R)- (3, 4-diphenylbut-1-yn-1-yl) triisopropylsilane (d34)<smiles></smiles>

Prepared following the general procedure showed above using styrene $(41.6 \mathrm{mg}, 0.4 \mathrm{mmol}, 2.0$ equiv), triisopropylsilylacetylene ( $36.4 \mathrm{mg}, 0.2 \mathrm{mmol}, 1.0$ equiv) and iodobenzene (244.8 $\mathrm{mg}, 1.2$ mmol, 6.0 equiv).The desired product was purified by column chromatography (petroleum ether) as colorless oil (35.4 mg, $49 \%)$.

${ }^{1} \mathrm{H}$ NMR (400 MHz, $\left.\mathrm{CDCl}_{3}\right): \delta$ 7.34-7.13 (m, $\left.10 \mathrm{H}\right), 3.96(\mathrm{dd}, J=8.4,6.0 \mathrm{~Hz}, 1 \mathrm{H}), 3.06-2.92(\mathrm{~m}$, 2H), 1.08-1.03 (m, 21H).

${ }^{13} \mathrm{C}$ NMR $\left(101 \mathrm{MHz}, \mathrm{CDCl}_{3}\right): \delta 141.5,139.0,129.6,128.4,128.1,127.8,126.8,126.5,109.2,84.5$,

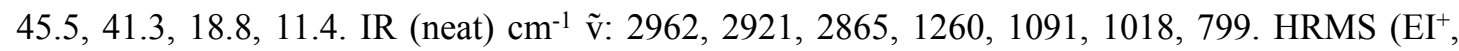
70eV): $\mathrm{C}_{25} \mathrm{H}_{34} \mathrm{Si}[\mathrm{M}]^{+}$: calcd: 362.2430 , found: $362.2440\left[{ }^{a}\right]_{\mathrm{D}}{ }^{25}=21.5\left(\mathrm{c}=0.73, \mathrm{CHCl}_{3}\right)$. HPLC (PC-3, 0.46*25 cm, $5 \mu \mathrm{m}, \mathrm{MeCN} / \mathrm{H}_{2} \mathrm{O}=70.0 / 30.0$ flow $0.7 \mathrm{~mL} / \mathrm{min}$, detection at $214 \mathrm{~nm}, 25^{\circ} \mathrm{C}$ ) retention time $=17.7 \mathrm{~min}$ (major) and $21.9 \mathrm{~min}$ (minor), ee $=96 \%$.

(R)- (4-(4-(tert-butyl) phenyl)-3-phenylbut-1-yn-1-yl)triisopropylsilane (d35)<smiles>CC(C)(C)c1ccc(CC(C#C[Se]C(=O)c2ccccc2)c2ccccc2)cc1</smiles>

Prepared following the general procedure showed above using styrene (41.6 mg, $0.4 \mathrm{mmol}, 2.0$ equiv), triisopropylsilylacetylene (36.4 mg, $0.2 \mathrm{mmol}, 1.0$ equiv) and 4-tert-butyliodobenzene (312.1 mg, $1.2 \mathrm{mmol}, 6.0$ equiv).The desired product was purified by column chromatography (petroleum ether) as colorless oil (45.1 $\mathrm{mg}, 54 \%$ ).

${ }^{1} \mathrm{H}$ NMR $\left(400 \mathrm{MHz}, \mathrm{CDCl}_{3}\right): \delta$ 7.44-7.42 (m, 2H), 7.38-7.36 (m, 2H), 7.33-7.26 (m, 3H), 7.18-7.16 (m, 2H), $4.00(\mathrm{dd}, J=9.2,5.6 \mathrm{~Hz}, 1 \mathrm{H}), 3.10-2.93(\mathrm{~m}, 2 \mathrm{H}), 1.35(\mathrm{~s}, 9 \mathrm{H}), 1.069-1.065(\mathrm{~m}, 21 \mathrm{H})$.

${ }^{13} \mathrm{C}$ NMR $\left(101 \mathrm{MHz}, \mathrm{CDCl}_{3}\right): \delta 149.2,141.8,136.1,129.2,128.5,127.8,126.8,125.0,109.2,84.5$, 45.1, 41.4, 34.5, 31.5, 18.8, 11.4. IR (neat) $\mathrm{cm}^{-1} \tilde{\mathrm{v}}: 3087,3061,3028,2942,2864,2171,1514,1493$, 1462, 1262, 1018, 883, 810, 755, 698, 675.HRMS (EI $\left.{ }^{+}, 70 \mathrm{eV}\right): \mathrm{C}_{29} \mathrm{H}_{42} \mathrm{Si}$. [M] $]^{+}$: calcd:, 418.3056 found:418.3048 [a $]_{\mathrm{D}}^{28}=258.6\left(\mathrm{c}=0.10, \mathrm{CHCl}_{3}\right)$. HPLC (PC-3, 0.46*25 cm, $5 \mu \mathrm{m}, \mathrm{MeCN} / \mathrm{H}_{2} \mathrm{O}=$ $70.0 / 30.0$ flow $0.7 \mathrm{~mL} / \mathrm{min}$, detection at $214 \mathrm{~nm}, 25{ }^{\circ} \mathrm{C}$ ) retention time $=20.0$ min (major) and $43.7 \mathrm{~min}$ (minor), ee $=92 \%$.

(R)-triisopropyl (4-(4-methoxyphenyl)-3-phenylbut-1-yn-1-yl) silane (d36) 
<smiles>COc1ccc(CC(C#C[Se]C)c2ccccc2)cc1</smiles>

Prepared following the general procedure showed above using styrene (41.6 mg, $0.4 \mathrm{mmol}, 2.0$ equiv), triisopropylsilylacetylene ( $36.4 \mathrm{mg}, 0.2 \mathrm{mmol}, 1.0$ equiv) and 4-iodomethoxybenzene (280.8 $\mathrm{mg}, 1.2 \mathrm{mmol}, 6.0$ equiv).The desired product was purified by column chromatography (petroleum ether: ethyl acetate $=50: 1)$ as colorless oil $(46.2 \mathrm{mg}, 59 \%)$.

${ }^{1} \mathrm{H}$ NMR (400 MHz, CDCl $): \delta$ 7.32-7.20 (m, 5H), 7.05-7.02 (m, 2H), 6.78-6.74 (m, 2H), $3.91(\mathrm{dd}$, $J=8.0,6.4 \mathrm{~Hz}, 1 \mathrm{H}), 3.77$ (s, 3H), 3.01-2.91 (m, 2H), 1.11-1.04 (m, 21H).

${ }^{13} \mathrm{C}$ NMR (101 MHz, $\left.\mathrm{CDCl}_{3}\right): \delta 158.3,141.5,131.1,130.6,128.4,127.9,126.8,113.5,109.4,84.4$, 55.4, 44.7, 41.5, 18.8, 11.4. IR (neat) $\mathrm{cm}^{-1} \tilde{\mathrm{v}}: 3063,3029,2941,2863,2170,1612,1512,1462,1246$, 1178, 1038, 882, 804, 698, 674. HRMS (EI+ $70 \mathrm{eV}): \mathrm{C}_{17} \mathrm{H}_{14} \mathrm{~F}_{3} \mathrm{~N}[\mathrm{M}]^{+}$: calcd: 392.2535, found: 392.2538. [ a $]_{\mathrm{D}}{ }^{25}=17.3\left(\mathrm{c}=1.27, \mathrm{CHCl}_{3}\right)$. HPLC (PC-3, 0.46*25 cm, $5 \mu \mathrm{m}, \mathrm{MeCN} / \mathrm{H}_{2} \mathrm{O}=$ $70.0 / 30.0$ flow $0.7 \mathrm{~mL} / \mathrm{min}$, detection at $214 \mathrm{~nm}, 25{ }^{\circ} \mathrm{C}$ ) retention time $=15.9 \mathrm{~min}$ (major) and $22.8 \mathrm{~min}$ (minor), ee $=92 \%$.

(R)- (4-([1, 1'-biphenyl]-4-yl)-3-phenylbut-1-yn-1-yl) triisopropylsilane (d37)<smiles>[SeH2+]C#CC(Cc1ccc(Br)cc1)c1ccccc1</smiles>

Prepared following the general procedure showed above using styrene $(41.6 \mathrm{mg}, 0.4 \mathrm{mmol}, 2.0$ equiv), triisopropylsilylacetylene ( $36.4 \mathrm{mg}, 0.2 \mathrm{mmol}, 1.0$ equiv) and 1-bromo-iodobenzene ( 339.5 $\mathrm{mg}, 1.2 \mathrm{mmol}, 6.0$ equiv).The desired product was purified by column chromatography (petroleum ether) as colorless oil (41.3mg, $47 \%$ ).

${ }^{1} \mathrm{H}$ NMR (400 MHz, CDCl $): \delta 7.36-7.33(\mathrm{~m}, 2 \mathrm{H}), 7.31-7.22(\mathrm{~m}, 5 \mathrm{H}), 7.00-6.98(\mathrm{~m}, 2 \mathrm{H}), 3.95(\mathrm{dd}$, $J=8.0,6.0 \mathrm{~Hz}, 1 \mathrm{H}), 3.03-2.92(\mathrm{~m}, 2 \mathrm{H}), 1.04-1.01(\mathrm{~m}, 21 \mathrm{H})$.

${ }^{13} \mathrm{C}$ NMR $\left(101 \mathrm{MHz}, \mathrm{CDCl}_{3}\right): \delta 141.0,137.8,131.4,131.1,128.5,127.8,127.0,120.4,85.0,44.7$, 41.0, 18.7, 11.4 .

IR (neat) $\mathrm{cm}^{-1} \tilde{\mathrm{v}}$ : 2941, 2891, 2863, 2170, 1489, 1462, 1260, 1072, 1013, 882, 799, 698, 675, 598. HRMS (EI $\left.{ }^{+}, 70 \mathrm{eV}\right): \mathrm{C}_{25} \mathrm{H}_{33} \mathrm{BrSi}[\mathrm{M}]^{+}:$calcd: 440.1535 , found:440.1527. [ a $]_{\mathrm{D}}{ }^{33}=23.9$ (c $=0.75$, $\mathrm{CHCl}_{3}$ ). HPLC (PC-3, 0.46*25 cm, $5 \mu \mathrm{m}, \mathrm{MeCN} / \mathrm{H}_{2} \mathrm{O}=90.0 / 10.0$ flow $0.7 \mathrm{~mL} / \mathrm{min}$, detection at $214 \mathrm{~nm}, 25^{\circ} \mathrm{C}$ ) retention time $=6.8 \mathrm{~min}$ (major) and $7.5 \mathrm{~min}$ (minor), ee $=90 \%$.

(R)- (4-([1, 1'-biphenyl]-4-yl)-3-phenylbut-1-yn-1-yl) triisopropylsilane (d38)<smiles>O=C([SeH]C#Cc1ccccc1)c1ccccc1</smiles>

Prepared following the general procedure showed above using styrene (41.6 mg, $0.4 \mathrm{mmol}, 2.0$ equiv), triisopropylsilylacetylene (36.4 mg, $0.2 \mathrm{mmol}, 1.0$ equiv) and 4-iodobiphenyl (336.1 mg, $1.2 \mathrm{mmol}, 6.0$ equiv).The desired product was purified by column chromatography (petroleum ether) 
as colorless oil (38.5 $\mathrm{mg}, 44 \%)$.

${ }^{1} \mathrm{H}$ NMR (400 MHz, CDCl $): \delta$ 7.57-7.55 (m, 2H), 7.47-7.40 (m, 4H), 7.38-7.29 (m, 5H) 7.26-7.21 (m, 3H), 4.00 (dd, $J=8.8,6.0 \mathrm{~Hz}, 1 \mathrm{H}), 3.11-2.99(\mathrm{~m}, 2 \mathrm{H}), 1.04-0.94(\mathrm{~m}, 21 \mathrm{H})$.

${ }^{13} \mathrm{C} \mathrm{NMR}\left(101 \mathrm{MHz}, \mathrm{CDCl}_{3}\right): \delta 141.5,141.3,139.4,138.1,130.0,128.8,128.5,127.8,127.2,126.9$, 109.1, 84.7, 45.2, 41.3, 18.8, 11.4. IR (neat) $\mathrm{cm}^{-1}$ ṽ: 3060, 3028, 2941, 2890, 2863, 2170, 1601, 1489, 1460, 1260, 1074, 1054, 1011, 883, 844, 814, 759, 696, 675. HRMS (EI $\left.{ }^{+}, 70 \mathrm{eV}\right): \mathrm{C}_{31} \mathrm{H}_{38} \mathrm{Si}$ $[\mathrm{M}]^{+}$: calcd: 438.2743, found:438.2730 [ $\left.{ }^{a}\right]_{\mathrm{D}}{ }^{33}=18.4\left(\mathrm{c}=2.60, \mathrm{CHCl}_{3}\right)$. HPLC (PC-3, 0.46*25 $\mathrm{cm}, 5 \mu \mathrm{m}, \mathrm{MeCN} / \mathrm{H}_{2} \mathrm{O}=80.0 / 20.0$ flow $0.7 \mathrm{~mL} / \mathrm{min}$, detection at $214 \mathrm{~nm}, 25^{\circ} \mathrm{C}$ ) retention time $=15.6 \mathrm{~min}$ (major) and $27.9 \mathrm{~min}$ (minor), ee $=93 \%$.

(R)-triisopropyl (4-(naphthalen-1-yl)-3-phenylbut-1-yn-1-yl) silane (d39)<smiles>[AsH2]C#CC(Cc1cccc2ccccc12)c1ccccc1</smiles>

Prepared following the general procedure showed above using styrene (41.6 mg, $0.4 \mathrm{mmol}, 2.0$ equiv), triisopropylsilylacetylene ( $36.4 \mathrm{mg}, 0.2 \mathrm{mmol}, 1.0$ equiv) and 1-iodonaphthalene ( $304.5 \mathrm{mg}$, $1.2 \mathrm{mmol}, 6.0$ equiv).The desired product was purified by column chromatography (petroleum ether: ethyl acetate $=100: 1)$ as colorless oil (34.6mg, $42 \%)$.

${ }^{1} \mathrm{H}$ NMR $\left(400 \mathrm{MHz}, \mathrm{CDCl}_{3}\right): \delta 8.10(\mathrm{~d}, J=8.4 \mathrm{~Hz}, 1 \mathrm{H}), 7.88-7.86(\mathrm{~m}, 1 \mathrm{H}), 7.73(\mathrm{~d}, J=7.6 \mathrm{~Hz}$, $1 \mathrm{H}), 7.54-7.46(\mathrm{~m}, 2 \mathrm{H}), 7.41-7.23(\mathrm{~m}, 7 \mathrm{H}), 4.12(\mathrm{dd}, J=8.8,5.6 \mathrm{~Hz}, 1 \mathrm{H}), 3.53-3.40(\mathrm{~m}, 2 \mathrm{H}), 1.02-$ $1.01(\mathrm{~m}, 21 \mathrm{H})$.

${ }^{13} \mathrm{C}$ NMR (101 MHz, $\left.\mathrm{CDCl}_{3}\right): \delta 142.0,135.0,134.0,132.0,129.0,128.6,128.2,127.7,127.3,126.9$, 126.0, 125.4 (d, $J=2.3 \mathrm{~Hz}), 123.6,109.3,84.6,42.9,40.4,18.8,11.4$. IR (neat) $\mathrm{cm}^{-1} \tilde{\mathrm{v}}: 3063,3030$, 2941, 2863, 2170, 1599, 1458, 1261, 1058, 1017, 995, 883, 790, 774, 697, 675. HRMS (EI+ $70 \mathrm{eV})$ : $\mathrm{C}_{17} \mathrm{H}_{14} \mathrm{~F}_{3} \mathrm{~N}[\mathrm{M}]^{+}$: calcd: 412.2586, found: 412.2584. [ $\mathrm{a}_{\mathrm{D}}{ }^{25}=10.8\left(\mathrm{c}=0.11, \mathrm{CHCl}_{3}\right)$. HPLC (PC3, $0.46 * 25 \mathrm{~cm}, 5 \mu \mathrm{m}, \mathrm{MeCN} / \mathrm{H}_{2} \mathrm{O}=70.0 / 30.0$, flow $0.7 \mathrm{~mL} / \mathrm{min}$, detection at $214 \mathrm{~nm}, 25^{\circ} \mathrm{C}$ ) retention time $=28.7 \mathrm{~min}$ (major) and $40.3 \mathrm{~min}$ (minor), ee $=94 \%$.

(R)-3-(2-phenyl-4-(triisopropylsilyl) but-3-yn-1-yl) pyridine (d40)<smiles>[AsH2]C#CC(Cc1cccnc1)c1ccccc1</smiles>

Prepared following the general procedure showed above using styrene (41.6 mg, $0.4 \mathrm{mmol}, 2.0$ equiv), triisopropylsilylacetylene ( $36.4 \mathrm{mg}, 0.2 \mathrm{mmol}, 1.0$ equiv) and 3-iodopyridine ( $246.0 \mathrm{mg}, 1.2$ mmol, 6.0 equiv).The desired product was purified by column chromatography (petroleum ether: ethyl acetate $=50: 1)$ as colorless oil $(43.5 \mathrm{mg}, 60 \%)$.

${ }^{1} \mathrm{H}$ NMR (400 MHz, $\left.\mathrm{CDCl}_{3}\right): \delta 8.45-8.34(\mathrm{~m}, 2 \mathrm{H}), 7.44(\mathrm{dt}, J=7.6,2.0 \mathrm{~Hz}, 1 \mathrm{H}), 7.32-7.31(\mathrm{~m}, 4 \mathrm{H})$ 7.28-7.23 (m, 1H), 7.16-7.13 (m, 1H), $4.00(\mathrm{dd}, J=8.4,5.6 \mathrm{~Hz}, 1 \mathrm{H}), 3.09-2.95(\mathrm{~m}, 2 \mathrm{H}), 1.10-1.03$ (m, 21H).

${ }^{13} \mathrm{C}$ NMR $\left(101 \mathrm{MHz}, \mathrm{CDCl}_{3}\right): \delta 150.8,148.0,140.6,137.1,134.1,128.6,127.8,127.2,123.0,108.2$, 
85.4, 42.3, 40.7, 18.7, 11.4. IR (neat) cm $\mathrm{cm}^{-1} \tilde{\mathrm{v}}$ : 3028, 2941, 2891, 2864, 2170, 1600, 1578, 1493, 1459, 1423, 1051, 1027, 994, 882, 789, 753, 714, 699, 663. HRMS (EI $\left.{ }^{+}, 70 \mathrm{eV}\right): \mathrm{C}_{24} \mathrm{H}_{33} \mathrm{NSi}[\mathrm{M}]^{+}$: calcd: 363.2382, found:363.2380 [a $]_{\mathrm{D}}{ }^{30}=33.2\left(\mathrm{c}=0.4, \mathrm{CHCl}_{3}\right)$.HPLC (OD-H, $0.46 * 25 \mathrm{~cm}, 5 \mu \mathrm{m}$, hexane / isopropanol $=99.4 / 0.6$, flow $0.8 \mathrm{~mL} / \mathrm{min}$, detection at $210 \mathrm{~nm}, 40^{\circ} \mathrm{C}$ ) retention time $=14.0 \mathrm{~min}$ (minor) and $15.8 \mathrm{~min}$ (major), ee $=93 \%$.

(R)-triisopropyl(3-phenyl-4-(thiophen-2-yl)but-1-yn-1-yl) silane (d41)<smiles>[SeH]C#CC(Cc1cccs1)c1ccccc1</smiles>

Prepared following the general procedure showed above using styrene $(41.6 \mathrm{mg}, 0.4 \mathrm{mmol}, 2.0$ equiv), triisopropylsilylacetylene ( $36.4 \mathrm{mg}, 0.2 \mathrm{mmol}, 1.0$ equiv) and 2-iodothiophene (252.0 $\mathrm{mg}$, $1.2 \mathrm{mmol}, 6.0$ equiv).The desired product was purified by column chromatography (petroleum ether: ethyl acetate $=50: 1)$ as colorless oil $(46.3 \mathrm{mg}, 63 \%)$.

${ }^{1} \mathrm{H}$ NMR $\left(400 \mathrm{MHz}, \mathrm{CDCl}_{3}\right): \delta 7.36-7.28(\mathrm{~m}, 4 \mathrm{H}), 7.25-7.21(\mathrm{~m}, 1 \mathrm{H}), 7.10(\mathrm{dd}, J=5.2,1.2 \mathrm{~Hz}, 1 \mathrm{H})$, $6.86(\mathrm{dd}, J=5.2,3.6 \mathrm{~Hz}, 1 \mathrm{H}), 6.75(\mathrm{dd}, J=3.2,0.4 \mathrm{~Hz}, 1 \mathrm{H}), 3.98(\mathrm{dd}, J=7.6,6.8 \mathrm{~Hz}, 1 \mathrm{H}), 3.30-$ $3.20(\mathrm{~m}, 2 \mathrm{H}), 1.08-1.04(\mathrm{~m}, 21 \mathrm{H})$.

${ }^{13} \mathrm{C}$ NMR $\left(101 \mathrm{MHz}, \mathrm{CDCl}_{3}\right): \delta 141.11,140.10,128.5,127.8,127.0,126.6,126.2,123.9,108.9$, 84.8, 41.6, 39.6, 18.8, 11.4.

IR (neat) $\mathrm{cm}^{-1}$ ṽ: 2941, 2863, 2170, 1460, 1245, 1054, 994, 882, 811, 758, 693, 663.

HRMS (EI+ $\left.{ }^{+}, 70 \mathrm{eV}\right): \mathrm{C}_{23} \mathrm{H}_{32} \mathrm{SSi}[\mathrm{M}]^{+}$: calcd: 368.1994 , found: 368.1998

$\left[{ }^{a}\right]_{\mathrm{D}}{ }^{26}=9.7\left(\mathrm{c}=0.23, \mathrm{CHCl}_{3}\right)$. HPLC $\left(\mathrm{PC}-3,0.46 * 25 \mathrm{~cm}, 5 \mu \mathrm{m}, \mathrm{MeCN} / \mathrm{H}_{2} \mathrm{O}=60.0 / 40.0\right.$ flow 0.7 $\mathrm{mL} / \mathrm{min}$, detection at $214 \mathrm{~nm}, 25^{\circ} \mathrm{C}$ ) retention time $=39.7 \mathrm{~min}$ (major) and $49.1 \mathrm{~min}$ (minor), ee $=84 \%$.

triisopropyl (((1R,4S)-3-phenylbicyclo[2.2.1] heptan-2-yl)ethynyl)silane (d42)<smiles>C(#CC1C2CCC(C2)C1c1ccccc1)c1ccccc1</smiles>

Prepared following the general procedure showed above using 2-norbornene (37.6 $\mathrm{mg}, 0.4 \mathrm{mmol}$, 2.0 equiv), triisopropylsilylacetylene ( $36.4 \mathrm{mg}, 0.2 \mathrm{mmol}, 1.0$ equiv) and iodobenzene ( $245.0 \mathrm{mg}$, $1.2 \mathrm{mmol}, 6.0$ equiv).The desired product was purified by column chromatography (petroleum ether) as colorless oil ( $9.1 \mathrm{mg}, 13 \%)$.

${ }^{1} \mathrm{H}$ NMR (400 MHz, $\left.\mathrm{CDCl}_{3}\right): \delta$ 7.60-7.52 (m, 4H), 7.44-7.30 (m, 5H), 2.74-2.73 (m, 1H), 2.69-2.66 $(\mathrm{m}, 1 \mathrm{H}), 2.55-2.54(\mathrm{~m}, 1 \mathrm{H}), 2-45-2.41(\mathrm{~m}, 1 \mathrm{H}), 2.08-2.02(\mathrm{~m}, 1 \mathrm{H}), 1.67-1.66(\mathrm{~m}, 1 \mathrm{H}), 1.43-1.40$ $(\mathrm{m}, 2 \mathrm{H}), 1.29-1.26(\mathrm{~m}, 2 \mathrm{H}), 1.11-1.10(\mathrm{~m}, 1 \mathrm{H})$.

${ }^{13} \mathrm{C}$ NMR (101 MHz, $\left.\mathrm{CDCl}_{3}\right): \delta 145.1,141.1,138.9,128.9,127.3,127.2,127.1,127.0,111.8,81.9$, 56.0, 43.9, 42.3, 42.2, 37.7, 30.8, 23.7, $18.9(\mathrm{~d}, \mathrm{~J}=1.0 \mathrm{~Hz}), 11.5$.

IR (neat) $\mathrm{cm}^{-1} \tilde{\mathrm{v}}: 2923.4,2864.2,2380.7,2348.0,2321.2,2297.7,2166.8,1465.1,1011.0,882.8$, 820.4, 760.9, 675.1, 659.9.

HRMS (EI+ $70 \mathrm{eV}$ ): $\mathrm{C}_{24} \mathrm{H}_{36} \mathrm{Si}[\mathrm{M}]^{+}$: calcd: 352.2586 , found: 352.2584

HPLC (PC-3, 0.46*25 cm, $5 \mu \mathrm{m}, \mathrm{MeCN} / \mathrm{H}_{2} \mathrm{O}=70.0 / 30.0$ flow $0.7 \mathrm{~mL} / \mathrm{min}$, detection at $214 \mathrm{~nm}$, $25^{\circ} \mathrm{C}$ ) retention time $=60.9 \min$ (major) and $66.5 \min$ (minor), ee $=2 \%$. 


\section{(3) Assignment of the absolute configuration}

The absolute configuration of product $\mathbf{d} \mathbf{4 3}$ was established by comparison of the sign of the optical rotation with reported compound prepared by Gregory $\mathrm{C}$. Fu. ${ }^{1}$
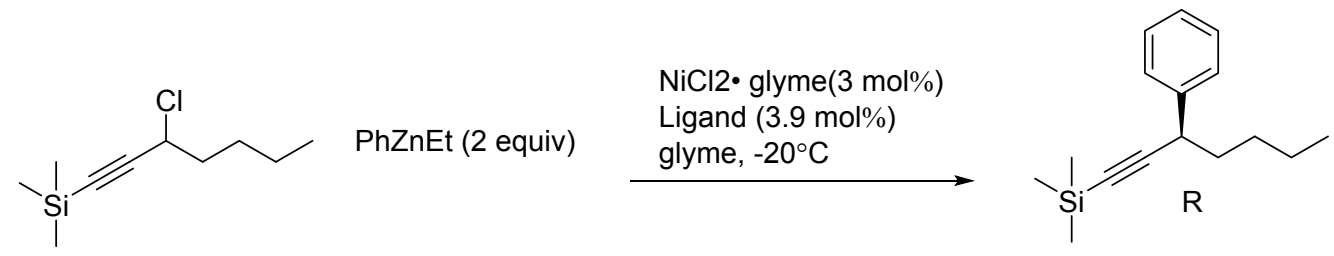<smiles>[CH]1Cc2ccccc2[C@H]2N=C(c3cccc(C4=N[C@H]5c6ccccc6C[C@H]5O4)n3)O[C@H]12</smiles>

ee $91 \%$

$[\alpha] \mathrm{D}^{22}=+12\left(\mathrm{c}=1.0 \mathrm{CHCl}_{3}\right)$<smiles>C=Cc1ccccc1</smiles><smiles>C#C[Si](C)(C)C</smiles><smiles>CCCI</smiles><smiles>CC(C)(C)O[N+](=O)[O-]</smiles><smiles>[R]C[C@H](C#C[Si](C)(C)C)c1ccccc1</smiles>

$$
\begin{gathered}
\mathbf{d 4 3} \\
\text { ee } 77 \% \\
{[\alpha] \mathrm{D}^{25}=+6.09\left(\mathrm{c}=0.30 \mathrm{CHCl}_{3}\right)}
\end{gathered}
$$

\section{Figure S1}

Prepared following the general procedure showed above using styrene $(20.0 \mathrm{mg}, 0.2 \mathrm{mmol}, 2.0$ equiv), ethynytrimethylsilane $(10.0 \mathrm{mg}, 0.1 \mathrm{mmol}, 1.0$ equiv) and 1-iodopropane (102.0 mg, 0.6 mmol, 6.0 equiv). The desired product was purified by column chromatography (petroleum ether) as colorless oil (15.9 $\mathrm{mg}, 65 \%)$.

${ }^{1} \mathrm{H}$ NMR (400 MHz, $\left.\mathrm{CDCl}_{3}\right): \delta 7.35-7.29(\mathrm{~m}, 4 \mathrm{H}), 7.25-7.20(\mathrm{~m}, 1 \mathrm{H}), 3.63(\mathrm{t}, J=7.2 \mathrm{~Hz}, 1 \mathrm{H}), 1.75-$ $1.69(\mathrm{~m}, 2 \mathrm{H}), 1.43-1.26(\mathrm{~m}, 4 \mathrm{H}), 0.88(\mathrm{t}, J=7.0 \mathrm{~Hz}, 1 \mathrm{H}), 0.18(\mathrm{~s}, 9 \mathrm{H})$.

${ }^{13} \mathrm{C} \mathrm{NMR}\left(101 \mathrm{MHz}, \mathrm{CDCl}_{3}\right): \delta 142.2,128.5,127.6,126.7,108.7,87.1,38.9,38.5,29.6,22.5,14.1$, 0.33. HRMS (EI+ $70 \mathrm{eV}): \mathrm{C}_{16} \mathrm{H}_{24} \mathrm{Si}[\mathrm{M}]^{+}$: calcd:244.1647, found: 244.1644. [ a $]_{\mathrm{D}}{ }^{25}=+6.1(\mathrm{c}=0.30$, $\mathrm{CHCl}_{3}$ ). IR (neat) $\mathrm{cm}^{-1} \tilde{\mathrm{v}}:$ 2958, 2933, 2860, 2172, 1538, 1493, 1249, 1046, 1032, 842, 758, 698. The ee was showed by GC ( column:VARIAN CHIRASIL-DEX CB): $65^{\circ} \mathrm{C}$, hold 2 min @ $2{ }^{\circ} \mathrm{C} / \mathrm{min}$, then $170{ }^{\circ} \mathrm{C}$, hold $10 \mathrm{~min}$; flow rate $10.0 \mathrm{psi}$, retention time $=42.8 \mathrm{~min}$ (minor) and $43.1 \mathrm{~min}$ (major), ee $=77 \%$

\section{Synthetic Applications}

(1) Removing the TIPS group in d1 
<smiles>FC(F)(F)CCC(C#C[SiH2]C(F)(F)F)c1ccc(-c2ccccc2)cc1</smiles>

ee $96 \%$

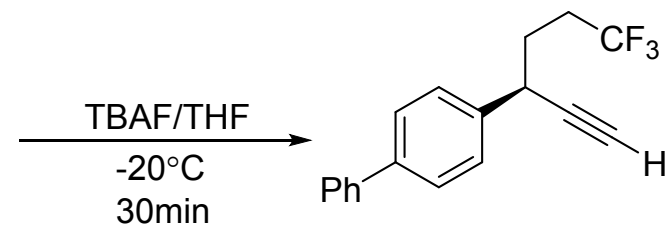

e1 yiled $90 \%$ ee $95 \%$

The d1 $(44.4 \mathrm{mg}, 0.1 \mathrm{mmol})$ was treated with $4 \mathrm{~mL} 1 \mathrm{M}$ THF solution of tetrabutylammonium fluoride. The mixture was stirred for $30 \mathrm{~min}$ at $-20^{\circ} \mathrm{C}$. After completion, the mixture was diluted with ethyl acetate, washed with brine, dried over $\mathrm{Na}_{2} \mathrm{SO}_{4}$. After concentration under vacuum, the residue was purified by column chromatography (petroleum ether) to give the product $\mathbf{e} \mathbf{1}$ as a white solid $(25.9 \mathrm{mg}, 90 \%$, ee $=95 \%)$

${ }^{1} \mathrm{H}$ NMR $\left(400 \mathrm{MHz}, \mathrm{CDCl}_{3}\right): \delta$ 7.59-7.57 (m, 4H), 7.45-7.43 (m, 4H), 7.37-7.33 (m, 1H), 3.81-3.77 (m, 1H), $2.37(\mathrm{~d}, J=2.4 \mathrm{~Hz}, 1 \mathrm{H}), 2.34-2.21(\mathrm{~m}, 2 \mathrm{H}), 2.13-1.97(\mathrm{~m}, 2 \mathrm{H})$.

${ }^{13} \mathrm{C}$ NMR (101 MHz, $\left.\mathrm{CDCl}_{3}\right): \delta 140.8,140.5,138.9,128.9,127.8,127.7,127.54$ (q, $\left.J=277.2 \mathrm{~Hz}\right)$, 127.52, 84.1, 72.6, 36.2, $31.6(\mathrm{q}, J=29.1 \mathrm{~Hz}), 30.4(\mathrm{q}, J=2.7 \mathrm{~Hz})$.

${ }^{19} \mathrm{~F}$ NMR $\left(376 \mathrm{MHz}, \mathrm{CDCl}_{3}\right): \delta-66.00(\mathrm{t}, J=10.7 \mathrm{~Hz}, 3 \mathrm{~F})$. IR (neat) $\mathrm{cm}^{-1} \tilde{\mathrm{v}}: 3311,3059,3033$, 2979, 2946, 2874, 2851, 2357, 1487, 1388, 1254, 1132, 1001, 837, 761, 642, 570. HRMS (EI+, $70 \mathrm{eV}): \mathrm{C}_{18} \mathrm{H}_{15} \mathrm{~F}[\mathrm{M}]^{+}$: calcd: 288.1126 , found: $288.1125 .[\alpha]_{\mathrm{D}}{ }^{25}=15.7(\mathrm{c}=0.21, \mathrm{CHCl} 3)$.

HPLC (OD-H, 0.46*25 cm, $5 \mu \mathrm{m}$, hexane:isopropanol $=99: 1$, flow $0.8 \mathrm{~mL} / \mathrm{min}$, detection at 210 $\mathrm{nm}, 40{ }^{\circ} \mathrm{C}$ ) retention time $=6.8 \mathrm{~min}$ (major) and $7.8 \mathrm{~min}$ (minor), ee $=95 \%$.

\section{(2) Preparation of f1}<smiles>FC(F)(F)CCC(C#C[SeH])c1ccc(-c2ccccc2)cc1</smiles>

d1

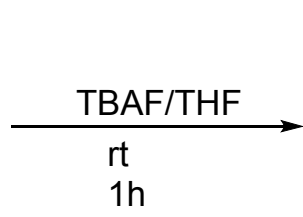

$\mathrm{Ph}^{-1}$<smiles>C=C(CCC(F)(F)F)c1ccc(-c2ccccc2)cc1</smiles>

f1

yield $78 \%$

The d1 $(44.4 \mathrm{mg}, 0.1 \mathrm{mmol})$ was treated with $4 \mathrm{~mL} 1 \mathrm{M}$ THF solution of tetrabutylammonium fluoride and stirred at $25{ }^{\circ} \mathrm{C}$ for $1 \mathrm{~h}$. After completion, the mixture was diluted with ethyl acetate, washed with brine, dried over $\mathrm{Na}_{2} \mathrm{SO}_{4}$. After concentration under vacuum, the residue was purified by column chromatography (petroleum ether) to give the product $\mathbf{f 1}$ as a white solid $(22.5 \mathrm{mg}, 78 \%)$ ${ }^{1} \mathrm{H}$ NMR (400 MHz, $\left.\mathrm{CDCl}_{3}\right): \delta$ 7.61-7.57 (m, 4H), 7.47-7.42 (m, 4H), 7.37-7.33 (m, 1H), $5.22(\mathrm{t}, J$ $=3.6 \mathrm{~Hz}, 2 \mathrm{H}), 2.74-2.68(\mathrm{~m}, 1 \mathrm{H}), 2.44-2.32(\mathrm{~m}, 2 \mathrm{H})$.

${ }^{13} \mathrm{C}$ NMR (101 MHz, $\left.\mathrm{CDCl}_{3}\right): \delta 208.1,140.7,140.0,134.4,129.0,127.5,127.4,127.2$ (q, $J=277.4$ $\mathrm{Hz}), 127.1,126.3,102.9,80.3,32.6(\mathrm{q}, J=28.7 \mathrm{~Hz}), 22.0(\mathrm{q}, J=3.1 \mathrm{~Hz})$.

${ }^{19} \mathrm{~F}$ NMR (376 MHz, $\mathrm{CDCl}_{3}$ ): $\delta-66.35$ (t, $\left.J=10.7 \mathrm{~Hz}, 3 \mathrm{~F}\right)$. HRMS (EI $\left.{ }^{+}, 70 \mathrm{eV}\right): \mathrm{C}_{18} \mathrm{H}_{15} \mathrm{~F}[\mathrm{M}]^{+}$: calcd: 288.1126 , found: 288.1123 . IR (neat) $\mathrm{cm}^{-1}$ ṽ: 3662, 2971, 2901, 1941, 1486, 1438, 1324, $1255,1234,1149,1102,1068,978,861,840,767,725,696,616$.

\section{(3) Gram-Scale Reaction}




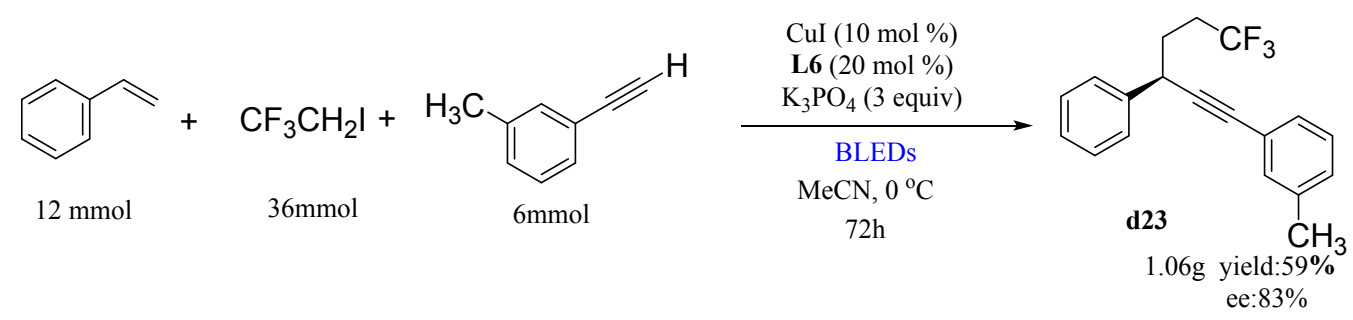

Under nitrogen atmosphere, L6 (1.2 mmol, $20 \mathrm{~mol} \%)$, CuI (0.6 mmol, $10 \mathrm{~mol} \%), \mathrm{K}_{3} \mathrm{PO}_{4}(18 \mathrm{mmol}$, 3.0 equiv), styrene ( $12 \mathrm{mmol}, 2.0$ equiv), 3-ethynyltoluene ( $6 \mathrm{mmol}, 1.0$ equiv) and 1, 1, 1-trifluoro2-iodoethane ( $36 \mathrm{mmol}, 6.0$ equiv). were added in a dried reaction vessel with $20 \mathrm{~mL} \mathrm{MeCN}$, and the reaction mixture was stirred at $0{ }^{\circ} \mathrm{C}$ under Blue LED for $72 \mathrm{~h}$. After completion, the reaction was quenched and the suspension was filtered through a pad of silica gel, rinsed with $\mathrm{CH}_{2} \mathrm{Cl}_{2}$. The eluent was evaporated in vacuo and the residual oil was purified by column chromatography (petroleum ether), the desired product was purified by column chromatography as colorless oil (1.06g, 59\%). HPLC (AD-H, 0.46*25 cm, $5 \mu \mathrm{m}, 100 \%$ hexane, flow $1 \mathrm{~mL} / \mathrm{min}$, detection at $210 \mathrm{~nm}, 40{ }^{\circ} \mathrm{C}$ ) retention time $=5.3 \mathrm{~min}$ (minor) and $5.8 \mathrm{~min}$ (major), ee $=83 \%$

\section{Mechanistic Investigations}

\section{(1)The reaction of TEMPO and under the standard conditions}

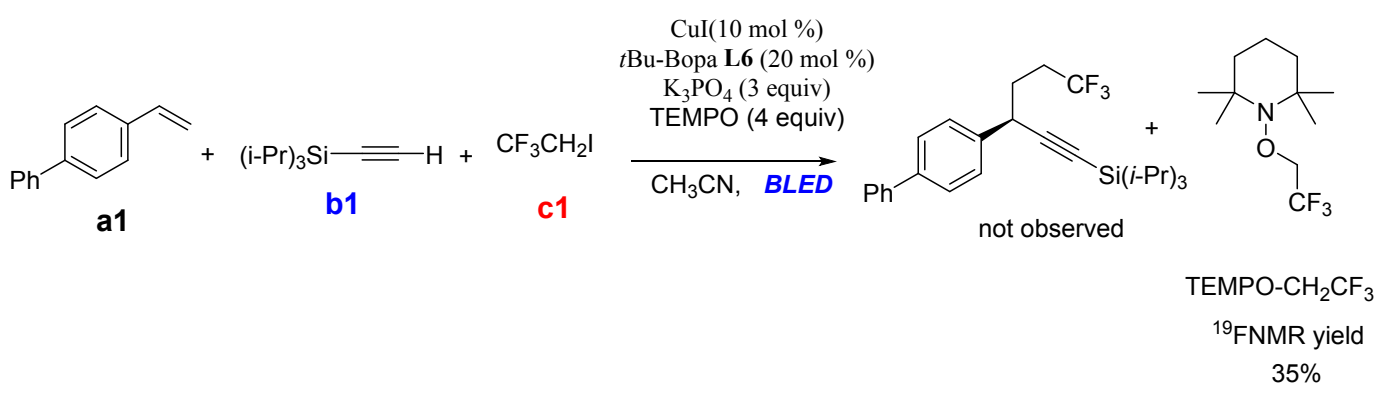

\section{Figure S2}

Under nitrogen atmosphere, L6 (8.4 mg, $0.02 \mathrm{mmol}, 20 \mathrm{~mol} \%$ ), CuI (1.9 mg, $0.01 \mathrm{mmol}, 10 \mathrm{~mol} \%$ ), $\mathrm{K}_{3} \mathrm{PO}_{4}$ (63.7 mg, $0.3 \mathrm{mmol}, 3.0$ equiv) and triisopropylsilylacetylene ( $0.1 \mathrm{mmol}, 1.0$ equiv), olefins ( $0.2 \mathrm{mmol}, 2.0$ equiv), and 1, 1, 1-trifluoro-2-iodoethane ( $0.6 \mathrm{mmol}, 6.0$ equiv) and TEMPO (56 $\mathrm{mg}, 0.4 \mathrm{mmol}, 4.0$ equiv) were added sequentially and the reaction mixture was stirred at $0{ }^{\circ} \mathrm{C}$ under BLED irradiation for $24 \mathrm{~h}$. After reaction completion by TLC, the reaction was quenched by water and the resulting mixture was extracted by ethyl acetate (EA). Volatile solvent was evaporated in vacuo. ${ }^{19} \mathrm{FNMR}$ analysis of the residual oil indicated that TEMPO- $\mathrm{CH}_{2} \mathrm{CF}_{3}$ was formed in 35\%. EIMS (m/z, relative intentsity): $239\left(\mathrm{M}^{+}, 11\right), 224$ (100), 156 (79), 124 (28), 109 (78), 69 (90), ${ }^{19} \mathrm{~F}$ NMR (376 MHz, $\left.\mathrm{CDCl}_{3}\right): \delta-71.71(\mathrm{~s}, 3 \mathrm{~F}){ }^{2}$

\section{(2) Considerations of the reaction's active intermediate.}



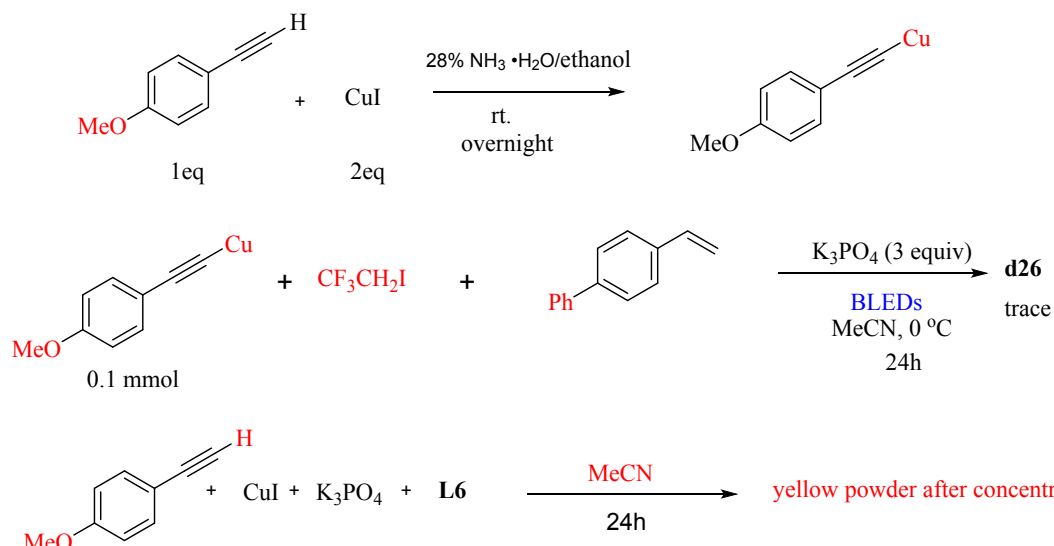

$\underset{24 \mathrm{~h}}{\mathrm{MeCN}}$ yellow powder after concentration

(3)
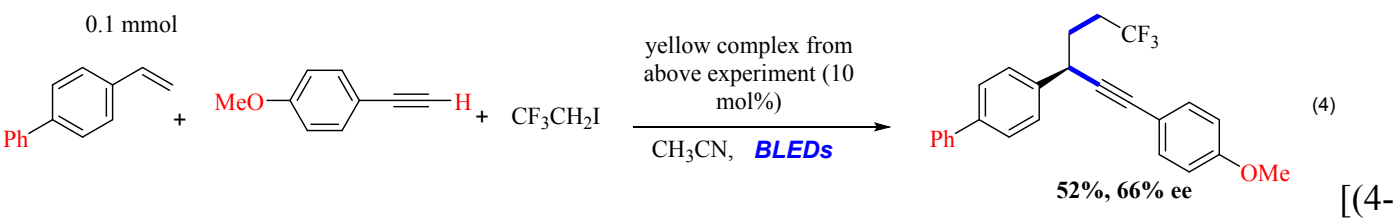

Methoxyphenyl)ethynyl]copper was obtained according to Jouvin's method (eq 1). ${ }^{3}$

Control experiment (eq 2): Under nitrogen atmosphere, stoichiometry [(4Methoxyphenyl)ethynyl]copper or catalytic amount [(4-Methoxyphenyl)ethynyl]copper, $\mathrm{K}_{3} \mathrm{PO}_{4}(63.7 \mathrm{mg}, 0.3 \mathrm{mmol}, 3.0$ equiv) and 3-ethynyltoluene (0.1 mmol-0.09 mmol), 4-vinyl-1,1'biphenyl ( $0.2 \mathrm{mmol}, 2.0$ equiv) and 1, 1, 1-trifluoro-2-iodoethane ( $0.6 \mathrm{mmol}, 6.0$ equiv) were added sequentially and the reaction mixture was stirred at $0{ }^{\circ} \mathrm{C}$. After completion, the reaction was quenched by water and the resulting mixture was extracted by ethyl acetate (EA). No desired product was obtained.

We prepared the Bopa-Cu-acetylide complex and isolated it as a crude yellow powder (eq 3).

When the above yellow complex was used as the catalyst under our standard conditions, it could smoothly catalyze the reaction with the desired product in $52 \%$ yield and $96 \%$ ee. This result was similar to the outcome using the in situ generated catalyst by combining CuI, L6, and acetylide (eq 4).

(3) UV-visible spectra of substrates and complexes 


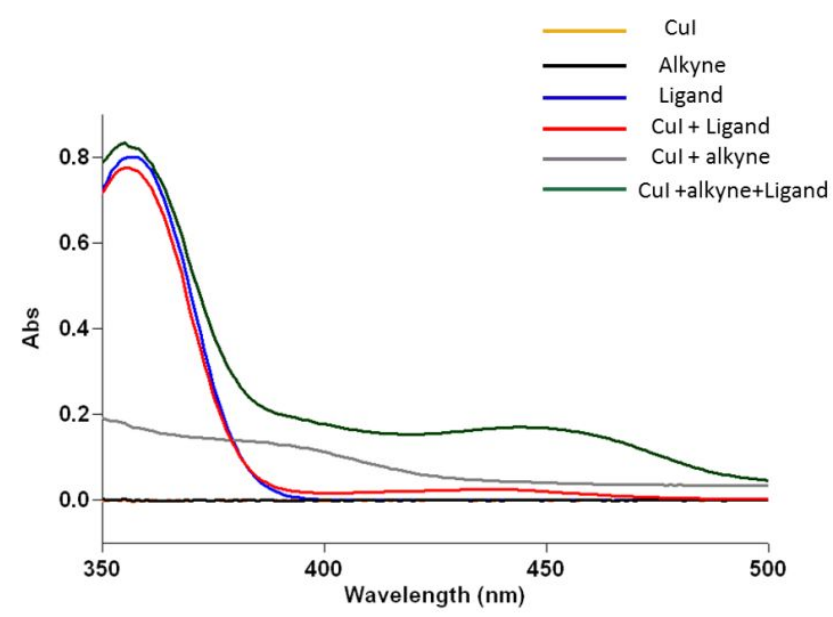

\section{Figure S3}

Organ, back and blue lines are respected CuI, alkyne (triisopropylsilyacetylene) and ligand (L6). Red line indicates mixture of $\mathrm{CuI}$ and ligand. Gray line indicates mixture of copper acetylide. Green line indicates mixture of $\mathrm{CuI}$, triisopropylsilyacetylene and L6. All the samples were used in 0.75 $\mathrm{mol} / \mathrm{L}$ solution freshly. In the range of $380-500 \mathrm{~nm}$, copper acetylide and copper acetylide-ligand showed obvious absorption.

\section{(4) Cyclic Woltammetry Analysis}

Cyclic Woltammetry analysis was performed by Shanghai Chen Hua CHI660D. Pt electrode, $\mathrm{Ag} / \mathrm{AgCl}$ and $\mathrm{Pt}$ wire were working electrode, reference electrode and auxiliary electride, respectively. Tetrabutylammonium hexafluorophosphata $\left(\mathrm{nBu}_{4} \mathrm{NPF}_{6}\right)$ was supporting electrolyte and electrolysis was conducted at ambient temperature.

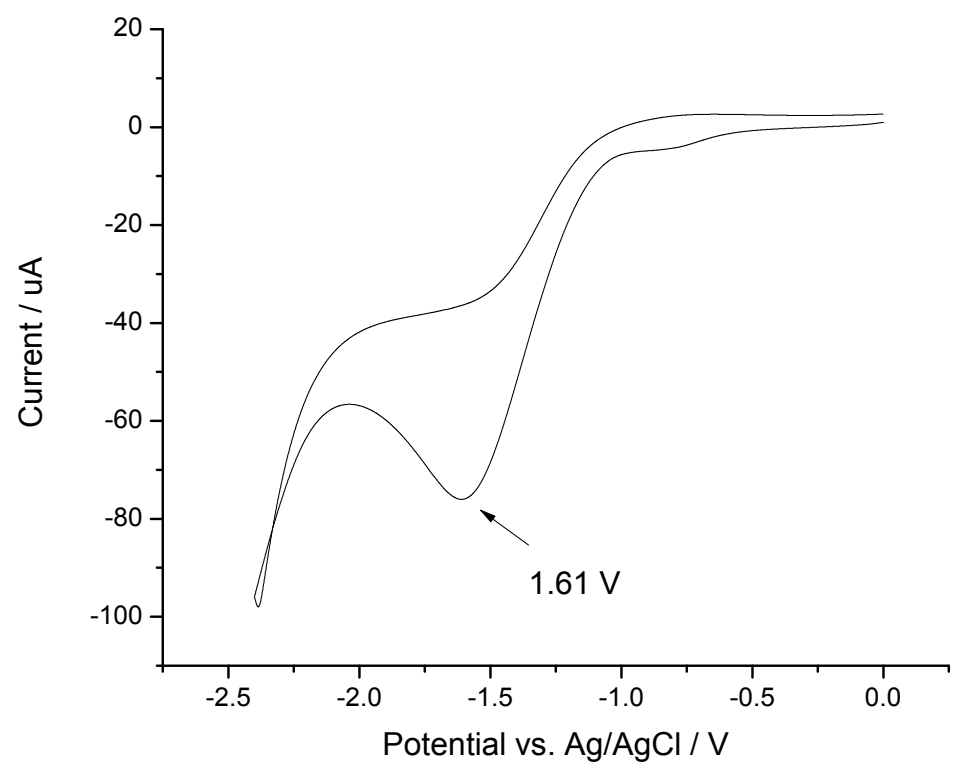

Figure S4 Cyclic woltammograms of $\mathrm{CF}_{3} \mathrm{CH}_{2} \mathrm{I}$, Scan rate: $0.1 \mathrm{~V} / \mathrm{s} . E_{1 / 2}=-1.61 \mathrm{~V}$ 


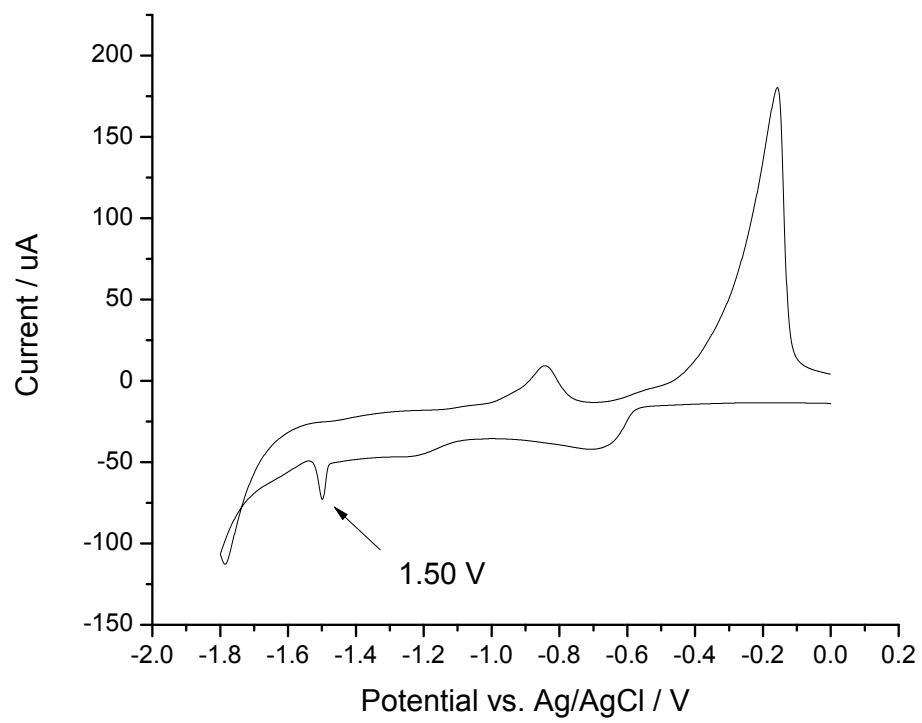

\section{Figure S5}

Cyclic woltammograms of copper acetylide, Scan rate: $0.1 \mathrm{~V} / \mathrm{s} . E_{1 / 2}=-1.50 \mathrm{~V}$

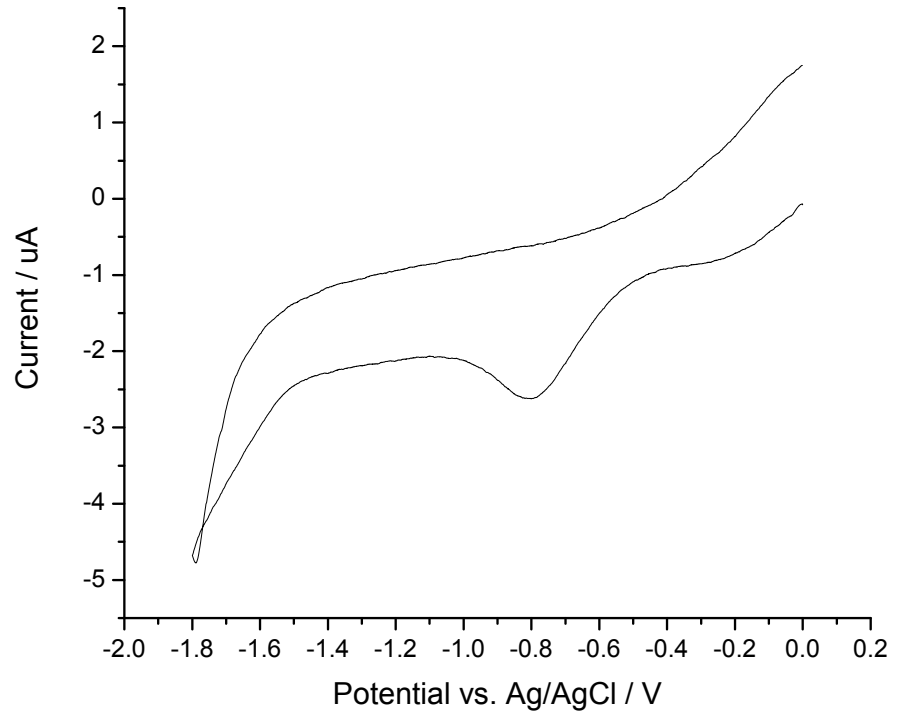

\section{Figure S6}

Cyclic woltammograms of L6, Scan rate: $0.1 \mathrm{~V} / \mathrm{s}$. No obvious signal was found. 


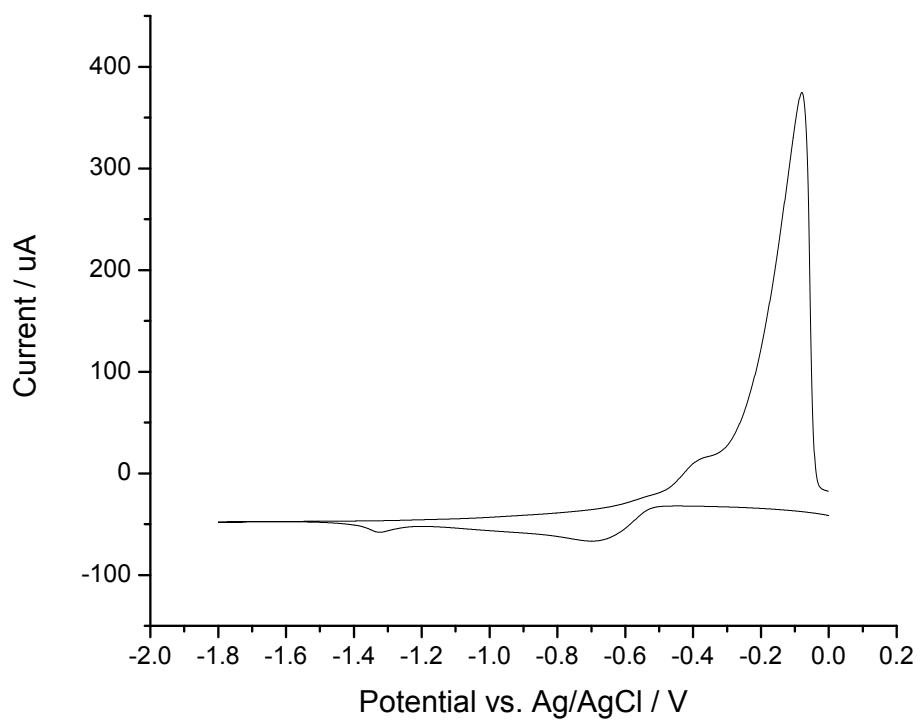

\section{Figure S7}

Cyclic woltammograms of $\mathrm{CuBr}$ and Ligand, Scan rate: $0.1 \mathrm{~V} / \mathrm{s}$.

As illustrated, the $\mathrm{CF}_{3} \mathrm{CH}_{2} \mathrm{I}$ was estimated a reversible reduction/ oxidation peak at $\mathrm{E}_{\text {red }}=-1.61 \mathrm{~V}$, while the copper acetylide showed a reduction peak at $\mathrm{E}_{\text {red }}=-1.50 \mathrm{~V}$. The alkyl halides do not generated the corresponding alkyl radical with copper acetylide.

\section{Preparation of the sample for cyclic woltammetry}

$\mathrm{CF}_{3} \mathrm{CH}_{2} \mathrm{I}$ in $\mathrm{MeCN}: \mathrm{CF}_{3} \mathrm{CH}_{2} \mathrm{I}(10.1 \mathrm{mg}, 0.05 \mathrm{M})$ and $\mathrm{nBu}_{4} \mathrm{NPF}_{6}(38.7 \mathrm{mg}, 0.1 \mathrm{M})$ were dissolved in $10 \mathrm{~mL} \mathrm{MeCN}$

L6 in MeCN: L6 (20.9 mg, $0.05 \mathrm{M})$ and $\mathrm{nBu}_{4} \mathrm{NPF}_{6}(38.7 \mathrm{mg}, 0.1 \mathrm{M})$ were dissolved in $10 \mathrm{~mL}$ $\mathrm{MeCN}$

$\mathrm{CuI}$ in $\mathrm{MeCN}$ : $\mathrm{CuI}(10.1 \mathrm{mg}, 0.05 \mathrm{M})$ and $\mathrm{nBu}_{4} \mathrm{NPF}_{6}(38.7 \mathrm{mg}, 0.1 \mathrm{M})$ were dissolved in $10 \mathrm{~mL}$ $\mathrm{MeCN}$.

$\mathrm{CuI}$ and ethynyltriisopropylsilane in $\mathrm{MeCN}$ : $\mathrm{CuI}(10.1 \mathrm{mg}, 0.05 \mathrm{M})$, ethynyltriisopropylsilane (9.1 $\mathrm{mg}, 0.05 \mathrm{M})$ and $\mathrm{nBu}_{4} \mathrm{NPF}_{6}(38.7 \mathrm{mg}, 0.1 \mathrm{M})$ were dissolved in $10 \mathrm{~mL} \mathrm{MeCN}$.

\section{Quantum Yield Measurement}

We conducted the quantum yield measurement of the photoinduced reaction by an optical power meter. The result was calculated based on the product yield and the photon flux of the light source ${ }^{4,5}$.
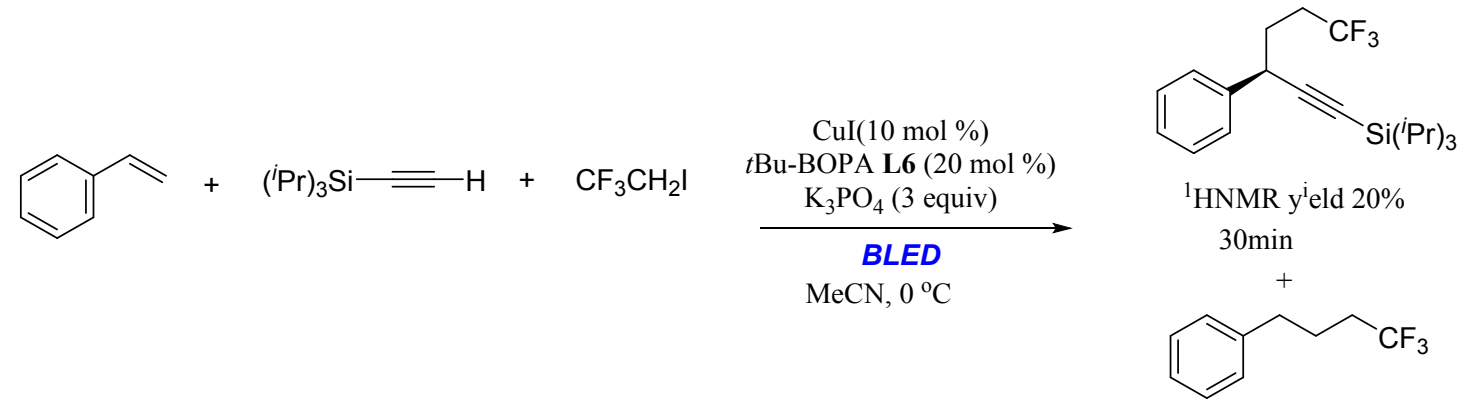

${ }^{1}$ HNMR yield $23 \%$ $30 \mathrm{~min}$

Experimental procedure 
Under nitrogen atmosphere, $\mathbf{L 6}(0.02 \mathrm{mmol}, 20 \mathrm{~mol} \%)$, $\mathrm{CuI}(0.01 \mathrm{mmol}, 10 \mathrm{~mol} \%), \mathrm{K}_{3} \mathrm{PO}_{4}(0.3$ mmol, 3.0 equiv), triisopropylsilylacetylene ( $36.4 \mathrm{mg}, 0.2 \mathrm{mmol}, 1.0$ equiv), styrene ( $41.6 \mathrm{mg}, 0.4$ mmol, 2.0 equiv) and 1-trifluoro-2-iodoethane $(251.9 \mathrm{mg}, 1.2 \mathrm{mmol}, 6.0$ equiv) were added in a dried reaction vessel with $2 \mathrm{~mL} \mathrm{MeCN}$, and the reaction mixture was stirred under Blue LED for $30 \mathrm{~min}$. After completion, the reaction was quenched and the suspension was filtered through a pad of silica gel, rinsed with $\mathrm{CH}_{2} \mathrm{Cl}_{2}$. Diethyl phthalate was internal standard for ${ }^{1} \mathrm{H}$ NMR.

The photon flux of the light source was determined by an optical power meter to be $528.0 \mathrm{~mW}$ (average of three experiments).

Photon flux $=\frac{P}{N_{A} \cdot h c / \lambda}=\frac{528.0 \times 10^{-3}}{6.02 \times 10^{23} \times 6.63 \times 10^{-34} \times 3 \times 10^{8} /\left(400 \times 10^{-9}\right)}=1.8 \times 10^{-6}$ einstein $\cdot \mathrm{s}^{-1}$

Thus, the overall quantum yield can be calculated by the equation (2). $\mathrm{f}$ is the fraction of light ( $\mathrm{f}>0.999$ ), and it is assumed that the light was absorbed by the copper acetylide-ligand. $\mathrm{T}$ is the reaction time $(30 \times 60 \mathrm{~s})$. We calculate a quantum yield of $\Phi=0.6 \%$.

$\Phi=\frac{\text { mol product }}{p \text { hoton flux.t.f }}=\frac{2.0 \times 10^{-5}}{1.8 \times 10^{-6} \times 30 \times 60}=0.6 \%$.

\section{Stern-Volmer Experiment}

To investigate the copper acetylide, copper-ligand or copper acetylide-ligand interacts with haloalkane, luminescence quenching experiments were performed ${ }^{4}$.

\section{Preparation of the sample}

Ligand, $\mathrm{CuI}$ and $\mathrm{K}_{3} \mathrm{PO}_{4}$ in MeCN: $\mathbf{L 6}$ (0.02 mmol, $20 \mathrm{~mol} \%$ ), $\mathrm{CuI}(0.01 \mathrm{mmol}, 10 \mathrm{~mol} \%), \mathrm{K}_{3} \mathrm{PO}_{4}$ $(0.3 \mathrm{mmol}, 3.0$ equiv) were added in a dried reaction vessel with $2 \mathrm{~mL} \mathrm{MeCN}$ and the reaction mixture was stirred at room temperature. After turned yellow, $10 \mu 1$ of the solution diluted with $2 \mathrm{~mL}$ $\mathrm{MeCN}$ was added 1-trifluoro-2-iodoethane (0.15-1.5 mM). Sterm-Volmer plot for the emission quenching of in situ generated $\mathrm{Cu}$-Ligand $\left(0.5 \times 10^{-3} \mathrm{mM}\right)$ is given in Figure $\mathrm{S} 8$

Triisopropylsilylacetylene, $\mathrm{CuI}$ and $\mathrm{K}_{3} \mathrm{PO}_{4}$ in $\mathrm{MeCN}$ : $\mathrm{CuI}(0.01 \mathrm{mmol}, 10 \mathrm{~mol} \%), \mathrm{K}_{3} \mathrm{PO}_{4}(0.3 \mathrm{mmol}$, 3.0 equiv), triisopropylsilylacetylene ( $18.2 \mathrm{mg}, 0.1 \mathrm{mmol}, 1.0$ equiv), were added in a dried reaction vessel with $2 \mathrm{~mL} \mathrm{MeCN}$ After turned yellow, $10 \mu \mathrm{l}$ of the solution diluted with $2 \mathrm{~mL} \mathrm{MeCN}$ was added 1-trifluoro-2-iodoethane(0.15-1.5 mM). Sterm-Volmer plot for the emission quenching of in situ generated copper acetylide $\left(0.5 \times 10^{-3} \mathrm{mM}\right)$ is given in Figure $\mathrm{S} 9$

Triisopropylsilylacetylene, CuI , Ligand and $\mathrm{K}_{3} \mathrm{PO}_{4}$ in MeCN: L6 (0.02 mmol, $\left.20 \mathrm{~mol} \%\right)$, CuI (0.01 mmol, $10 \mathrm{~mol} \%), \mathrm{K}_{3} \mathrm{PO}_{4}$ (0.3 mmol, 3.0 equiv), triisopropylsilylacetylene (18.2 mg, $0.1 \mathrm{mmol}, 1.0$ equiv), were added in a dried reaction vessel with $2 \mathrm{~mL} \mathrm{MeCN}$ and the reaction mixture was stirred 30min. After turned yellow, the reaction was added 1-trifluoro-2-iodoethane (0.02-0.3 M). StermVolmer plot for the emission quenching of in situ generated copper acetylide-ligand $(0.5 \mathrm{mM})$ is given in Figure S10 


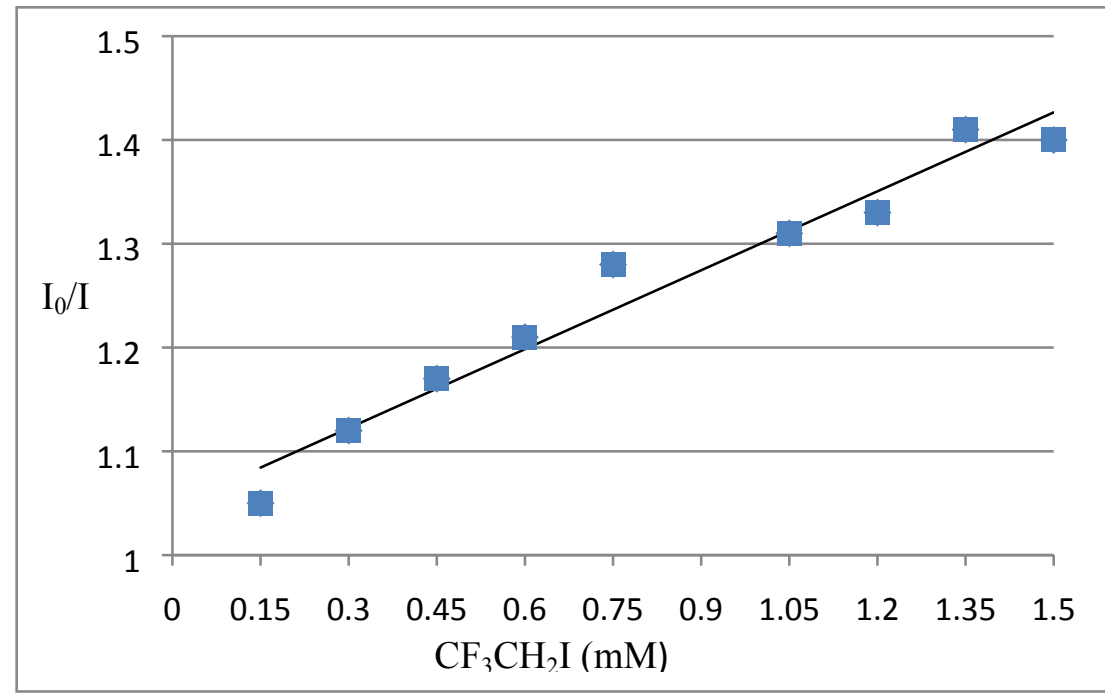

Figure S8 Stern-Volmer plots for opper acetylide. Stern-Volmer constant was $0.3 \mathrm{M}^{-1}$

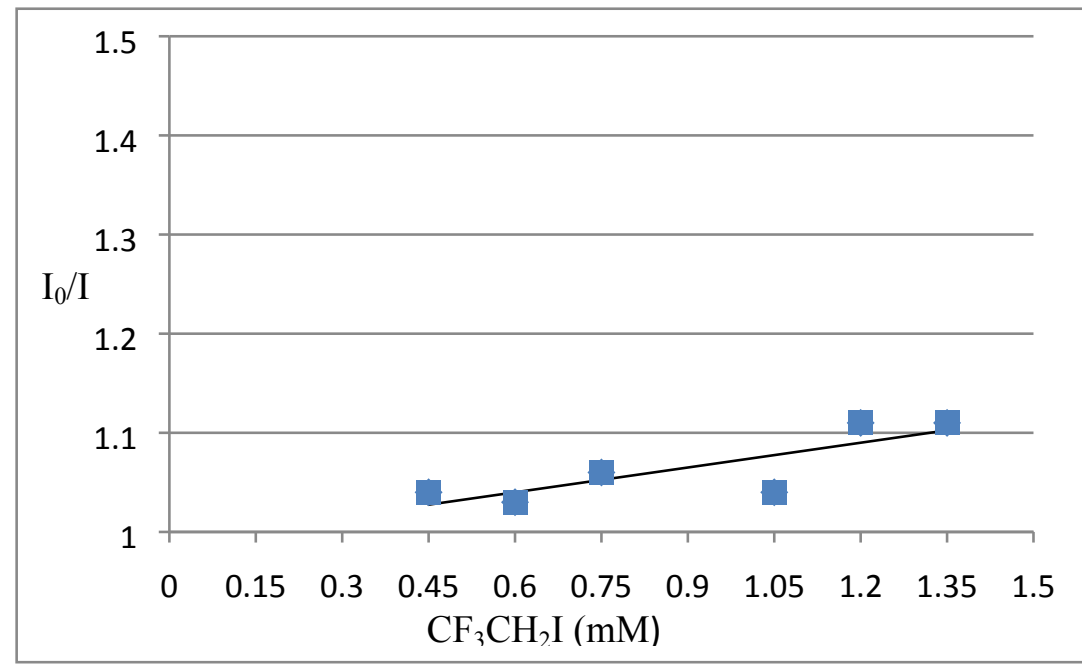

Figure S9 Stern-Volmer plots for opper-ligand. Stern-Volmer constant was $0.1 \mathrm{M}^{-1}$

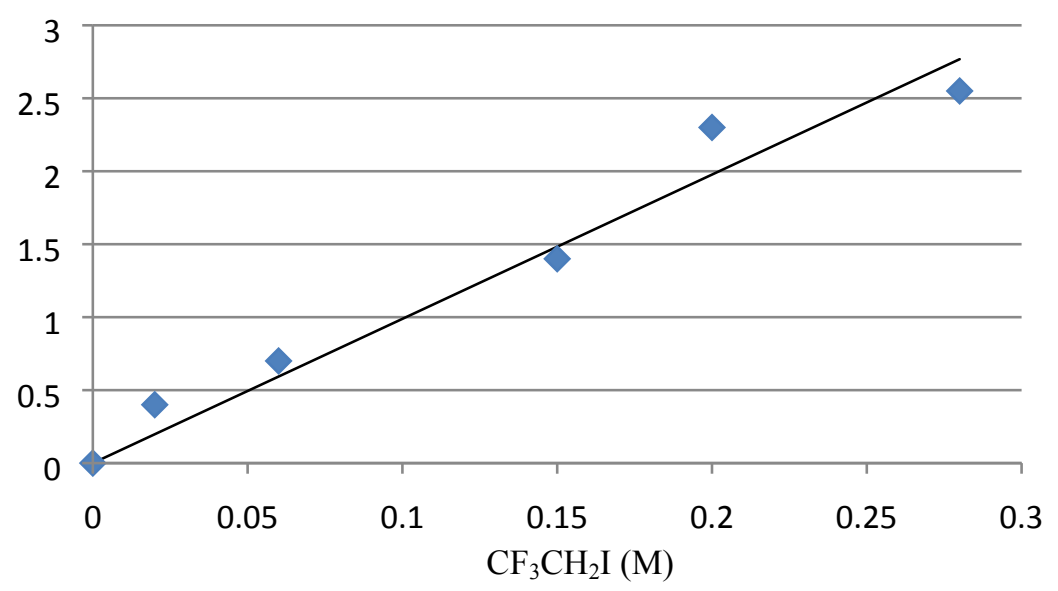

Figure S10 Stern-Volmer plots for copper acetylide-ligand. Stern-Volmer constant was $9.9 \mathrm{M}^{-1}$ Stern-Volmer plot for the transiently formed intermediate (copper-ligand, copper acetylide and 
copper acetylide-ligand) quenched by $\mathrm{CF}_{3} \mathrm{CH}_{2} \mathrm{I}$ were showed in Figure $\mathrm{S} 8, \mathrm{~S} 9$ and $\mathrm{S} 10$. Based on Stern-Volmer constants, copper acetylide and copper ligand was also quenched by the quencher. But compared the Stern-Volmer constants, it suggested that the excited-state copper acetylideligand quenched by the $\mathrm{CF}_{3} \mathrm{CH}_{2} \mathrm{I}$.

\section{References}

(1) Smith, S.W.; Fu, G.C.; Nickel-Catalyzed Asymmetric Cross-Couplings of Racemic Propargylic Halides with Arylzinc Reagents J. Am. Chem. Soc. 2008, 130, 12645-12647.

(2) Nian, N.Y; Hao, Z; Chong, H. X; Wei, D; Rui, W; Dong, M. P; Ze, B. Z; Jian, N. X Copper/Silver Cocatalyzed Oxidative Coupling of Vinylarenes with $\mathrm{ICH}_{2} \mathrm{CF}_{3}$ or $\mathrm{ICH}_{2} \mathrm{CHF}_{2}$ Leading to $\beta$ $\mathrm{CF}_{3} / \mathrm{CHF}_{2}$-Substituted Ketones .Org.Lett. 2016, 18, 1780-1783.

(3) Kévin. J.; Jérémy. H.; Gwilherm. E. Click-alkynlation of N- and P-nucleophiles by oxidative cross-coupling with alkynylcopper reagent: a general synthesis of ynamides and alkynylphosphonates Chem. Sci. 2012, 3, 756-760

(4) Li, Z; Lei, J. Visible-Light-Induced Organocatalytic Borylation of Aryl Chlorides $\quad J$. Am. Chem. Soc. 2019, 141, 9124-9128.

(5) Megan. A. C. ; Tehsik, P. Y. Characterizing chain processes in visible light photoredox catalysis Chem. Sci. 2015, 6, 5426. 
8 Copies of NMR Spectra and HPLC Chromatographs

(R)- (3-([1, 1'-biphenyl]-4-yl)-6, 6, 6-trifluorohex-1-yn-1-yl) triisopropylsilane (d1) d1 - ${ }^{1}$ H NMR

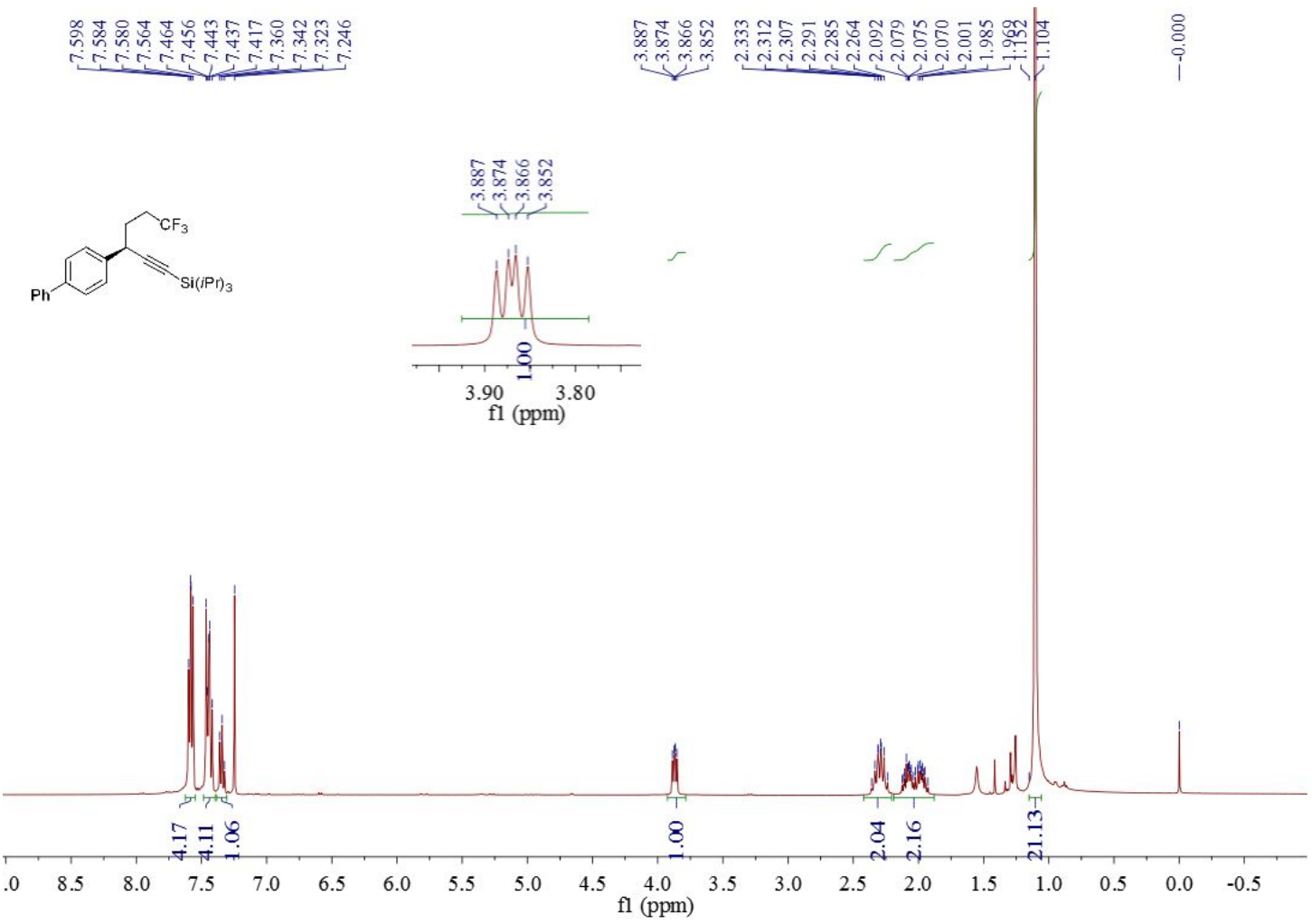

d1 $-{ }^{13}$ C NMR
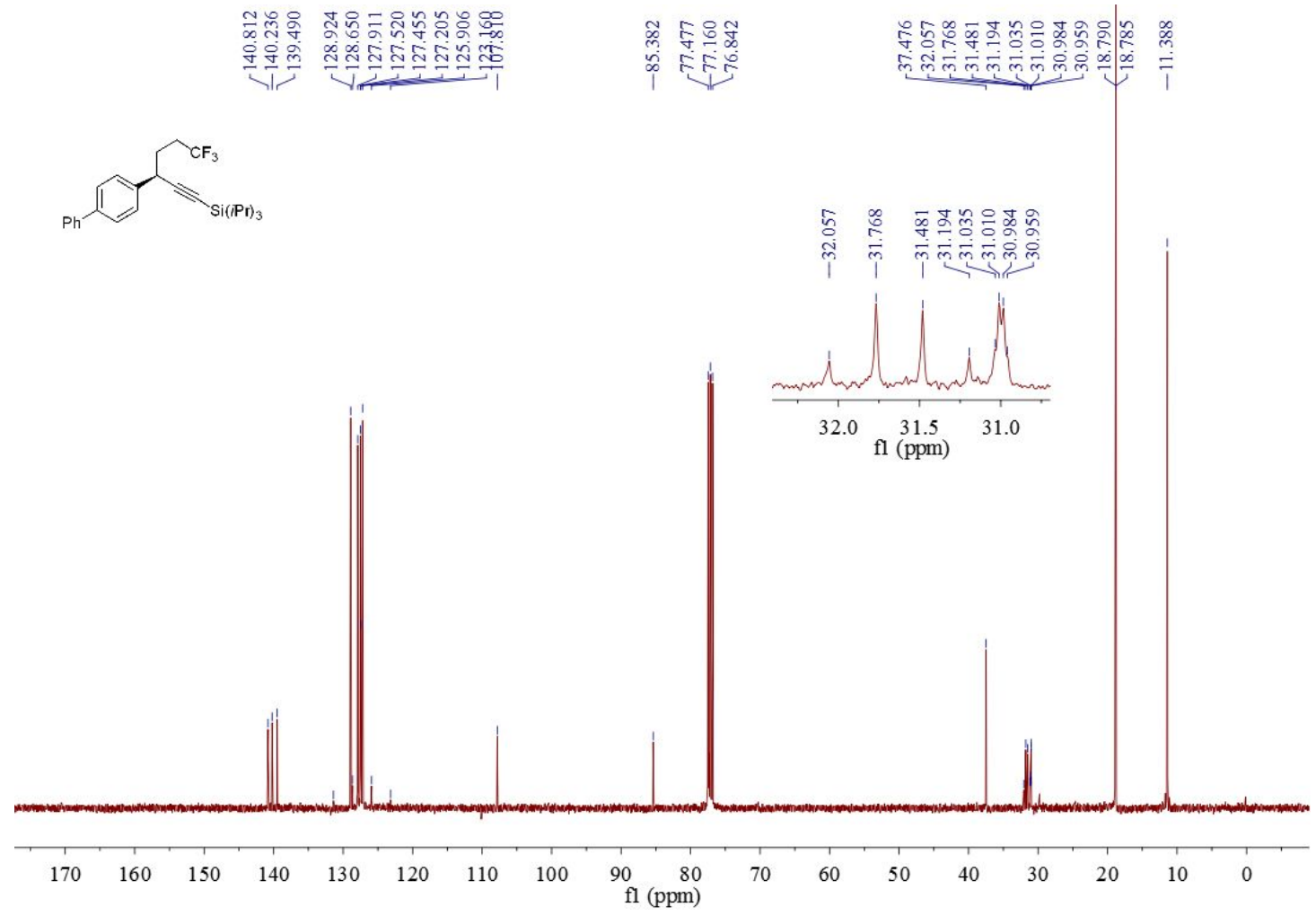


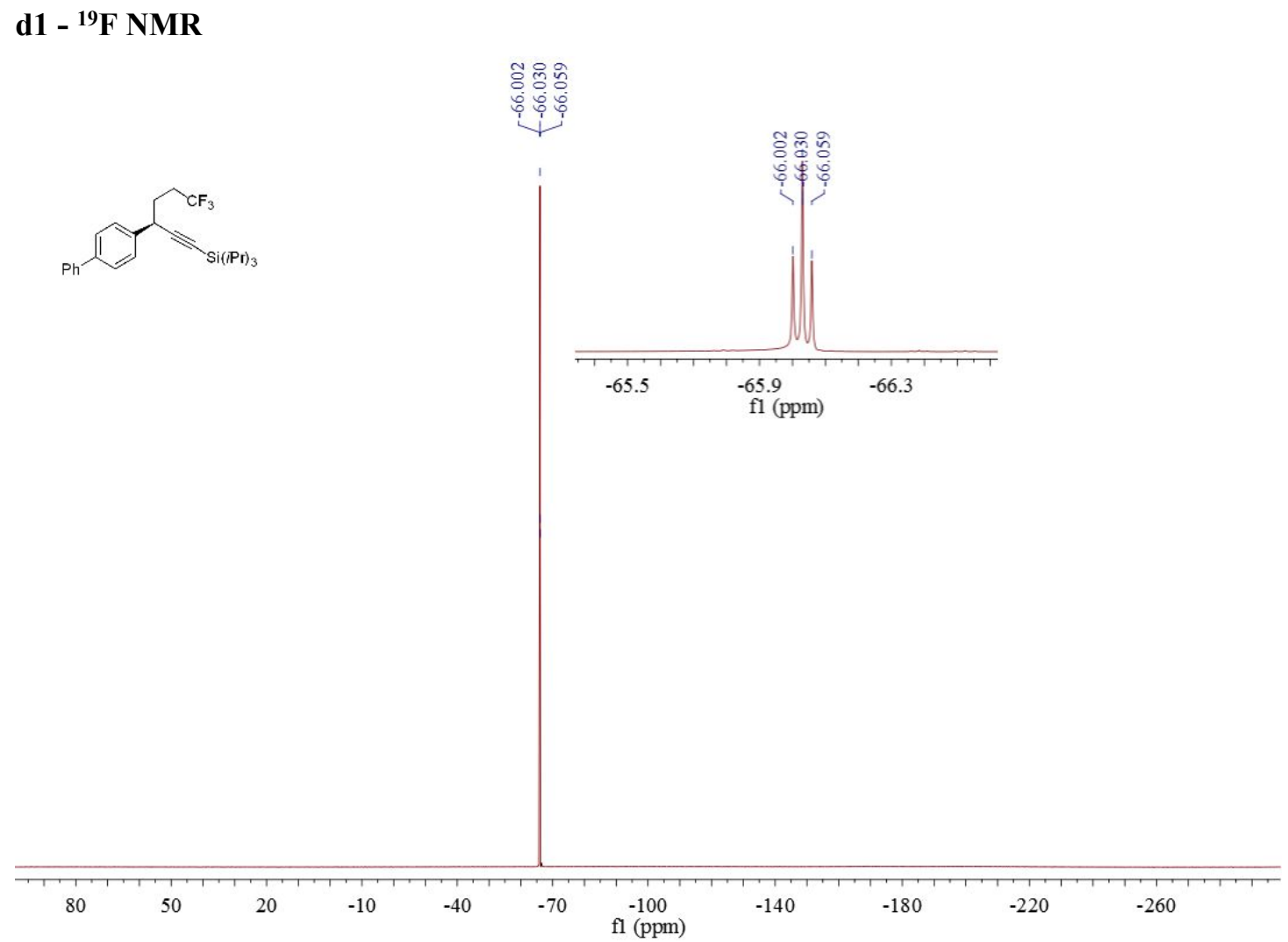

\section{d1 - HPLC}

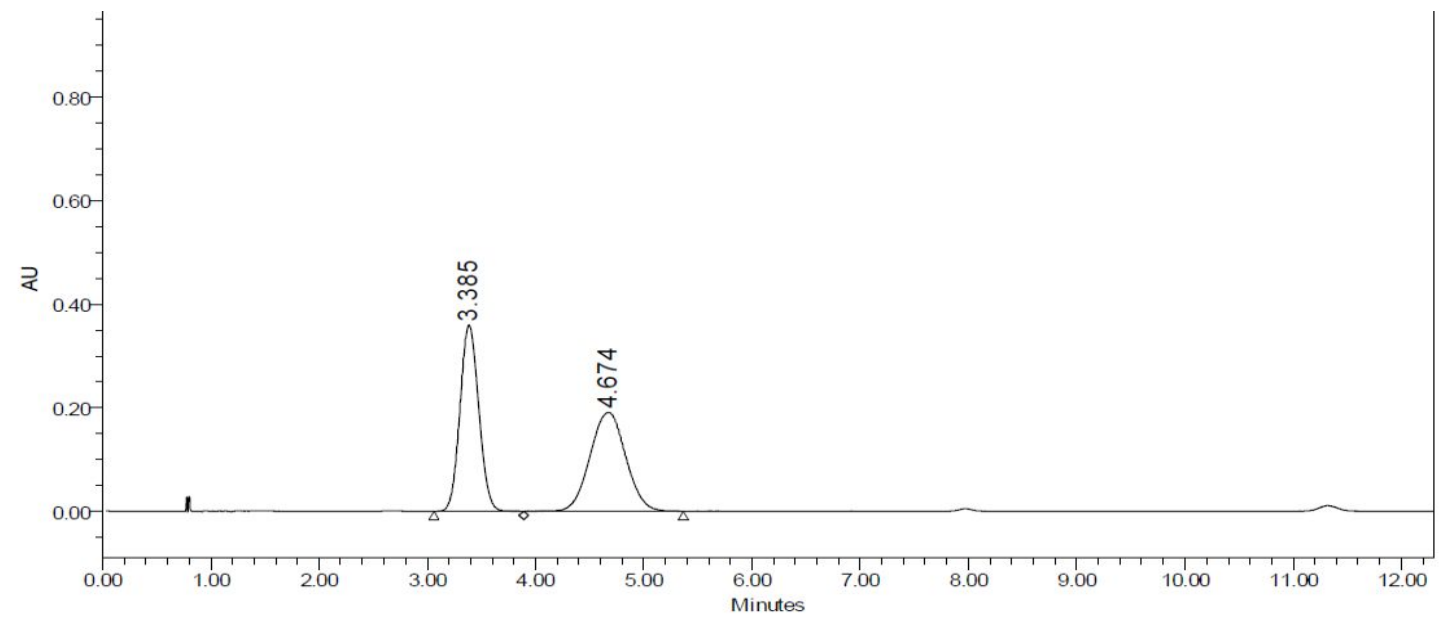

\begin{tabular}{|c|c|l|c|c|c|c|}
\hline & RT & Peak Type & Height & $\begin{array}{c}\text { Width } \\
(\mathrm{sec})\end{array}$ & Area & $\%$ Area \\
\hline 1 & 3.385 & Unknown & 358627 & 49.900 & 4339035 & 49.93 \\
\hline 2 & 4.674 & Unknown & 190471 & 88.350 & 4351831 & 50.07 \\
\hline
\end{tabular}




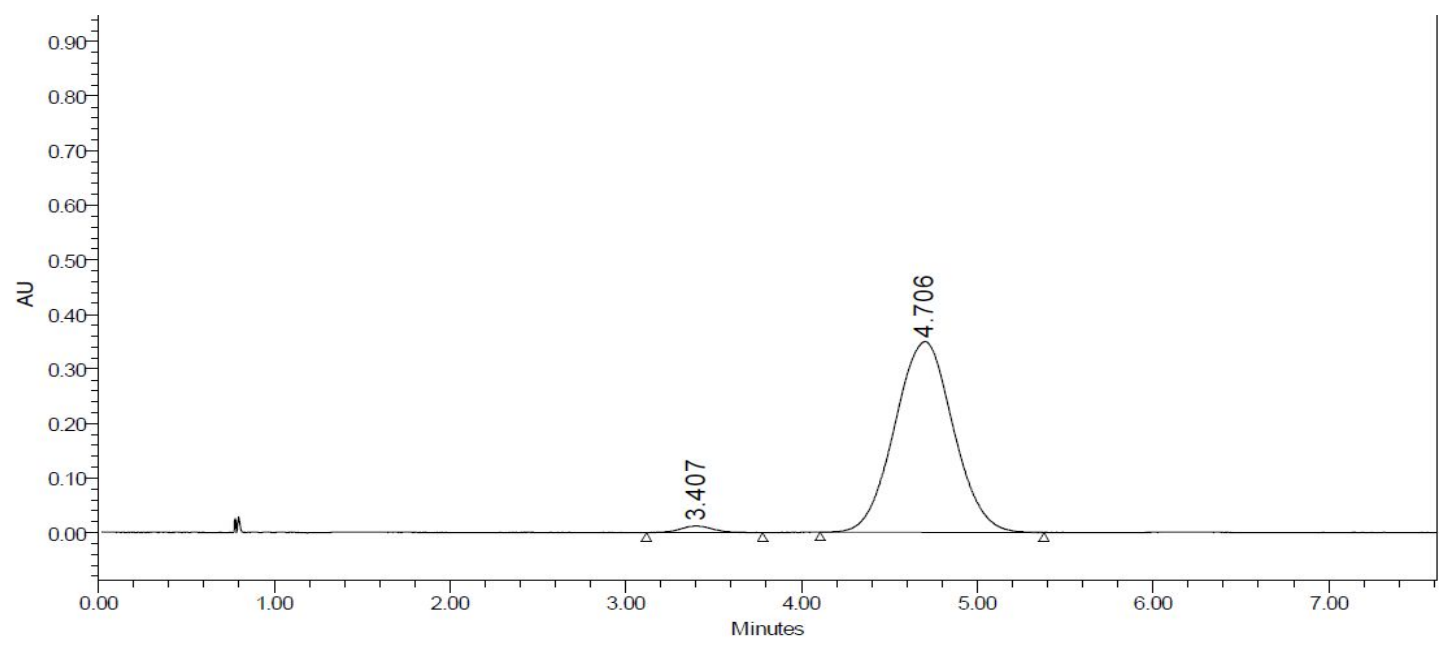

\begin{tabular}{|r|c|l|r|r|r|r|}
\hline & RT & Peak Type & Height & $\begin{array}{c}\text { Width } \\
(\mathrm{sec})\end{array}$ & \multicolumn{1}{|c|}{ Area } & \% Area \\
\hline 1 & 3.407 & Unknown & 12108 & 39.550 & 153151 & 1.85 \\
\hline 2 & 4.706 & Unknown & 349474 & 76.400 & 8109248 & 98.15 \\
\hline
\end{tabular}

(R)-triisopropyl (6, 6, 6-trifluoro-3-phenylhex-1-yn-1-yl) silane (d2) d2 - ${ }^{1}$ H NMR

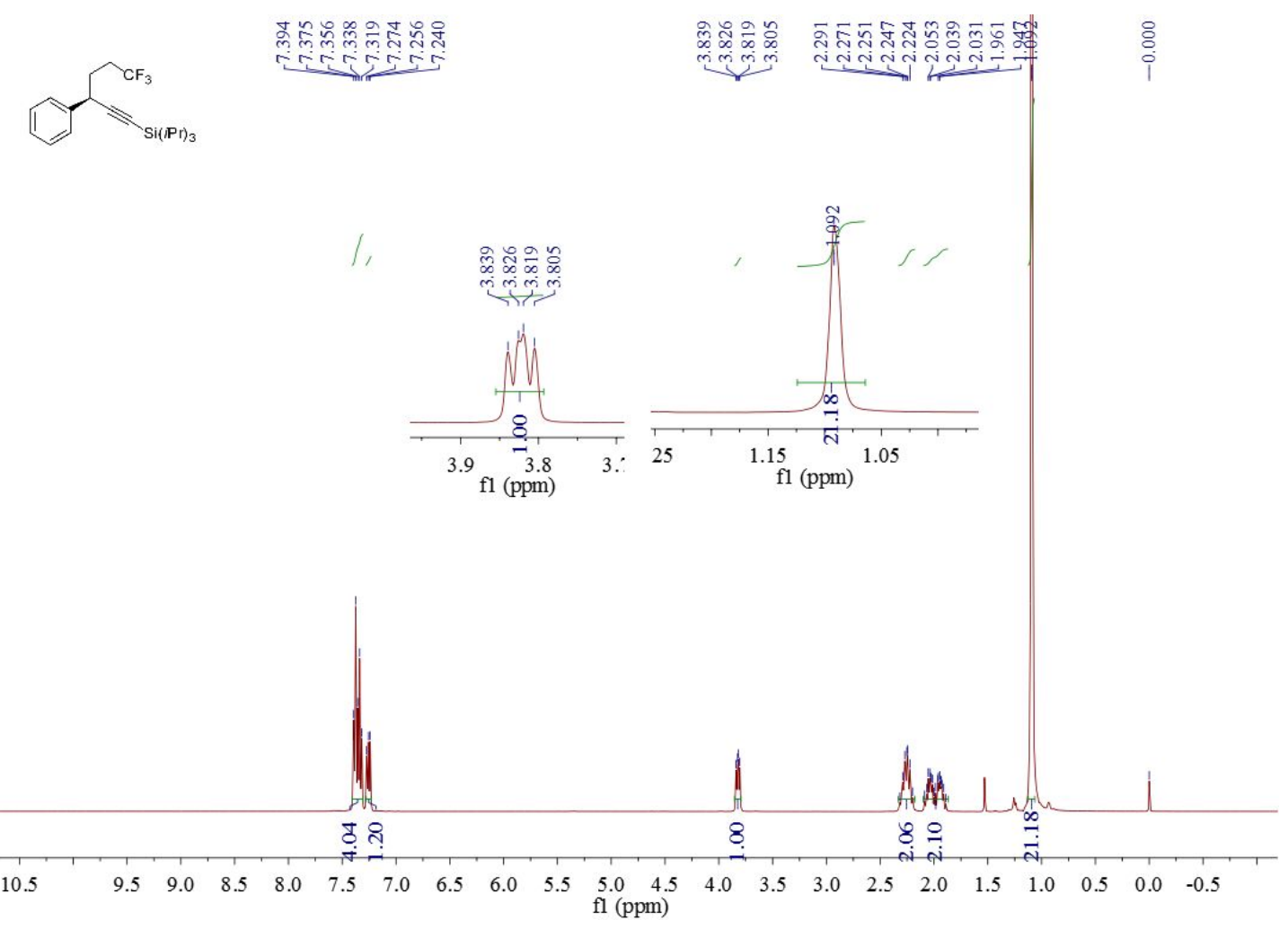


d2 $-{ }^{13}$ C NMR

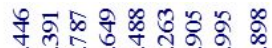

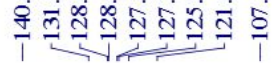

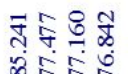

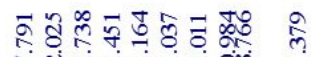

कहि

लेंलेलंलें

$\overbrace{\mathrm{SiPr}_{3}}^{\mathrm{CF}_{3}}$

ลิ

लेल लंल ले
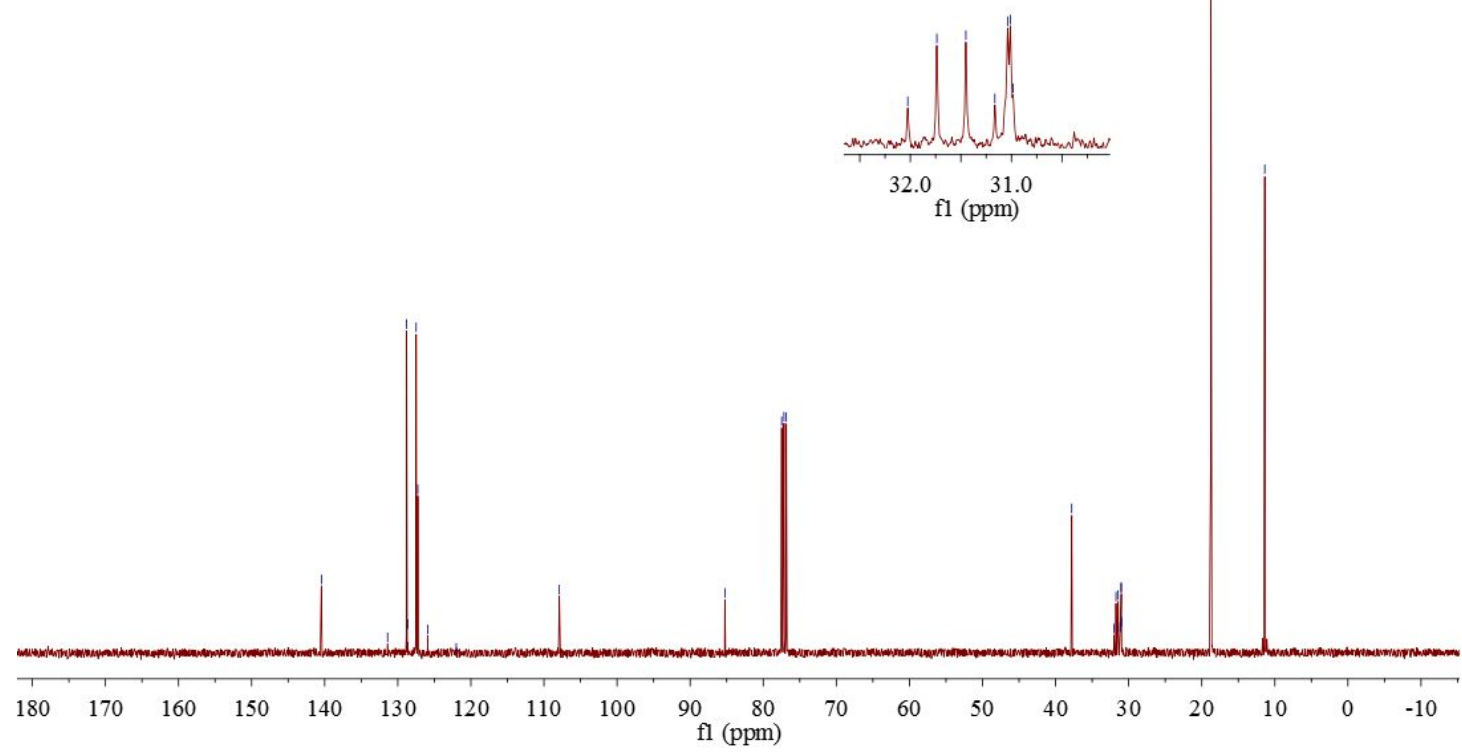

\section{d2 - ${ }^{19}$ F NMR}

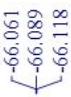

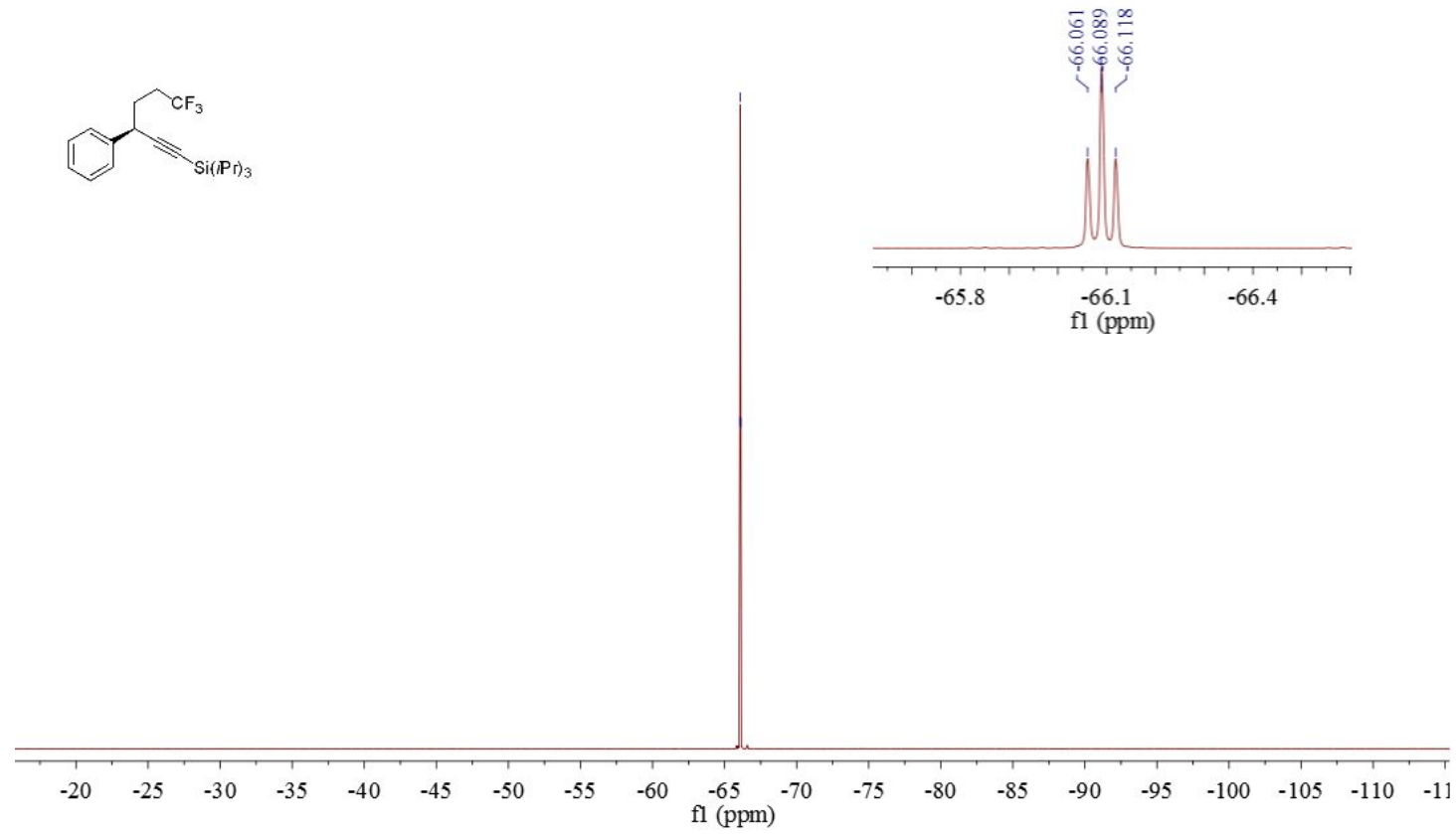




\section{d2 - HPLC}

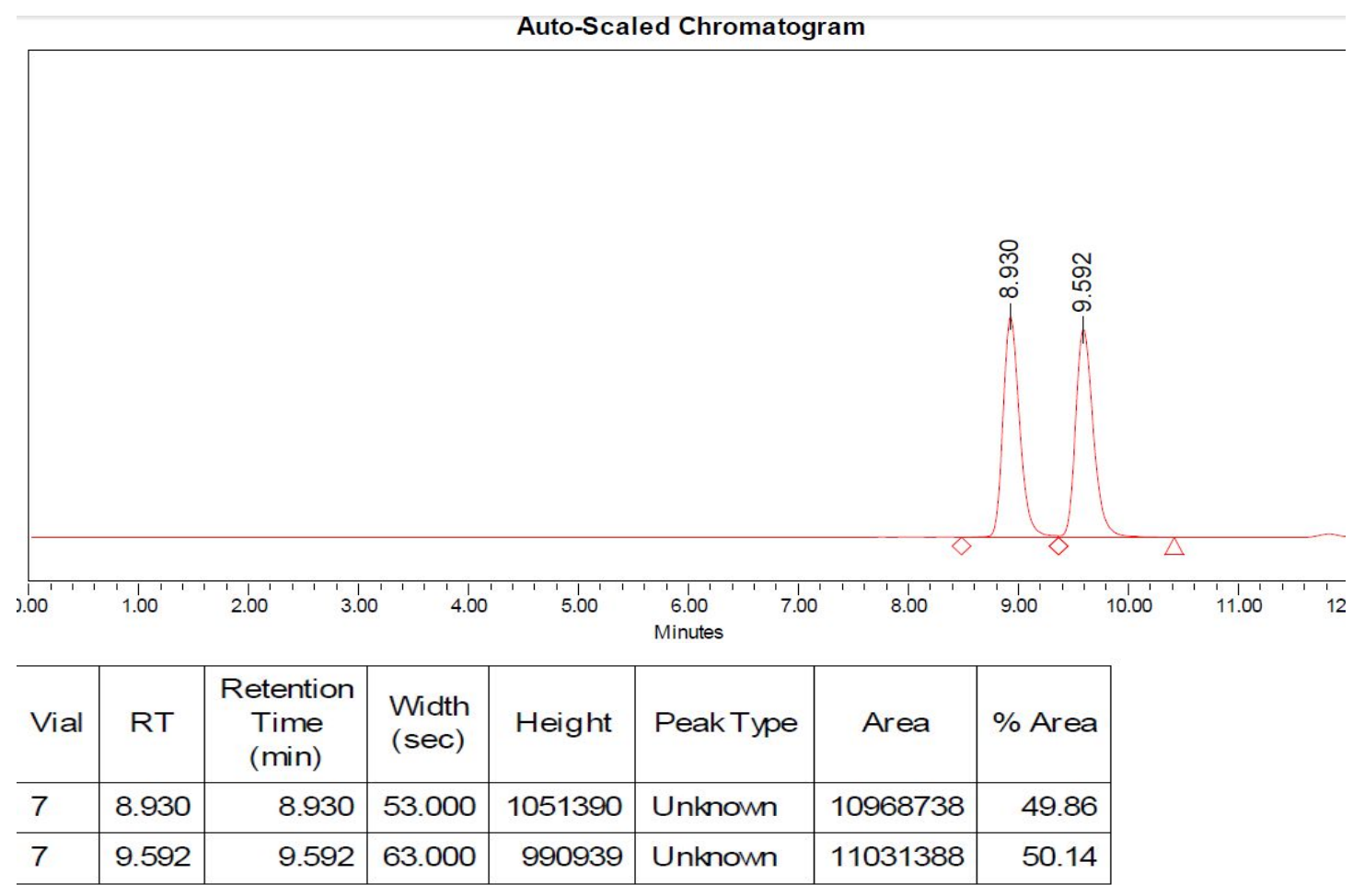

Auto-Scaled Chromatogram

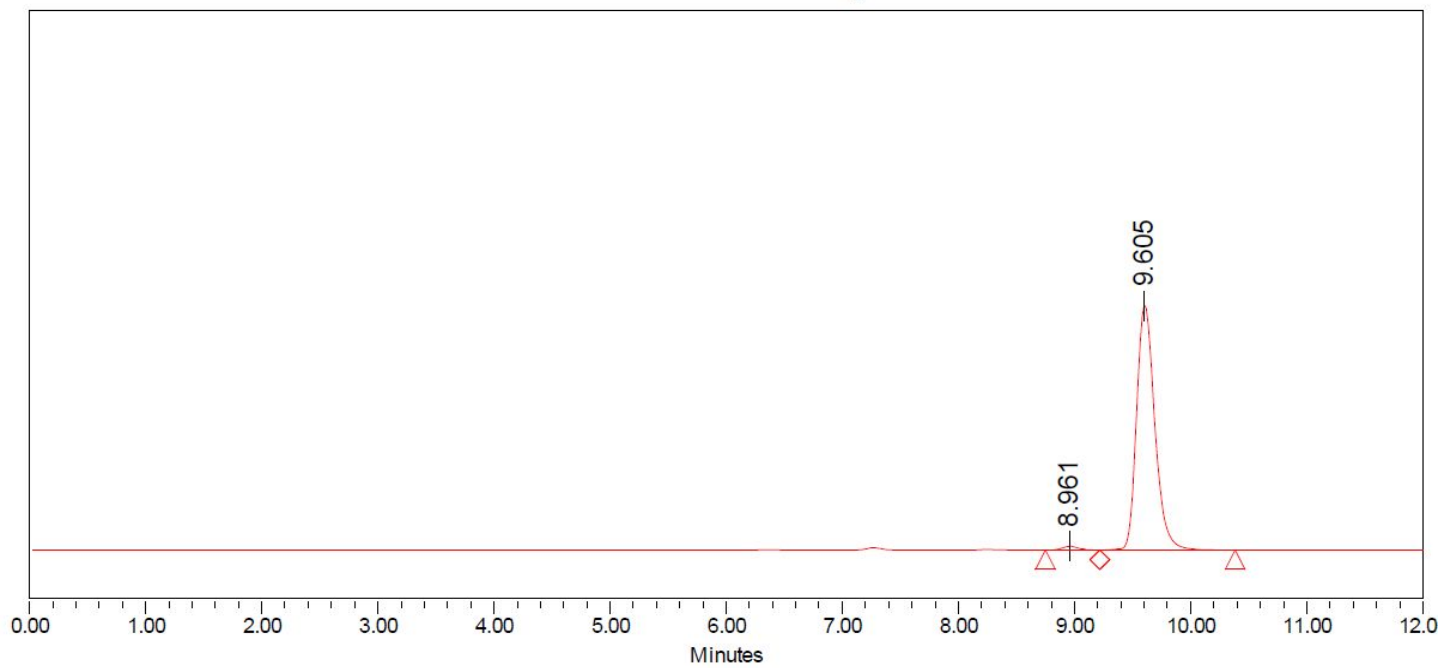

\begin{tabular}{l|r|r|r|r|r|r|r|}
\hline Vial & RT & $\begin{array}{c}\text { Retention } \\
\text { Time } \\
(\mathrm{min})\end{array}$ & $\begin{array}{l}\text { Width } \\
(\mathrm{sec})\end{array}$ & Height & PeakType & Area & \% Area \\
\hline 26 & 8.961 & 8.961 & 28.000 & 14355 & Unknown & 141862 & 1.37 \\
\hline 26 & 9.605 & 9.605 & 70.000 & 938273 & Unknown & 10177560 & 98.63 \\
\hline
\end{tabular}


(R)- (3-(4-chlorophenyl)-6, 6, 6-trifluorohex-1-yn-1-yl) triisopropylsilane (d3) d3 - ${ }^{1}$ H NMR

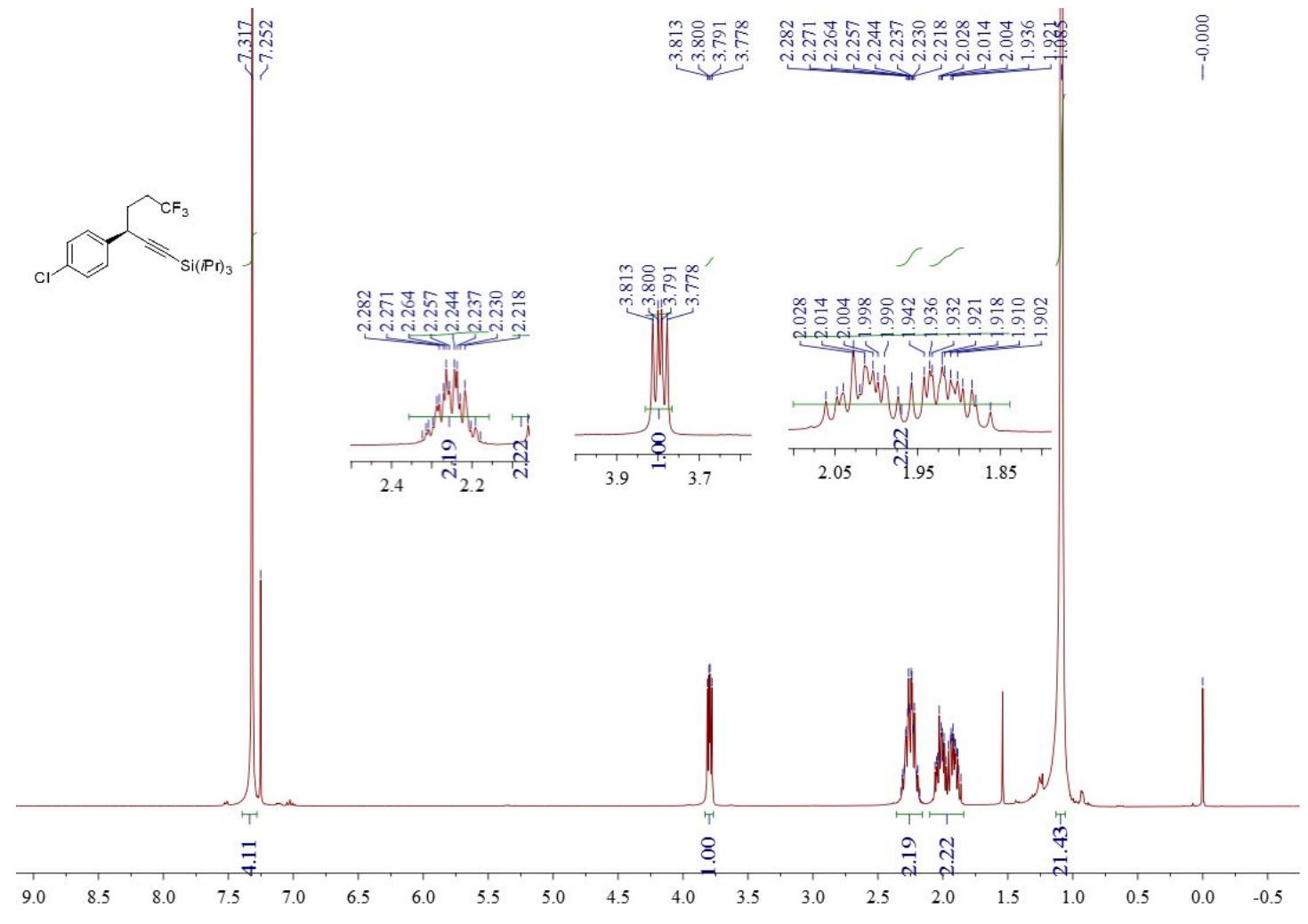

d3 $-{ }^{13}$ C NMR

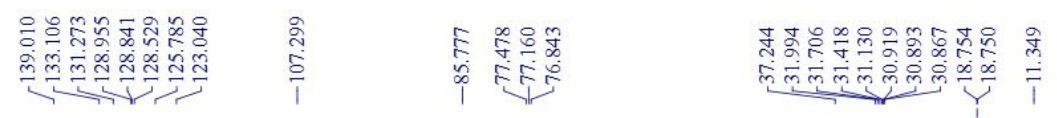
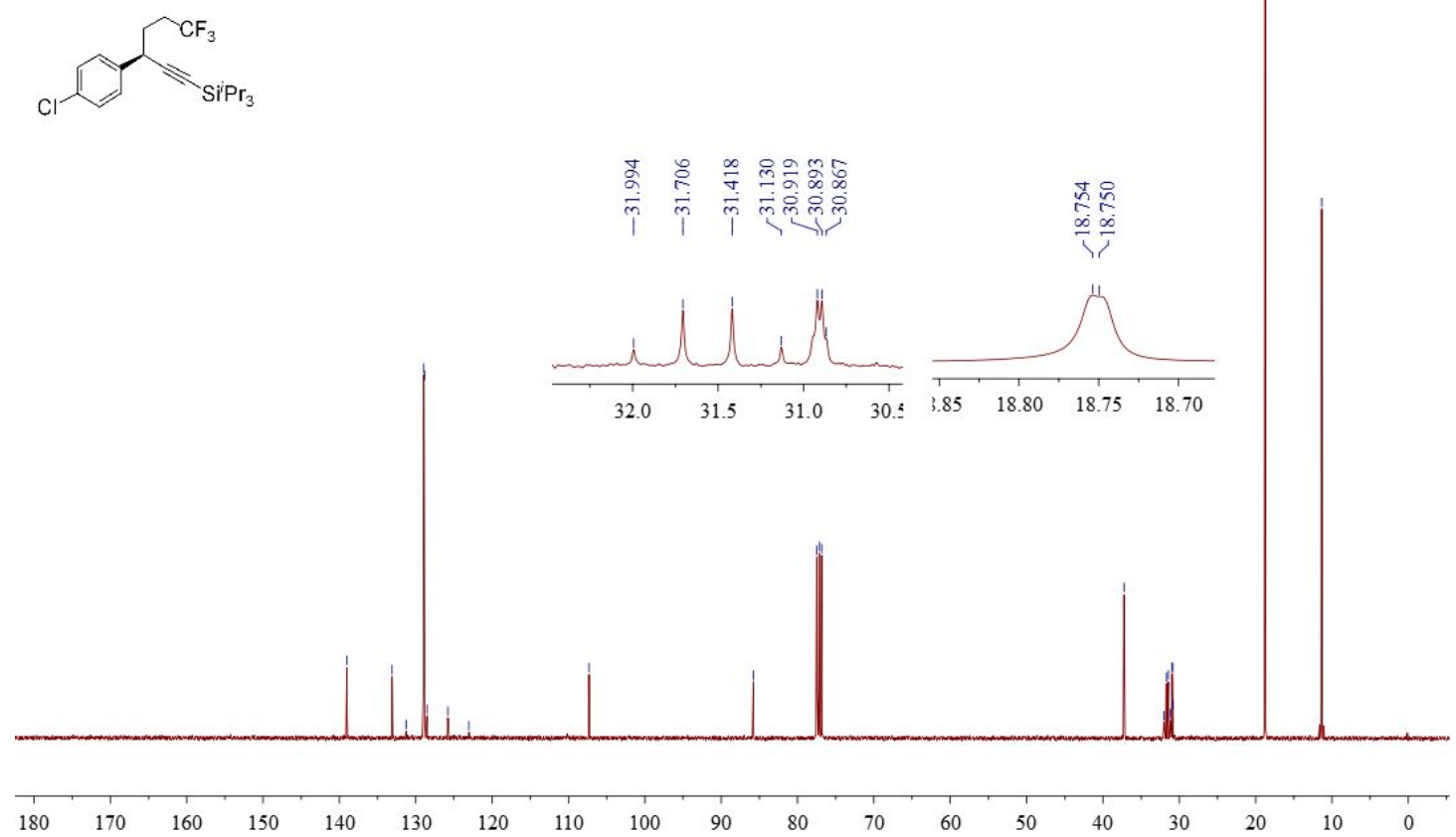
d3 - ${ }^{19}$ F NMR

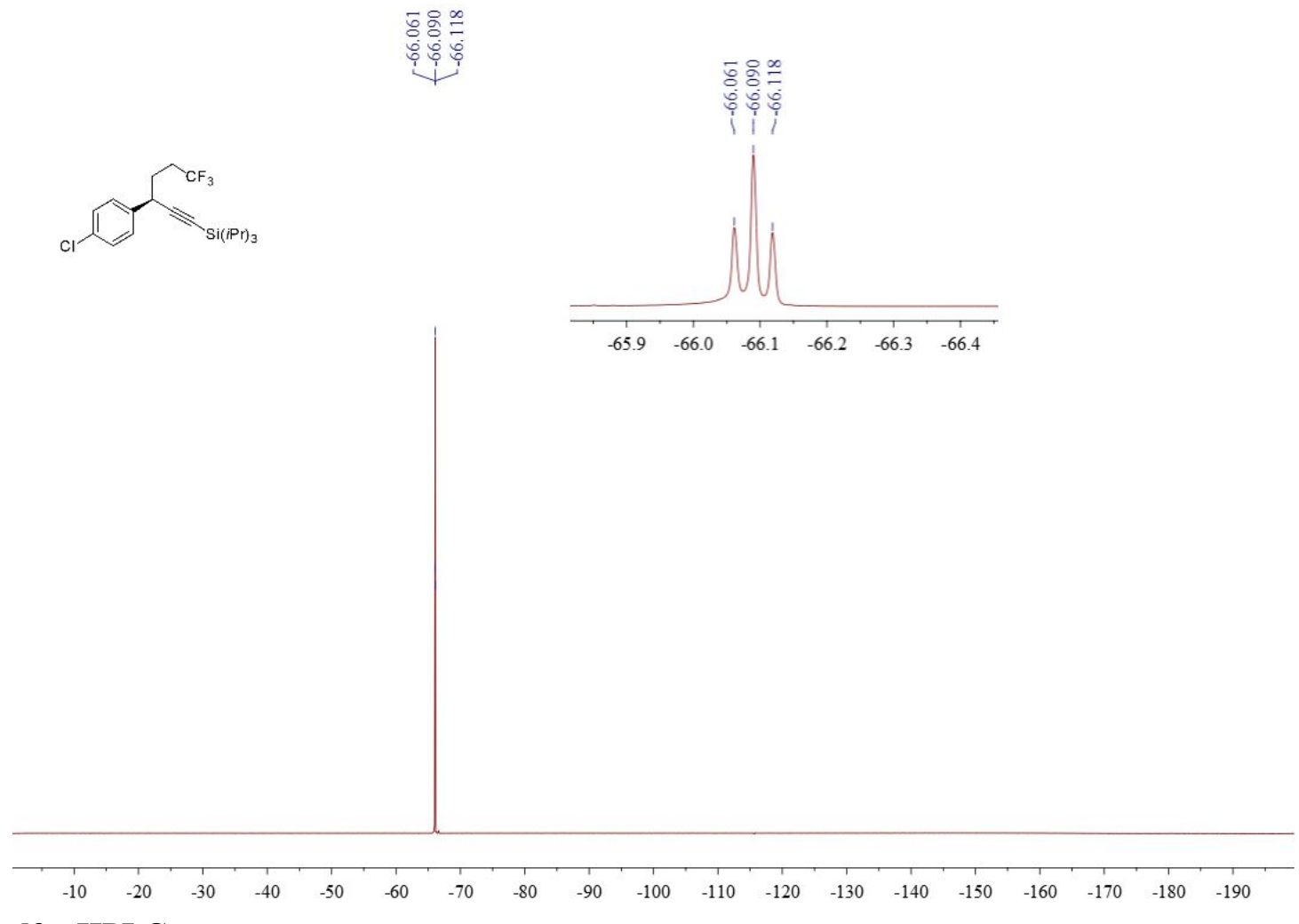

d3 - HPLC

Auto-Scaled Chromatogram

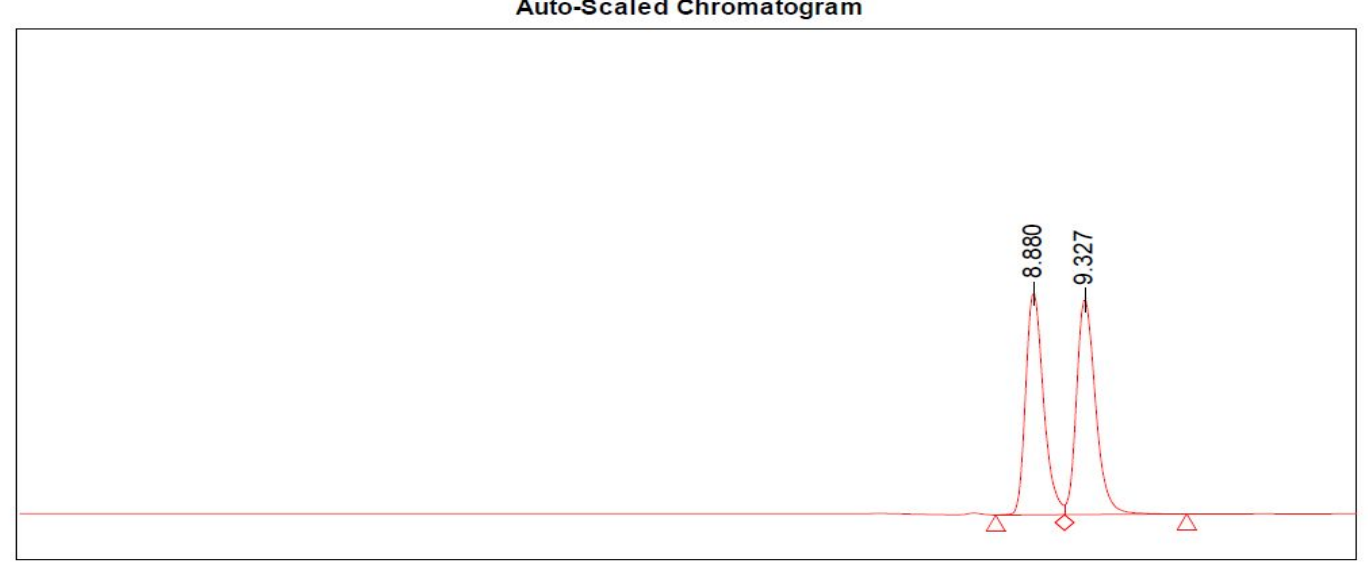

\begin{tabular}{|l|c|r|r|r|l|l|r|}
\hline Vial & RT & $\begin{array}{c}\text { Retention } \\
\text { Time } \\
(\mathrm{min})\end{array}$ & $\begin{array}{l}\text { Width } \\
(\mathrm{sec})\end{array}$ & Height & PeakType & Area & \% Area \\
\hline 8 & 8.880 & 8.880 & 36.000 & 2651255 & Unknown & 28845137 & 49.30 \\
\hline 8 & 9.327 & 9.327 & 64.000 & 2572451 & Unknown & 29670145 & 50.70 \\
\hline
\end{tabular}




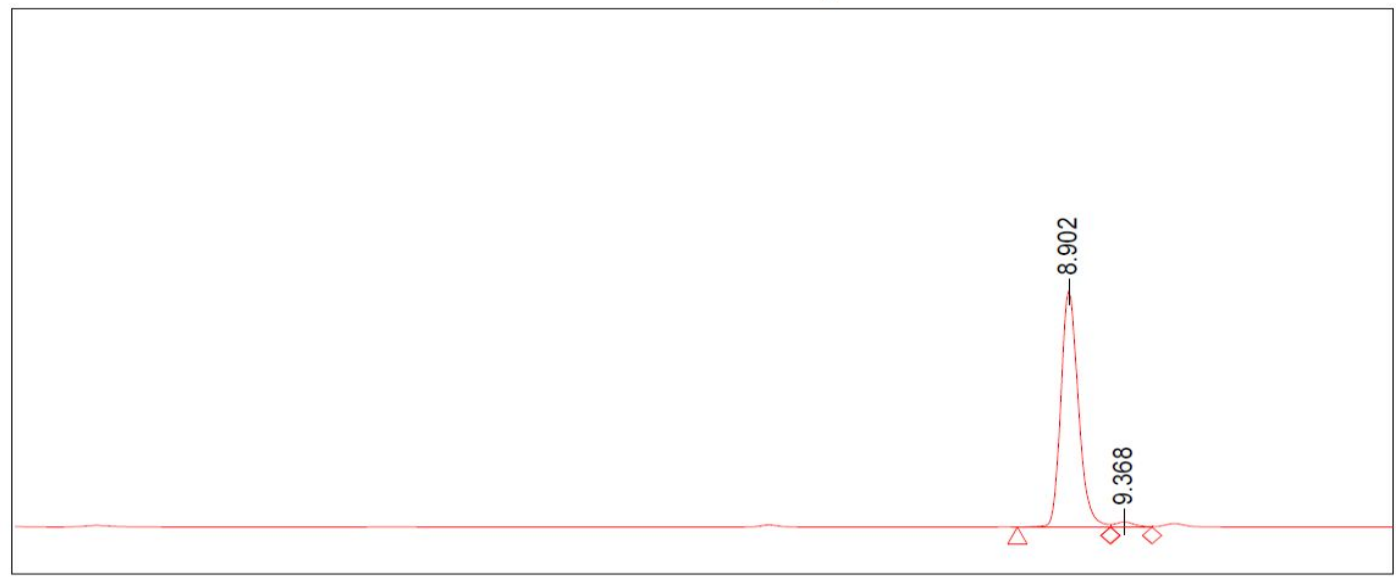

\begin{tabular}{l|r|r|r|r|l|r|r|}
\hline Vial & RT & $\begin{array}{c}\text { Retention } \\
\text { Time } \\
(\mathrm{min})\end{array}$ & $\begin{array}{l}\text { Width } \\
(\mathrm{sec})\end{array}$ & Height & PeakType & Area & \% Area \\
\hline 27 & 8.902 & 8.902 & 47.000 & 887059 & Unknown & 9035676 & 97.79 \\
\hline 27 & 9.368 & 9.368 & 21.000 & 18496 & Unknown & 204452 & 2.21 \\
\hline
\end{tabular}

(R)- (3-(4-bromophenyl)-6, 6, 6-trifluorohex-1-yn-1-yl) triisopropylsilane (d4) d4 - ${ }^{1}$ H NMR

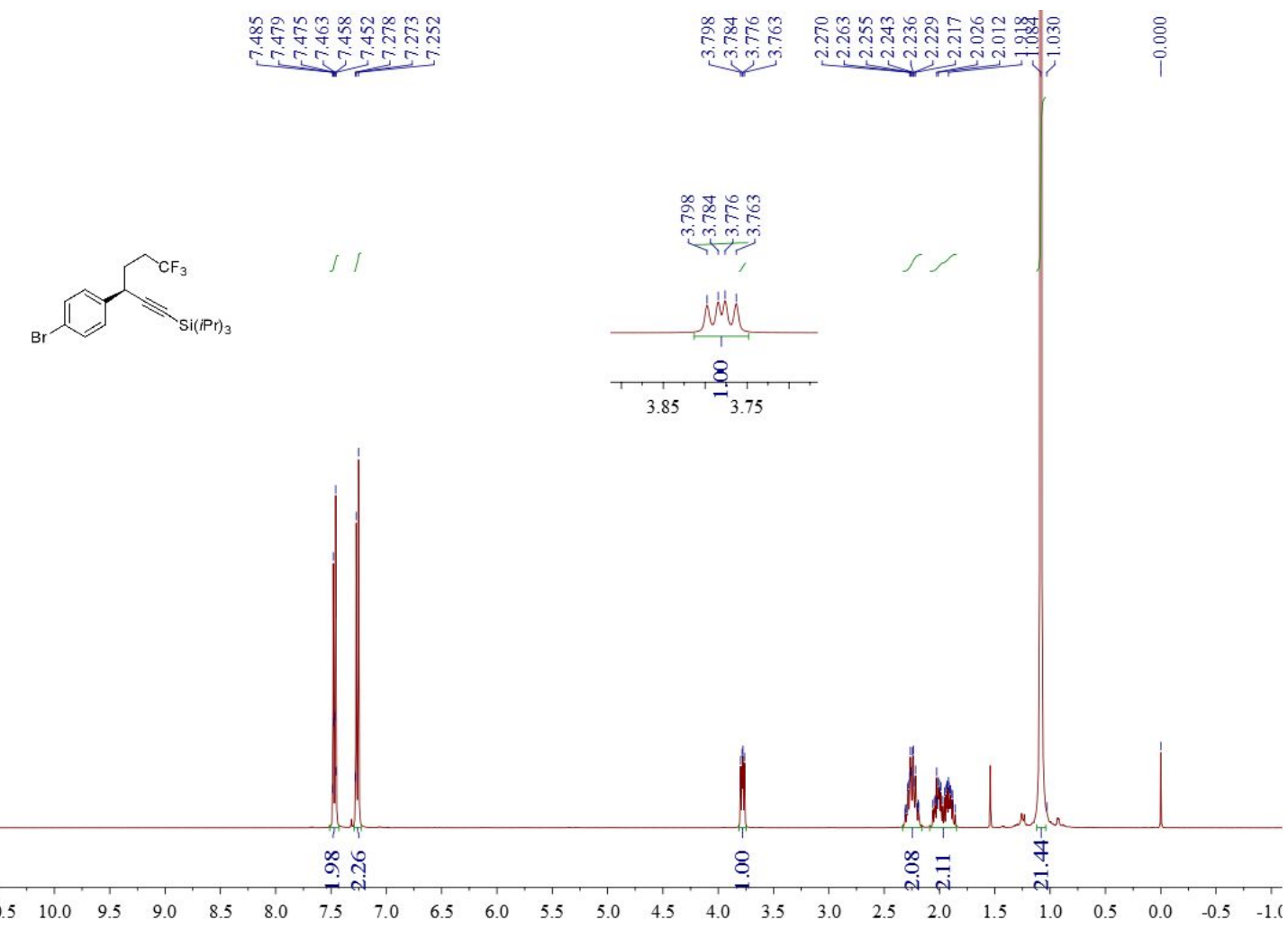


d4 - ${ }^{13}$ C NMR

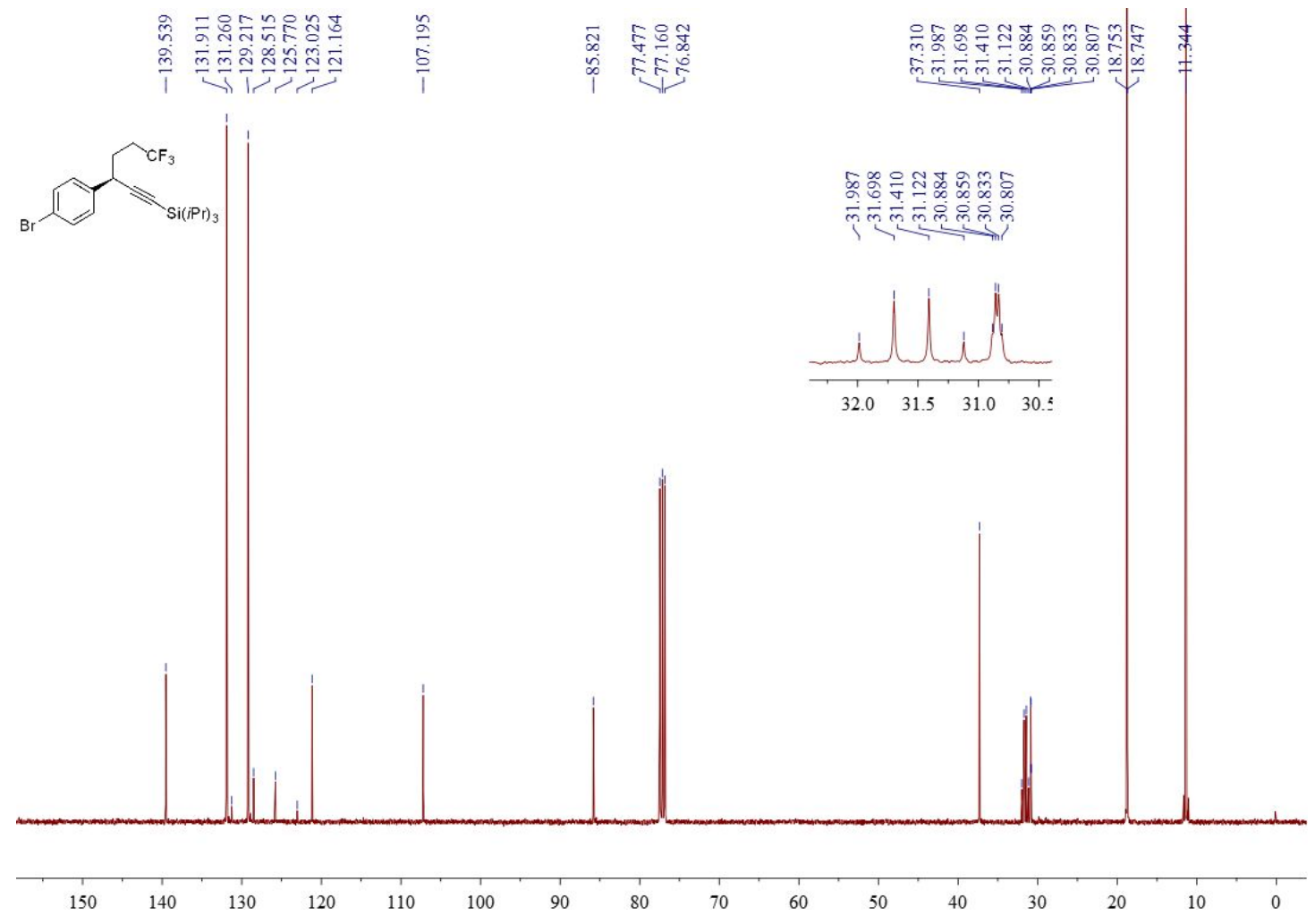

\section{d4 - ${ }^{19}$ F NMR}

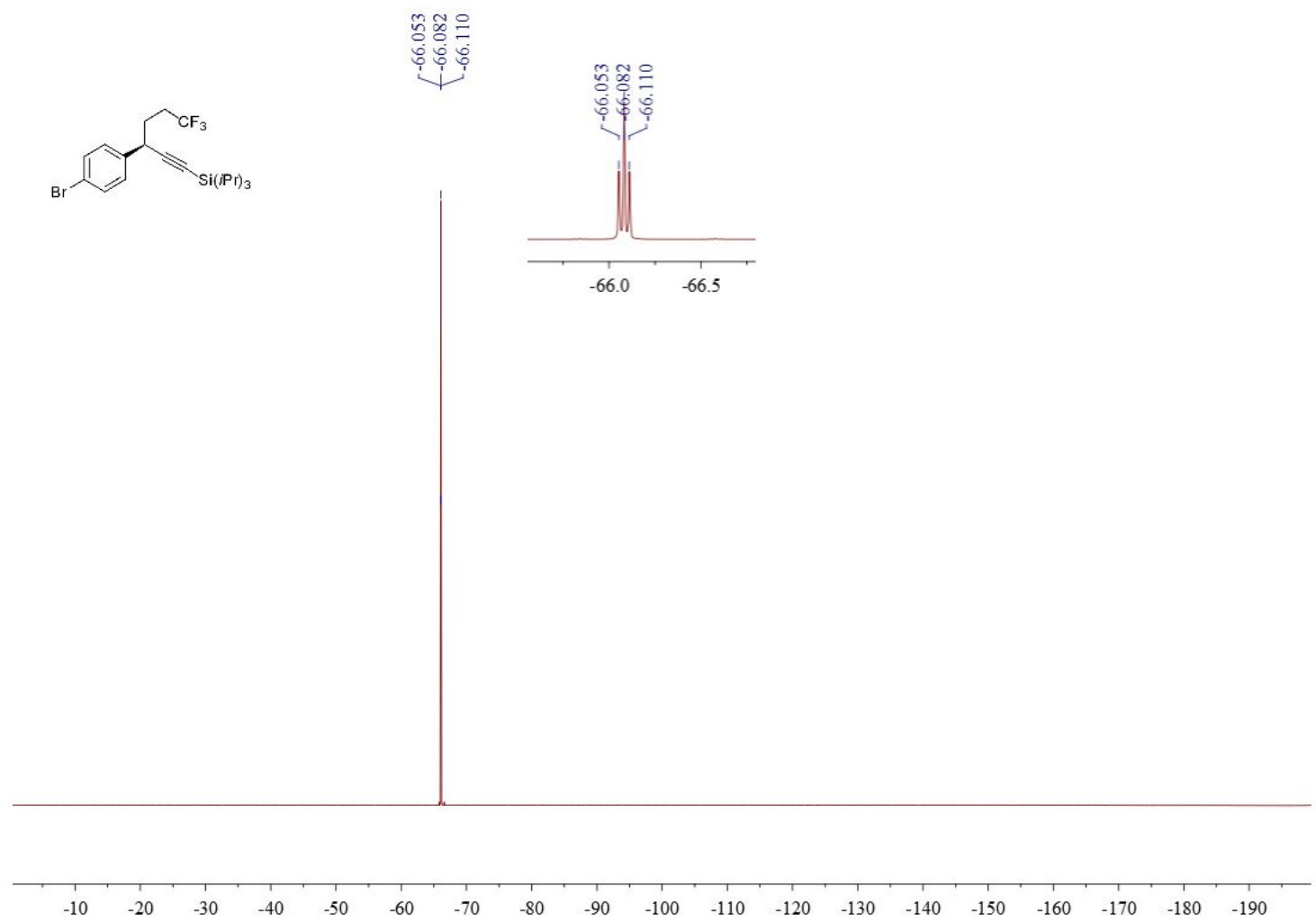




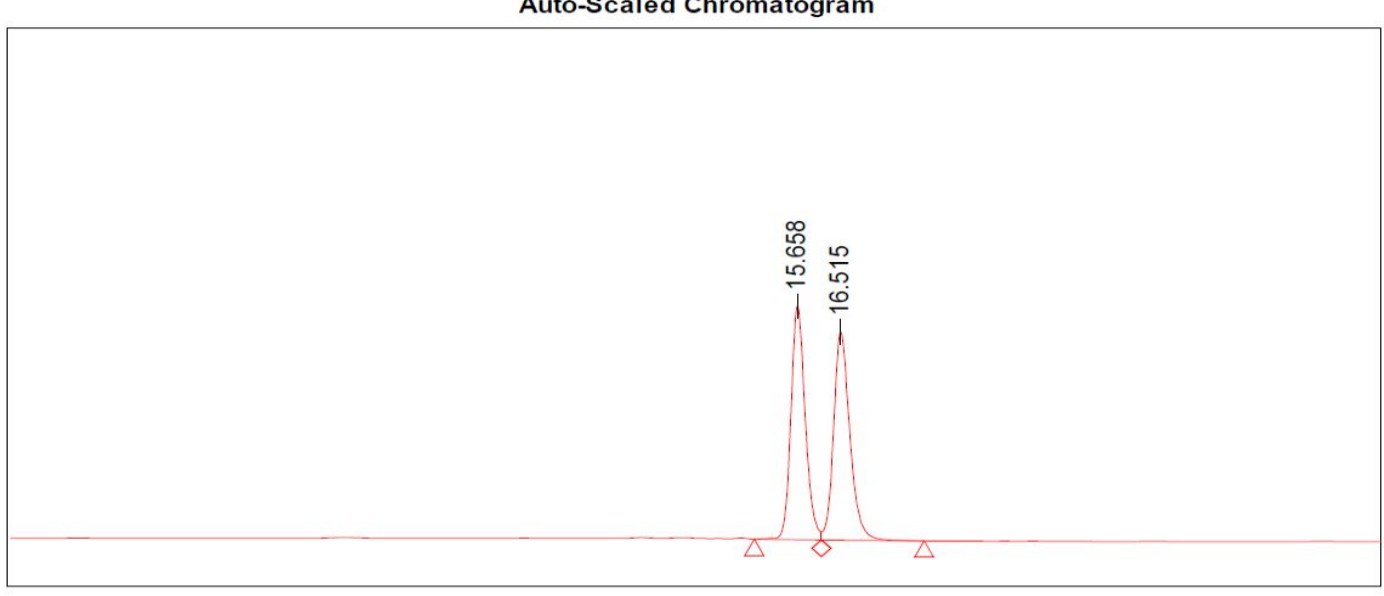

\begin{tabular}{|c|c|c|c|c|c|c|c|}
\hline Vial & RT & $\begin{array}{c}\text { Retention } \\
\text { Time } \\
(\mathrm{min})\end{array}$ & $\begin{array}{c}\text { Width } \\
(\mathrm{sec})\end{array}$ & Height & PeakType & Area & \% Area \\
\hline 10 & 15.658 & 15.658 & 80.000 & 1260182 & Unknown & 24779106 & 49.69 \\
\hline 10 & 16.515 & 16.515 & 122.000 & 1121692 & Unknown & 25089696 & 50.31 \\
\hline
\end{tabular}

Auto-Scaled Chromatogram

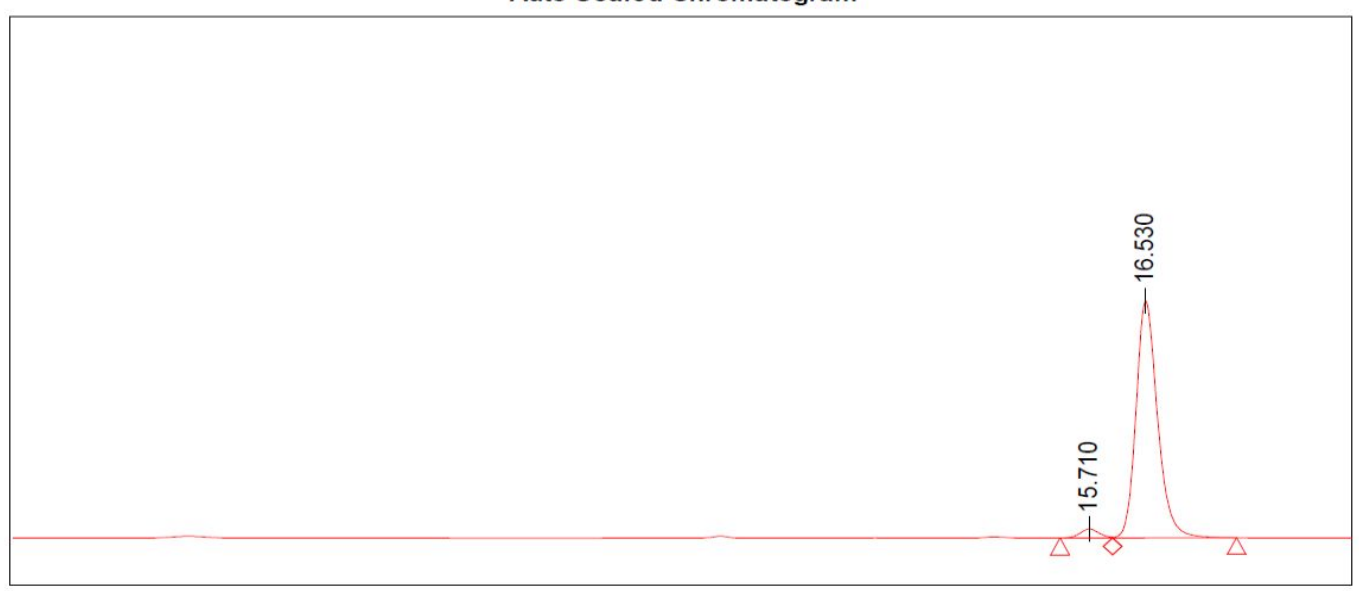

\begin{tabular}{|l|r|r|r|r|r|r|r|}
\hline Vial & RT & $\begin{array}{c}\text { Retention } \\
\text { Time } \\
(\mathrm{min})\end{array}$ & $\begin{array}{l}\text { Width } \\
(\mathrm{sec})\end{array}$ & Height & Peak Type & Area & \% Area \\
\hline 30 & 15.710 & 15.710 & 46.000 & 22877 & Unknown & 434675 & 3.10 \\
\hline 30 & 16.530 & 16.530 & 108.000 & 625986 & Unknown & 13576086 & 96.90 \\
\hline
\end{tabular}


(R)-triisopropyl (6, 6, 6-trifluoro-3-(p-tolyl) hex-1-yn-1-yl) silane (d5)

\section{d5 - ${ }^{1}$ H NMR}

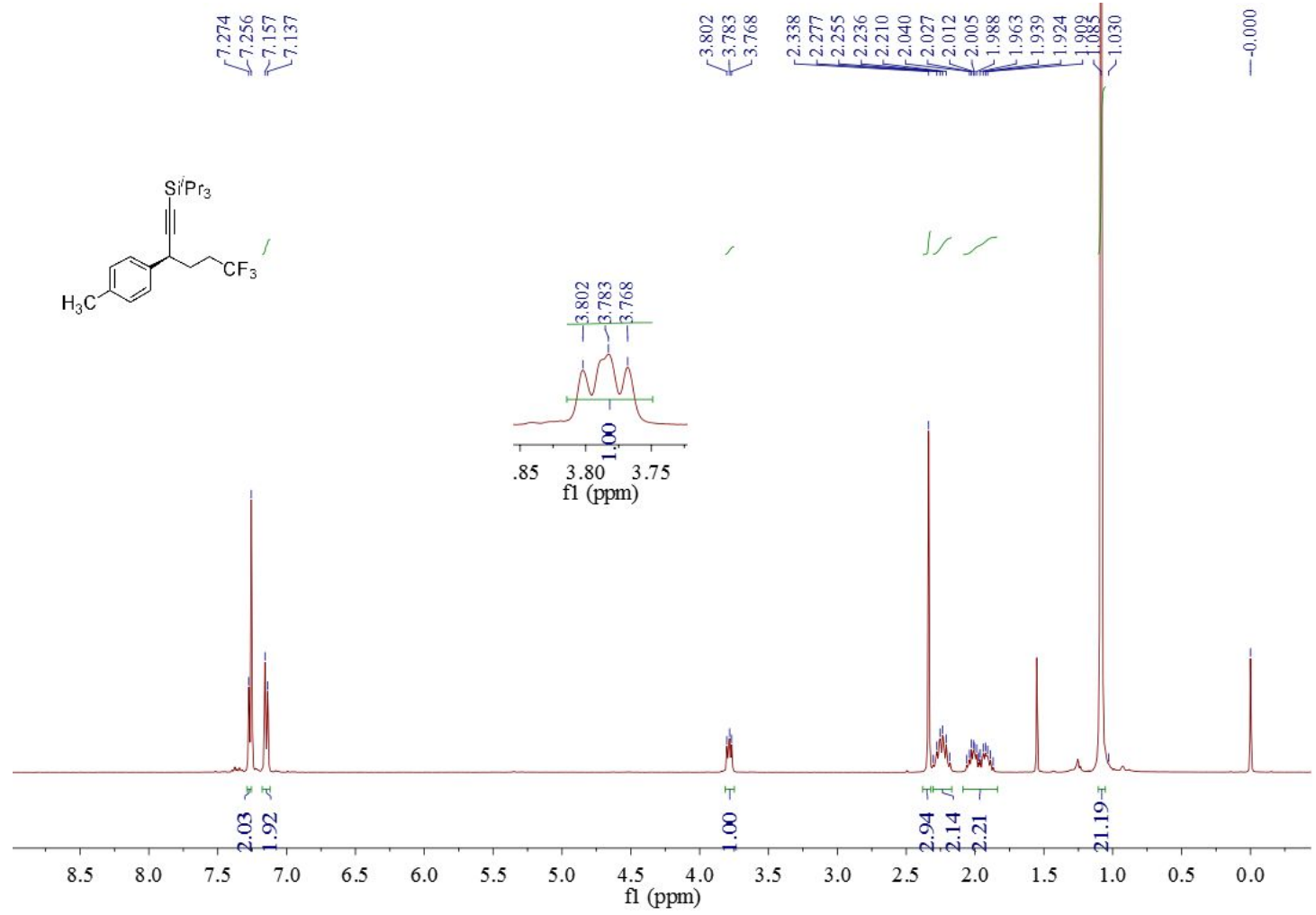

d5 - ${ }^{13}$ C NMR

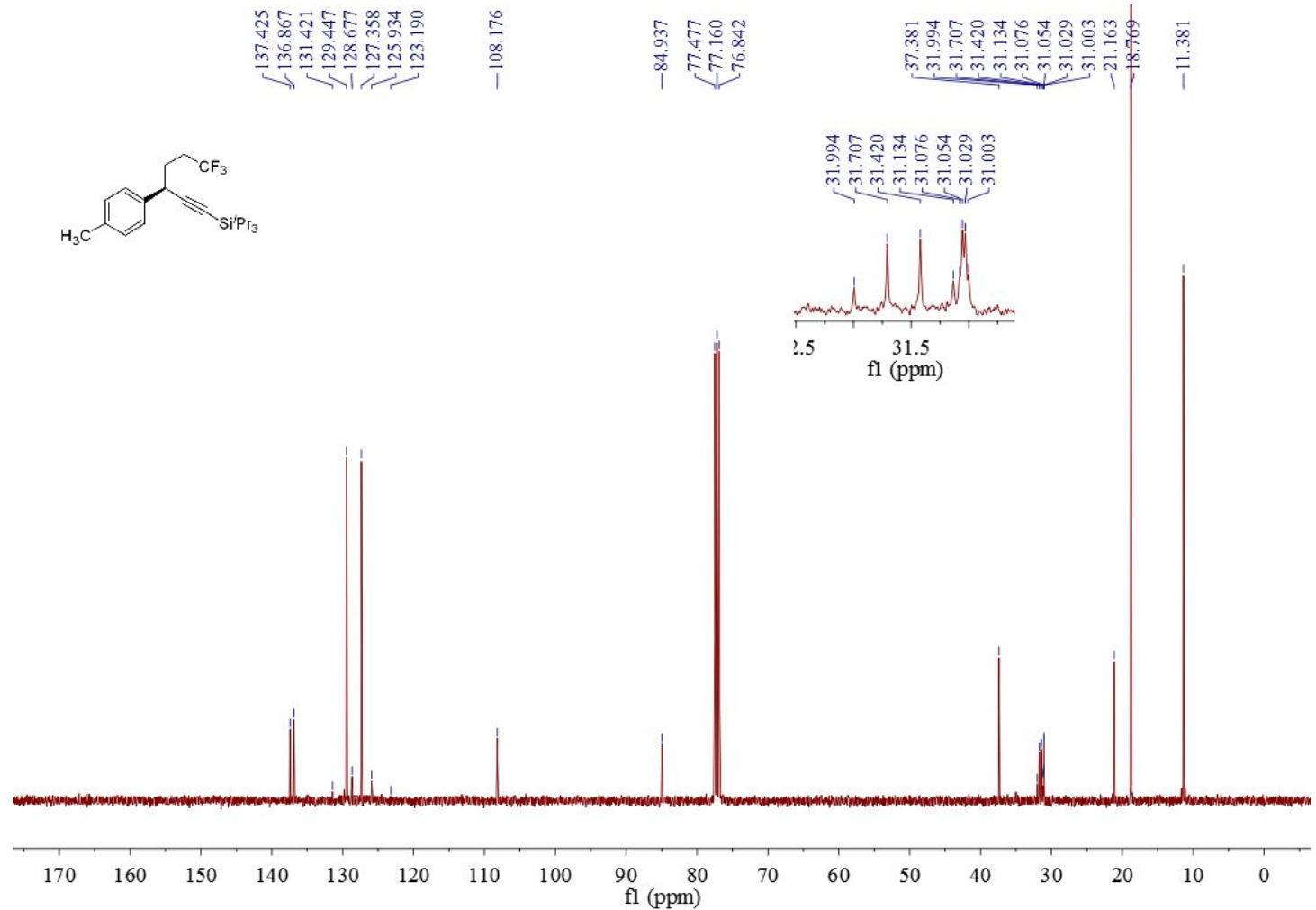


<smiles>CCCc1ccc(C)cc1</smiles>

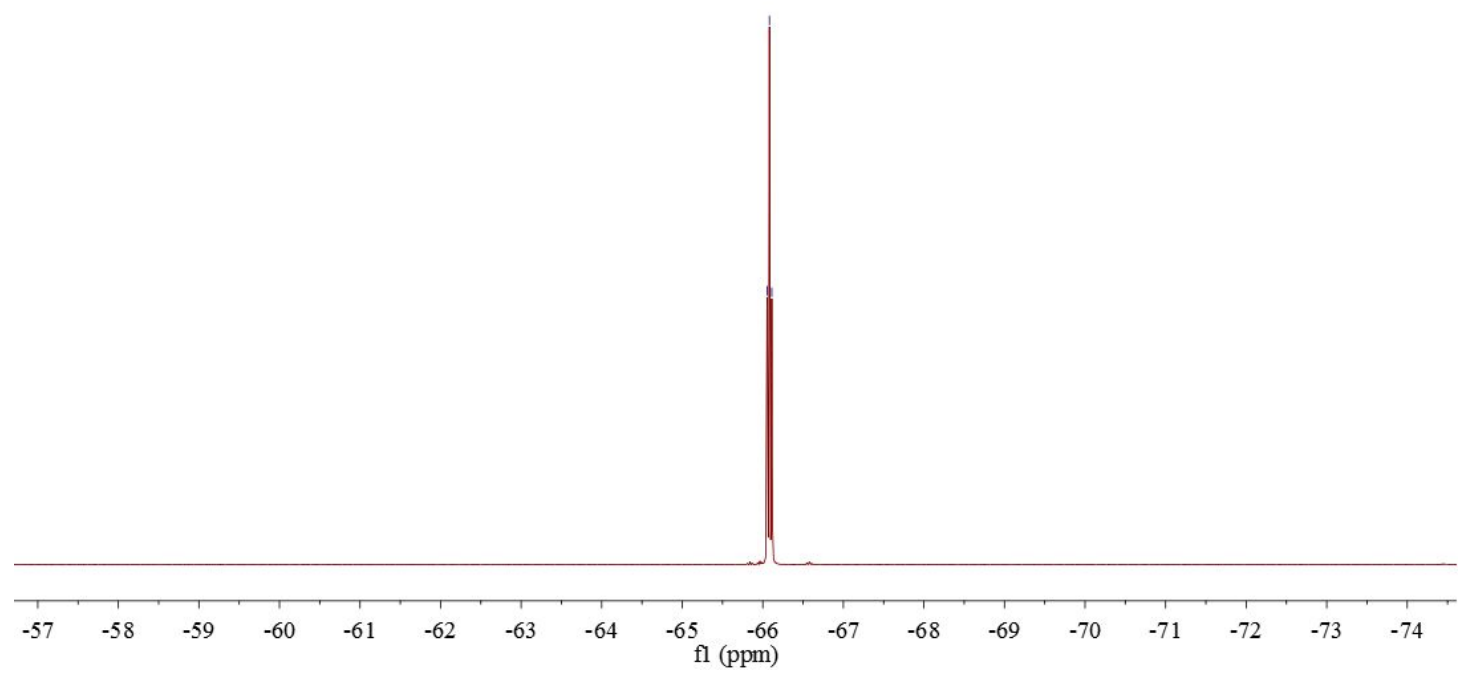

d5 - HPLC

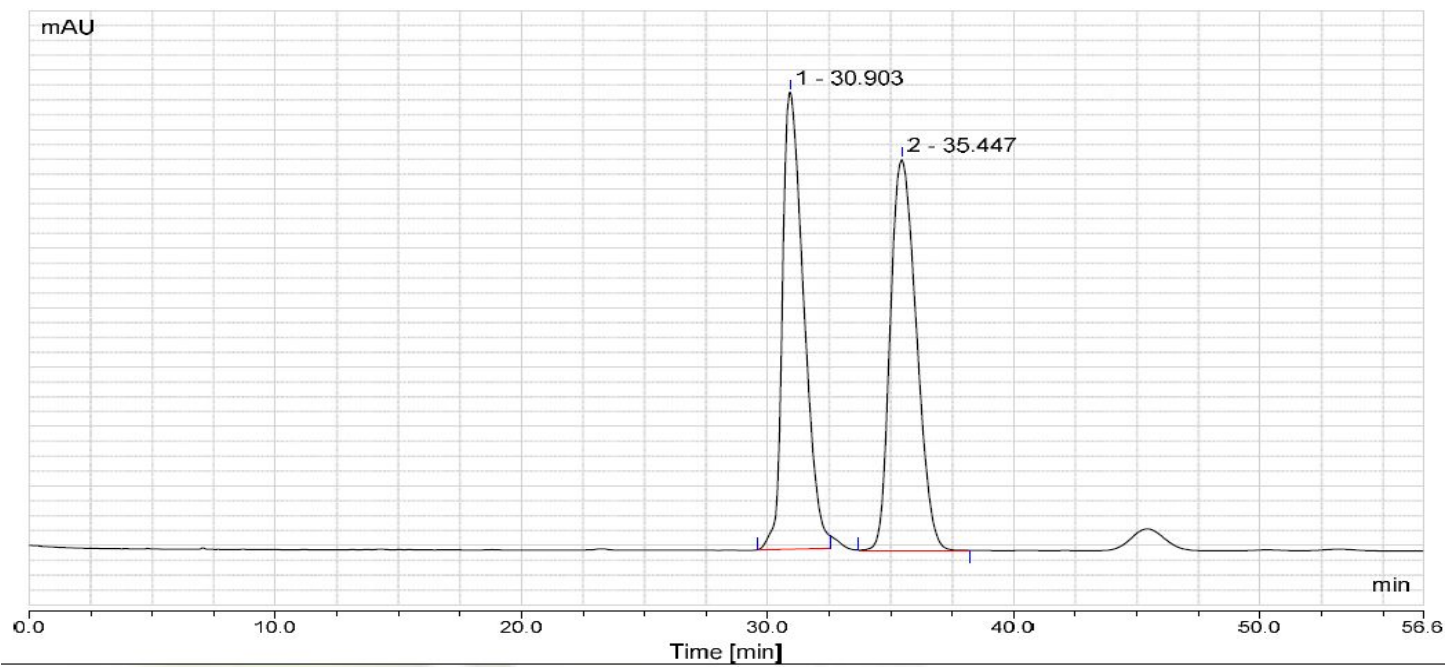

\begin{tabular}{|c|c|c|c|c|c|}
\hline \multicolumn{6}{|c|}{ Integration Results } \\
\hline No. & $\begin{array}{l}\text { Retention Time } \\
\text { min }\end{array}$ & $\begin{array}{c}\text { Retention Time } \\
\text { min }\end{array}$ & $\begin{array}{c}\text { Area } \\
m A U^{*} \min \end{array}$ & $\begin{array}{c}\text { Height } \\
\text { mAU }\end{array}$ & $\begin{array}{c}\text { Relative Area } \\
\%\end{array}$ \\
\hline 1 & 31 & 30.903 & 1540.288 & 1538.907 & 48.83 \\
\hline 2 & 35 & 35.447 & 1613.968 & 1315.405 & 51.17 \\
\hline \multicolumn{2}{|c|}{ Total: } & 3154.256 & 2854.312 & 100.00 & \\
\hline
\end{tabular}




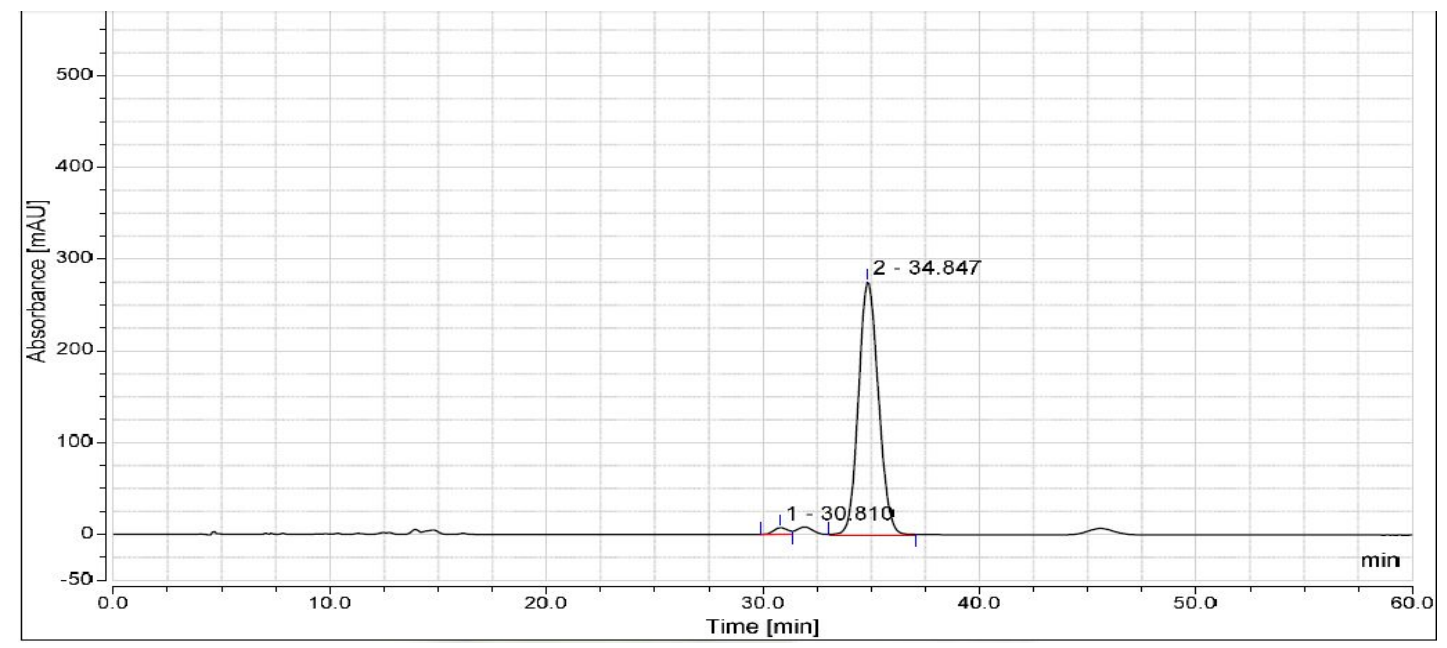

\begin{tabular}{|c|c|c|c|c|c|}
\hline \multicolumn{6}{|c|}{ Integration Results } \\
\hline No. & $\begin{array}{l}\text { Retention Time } \\
\text { min }\end{array}$ & $\begin{array}{l}\text { Retention Time } \\
\text { min }\end{array}$ & $\begin{array}{c}\text { Area } \\
m A U^{*} \min \end{array}$ & $\begin{array}{c}\text { Height } \\
\text { mAU }\end{array}$ & $\begin{array}{c}\text { Relative Area } \\
\%\end{array}$ \\
\hline 1 & 31 & 30.810 & 5.626 & 7.375 & 1.87 \\
\hline 2 & 35 & 34.847 & 294.591 & 276.211 & 98.13 \\
\hline \multicolumn{2}{|c|}{ Total: } & 300.217 & 283.586 & 100.00 & \\
\hline
\end{tabular}

(R)- (3-(4-(tert-butyl) phenyl)-6, 6, 6-trifluorohex-1-yn-1-yl) triisopropylsilane (d6) d6 - ${ }^{1}$ H NMR

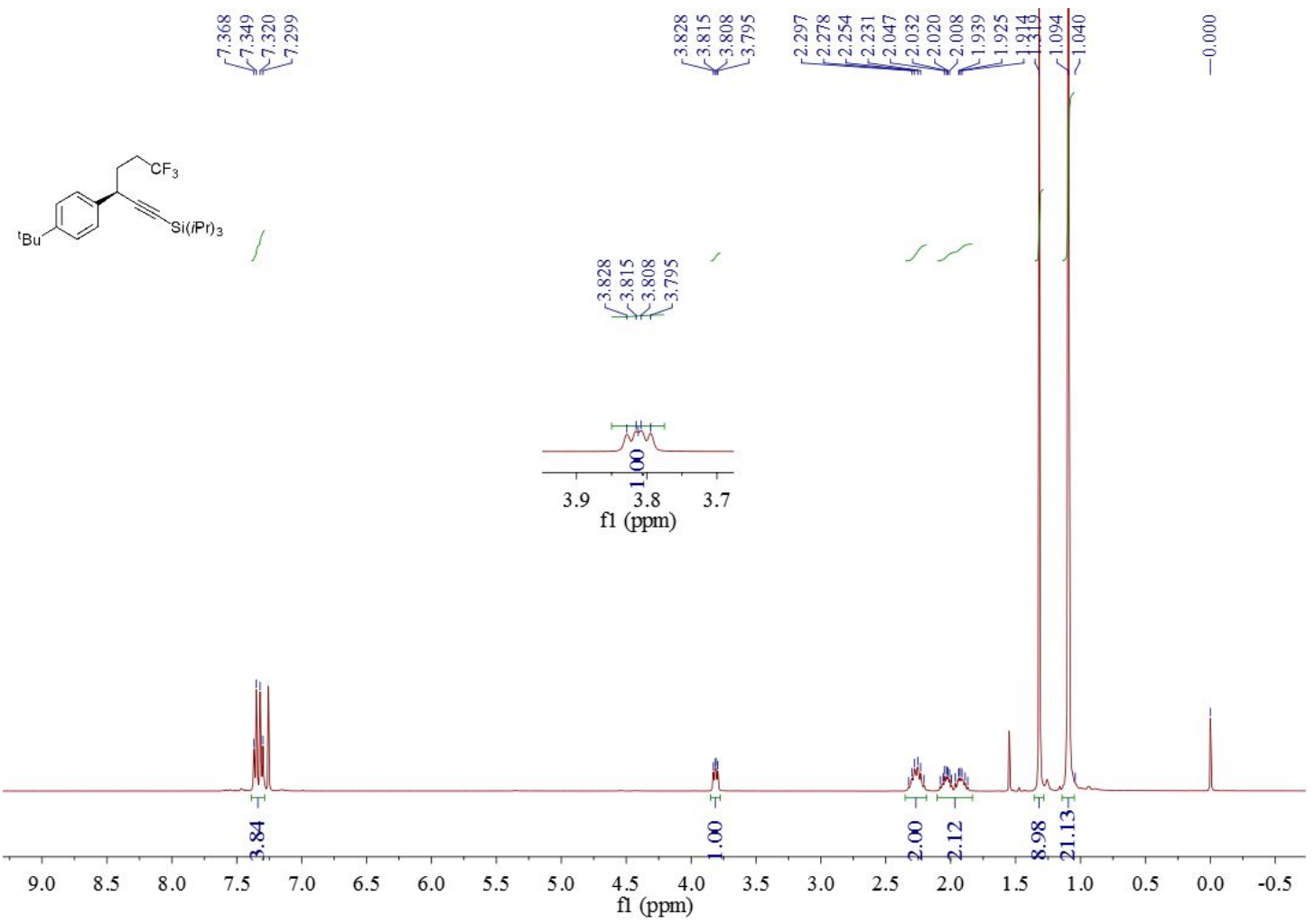




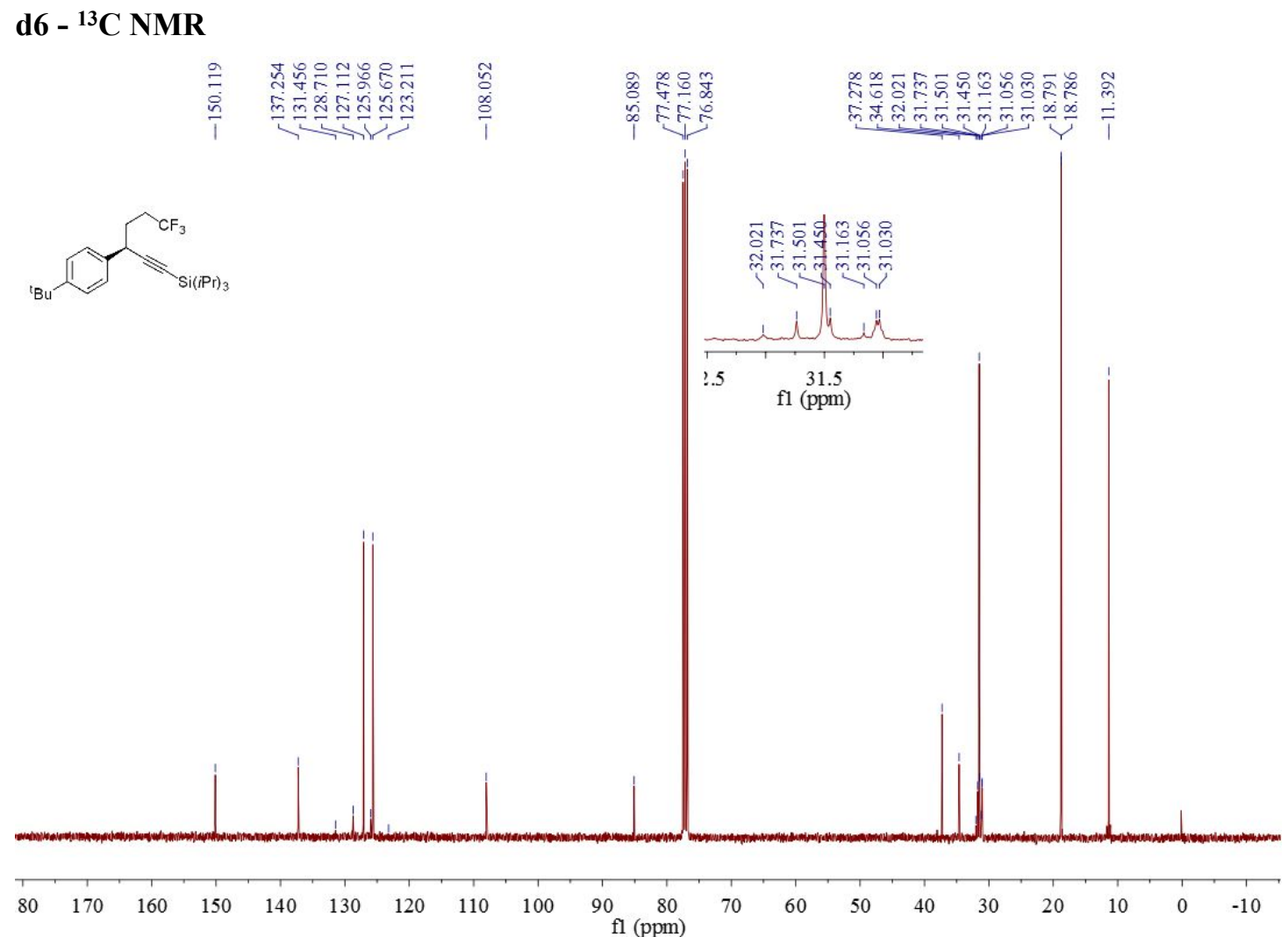

\section{d6 - ${ }^{19}$ F NMR}

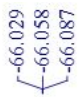
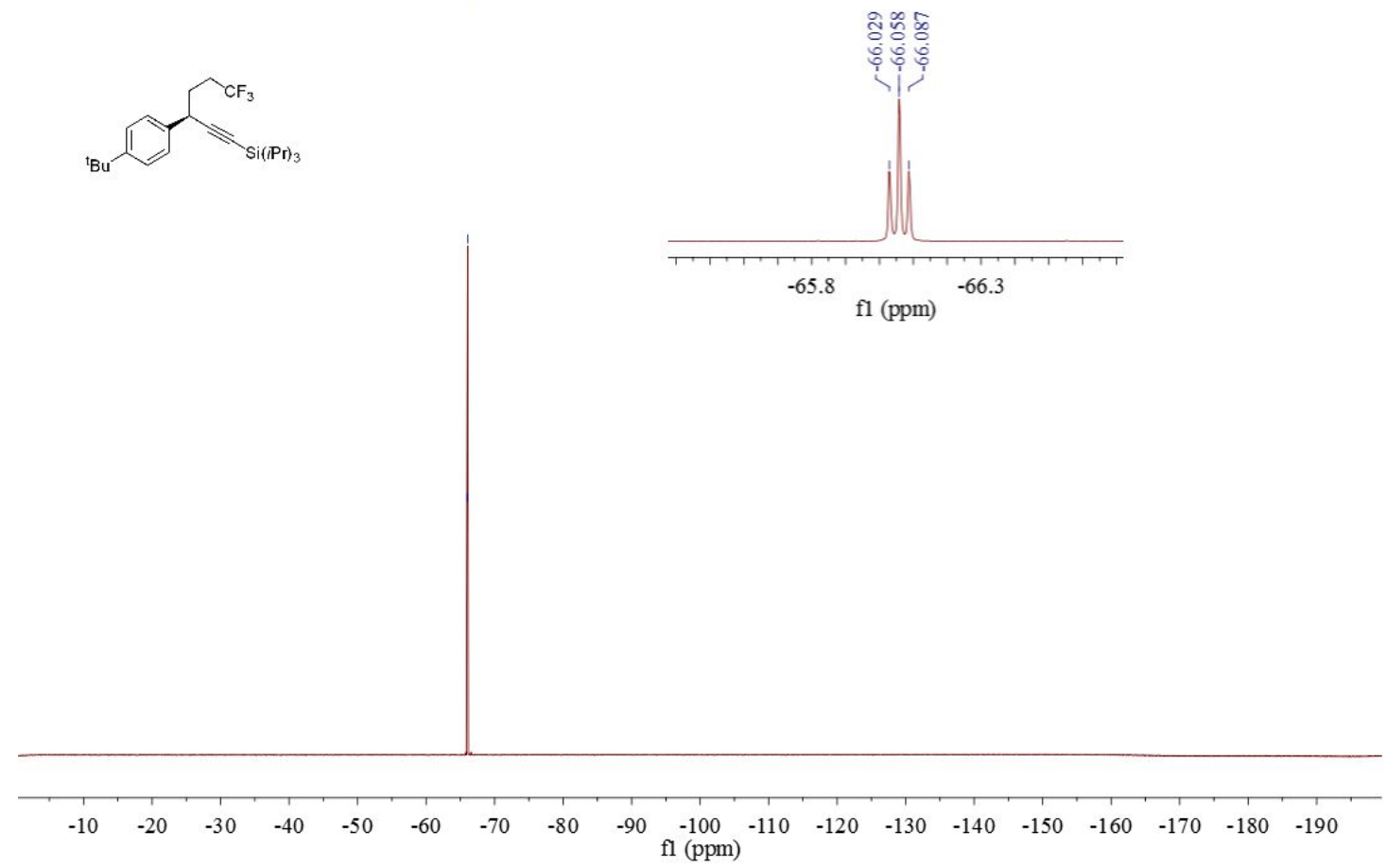
d6 - HPLC

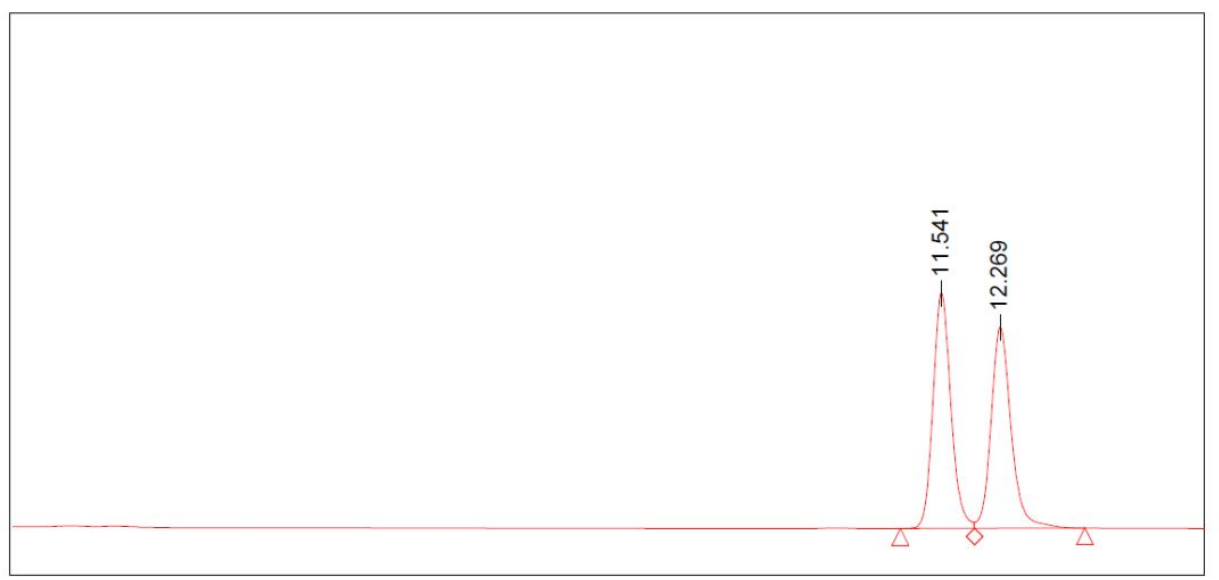

\begin{tabular}{l|c|c|c|c|l|l|r|}
\hline Vial & RT & $\begin{array}{c}\text { Retention } \\
\text { Time } \\
(\mathrm{min})\end{array}$ & $\begin{array}{c}\text { Width } \\
(\mathrm{sec})\end{array}$ & Height & Peak Type & Area & \% Area \\
\hline 11 & 11.541 & 11.541 & 55.000 & 543859 & Unknown & 8362112 & 51.09 \\
\hline 11 & 12.269 & 12.269 & 82.000 & 464505 & Unknown & 8003887 & 48.91 \\
\hline
\end{tabular}

Auto-Scaled Chromatogram

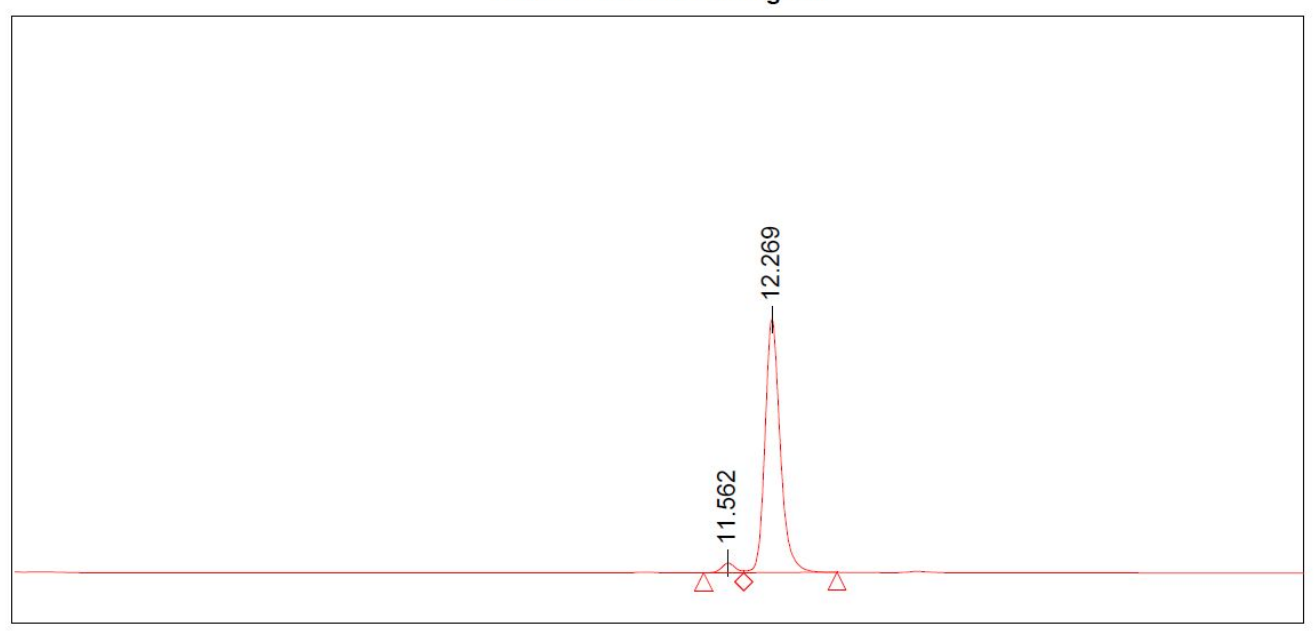

Peak Results

\begin{tabular}{|l|c|r|r|r|l|r|r|}
\hline Vial & RT & $\begin{array}{c}\text { Retention } \\
\text { Time } \\
(\mathrm{min})\end{array}$ & $\begin{array}{l}\text { Width } \\
(\mathrm{sec})\end{array}$ & Height & Peak Type & Area & \% Area \\
\hline 31 & 11.562 & 11.562 & 39.000 & 25716 & Unknown & 388775 & 3.20 \\
\hline 31 & 12.269 & 12.269 & 90.000 & 687836 & Unknown & 11742733 & 96.80 \\
\hline
\end{tabular}


(R)-triisopropyl(6 , 6, 6-trifluoro-3-(4-methoxyphenyl)hex-1-yn-1-yl)silane (d7) d7 - ${ }^{1}$ H NMR

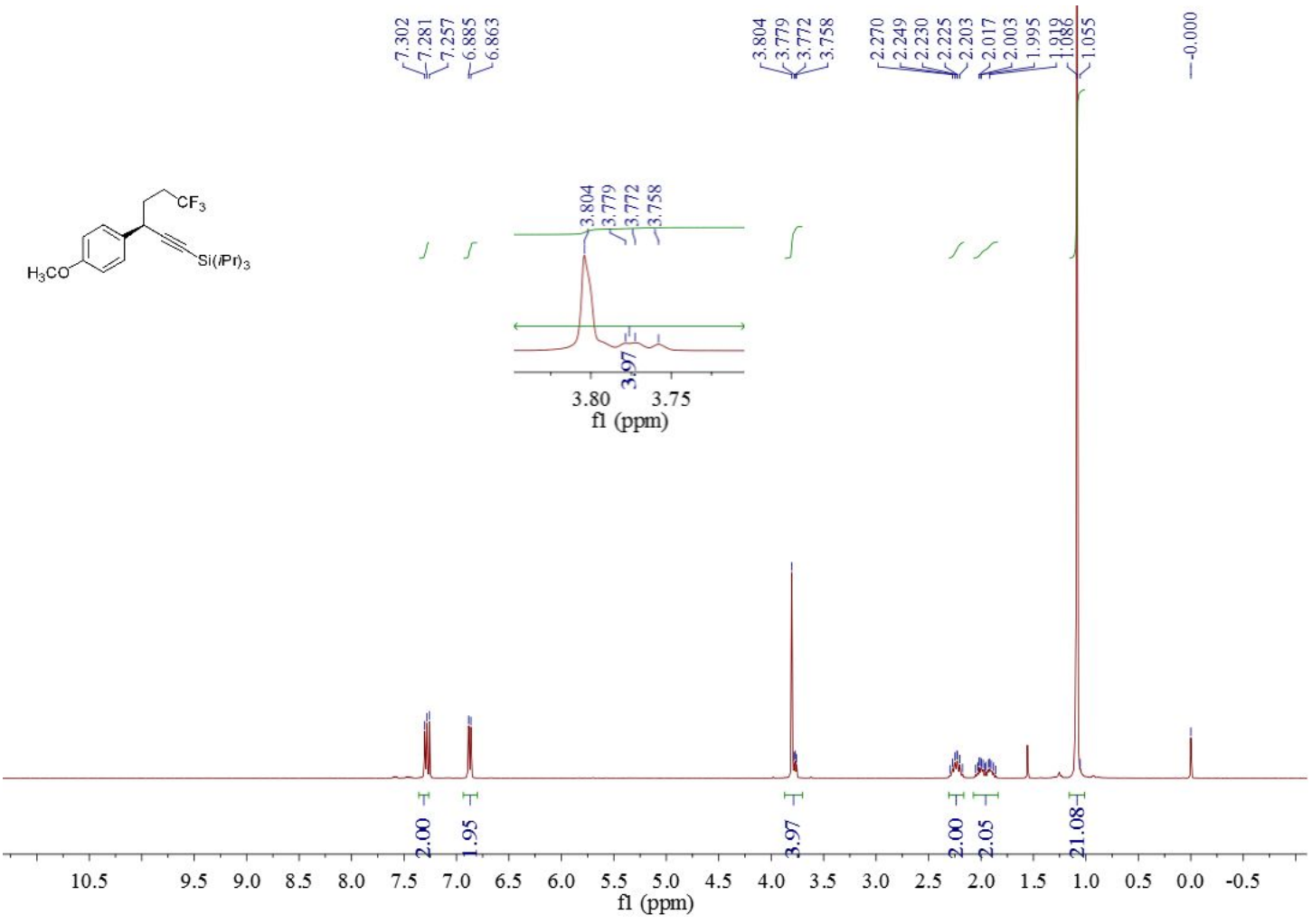

d7 $-{ }^{13}$ C NMR

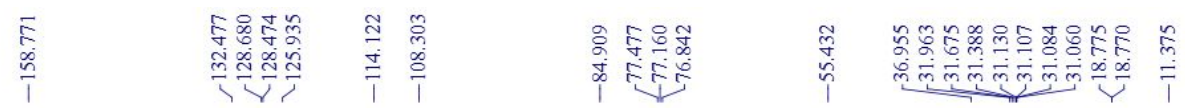

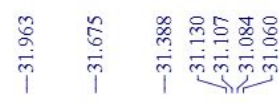
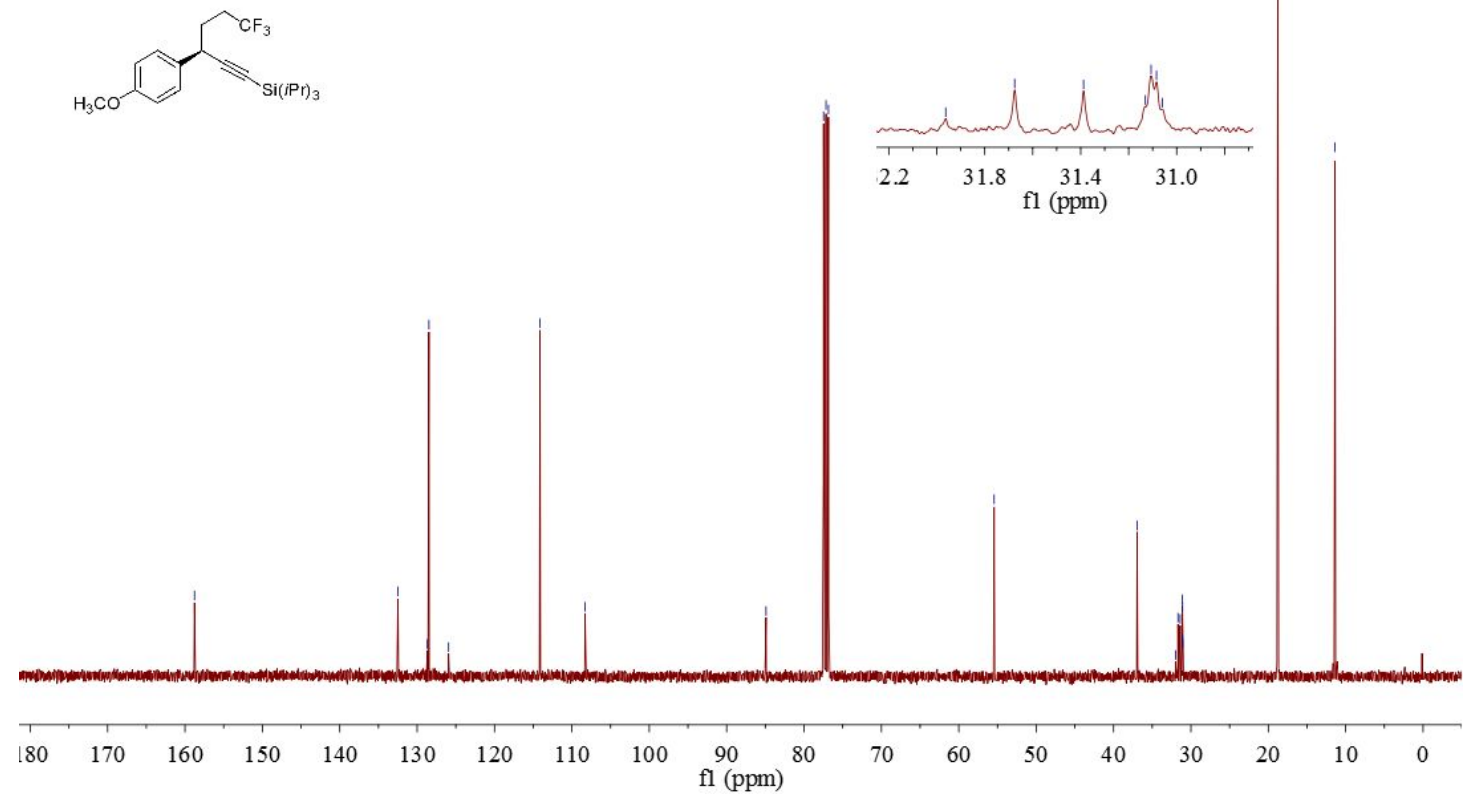

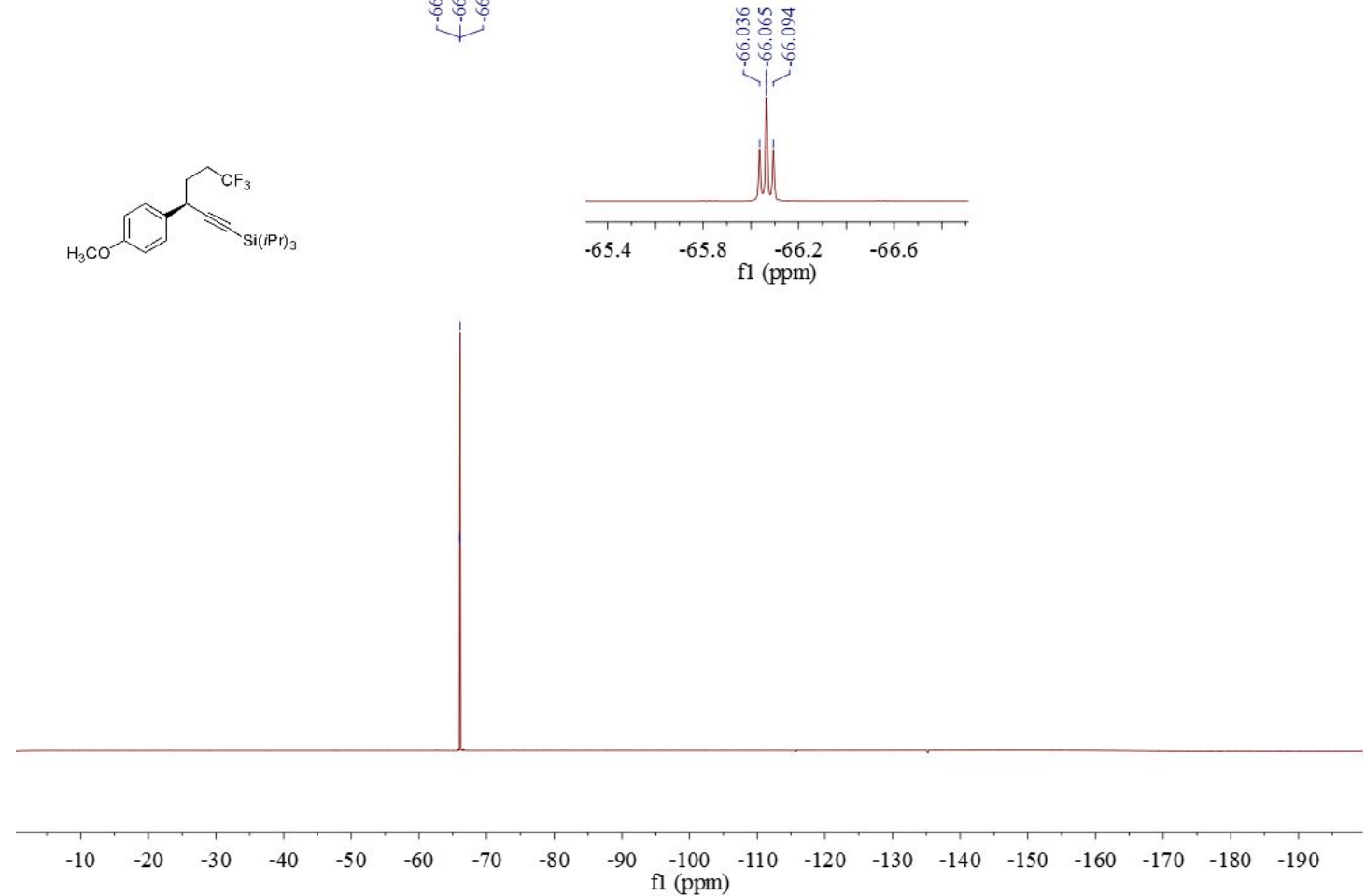

d7 - HPLC

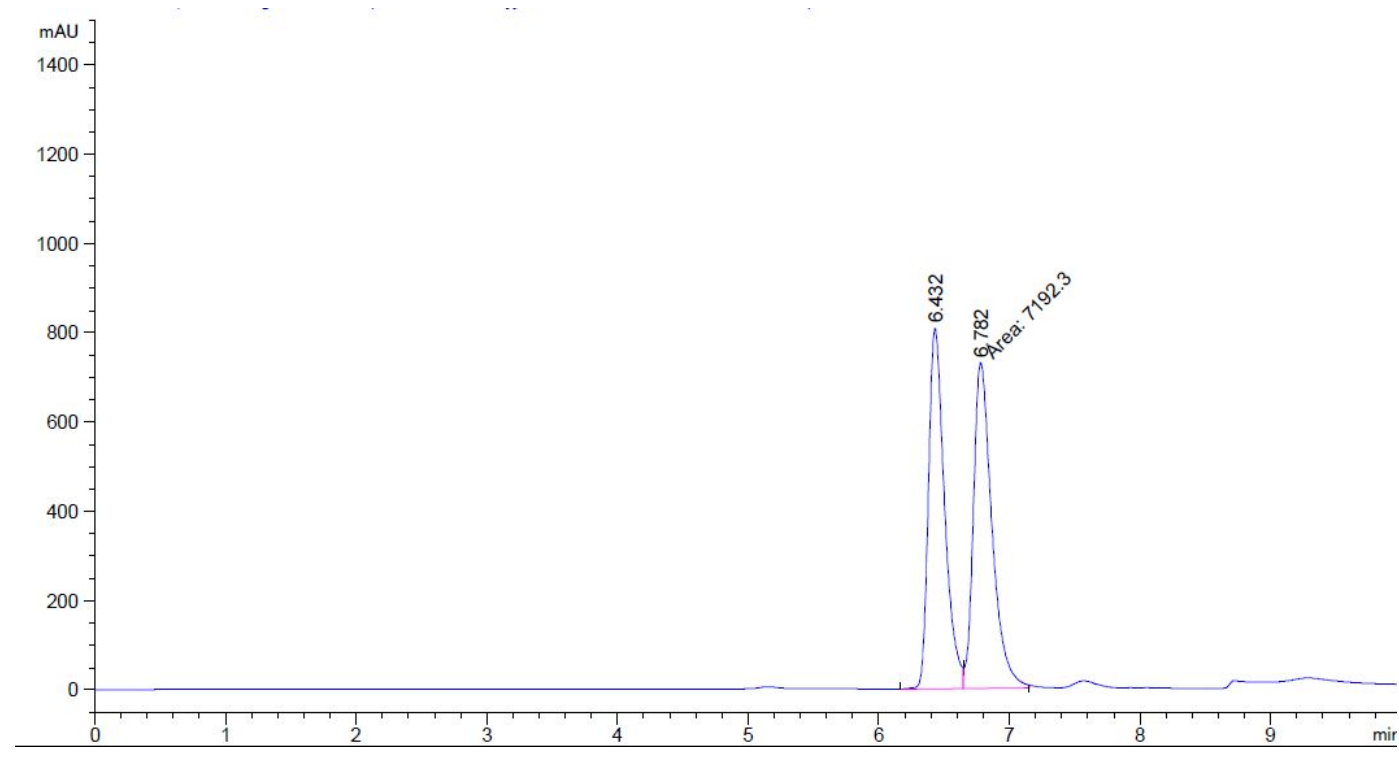

Peak RetTime Type Width Area Height Area

$\begin{array}{llll}\# & {[\mathrm{~min}]} & {[\mathrm{min}]} & {[\mathrm{mAU} * \mathrm{~s}]}\end{array}$

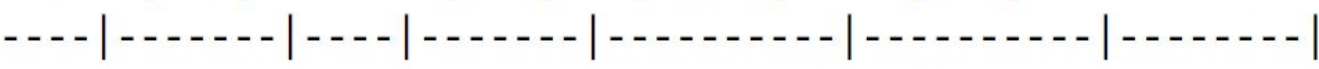

$\begin{array}{lllllll}1 & 6.432 & \text { BV } & 0.1299 & 6920.86572 & 808.09570 & 49.0384\end{array}$

$2 \quad 6.782$ MF $\quad 0.16407192 .29785 \quad 731.07892 \quad 50.9616$

Totals :

$1.41132 \mathrm{e} 4 \quad 1539.17462$ 

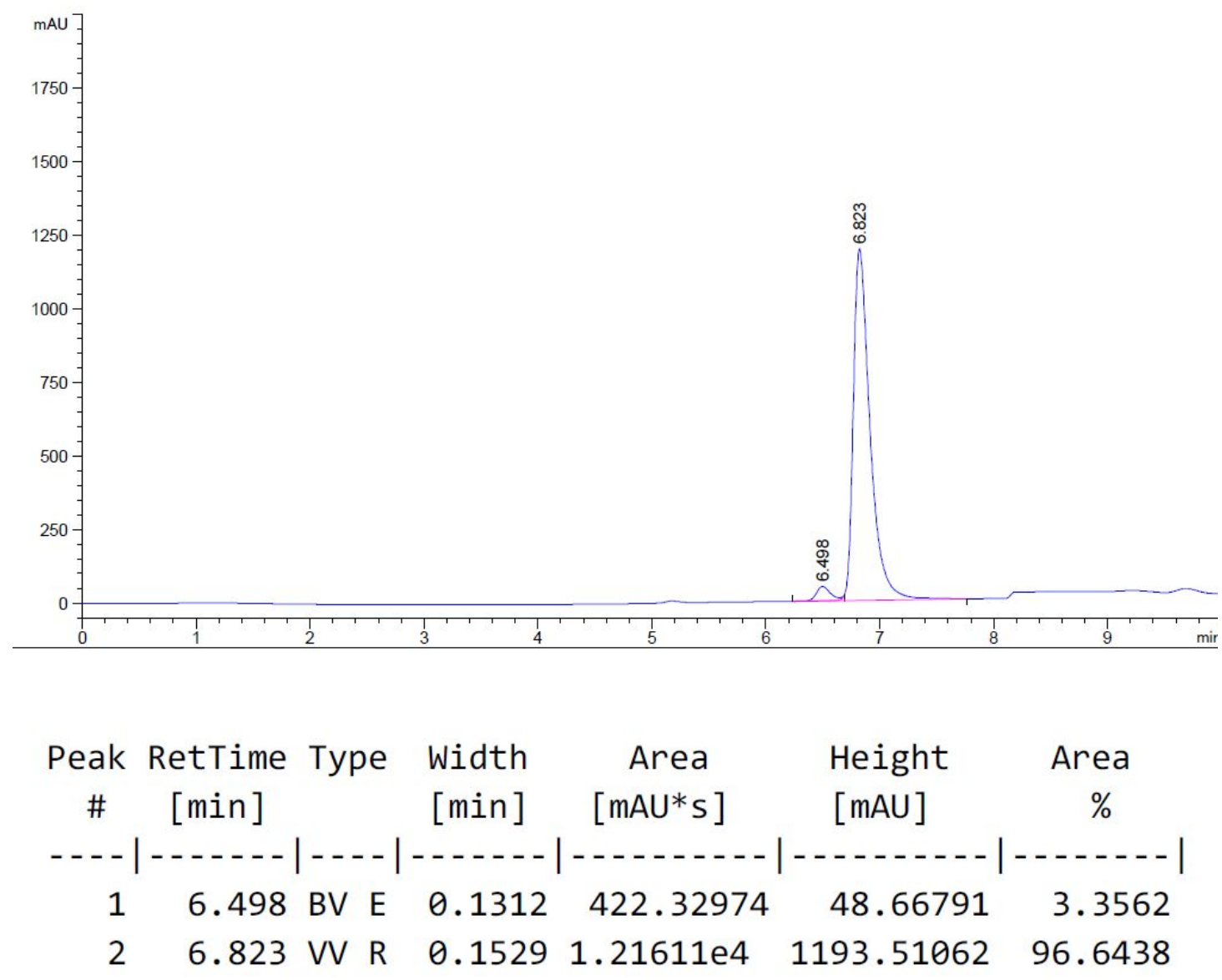

Totals :

$1.25834 \mathrm{e} 4 \quad 1242.17853$ 
(R)-triisopropyl (6, 6, 6-trifluoro-3-(4-(trifluoromethyl)phenyl)hex-1-yn-1-yl) silane (d8) d8 - ${ }^{1}$ H NMR

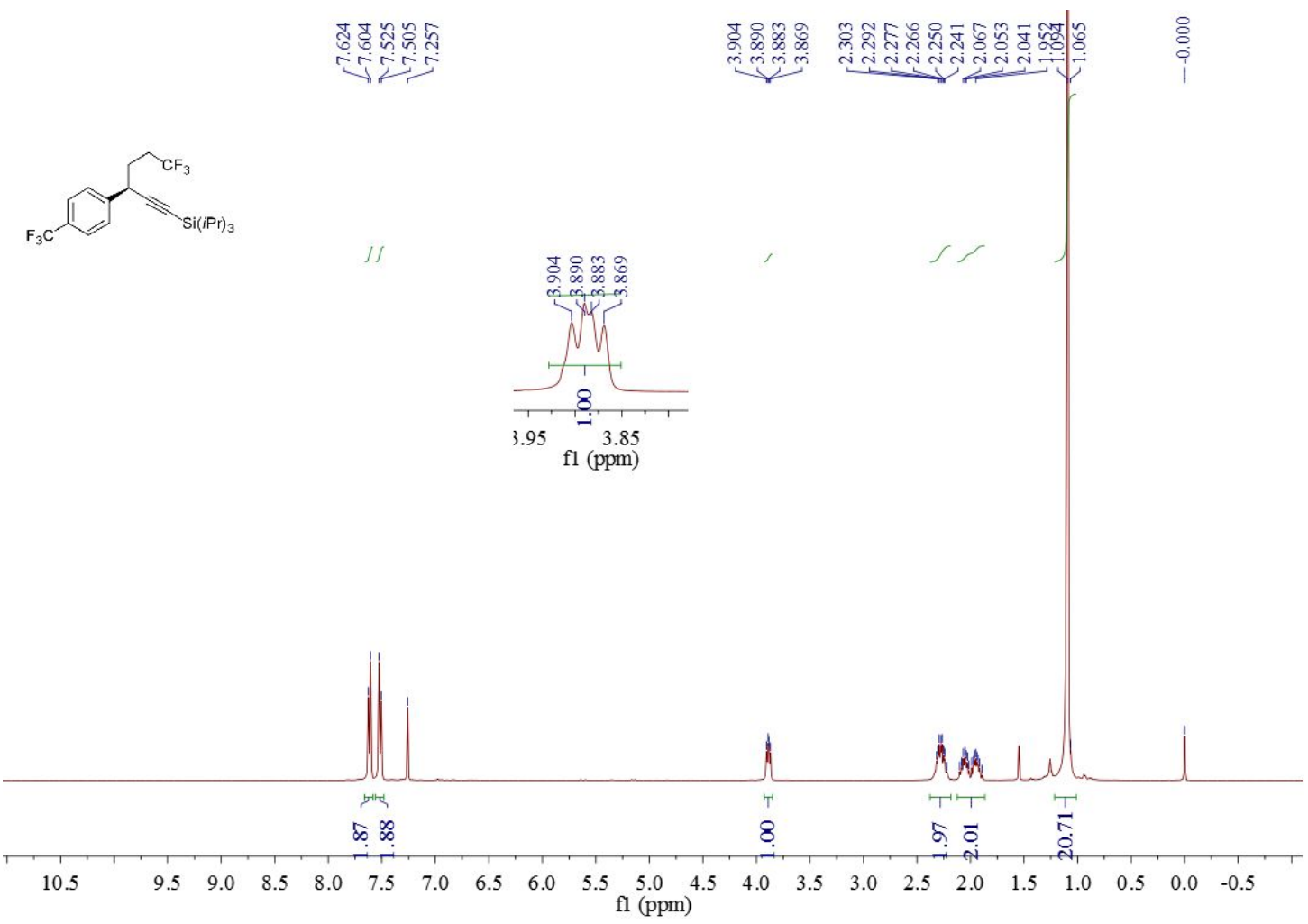

d8 $-{ }^{13}$ C NMR

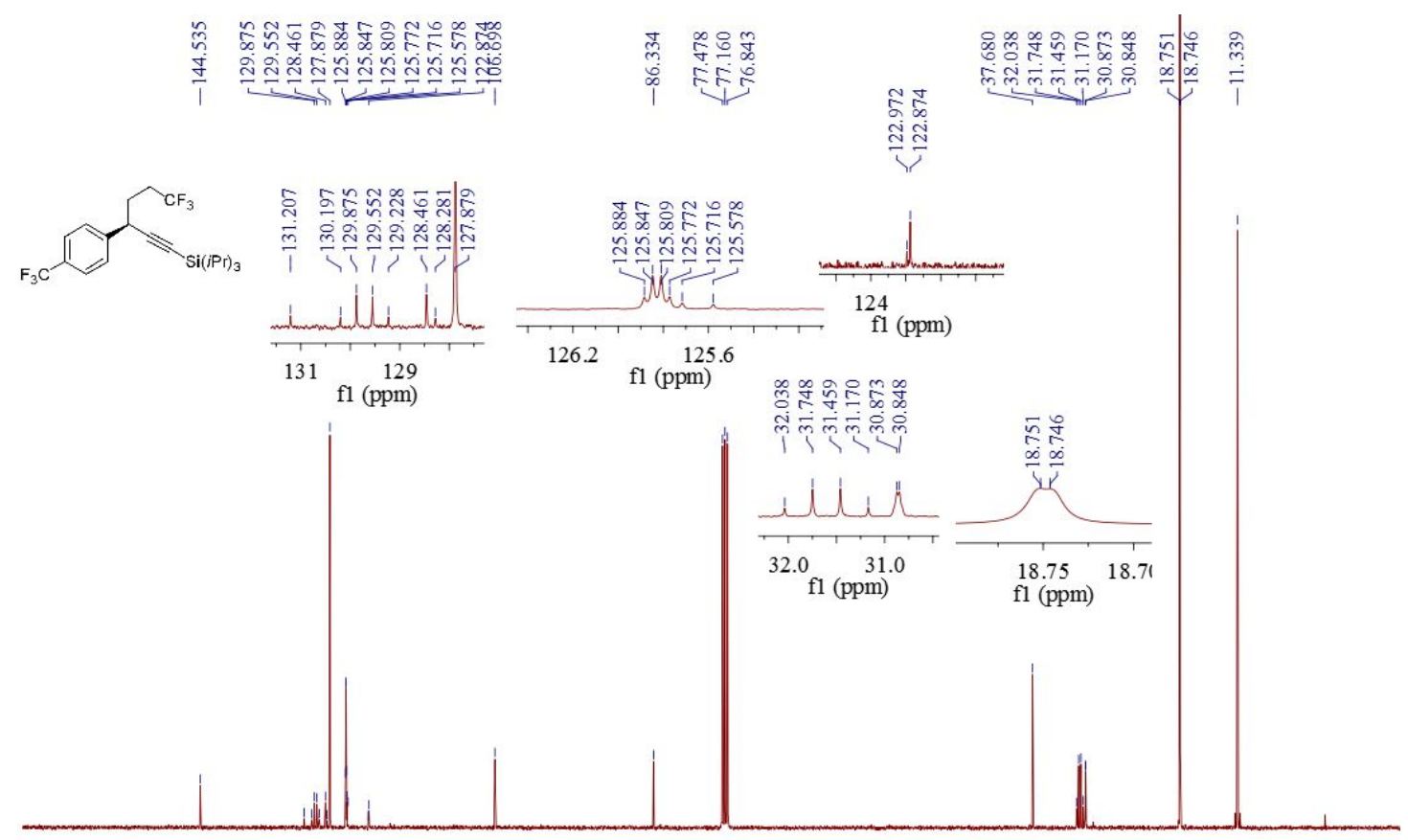

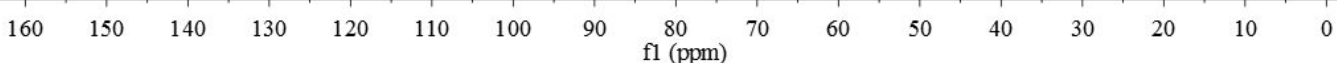




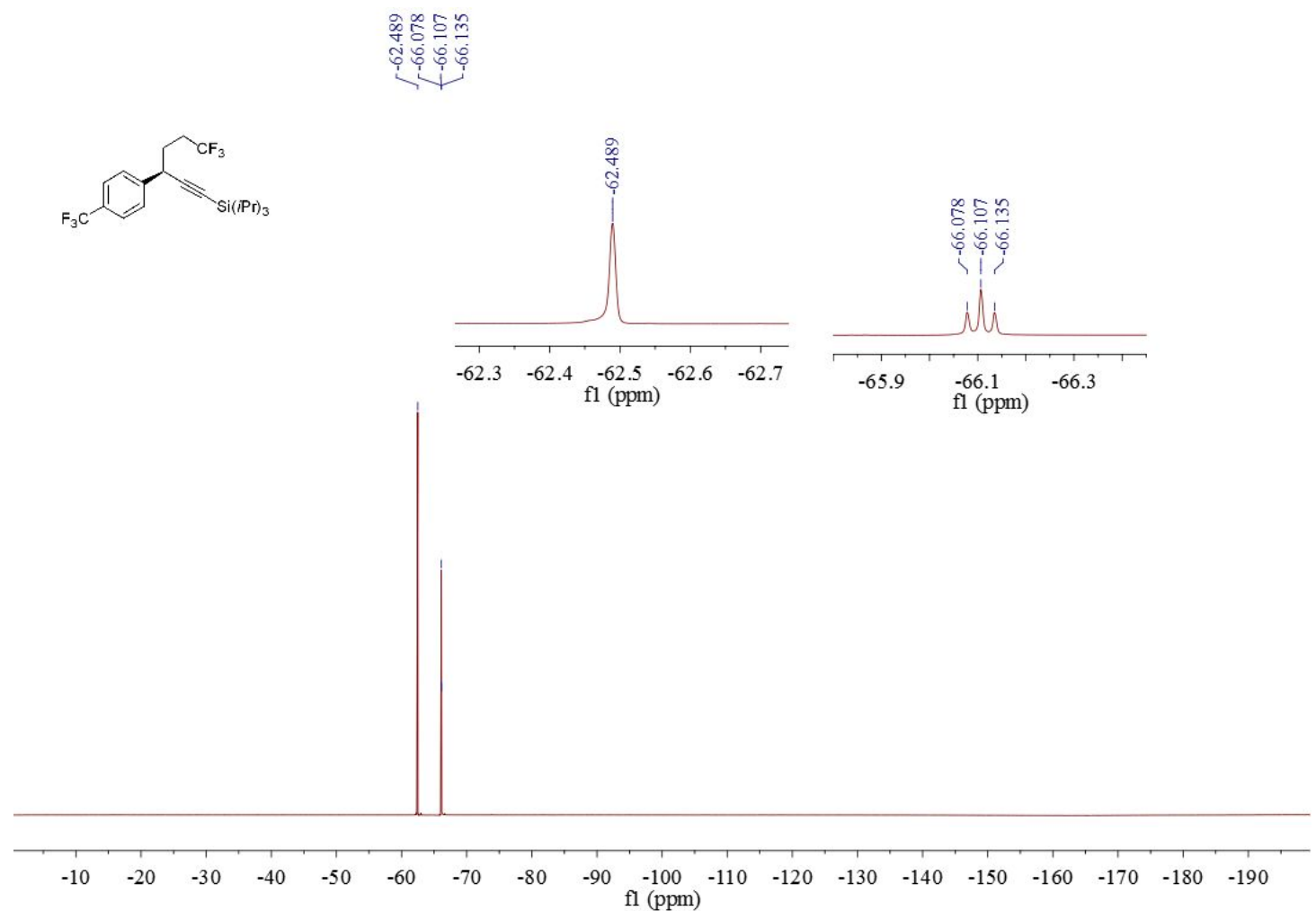

d8 - HPLC

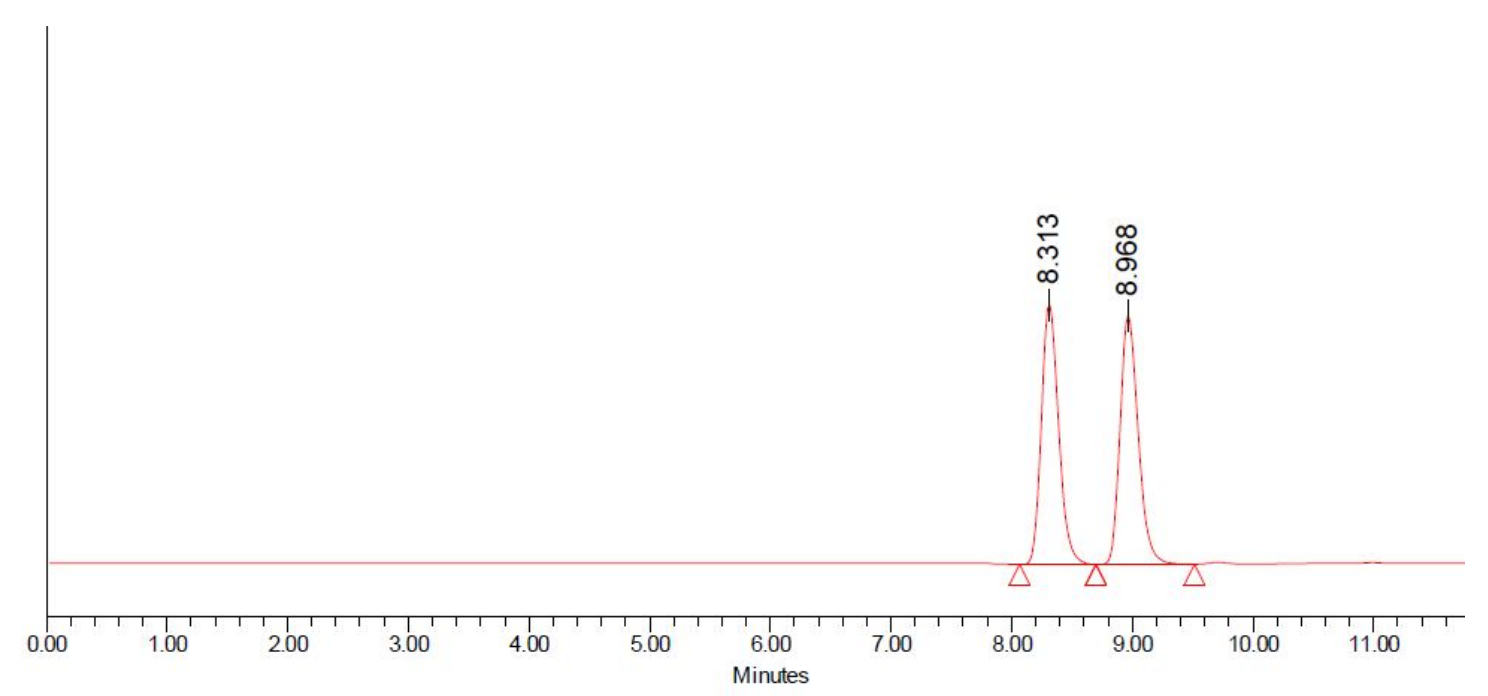

\begin{tabular}{|l|c|c|c|c|l|r|r|}
\hline Vial & RT & $\begin{array}{c}\text { Retention } \\
\text { Time } \\
(\mathrm{min})\end{array}$ & $\begin{array}{c}\text { Width } \\
(\mathrm{sec})\end{array}$ & Height & Peak Type & Area & \% Area \\
\hline 6 & 8.313 & 8.313 & 38.000 & 989362 & Unknown & 9783742 & 49.79 \\
\hline 6 & 8.968 & 8.968 & 49.000 & 945167 & Unknown & 9864920 & 50.21 \\
\hline
\end{tabular}




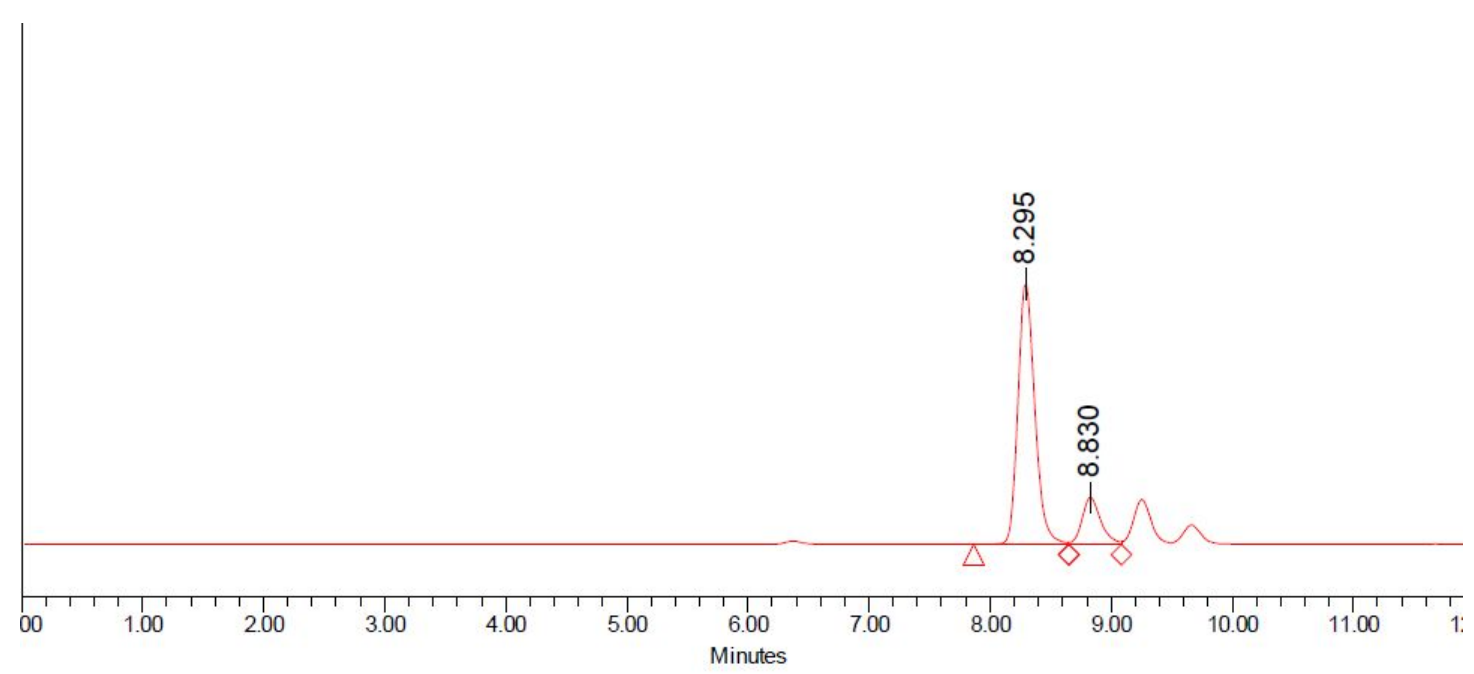

\begin{tabular}{l|c|r|r|r|r|r|r|}
\hline Vial & RT & $\begin{array}{c}\text { Retention } \\
\text { Time } \\
(\mathrm{min})\end{array}$ & $\begin{array}{c}\text { Width } \\
(\mathrm{sec})\end{array}$ & Height & Peak Type & Area & $\%$ Area \\
\hline 29 & 8.295 & 8.295 & 47.000 & 858152 & Unknown & 8317533 & 83.41 \\
\hline 29 & 8.830 & 8.830 & 26.000 & 154667 & Unknown & 1653936 & 16.59 \\
\hline
\end{tabular}

(R)- (3-(3-bromophenyl)-6, 6, 6-trifluorohex-1-yn-1-yl)triisopropylsilane (d9) d9 - ${ }^{1}$ H NMR

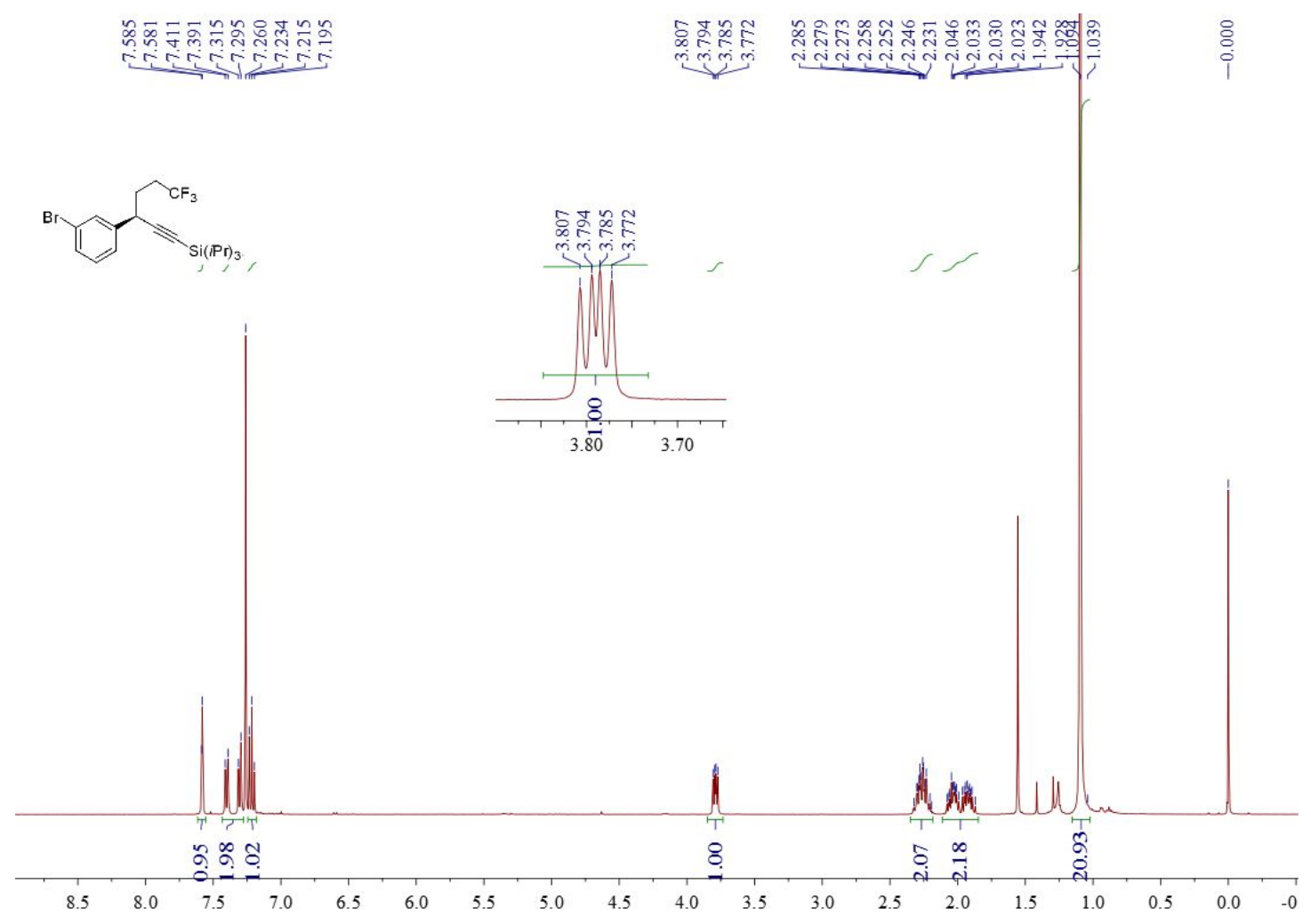


d9 - ${ }^{13}$ C NMR
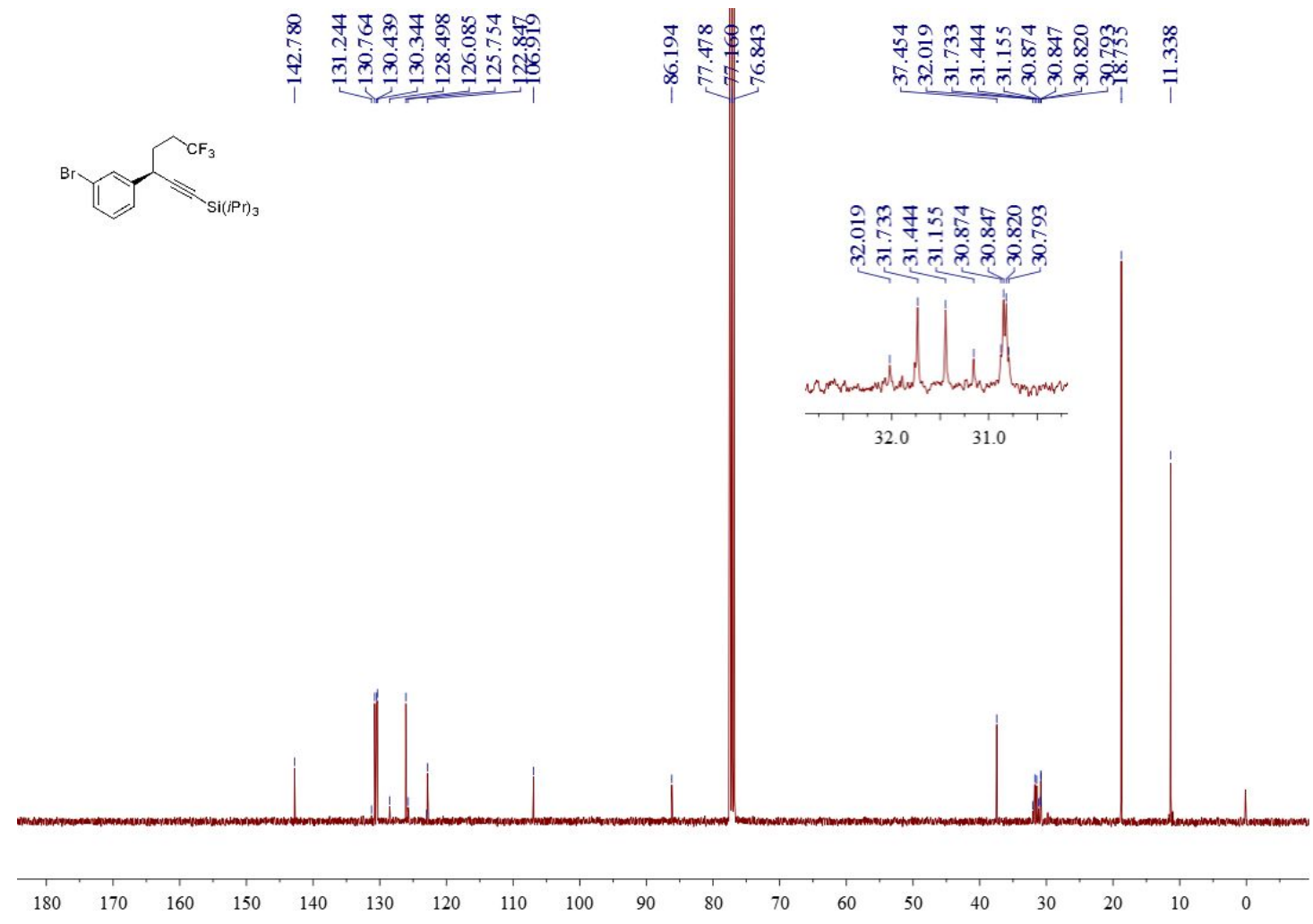
d9 - ${ }^{19}$ F NMR

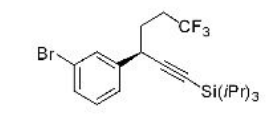

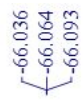

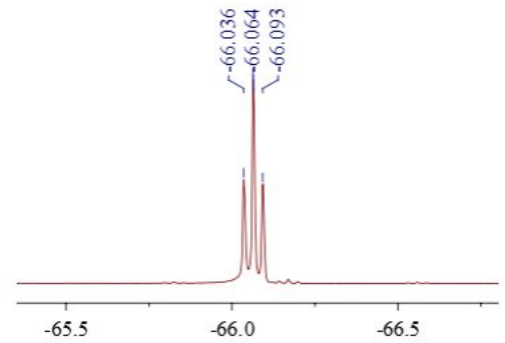

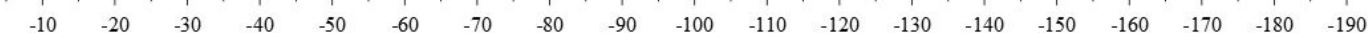




\section{d9 - HPLC}

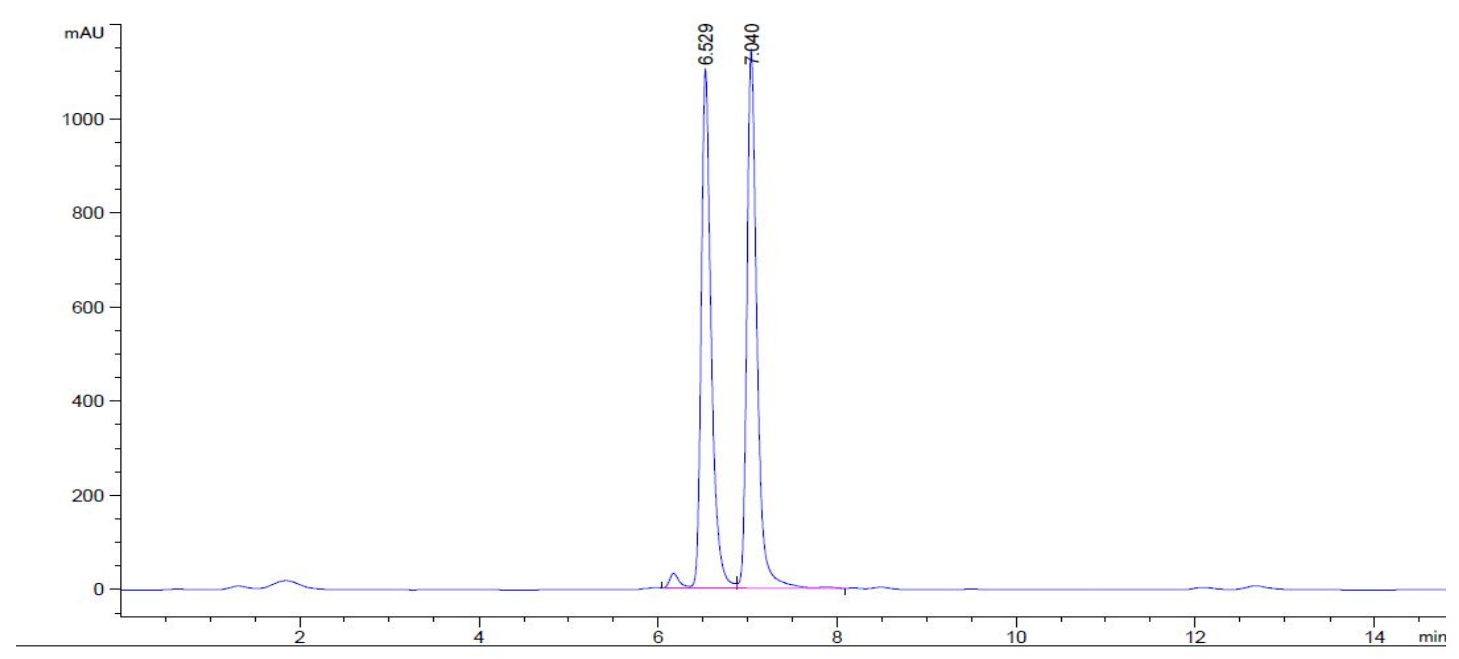

$$
\begin{aligned}
& \text { Peak RetTime Type width Area Height Area } \\
& \text { \# [min] [min] [mAU*s] [mAU] }
\end{aligned}
$$

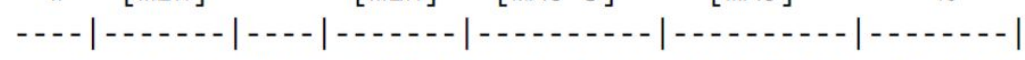

$$
\begin{aligned}
& \begin{array}{lllllll}
1 & 6.529 \text { VV R } & 0.1147 & 8691.46582 & 1100.49866 & 49.4214
\end{array} \\
& 2 \quad 7.040 \text { VV R } 0.1155 \quad 8894.97070 \quad 1141.42310 \quad 50.5786
\end{aligned}
$$

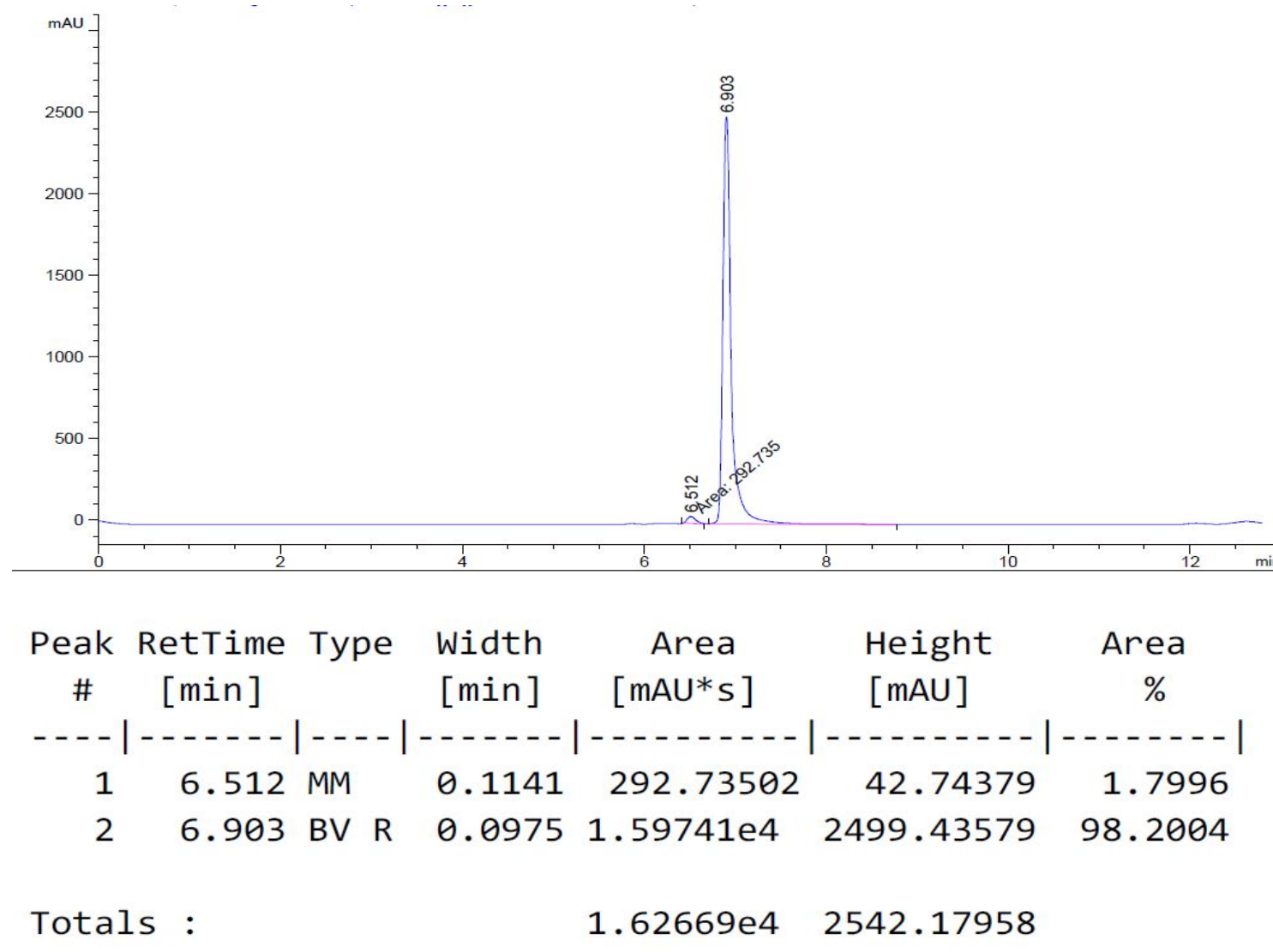

(R)- (3-(2-chlorophenyl)-6,6,6-trifluorohex-1-yn-1-yl)triisopropylsilane (d10) 


\section{d10 - ${ }^{1} \mathrm{H}$ NMR}

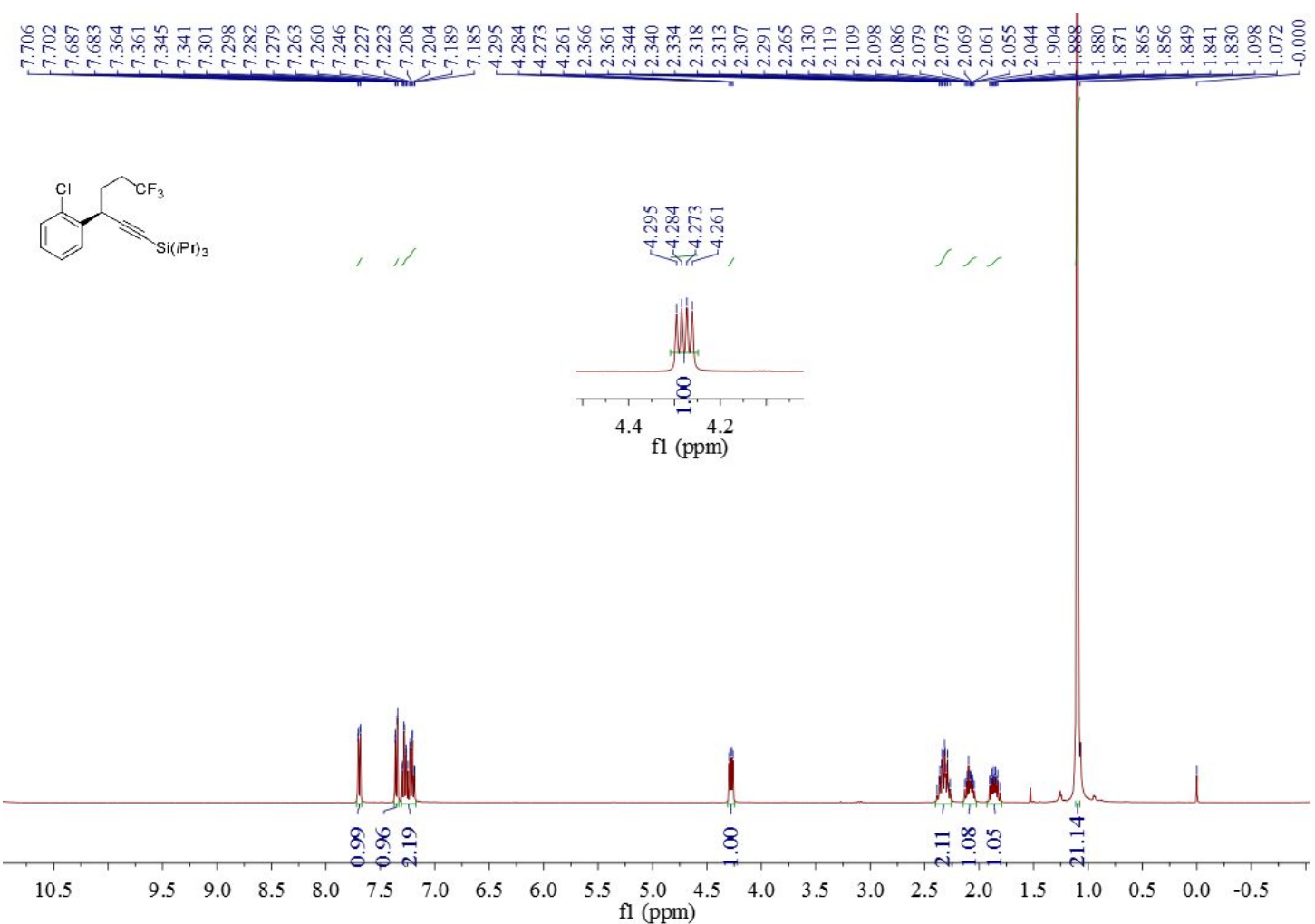

\section{d10 - ${ }^{13}$ C NMR}

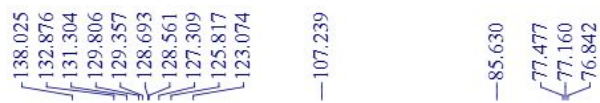

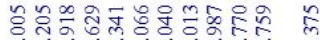

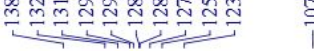<smiles>CCCCc1ccccc1Cl</smiles>

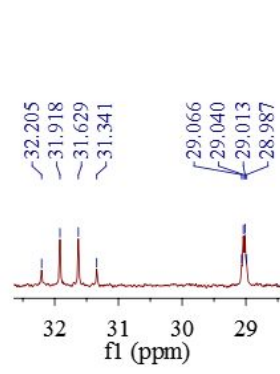

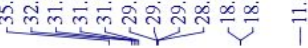

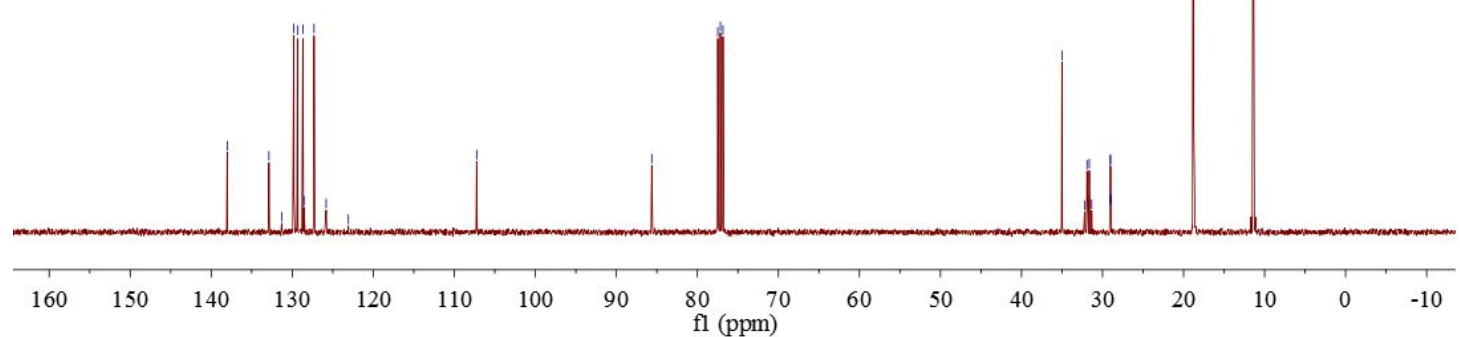

d10 - ${ }^{19}$ F NMR 


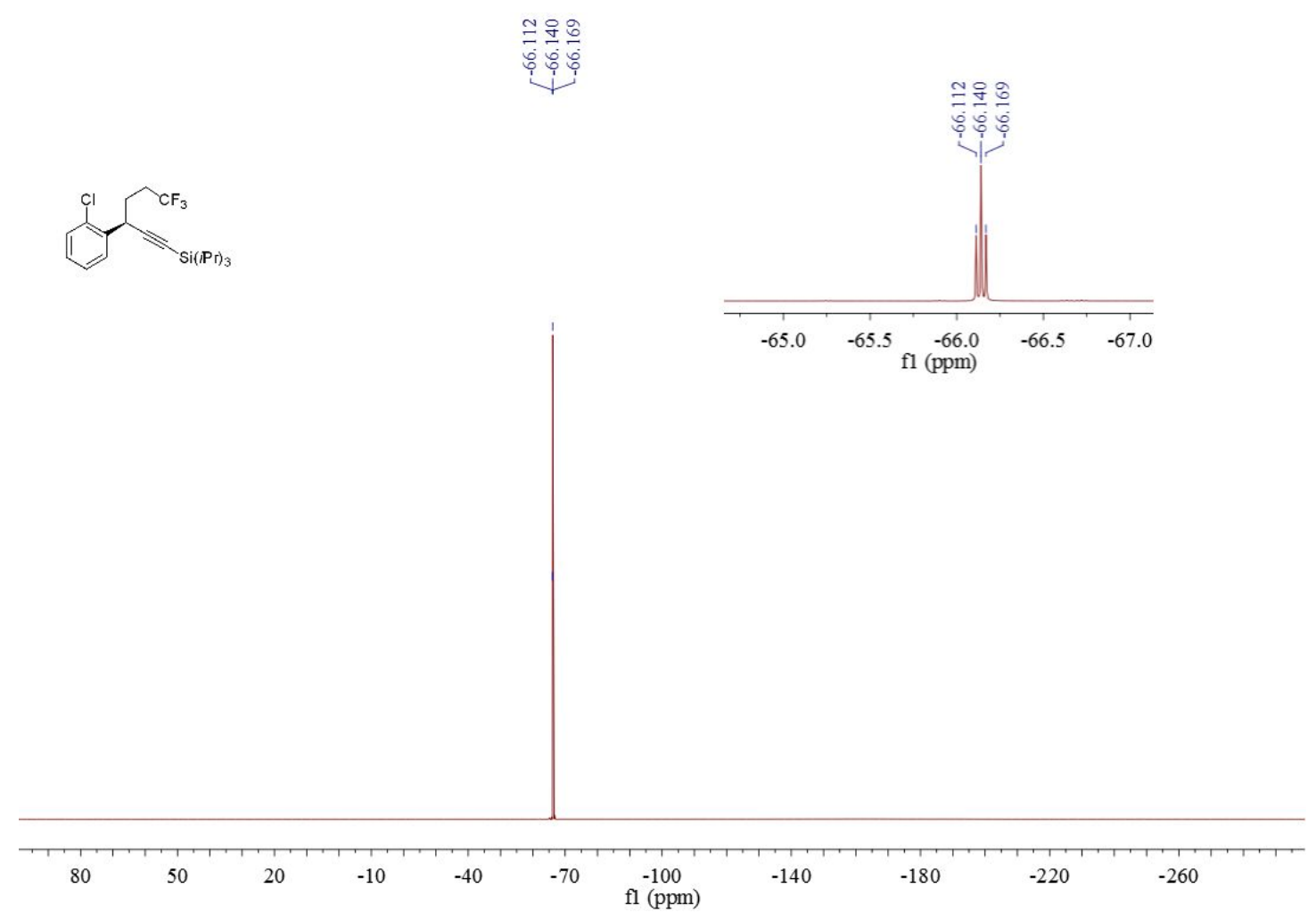

d10 - HPLC

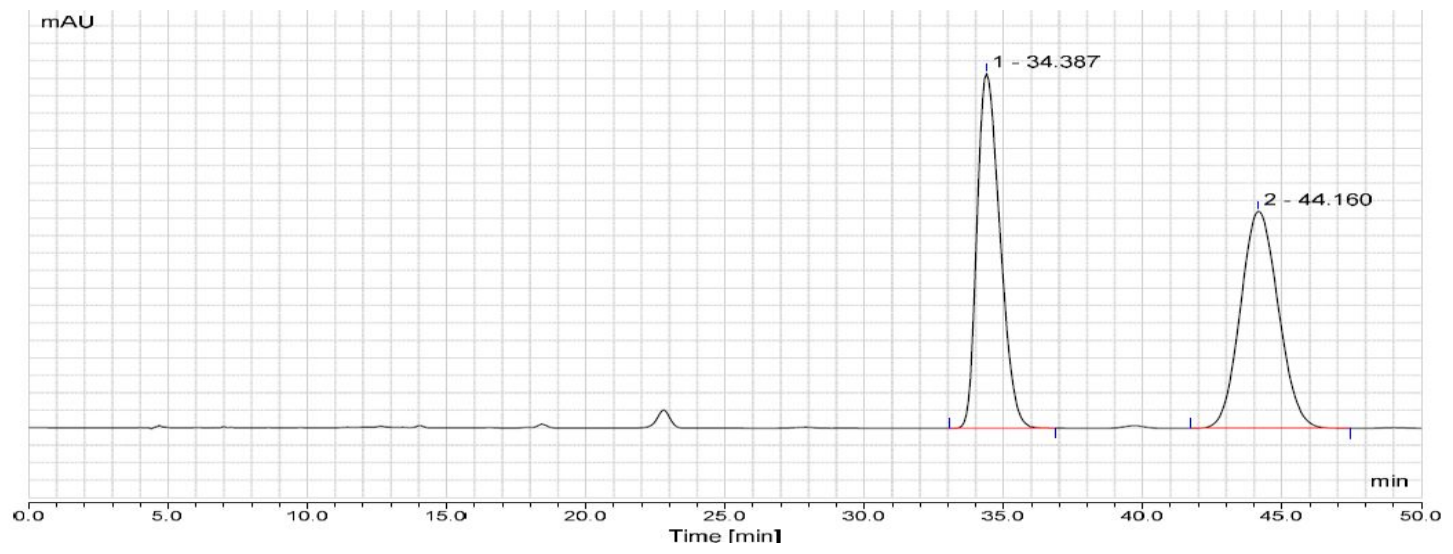

\begin{tabular}{|c|c|c|c|c|c|}
\hline \multicolumn{6}{|c|}{ Integration Results } \\
\hline No. & \begin{tabular}{|l|} 
Retention Time \\
min \\
\end{tabular} & $\begin{array}{c}\text { Retention Time } \\
\text { min }\end{array}$ & $\begin{array}{c}\text { Area } \\
\mathrm{mAU}^{*} \mathrm{~min}\end{array}$ & $\begin{array}{c}\text { Height } \\
\text { mAU }\end{array}$ & $\begin{array}{c}\text { Relative Area } \\
\%\end{array}$ \\
\hline 1 & 34 & 34.387 & 485.432 & 507.590 & 49.75 \\
\hline 2 & 44 & 44.160 & 490.367 & 310.580 & 50.25 \\
\hline \multicolumn{2}{|c|}{ Total: } & 975.799 & 818.169 & 100.00 & \\
\hline
\end{tabular}




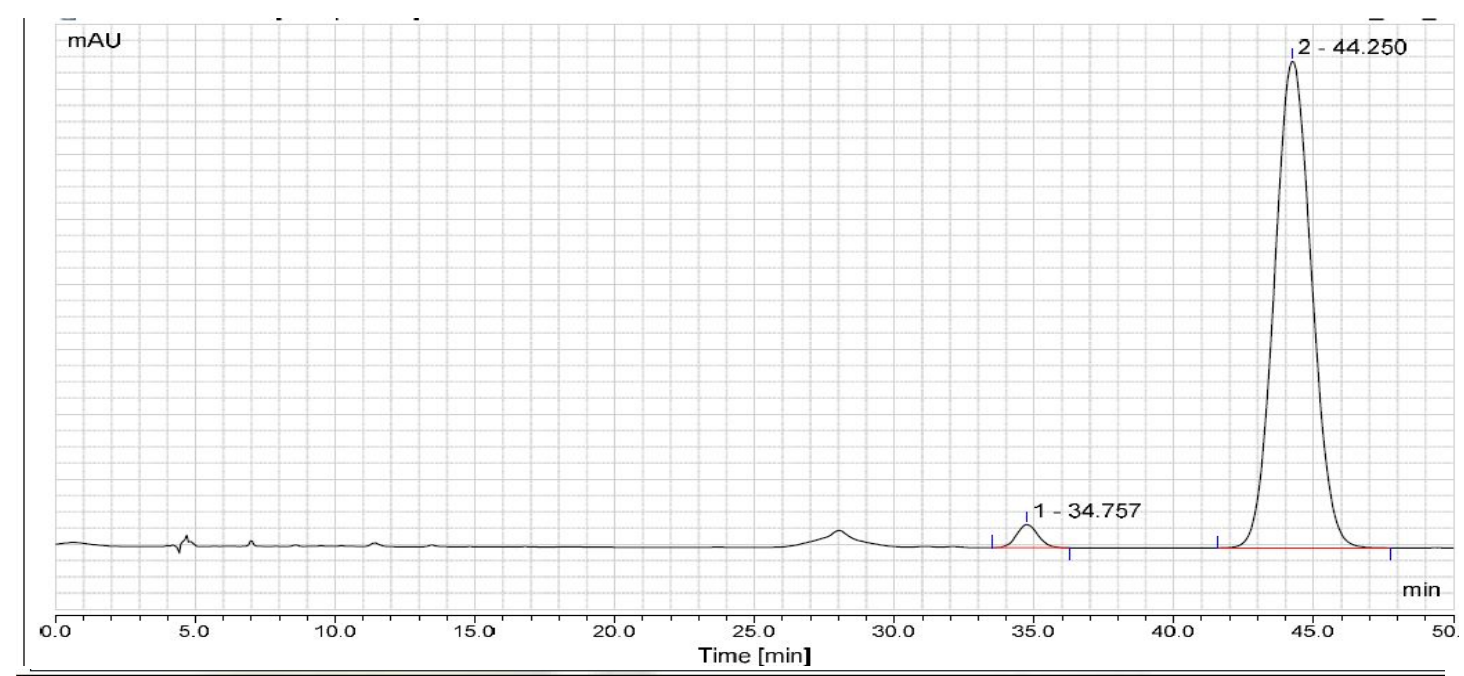

\begin{tabular}{|c|c|c|c|c|c|}
\hline \multicolumn{6}{|c|}{ Integration Results } \\
\hline No. & $\begin{array}{l}\text { Retention Time } \\
\text { min }\end{array}$ & $\begin{array}{l}\text { Retention Time } \\
\text { min }\end{array}$ & $\begin{array}{c}\text { Area } \\
m A U^{*} \min \end{array}$ & $\begin{array}{c}\text { Height } \\
\text { mAU }\end{array}$ & $\begin{array}{c}\text { Relative Area } \\
\%\end{array}$ \\
\hline $\begin{array}{l}1 \\
2\end{array}$ & $\begin{array}{l}35 \\
44\end{array}$ & $\begin{array}{l}34.757 \\
44250\end{array}$ & 6.310 & 7.141 & 2.66 \\
\hline \multicolumn{2}{|c|}{ Total: } & 237.500 & 156.901 & 100.00 & \\
\hline
\end{tabular}

(R)- triisopropyl (6,6,6-trifluoro-3-(2-fluorophenyl) hex-1-yn-1-yl) silane (d11)

\section{d11 - ${ }^{1} \mathrm{H}$ NMR}

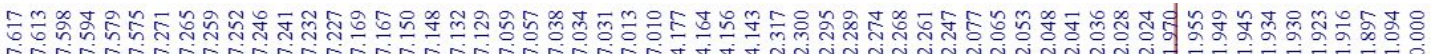

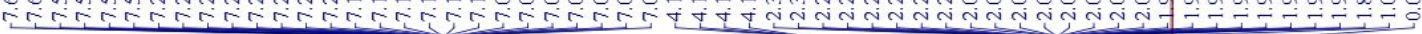
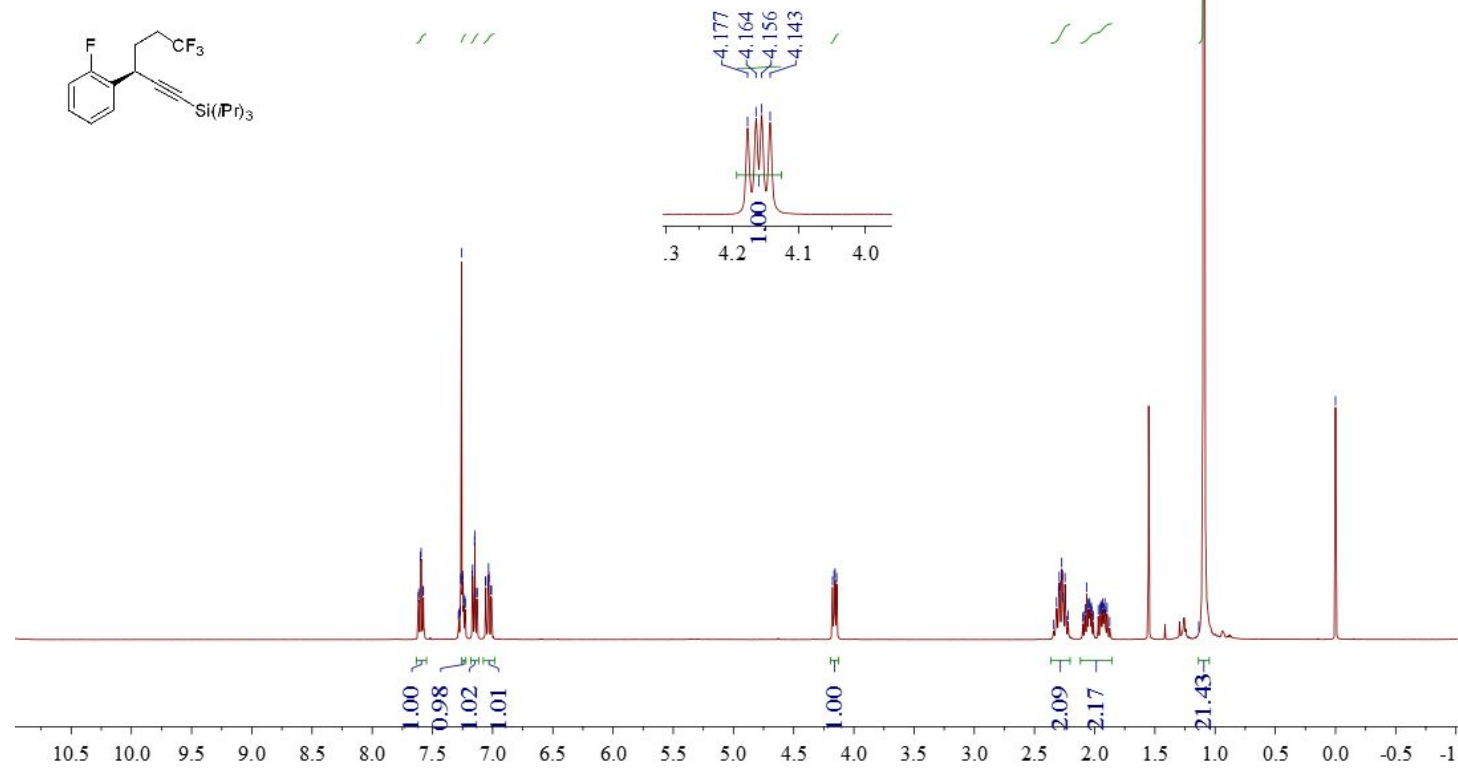
d11 - ${ }^{13}$ C NMR

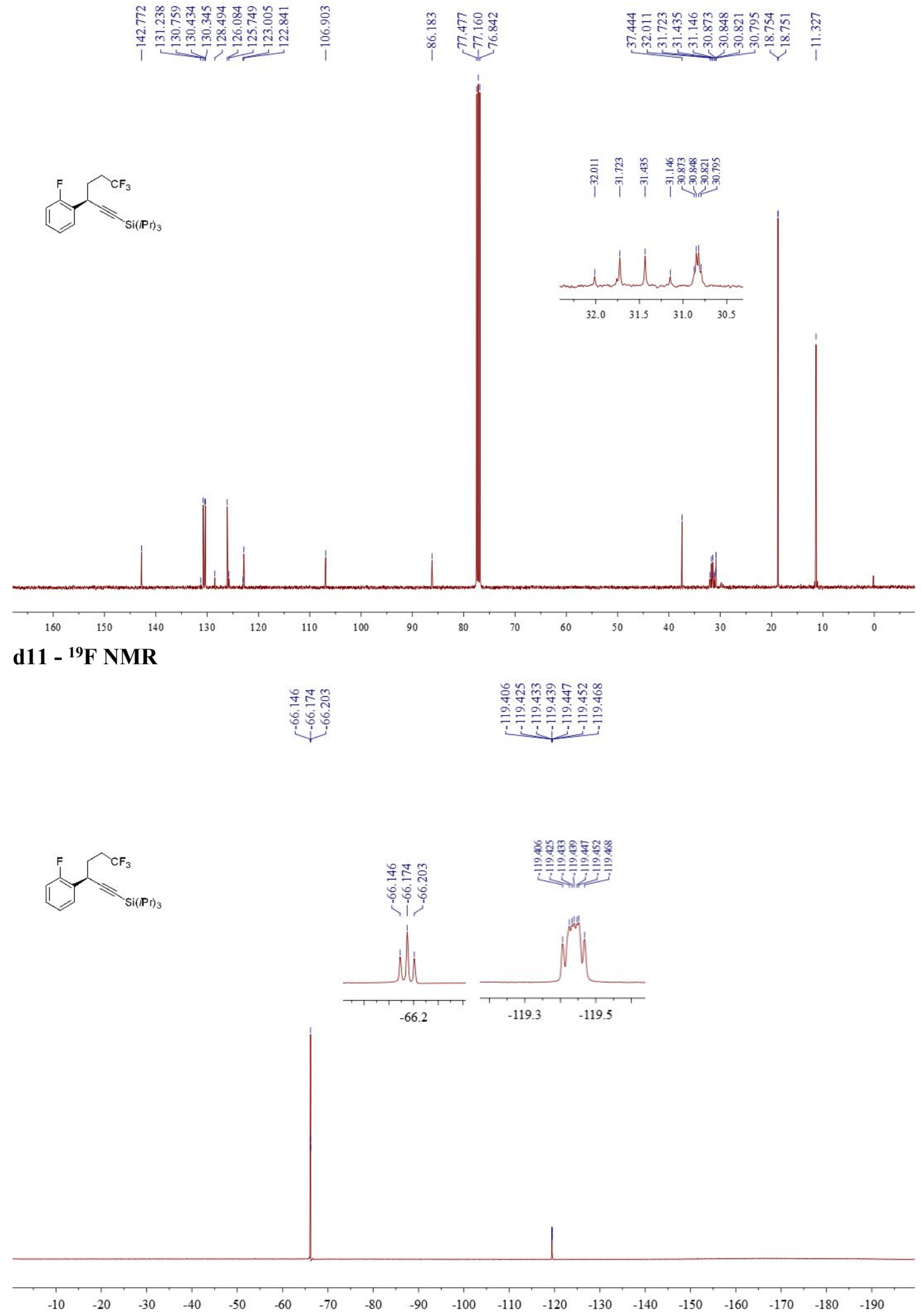




\section{d11 - HPLC}

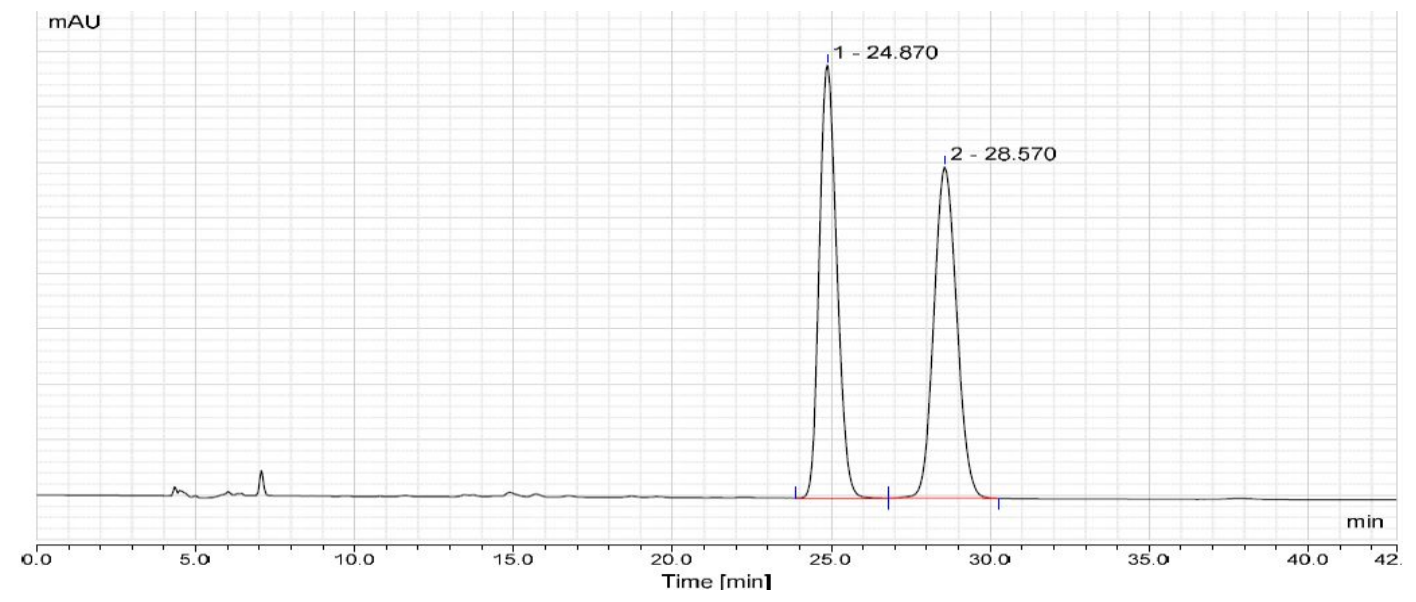

\begin{tabular}{|c|c|c|c|c|c|}
\hline \multicolumn{6}{|c|}{ Integration Results } \\
\hline No. & $\begin{array}{l}\text { Retention Time } \\
\text { min }\end{array}$ & $\begin{array}{c}\text { Retention Time } \\
\text { min }\end{array}$ & $\begin{array}{c}\text { Area } \\
m A U^{*} \min \end{array}$ & $\begin{array}{c}\text { Height } \\
\text { mAU }\end{array}$ & $\begin{array}{c}\text { Relative Area } \\
\%\end{array}$ \\
\hline 1 & 25 & 24.870 & 124.120 & 195.140 & 49.97 \\
\hline 2 & 29 & 28.570 & 124.272 & 149.377 & 50.03 \\
\hline \multicolumn{2}{|c|}{ Total: } & 248.392 & 344.517 & 100.00 & \\
\hline
\end{tabular}

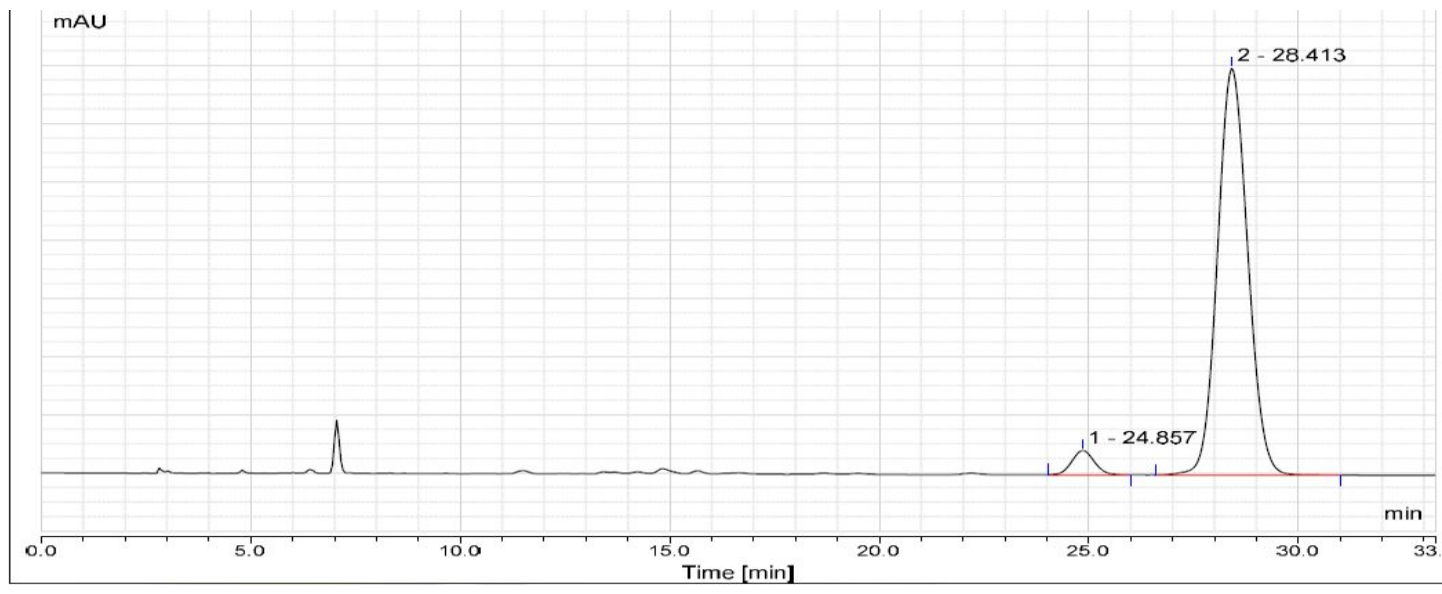

\begin{tabular}{|c|c|c|c|c|c|}
\hline \multicolumn{6}{|c|}{ Integration Results } \\
\hline$\overline{\text { No. }}$ & $\begin{array}{l}\text { Retention Time } \\
\text { min }\end{array}$ & $\begin{array}{c}\text { Retention Time } \\
\text { min }\end{array}$ & $\begin{array}{c}\text { Area } \\
m A U^{*} \min \end{array}$ & $\begin{array}{c}\text { Height } \\
\text { mAU }\end{array}$ & $\begin{array}{c}\text { Relative Area } \\
\%\end{array}$ \\
\hline 1 & 25 & 24.857 & 5.112 & 8.326 & 4.18 \\
\hline 2 & 28 & 28.413 & 117.189 & 139.824 & 95.82 \\
\hline \multicolumn{2}{|c|}{ Total: } & 122.300 & 148.150 & 100.00 & \\
\hline
\end{tabular}


(R)- triisopropyl(6,6,6-trifluoro-3-(naphthalen-2-yl)hex-1-yn-1-yl) silane (d12) d12 - ${ }^{1}$ H NMR
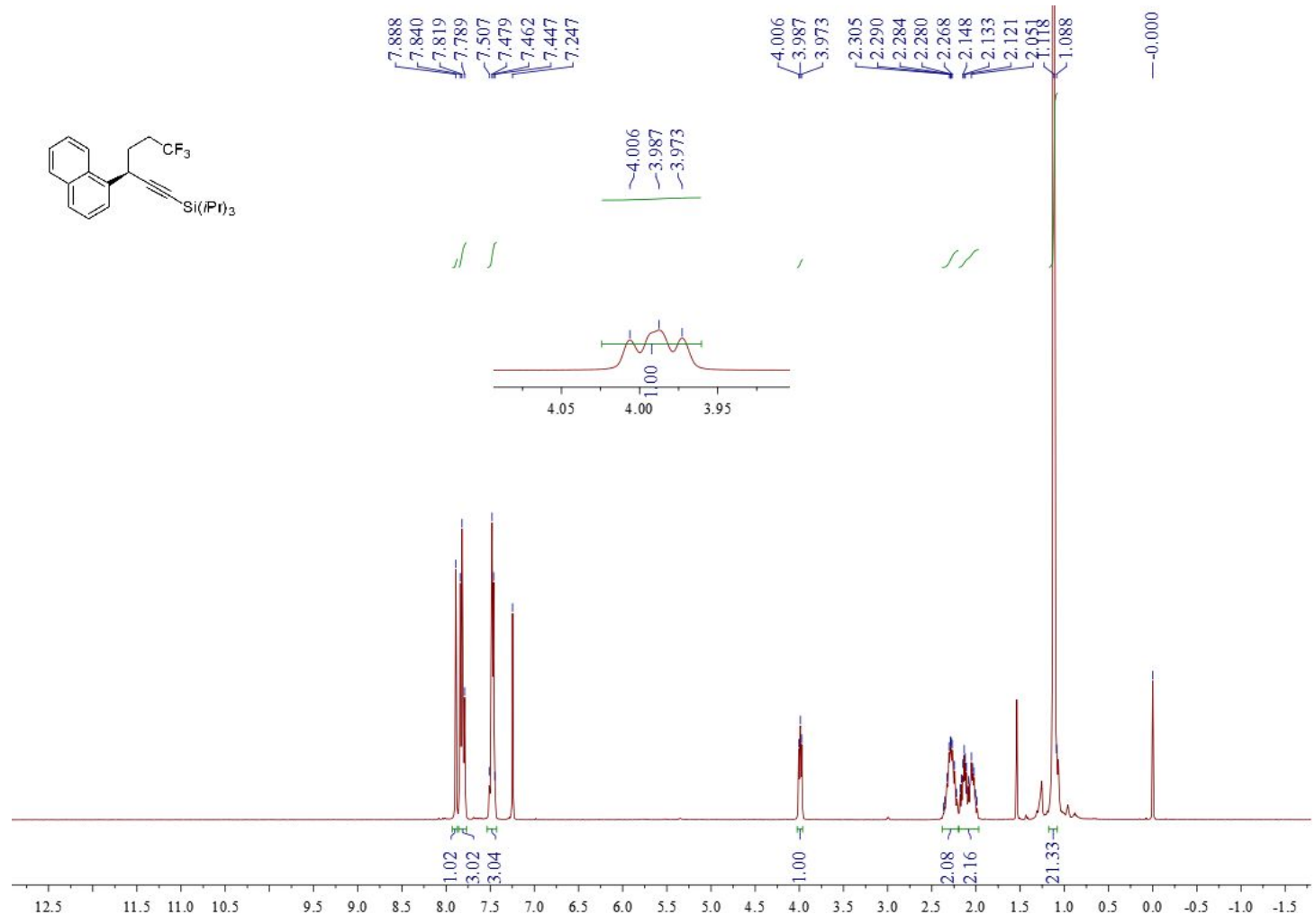
d12 - ${ }^{13}$ C NMR

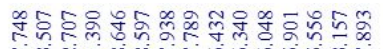

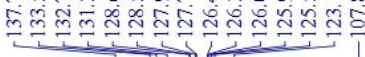

\section{$\infty$}

$\infty$ ह5<smiles>FC(F)(F)CCC(C#C[AlH2])c1cccc2ccccc12</smiles>
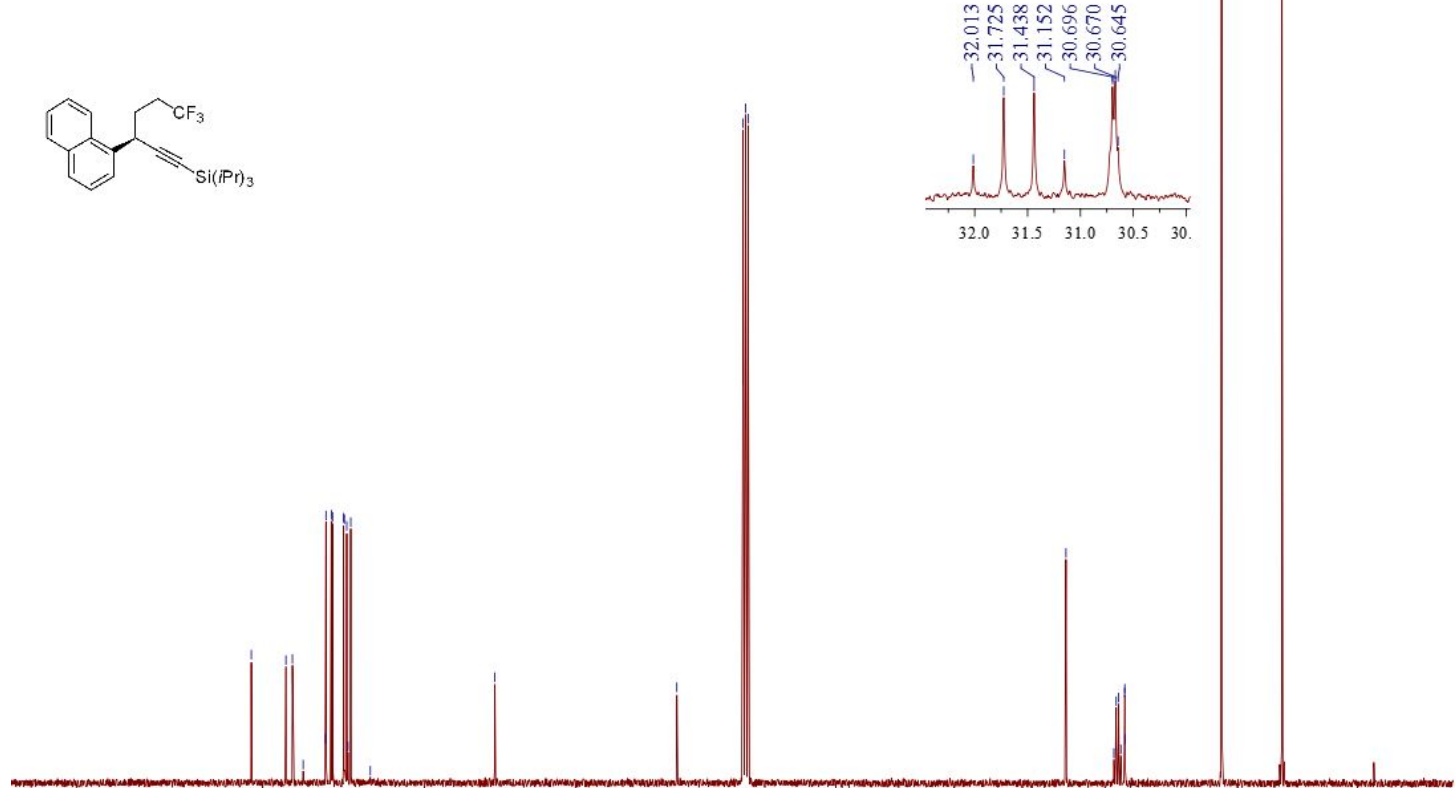

160

$150 \quad 140$

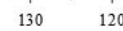

$110 \quad 100$ 
d12 - ${ }^{19}$ F NMR

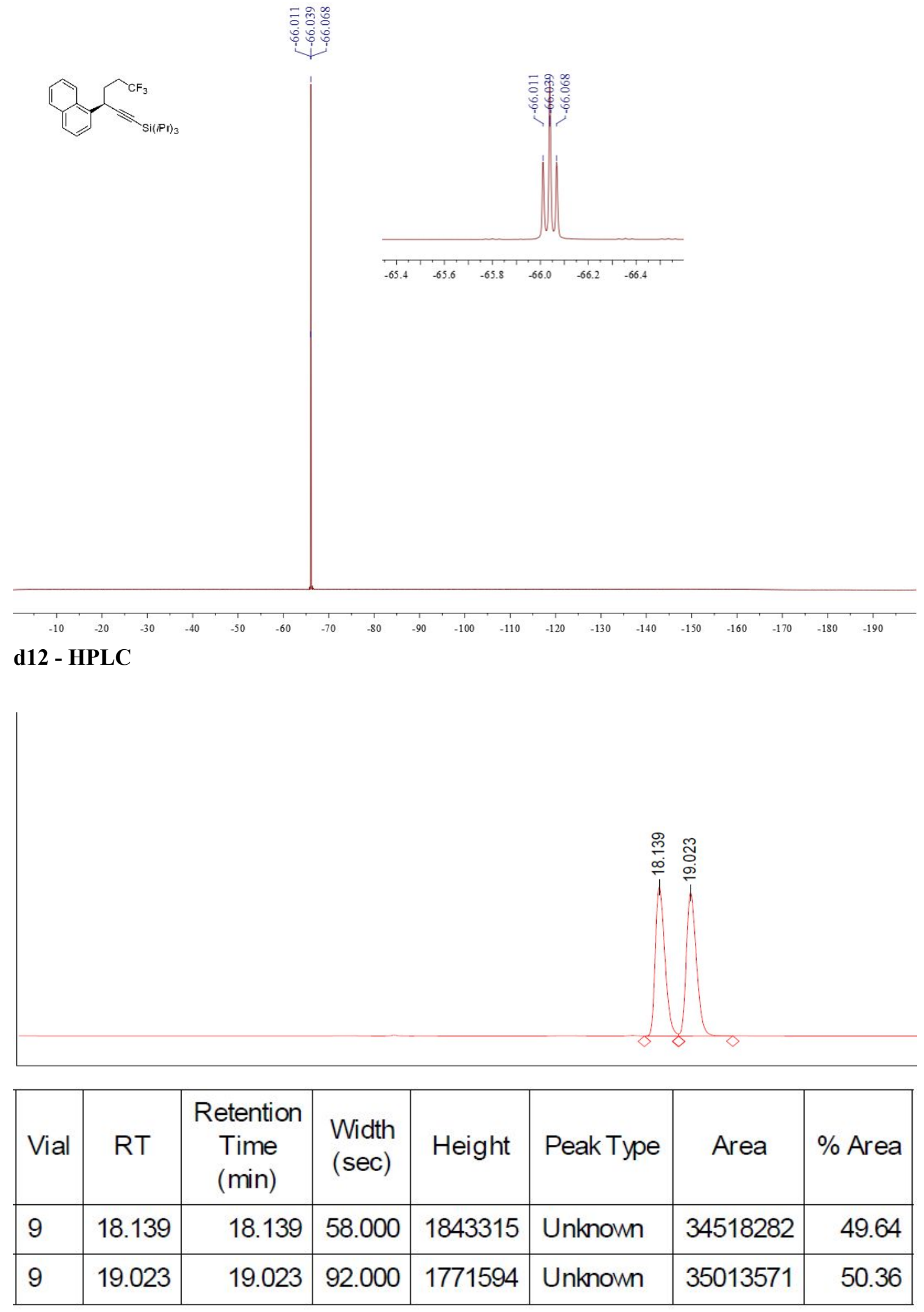




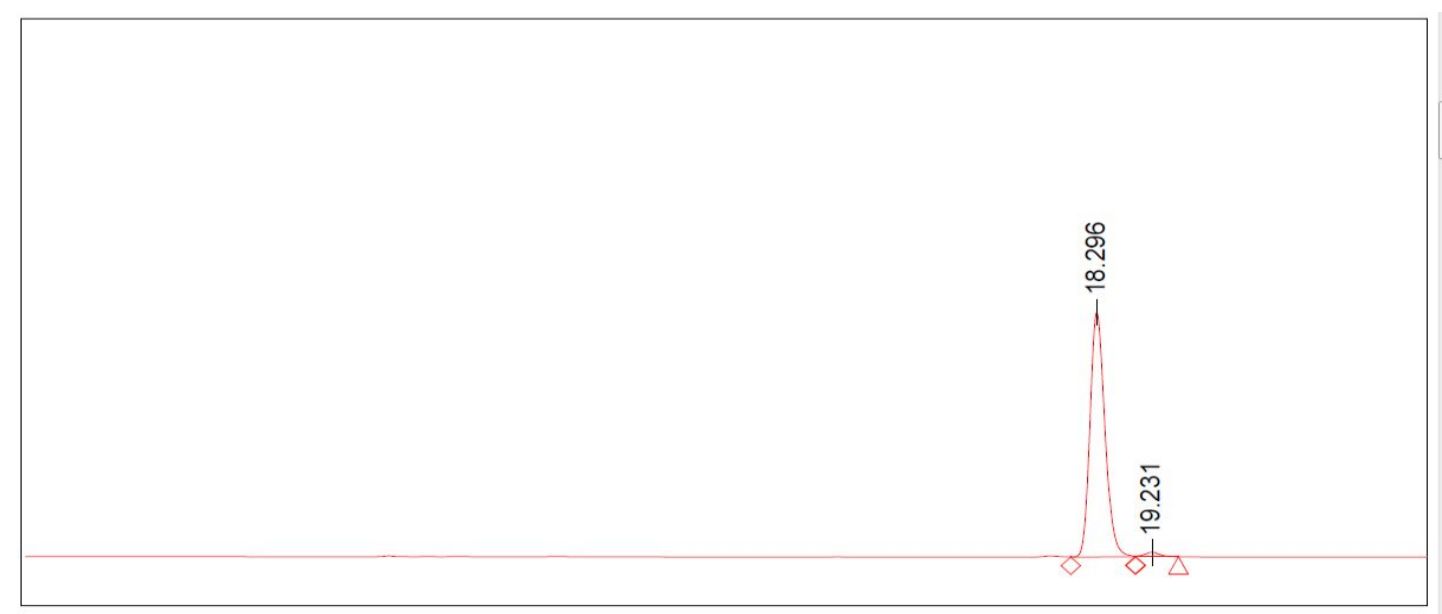

\begin{tabular}{|l|r|r|r|r|l|r|r|}
\hline Vial & RT & $\begin{array}{c}\text { Retention } \\
\text { Time } \\
\text { (min) }\end{array}$ & $\begin{array}{l}\text { Width } \\
(\mathrm{sec})\end{array}$ & Height & Peak Type & Area & \% Area \\
\hline 25 & 18.296 & 18.296 & 66.000 & 596098 & Unknown & 10494831 & 98.19 \\
\hline 25 & 19.231 & 19.231 & 44.000 & 10502 & Unknown & 193408 & 1.81 \\
\hline
\end{tabular}

(R)- triisopropyl (6, 6, 6-trifluoro-3-(thiophen-2-yl) hex-1-yn-1-yl) silane (d13) d13 - 'H NMR

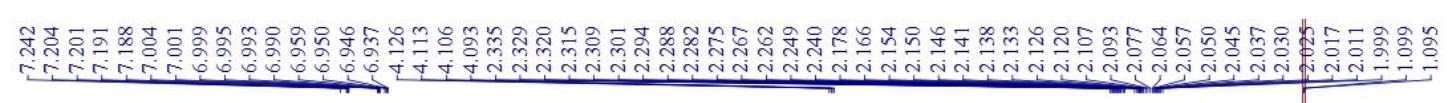
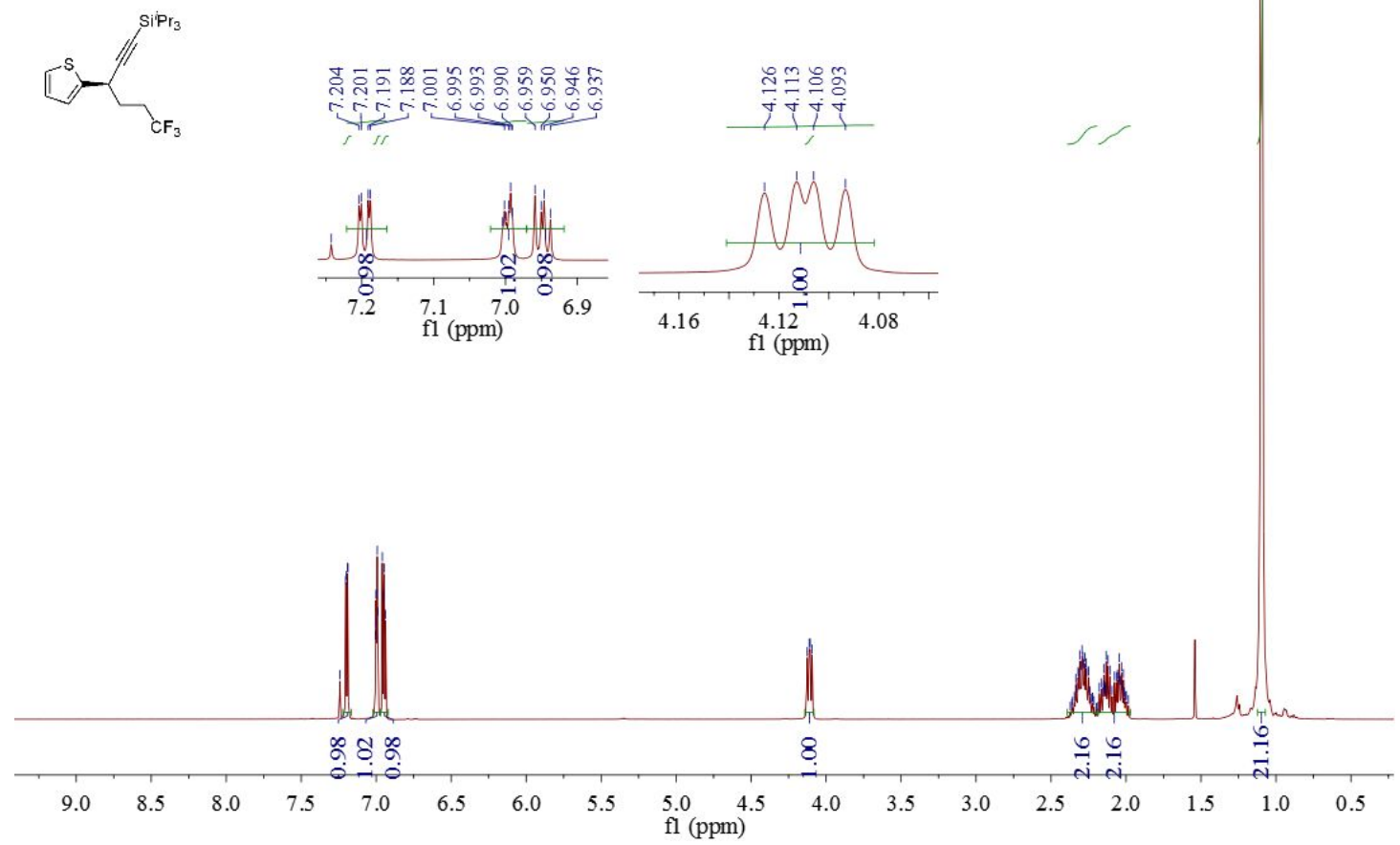


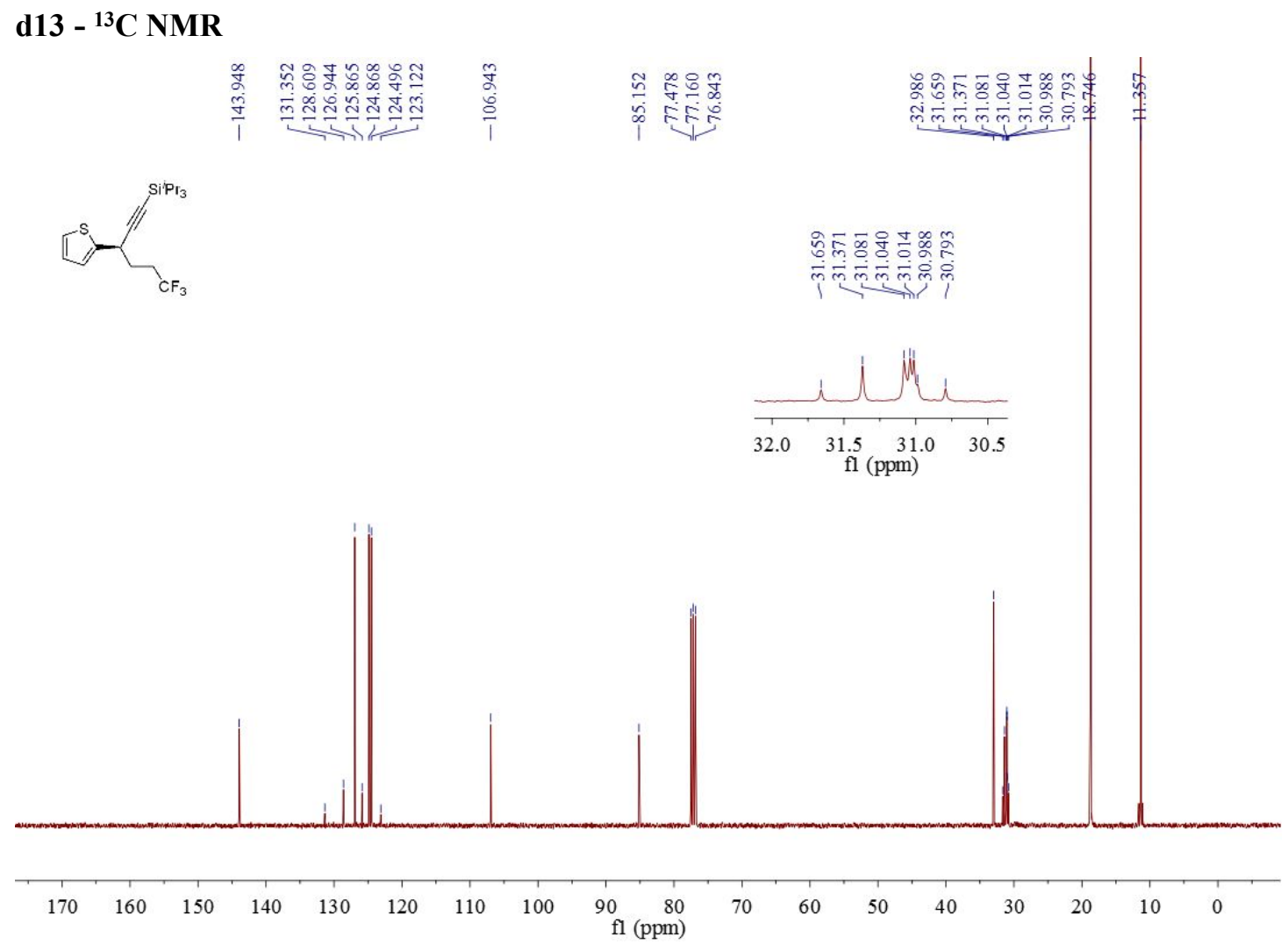

d13 - ${ }^{19}$ F NMR

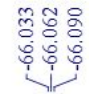

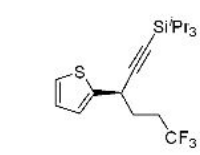

$\begin{array}{lllllll}-60.5 & -61.5 & -62.5 & -63.5 & -64.5 & -65.5\end{array}$ 
d13 - HPLC

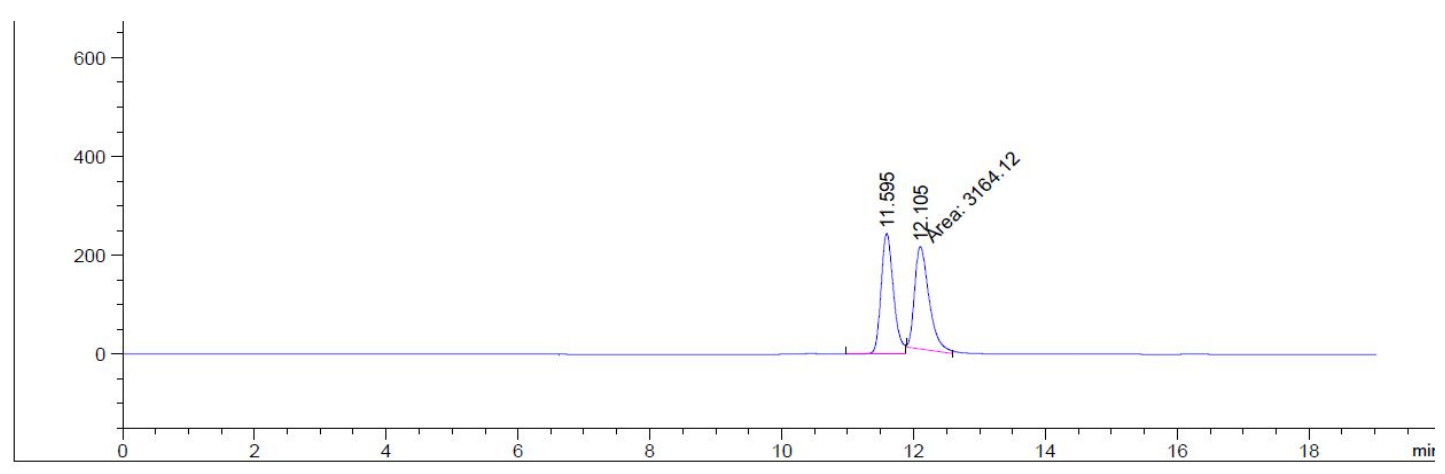

\begin{tabular}{|c|c|c|c|c|c|c|}
\hline $\begin{array}{c}\text { eak } \\
\#\end{array}$ & $\begin{array}{c}\text { RetTime } \\
{[\min ]}\end{array}$ & Type & $\begin{array}{l}\text { Width } \\
\text { [min] }\end{array}$ & $\begin{array}{c}\text { Area } \\
{\left[\mathrm{mAU}^{*} \mathrm{~s}\right]}\end{array}$ & $\begin{array}{l}\text { Height } \\
{[\mathrm{mAU}]}\end{array}$ & $\begin{array}{c}\text { Area } \\
\%\end{array}$ \\
\hline & & & & & & \\
\hline 1 & & & & 3198 & 07 & 20 \\
\hline 2 & & & & 3164.11646 & 207.45180 & 7286 \\
\hline
\end{tabular}

Totals :

$6362.84912 \quad 451.40787$
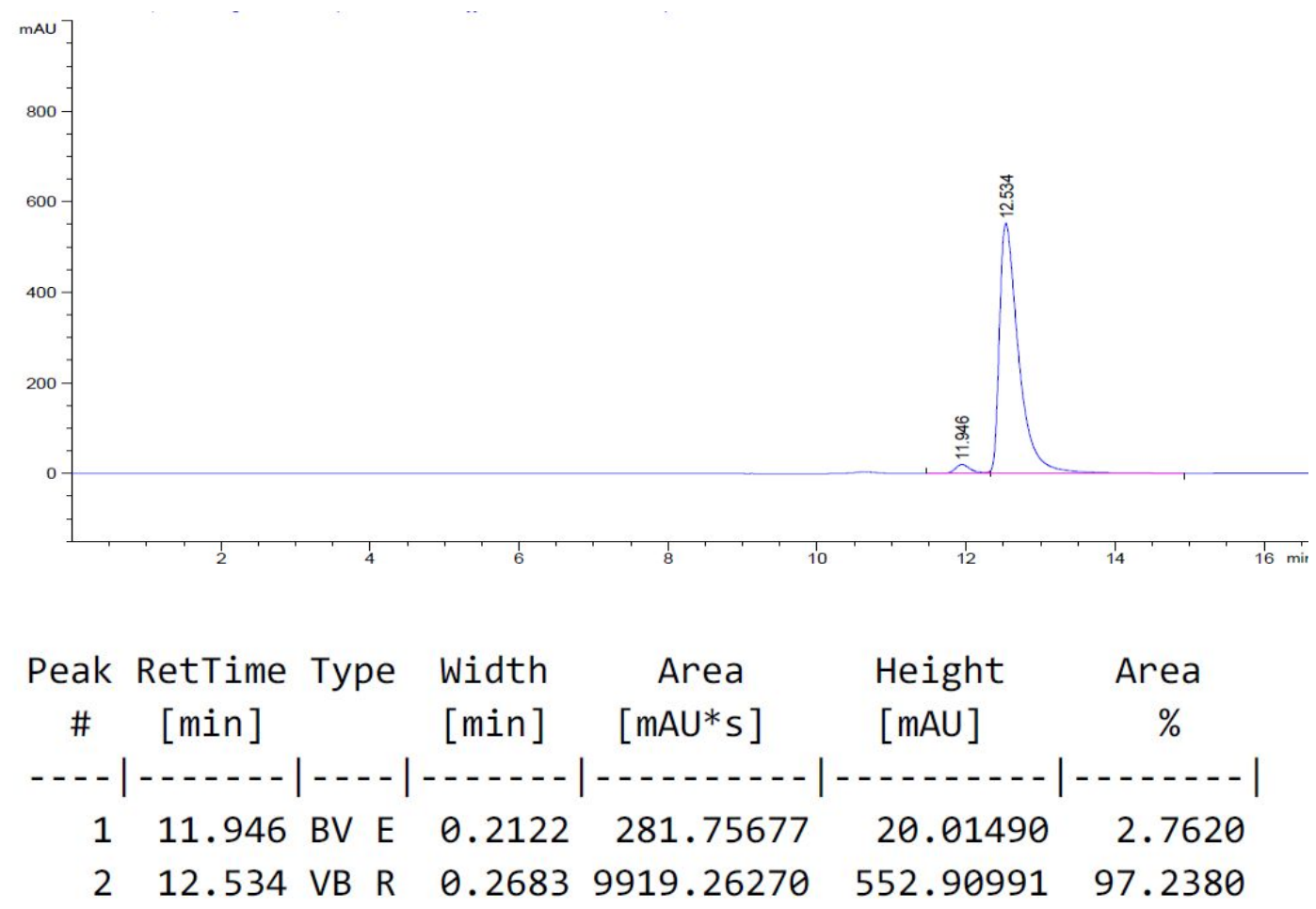

Totals :

$1.02010 \mathrm{e} 4 \quad 572.92482$ 
(R)- (3-([1, 1'-biphenyl]-4-yl)-6, 6, 6-trifluorohex-1-yn-1-yl) trimethylsilane (d14) d14 - ${ }^{1}$ H NMR

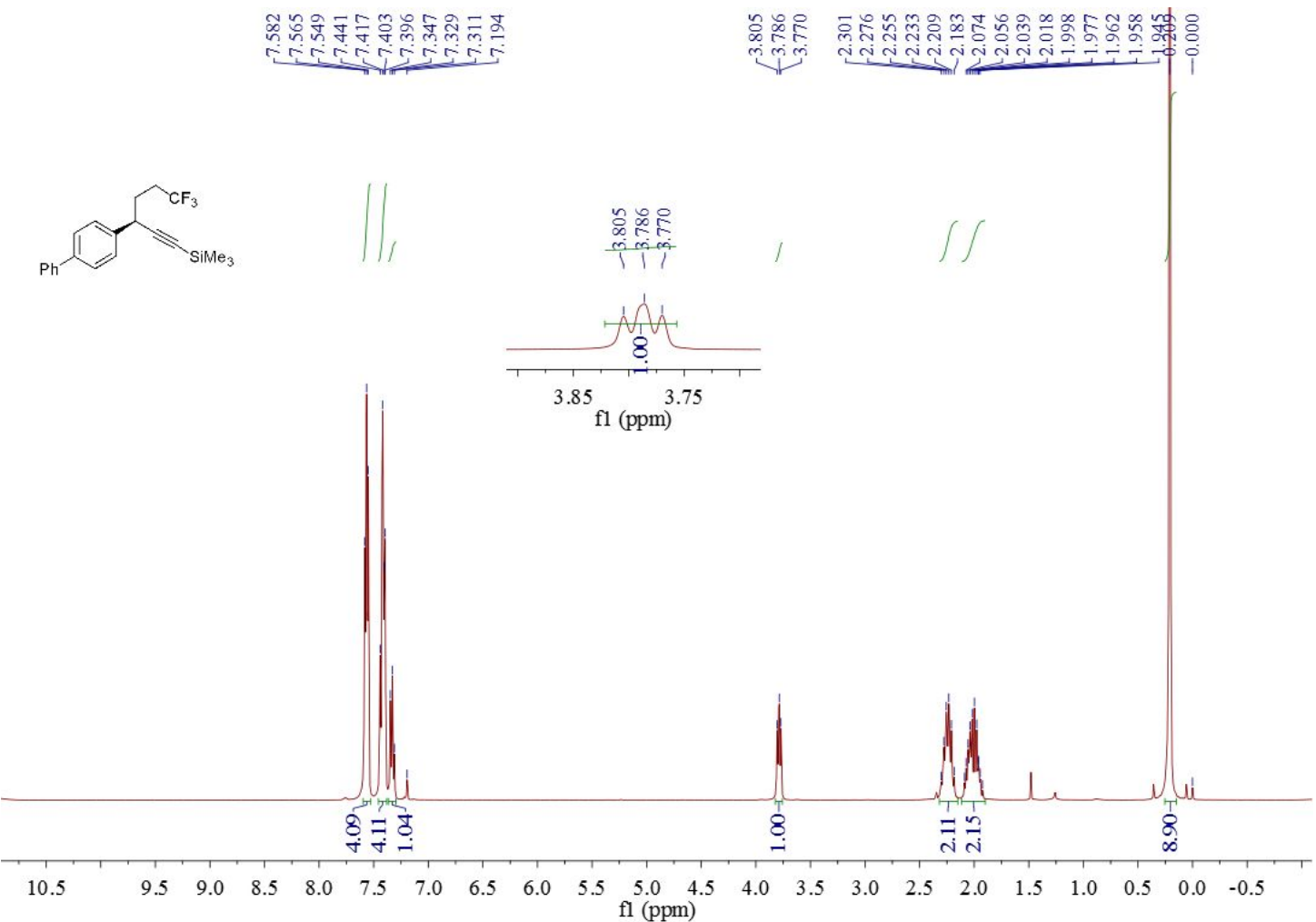

d14 - ${ }^{13}$ C NMR

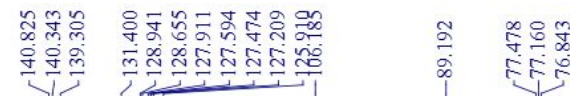

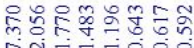

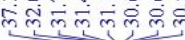

ले

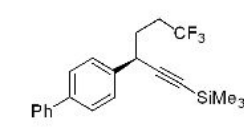

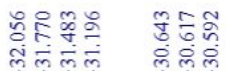

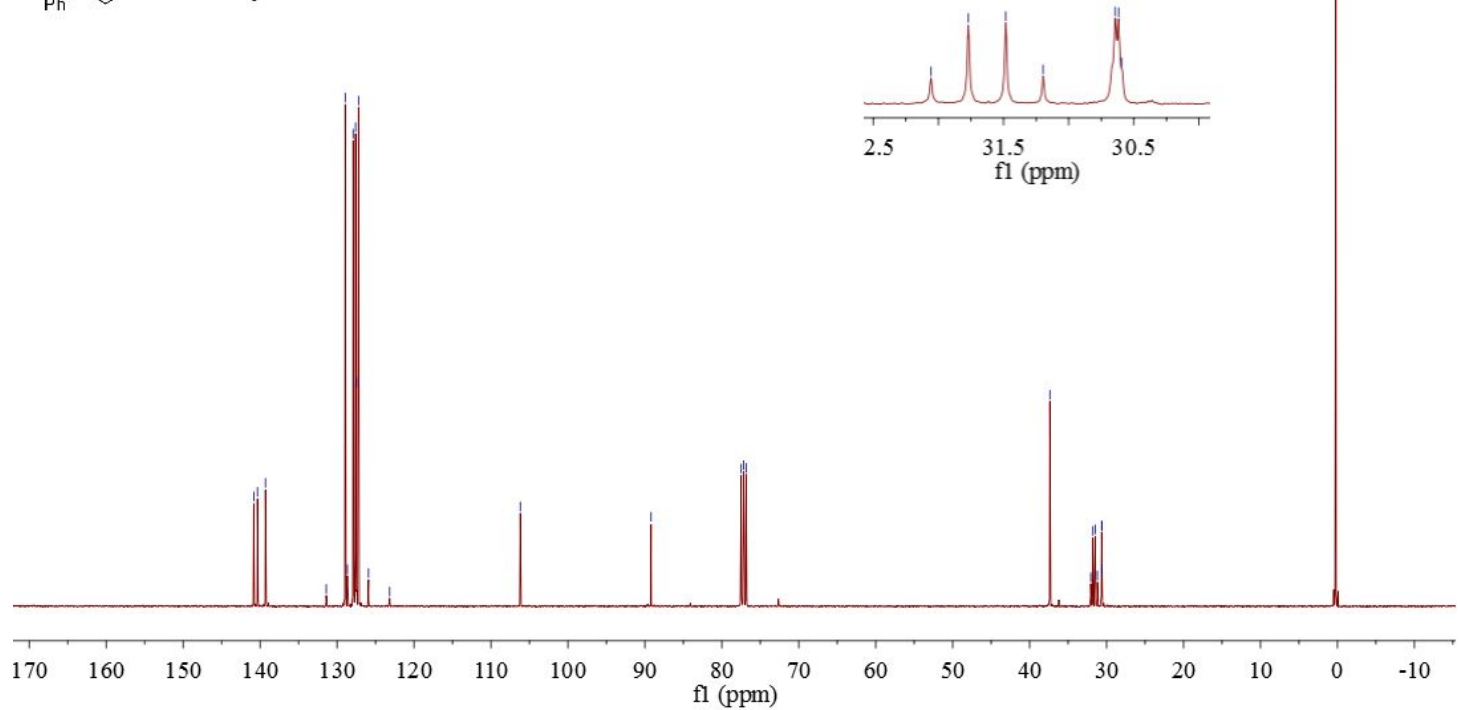




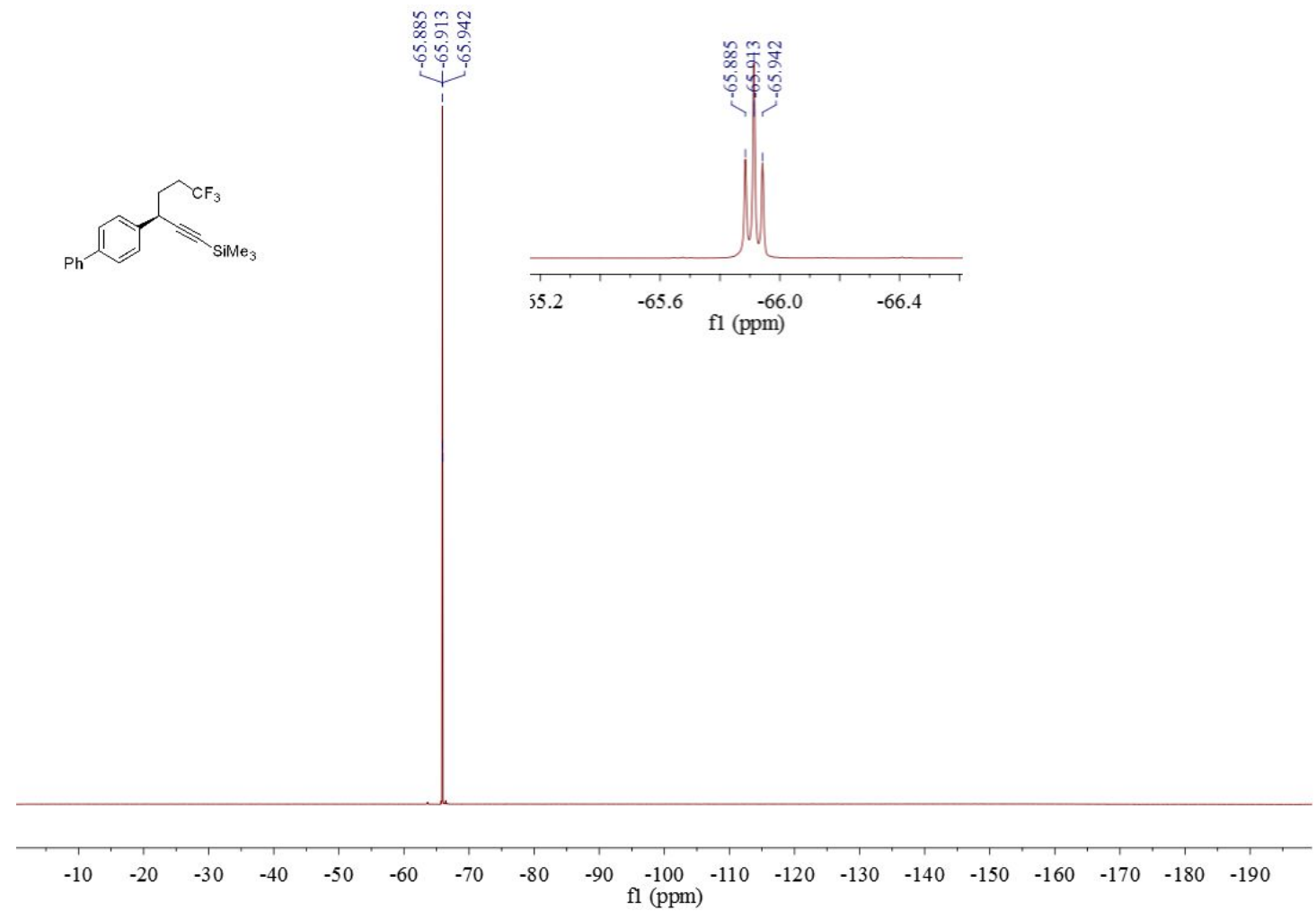

\section{d14 - HPLC}

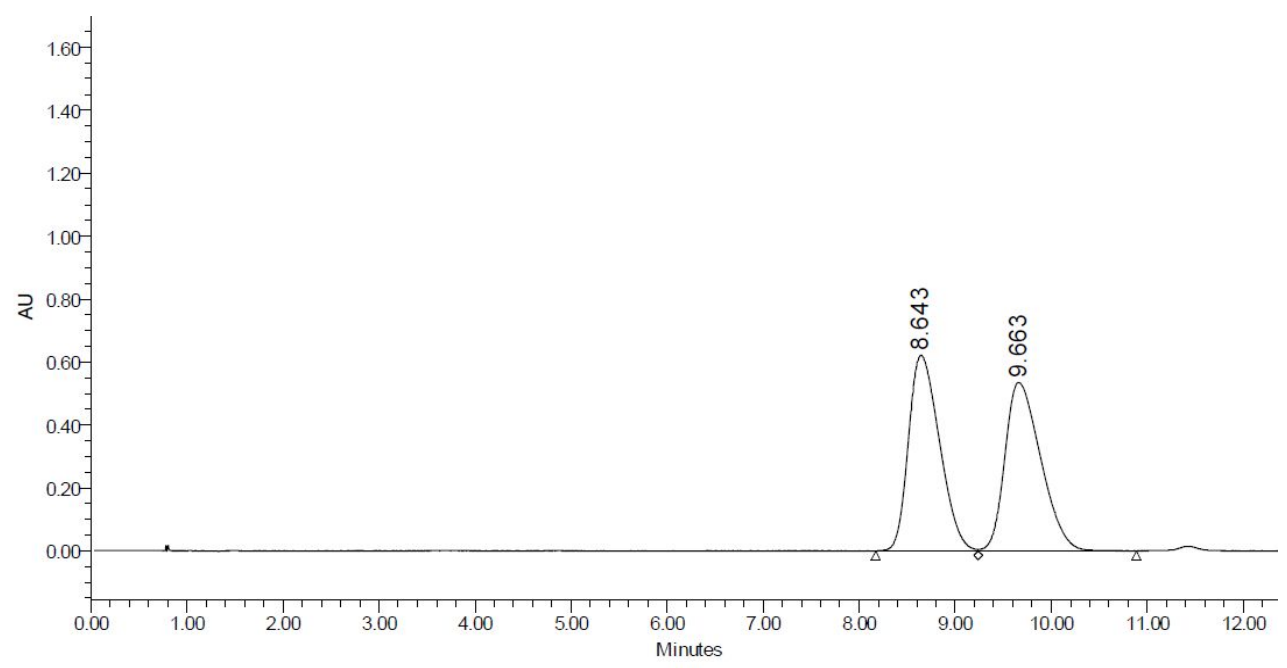

\begin{tabular}{|c|c|l|c|c|c|c|}
\hline & RT & Peak Type & Height & $\begin{array}{c}\text { Width } \\
(\mathrm{sec})\end{array}$ & Area & $\%$ Area \\
\hline 1 & 8.643 & Unknown & 621470 & 63.950 & 13874210 & 49.90 \\
\hline 2 & 9.663 & Unknown & 534926 & 98.800 & 13929007 & 50.10 \\
\hline
\end{tabular}




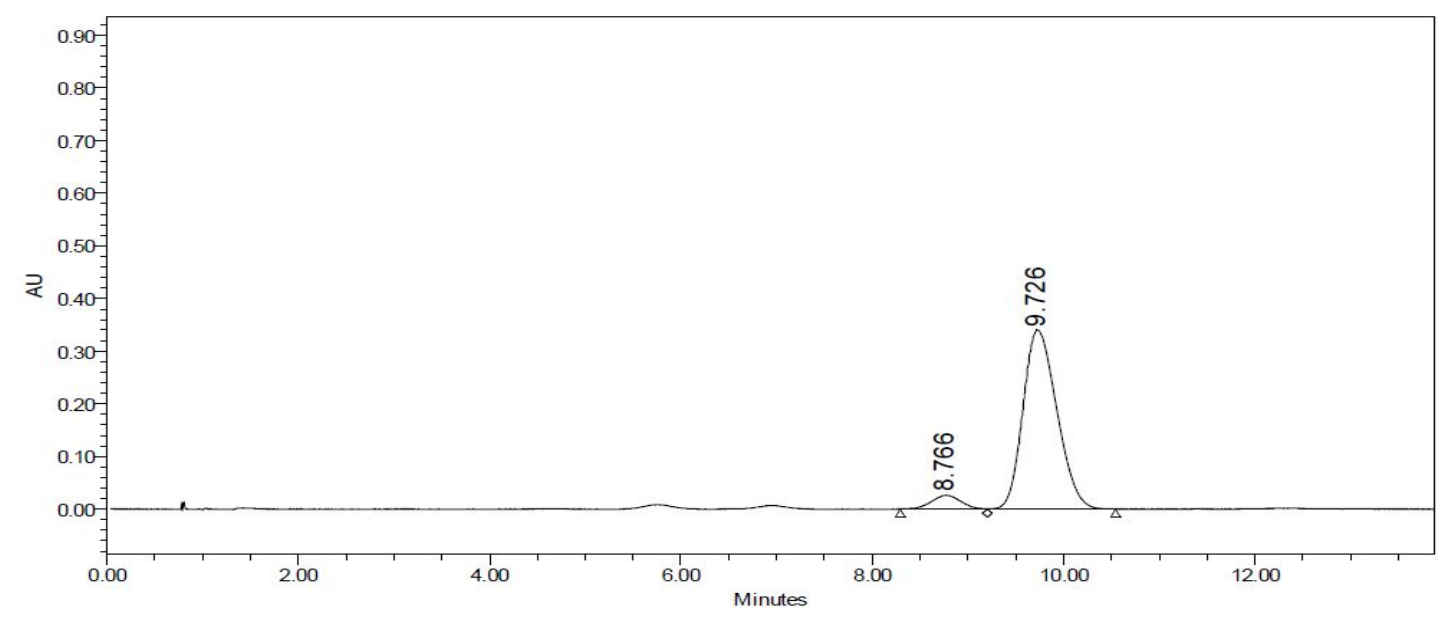

\begin{tabular}{|r|c|l|r|r|r|r|}
\hline & RT & Peak Type & Height & $\begin{array}{r}\text { Width } \\
(\mathrm{sec})\end{array}$ & Area & $\%$ Area \\
\hline 1 & 8.766 & Unknown & 25988 & 54.750 & 565013 & 6.34 \\
\hline 2 & 9.726 & Unknown & 340263 & 80.450 & 8346314 & 93.66 \\
\hline
\end{tabular}

(R)- (3-([1, 1'-biphenyl]-4-yl)-6, 6, 6-trifluorohex-1-yn-1-yl) triethylsilane (d15) d15 - ${ }^{1}$ H NMR

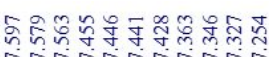

ing
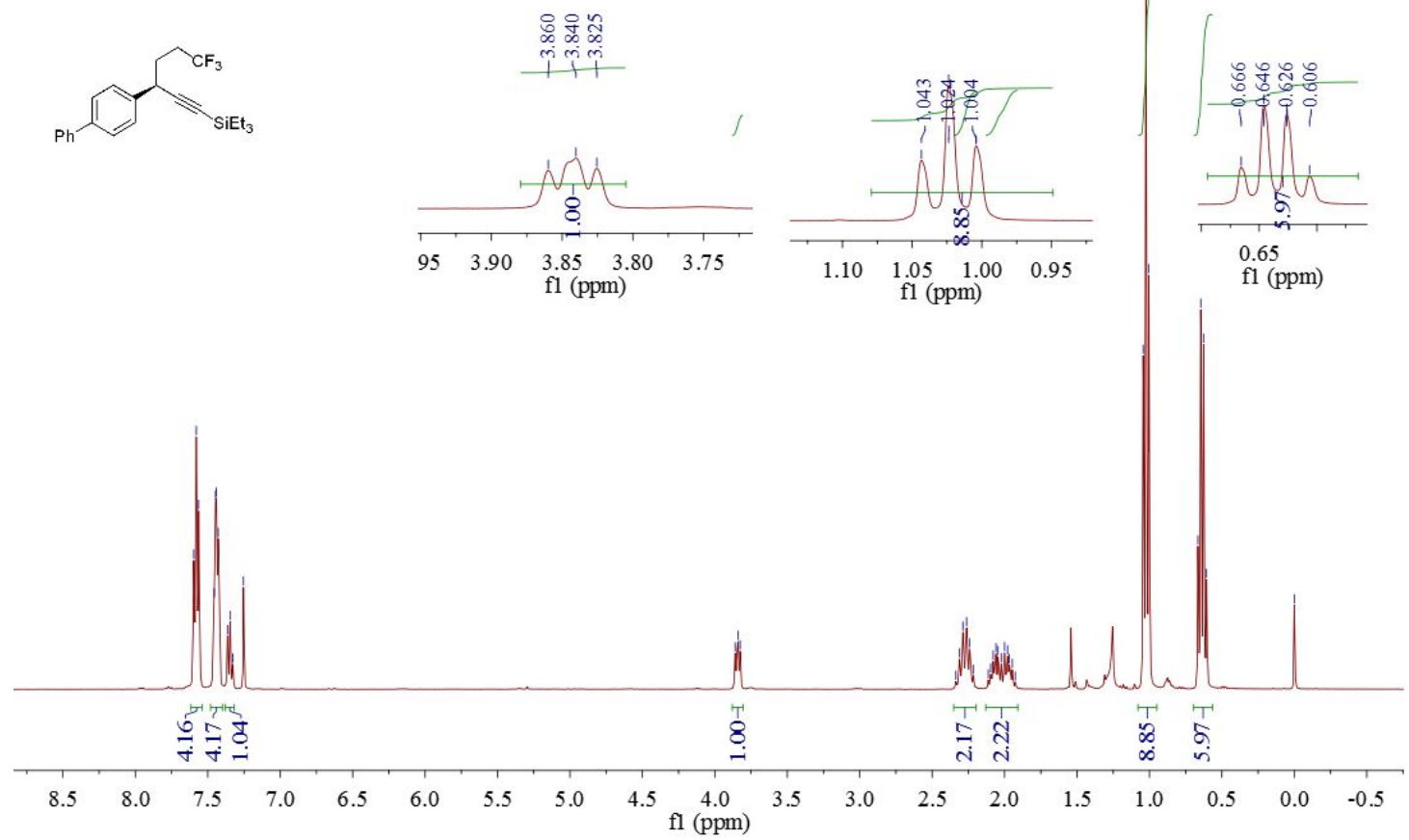
d15 - ${ }^{13}$ C NMR

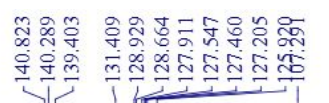

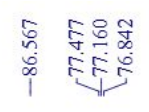

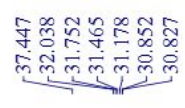

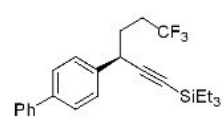

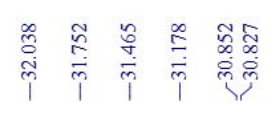

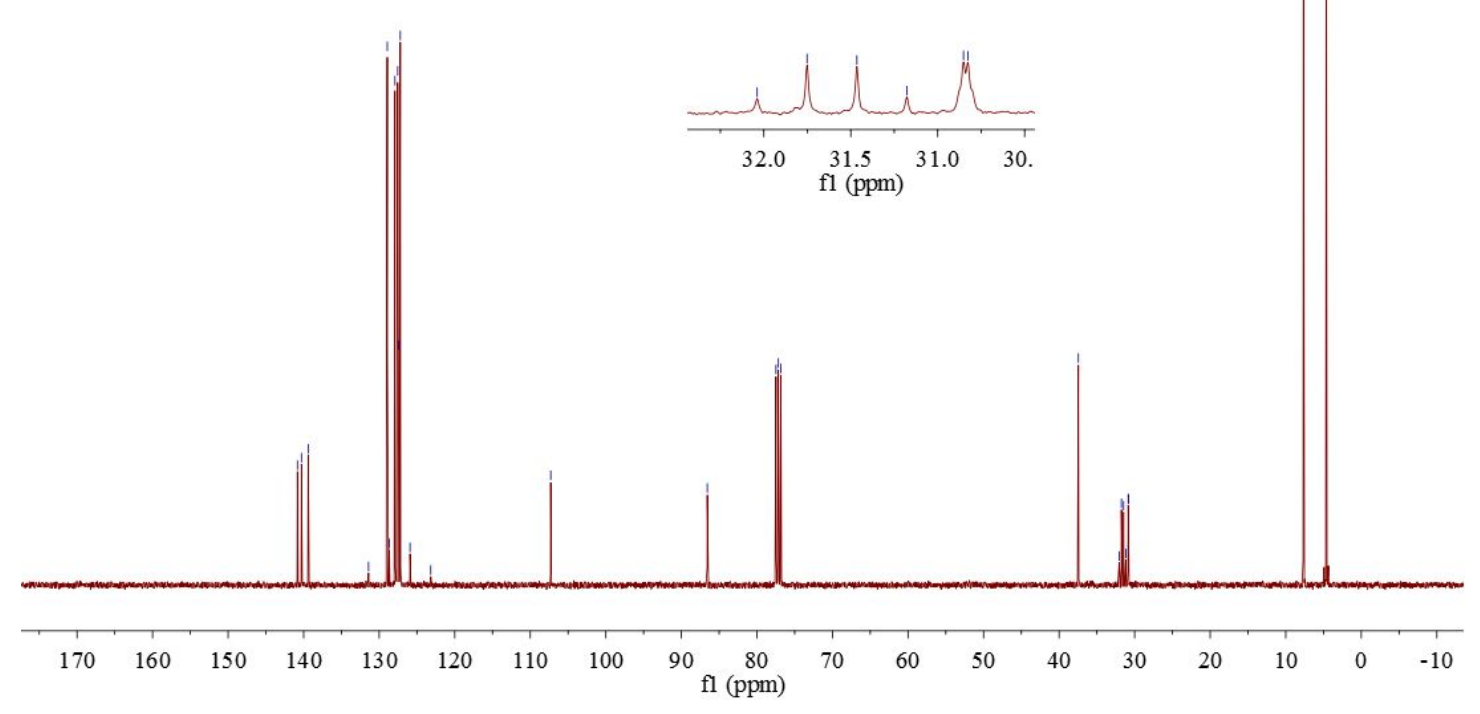

d15 - ${ }^{19}$ F NMR

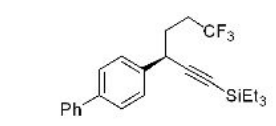

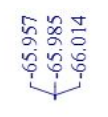
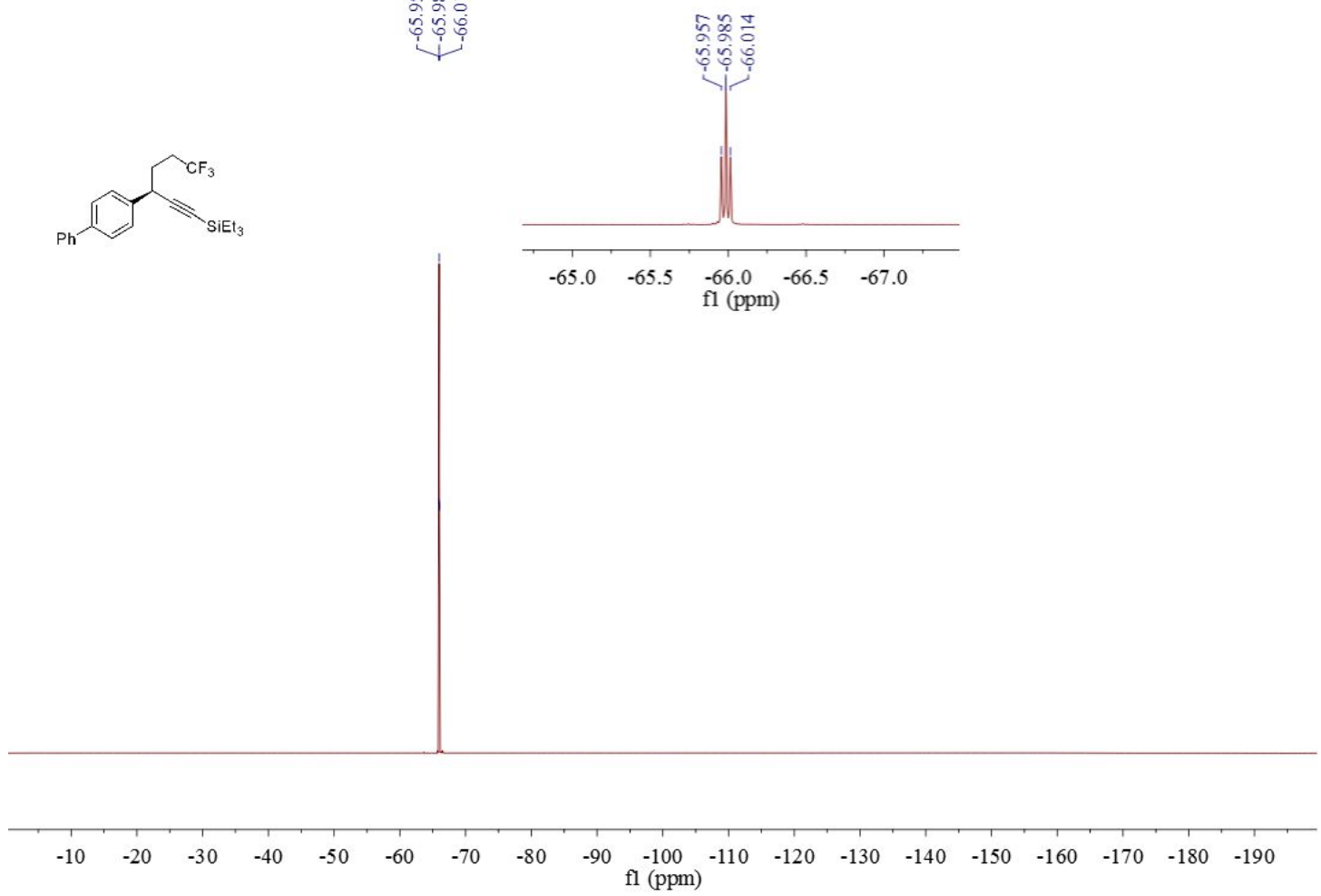

S71 


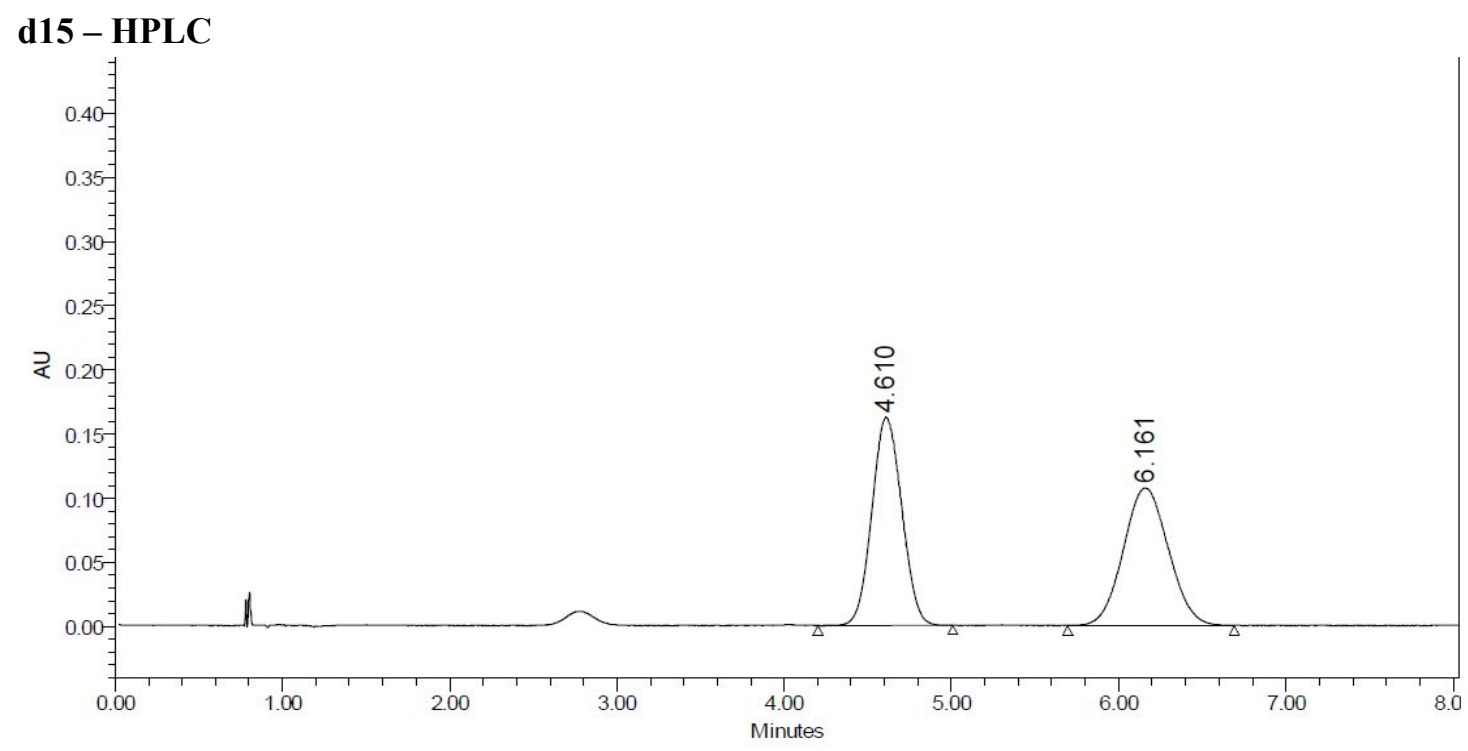

\begin{tabular}{|c|c|l|c|c|c|c|}
\hline & RT & Peak Type & Height & $\begin{array}{c}\text { Width } \\
(\mathrm{sec})\end{array}$ & Area & $\%$ Area \\
\hline 1 & 4.610 & Unknown & 162141 & 48.450 & 2014486 & 50.00 \\
\hline 2 & 6.161 & Unknown & 107318 & 59.750 & 2014262 & 50.00 \\
\hline
\end{tabular}

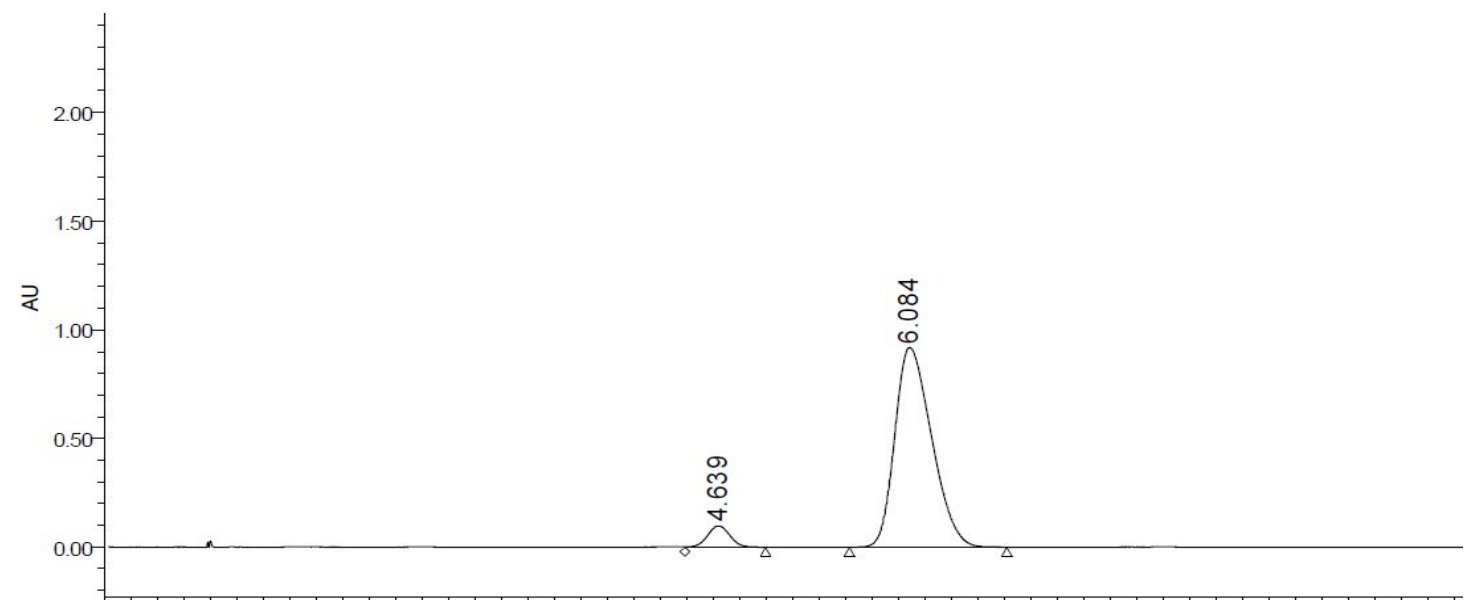

\begin{tabular}{|r|c|l|r|r|r|r|}
\hline & RT & Peak Type & Height & $\begin{array}{c}\text { Width } \\
(\mathrm{sec})\end{array}$ & Area & \% Area \\
\hline 1 & 4.639 & Unknown & 97874 & 36.500 & 1129778 & 5.96 \\
\hline 2 & 6.084 & Unknown & 917544 & 71.450 & 17836824 & 94.04 \\
\hline
\end{tabular}


(R)-4-(1, 1, 1-trifluorodec-5-yn-4-yl)-1, 1'-biphenyl (d16)

d16 - ${ }^{1} H$ NMR

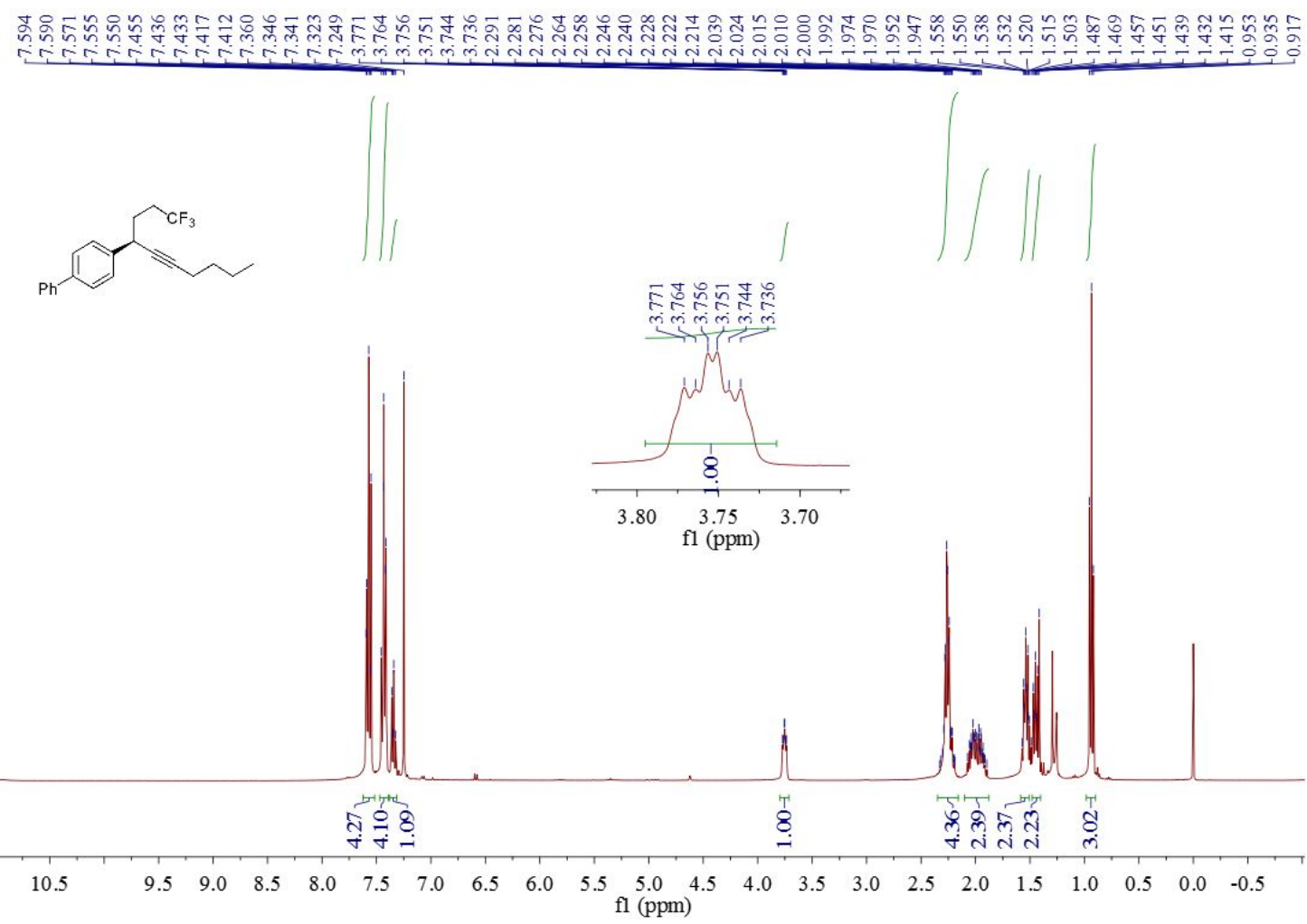

d16 - ${ }^{13}$ C NMR

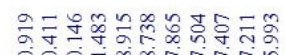

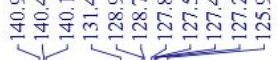

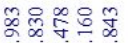

क्षिह5

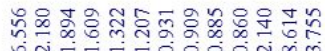

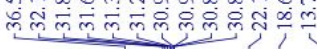

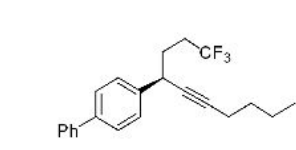

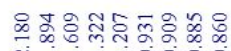

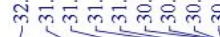
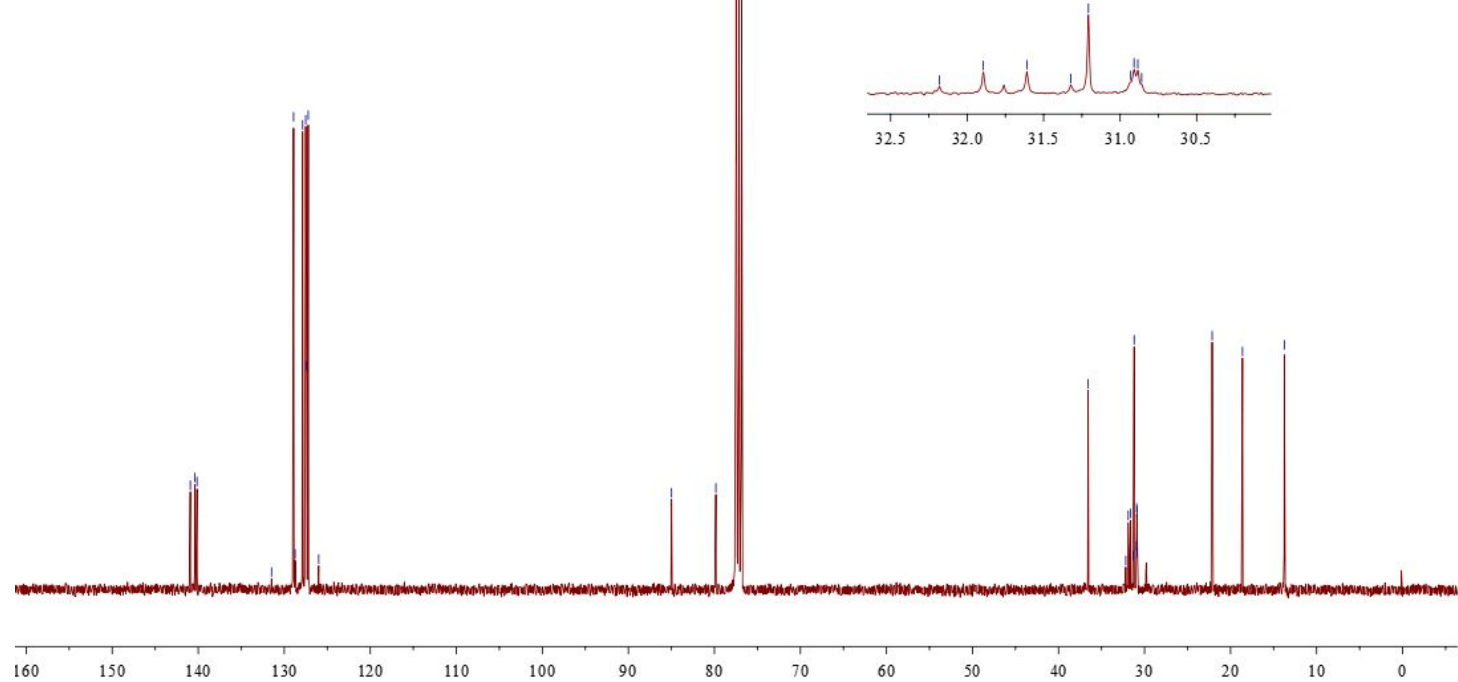


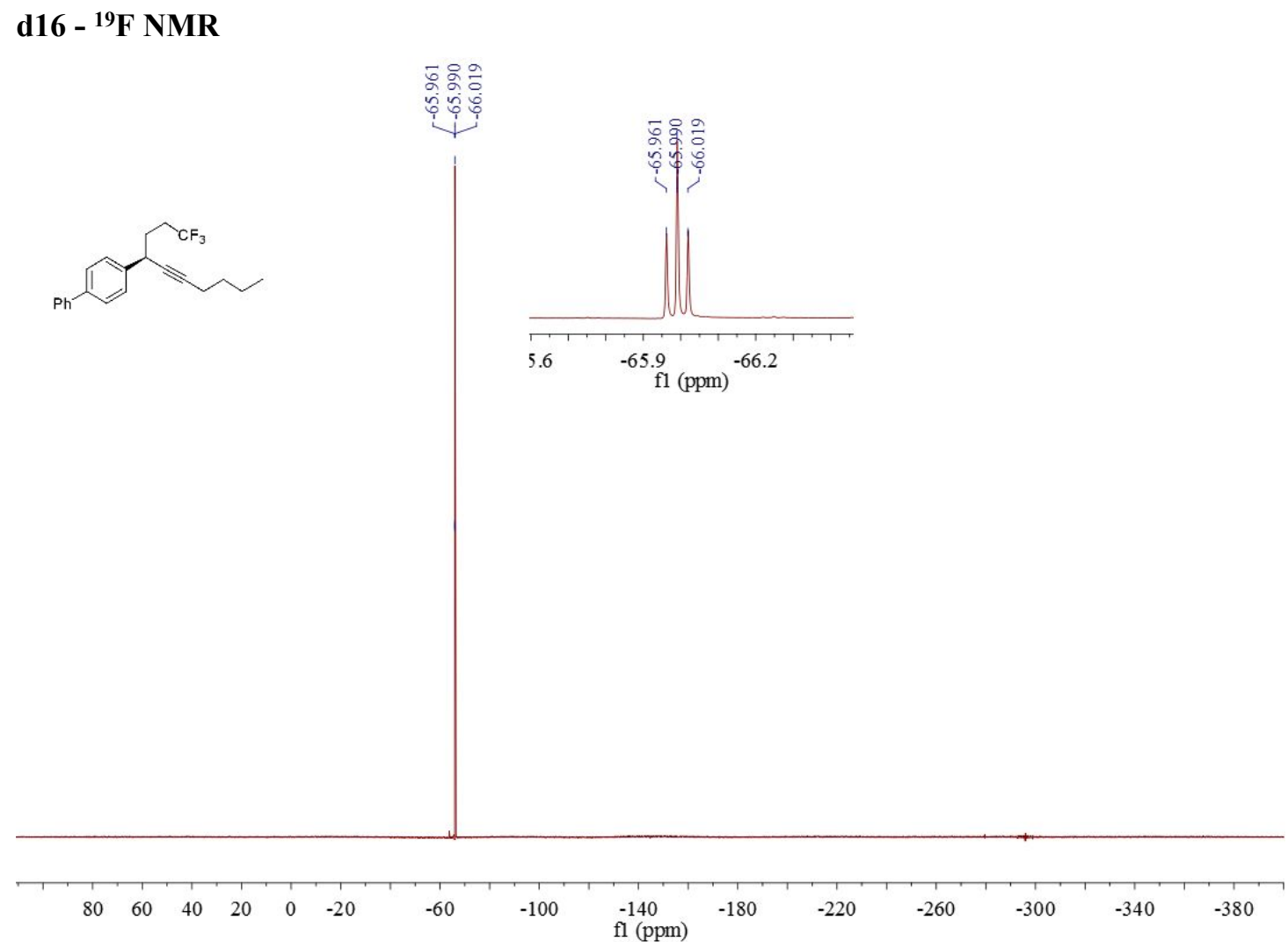

\section{d16 - HPLC}

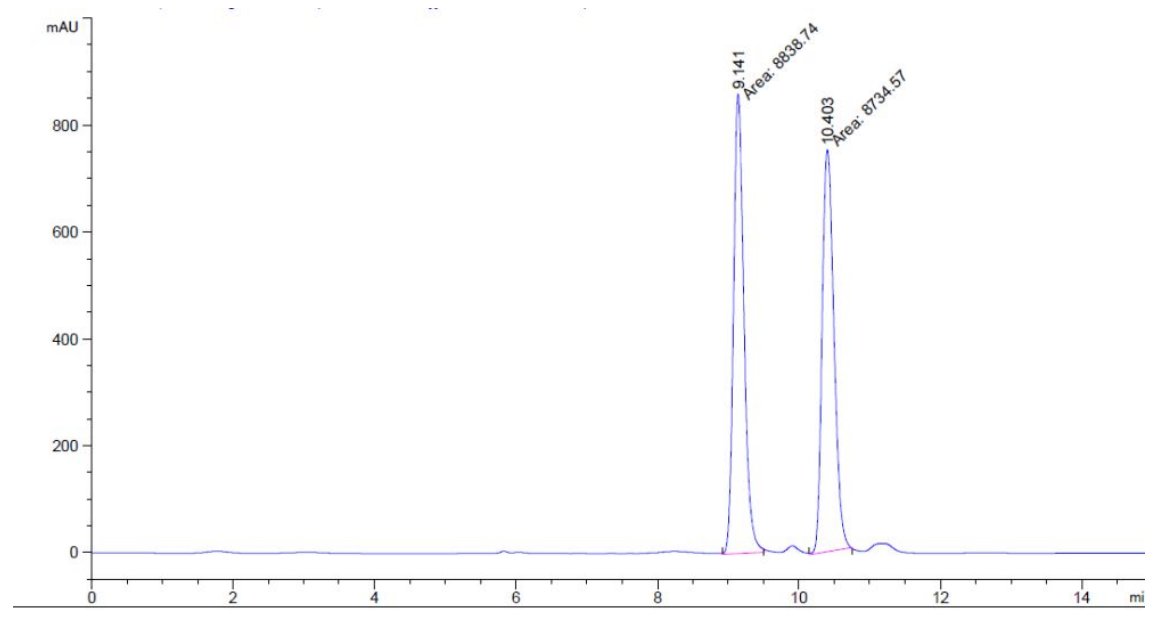

$$
\begin{aligned}
& \text { Peak RetTime Type Width Area Height Area } \\
& \text { \# }[\mathrm{min}] \quad[\mathrm{min}] \quad[\mathrm{mAU} * \mathrm{~s}] \quad[\mathrm{mAU}] \quad \%
\end{aligned}
$$

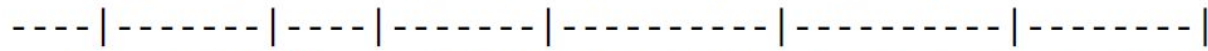

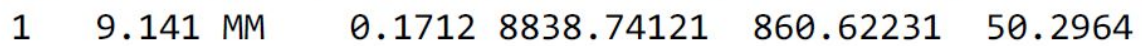

$$
\begin{aligned}
& \begin{array}{lllllll}
2 & 10.403 & \text { MM } & 0.1934 & 8734.57031 & 752.70093 & 49.7036
\end{array}
\end{aligned}
$$

Totals :

$1.75733 \mathrm{e} 4 \quad 1613.32324$ 


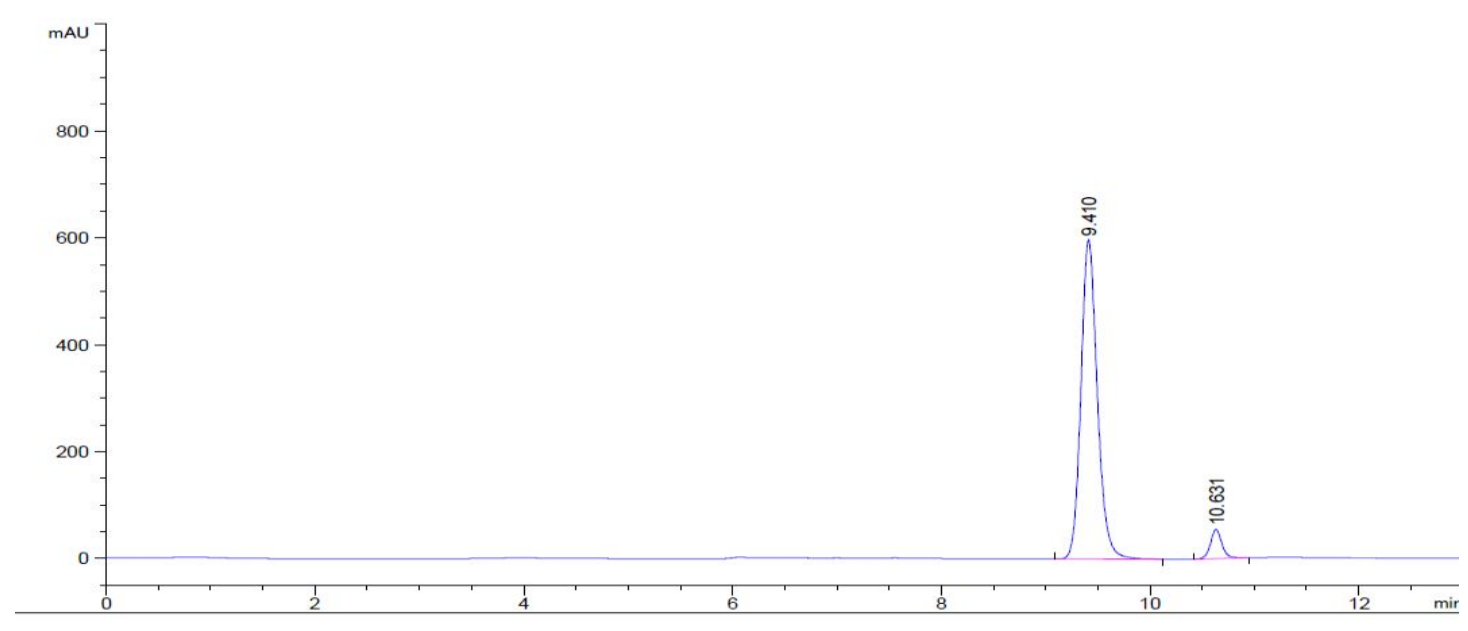

\begin{tabular}{|c|c|c|c|c|c|c|}
\hline $\begin{array}{c}\text { Peak } \\
\#\end{array}$ & $\begin{array}{c}\text { RetTime } \\
\text { [min] }\end{array}$ & Type & $\begin{array}{l}\text { Width } \\
\text { [min] }\end{array}$ & $\begin{array}{c}\text { Area } \\
{\left[\mathrm{mAU}^{*} \mathrm{~s}\right]}\end{array}$ & $\begin{array}{l}\text { Height } \\
{[\mathrm{mAU}]}\end{array}$ & $\begin{array}{c}\text { Area } \\
\%\end{array}$ \\
\hline & & & & & & \\
\hline 1 & & $B$ & & 6506.37695 & 596.6 & 93 \\
\hline & 10.631 & & 7 & 415.32175 & 54.61989 & 003 \\
\hline
\end{tabular}

Totals :

$6921.69870 \quad 651.25167$

(R)-4-(1, 1, 1-trifluoro-9-phenylnon-5-yn-4-yl)-1, 1'-biphenyl (d17) d17 - ${ }^{1}$ H NMR

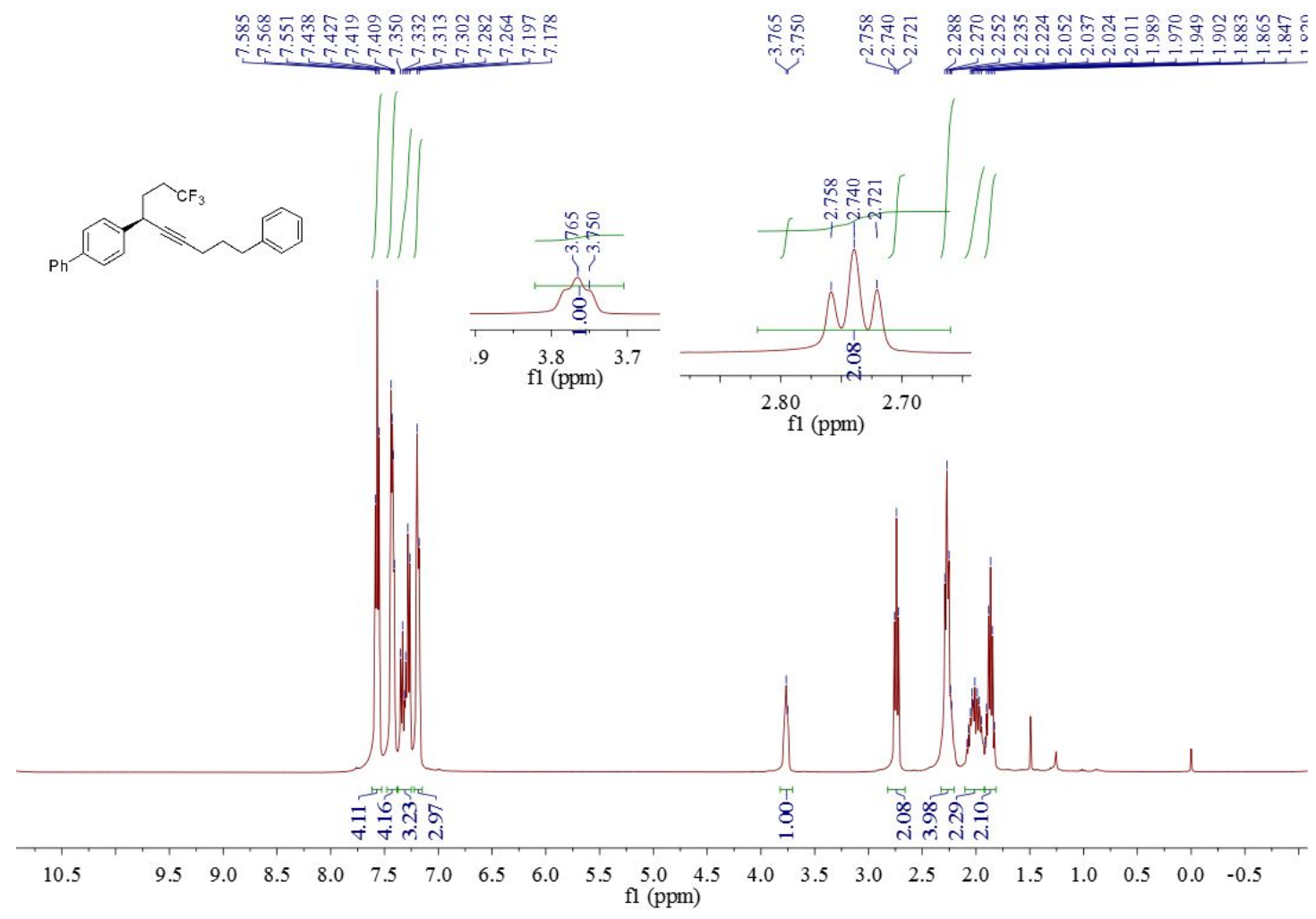


d17 - ${ }^{13}$ C NMR

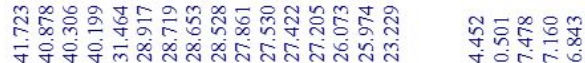

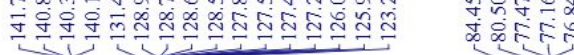

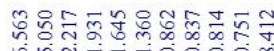

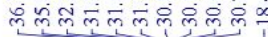

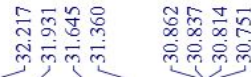
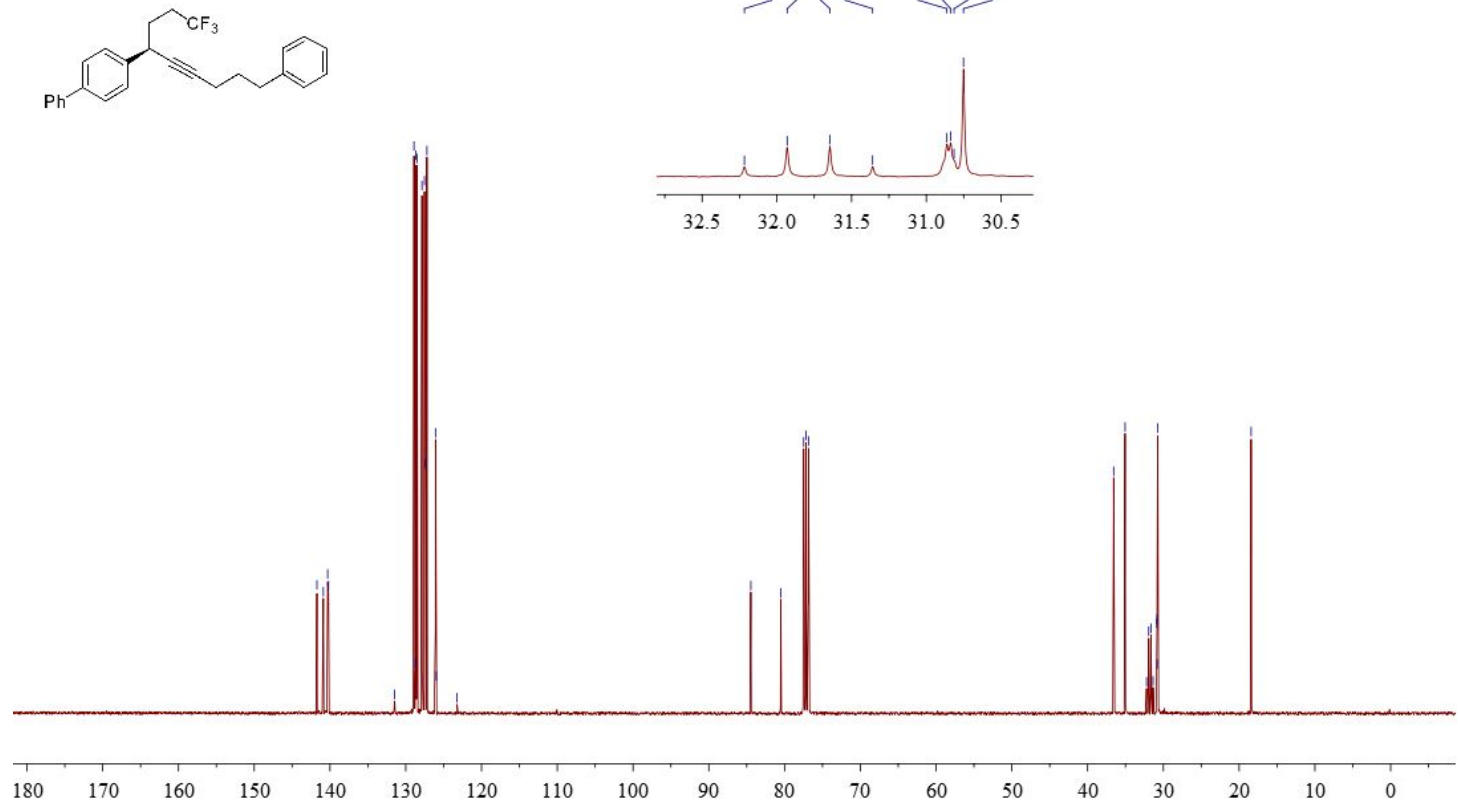

d17 - ${ }^{19}$ F NMR
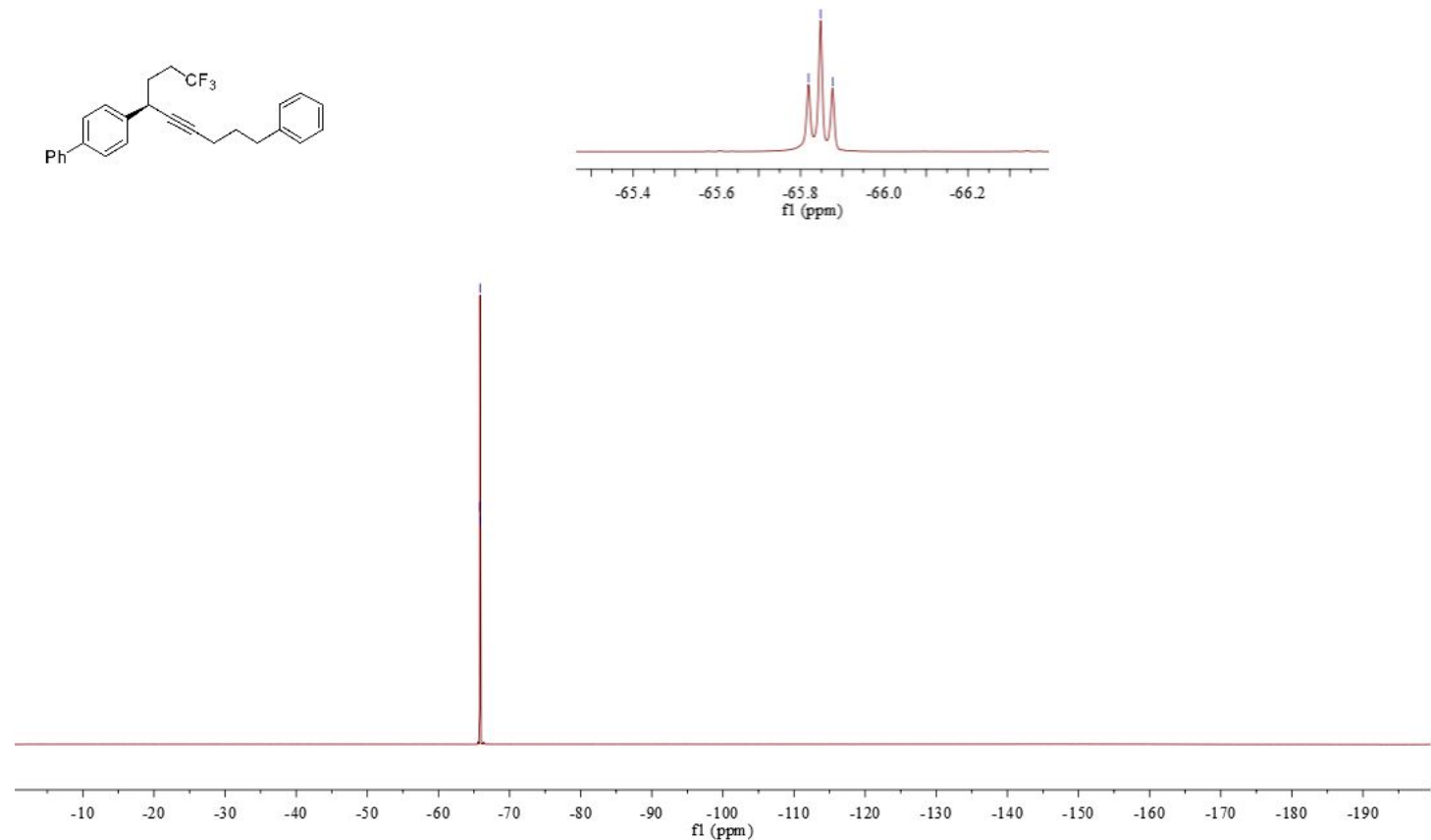


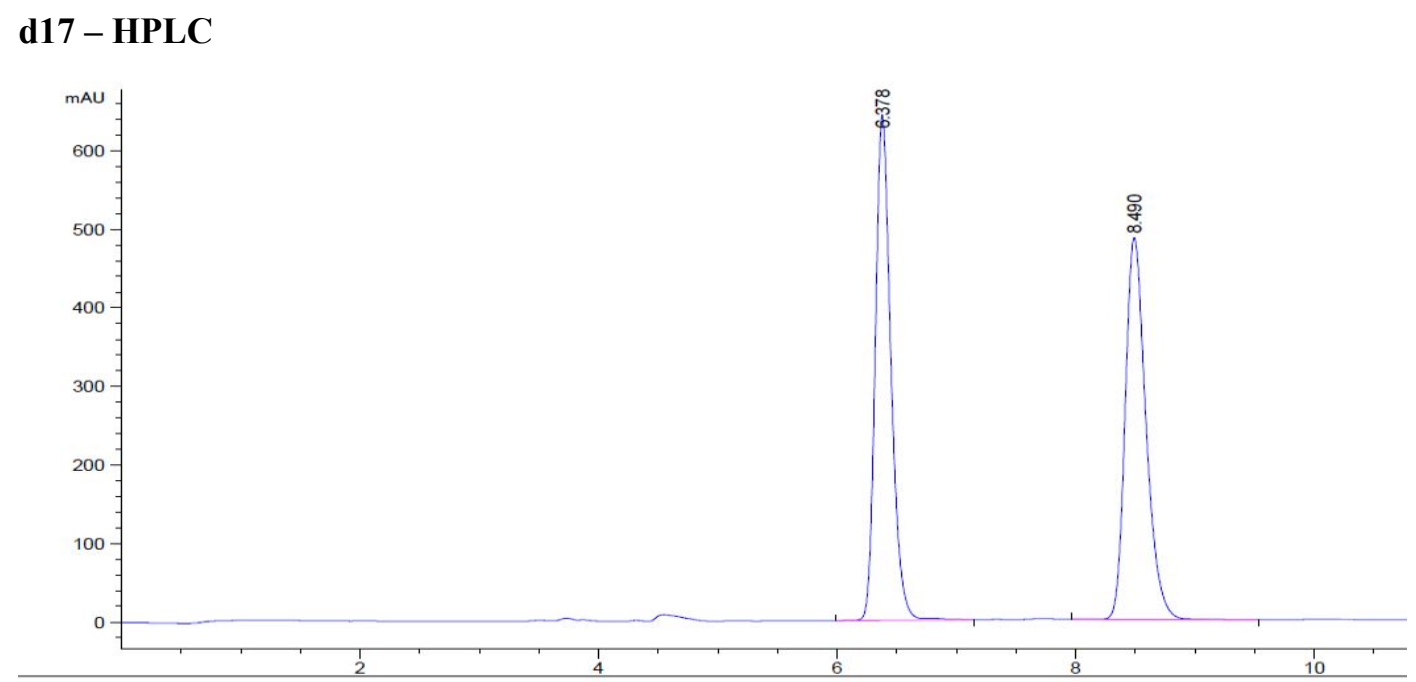

\begin{tabular}{|c|c|c|c|c|c|c|}
\hline $\begin{array}{c}\text { Peak } \\
\#\end{array}$ & $\begin{array}{c}\text { RetTime } \\
\text { [min] }\end{array}$ & Type & $\begin{array}{l}\text { Width } \\
\text { [min] }\end{array}$ & $\begin{array}{c}\text { Area } \\
{\left[\mathrm{mAU}^{*} \mathrm{~s}\right]}\end{array}$ & $\begin{array}{l}\text { Height } \\
{[\mathrm{mAU}]}\end{array}$ & $\begin{array}{c}\text { Area } \\
\%\end{array}$ \\
\hline & & & & & 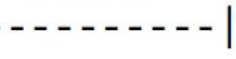 & --- \\
\hline 1 & 6.378 & $B V R$ & 0.1375 & 5845.96875 & 643.57672 & 49.9037 \\
\hline 2 & 8.490 & BB & 0.1849 & 5868.53955 & 486.08835 & 50.0963 \\
\hline
\end{tabular}

Totals :

$1.17145 \mathrm{e} 4 \quad 1129.66507$

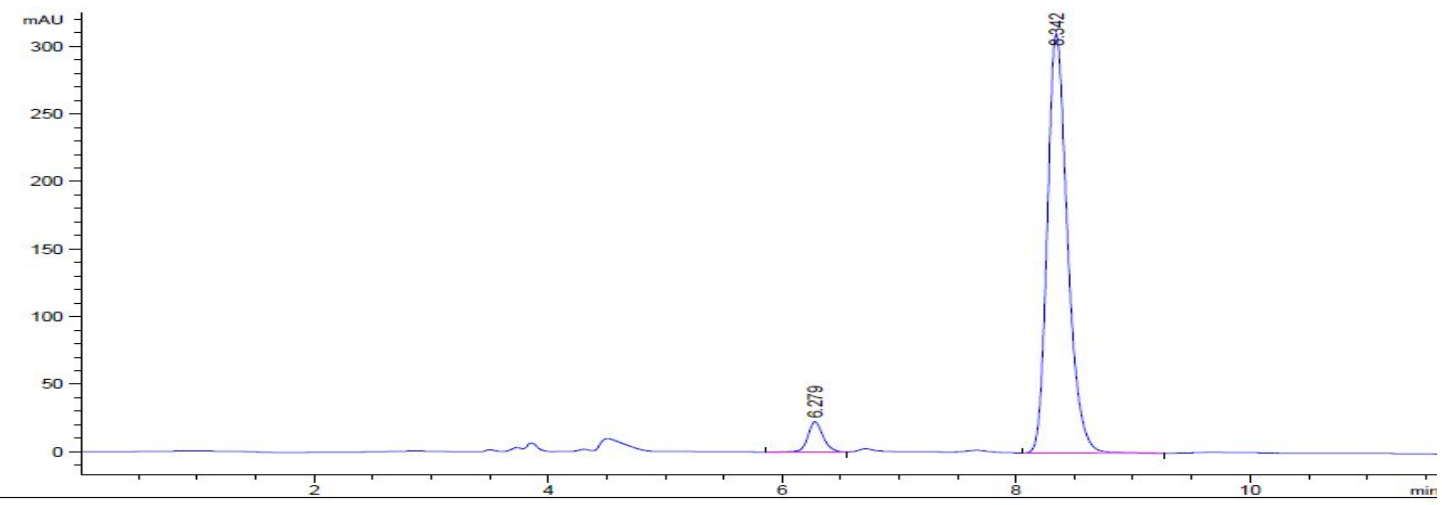

\begin{tabular}{|c|c|c|c|c|c|c|}
\hline $\begin{array}{c}\text { Peak } \\
\text { \# }\end{array}$ & $\begin{array}{c}\text { RetTime } \\
\text { [min] }\end{array}$ & Type & $\begin{array}{l}\text { Width } \\
\text { [min] }\end{array}$ & $\begin{array}{c}\text { Area } \\
{\left[\mathrm{mAU}^{*} \mathrm{~s}\right]}\end{array}$ & $\begin{array}{l}\text { Height } \\
{[\mathrm{mAU}]}\end{array}$ & $\begin{array}{c}\text { Area } \\
\%\end{array}$ \\
\hline & & & & & & \\
\hline 1 & & 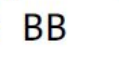 & & 203. & 94 & \\
\hline 2 & 342 & B & 42 & 3725.47827 & 243 & 94 \\
\hline
\end{tabular}

Totals :

$3928.72299 \quad 332.45337$ 

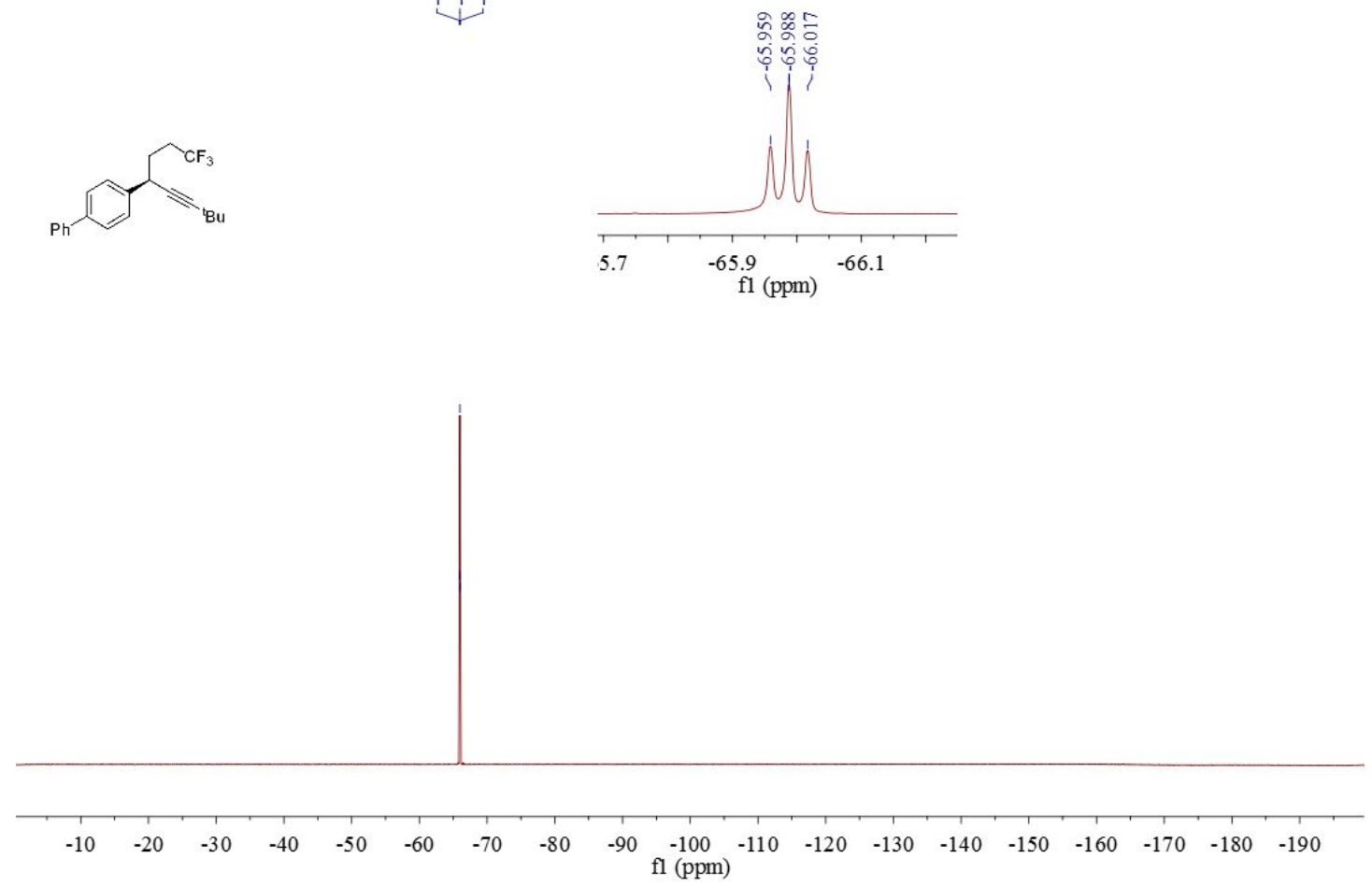

$$
\text { d18 - HPLC }
$$

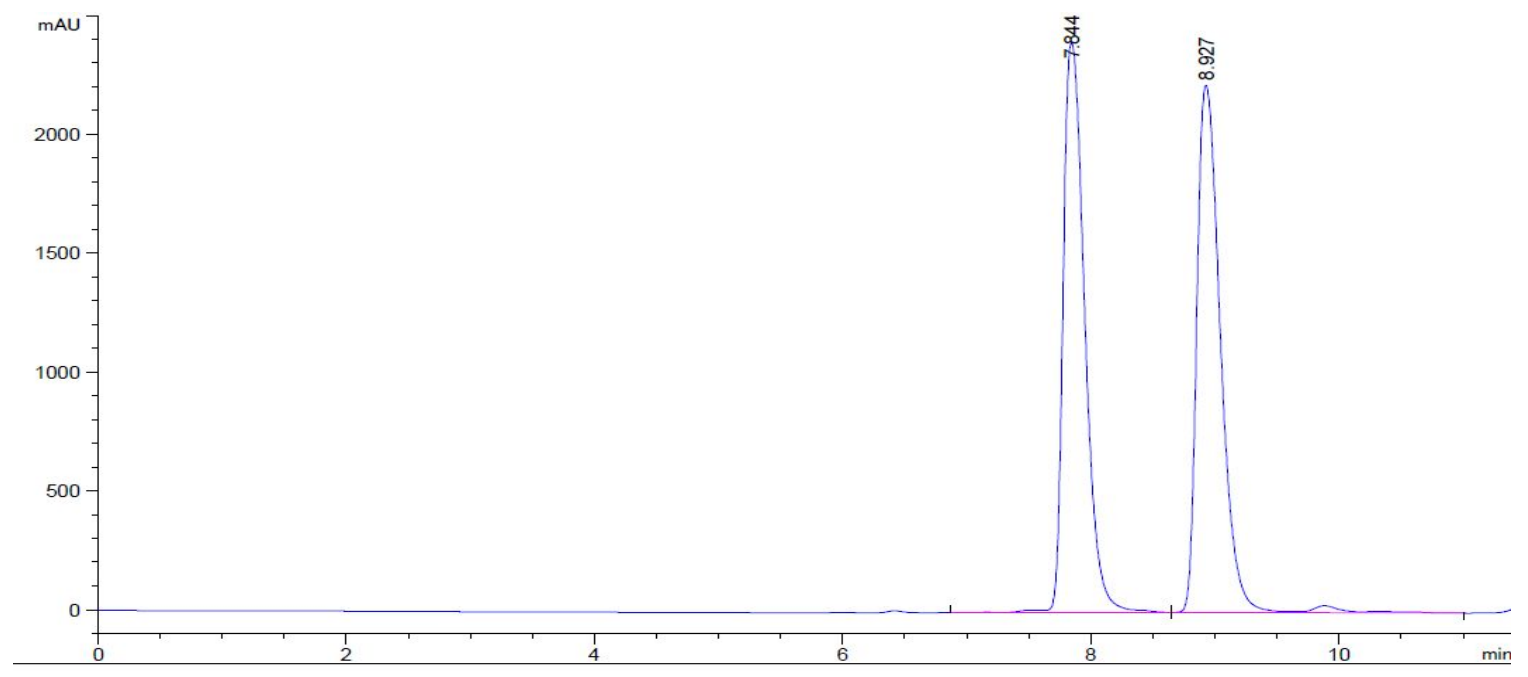

Peak RetTime Type Width Area Height Area

\begin{tabular}{ccccccc|}
$\#$ & {$[\mathrm{~min}]$} & {$[\mathrm{min}]$} & {$[\mathrm{mAU} * \mathrm{~s}]$} & {$[\mathrm{mAU}]$} & $\%$ \\
\hline 1 & 7.844 & VB R & 0.1869 & $2.89015 \mathrm{e} 4$ & 2397.35034 & 49.3027 \\
2 & 8.927 & BV R & 0.2038 & $2.97190 \mathrm{e} 4$ & 2218.81006 & 50.6973
\end{tabular}

Totals :

$5.86205 \mathrm{e} 4 \quad 4616.16040$ 


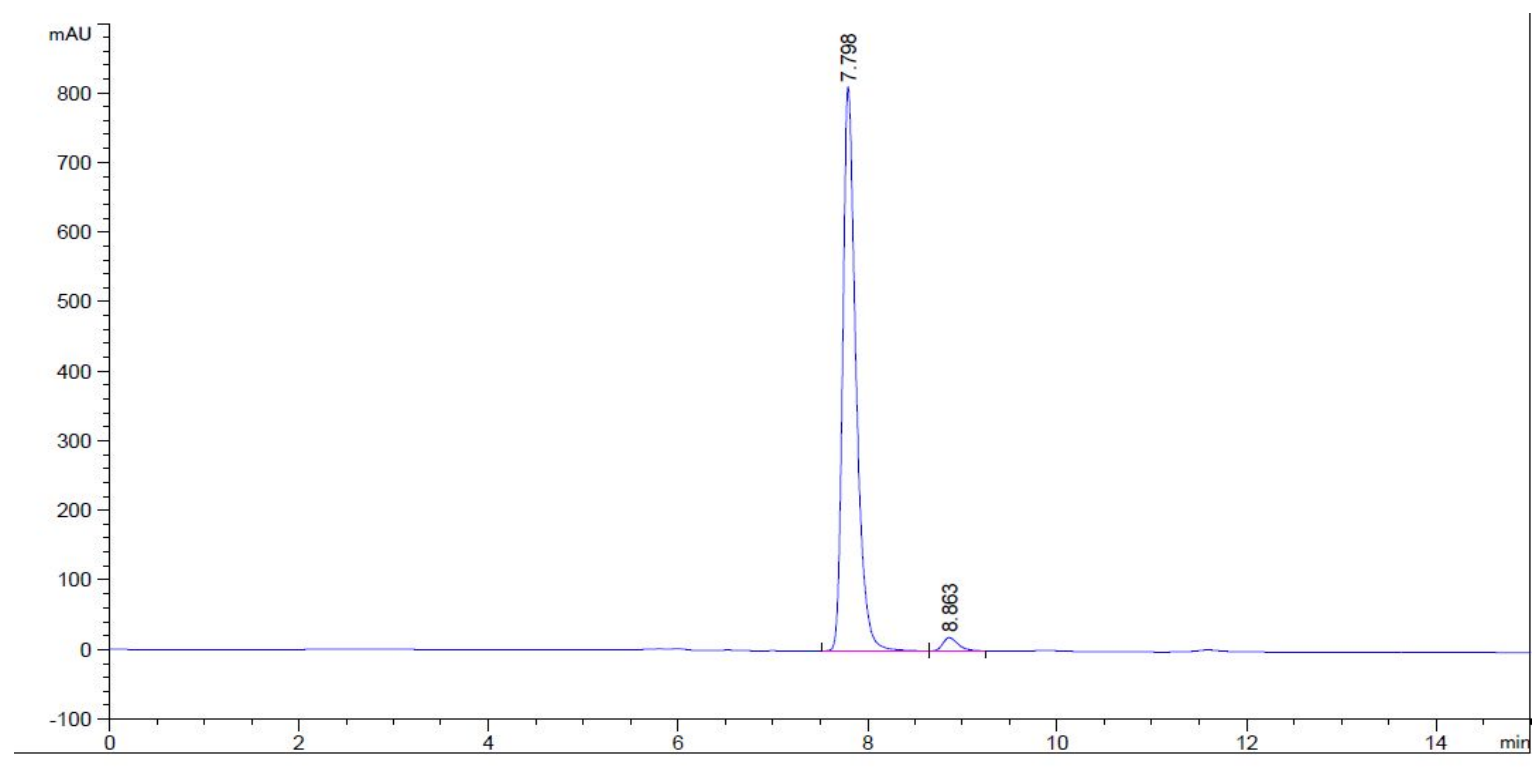

\begin{tabular}{|c|c|c|c|c|c|c|}
\hline $\begin{array}{c}\text { Peak } \\
\#\end{array}$ & $\begin{array}{c}\text { RetTime } \\
\text { [min] }\end{array}$ & Type & $\begin{array}{l}\text { Width } \\
\text { [min] }\end{array}$ & $\begin{array}{c}\text { Area } \\
{\left[\mathrm{mAU}^{*} \mathrm{~s}\right]}\end{array}$ & $\begin{array}{l}\text { Height } \\
{[\mathrm{mAU}]}\end{array}$ & $\begin{array}{c}\text { Area } \\
\%\end{array}$ \\
\hline & & & & - - - - & $\ldots$ & - \\
\hline 1 & 7 & & 0.1 & 7911.7 & 810.6 & 97. \\
\hline 2 & 8.863 & $B$ & 48 & 210.67918 & 19.23439 & 2.5938 \\
\hline
\end{tabular}

Totals :

$8122.40428 \quad 829.92281$

(R)-4-(1, 1, 1-trifluoro-7-methyloct-5-yn-4-yl)-1, 1'-biphenyl (d19) d19 - ${ }^{1}$ H NMR

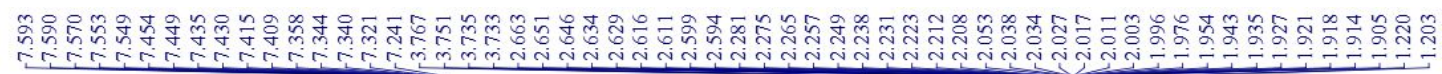
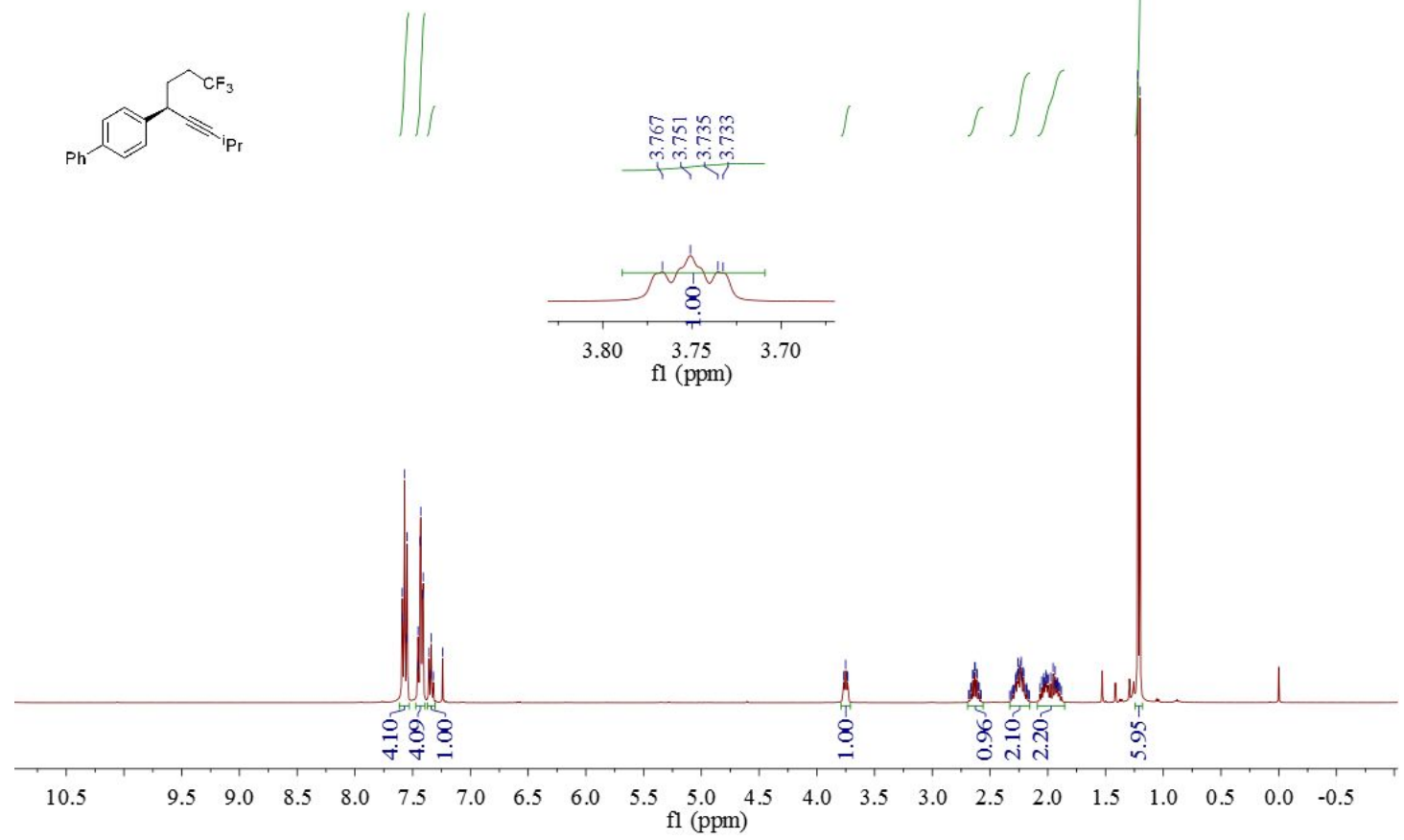
d19 - ${ }^{13}$ C NMR

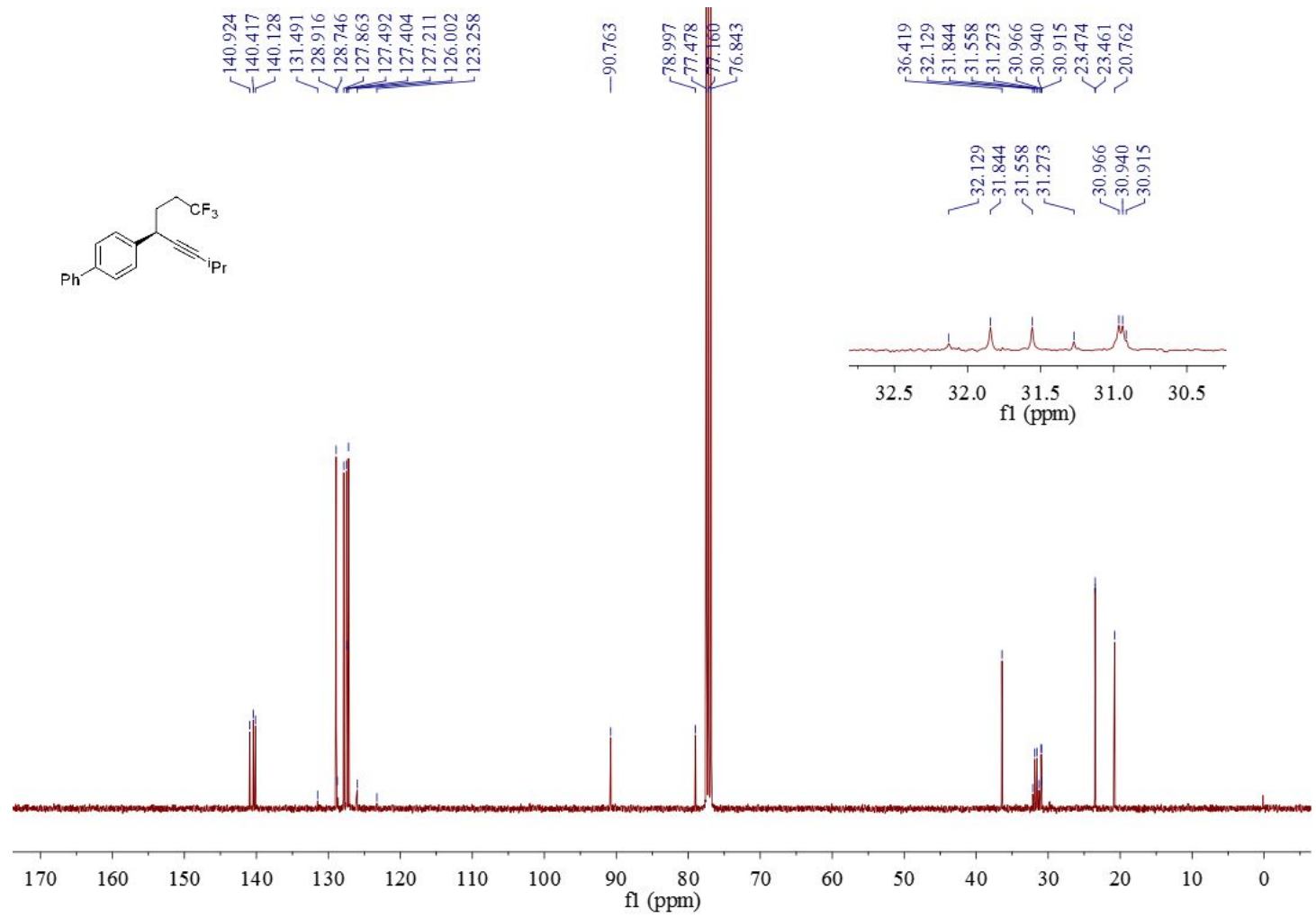

\section{d19 - ${ }^{19}$ F NMR}<smiles>CC#CC(CCC)c1ccc(P)cc1</smiles>

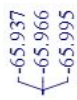
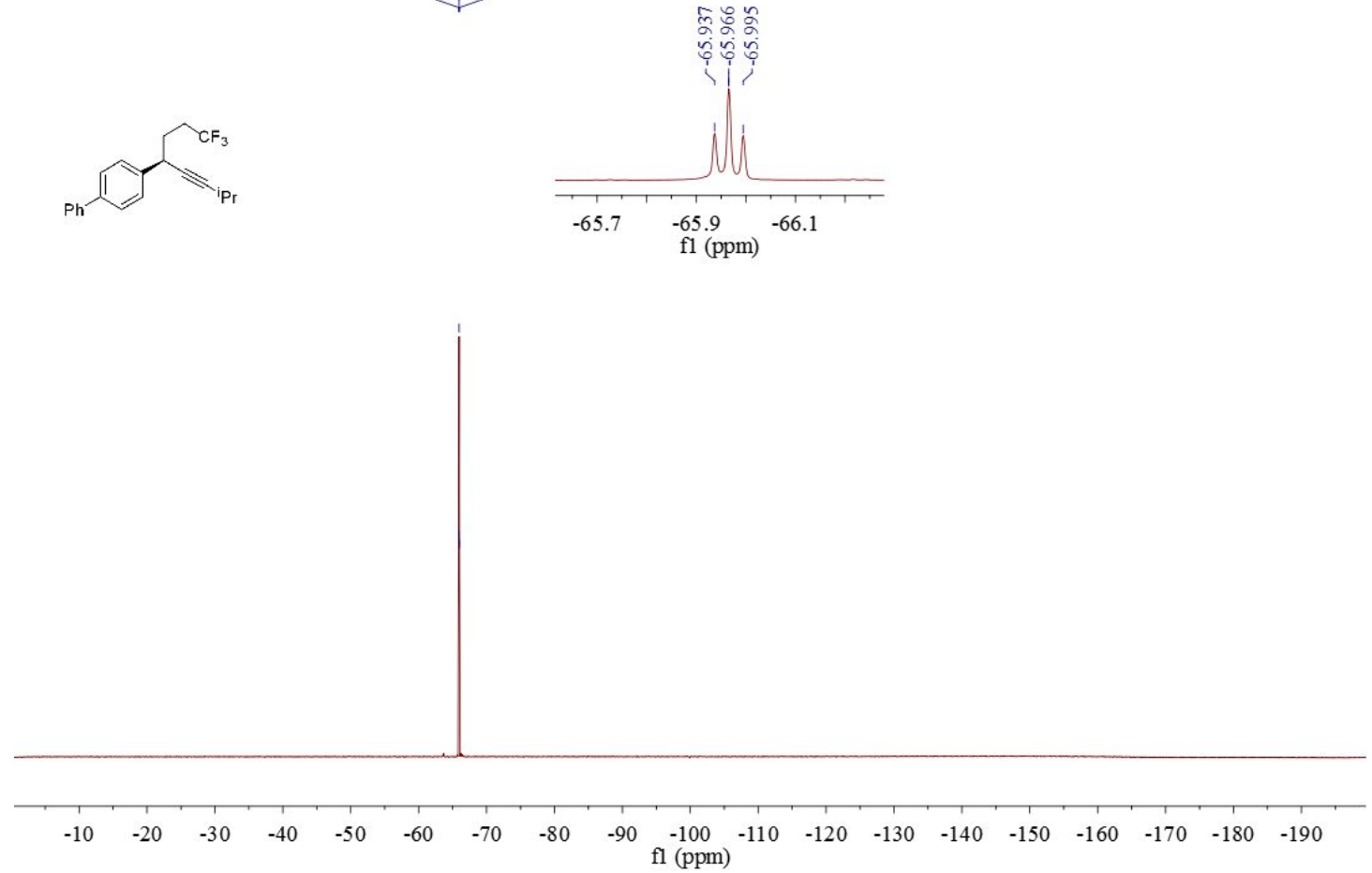

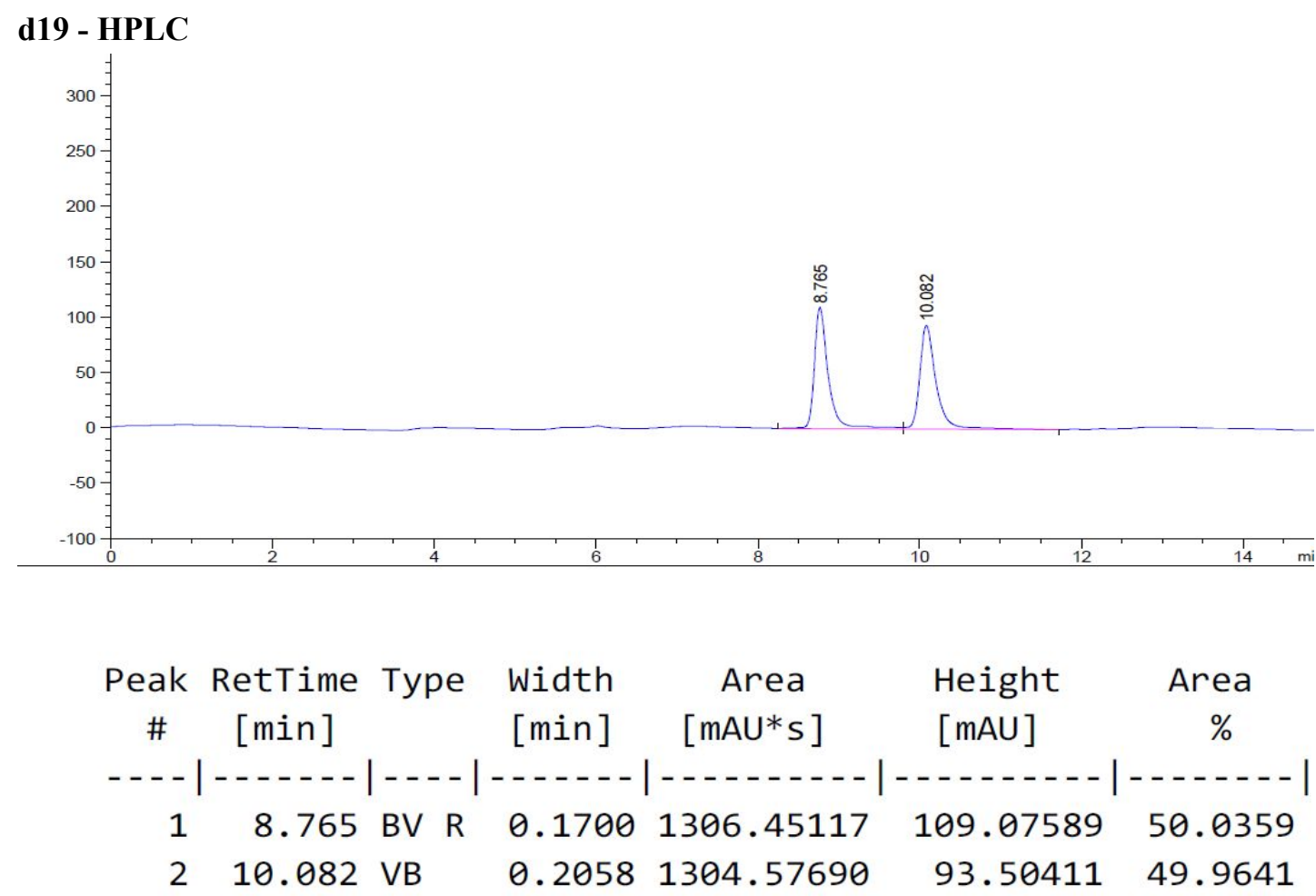

Totals :

$2611.02808 \quad 202.58000$

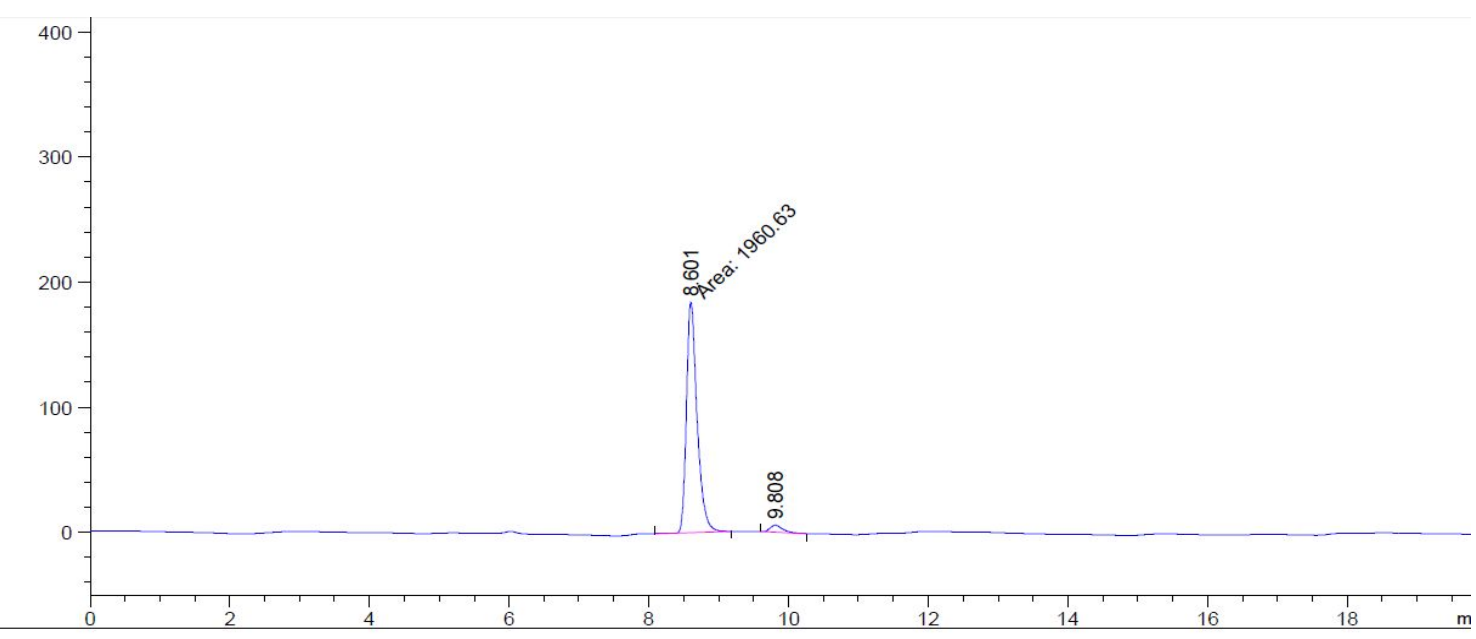

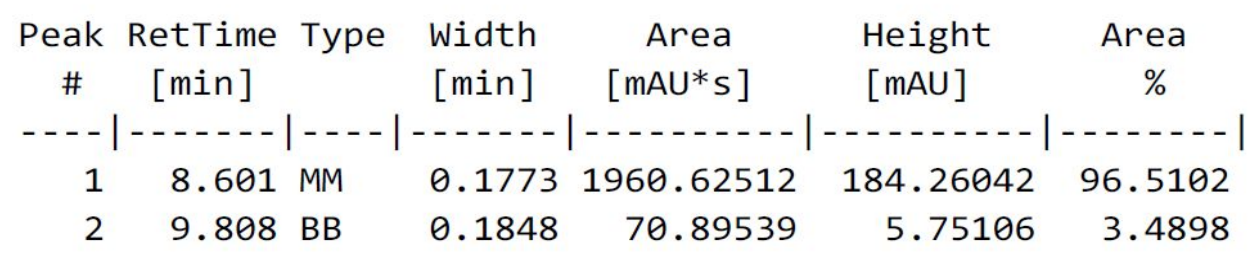

Totals :

$2031.52051 \quad 190.01149$ 
(R)-4-(1, 1, 1-trifluoro-8-methylnon-5-yn-4-yl)-1, 1'-biphenyl (d20) d20 - ${ }^{1}$ H NMR

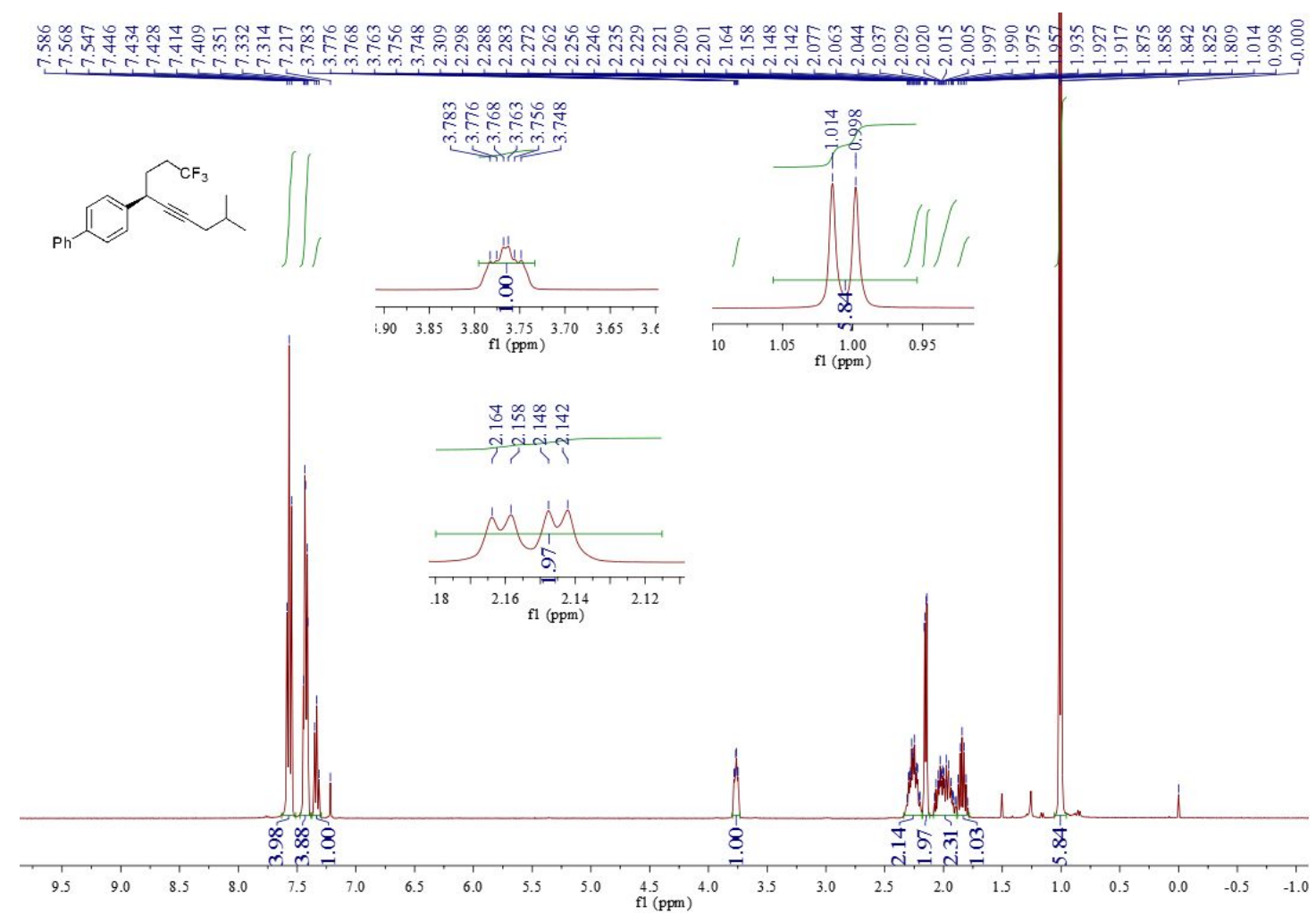

d20 - ${ }^{13}$ C NMR

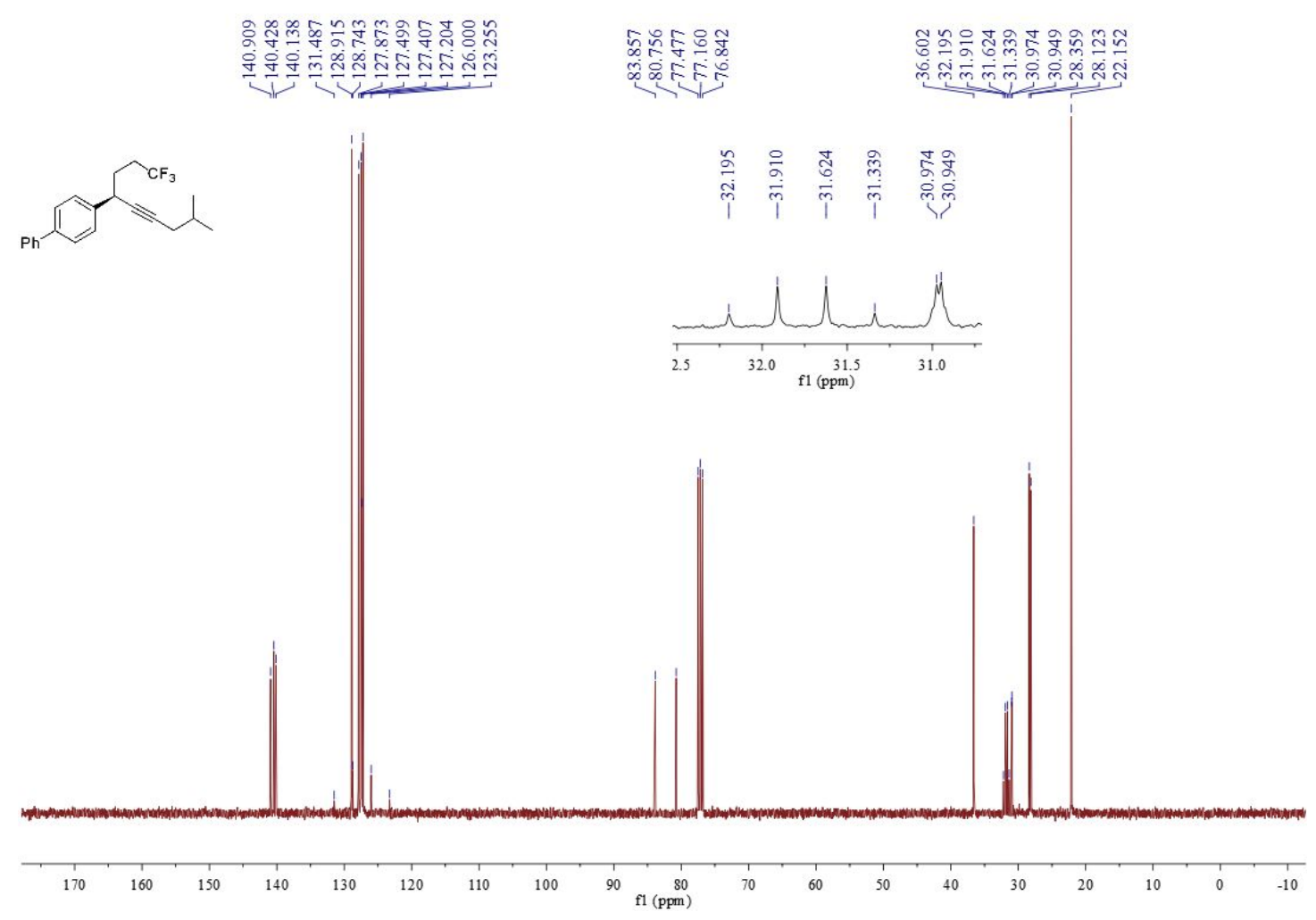




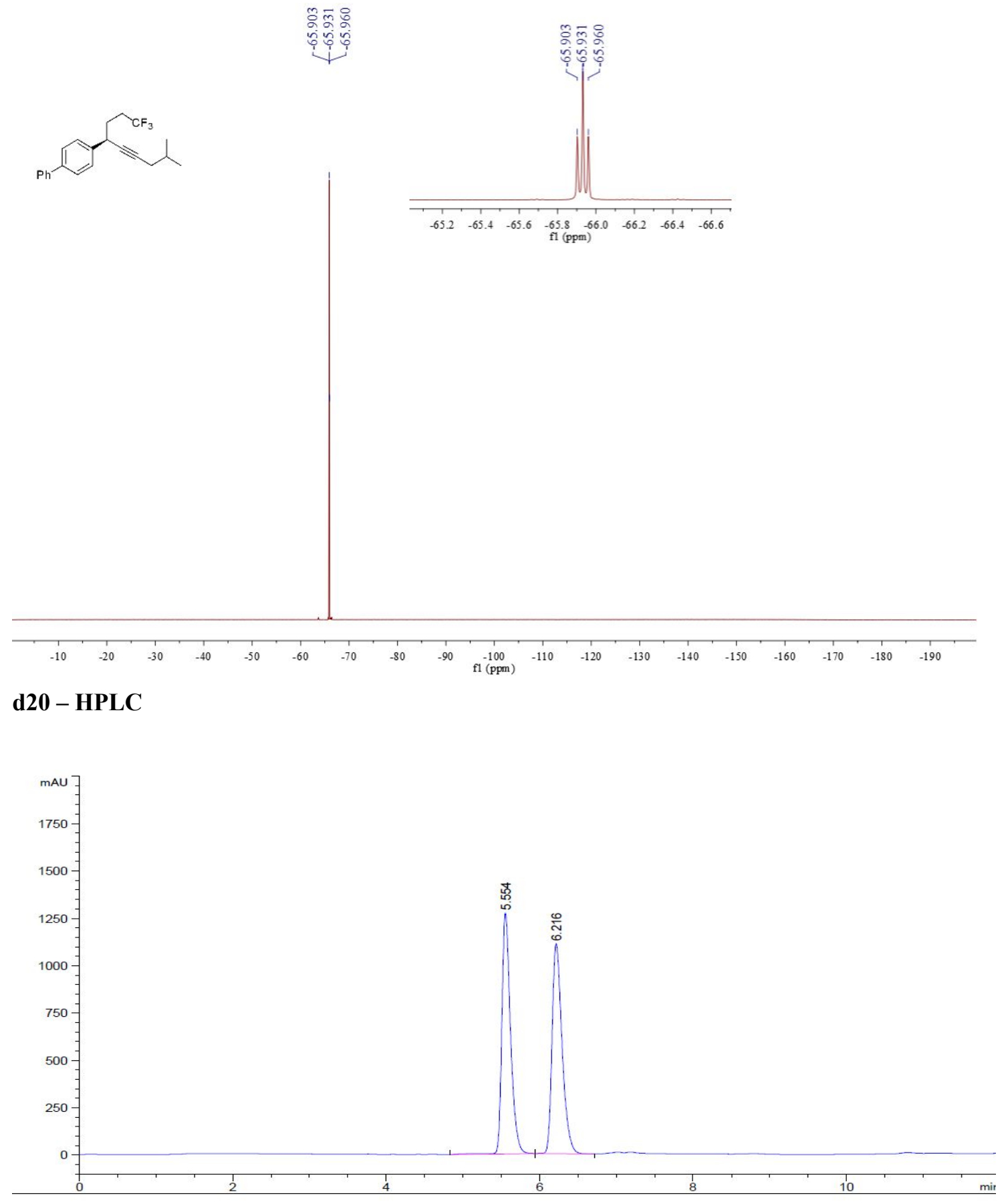

\begin{tabular}{|c|c|c|c|c|c|c|}
\hline $\begin{array}{c}\text { Peak } \\
\quad \#\end{array}$ & $\begin{array}{c}\text { RetTime } \\
\text { [min] }\end{array}$ & Type & $\begin{array}{l}\text { Width } \\
\text { [min] }\end{array}$ & $\begin{array}{c}\text { Area } \\
{\left[\mathrm{mAU}^{*} \mathrm{~s}\right]}\end{array}$ & $\begin{array}{l}\text { Height } \\
{[\mathrm{mAU}]}\end{array}$ & $\begin{array}{c}\text { Area } \\
\%\end{array}$ \\
\hline & & & & & & \\
\hline 1 & 5.5 & VB $R$ & 0.1177 & 9960.11426 & 1270.91724 & 49.7966 \\
\hline 2 & 6.216 & BB & 0.1387 & $1.00415 \mathrm{e} 4$ & 1108.57605 & 50.2034 \\
\hline
\end{tabular}

Totals :

$2.00016 \mathrm{e} 4 \quad 2379.49329$ 


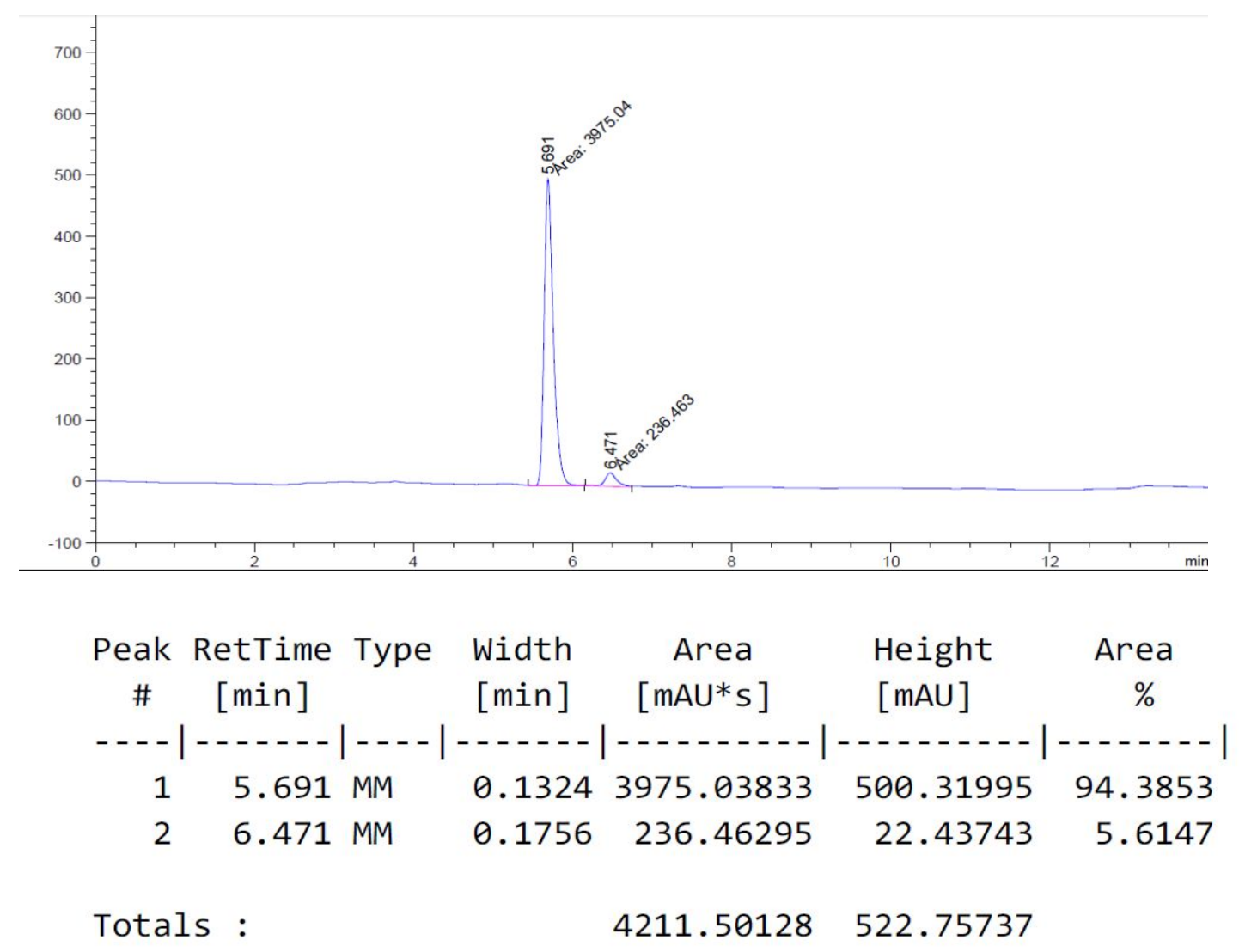

(R)-4-(1-cyclopropyl-6, 6, 6-trifluorohex-1-yn-3-yl)-1, 1'-biphenyl (d21) d21 - ${ }^{1}$ H NMR

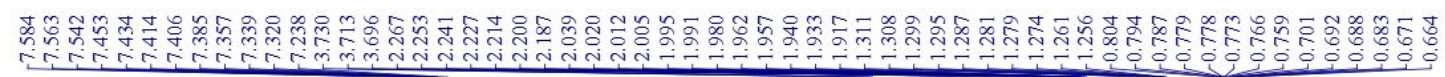

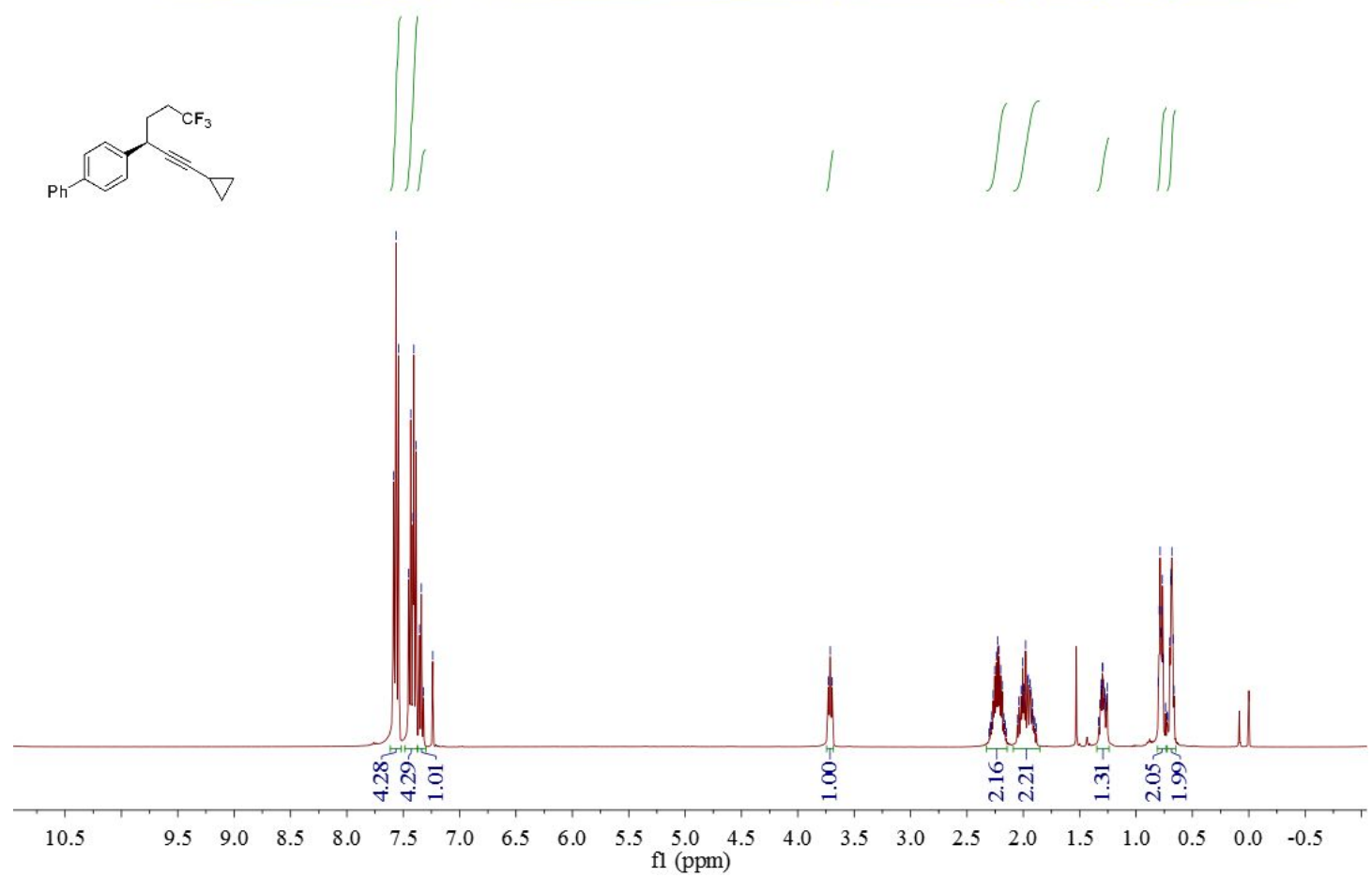


d21 - ${ }^{13}$ C NMR

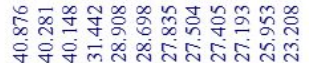

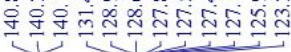

के

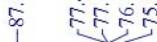

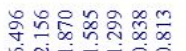

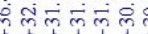

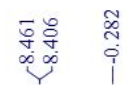

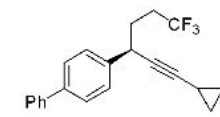

nำ

ल्रलm ल्र
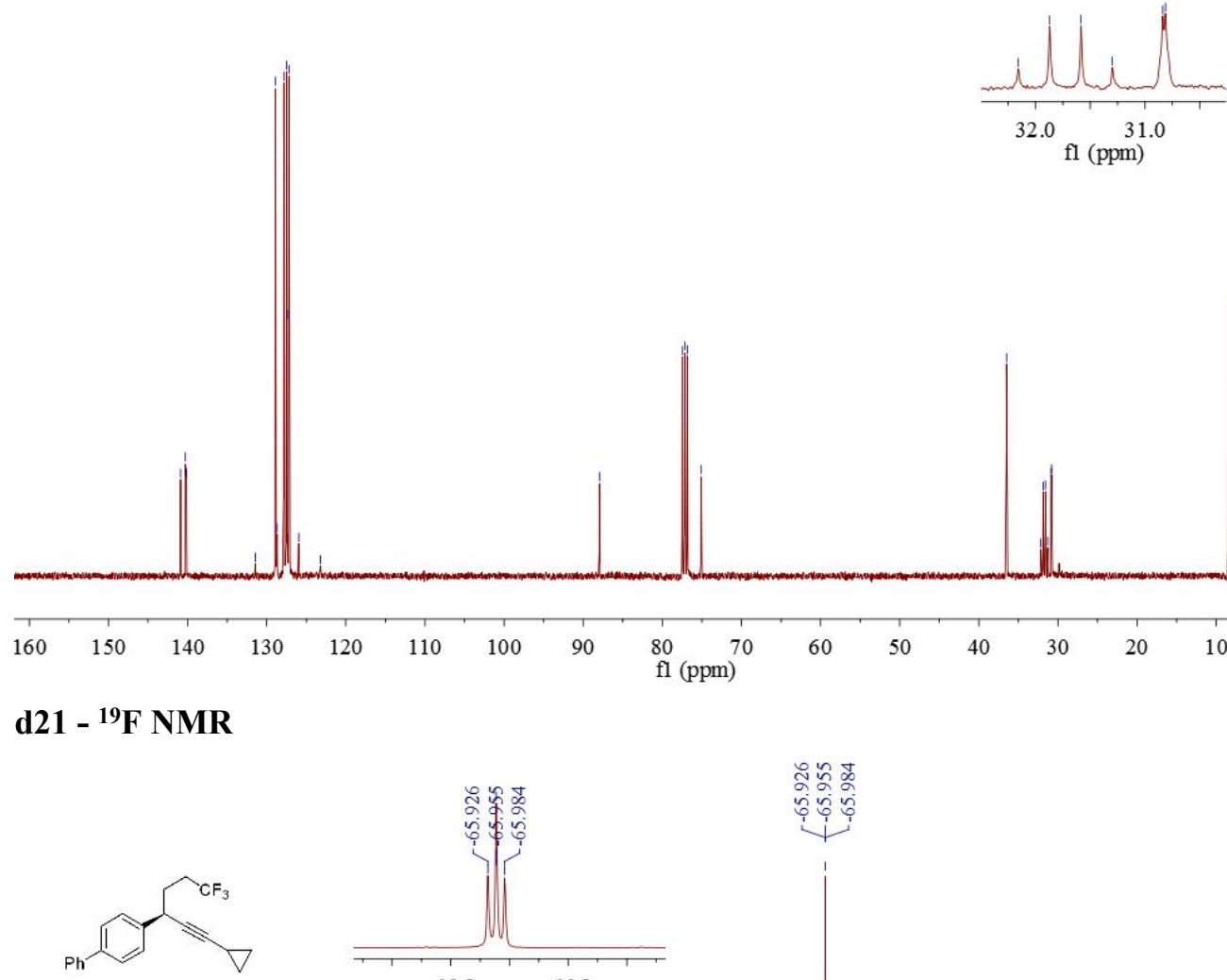

$\mathrm{fl}(\mathrm{ppm})$
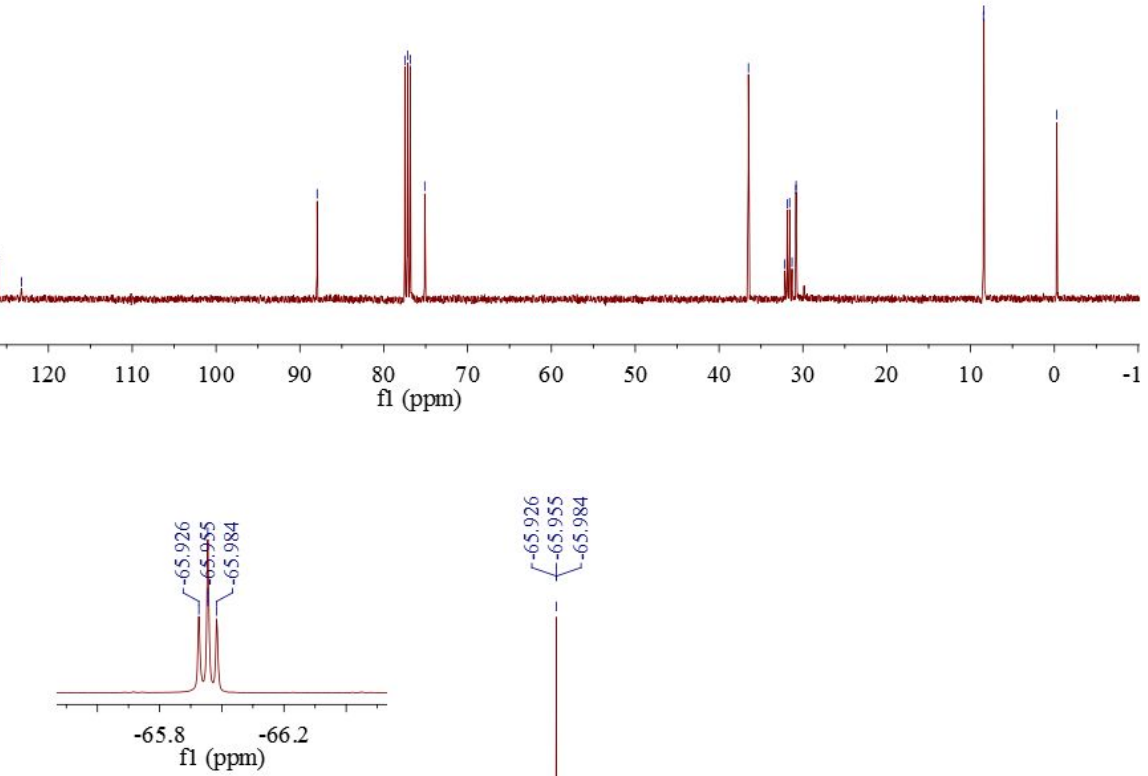

สำू

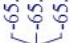
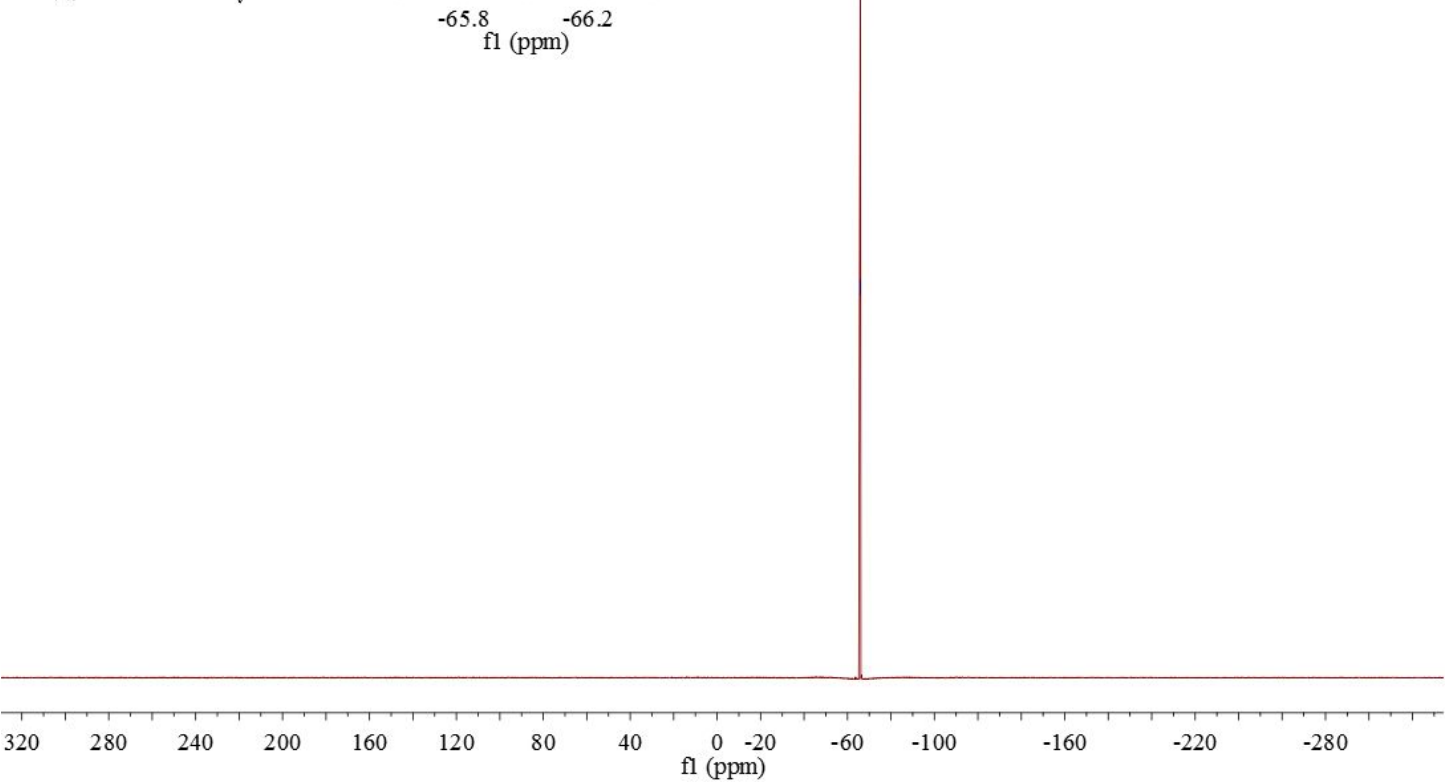
d21 - HPLC
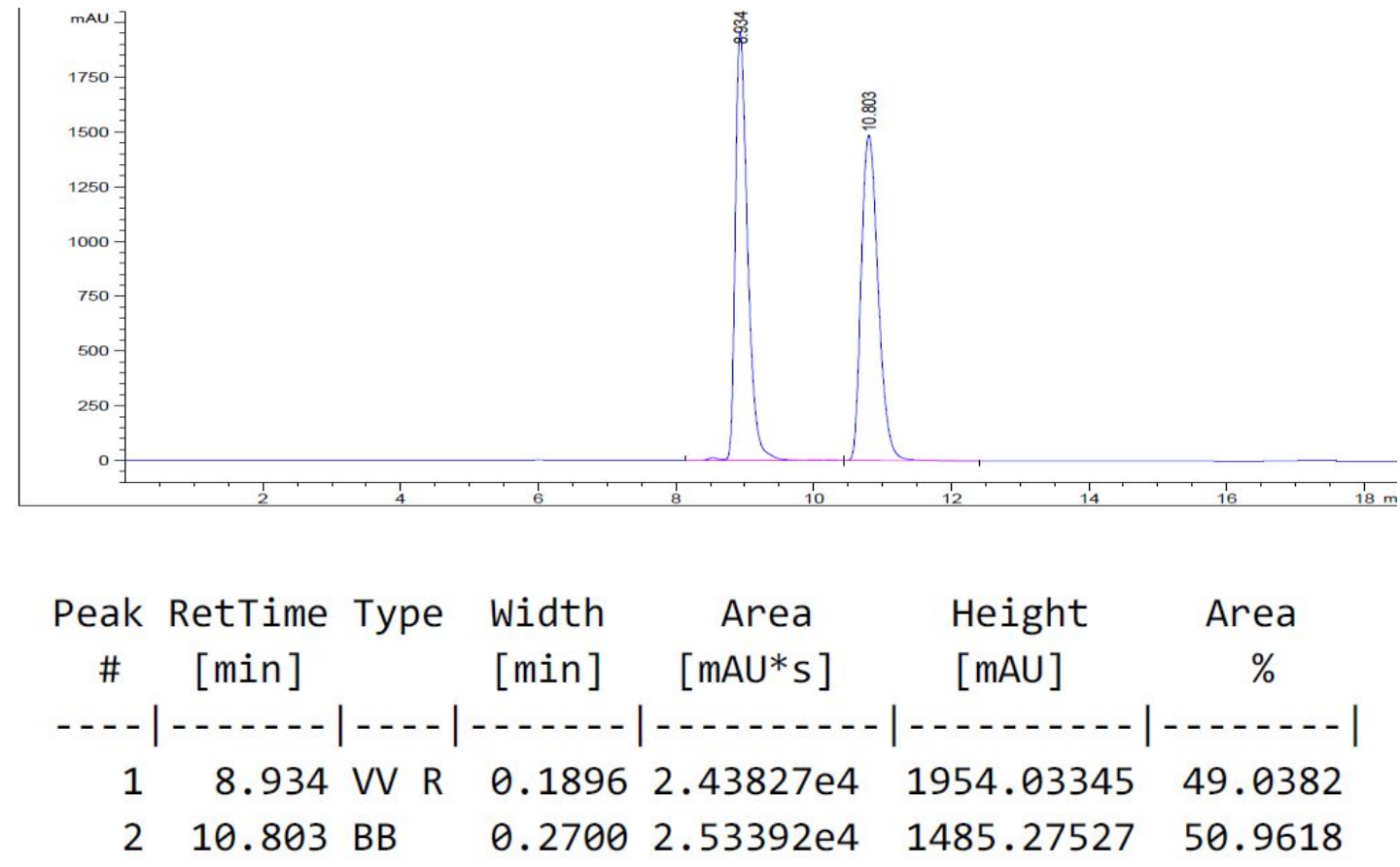

Totals :

4.97220e4 3439.30872

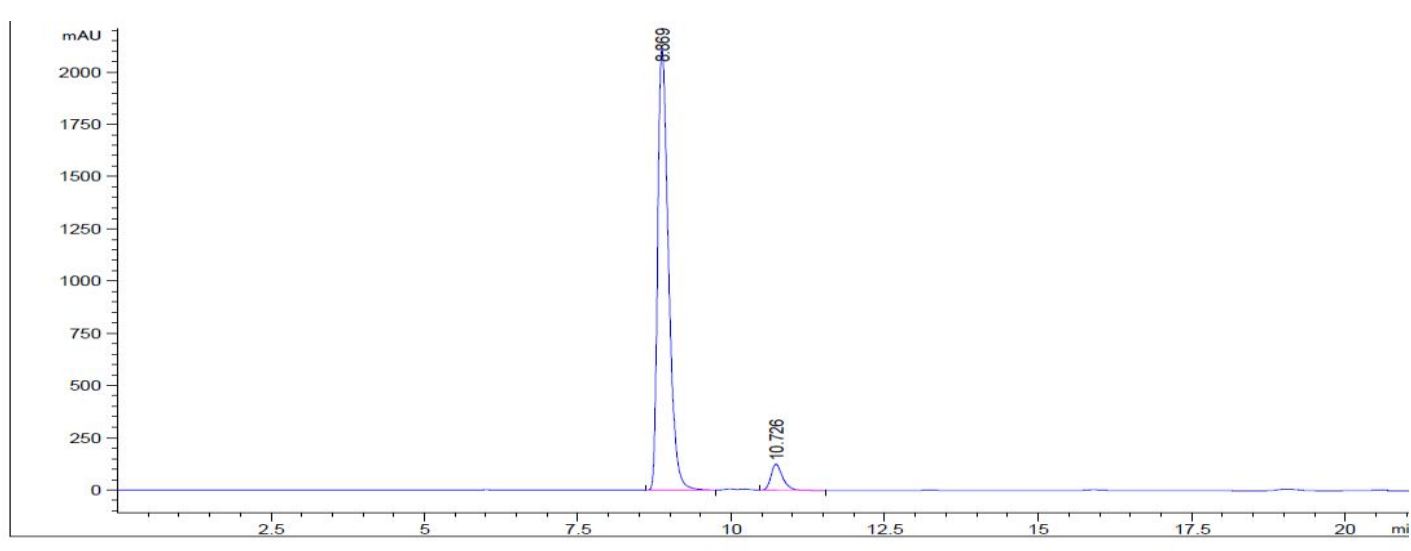

\begin{tabular}{|c|c|c|c|c|c|c|}
\hline $\begin{array}{l}\text { eak } \\
\#\end{array}$ & $\begin{array}{c}\text { RetTime } \\
\text { [min] }\end{array}$ & Type & $\begin{array}{l}\text { Width } \\
\text { [min] }\end{array}$ & $\begin{array}{c}\text { Area } \\
{\left[\mathrm{mAU}^{*} \mathrm{~s}\right]}\end{array}$ & $\begin{array}{l}\text { Height } \\
{[\mathrm{mAU}]}\end{array}$ & $\begin{array}{c}\text { Area } \\
\%\end{array}$ \\
\hline & & & & & & \\
\hline 1 & & D & 98 & $2.59569 \mathrm{e} 4$ & 2106 & 94. \\
\hline 2 & 26 & 1 & 7 & 1627. & 550 & 9013 \\
\hline
\end{tabular}

Totals :

$2.75847 e 4 \quad 2230.46558$ 
(R)-3-(6, 6, 6-trifluoro-3-phenylhex-1-yn-1-yl) pyridine (d22)

\section{d22 - ${ }^{1}$ H NMR}

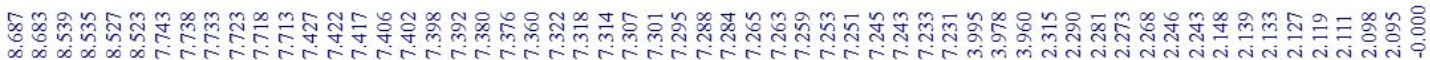
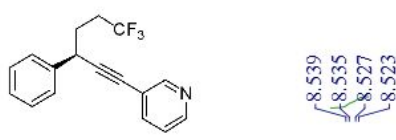

|cing
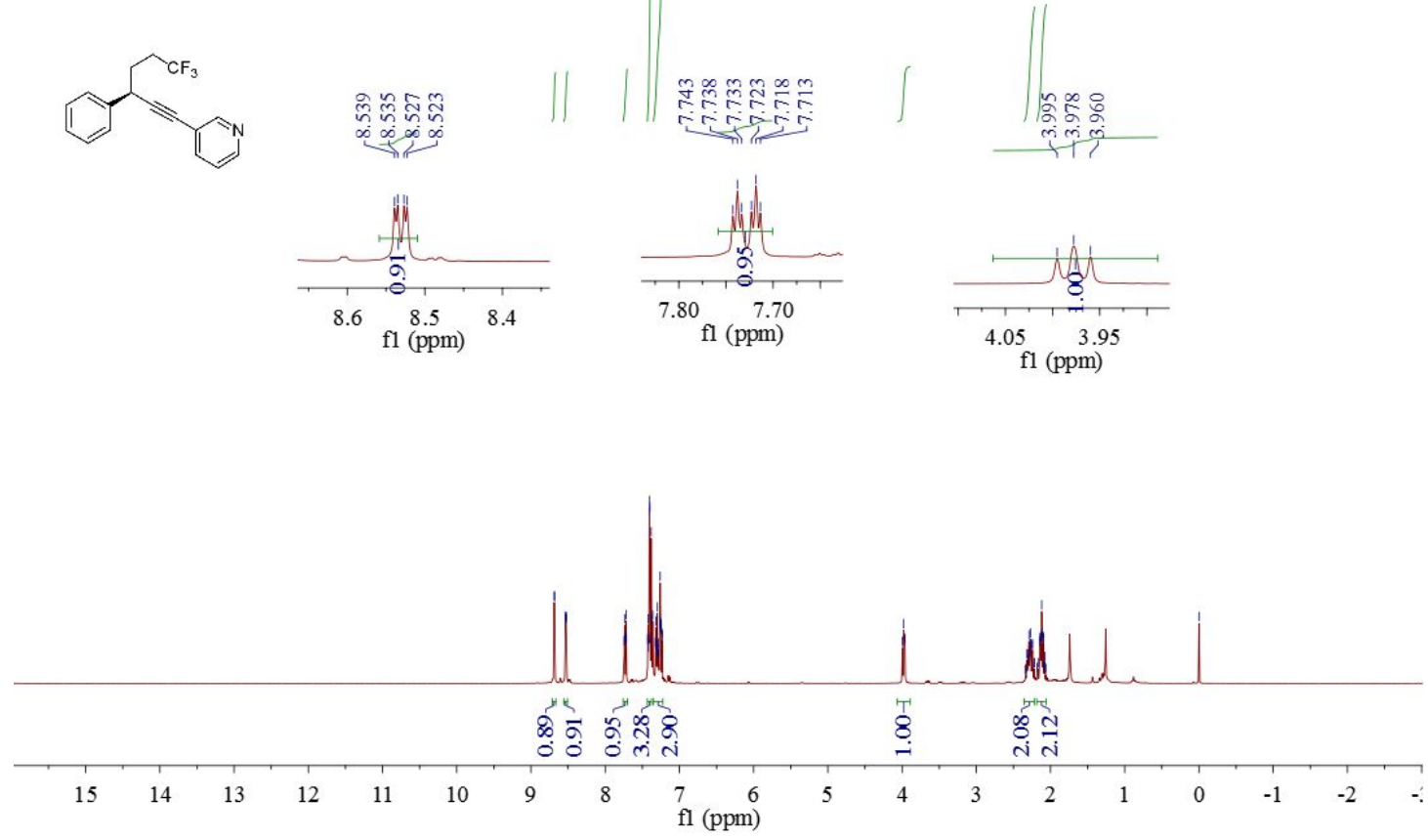

d22 $-{ }^{13}$ C NMR

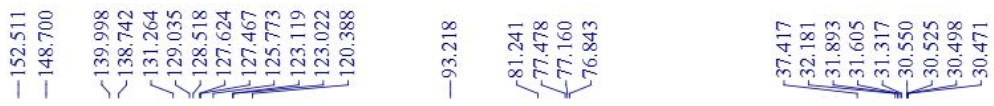
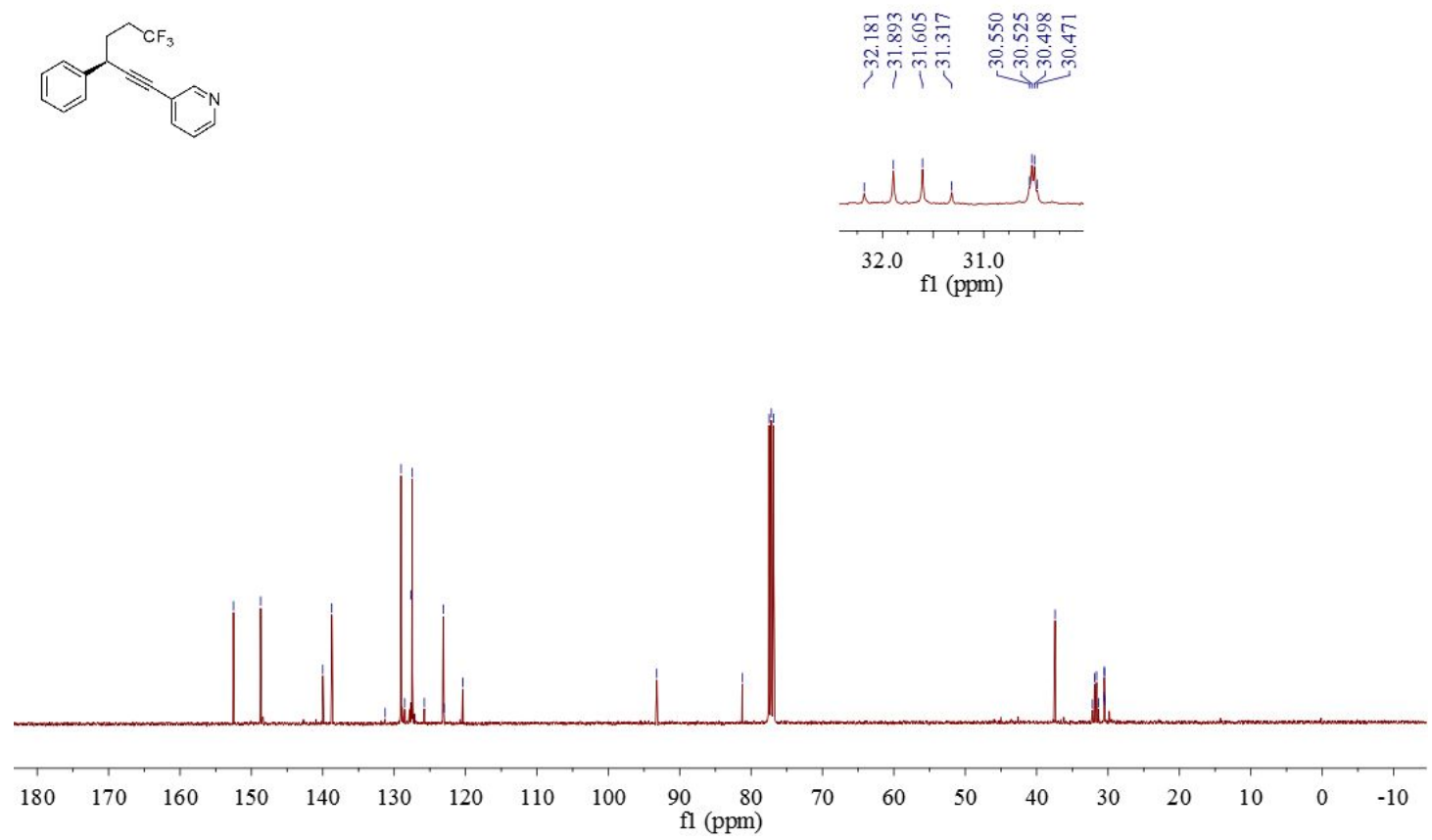
d22 - ${ }^{19}$ F NMR
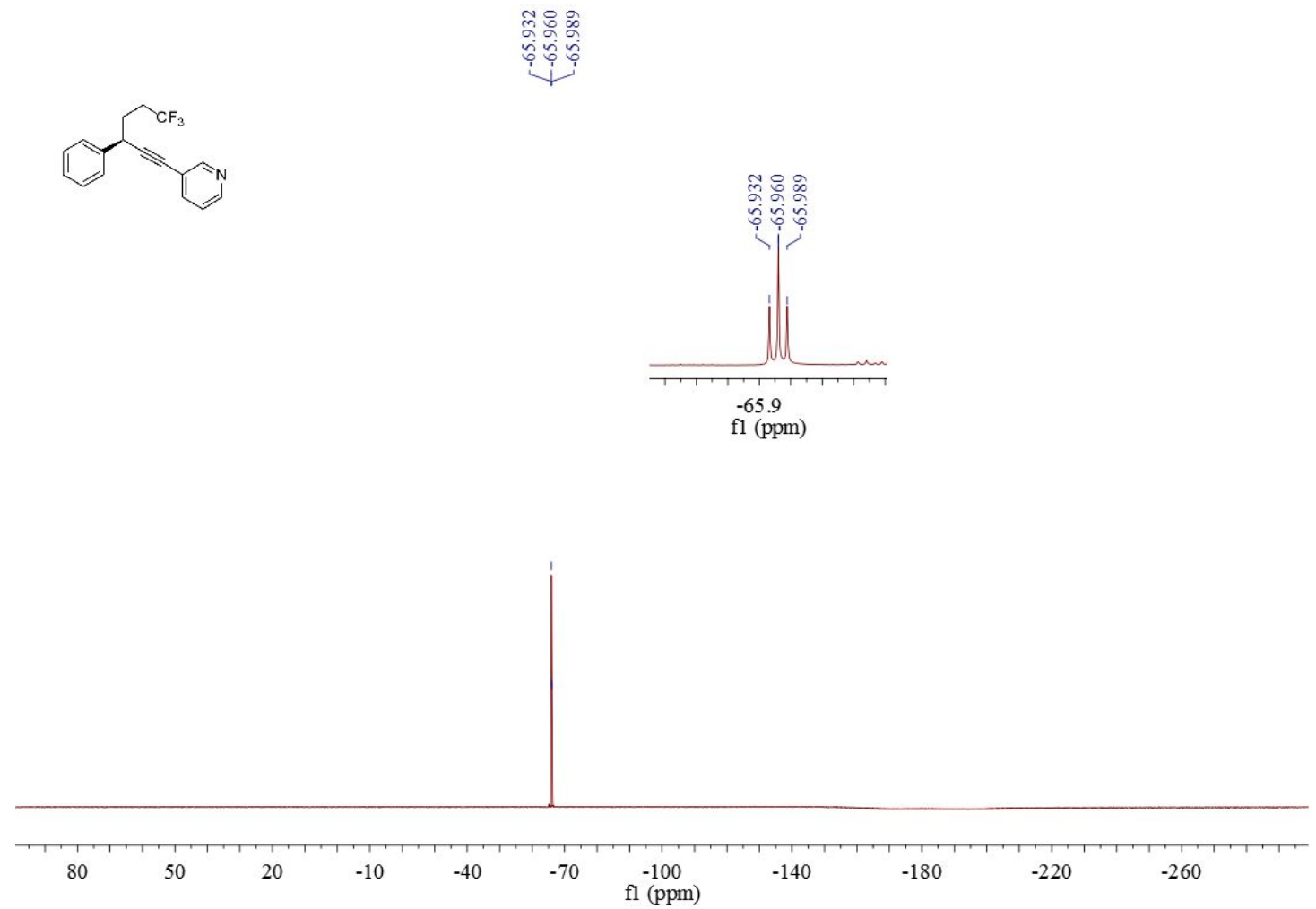

\section{d22 - HPLC}

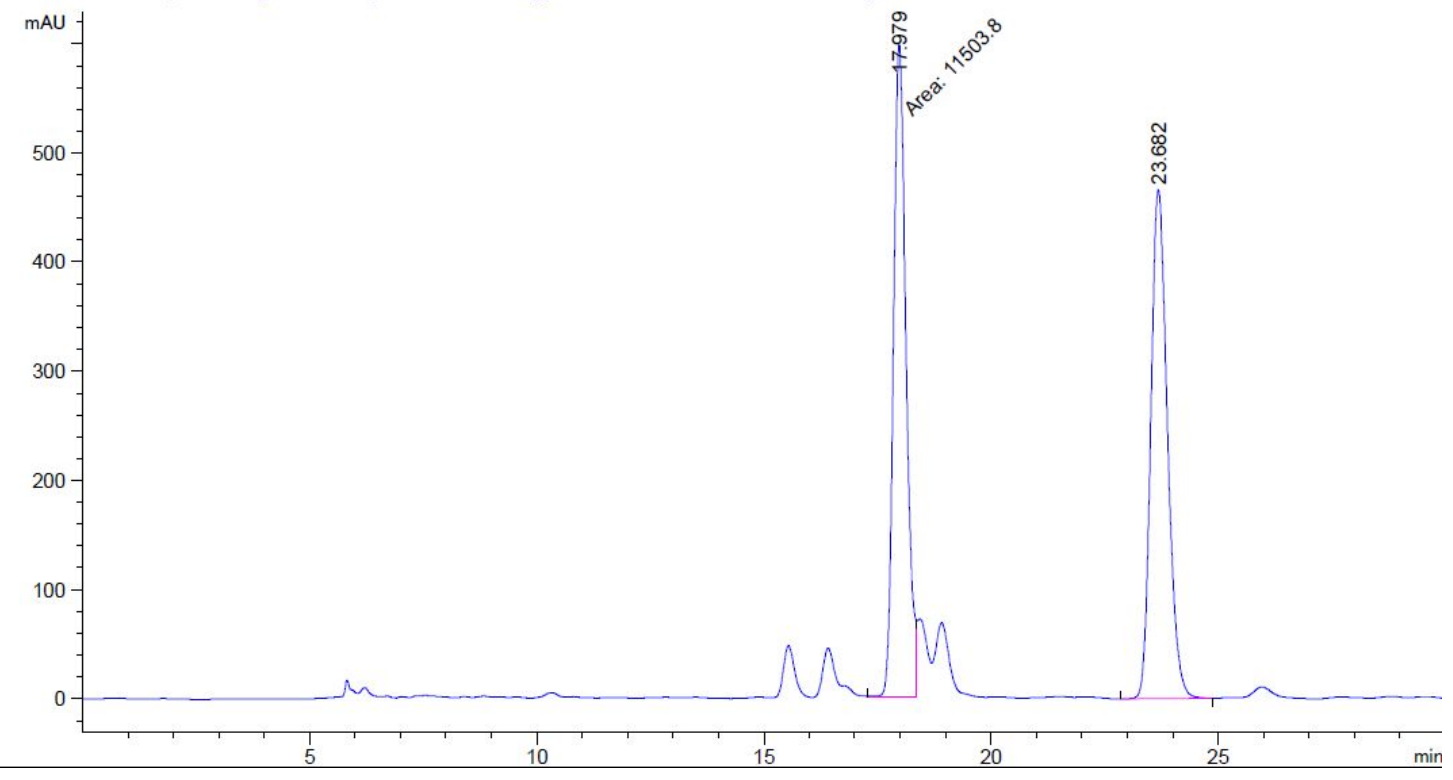




\begin{tabular}{|c|c|c|c|c|c|c|}
\hline $\begin{array}{c}\text { Peak } \\
\quad \#\end{array}$ & $\begin{array}{c}\text { RetTime } \\
\text { [min] }\end{array}$ & Type & $\begin{array}{l}\text { Width } \\
\text { [min] }\end{array}$ & $\begin{array}{c}\text { Area } \\
{\left[\mathrm{mAU}^{*} \mathrm{~s}\right]}\end{array}$ & $\begin{array}{l}\text { Height } \\
{[\mathrm{mAU}]}\end{array}$ & $\begin{array}{c}\text { Area } \\
\%\end{array}$ \\
\hline & & & & & & I \\
\hline 1 & 17.97 & MF & 0.3209 & $1.15038 \mathrm{e} 4$ & 597.42432 & 49.2775 \\
\hline 2 & 23.68 & BB & 0.3903 & $1.18411 \mathrm{e} 4$ & 466.28647 & 50.7225 \\
\hline
\end{tabular}

Totals :

$2.33449 \mathrm{e} 4 \quad 1063.71078$

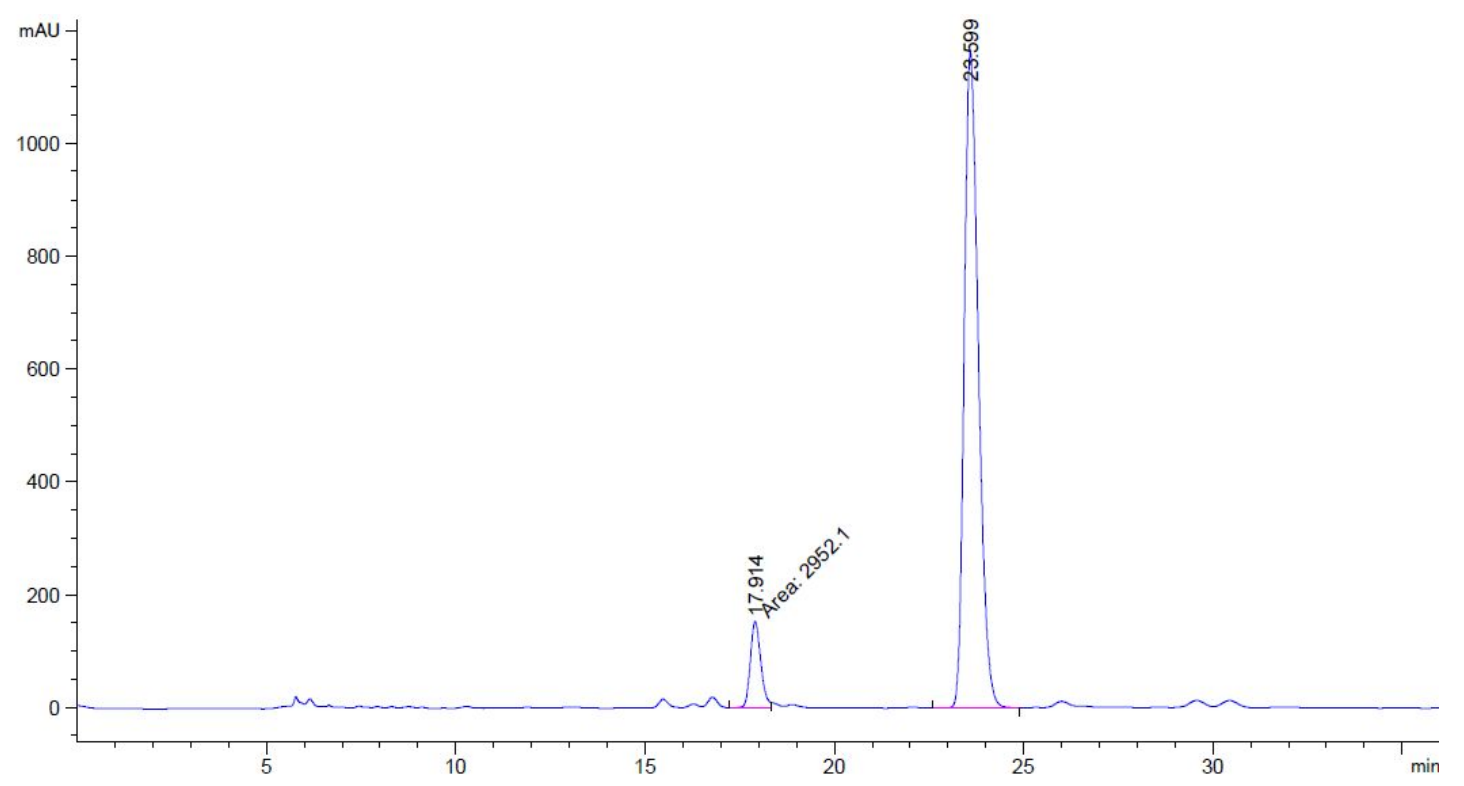

Peak RetTime Type Width Area Height Area

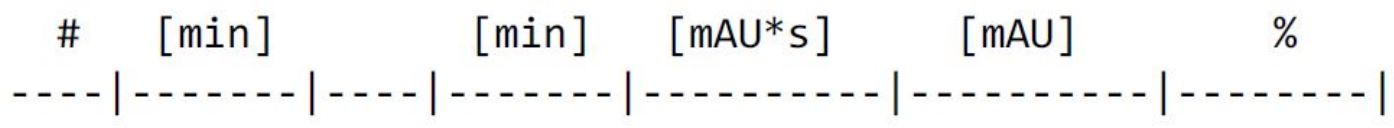

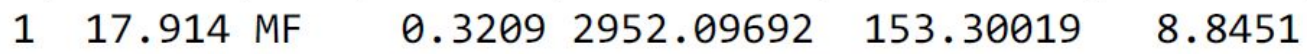

$\begin{array}{lllllll}2 & 23.599 & \text { BB } & 0.4072 & 3.04235 \mathrm{e} 4 & 1162.85022 & 91.1549\end{array}$

Totals :

$3.33756 \mathrm{e} 4 \quad 1316.15041$ 
(R)-1-methyl-3-(6, 6, 6-trifluoro-3-phenylhex-1-yn-1-yl) benzene (d23)

\section{d23 - ${ }^{1}$ H NMR}

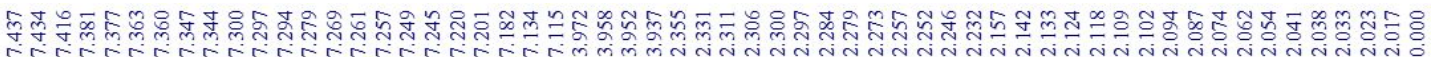

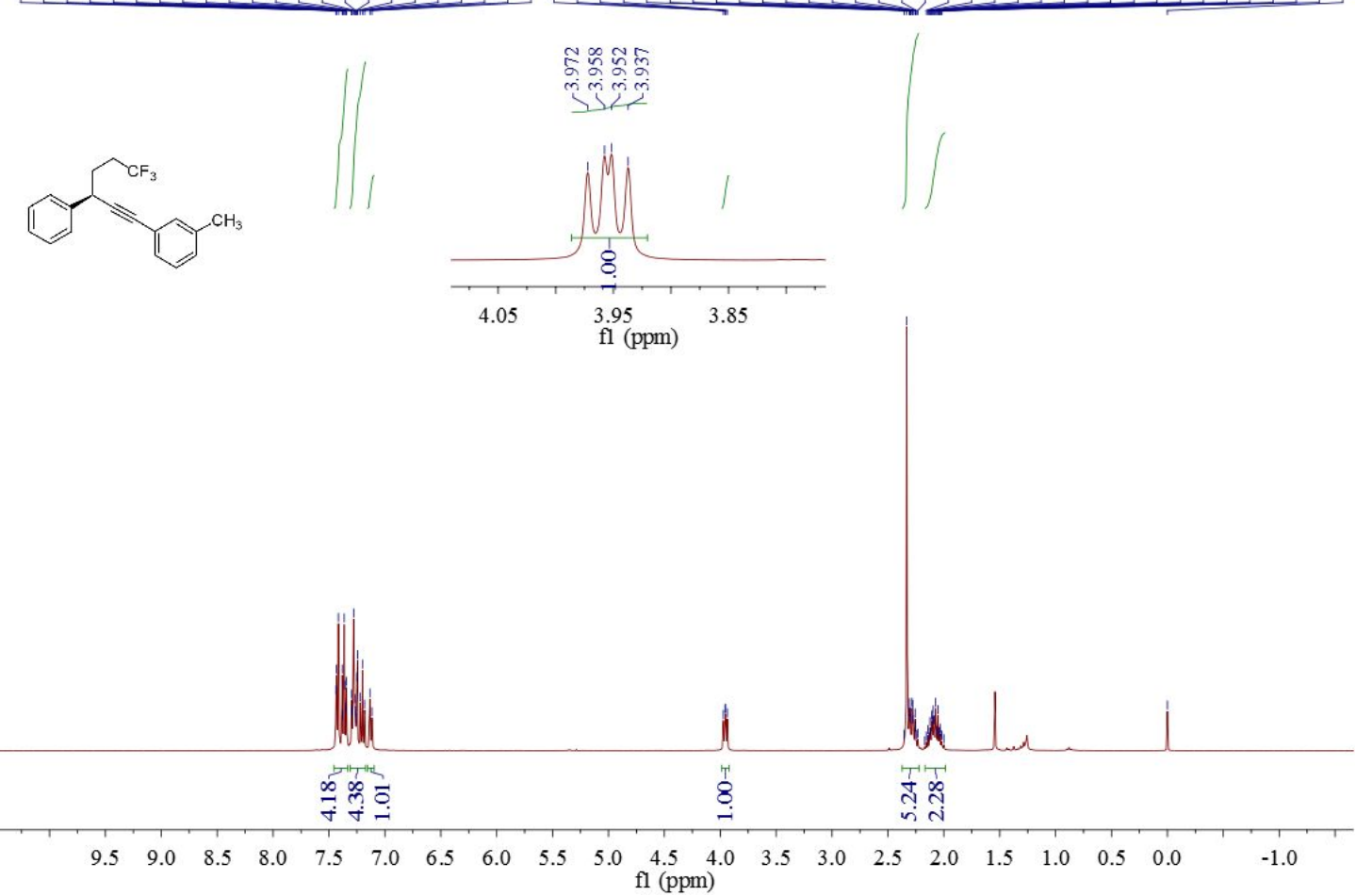

d23 - ${ }^{13}$ C NMR

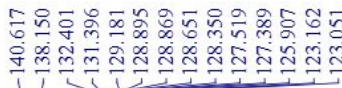

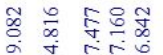

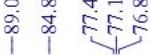

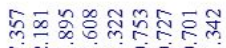

लिलmंmंघंले
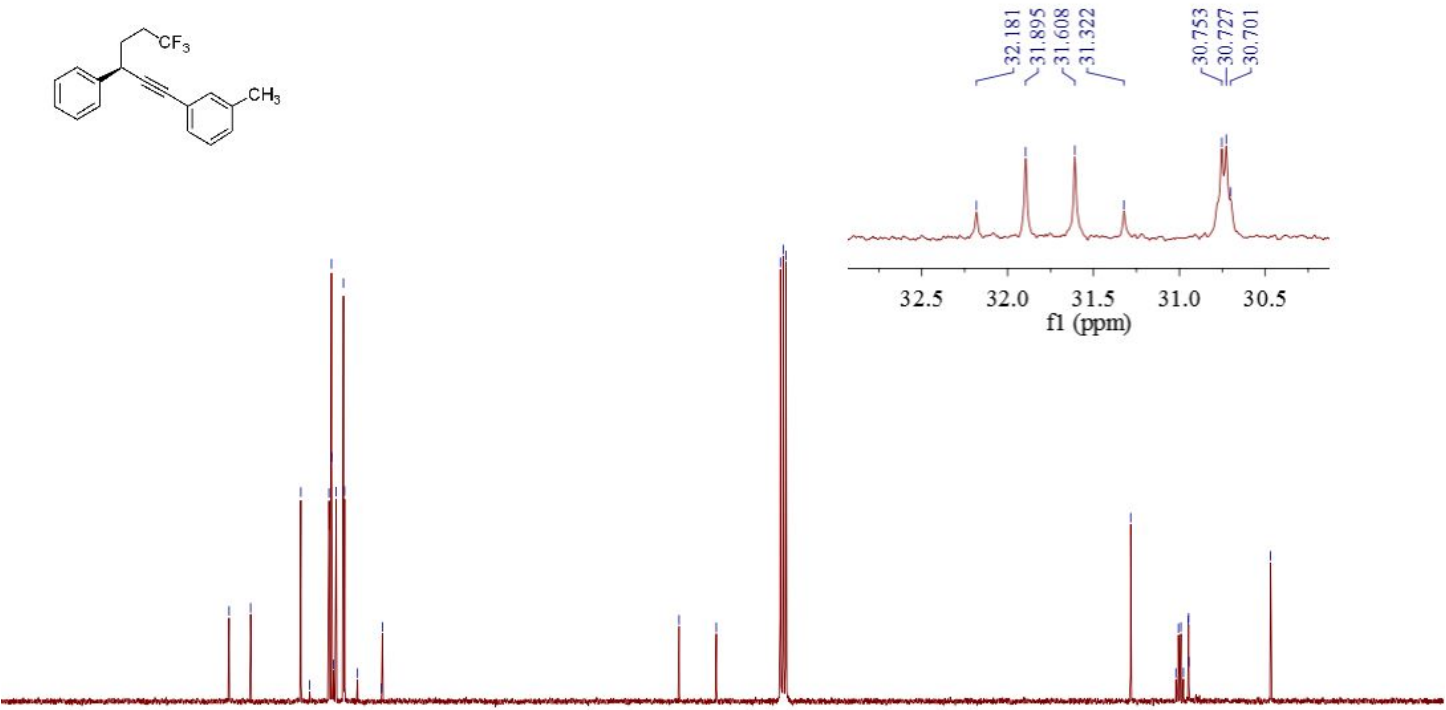

160

$\begin{array}{llllll}140 & 130 & 120 & 110 & 100 & 90 \quad \begin{array}{r}80 \\ \mathrm{fl}(\mathrm{ppm})\end{array}\end{array}$

$70 \quad 60$

10 

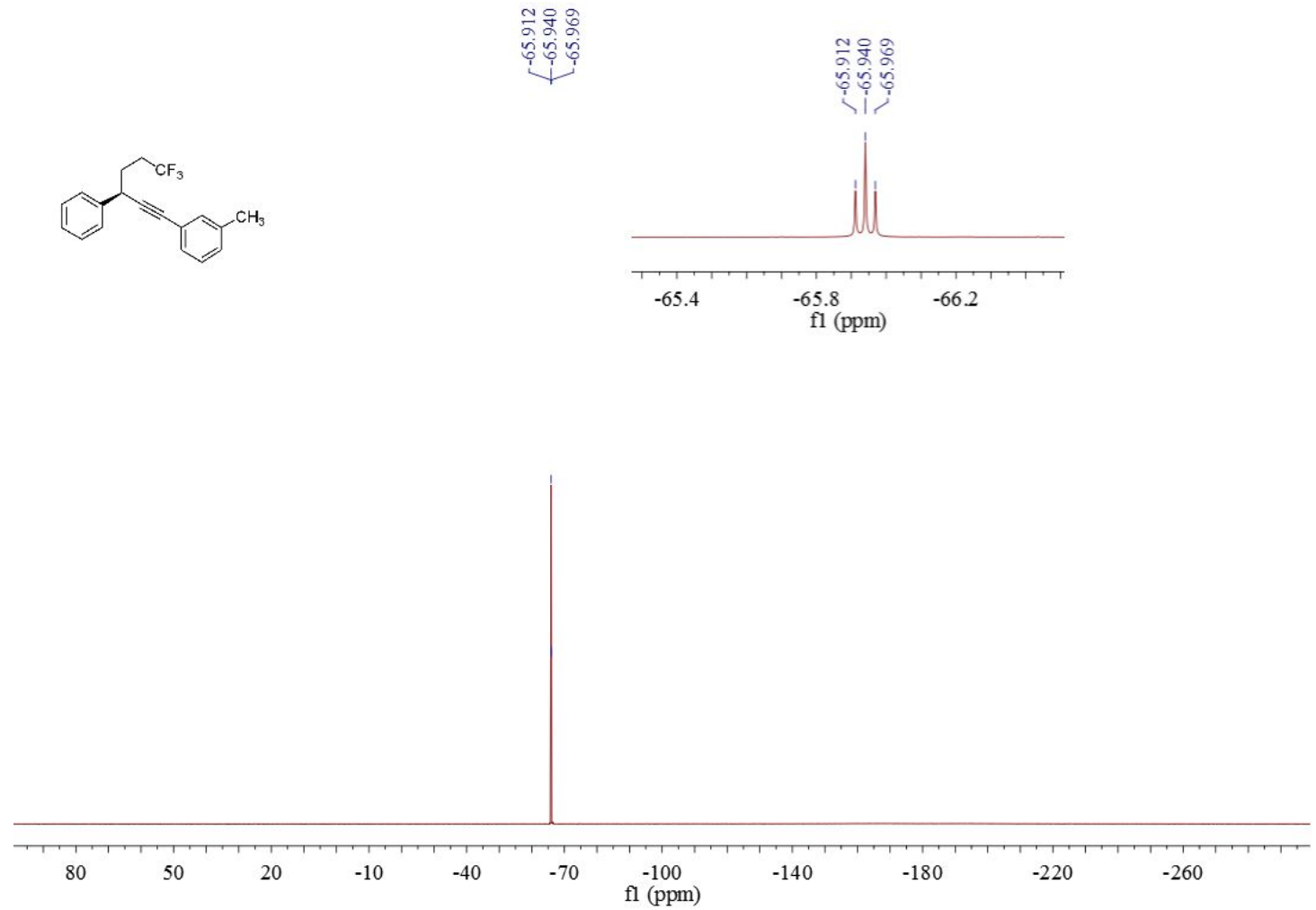

\section{d23 - HPLC}

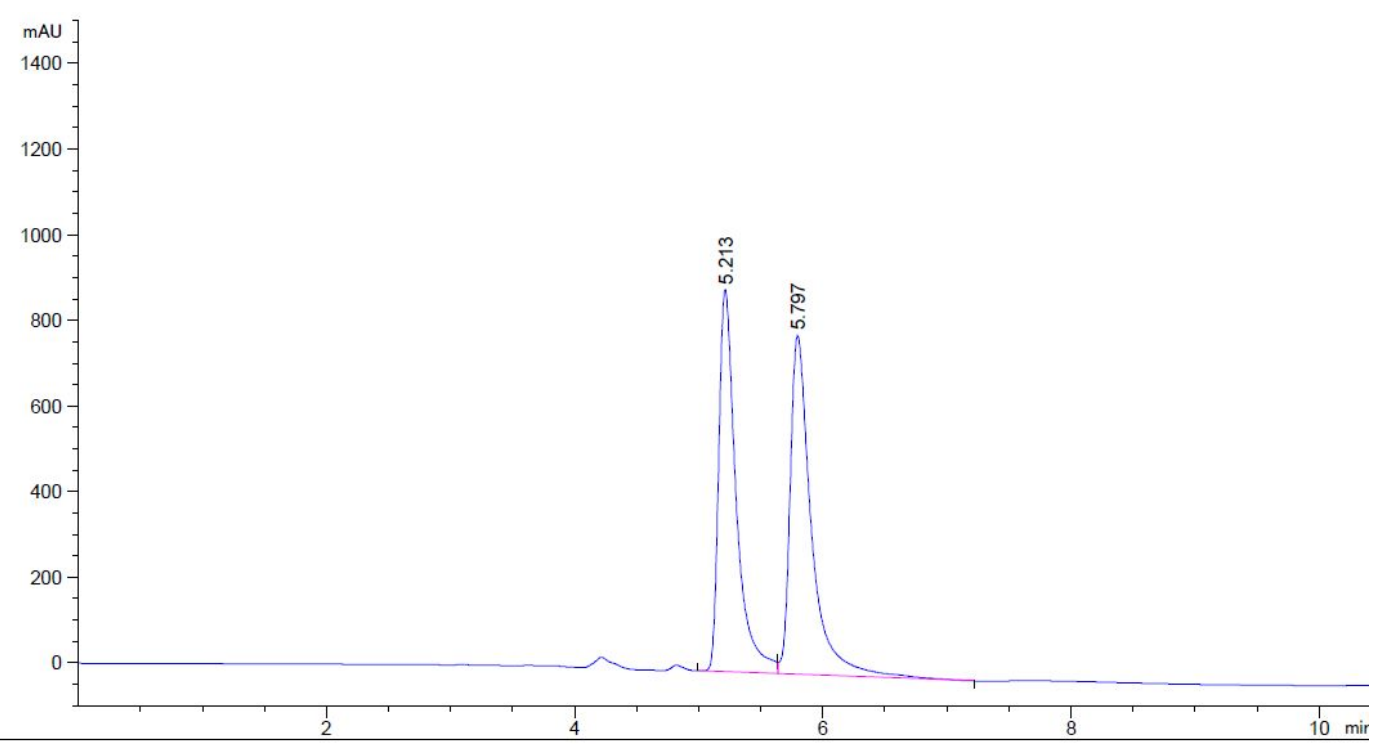

Peak RetTime Type width Area Height Area

\begin{tabular}{ccccccc}
$\#$ & {$[\mathrm{~min}]$} & {$[\mathrm{min}]$} & {$\left[\mathrm{mAU}{ }^{*} \mathrm{~s}\right]$} & {$[\mathrm{mAU}]$} & $\%$ \\
\hline 1 & 5.213 & BV & 0.1449 & 8711.53516 & 892.02856 & 47.9173 \\
2 & 5.797 VB & 0.1767 & 9468.83301 & 790.49377 & 52.0827
\end{tabular}

Totals :

$1.81804 \mathrm{e} 4 \quad 1682.52234$ 


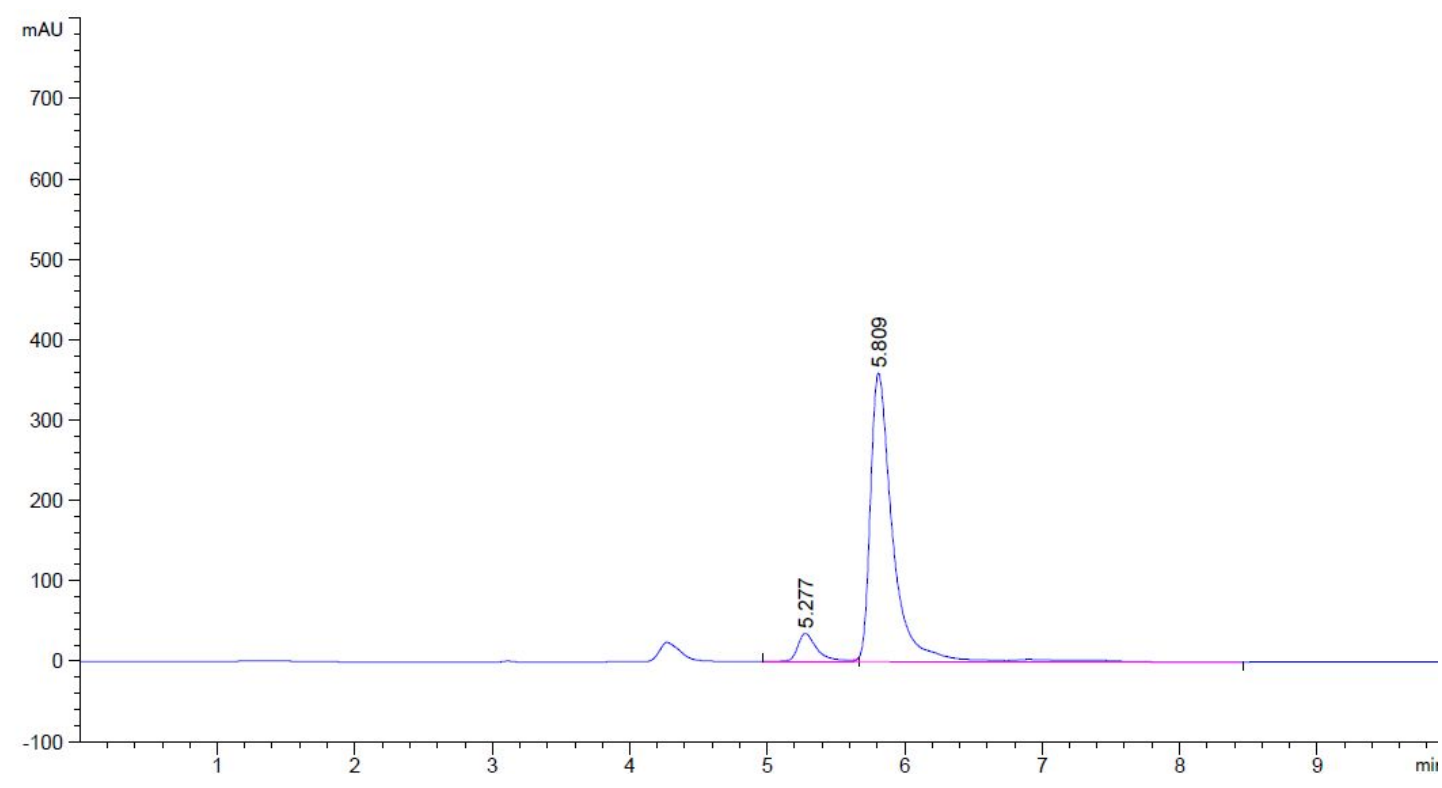

\begin{tabular}{|c|c|c|c|c|c|c|}
\hline $\begin{array}{c}\text { Peak } \\
\#\end{array}$ & $\begin{array}{c}\text { RetTime } \\
\text { [min] }\end{array}$ & Type & $\begin{array}{l}\text { Width } \\
\text { [min] }\end{array}$ & $\begin{array}{c}\text { Area } \\
{\left[\mathrm{mAU}^{*} \mathrm{~s}\right]}\end{array}$ & $\begin{array}{l}\text { Height } \\
{[\mathrm{mAU}]}\end{array}$ & $\begin{array}{c}\text { Area } \\
\%\end{array}$ \\
\hline & & & & & & - - \\
\hline 1 & 5.277 & BV E & 1467 & 349.85919 & 34.97495 & 7.8034 \\
\hline 2 & 5.809 & VV $R$ & 0.1670 & 4133.54932 & 359.18781 & 92.1966 \\
\hline
\end{tabular}

Totals :

$4483.40851 \quad 394.16275$

(R)-1-chloro-2-(6, 6, 6-trifluoro-3-phenylhex-1-yn-1-yl) benzene (d24) d24 - ${ }^{1}$ H NMR

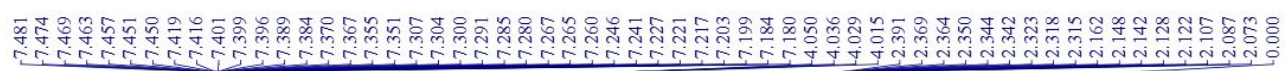
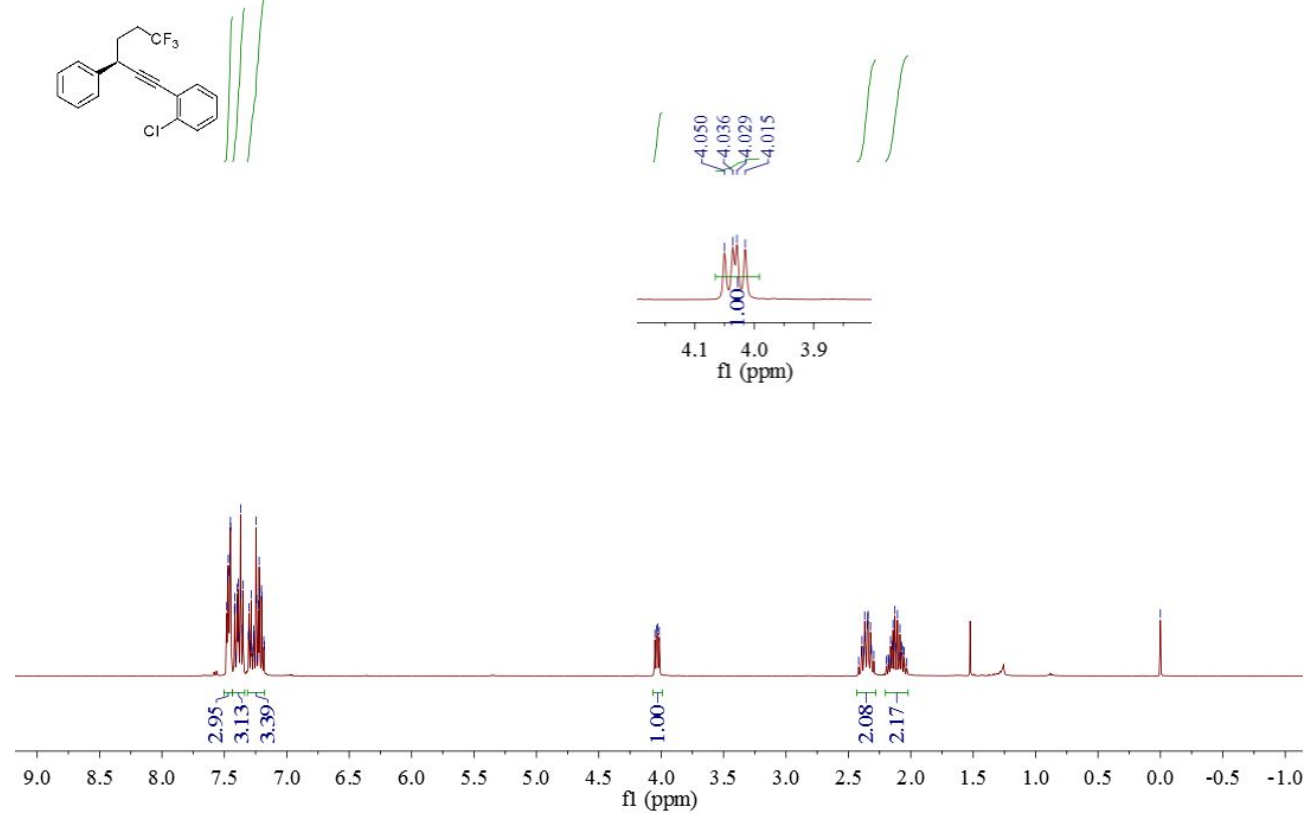
d24 - ${ }^{13}$ C NMR

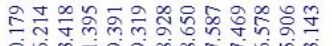

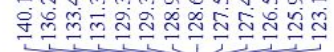

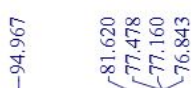

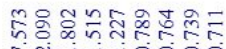

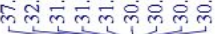

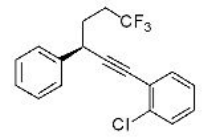

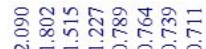

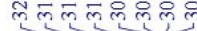

$\frac{|d|}{32 \quad 31}$

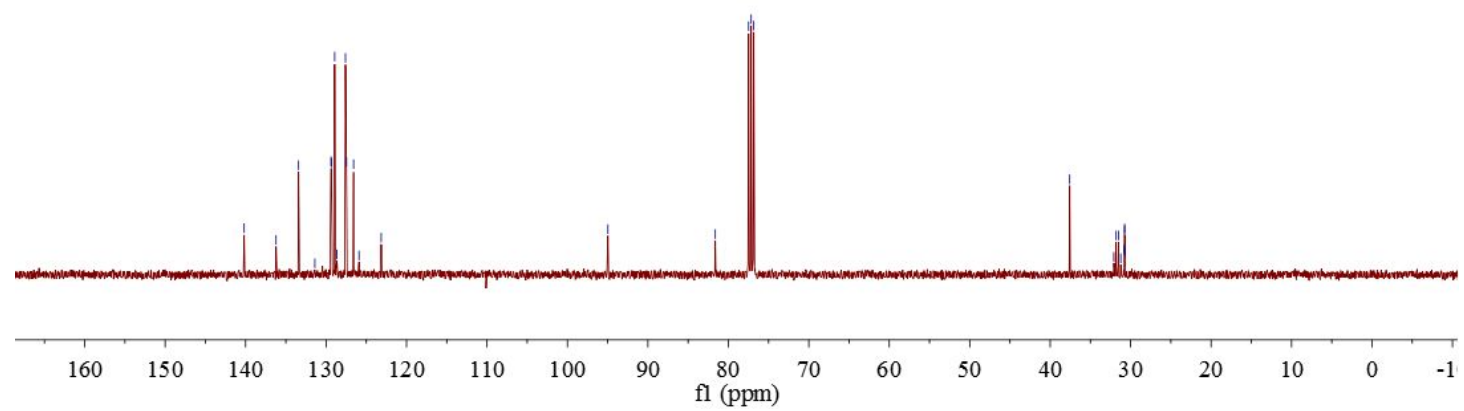

\section{d24 - ${ }^{19}$ F NMR}
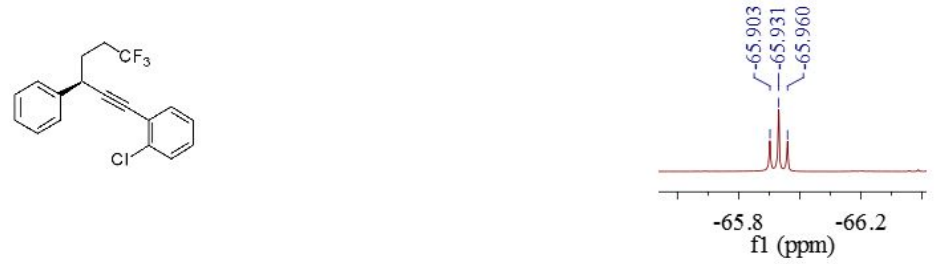

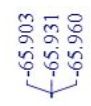

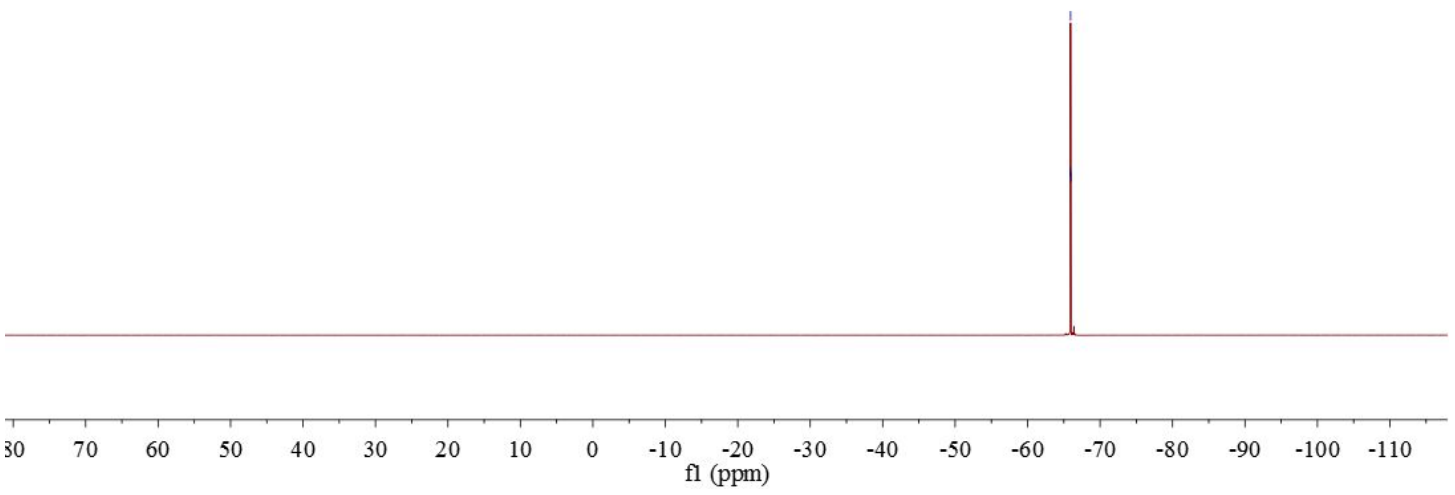




\section{d24 - HPLC}

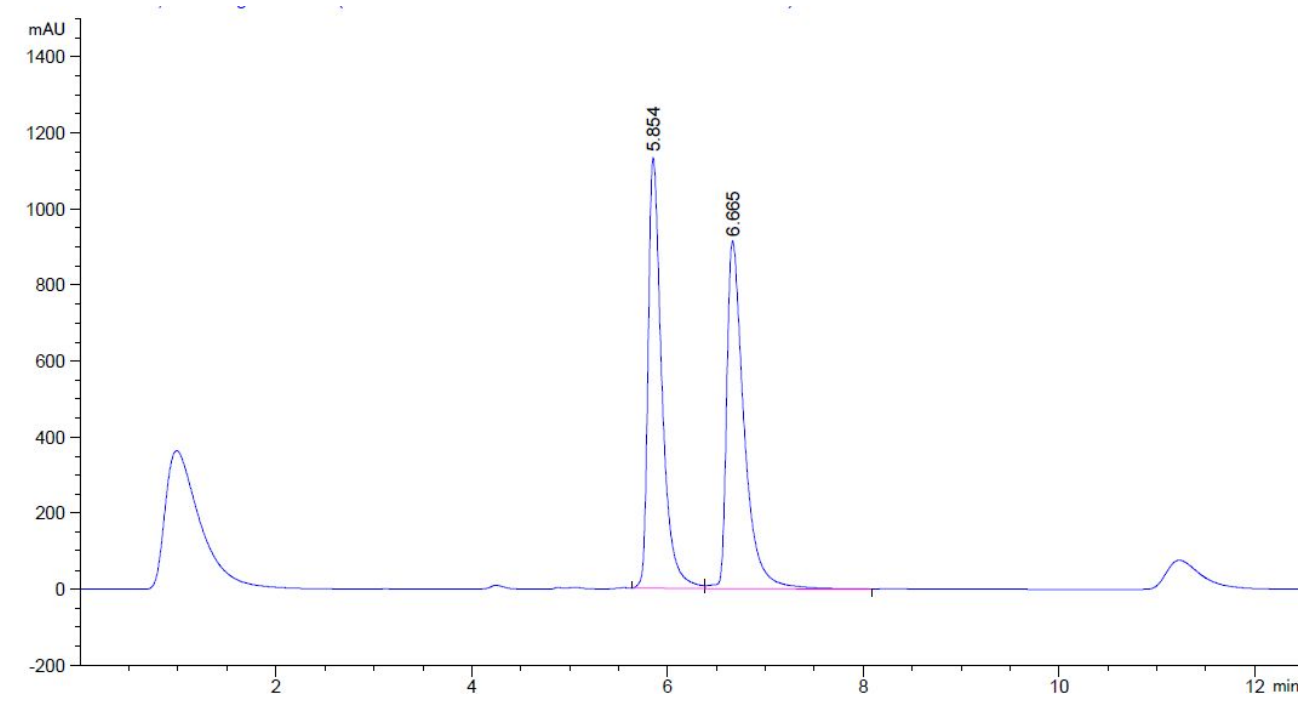

Peak RetTime Type width Area Height Area

$\begin{array}{lllll}\# & {[\mathrm{~min}]} & {[\mathrm{min}]} & {[\mathrm{mAU} * \mathrm{~s}]} & {[\mathrm{mAU}]}\end{array}$

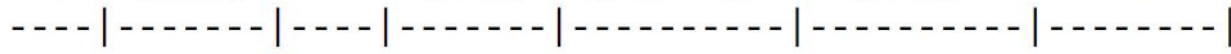

$\begin{array}{llllll}1 & 5.854 \text { BV } \quad 0.1468 & 1.10590 \mathrm{e} 4 & 1134.04285 & 49.6475\end{array}$

$\begin{array}{llllll}2 & 6.665 \text { VB } & 0.1832 & 1.12160 \mathrm{e} 4 & 914.07184 & 50.3525\end{array}$

Totals : $\quad 2.22750 \mathrm{e} 4 \quad 2048.11469$

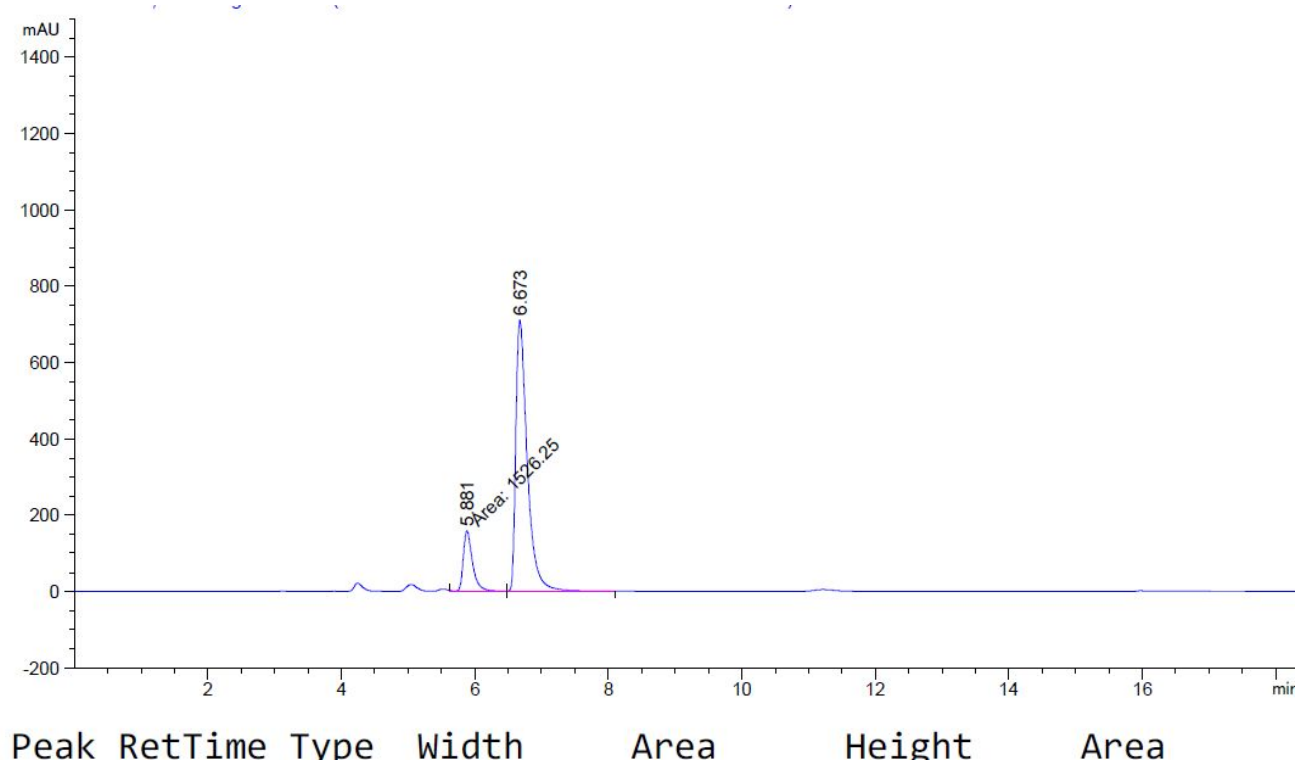

Peak RetTime Type Width Area Height Area

$\begin{array}{llll}\# & {[\mathrm{~min}]} & {[\mathrm{min}]} & {[\mathrm{mAU} * \mathrm{~s}]}\end{array}$

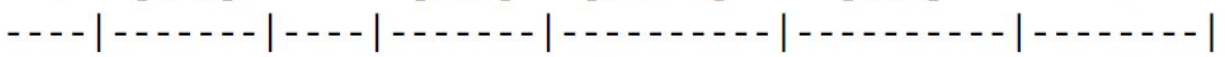

$\begin{array}{lllllll}1 & 5.881 & \mathrm{FM} & 0.1608 & 1526.25452 & 158.15327 & 15.2888\end{array}$

$\begin{array}{lllllll}2 & 6.673 \text { VB } & 0.1789 & 8456.57910 & 710.23492 & 84.7112\end{array}$

Totals : $\quad 9982.83362 \quad 868.38820$ 
(R)-2-(6, 6, 6-trifluoro-3-phenylhex-1-yn-1-yl) thiophene (d25)

\section{d25 - ${ }^{1}$ H NMR}

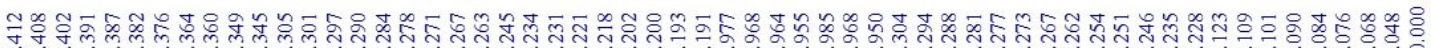

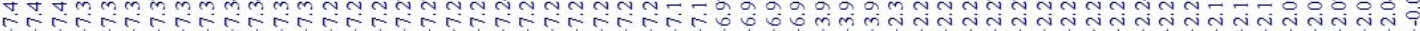
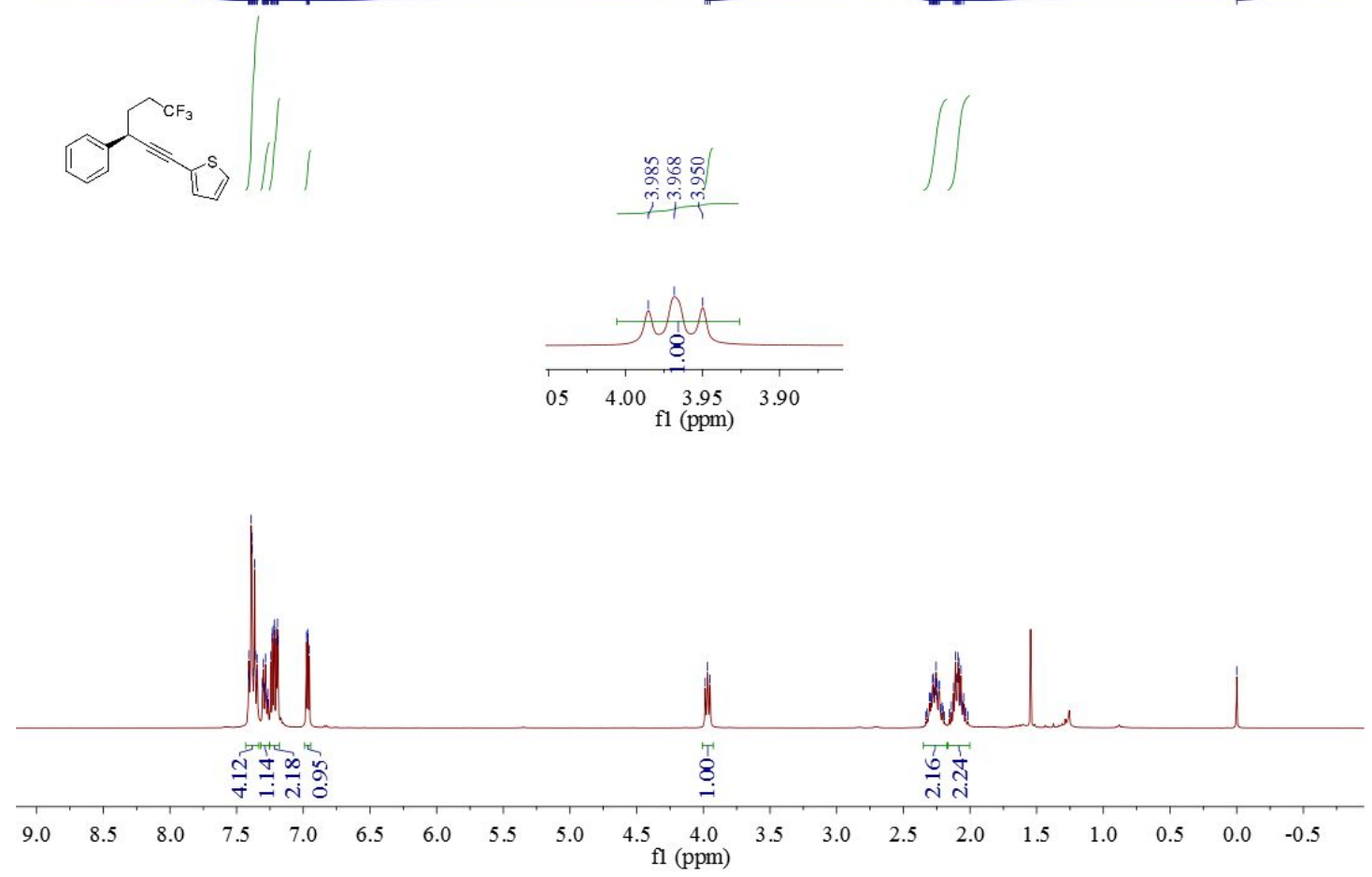

d25 - ${ }^{13}$ C NMR

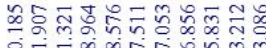

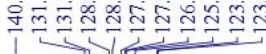

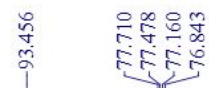

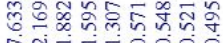

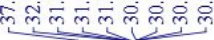
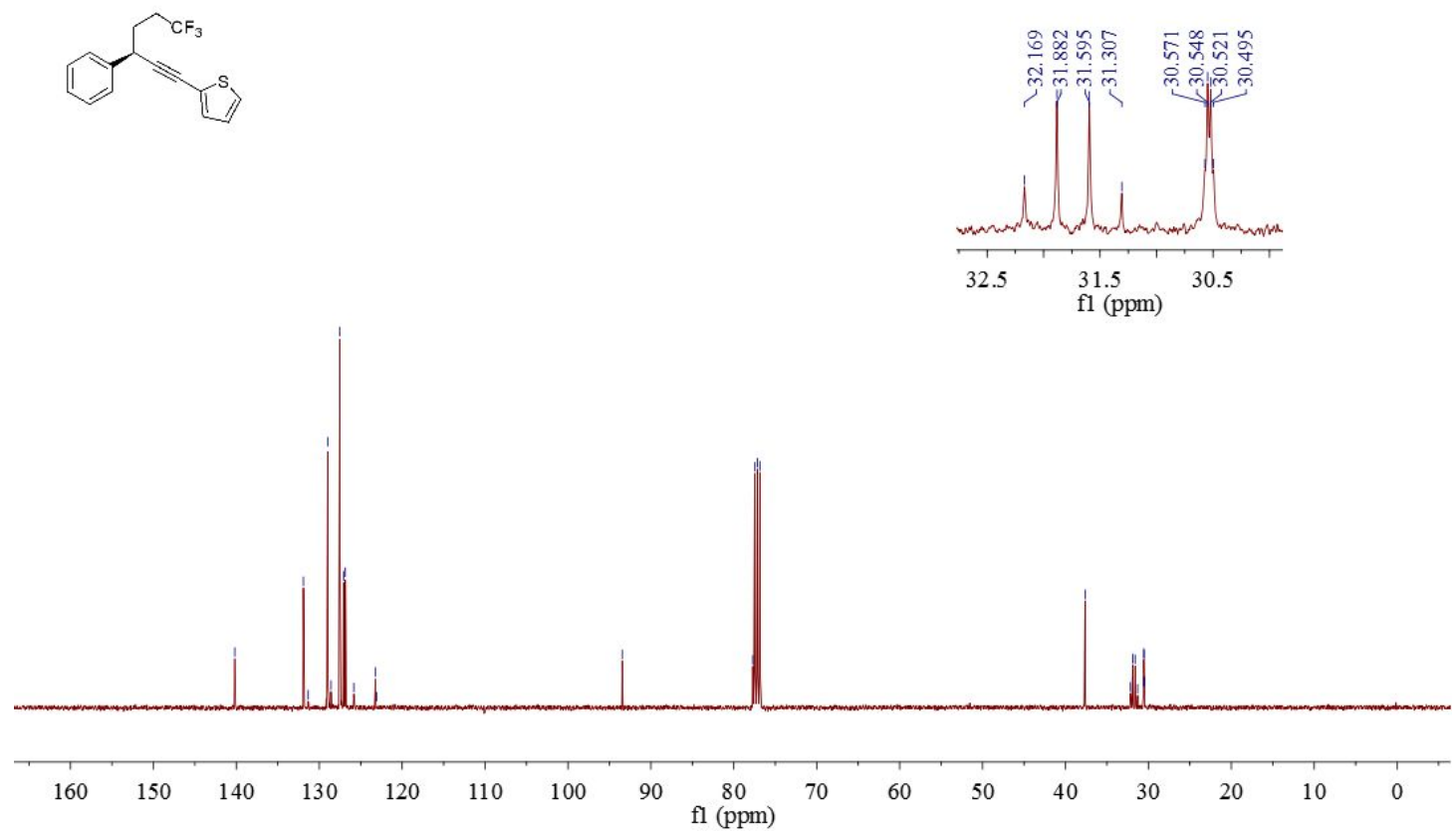


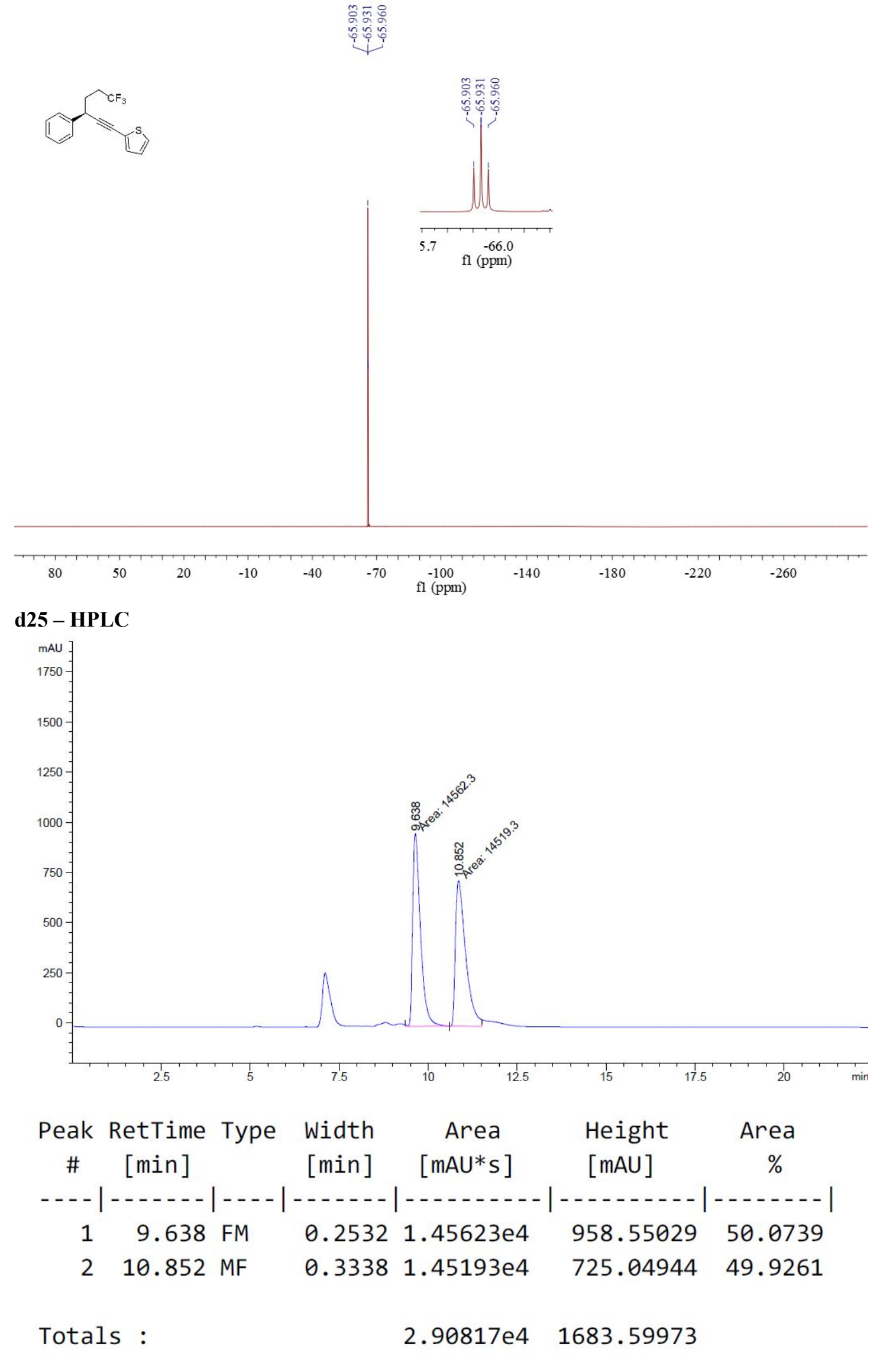




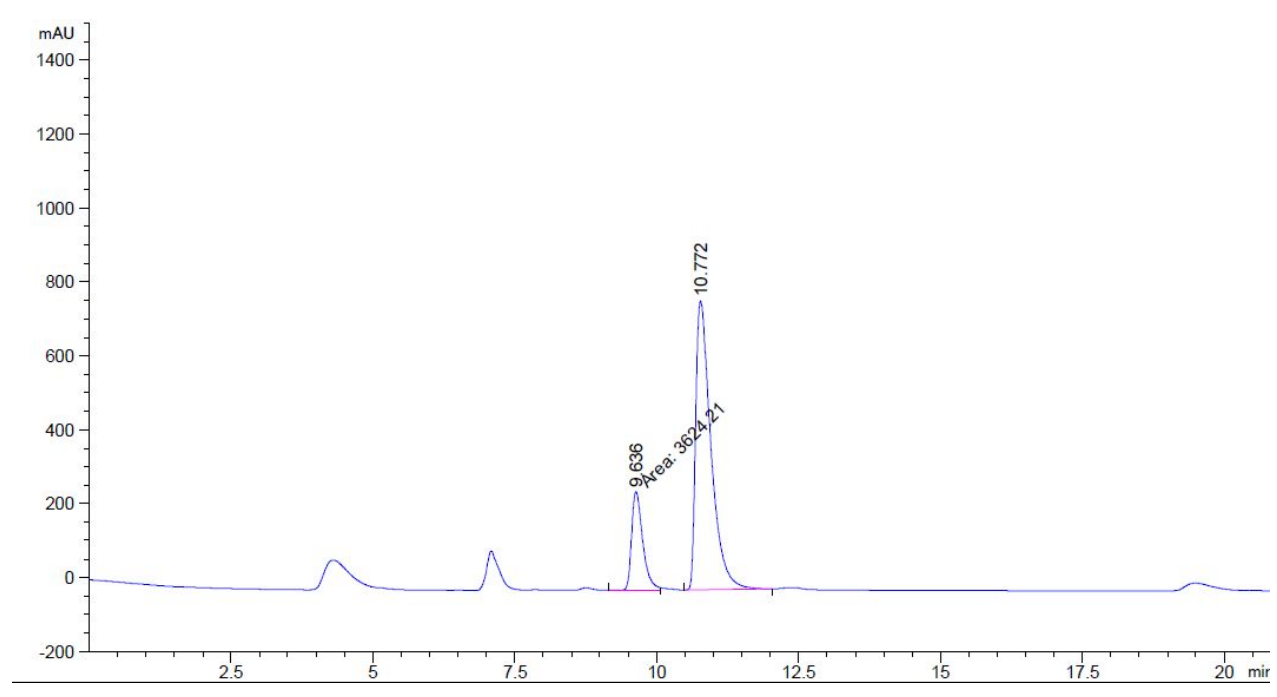

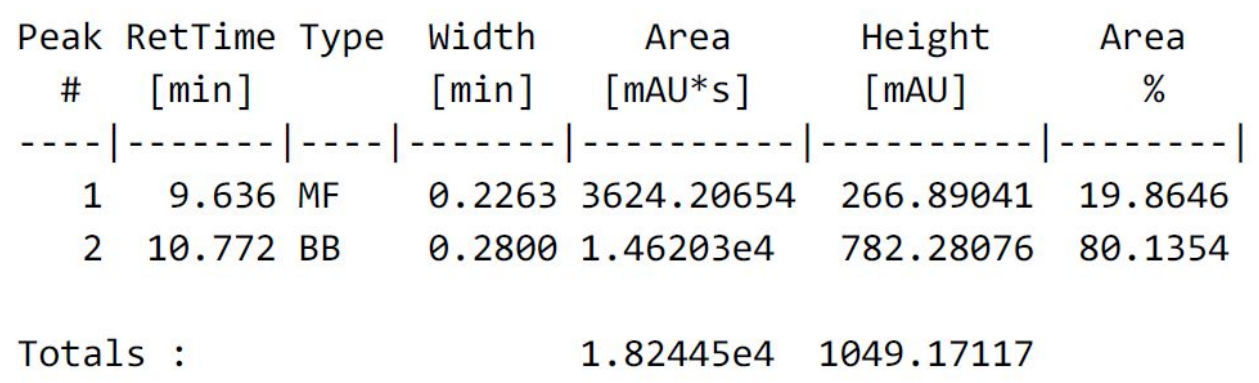

(R)-4-(6, 6, 6-trifluoro-1-(4-methoxyphenyl)hex-1-yn-3-yl)-1,1'-biphenyl (d26) d26 - ${ }^{1} \mathrm{H}$ NMR

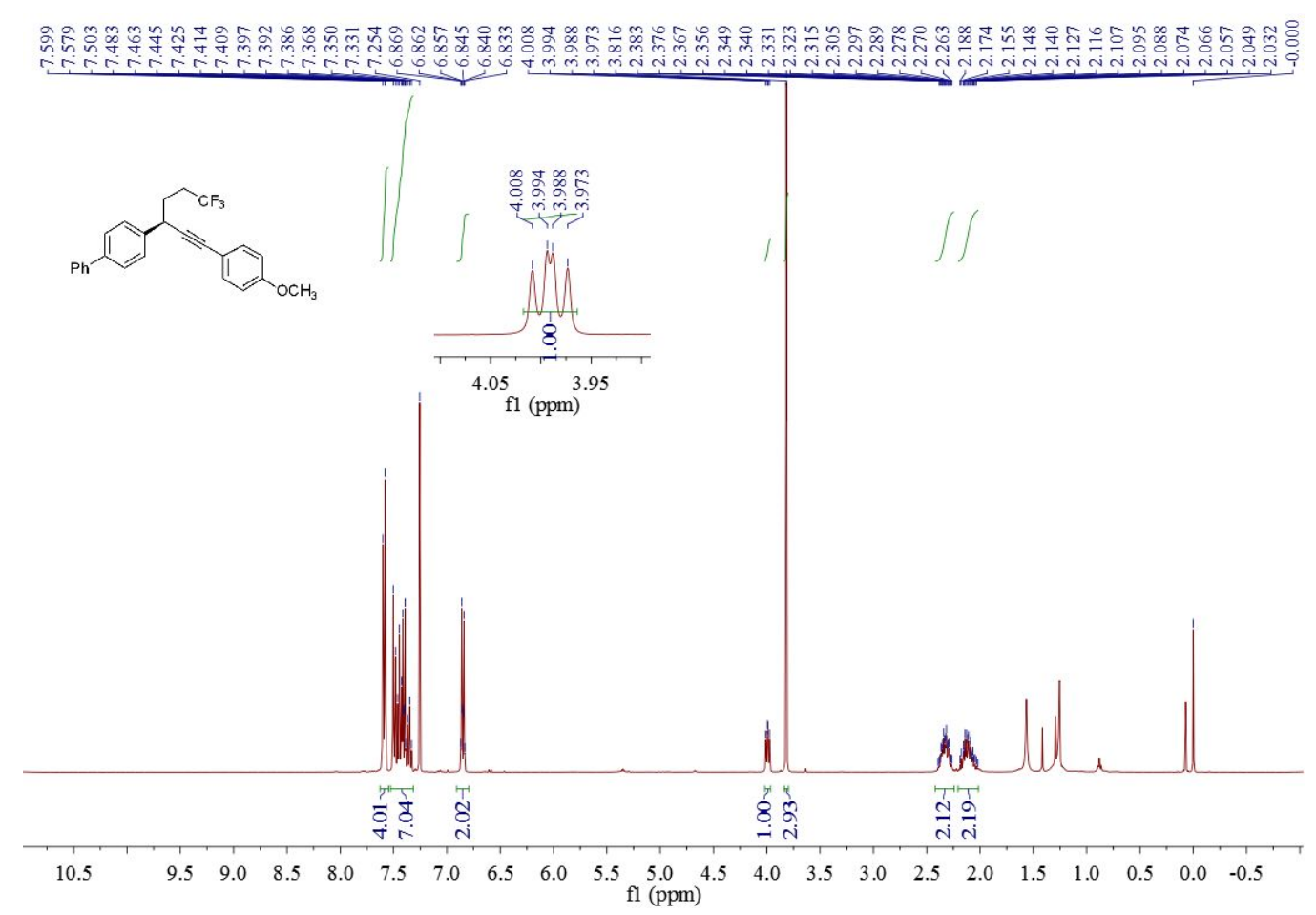


d26 - ${ }^{13}$ C NMR

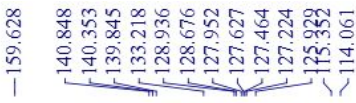<smiles>COc1ccc(C#CC(CC(F)(F)F)c2ccc(-c3ccccc3)cc2)cc1</smiles>

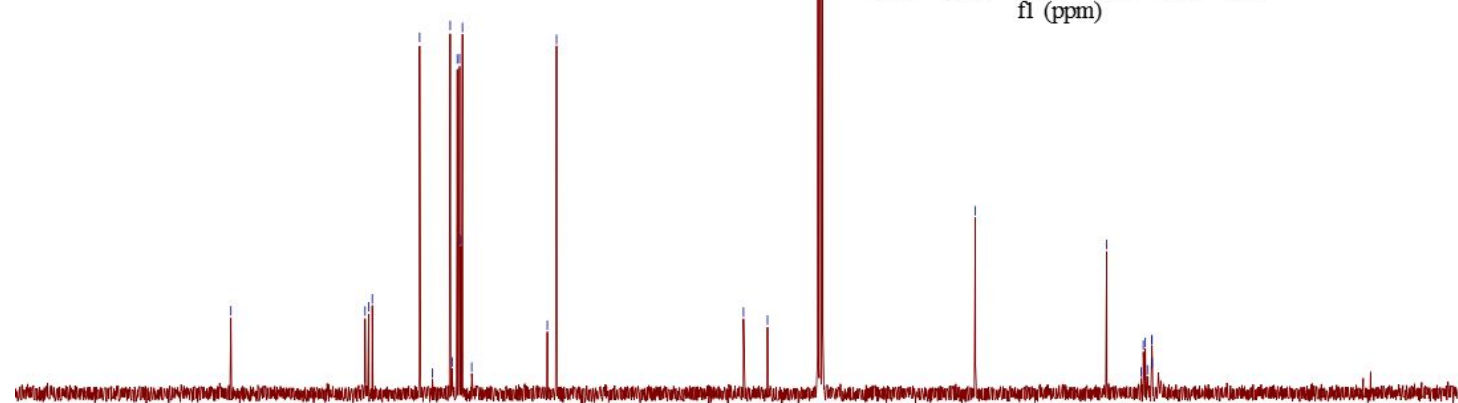

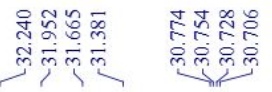

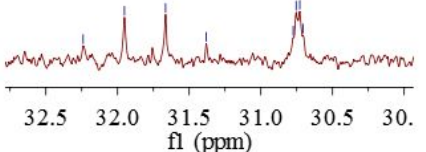

$\begin{array}{lllllllllllllllllll}180 & 170 & 160 & 150 & 140 & 130 & 120 & 110 & 100 & \underset{\mathrm{fl}(\mathrm{ppm})}{90} 80 & 70 & 60 & 50 & 40 & 30 & 20 & 10 & 0 & -10\end{array}$

\section{d26 - ${ }^{19}$ F NMR}

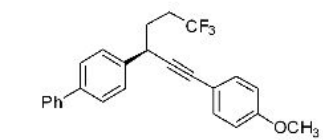

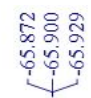

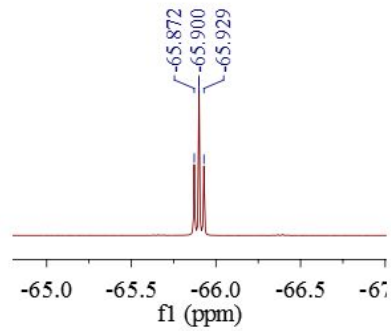

$\begin{array}{lllllllllllllllllll}-10 & -20 & -30 & -40 & -50 & -60 & -70 & -80 & -90 & \begin{array}{c}-100 \\ \mathrm{fl}(\mathrm{ppm})\end{array} & -110 & -120 & -130 & -140 & -150 & -160 & -170 & -180 & -190\end{array}$ 
d26 - HPLC

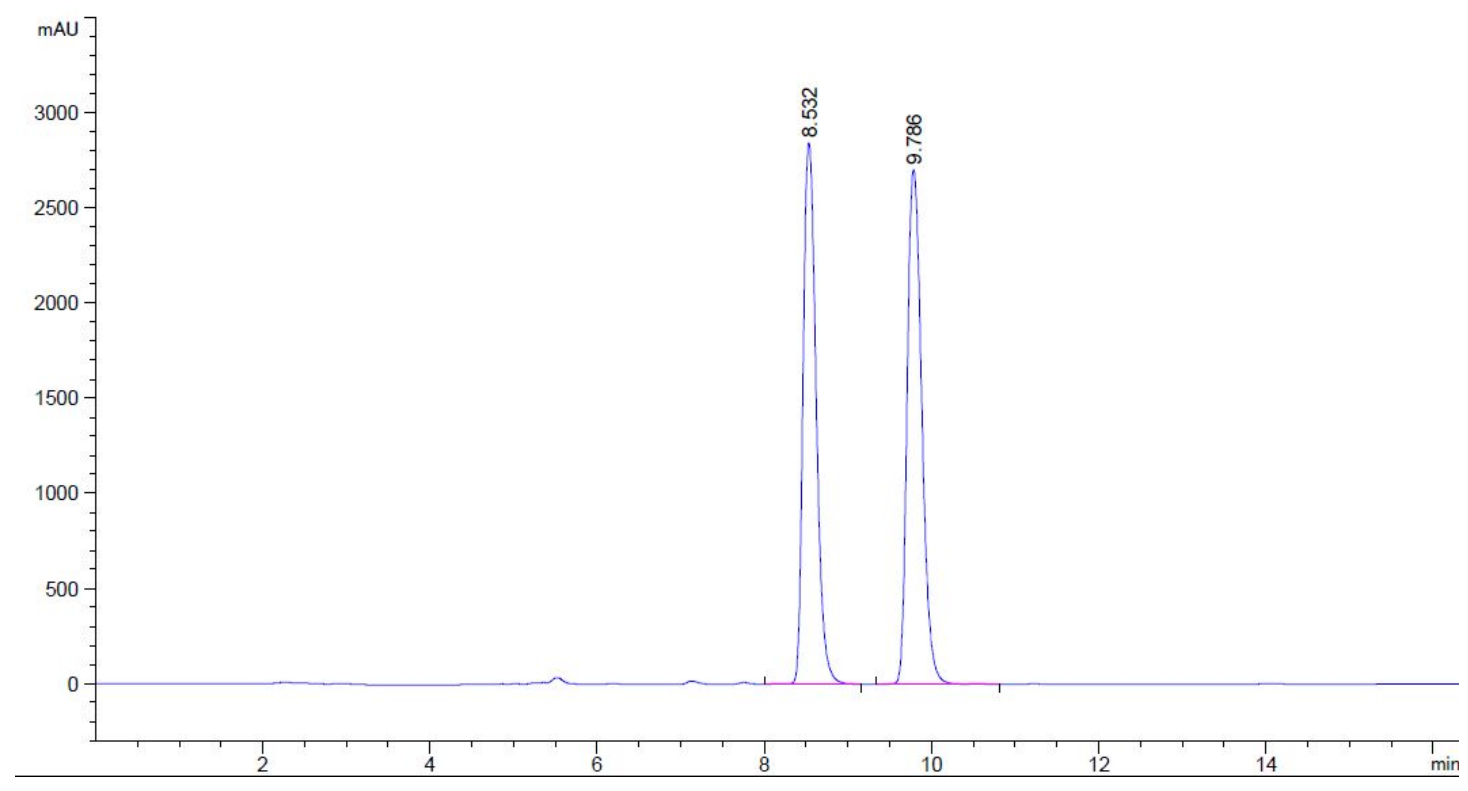

Peak RetTime Type width Area Height Area

$\begin{array}{lllll}\# & {[\mathrm{~min}]} & {[\mathrm{min}]} & {[\mathrm{mAU} * \mathrm{~s}]} & {[\mathrm{mAU}]}\end{array}$

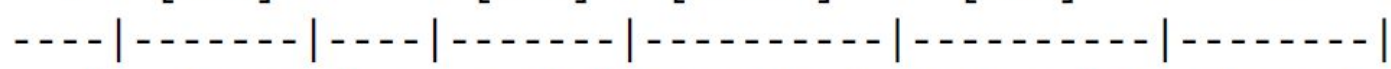

$\begin{array}{llllllll}1 & 8.532 & \text { VB R } & 0.1709 & 3.09431 \mathrm{e} & 2845.07422 & 48.5375\end{array}$

$\begin{array}{lllllll}2 & 9.786 & \text { BV R } & 0.1904 & 3.28079 \mathrm{e} 4 & 2703.92505 & 51.4625\end{array}$

Totals : $\quad 6.37510 \mathrm{e} 4 \quad 5548.99927$

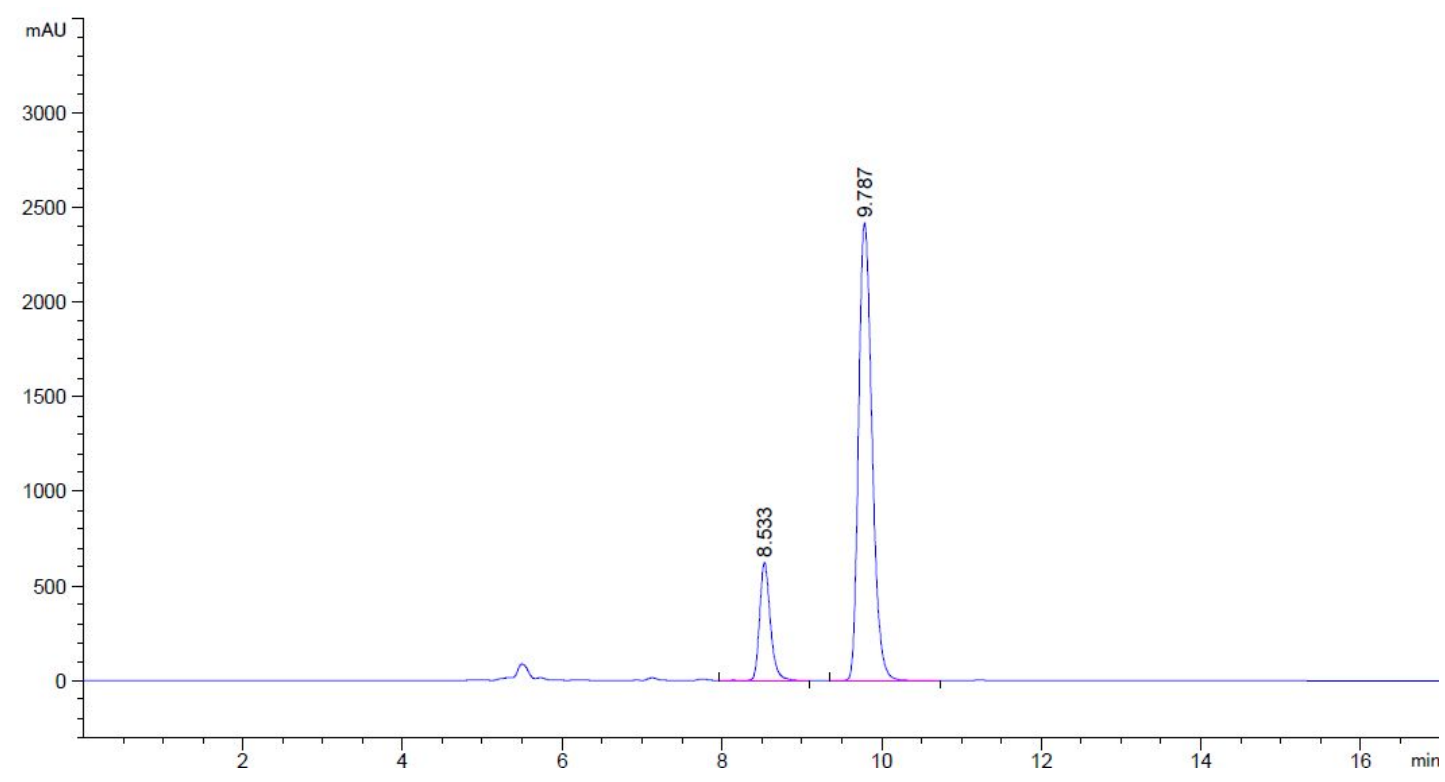




$$
\begin{array}{cccccc}
\begin{array}{c}
\text { Peak RetTime Type } \\
\text { \# }
\end{array} \begin{array}{c}
\text { Width } \\
{[\mathrm{min}]}
\end{array} & \begin{array}{c}
\text { Area } \\
{[\mathrm{min}]}
\end{array} & \begin{array}{c}
\text { Height } \\
{[\mathrm{mAU} \text { s }]}
\end{array} & \begin{array}{c}
\text { Area } \\
{[\mathrm{mAU}]}
\end{array} & \% \\
\hline 1 & 8.533 \text { VB R } & 0.1413 & 5796.35645 & 624.39923 & 16.9760 \\
2 & 9.787 \text { BB } & 0.1837 & 2.83481 \mathrm{e} 4 & 2419.17310 & 83.0240
\end{array}
$$

Totals : $\quad 3.41445 \mathrm{e} 4 \quad 3043.57233$

(R)- (3-([1, 1'-biphenyl]-4-yl)-6, 6-difluorohex-1-yn-1-yl) triisopropylsilane (d27) d27 - ${ }^{1}$ H NMR

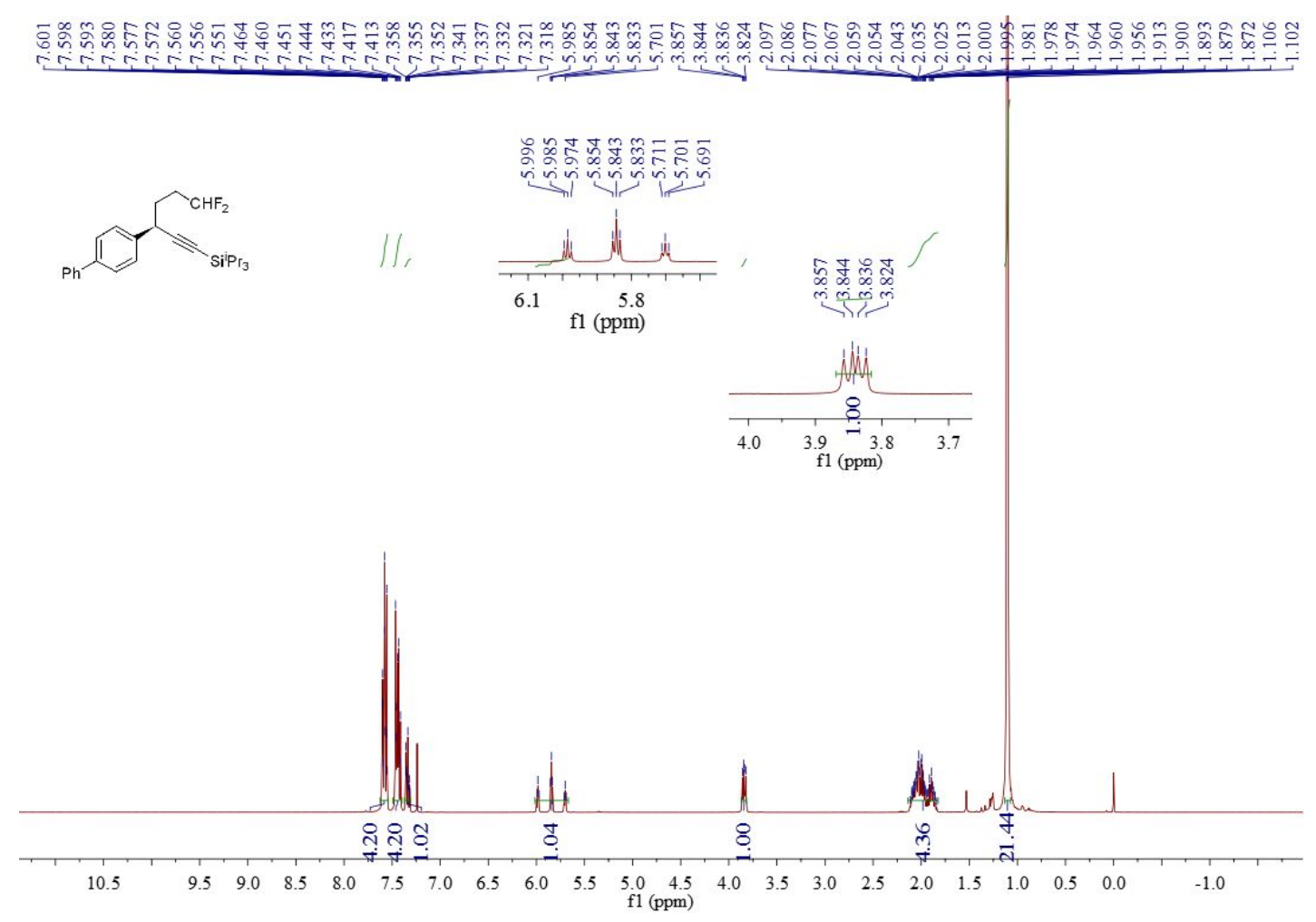




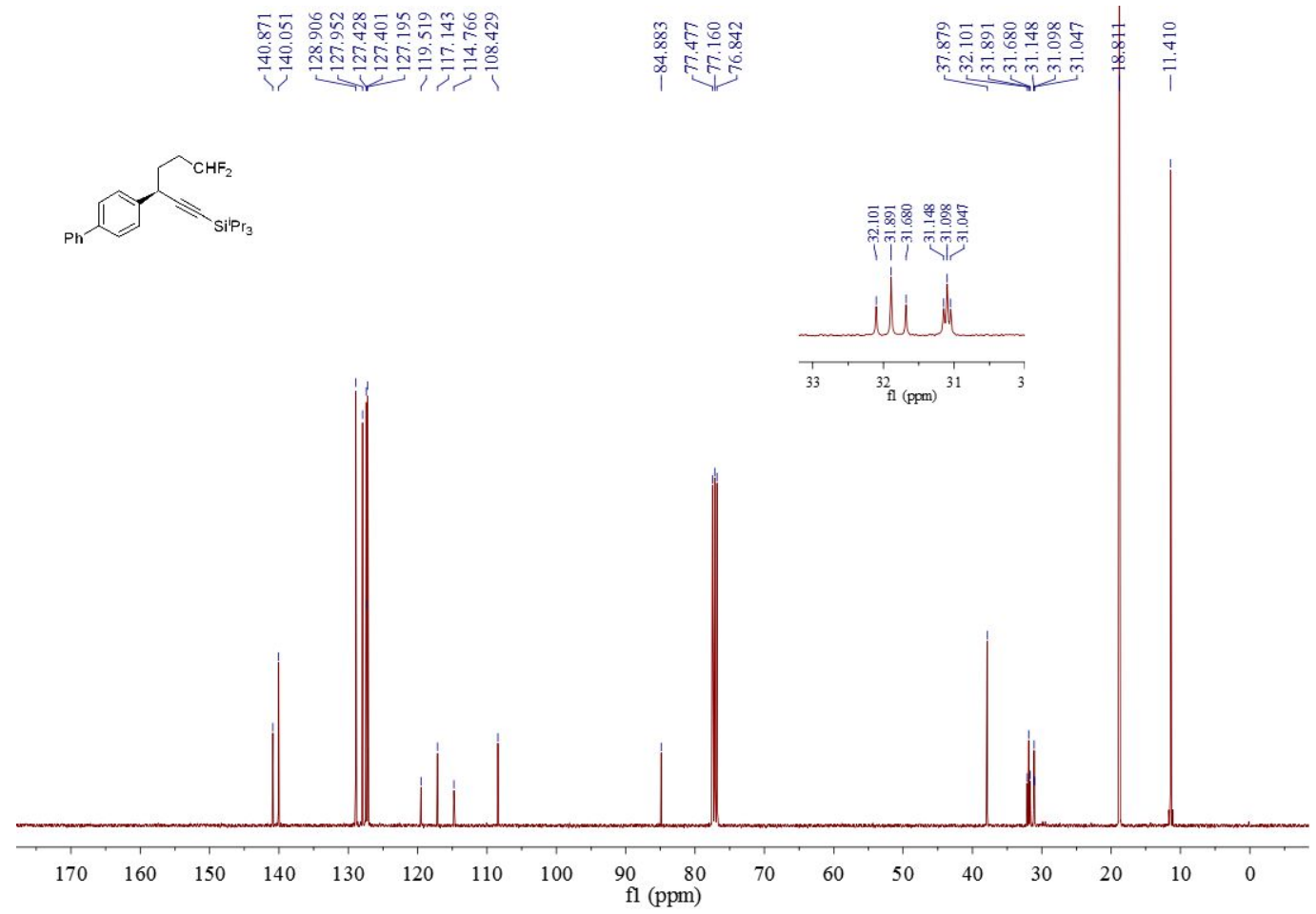

d27 - ${ }^{19}$ F NMR
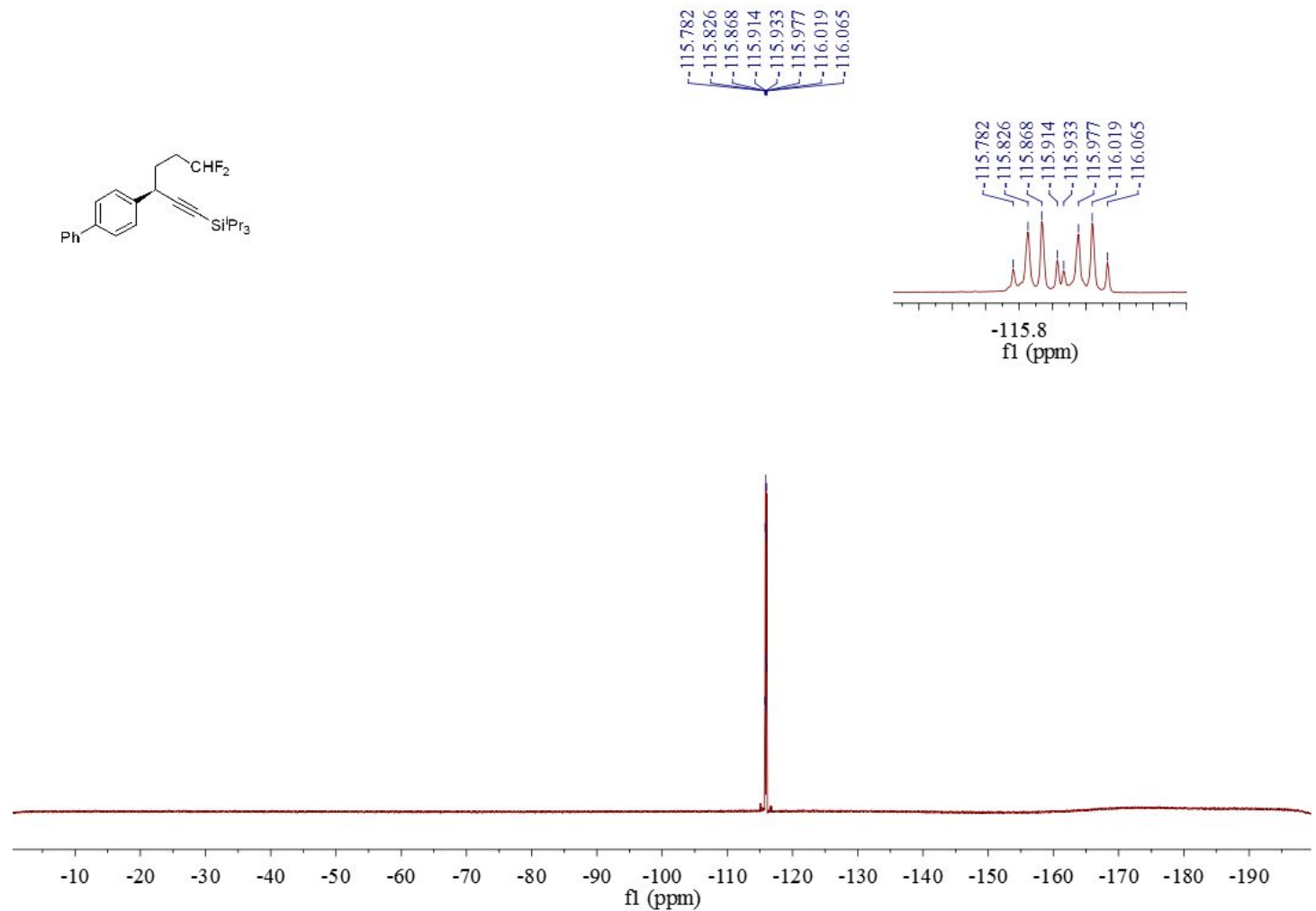


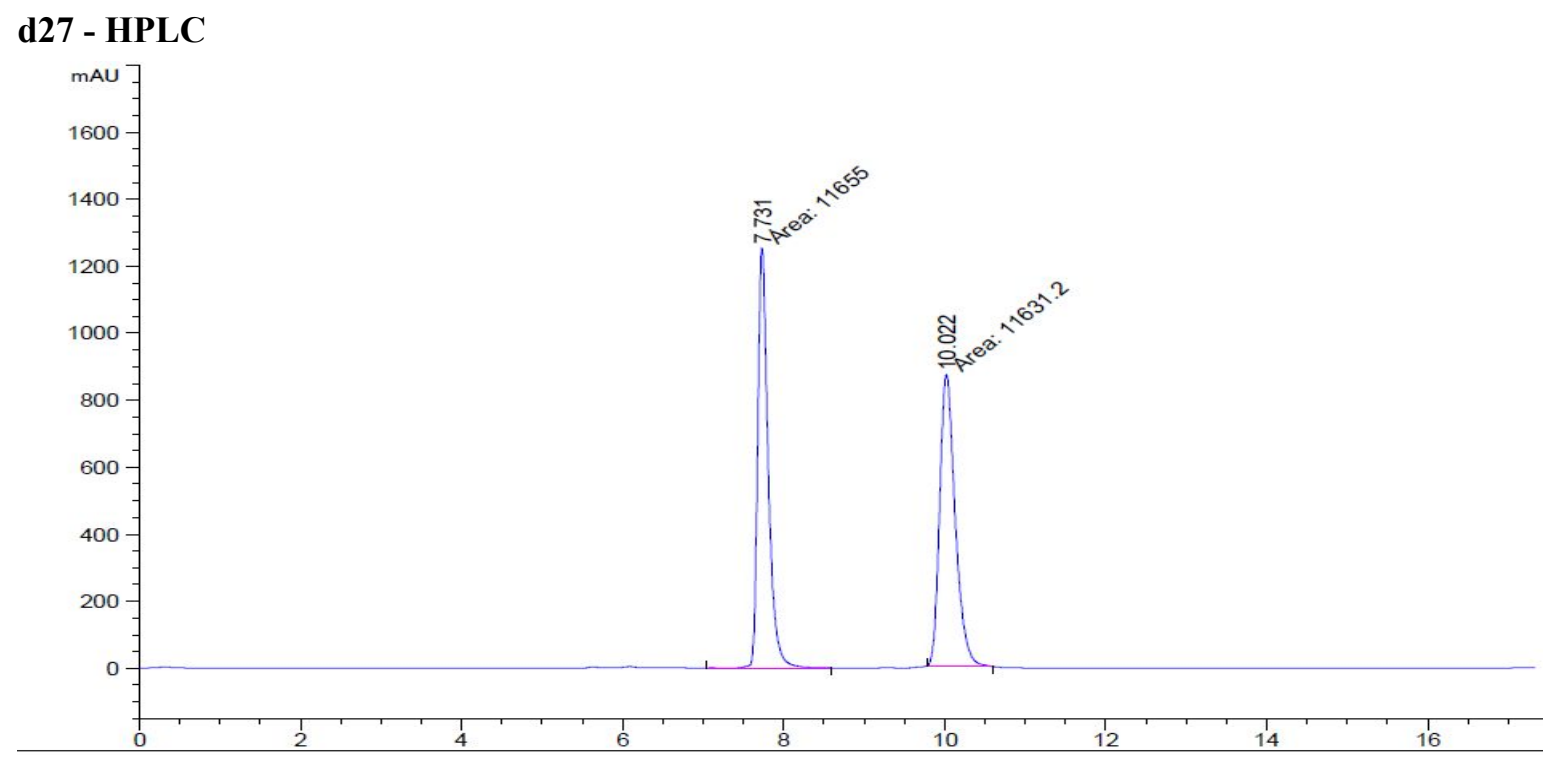

$$
\begin{array}{cccccc}
\begin{array}{c}
\text { Peak RetTime Type } \\
\text { W }
\end{array} & \begin{array}{c}
\text { Width } \\
{[\mathrm{min}]}
\end{array} & \begin{array}{c}
\text { Area } \\
{[\mathrm{min}]}
\end{array} & \begin{array}{c}
\text { Height } \\
{[\mathrm{mAU} \text { s }]}
\end{array} & \begin{array}{c}
\text { Area } \\
{[\mathrm{mAU}]}
\end{array} & \% \\
\hdashline 1 & 7.731 \mathrm{MM} & 0.1547 & 1.16550 \mathrm{e} 4 & 1255.65186 & 50.0509 \\
2 & 10.022 \mathrm{MM} & 0.2226 & 1.16312 \mathrm{e} 4 & 870.77301 & 49.9491
\end{array}
$$

\begin{tabular}{|c|c|c|c|c|c|c|}
\hline $\begin{array}{c}\text { Peak } \\
\quad \#\end{array}$ & $\begin{array}{c}\text { RetTime } \\
\text { [min] }\end{array}$ & Type & $\begin{array}{l}\text { Width } \\
\text { [min] }\end{array}$ & $\begin{array}{c}\text { Area } \\
{\left[\mathrm{mAU}^{*} \mathrm{~s}\right]}\end{array}$ & $\begin{array}{l}\text { Height } \\
{[\mathrm{mAU}]}\end{array}$ & $\begin{array}{c}\text { Area } \\
\%\end{array}$ \\
\hline & & & & & & \\
\hline 1 & 7 & $\sqrt{1}$ & 5 & $1.40991 \mathrm{e} 4$ & 155 & 96.9624 \\
\hline 2 & $a f r r a t$ & V & 35 & 441.68723 & 283 & 3.0376 \\
\hline
\end{tabular}
Totals :
$2.32862 \mathrm{e} 4 \quad 2126.42487$

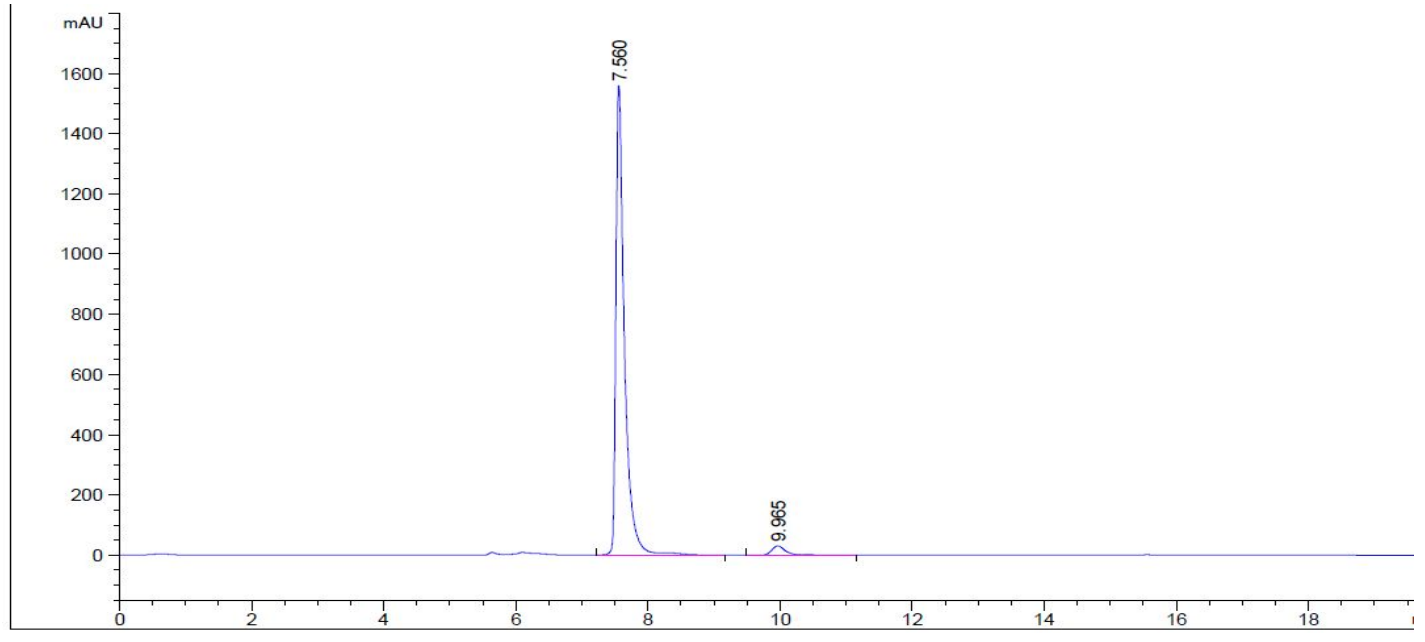

Totals :

$1.45408 \mathrm{e} 4 \quad 1590.04131$ 
(R)- (3-([1, 1'-biphenyl]-4-yl)-6-fluorohex-1-yn-1-yl) triisopropylsilane (d28) d28 - ${ }^{1}$ H NMR

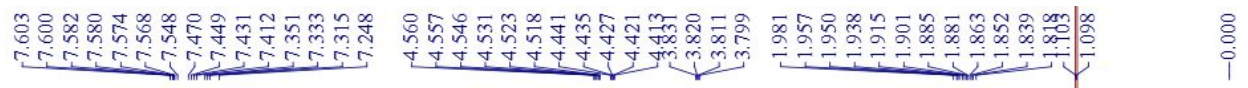
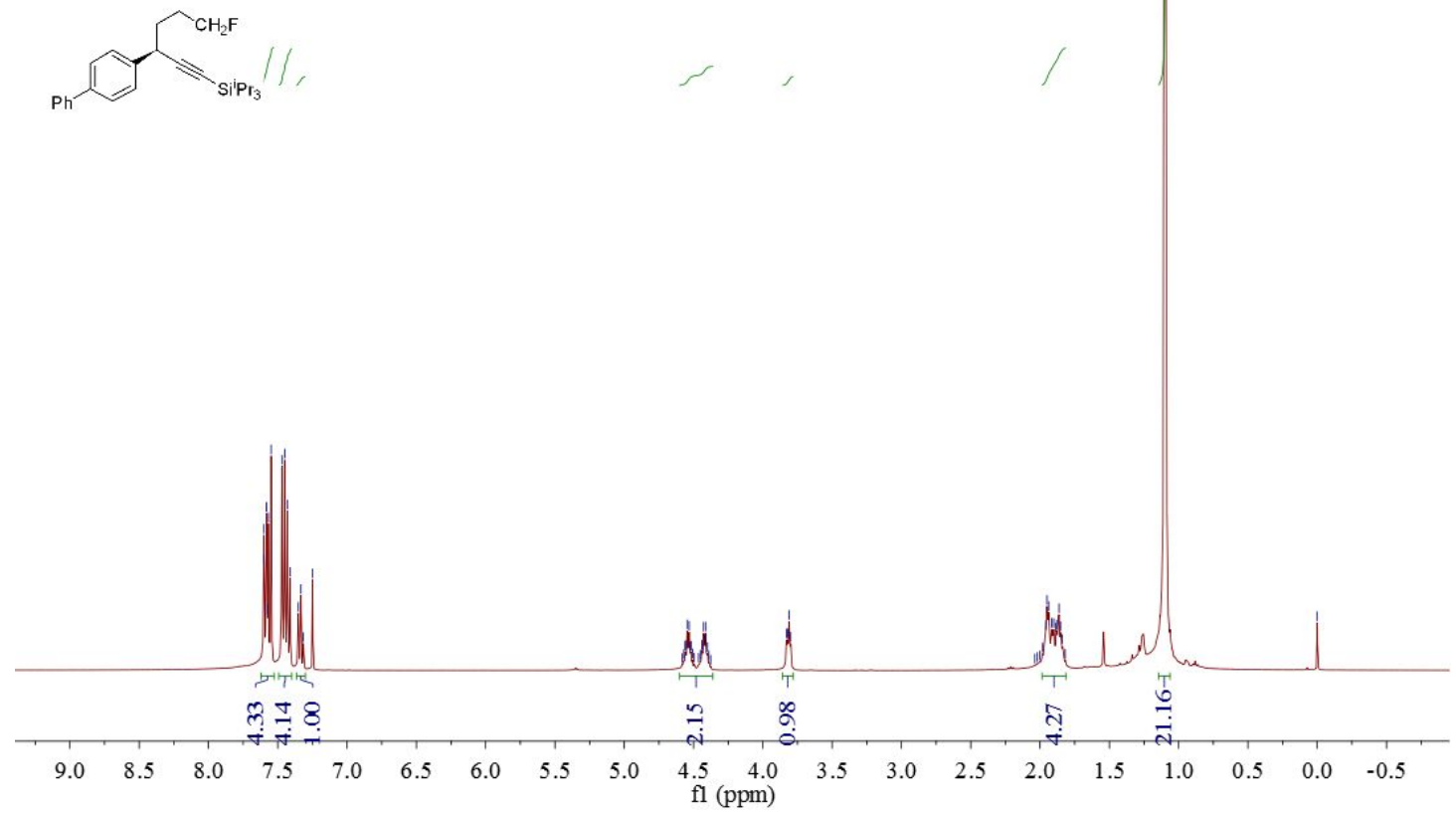

d28 - ${ }^{13}$ C NMR

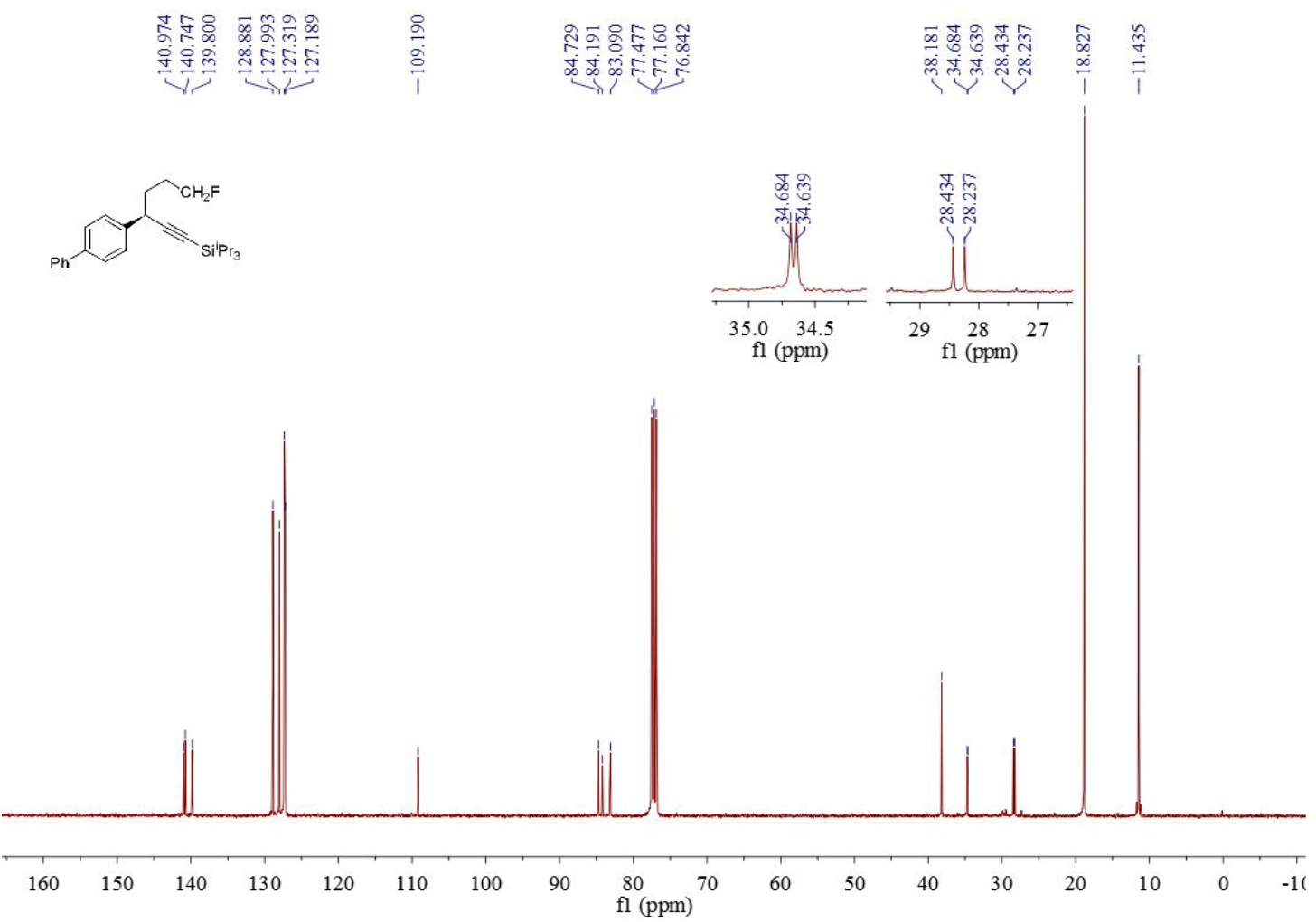



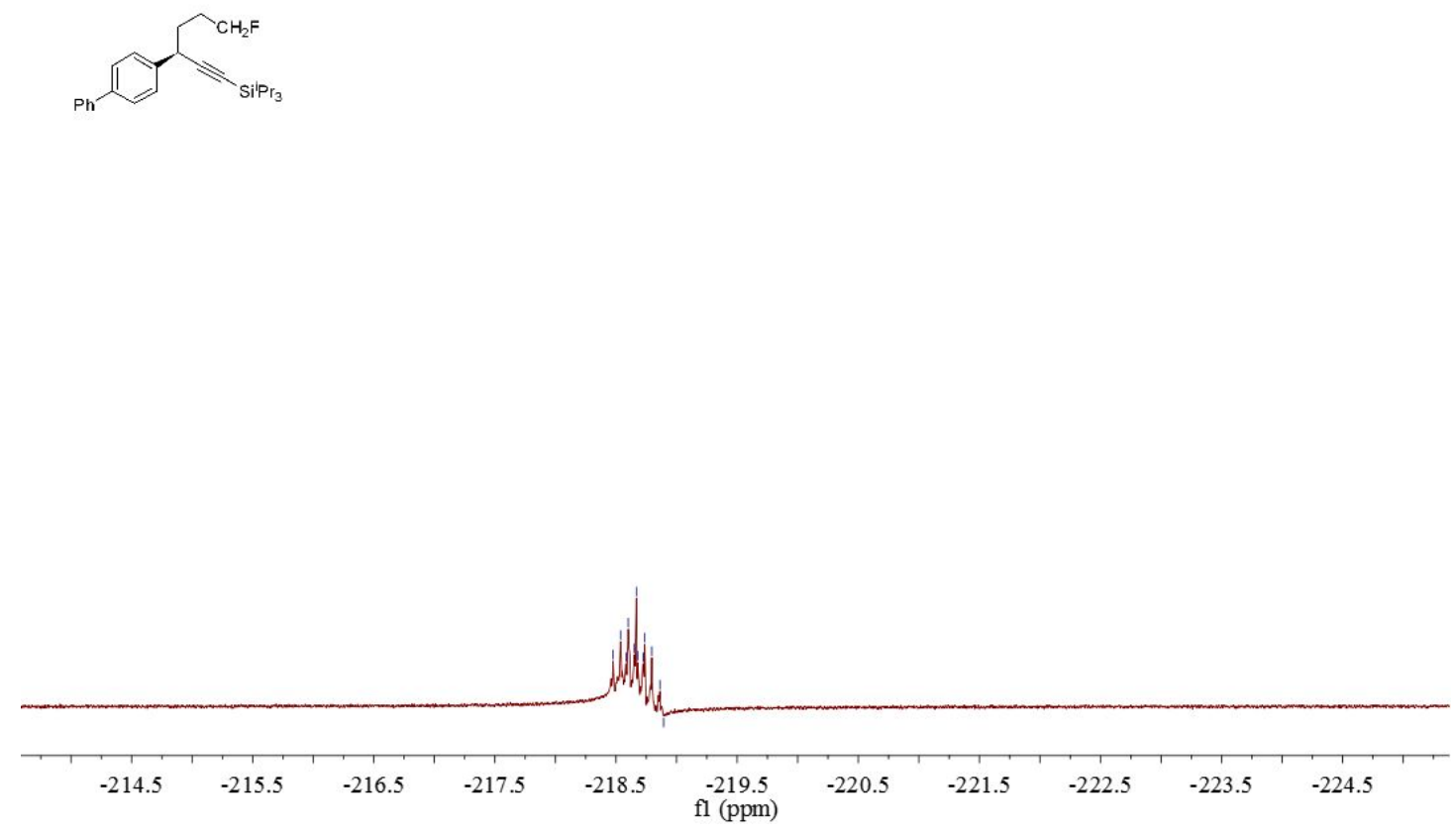

d28 - HPLC

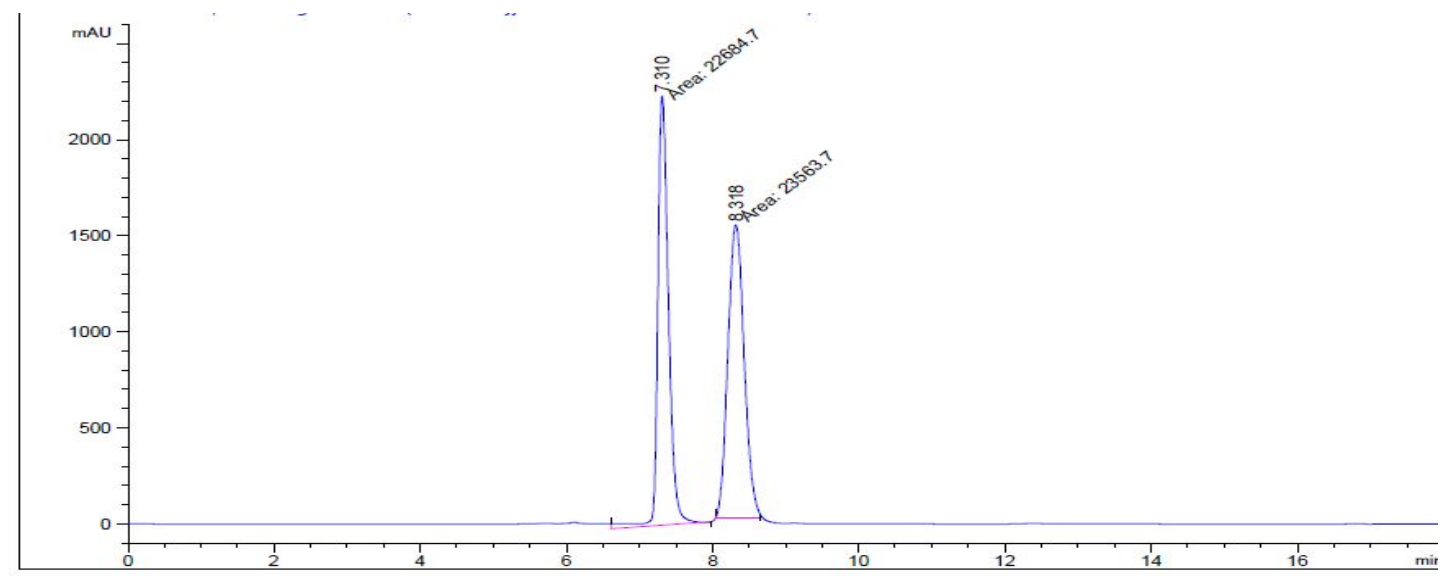

\begin{tabular}{|c|c|c|c|c|c|c|}
\hline $\begin{array}{c}\text { Peak } \\
\#\end{array}$ & $\begin{array}{c}\text { RetTime } \\
\text { [min] }\end{array}$ & Type & $\begin{array}{l}\text { Width } \\
\text { [min] }\end{array}$ & $\begin{array}{c}\text { Area } \\
{\left[\mathrm{mAU}^{*} \mathrm{~s}\right]}\end{array}$ & $\begin{array}{l}\text { Height } \\
{[\mathrm{mAU}]}\end{array}$ & $\begin{array}{c}\text { Area } \\
\%\end{array}$ \\
\hline & 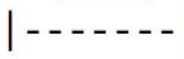 & & $-\ldots$ & | - - - - & | - - - - & $----\cdots$ \\
\hline 1 & 7.310 & $\mathrm{MM}$ & 0.1695 & $2.26847 e 4$ & 2231.17651 & 49.0497 \\
\hline 2 & 8.318 & MM & 0.2578 & $2.35637 \mathrm{e} 4$ & 1523.48657 & 50.9503 \\
\hline Tota. & & & & $4.62484 \mathrm{e} 4$ & 3754.66309 & \\
\hline
\end{tabular}



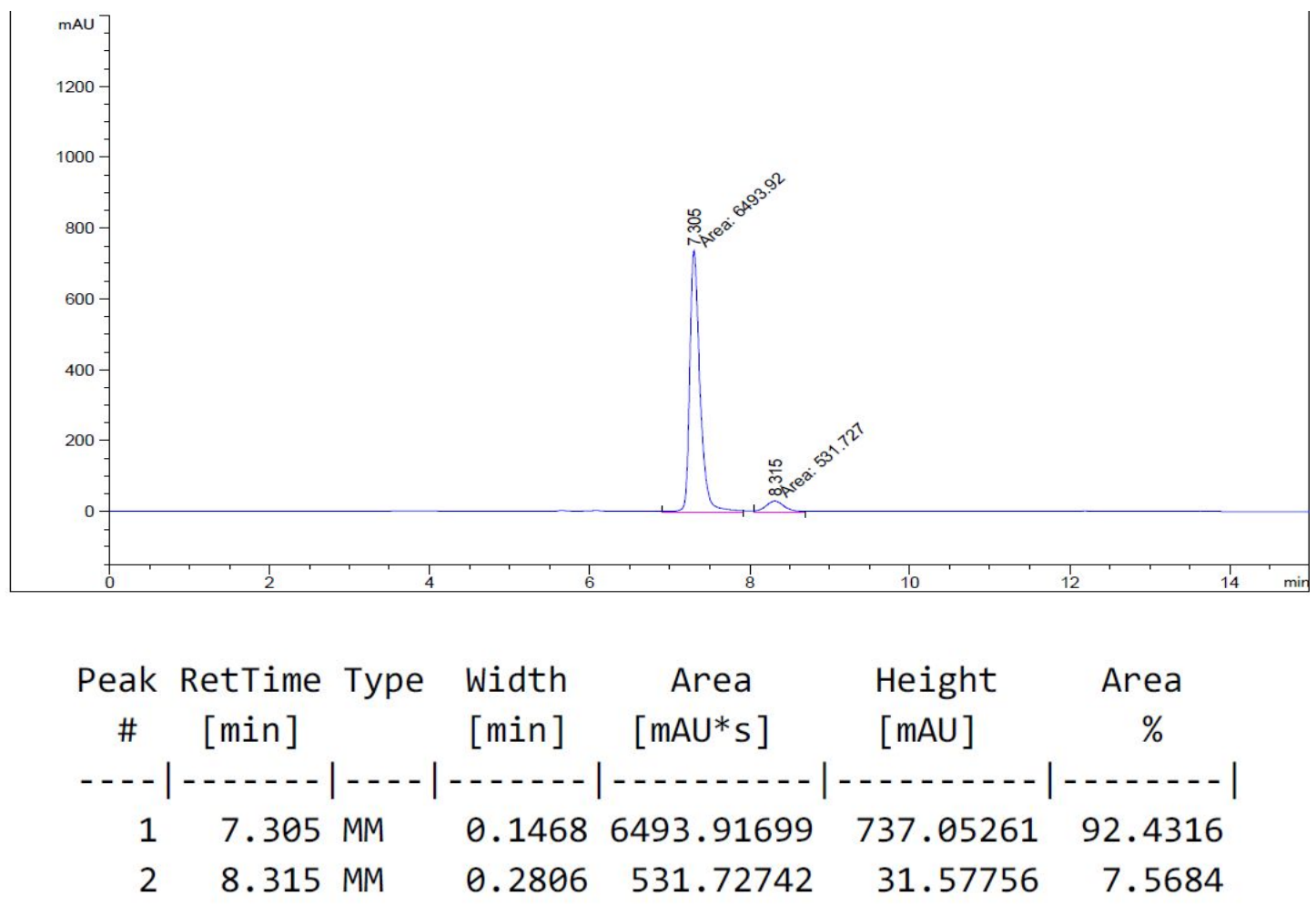

Totals :

7025.64441768 .63017

(R)- (3-([1, 1'-biphenyl]-4-yl)-6, 6, 7, 7-tetrafluorohept-1-yn-1-yl) triisopropylsilane (d29) d29 - ${ }^{1}$ H NMR

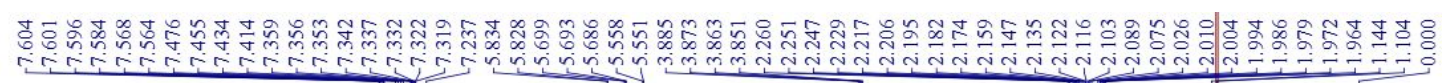
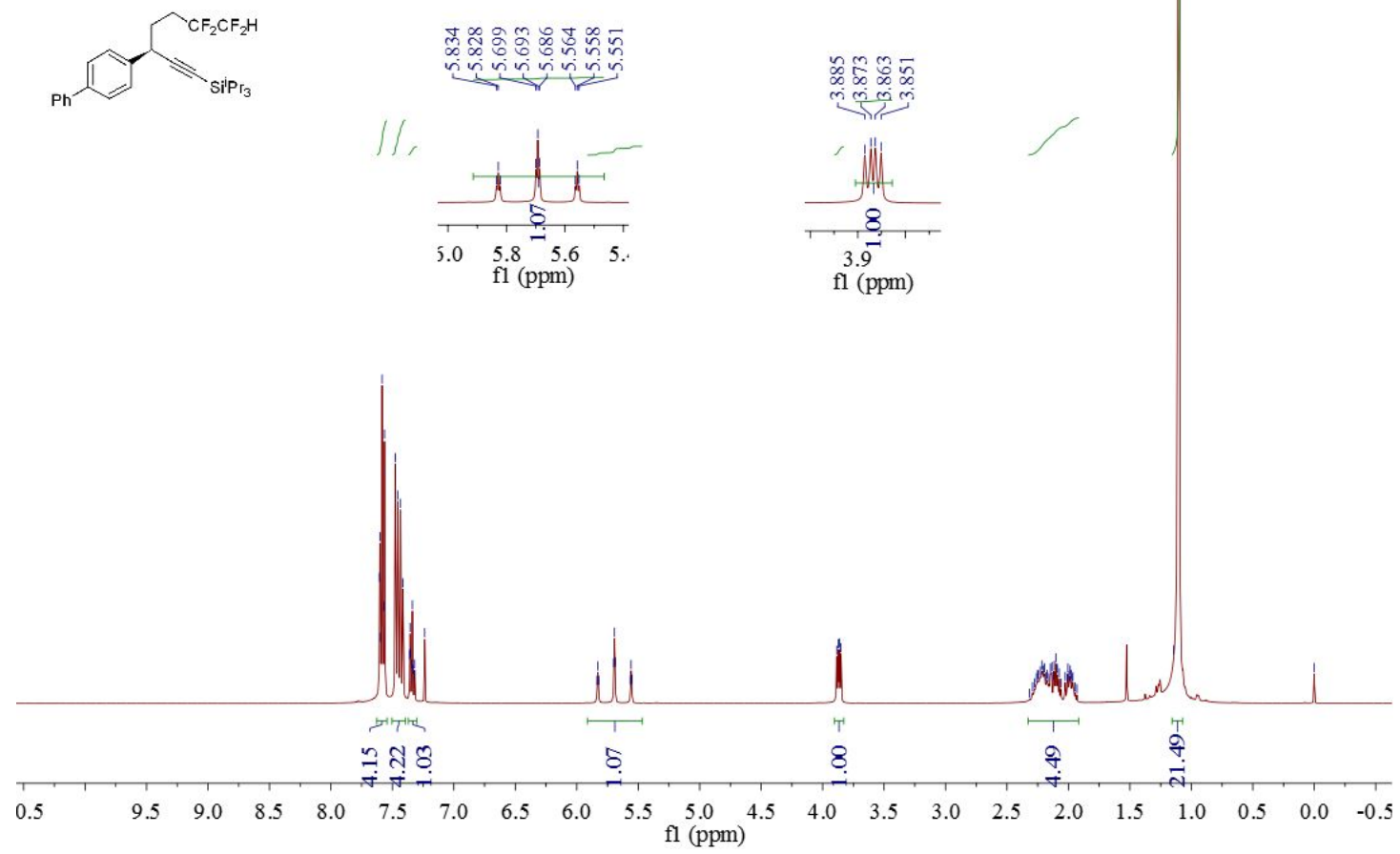

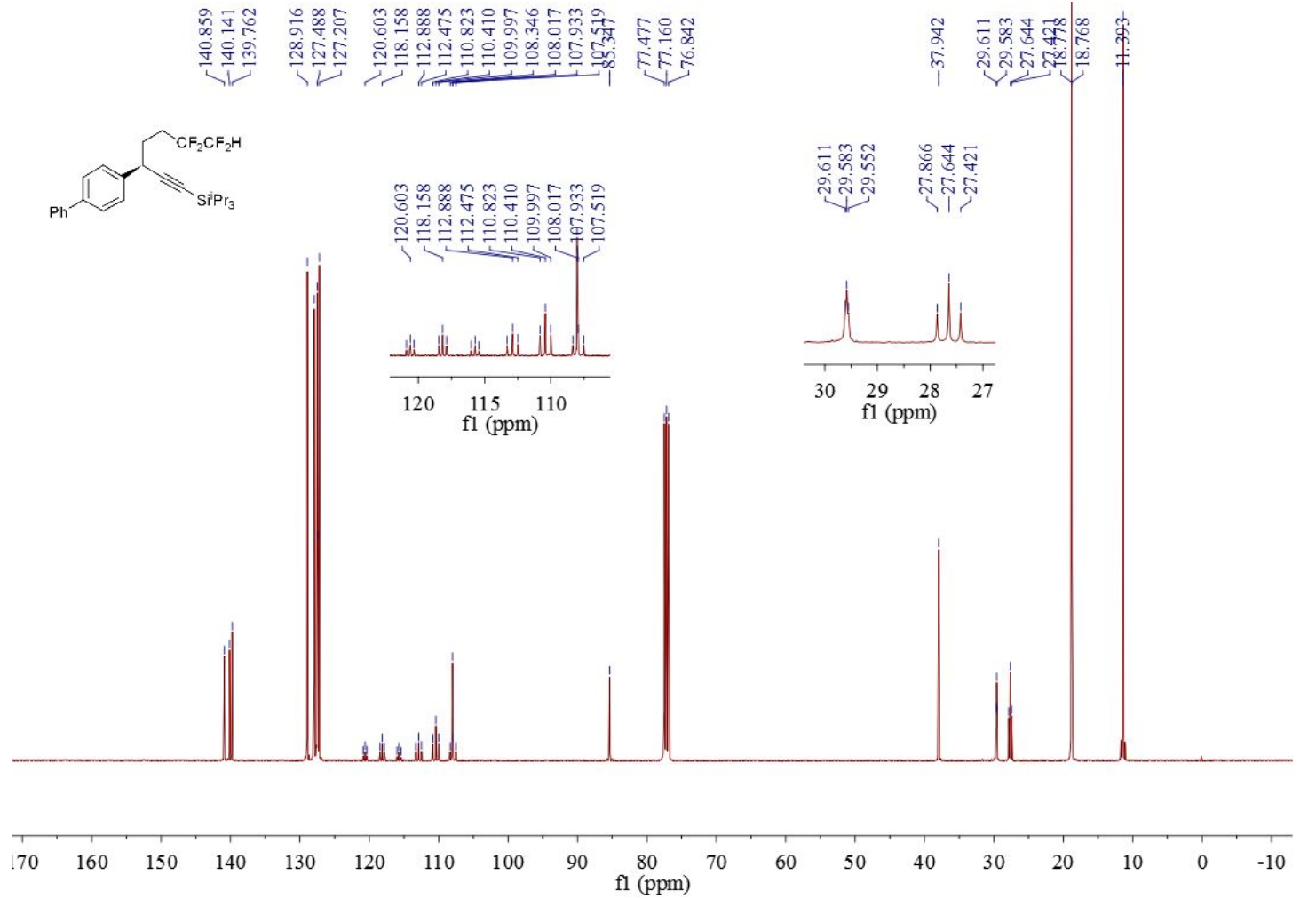

\section{d29 - ${ }^{19}$ F NMR}

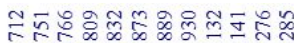

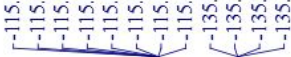
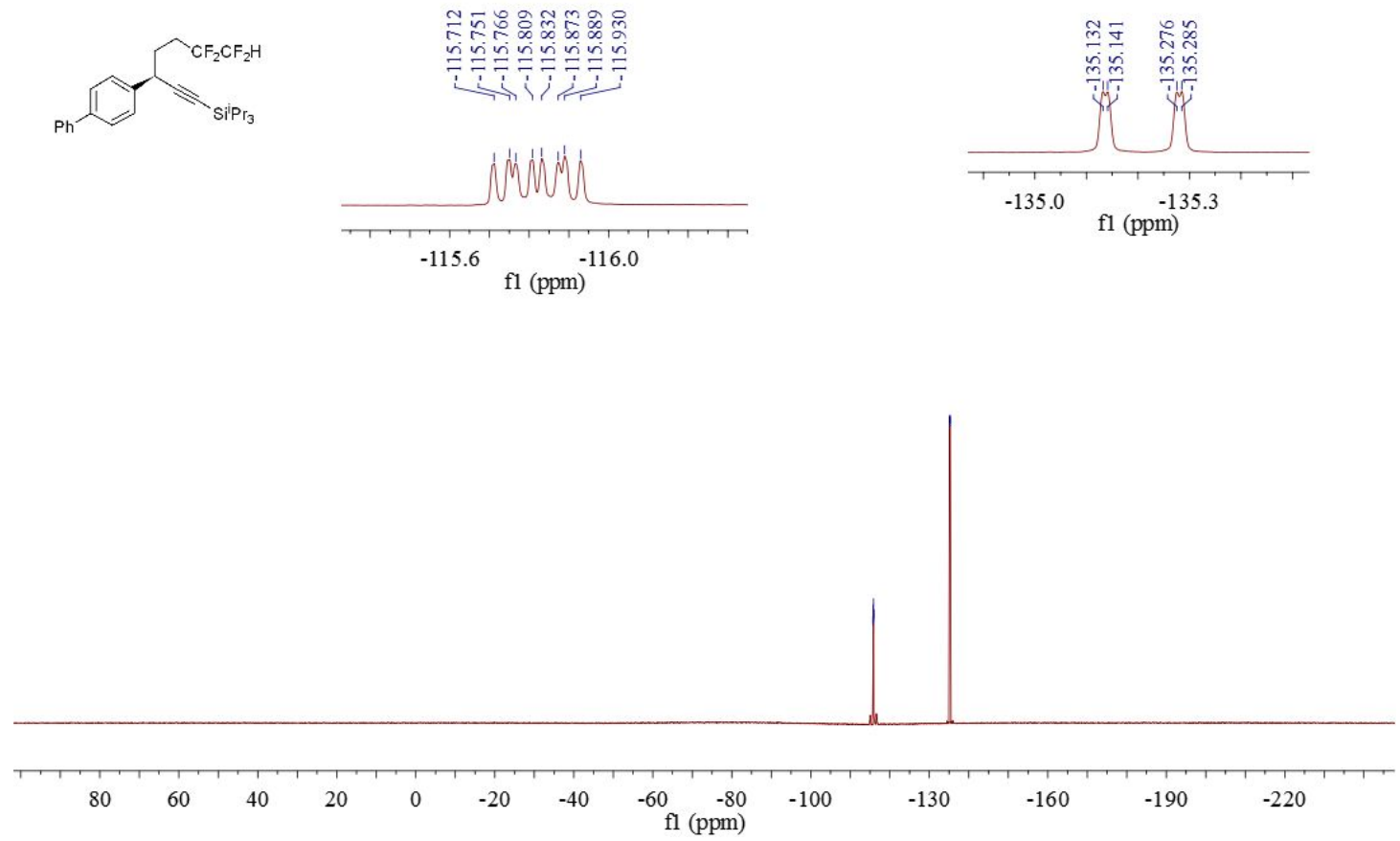


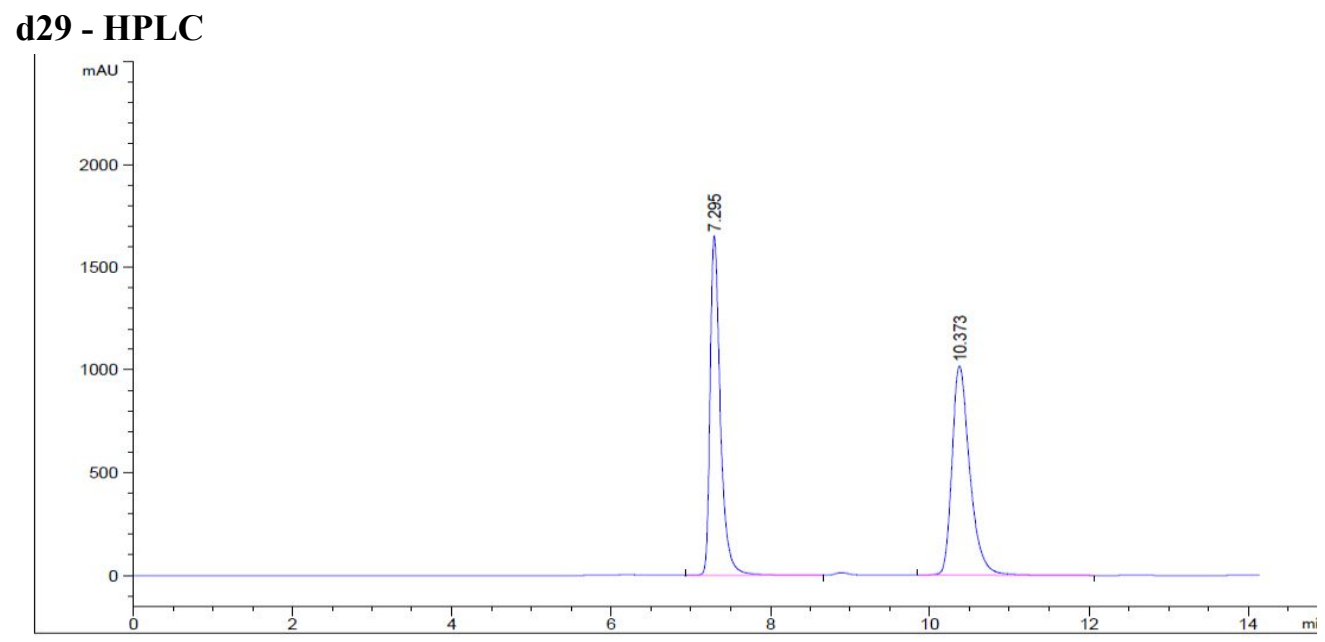

$$
\begin{aligned}
& \text { Peak RetTime Type width Area Height Area } \\
& \begin{array}{lllll}
\# & {[\mathrm{~min}]} & {[\mathrm{min}]} & {[\mathrm{mAU} * \mathrm{~s}]} & {[\mathrm{mAU}]}
\end{array}
\end{aligned}
$$

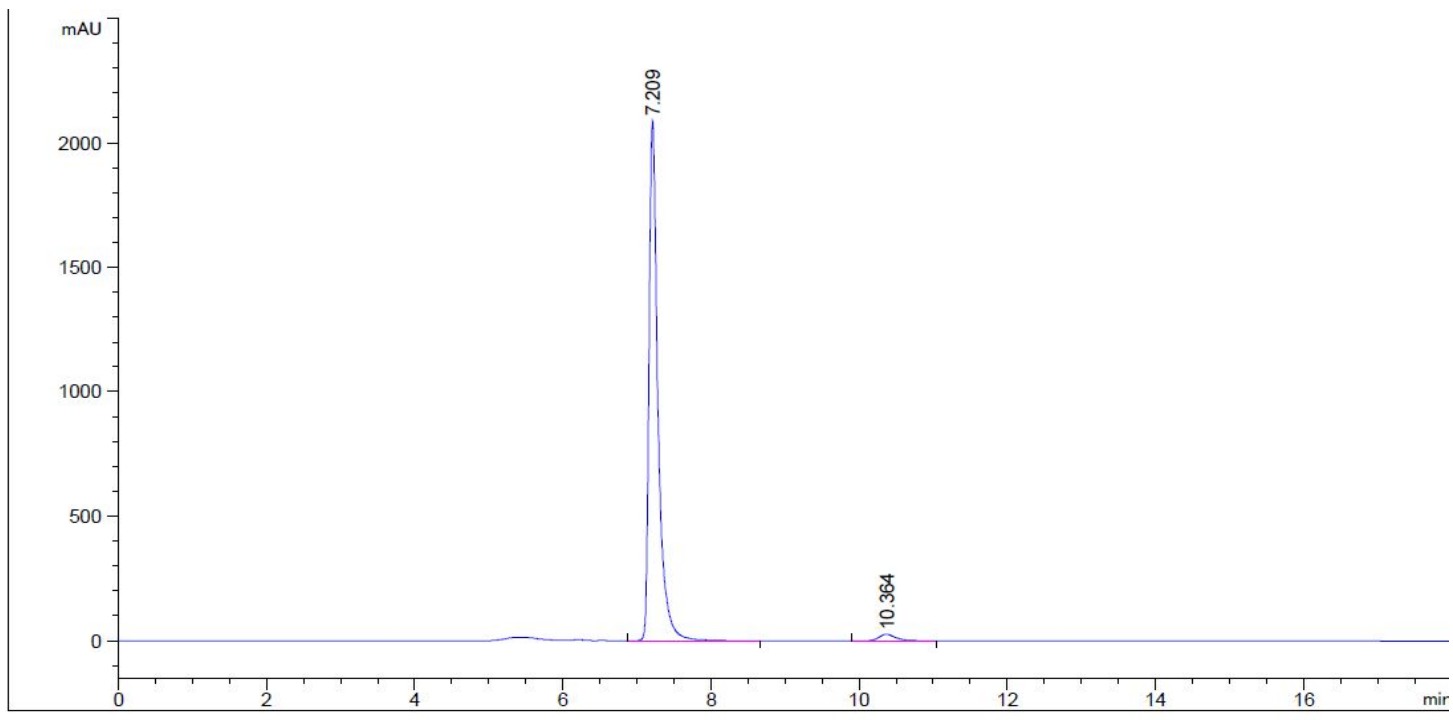

$$
\begin{array}{cccccc}
\begin{array}{c}
\text { Peak RetTime Type } \\
\#
\end{array} & \text { width } & \text { Area } & \text { Height } & \text { Area } \\
\text { [min] } & {[\text { min }]} & {[\text { mAU*s }]} & {[\text { mAU }]} & \% \\
1 & 7.209 \text { BB } & 0.1266 & 1.76584 e 4 & 2089.01025 & 97.8258 \\
2 & 10.364 \text { BB } & 0.2325 & 392.45734 & 25.50155 & 2.1742
\end{array}
$$

Totals : $\quad 1.80508 \mathrm{e} 4 \quad 2114.51180$ 
(R)- (3-([1, 1'-biphenyl]-4-yl)-5, 5, 6, 6, 6-pentafluorohex-1-yn-1-yl) triisopropylsilane (d30) d30 - ${ }^{1}$ H NMR

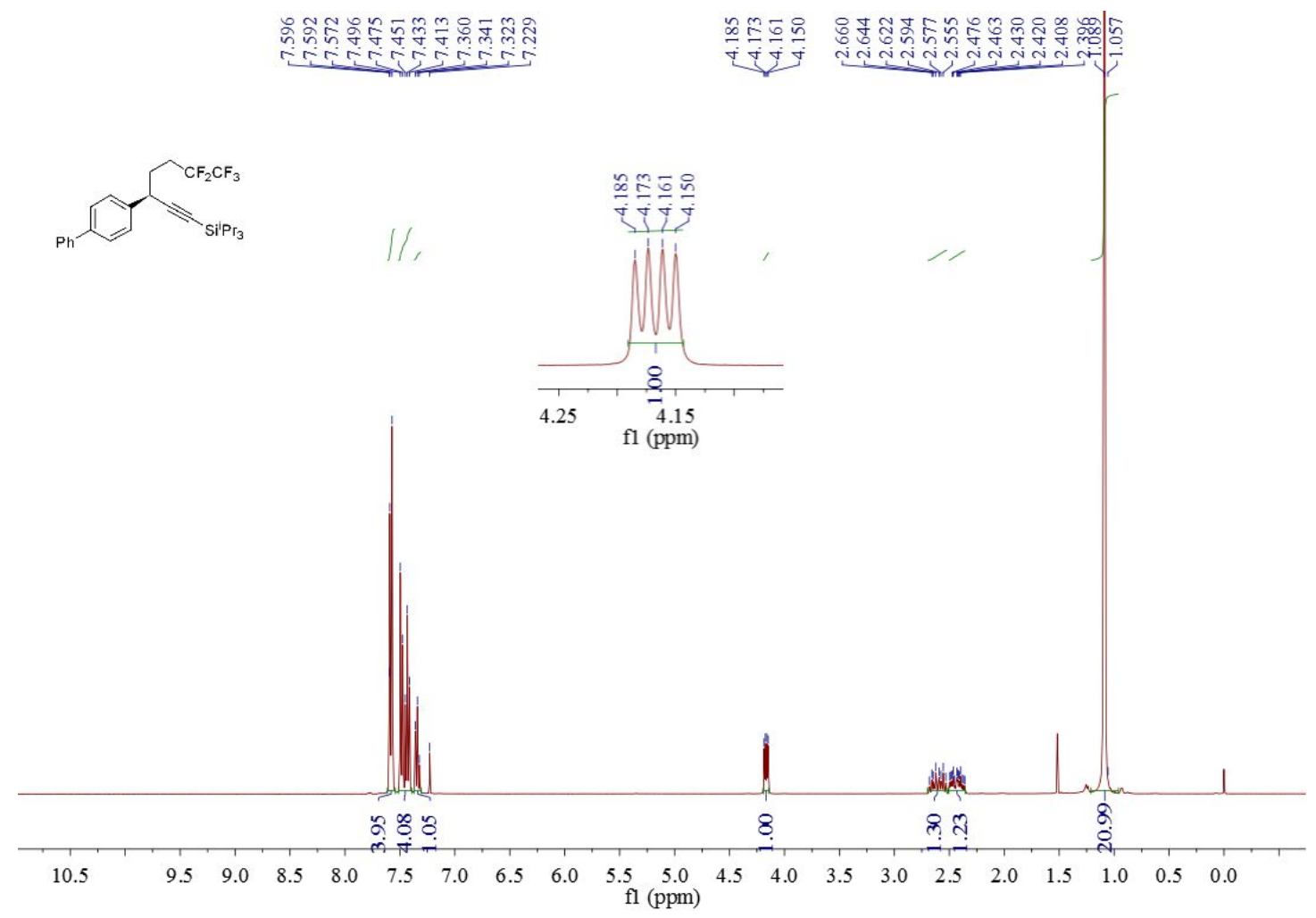

d30 - ${ }^{13}$ C NMR

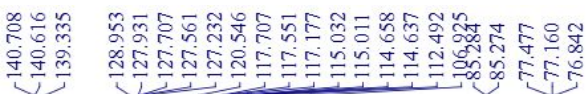

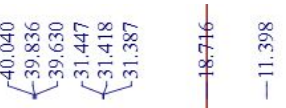


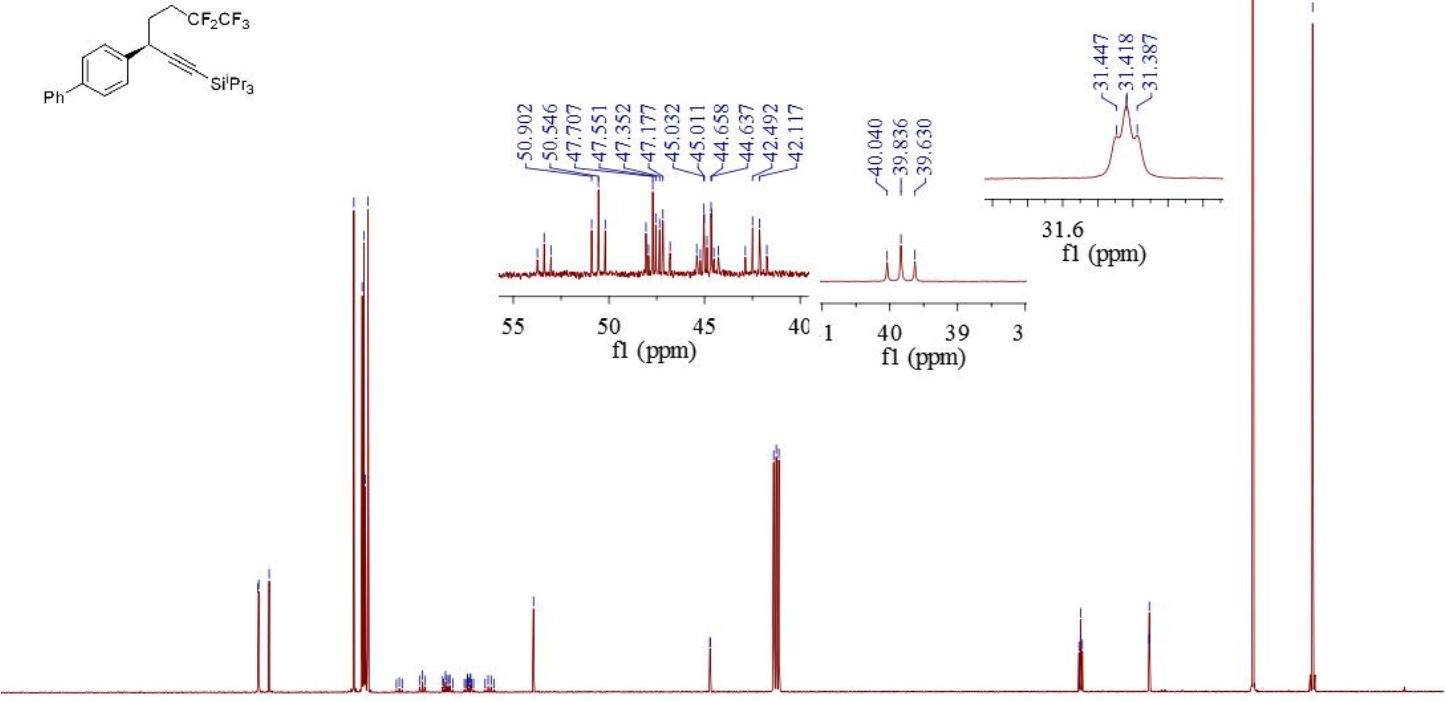

$\begin{array}{llllllll}160 & 150 & 140 & 130 & 120 & 110 & 100 & 90\end{array}$

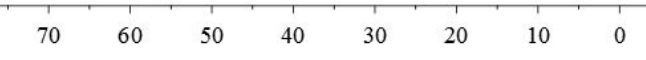


d30 - ${ }^{19}$ F NMR

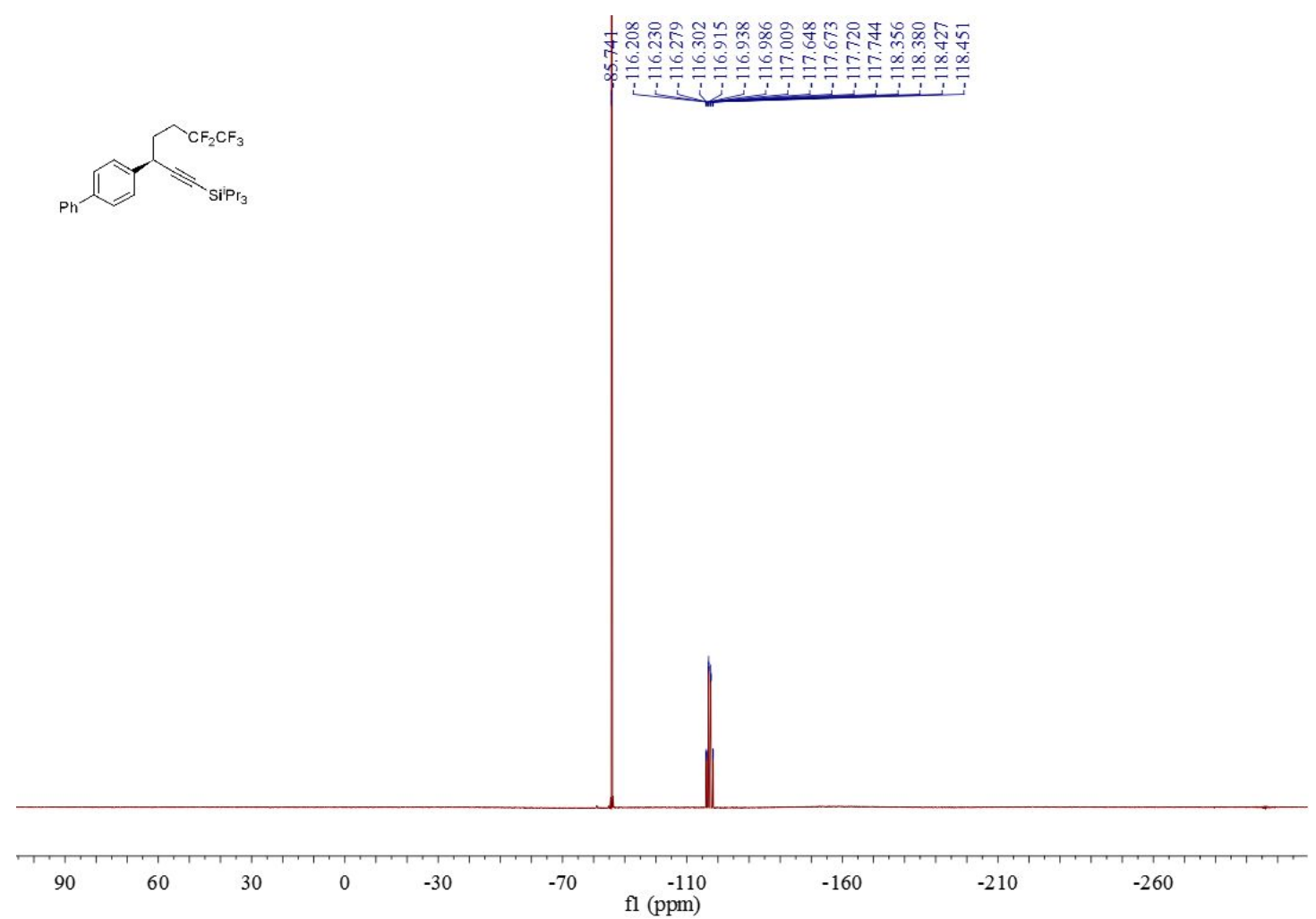

d30 - HPLC

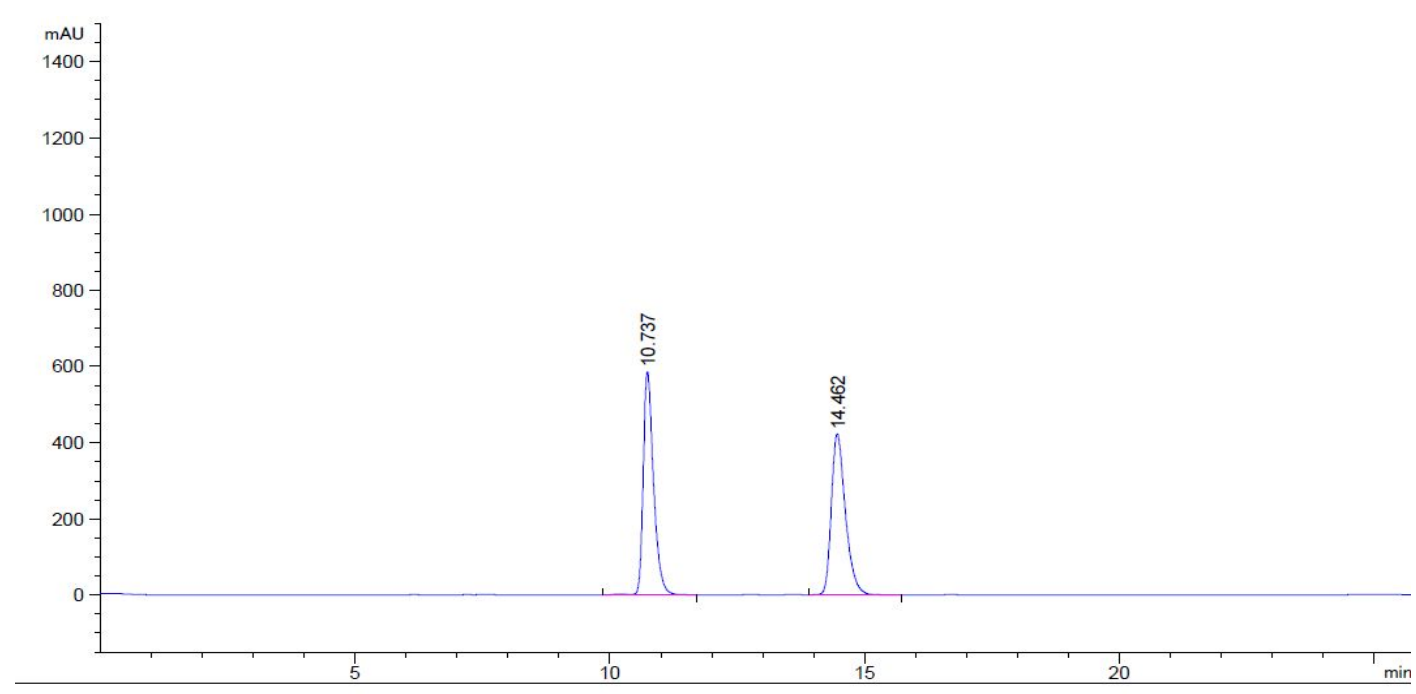

\begin{tabular}{|c|c|c|c|c|c|c|}
\hline $\begin{array}{c}\text { Peak } \\
\quad \#\end{array}$ & $\begin{array}{c}\text { RetTime } \\
\text { [min] }\end{array}$ & Type & $\begin{array}{l}\text { Width } \\
\text { [min] }\end{array}$ & $\begin{array}{c}\text { Area } \\
{\left[\mathrm{mAU}^{*} \mathrm{~s}\right]}\end{array}$ & $\begin{array}{l}\text { Height } \\
{[\mathrm{mAU}]}\end{array}$ & $\begin{array}{c}\text { Area } \\
\%\end{array}$ \\
\hline & & & & & $\ldots$ & \\
\hline 1 & 10.737 & VB $R$ & 0.2106 & 8294.10156 & 587.24469 & 50.4133 \\
\hline 2 & 14.462 & VB & 910 & 8158.12256 & 423.00674 & 49.5867 \\
\hline
\end{tabular}

Totals :

$1.64522 \mathrm{e} 4 \quad 1010.25143$ 


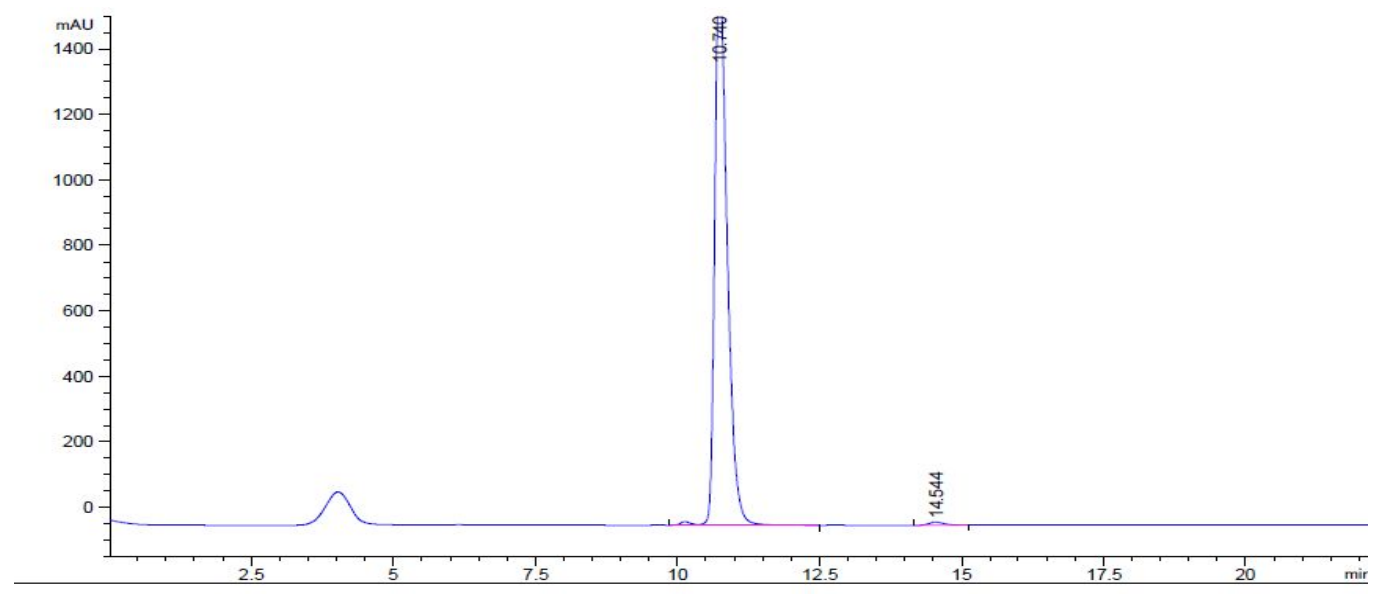

$$
\begin{aligned}
& \text { Peak RetTime Type Width Area Height Area }
\end{aligned}
$$

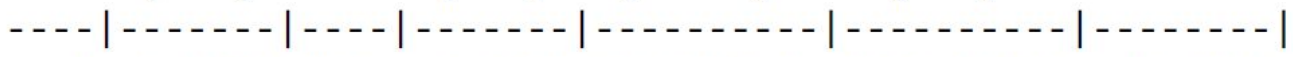

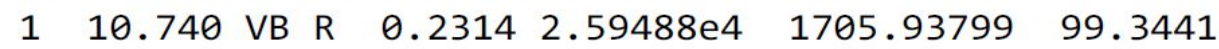

$$
\begin{aligned}
& \begin{array}{llllll}
2 & 14.544 \text { BB } & 0.2770 & 171.32129 & 9.42591 & 0.6559
\end{array}
\end{aligned}
$$

\# $[\mathrm{min}] \quad[\mathrm{min}] \quad\left[\mathrm{mAU}^{*} \mathrm{~s}\right] \quad[\mathrm{mAU}] \quad \%$

$$
\text { Totals : } \quad 2.61201 \mathrm{e} 4 \quad 1715.36390
$$

(R)- (3-([1, 1'-biphenyl]-4-yl)-5-fluoropent-1-yn-1-yl) triisopropylsilane (d31) d31 - ' 1 H NMR

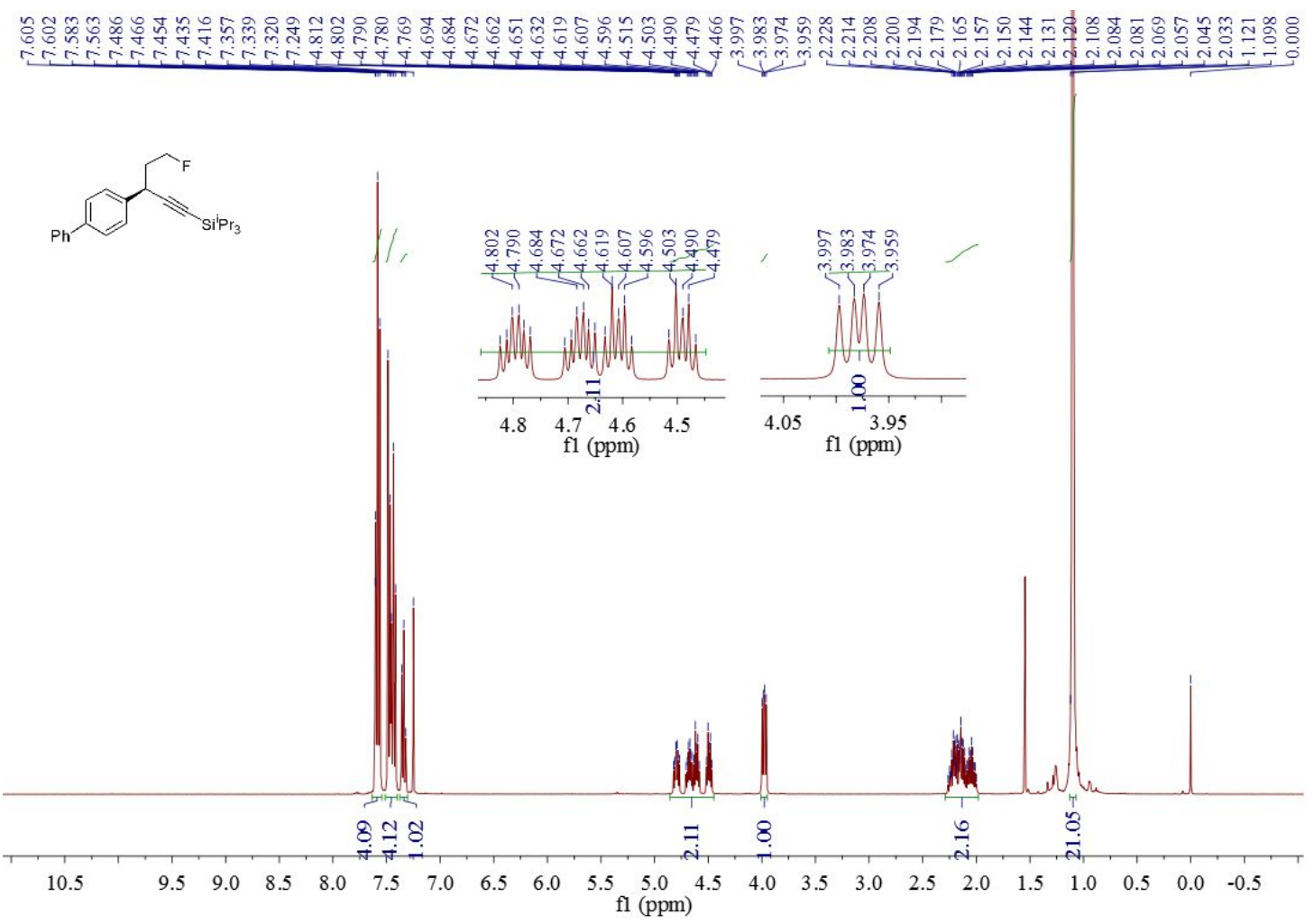


d31 - ${ }^{13}$ C NMR

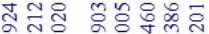

守守守

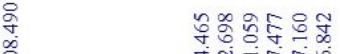

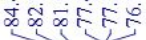
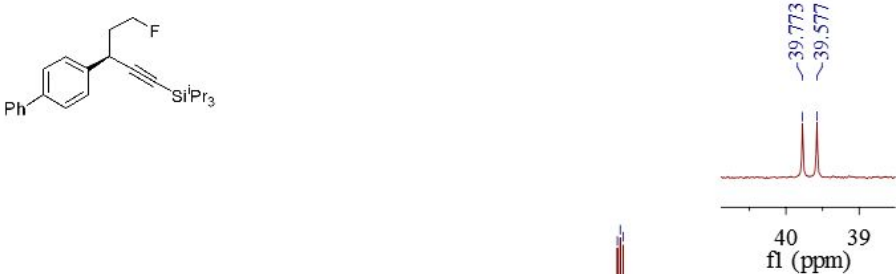

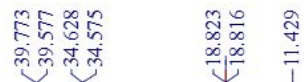

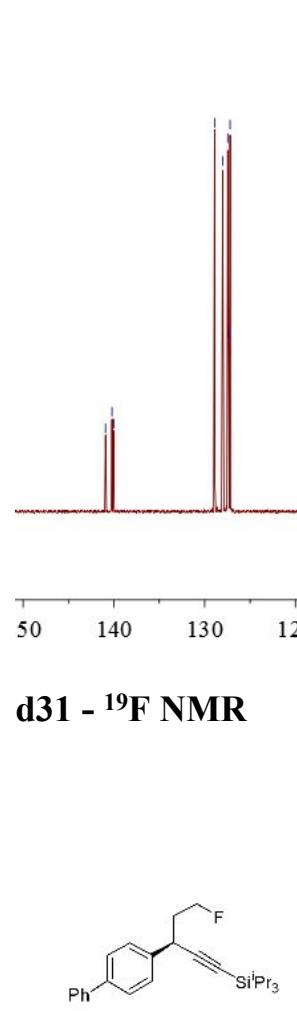

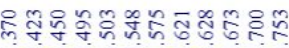

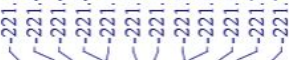

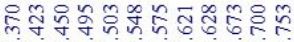

สิส
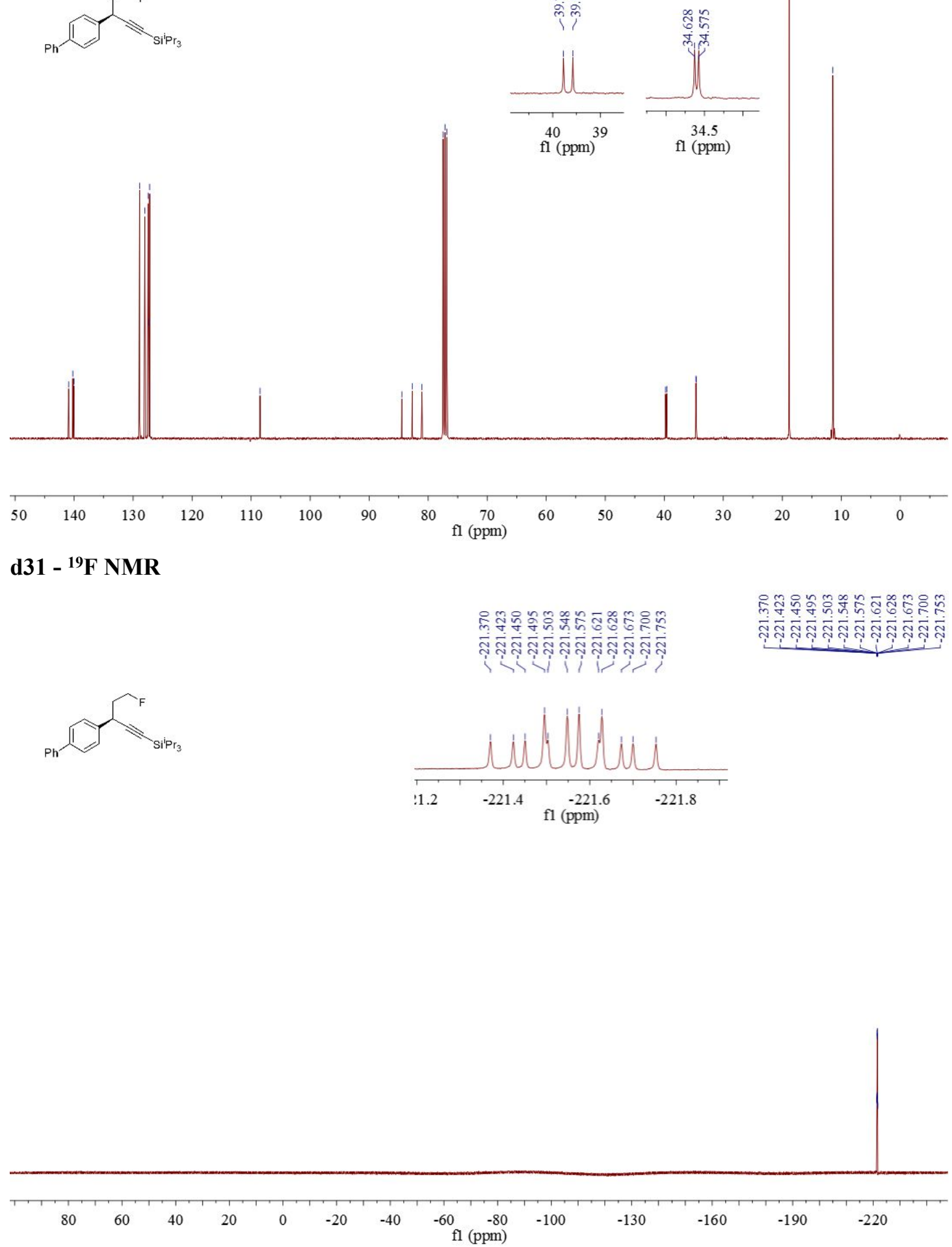


\section{d31 - HPLC}
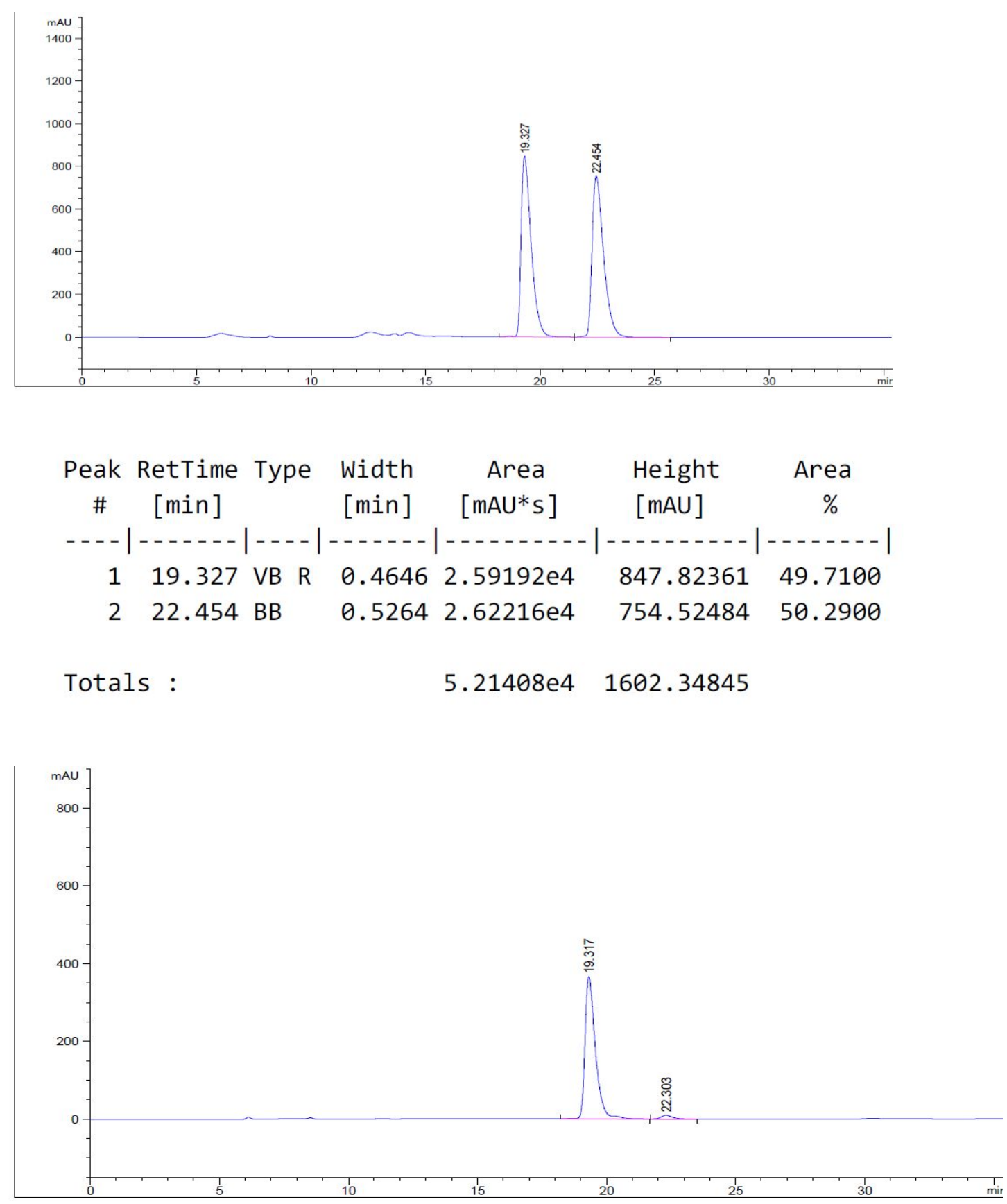

\begin{tabular}{|c|c|c|c|c|c|c|}
\hline eak & $\begin{array}{c}\text { RetTime } \\
\text { [min] }\end{array}$ & Type & $\begin{array}{l}\text { Width } \\
\text { [min] }\end{array}$ & $\begin{array}{c}\text { Area } \\
{\left[\mathrm{mAU}^{*} \mathrm{~s}\right]}\end{array}$ & $\begin{array}{l}\text { Height } \\
{[\mathrm{mAU}]}\end{array}$ & $\begin{array}{c}\text { Area } \\
\%\end{array}$ \\
\hline & & & & & & \\
\hline 1 & & & & 1.02 & 84 & 97 \\
\hline 2 & 22.303 & BB & 529 & 292.40869 & 9.74975 & 7782 \\
\hline
\end{tabular}

Totals : $\quad 1.05251 \mathrm{e} 4 \quad 375.55459$ 
(R)- (3- ([1, 1'-biphenyl] - 4 -yl) oct-1-yn-1-yl) triisopropylsilane (d32) d32 - ${ }^{1}$ H NMR

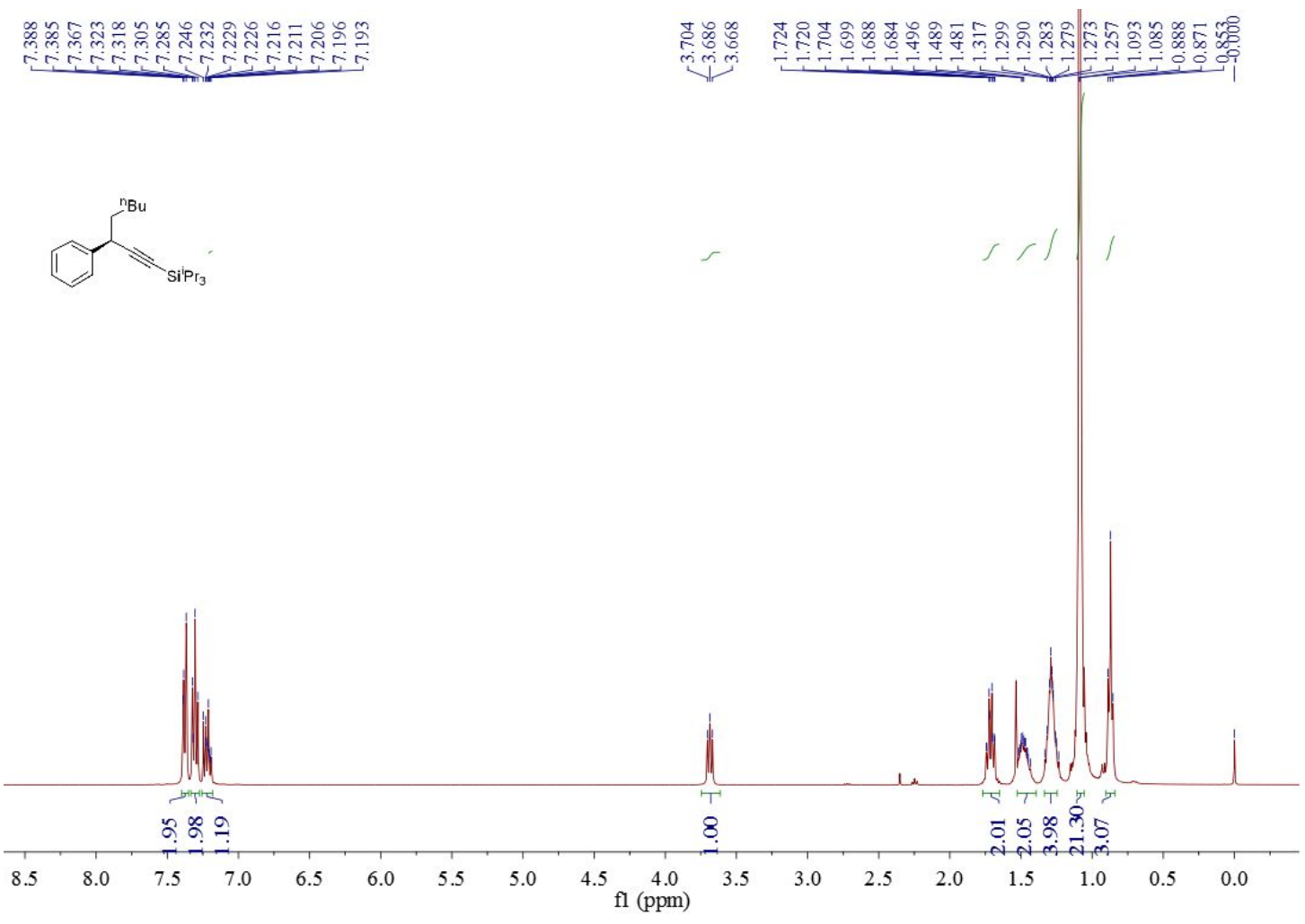

d32 - ${ }^{13}$ C NMR

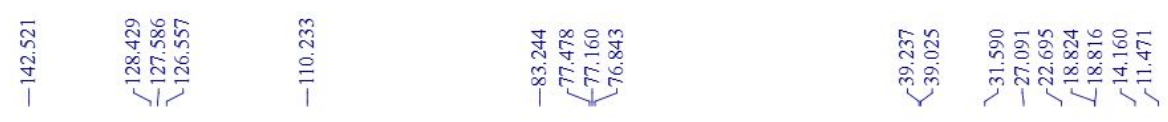
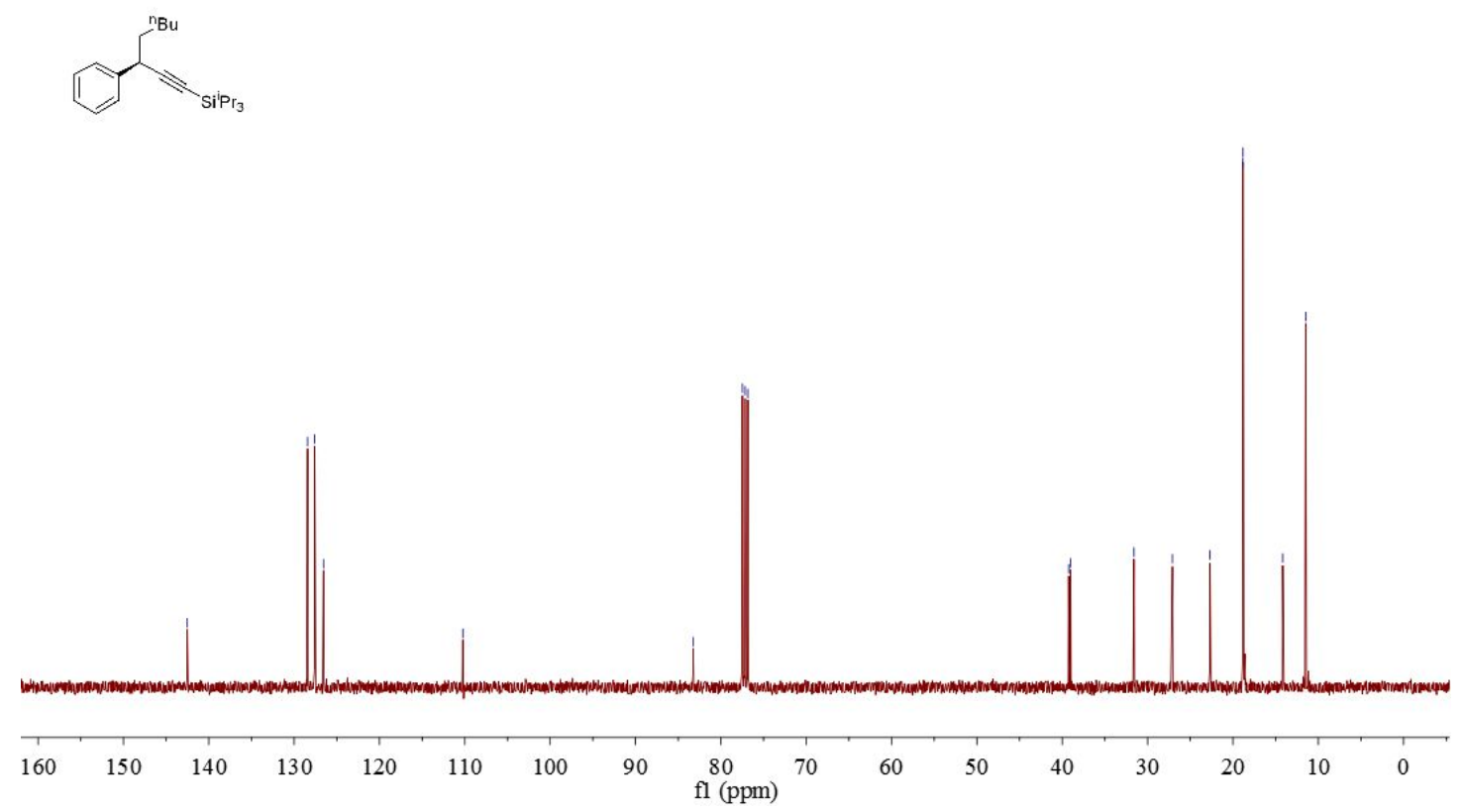
d32 - HPLC

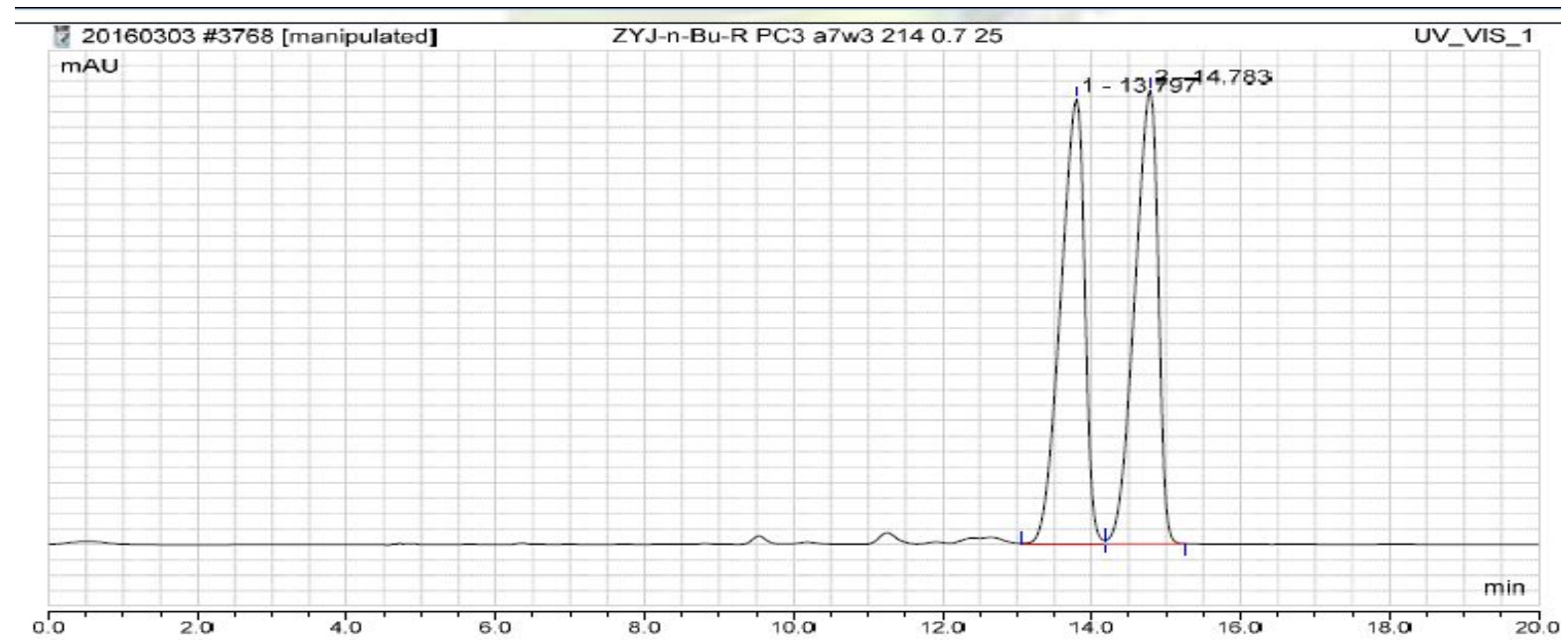

Time [min]

\begin{tabular}{|c|c|c|c|c|c|}
\hline \multicolumn{6}{|c|}{ Integration Results } \\
\hline No. & $\begin{array}{l}\text { Retention Time } \\
\text { min }\end{array}$ & $\begin{array}{c}\text { Retention Time } \\
\text { min }\end{array}$ & $\begin{array}{c}\text { Area } \\
\mathrm{mAU}{ }^{*} \min \end{array}$ & $\begin{array}{c}\text { Height } \\
\text { mAU }\end{array}$ & $\begin{array}{c}\text { Relative Area } \\
\%\end{array}$ \\
\hline 1 & 14 & 13.797 & 542.147 & 1442.039 & 50.46 \\
\hline 2 & 15 & 14.783 & 532.324 & 1468.049 & 49.54 \\
\hline \multicolumn{2}{|c|}{ Total: } & 1074.471 & 2910.088 & 100.00 & \\
\hline
\end{tabular}

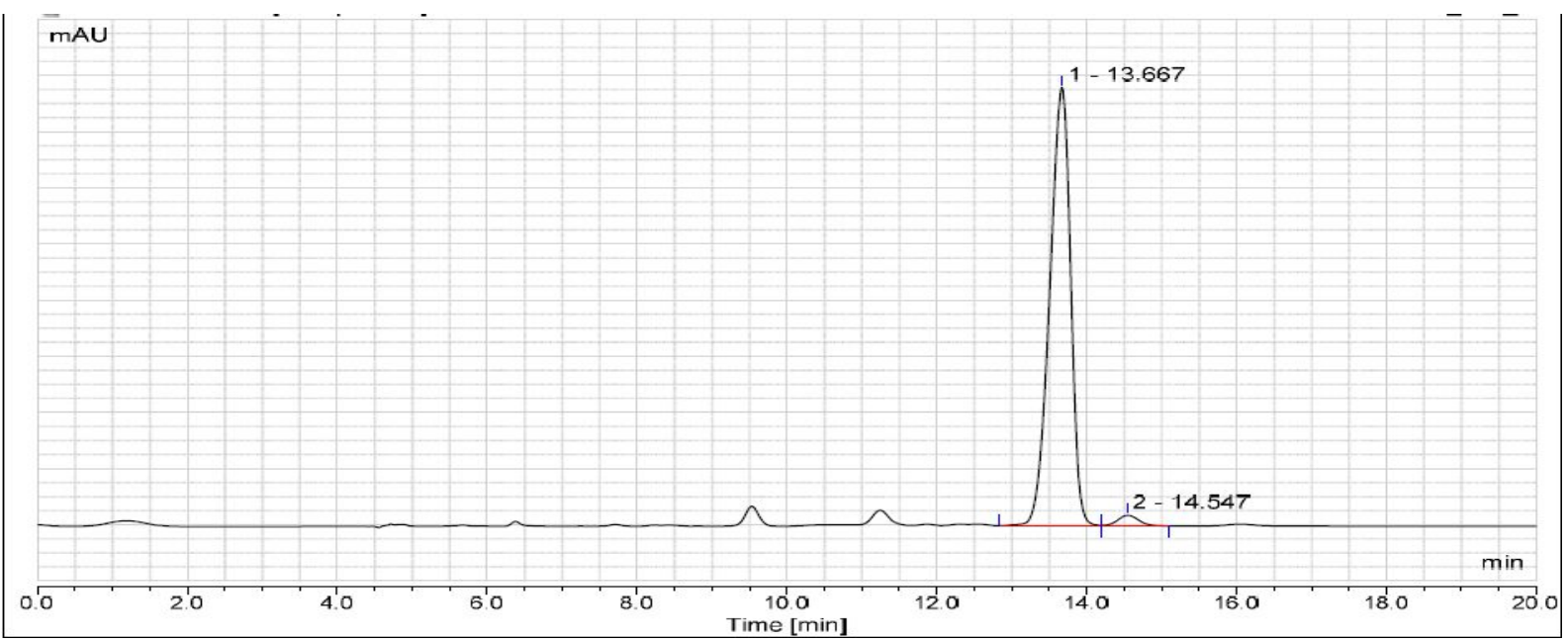

Time [min]

\begin{tabular}{|l|l|c|c|c|c|}
\hline \multicolumn{5}{|l|}{ Integration Results } \\
\cline { 1 - 4 } No. & $\begin{array}{l}\text { Retention Time } \\
\text { min }\end{array}$ & $\begin{array}{c}\text { Retention Time } \\
\text { min }\end{array}$ & $\begin{array}{c}\text { Area } \\
\text { mAU*min }\end{array}$ & $\begin{array}{c}\text { Height } \\
\mathrm{mAU}\end{array}$ & $\begin{array}{c}\text { Relative Area } \\
\%\end{array}$ \\
\hline 1 & 14 & 13.667 & 251.703 & 781.177 & 97.52 \\
2 & 15 & 14.547 & 6.408 & 19.301 & 2.48
\end{tabular}


(R)-triisopropyl (5-methyl-3- phenylhex-1-yn-1-yl) silane (d33)

\section{d33 - ${ }^{1}$ H NMR}

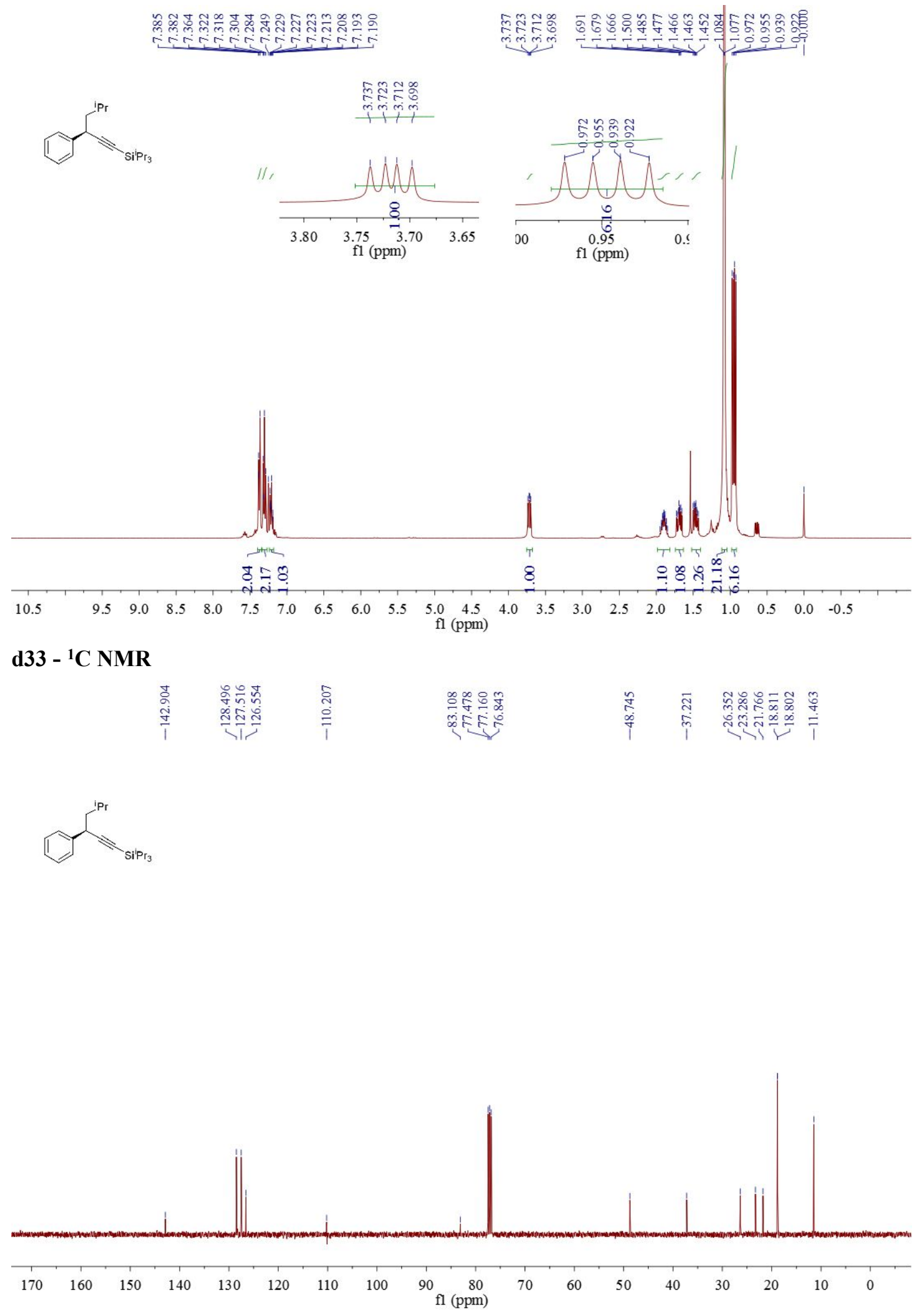


d33 - HPLC

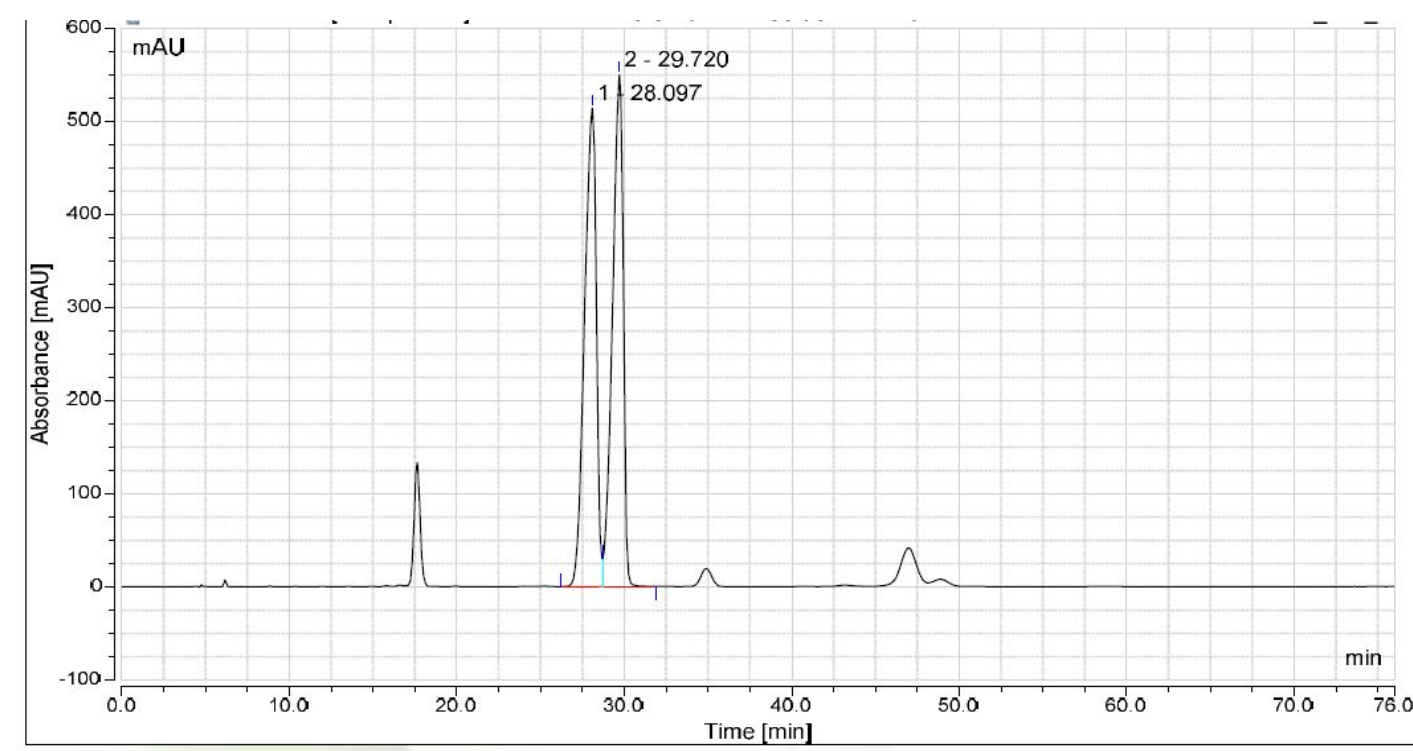

\begin{tabular}{|c|c|c|c|c|c|}
\hline \multicolumn{6}{|c|}{ Integration Results } \\
\hline No. & \begin{tabular}{|l} 
Retention Time \\
min
\end{tabular} & $\begin{array}{c}\text { Retention Time } \\
\text { min }\end{array}$ & $\begin{array}{c}\text { Area } \\
m A U^{*} \min \end{array}$ & $\begin{array}{l}\text { Height } \\
\text { mAU }\end{array}$ & $\begin{array}{c}\text { Relative Area } \\
\%\end{array}$ \\
\hline 1 & 28 & 28.097 & 419.984 & 513.998 & 50.67 \\
\hline 2 & 30 & 29.720 & 408.891 & 549.839 & 49.33 \\
\hline \multicolumn{2}{|c|}{ Total: } & 828.875 & 1063.837 & 100.00 & \\
\hline
\end{tabular}

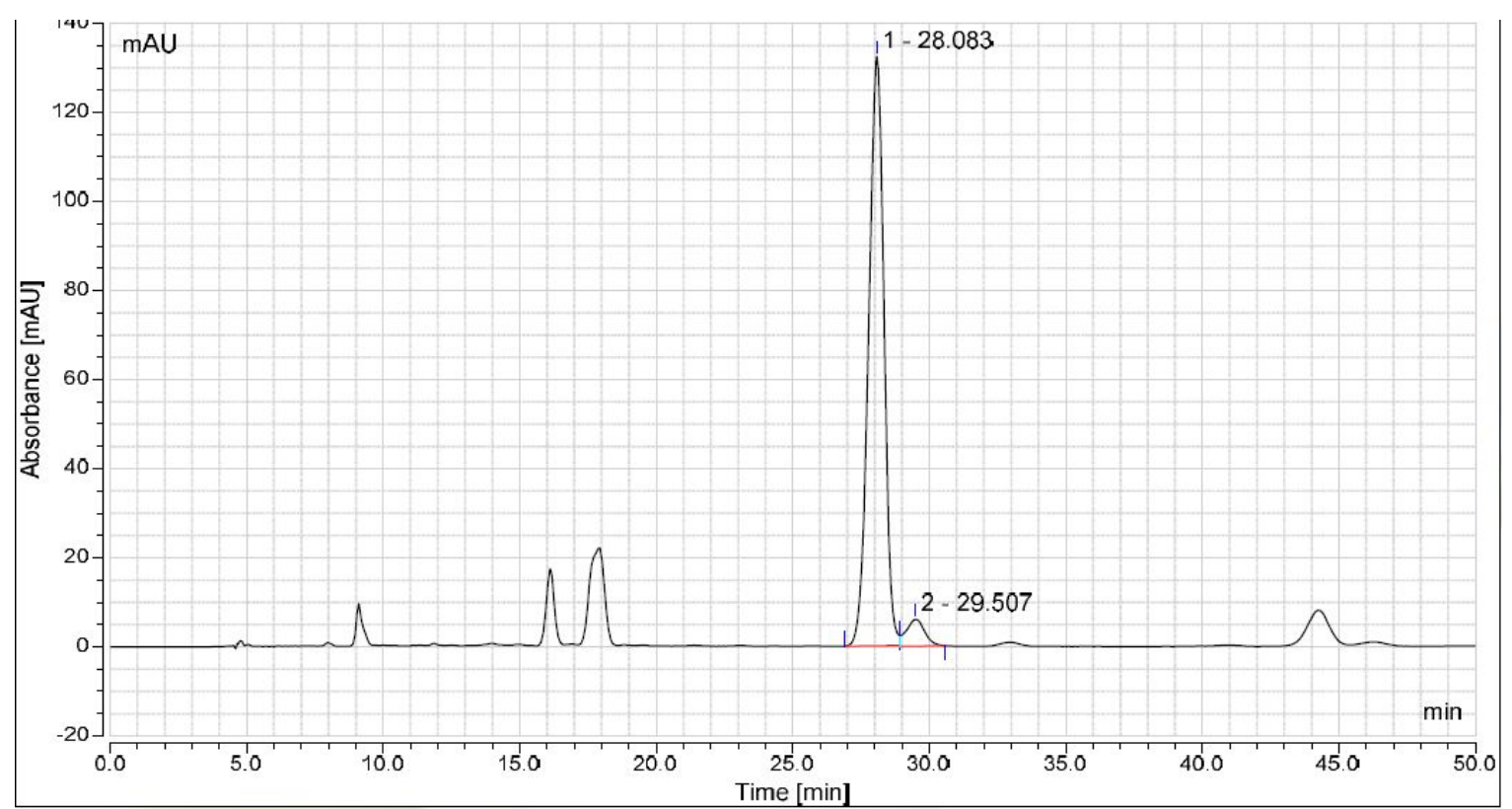

\begin{tabular}{|c|c|c|c|c|c|}
\hline \multicolumn{6}{|c|}{ Integration Results } \\
\hline No. & $\begin{array}{l}\text { Retention Time } \\
\text { min }\end{array}$ & $\begin{array}{c}\text { Retention Time } \\
\text { min }\end{array}$ & $\begin{array}{c}\text { Area } \\
\mathrm{mAU} U^{*} \min \end{array}$ & $\begin{array}{l}\text { Height } \\
\text { mAU }\end{array}$ & $\begin{array}{c}\text { Relative Area } \\
\%\end{array}$ \\
\hline$\overline{1}$ & 28 & 28.083 & 85.918 & 132.311 & 94.82 \\
\hline$\underline{2}$ & 30 & 29.507 & 4.691 & 6.043 & 5.18 \\
\hline \multicolumn{2}{|c|}{ Total: } & 90.609 & 138.354 & 100.00 & \\
\hline
\end{tabular}




\section{d34 - HPLC}

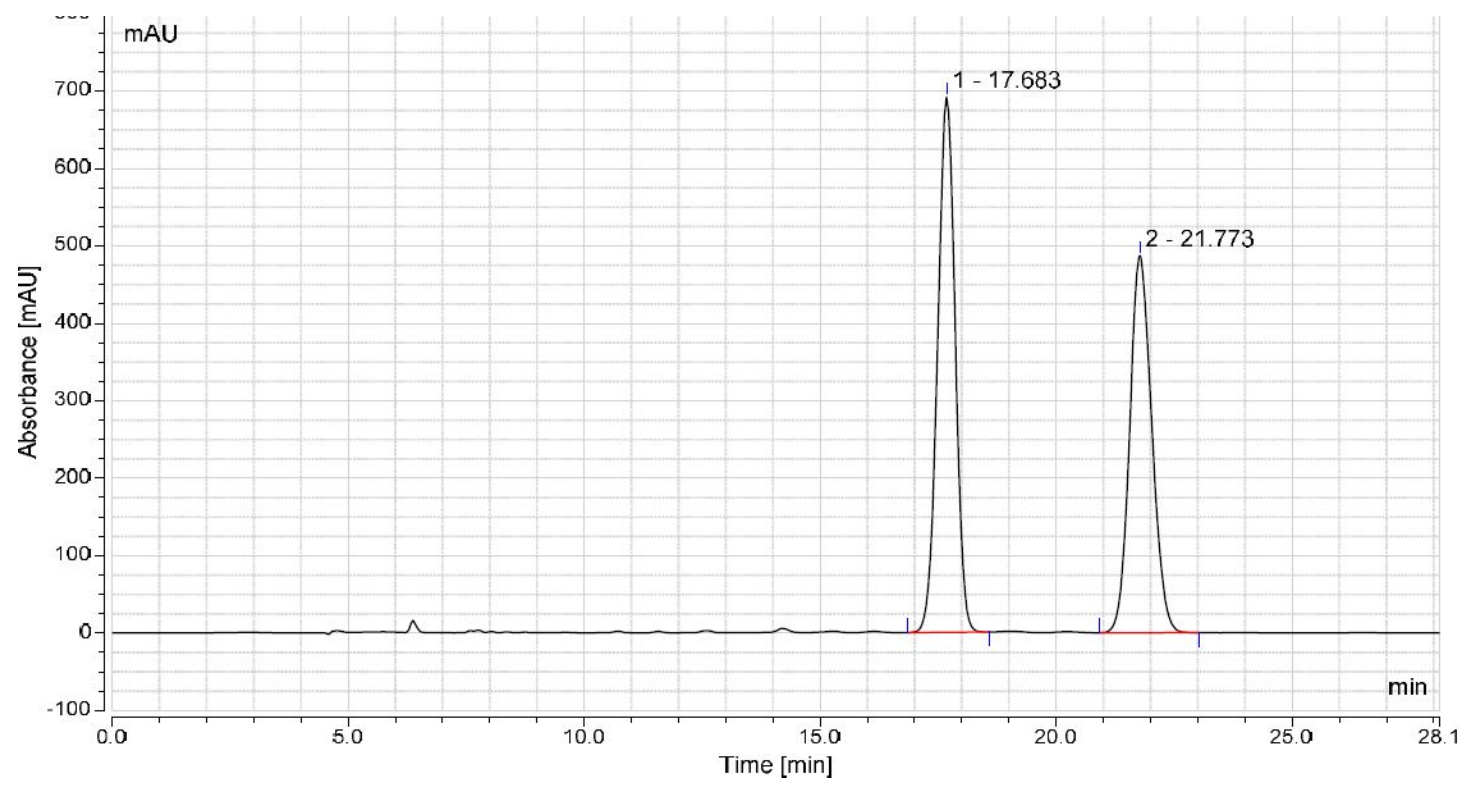

\section{Integration Results}

\begin{tabular}{l|l|c|c|c|c}
\hline No. & $\begin{array}{l}\text { Retention Time } \\
\text { min }\end{array}$ & $\begin{array}{c}\text { Retention Time } \\
\text { min }\end{array}$ & $\begin{array}{c}\text { Area } \\
\mathrm{mAU} U^{*} \text { in }\end{array}$ & $\begin{array}{c}\text { Height } \\
\mathrm{mAU}\end{array}$ & $\begin{array}{c}\text { Relative Area } \\
\%\end{array}$ \\
\hline 1 & 18 & 17.683 & 297.493 & 691.492 & 52.93 \\
2 & 22 & 21.773 & 264.509 & 487.174 & 47.07 \\
\hline
\end{tabular}

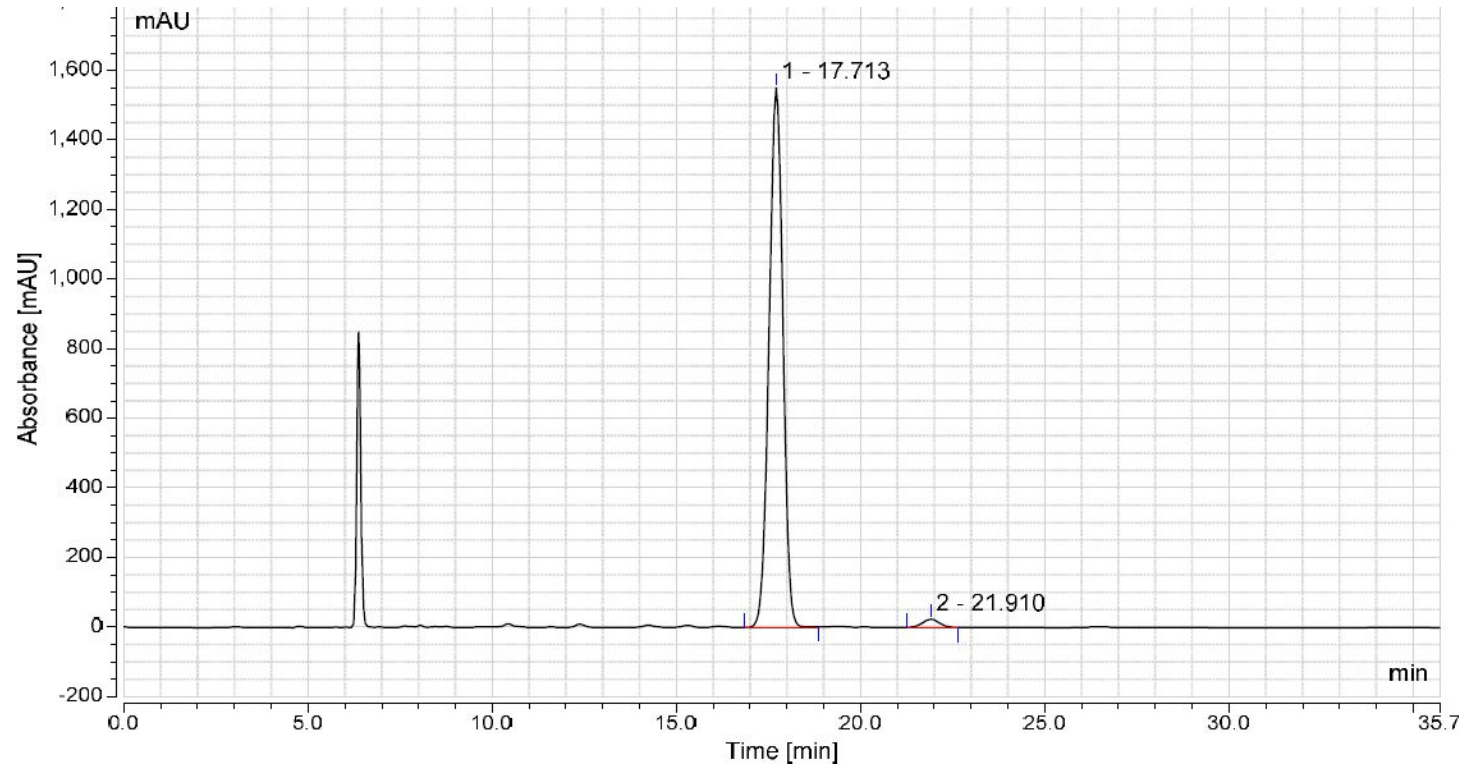

\section{Integration Results}

\begin{tabular}{|c|c|c|c|c|c|}
\hline No. & $\begin{array}{l}\text { Retention Time } \\
\text { min }\end{array}$ & $\begin{array}{c}\text { Retention Time } \\
\text { min }\end{array}$ & $\begin{array}{c}\text { Area } \\
\mathrm{mAU^{* } \operatorname { m i n }}\end{array}$ & $\begin{array}{l}\text { Height } \\
\text { mAU }\end{array}$ & $\begin{array}{c}\text { Relative Area } \\
\% \\
\end{array}$ \\
\hline 1 & 18 & 17.713 & 677.003 & 1551.630 & 98.22 \\
\hline 2 & 22 & 21.910 & 12.264 & 23.191 & 1.78 \\
\hline
\end{tabular}


(R)- (4-(4-(tert-butyl) phenyl)-3-phenylbut-1-yn-1-yl)triisopropylsilane (d35)

\section{d35 - ${ }^{1}$ H NMR}

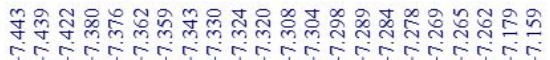

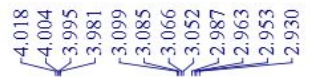<smiles>CC(C)CCCc1ccccc1-c1ccccc1</smiles>

\section{$\| / 1$}
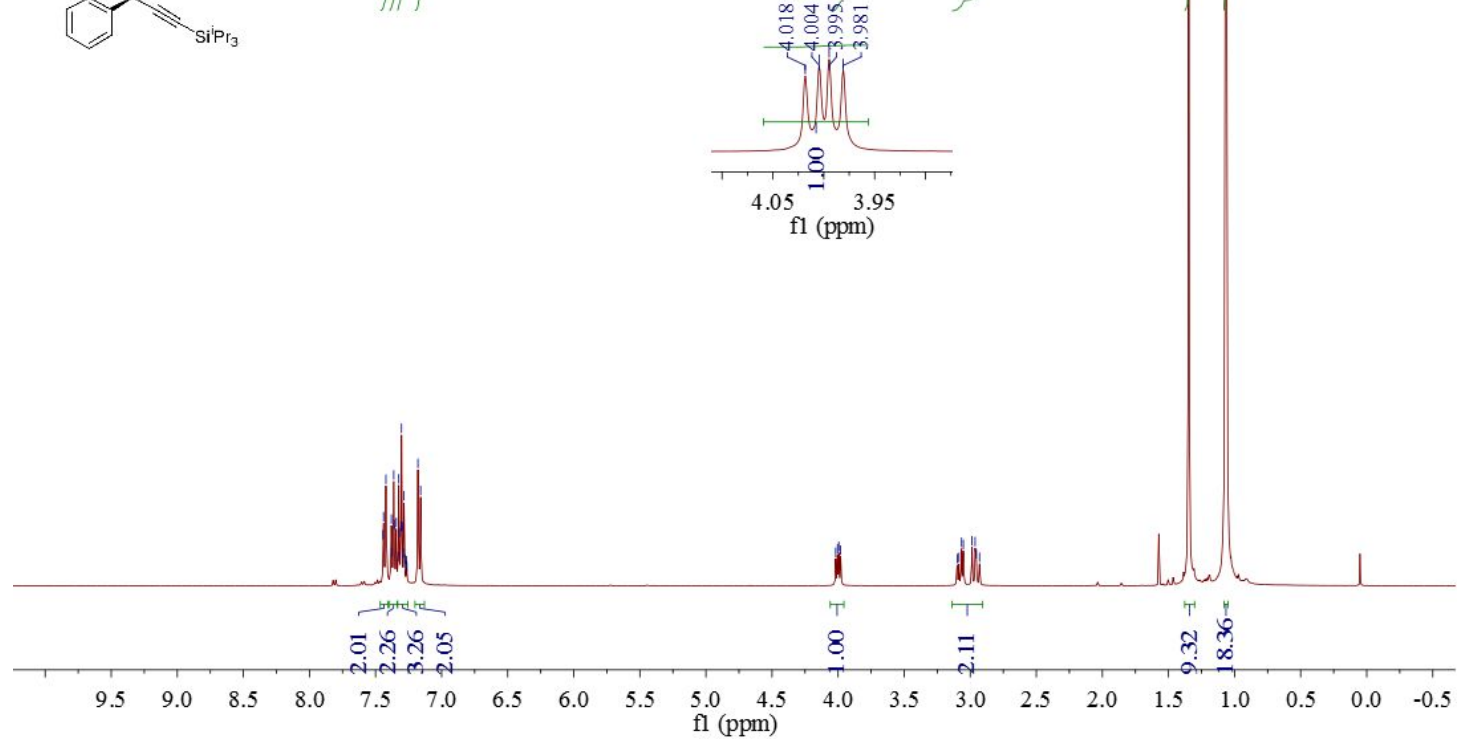

d35 - ${ }^{1}$ C NMR

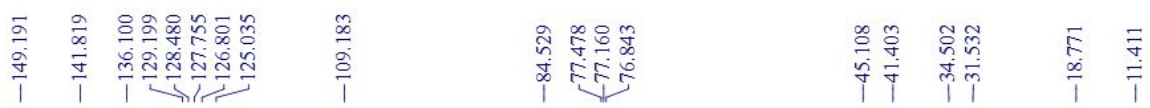
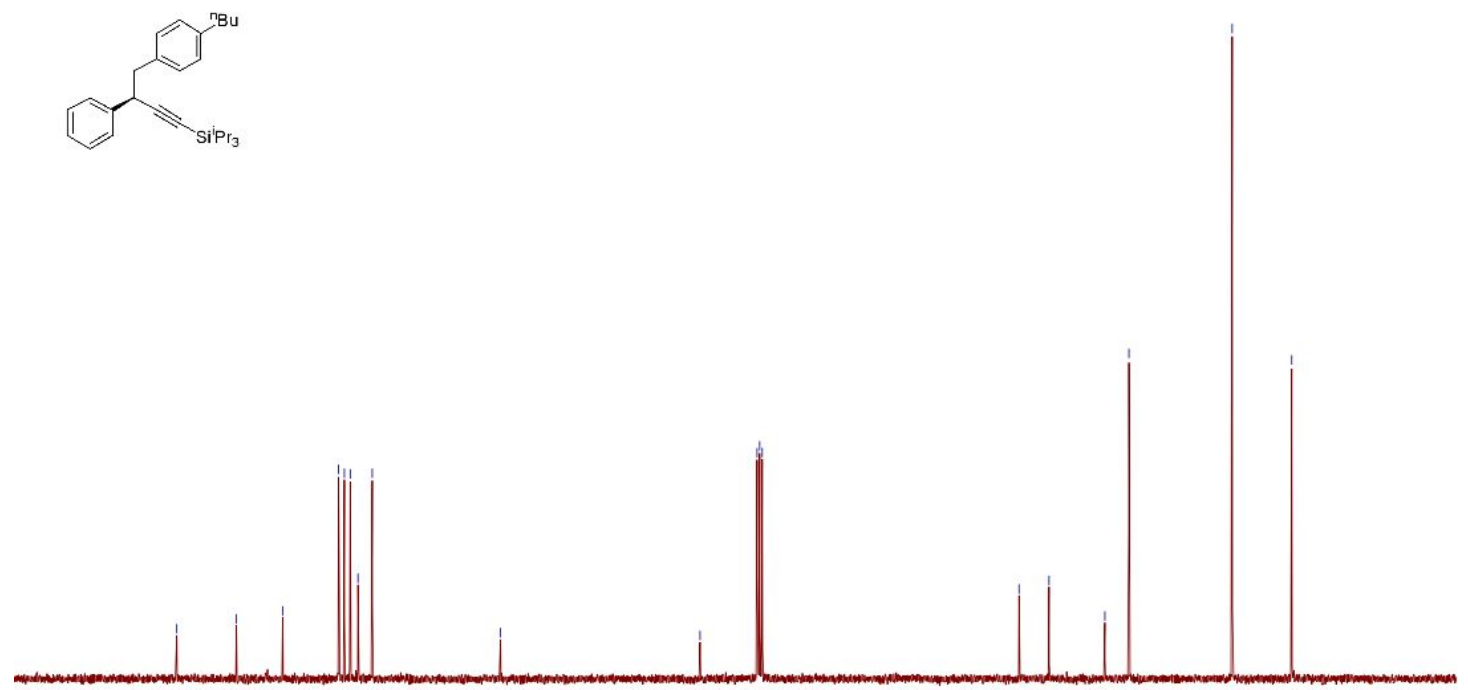

16

$\begin{array}{rrrrrrrrrrrrr}1 & 1 & 1 & 1 & 1\end{array}$




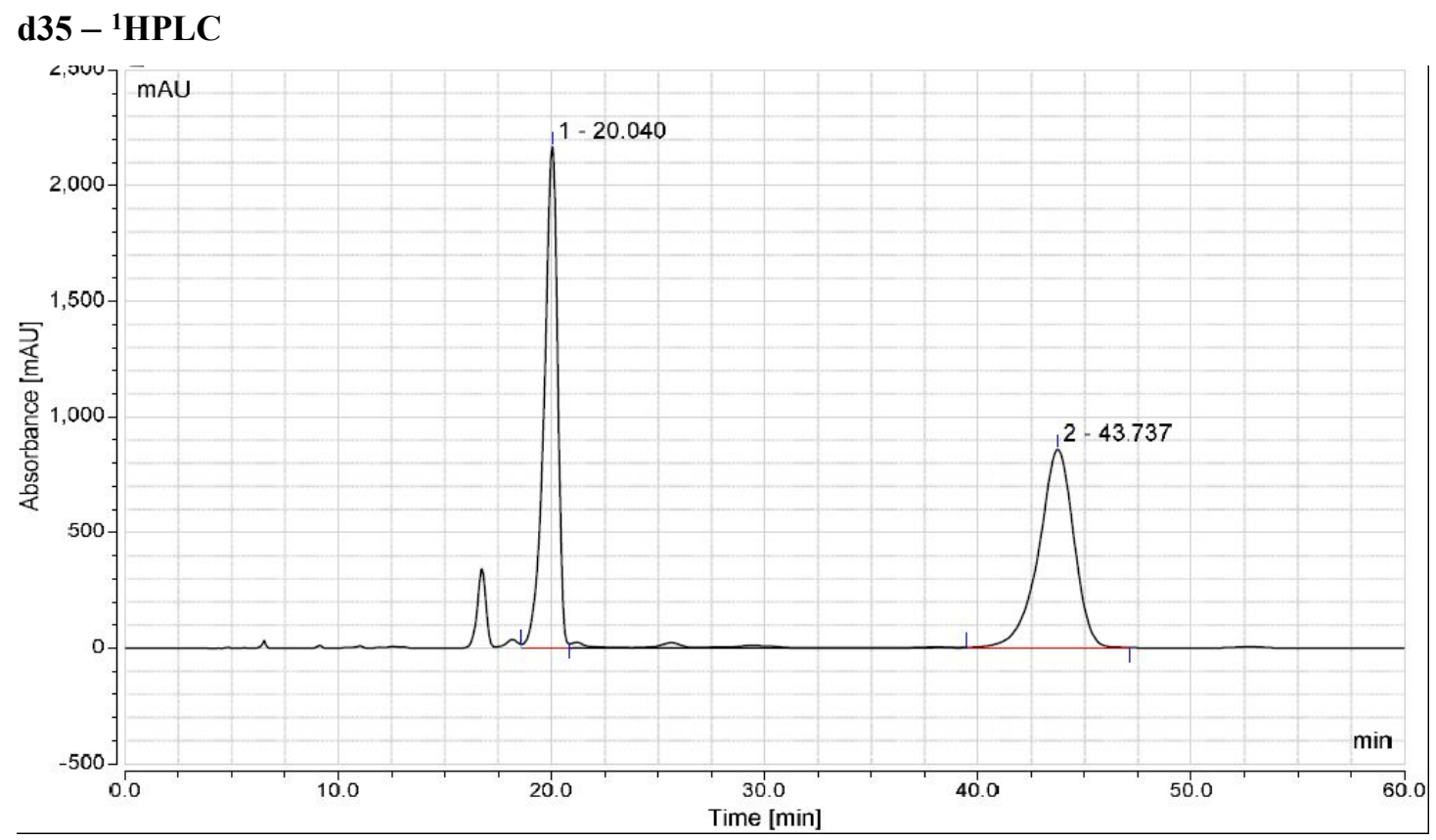

\begin{tabular}{|l|c|c|c|c}
\hline \multicolumn{4}{|l|}{ Integration Results } \\
\hline No. & $\begin{array}{c}\text { Retention Time } \\
\text { min }\end{array}$ & $\begin{array}{c}\text { Area } \\
\text { mAU*min }\end{array}$ & $\begin{array}{c}\text { Height } \\
\text { mAU }\end{array}$ & $\begin{array}{c}\text { Relative Area } \\
\%\end{array}$ \\
\hline 1 & 20.040 & 1589.0934 & 2169.2119 & 49.241 \\
2 & 43.737 & 1638.0815 & 858.6451 & 50.759 \\
\hline Total: & $\mathbf{3 2 2 7 . 1 7 5}$ & $\mathbf{1 4 0 1 . 9 9 8}$ & $\mathbf{1 0 0 . 0 0 0}$ \\
\hline
\end{tabular}

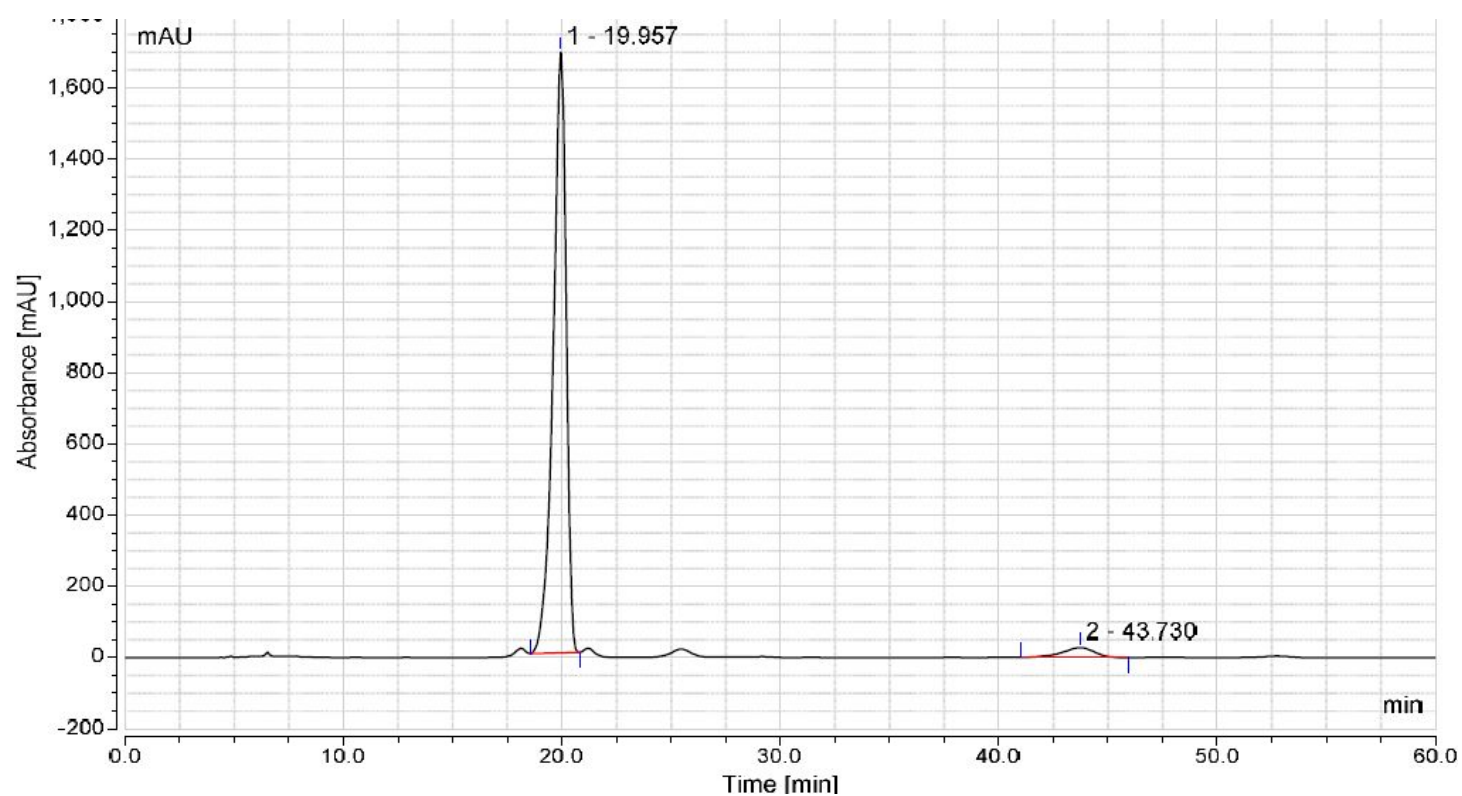

\section{Integration Results}

\begin{tabular}{|l|c|c|c|c}
\hline No. & $\begin{array}{c}\text { Retention Time } \\
\mathrm{min}\end{array}$ & $\begin{array}{c}\text { Area } \\
\mathrm{mAU}^{*} \mathrm{~min}\end{array}$ & $\begin{array}{c}\text { Height } \\
\mathrm{mAU}\end{array}$ & $\begin{array}{c}\text { Relative Area } \\
\%\end{array}$ \\
\hline 1 & 19.957 & 1162.0967 & 1686.7091 & 95.843 \\
2 & 43.730 & 50.4096 & 27.9517 & 4.157 \\
\hline \multicolumn{2}{l|}{ Total: } & $\mathbf{1 2 1 2 . 5 0 6}$ & $\mathbf{1 4 0 1 . 9 9 8}$ & $\mathbf{1 0 0 . 0 0 0}$
\end{tabular}


(R)-triisopropyl (4-(4-methoxyphenyl)-3-phenylbut-1-yn-1-yl) silane (d36) d36 - ' ${ }^{1}$ H NMR

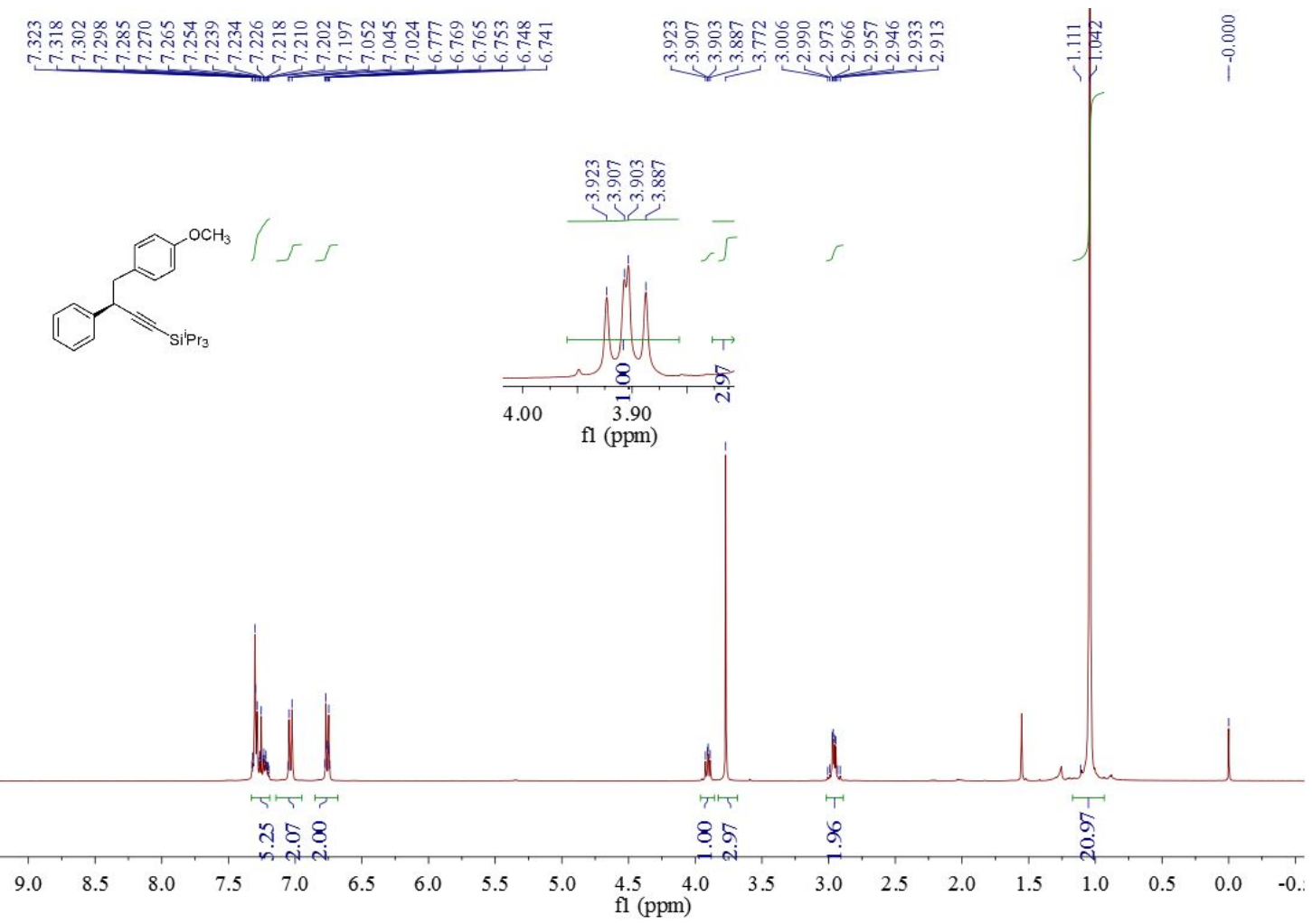

d36 - ${ }^{1}$ C NMR

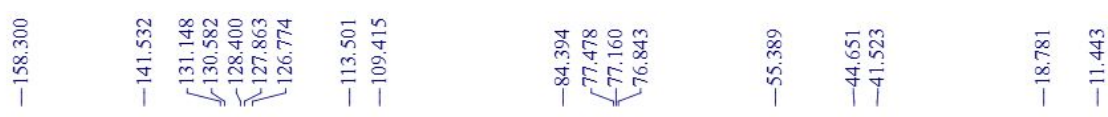
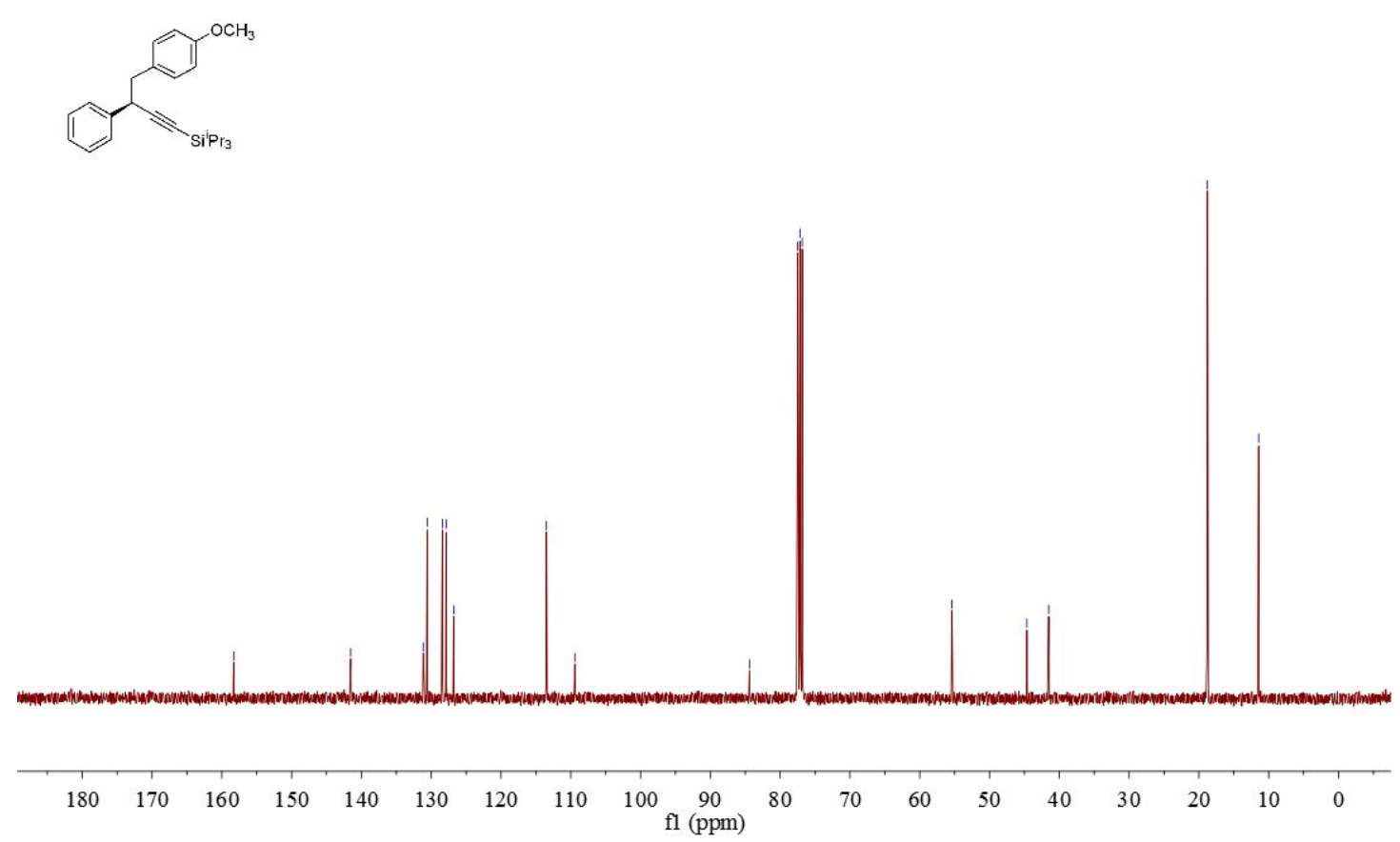


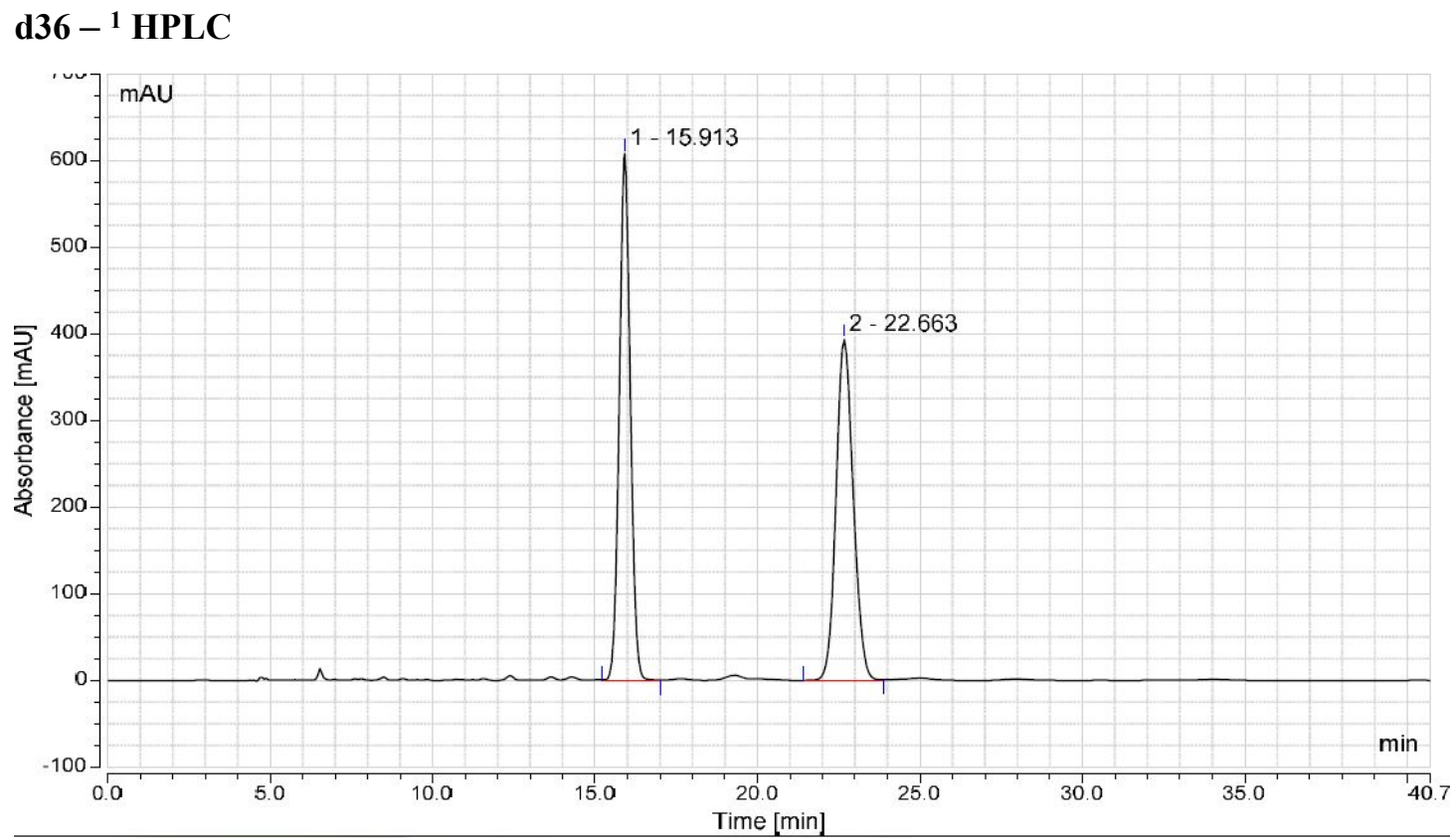

\begin{tabular}{|l|l|c|c|c|c|}
\hline \multicolumn{6}{|l|}{ Integration Results } \\
\hline No. & $\begin{array}{l}\text { Retention Time } \\
\text { min }\end{array}$ & $\begin{array}{c}\text { Retention Time } \\
\text { min }\end{array}$ & $\begin{array}{c}\text { Area } \\
\text { mAU*min }\end{array}$ & $\begin{array}{c}\text { Height } \\
\text { mAU }\end{array}$ & $\begin{array}{c}\text { Relative Area } \\
\%\end{array}$ \\
\hline 1 & 16 & 15.913 & 238.939 & 607.974 & 49.81 \\
2 & 23 & 22.663 & 240.777 & 393.570 & 50.19 \\
\hline \multicolumn{2}{|l|}{ Total: } & $\mathbf{4 7 9 . 7 1 6}$ & 1001.544 & 100.00 & \\
\end{tabular}

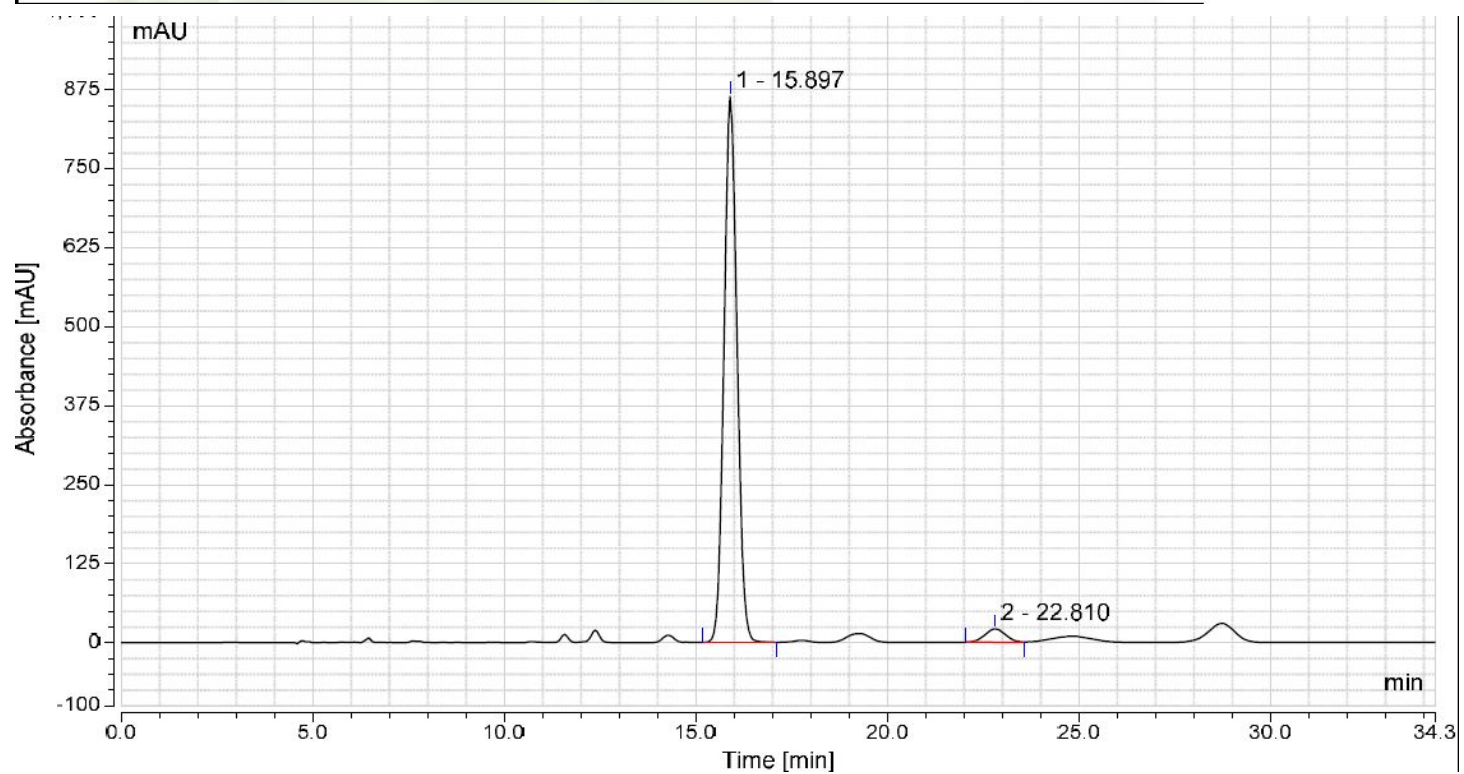

\section{Integration Results}

\begin{tabular}{l|l|c|c|c|c}
\hline No. & $\begin{array}{l}\text { Retention Time } \\
\text { min }\end{array}$ & $\begin{array}{c}\text { Retention Time } \\
\min \end{array}$ & $\begin{array}{c}\text { Area } \\
\mathrm{mAU}^{*} \min \end{array}$ & $\begin{array}{c}\text { Height } \\
\mathrm{mAU}\end{array}$ & $\begin{array}{c}\text { Relative Area } \\
\%\end{array}$ \\
\hline 1 & 16 & 15.897 & 340.494 & 864.531 & 96.22 \\
2 & 23 & 22.810 & 13.360 & 21.171 & 3.78 \\
\hline \multicolumn{2}{l|}{ Total: } & $\mathbf{3 5 3 . 8 5 4}$ & $\mathbf{8 8 5 . 7 0 2}$ & $\mathbf{1 0 0 . 0 0}$
\end{tabular}


(R)- (4-([1, 1'-biphenyl]-4-yl)-3-phenylbut-1-yn-1-yl) triisopropylsilane (d37) d37 - ${ }^{1} \mathrm{H}$ NMR

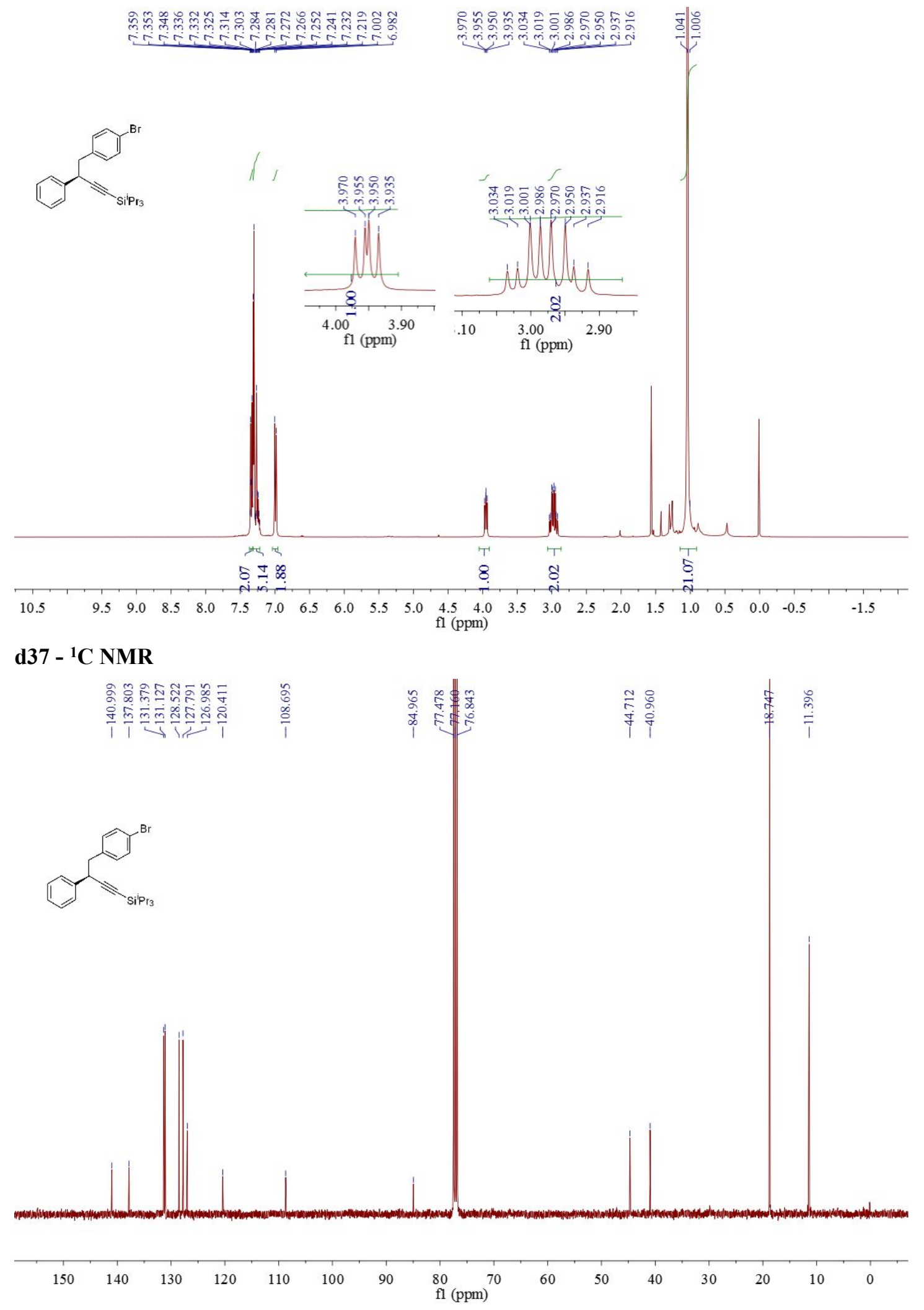




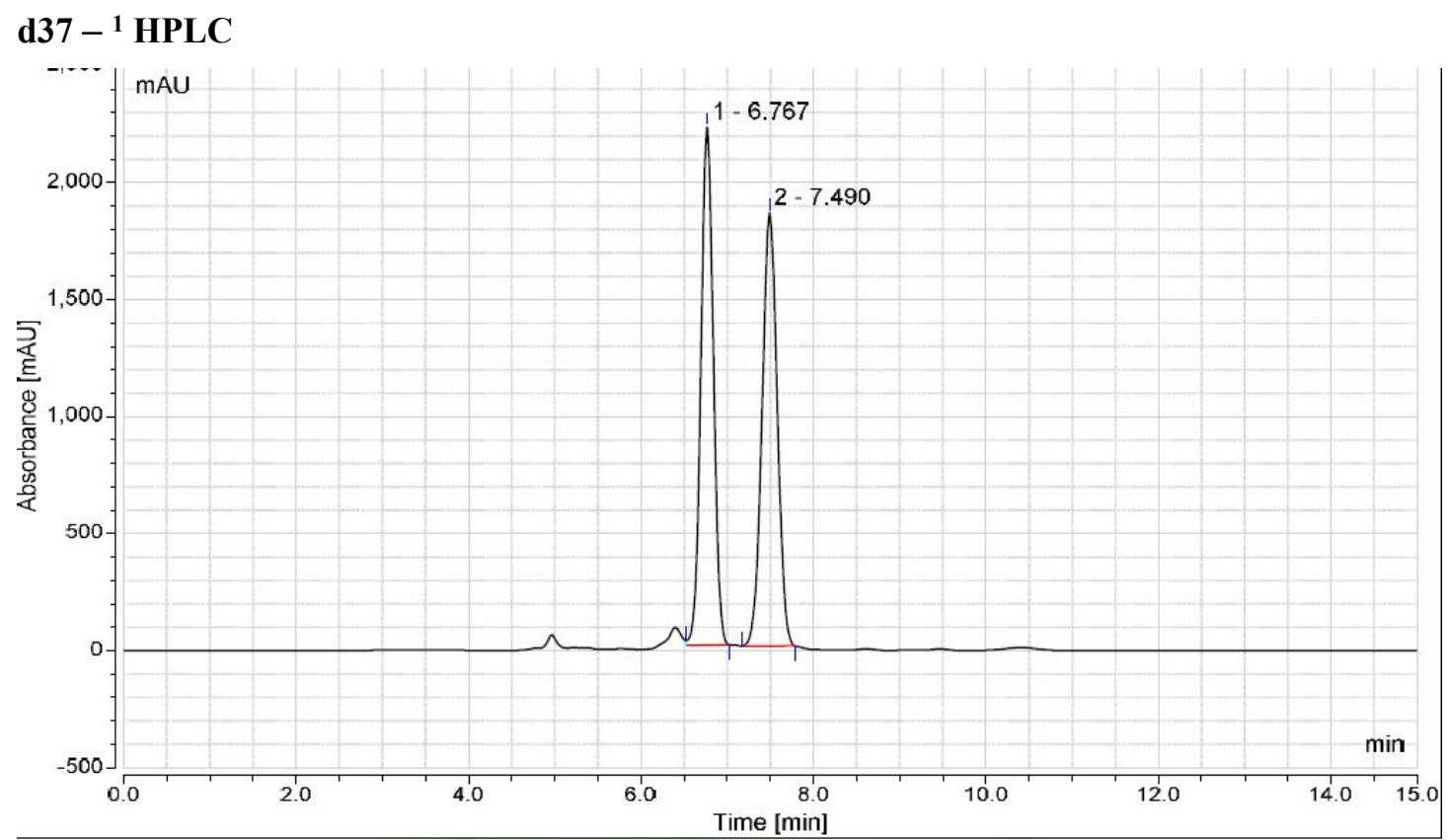

\section{Integration Results}

\begin{tabular}{|l|c|c|c|c|}
\hline No. & $\begin{array}{c}\text { Retention Time } \\
\mathrm{min}\end{array}$ & $\begin{array}{c}\text { Area } \\
\mathrm{mAU}^{*} \mathrm{~min}\end{array}$ & $\begin{array}{c}\text { Height } \\
\mathrm{mAU}\end{array}$ & $\begin{array}{c}\text { Relative Area } \\
\%\end{array}$ \\
\hline 1 & 6.767 & 369.8806 & 2212.8497 & 49.498 \\
2 & 7.490 & 377.3902 & 1849.9887 & 50.502 \\
\hline \multicolumn{2}{l}{ Total: } & $\mathbf{7 4 7 . 2 7 1}$ & $\mathbf{1 4 0 1 . 9 9 8}$ & $\mathbf{1 0 0 . 0 0 0}$
\end{tabular}

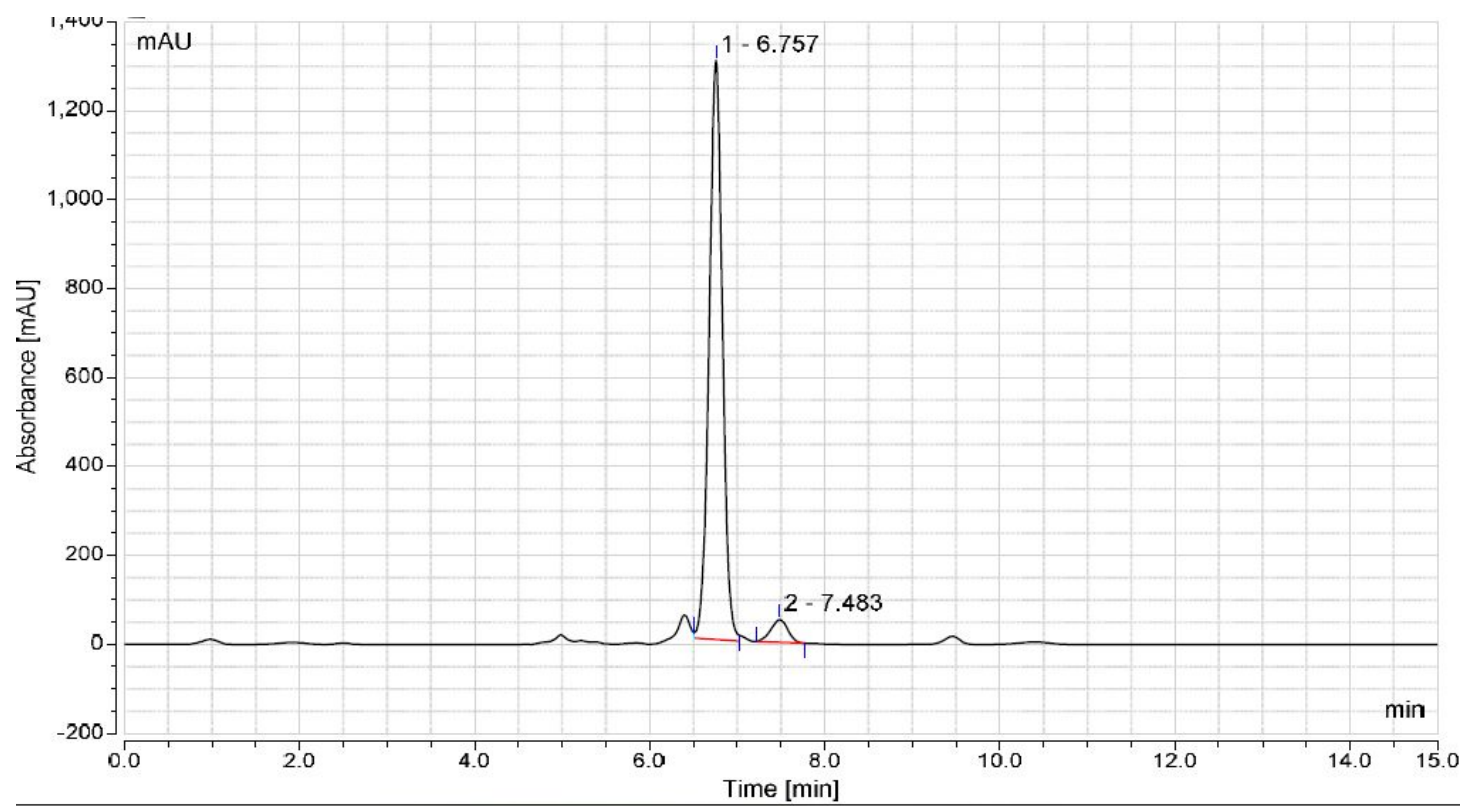

\section{Integration Results}

\begin{tabular}{l|c|c|c|c|}
\hline No. & $\begin{array}{c}\text { Retention Time } \\
\text { min }\end{array}$ & $\begin{array}{c}\text { Area } \\
\mathrm{mAU}^{*} \min \end{array}$ & $\begin{array}{c}\text { Height } \\
\mathrm{mAU}\end{array}$ & $\begin{array}{c}\text { Relative Area } \\
\%\end{array}$ \\
\hline 1 & 6.757 & 228.4536 & 1302.0914 & 95.209 \\
2 & 7.483 & 11.4959 & 50.8579 & 4.791 \\
\hline \multicolumn{2}{l}{ Total: } & $\mathbf{2 3 9 . 9 5 0}$ & $\mathbf{1 4 0 1 . 9 9 8}$ & $\mathbf{1 0 0 . 0 0 0}$
\end{tabular}


(R)- (4-([1, 1'-biphenyl]-4-yl)-3-phenylbut-1-yn-1-yl) triisopropylsilane (d38) d38 - ${ }^{1} H$ NMR

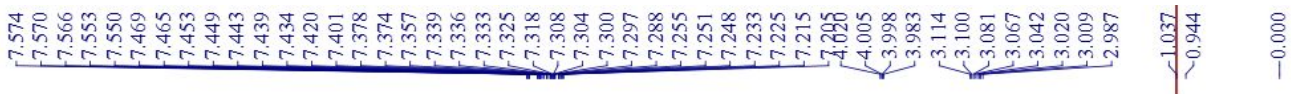
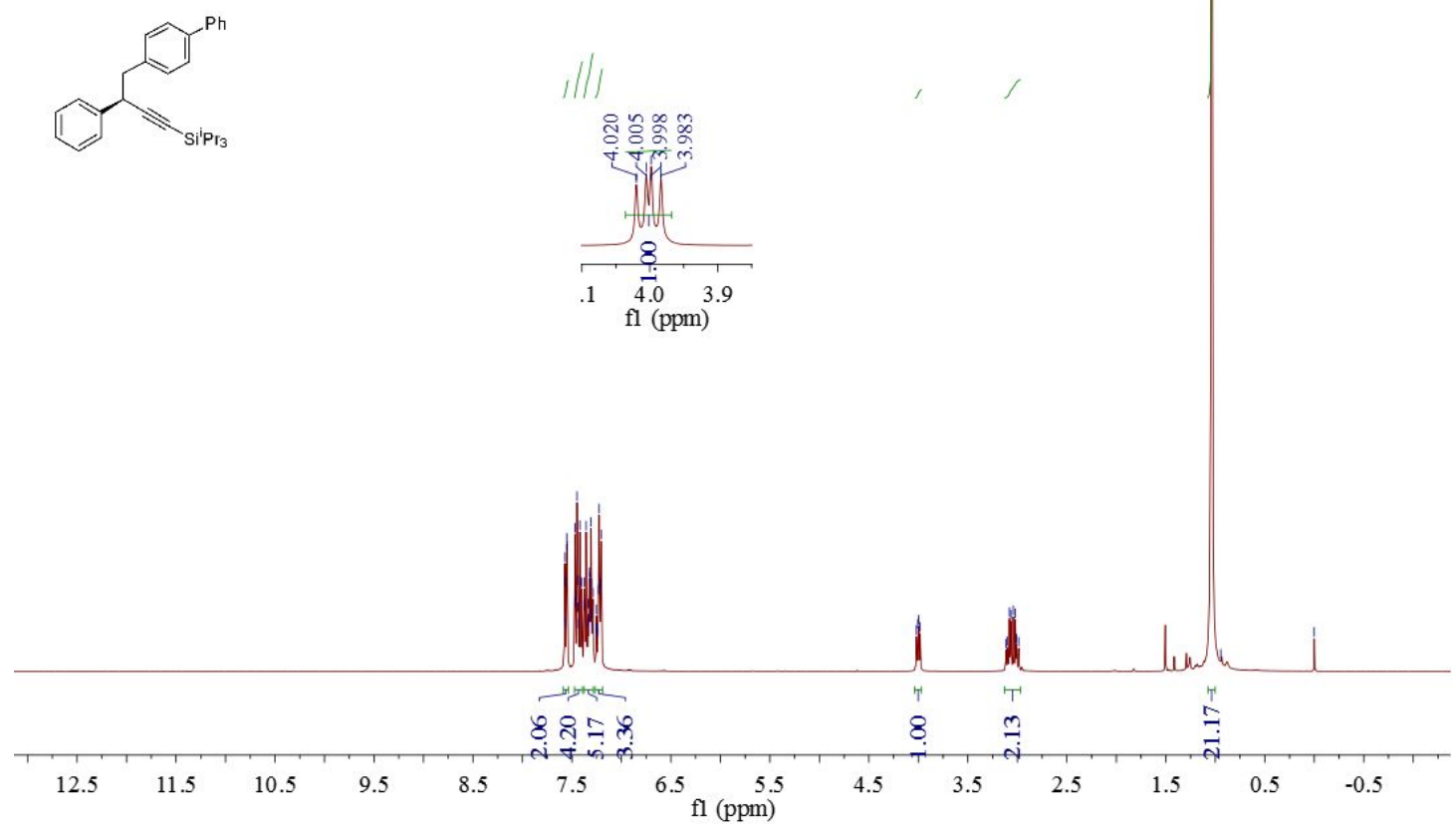

d38 - ${ }^{1}$ C NMR

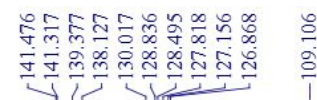

字造

कहि

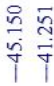

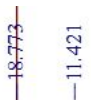
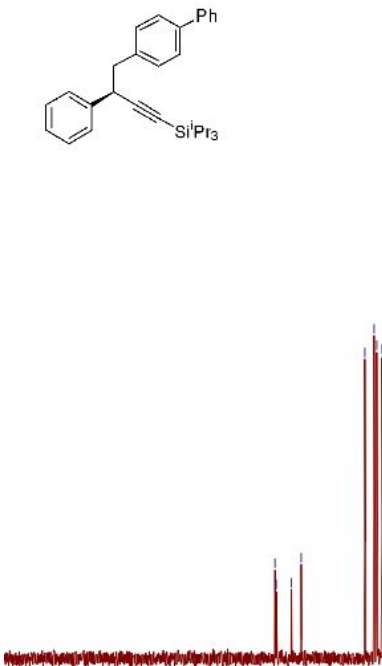

$\begin{array}{lllllllll}170 & 160 & 150 & 140 & 130 & 120 & 110 & 100 & 90 \\ \mathrm{fl}(\mathrm{ppm})\end{array}$

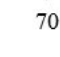

60

40

$30 \quad 20$

100 


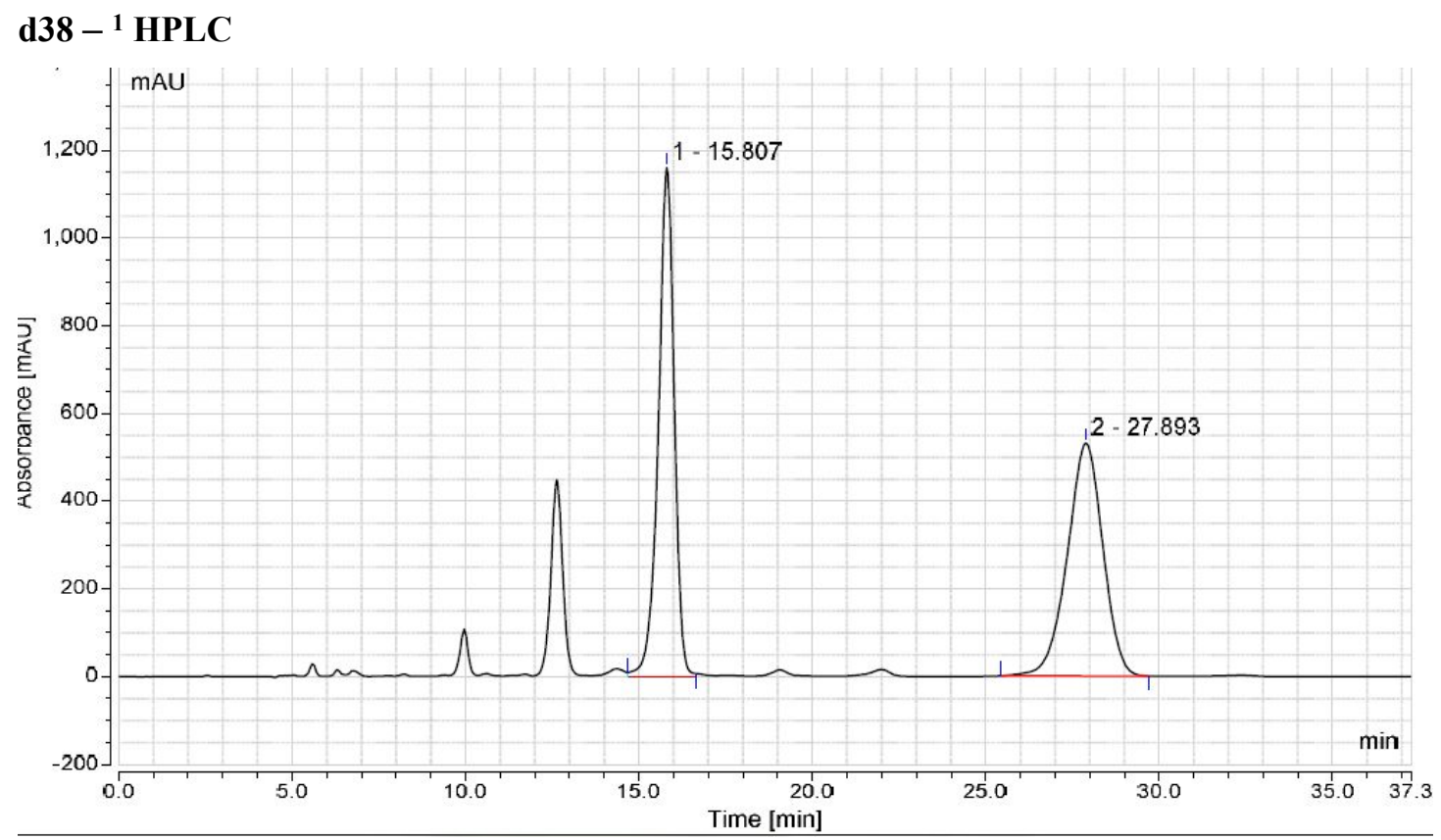

Integration Results

\begin{tabular}{l|c|c|c|c}
\hline No. & $\begin{array}{c}\text { Retention Time } \\
\mathrm{min}\end{array}$ & $\begin{array}{c}\text { Area } \\
\mathrm{mAU}^{*} \min \end{array}$ & $\begin{array}{c}\text { Height } \\
\mathrm{mAU}\end{array}$ & $\begin{array}{c}\text { Relative Area } \\
\%\end{array}$ \\
\hline 1 & 15.807 & 620.9918 & 1162.1739 & 49.613 \\
2 & 27.893 & 630.6683 & 531.1721 & 50.387 \\
\hline \multicolumn{2}{l}{ Total: } & $\mathbf{1 2 5 1 . 6 6 0}$ & $\mathbf{1 4 0 1 . 9 9 8}$ & $\mathbf{1 0 0 . 0 0 0}$
\end{tabular}

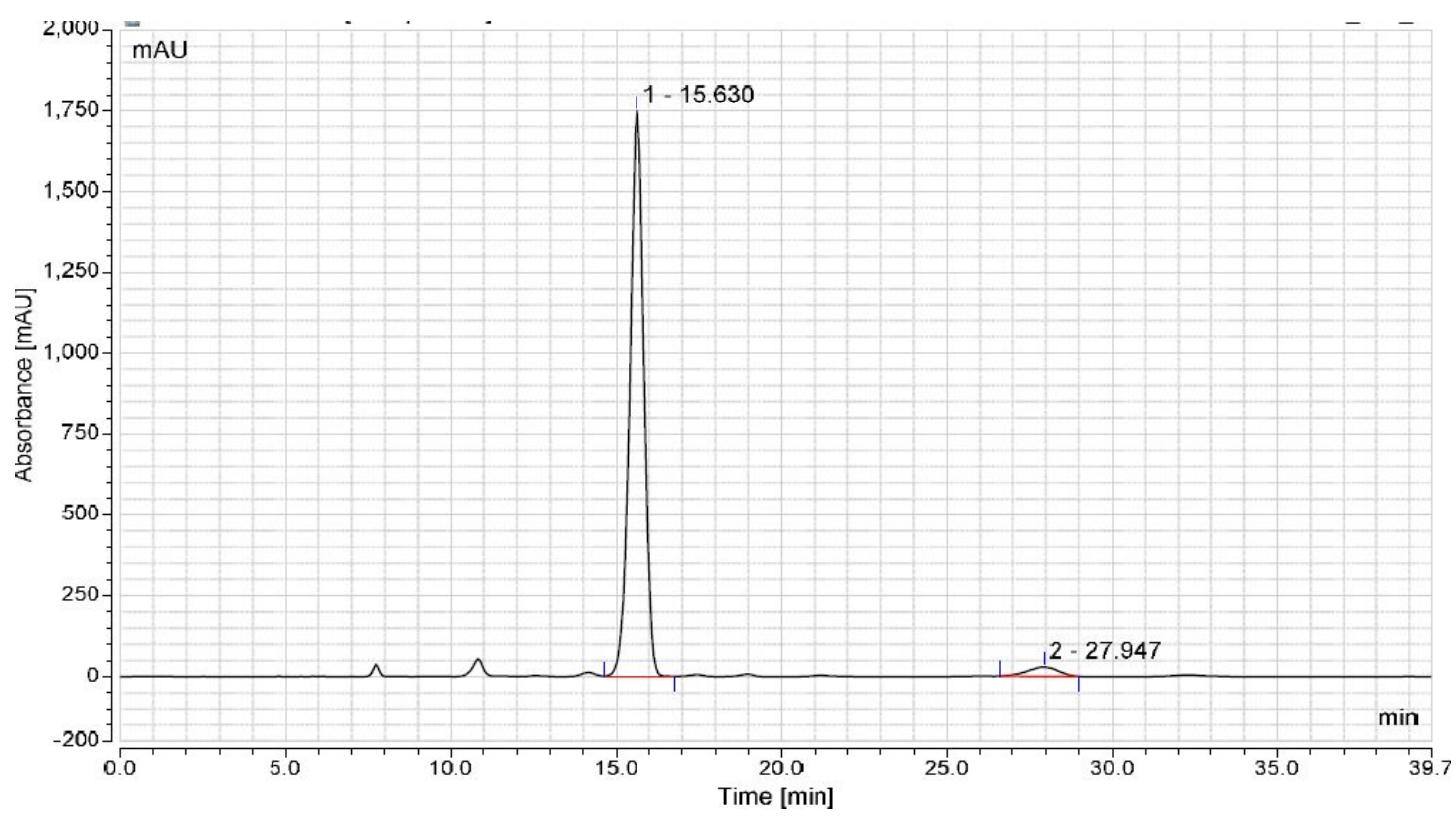

Integration Results

\begin{tabular}{l|c|c|c|c|}
\hline No. & $\begin{array}{c}\text { Retention Time } \\
\mathrm{min}\end{array}$ & $\begin{array}{c}\text { Area } \\
\mathrm{mAU}^{*} \min \end{array}$ & $\begin{array}{c}\text { Height } \\
\mathrm{mAU}\end{array}$ & $\begin{array}{c}\text { Relative Area } \\
\%\end{array}$ \\
\hline 1 & 15.630 & 892.1357 & 1748.9826 & 96.635 \\
2 & 27.947 & 31.0634 & 28.7048 & 3.365 \\
\hline \multicolumn{2}{l}{ Total: } & $\mathbf{9 2 3 . 1 9 9}$ & $\mathbf{1 4 0 1 . 9 9 8}$ & $\mathbf{1 0 0 . 0 0 0}$
\end{tabular}


(R)-triisopropyl (4-(naphthalen-1-yl)-3-phenylbut-1-yn-1-yl) silane (d39) d39 - ${ }^{1}$ H NMR

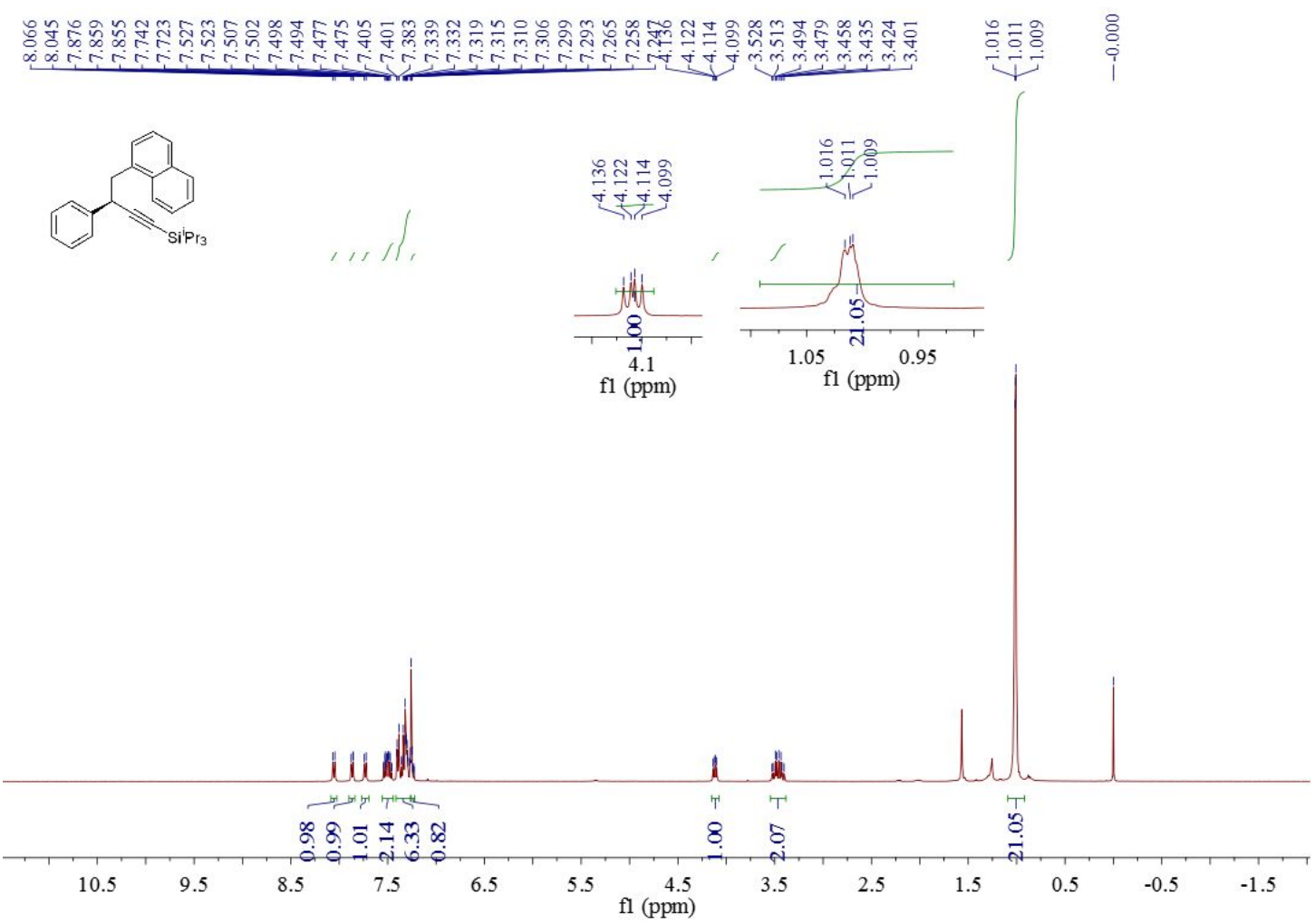

d39 - ' C NMR

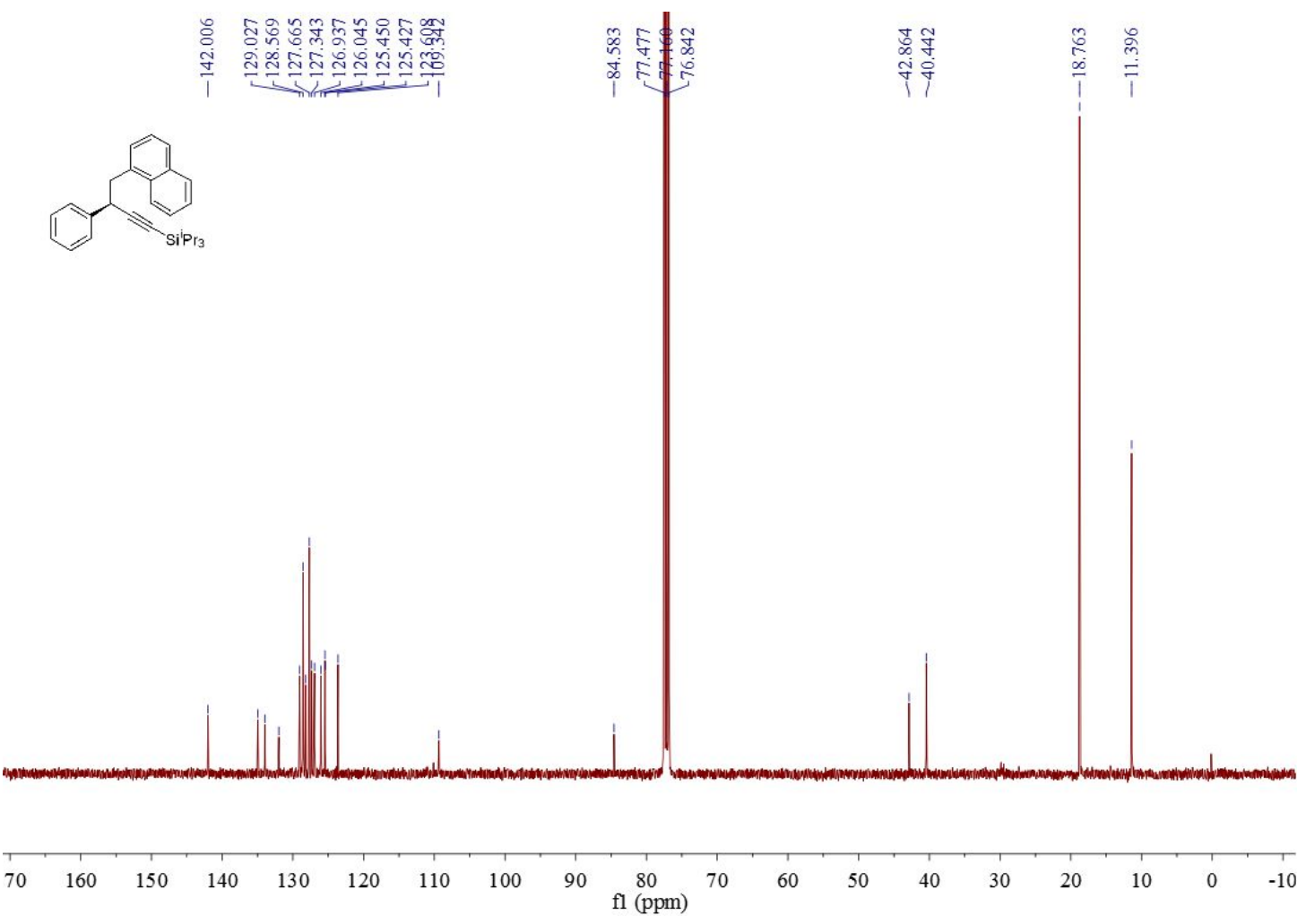




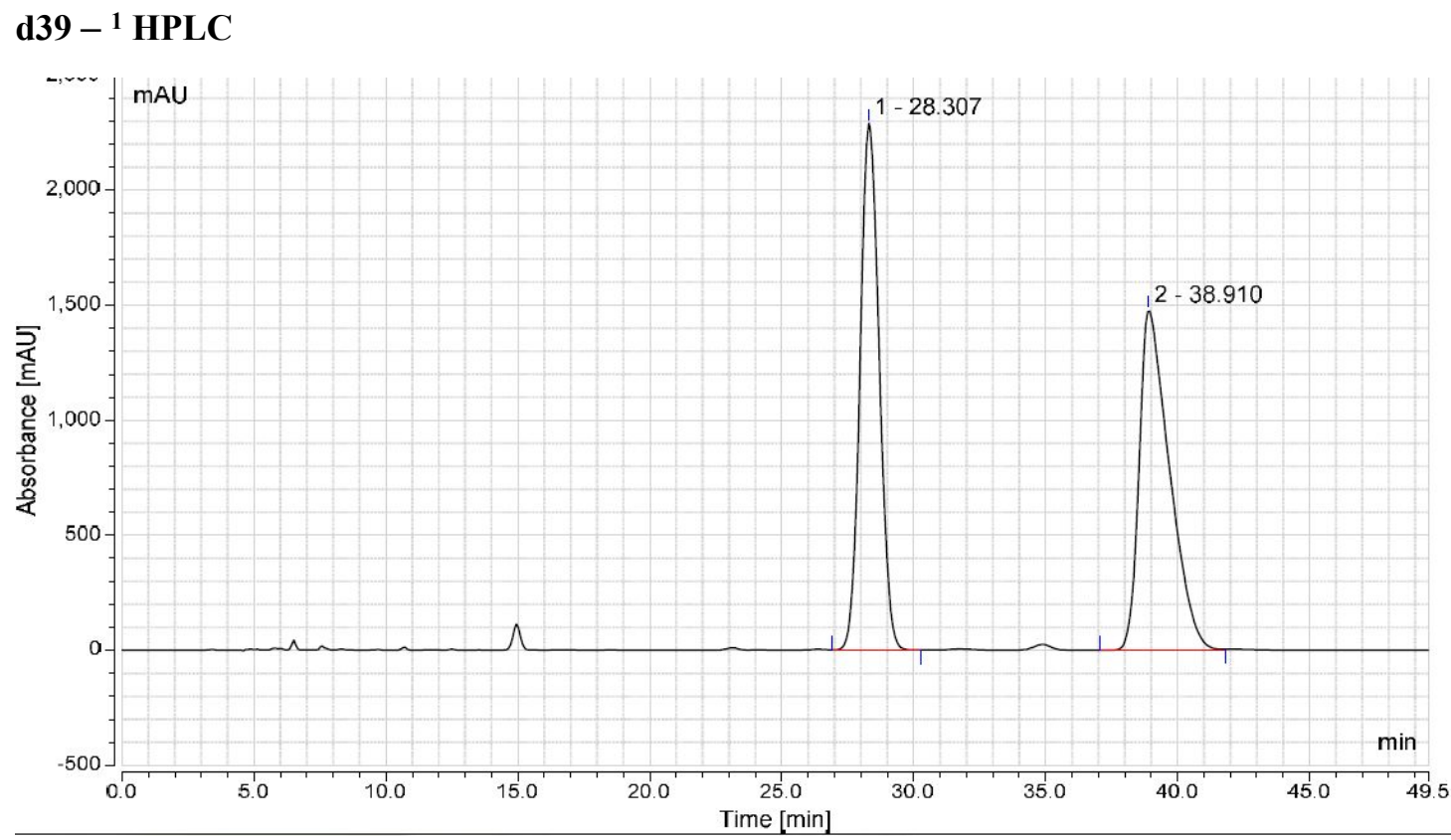

\begin{tabular}{|c|c|c|c|c|c|}
\hline \multicolumn{6}{|c|}{ Integration Results } \\
\hline No. & \begin{tabular}{|l|} 
Retention Time \\
min \\
\end{tabular} & $\begin{array}{c}\text { Retention Time } \\
\text { min }\end{array}$ & $\begin{array}{c}\text { Area } \\
\mathrm{mAU^{* } \operatorname { m i n }}\end{array}$ & $\begin{array}{l}\text { Height } \\
\text { mAU }\end{array}$ & $\begin{array}{c}\text { Relative Area } \\
\%\end{array}$ \\
\hline 1 & 28 & 28.307 & 1921.014 & 2290.865 & 49.47 \\
\hline 2 & 39 & 38.910 & 1961.981 & 1478.261 & 50.53 \\
\hline \multicolumn{2}{|c|}{ Total: } & 3882.995 & 3769.126 & 100.00 & \\
\hline
\end{tabular}

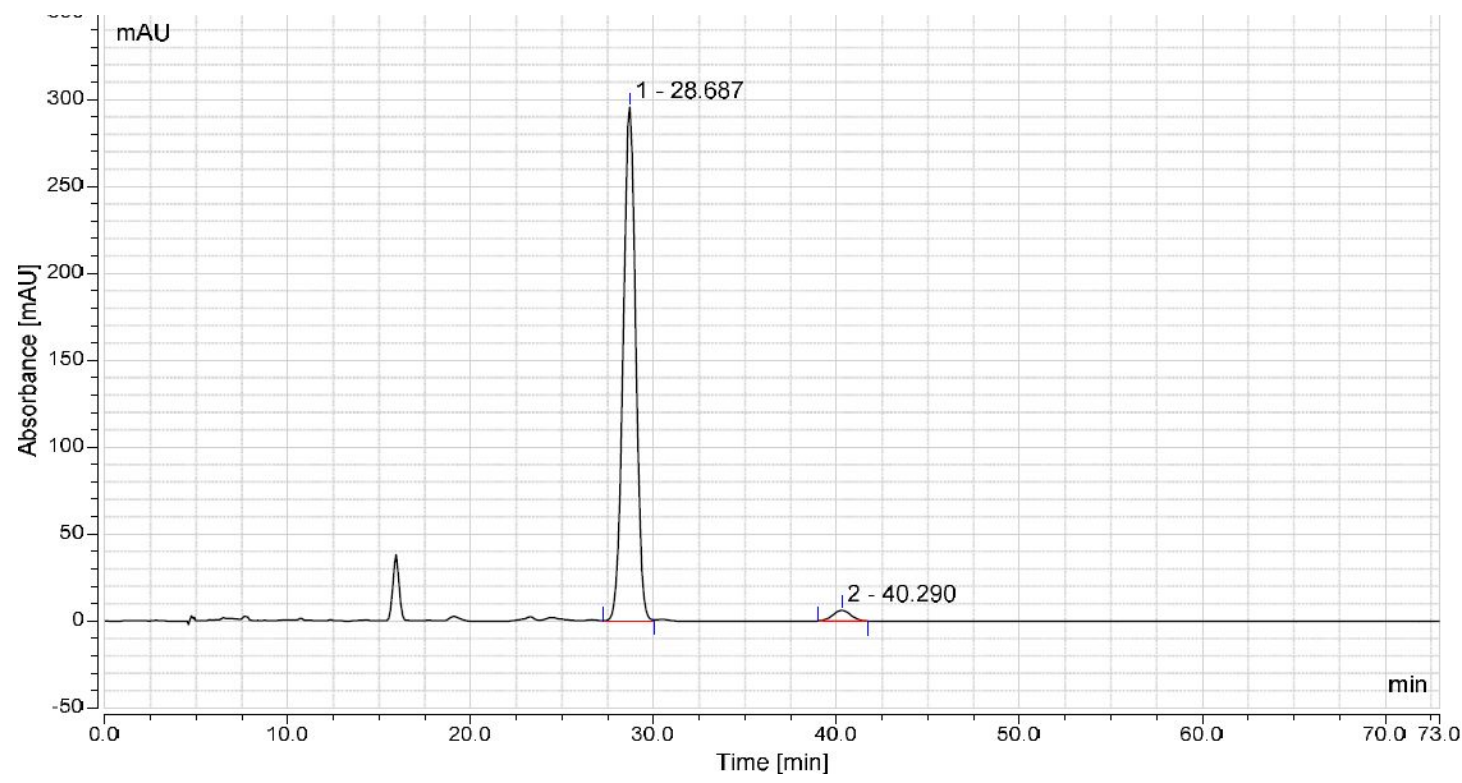

\section{Integration Results}

\begin{tabular}{|l|l|c|c|c|c|}
\hline No. & $\begin{array}{l}\text { Retention Time } \\
\text { min }\end{array}$ & $\begin{array}{c}\text { Retention Time } \\
\min \end{array}$ & $\begin{array}{c}\text { Area } \\
\text { mAU*min }\end{array}$ & $\begin{array}{c}\text { Height } \\
\text { mAU }\end{array}$ & $\begin{array}{c}\text { Relative Area } \\
\%\end{array}$ \\
\hline 1 & 29 & 28.687 & 235.015 & 295.754 & 97.13 \\
2 & 40 & 40.290 & 6.947 & 6.138 & 2.87
\end{tabular}


(R)-3-(2-phenyl-4-(triisopropylsilyl) but-3-yn-1-yl) pyridine (d40) d40 - ${ }^{1}$ H NMR

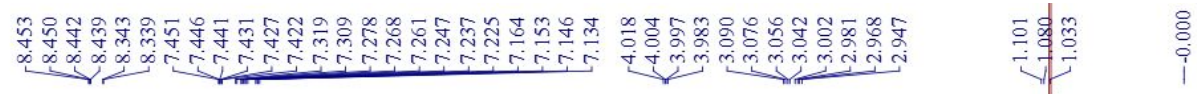

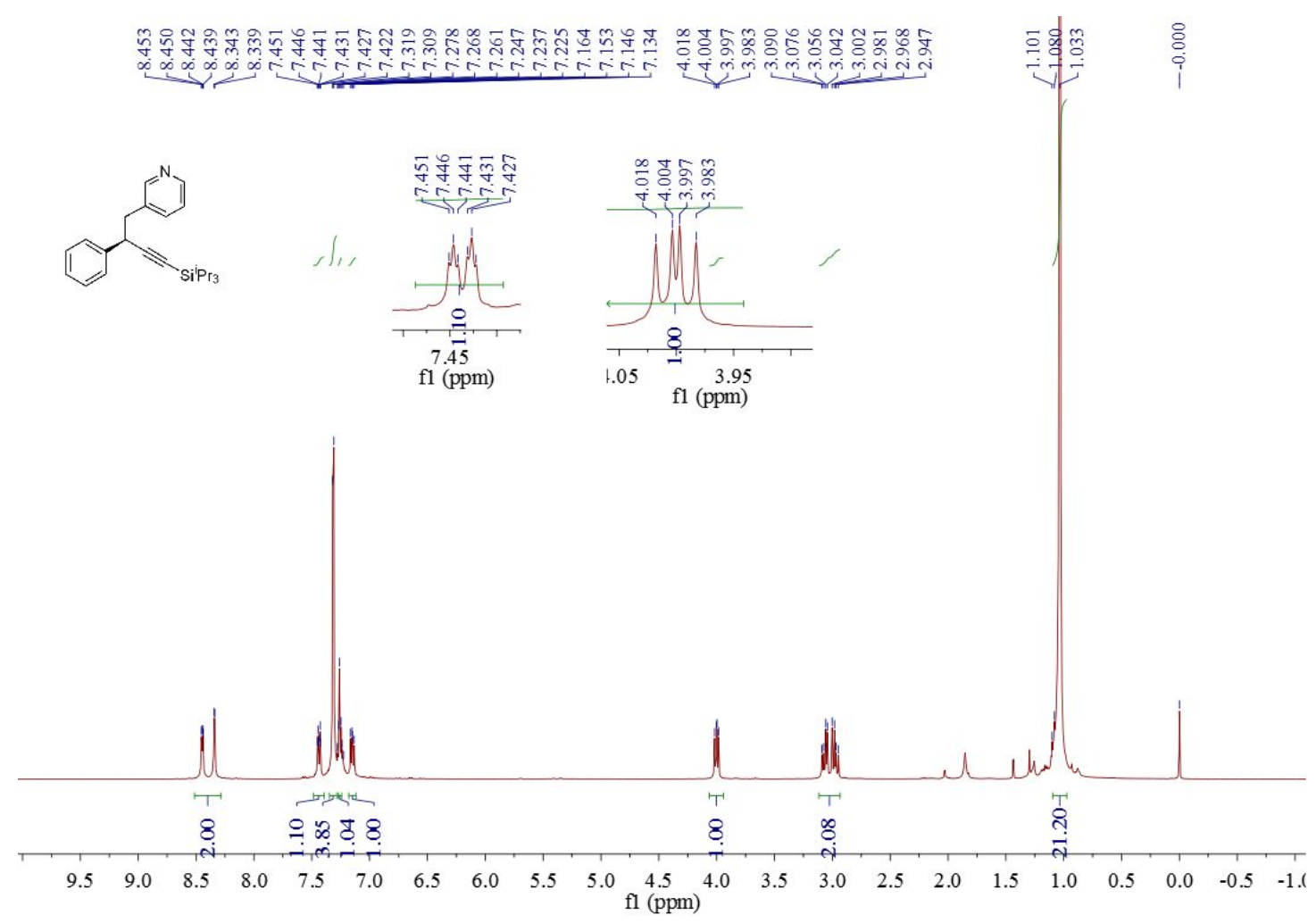

d40 - ${ }^{1}$ C NMR

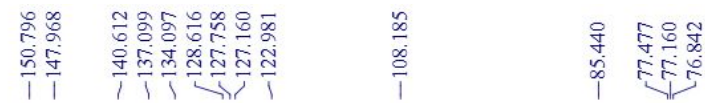

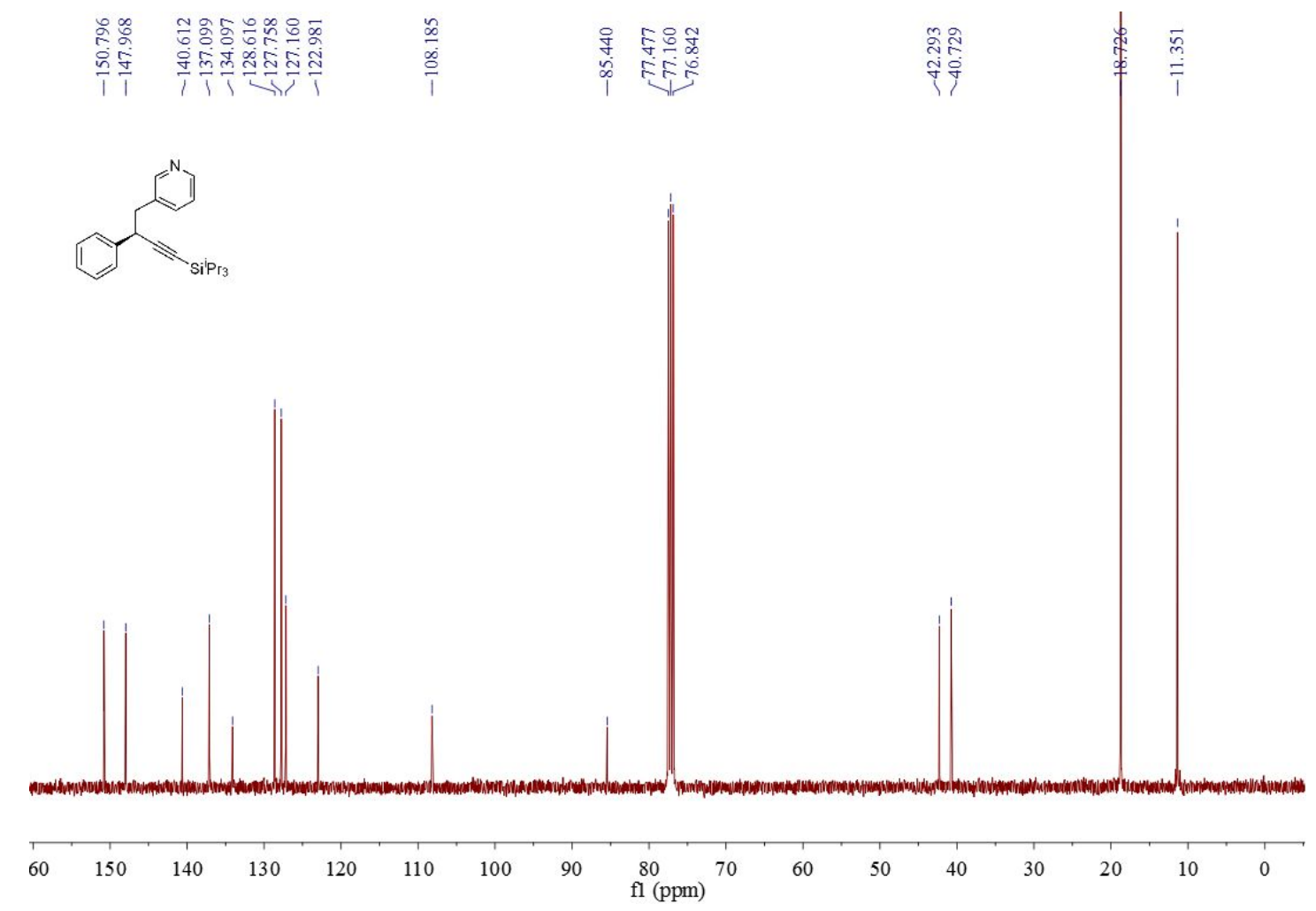

(ppm) 

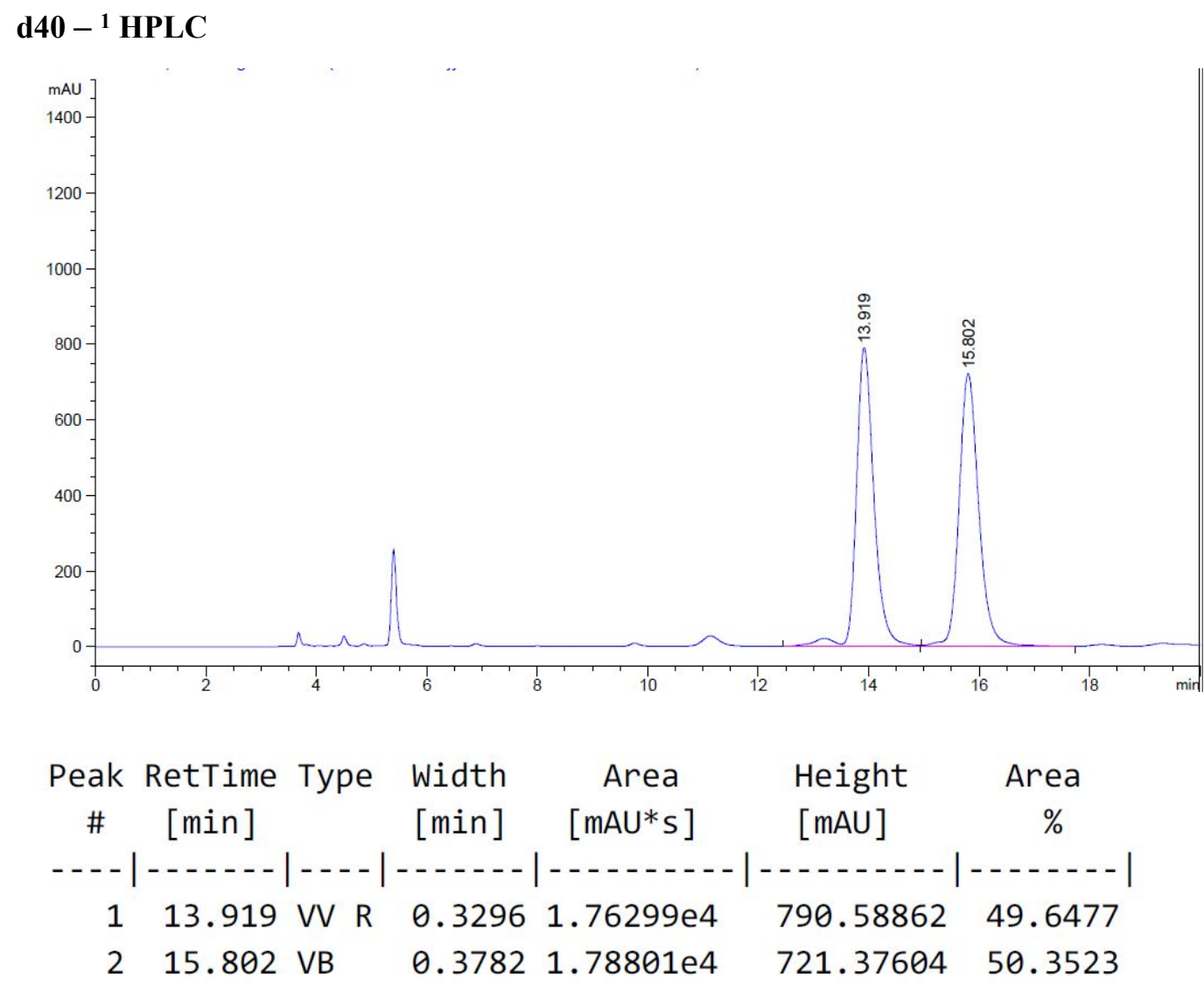

Totals :

$3.55100 \mathrm{e} 4 \quad 1511.96466$

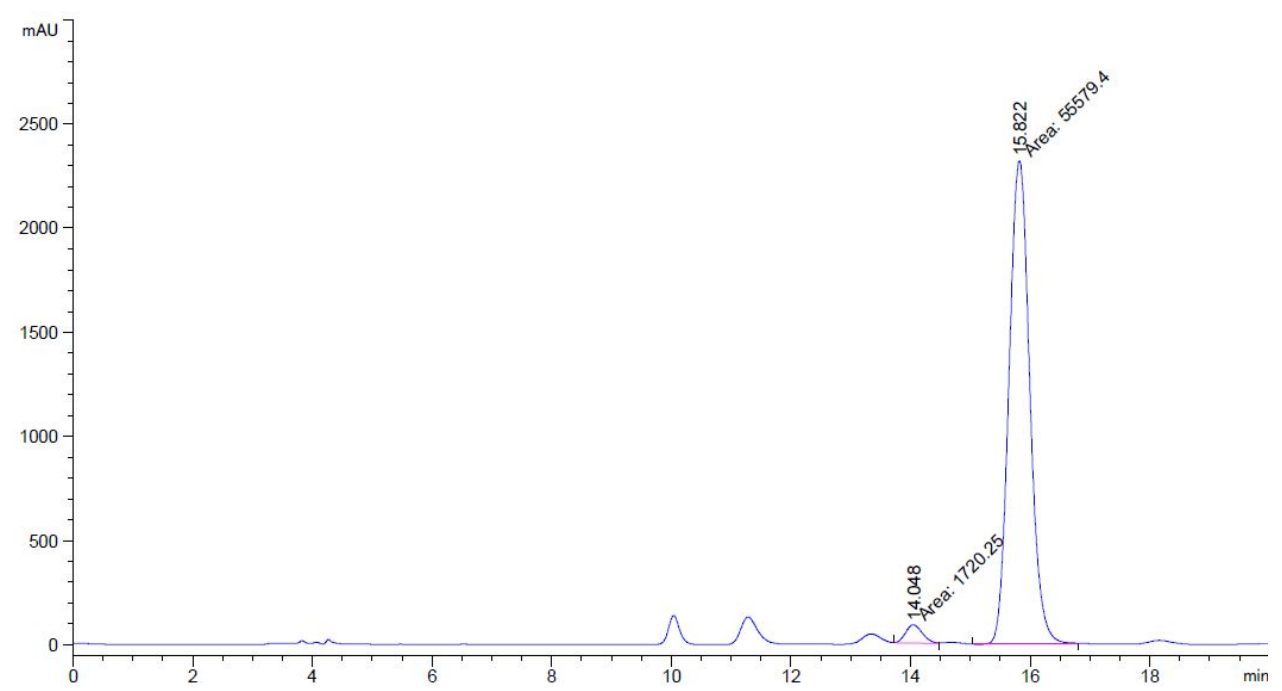

Peak RetTime Type Width Area Height Area

\begin{tabular}{rlrlrrr}
$\#$ & {$[\mathrm{~min}]$} & {$[\mathrm{min}]$} & {$[\mathrm{mAU} * \mathrm{~s}]$} & \multicolumn{1}{c}{$[\mathrm{mAU}]$} & $\%$ \\
\hline 1 & 14.048 & MM & 0.3259 & 1720.25183 & 87.96976 & 3.0022 \\
2 & 15.822 MM & 0.3999 & $5.55794 \mathrm{e} 4$ & 2316.41162 & 96.9978
\end{tabular}

Totals :

$5.72996 \mathrm{e} 4 \quad 2404.38139$ 
(R)-triisopropyl(3-phenyl-4-(thiophen-2-yl)but-1-yn-1-yl) silane (d41) d41 - ${ }^{1}$ H NMR

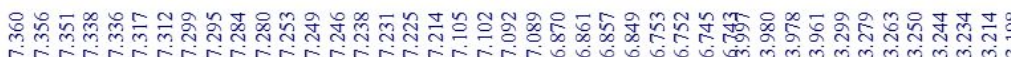

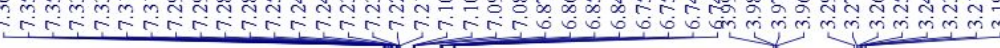<smiles>C[C@H](C=Cc1ccccc1)C1=CC=C1</smiles>
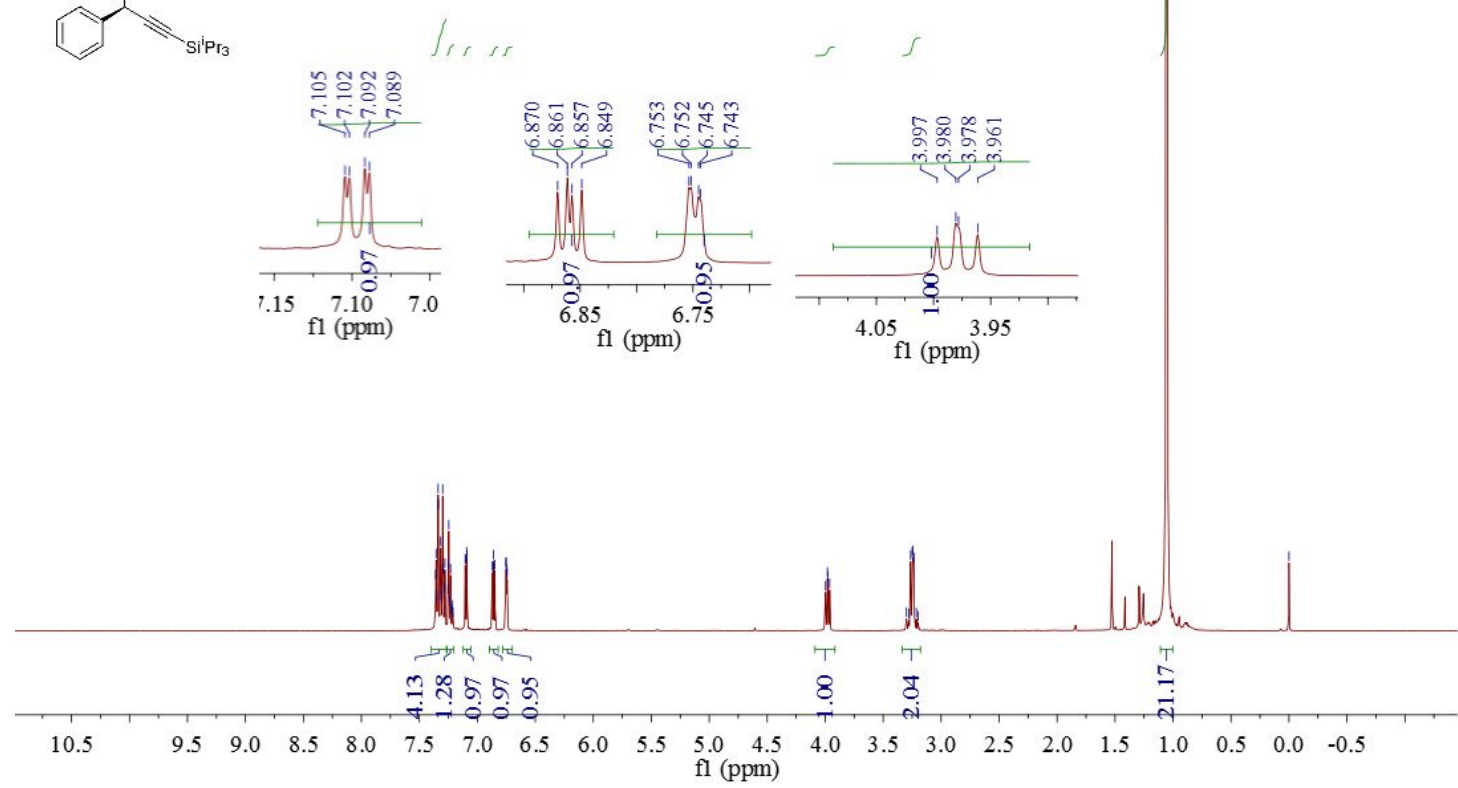

d41 - ${ }^{1}$ C NMR
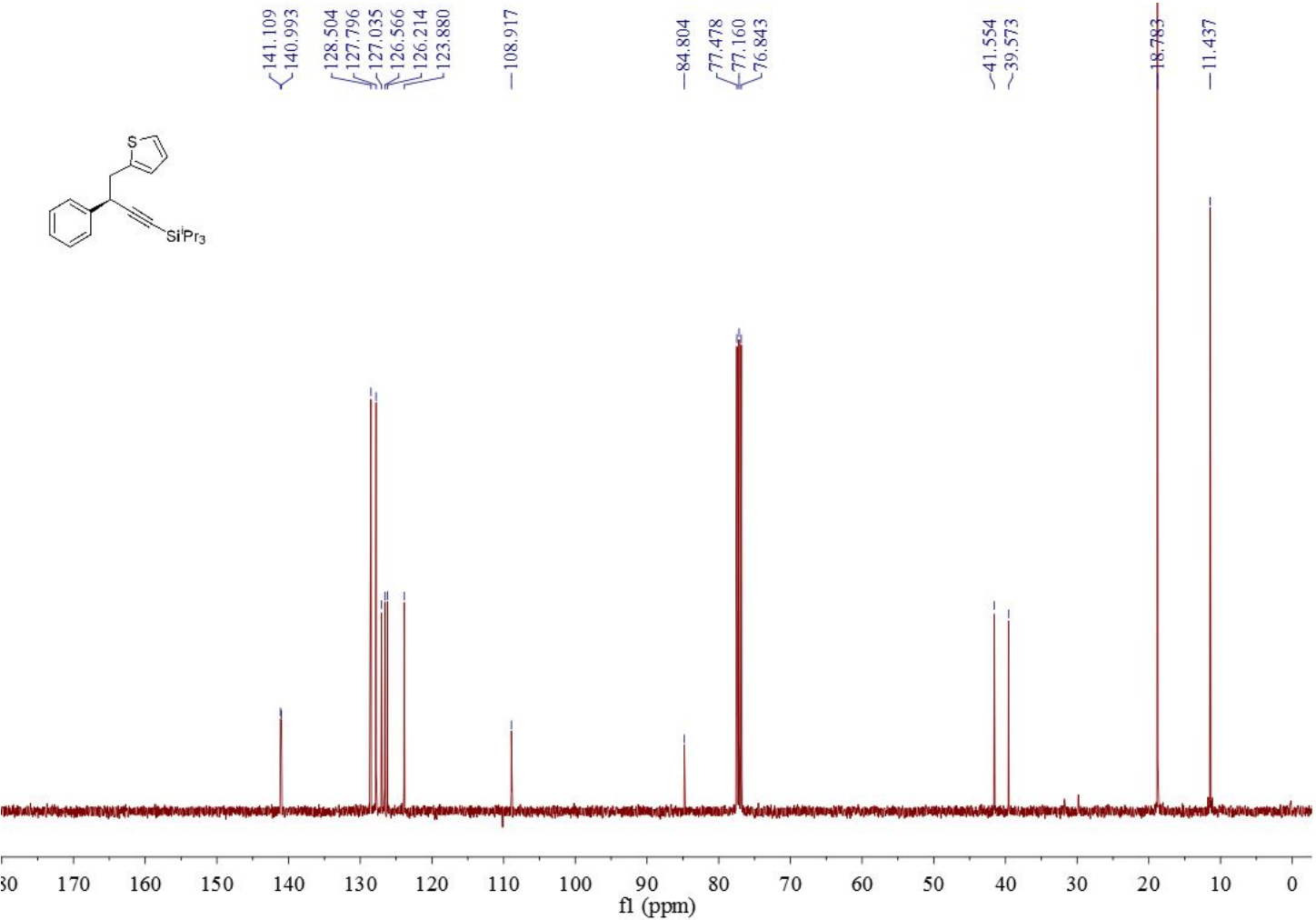


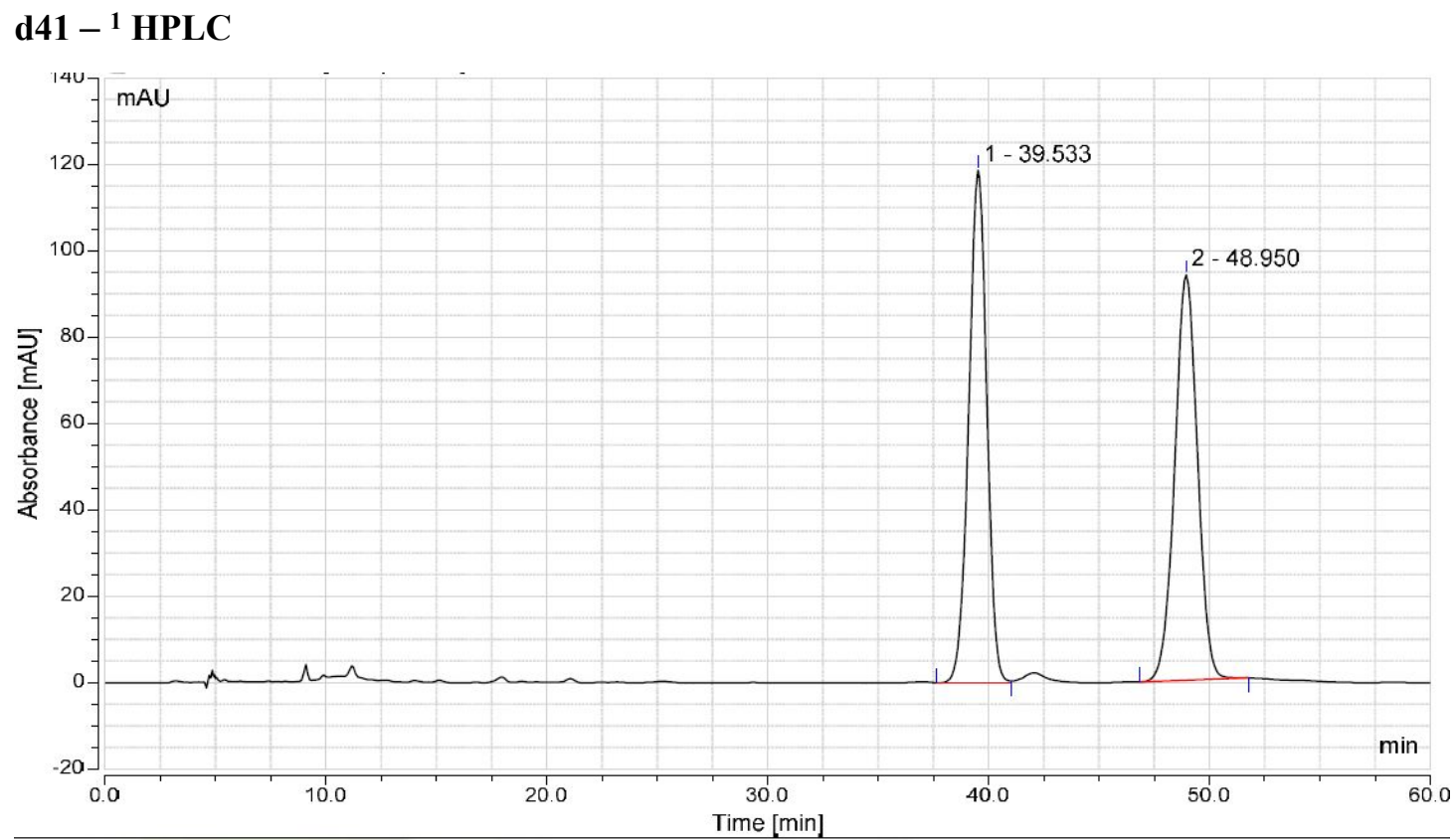

\section{Integration Results}

\begin{tabular}{l|l|c|c|c|c}
\hline No. & $\begin{array}{l}\text { Retention Time } \\
\min \end{array}$ & $\begin{array}{c}\text { Retention Time } \\
\mathrm{min}\end{array}$ & $\begin{array}{c}\text { Area } \\
\mathrm{mAU} \mathrm{U}^{*} \mathrm{~min}\end{array}$ & $\begin{array}{c}\text { Height } \\
\mathrm{mAU}\end{array}$ & $\begin{array}{c}\text { Relative Area } \\
\%\end{array}$ \\
\hline 1 & 40 & 39.533 & 112.082 & 118.660 & 50.01 \\
2 & 49 & 48.950 & 112.050 & 93.916 & 49.99 \\
\hline \multicolumn{2}{l|}{ Total: } \\
\hline
\end{tabular}

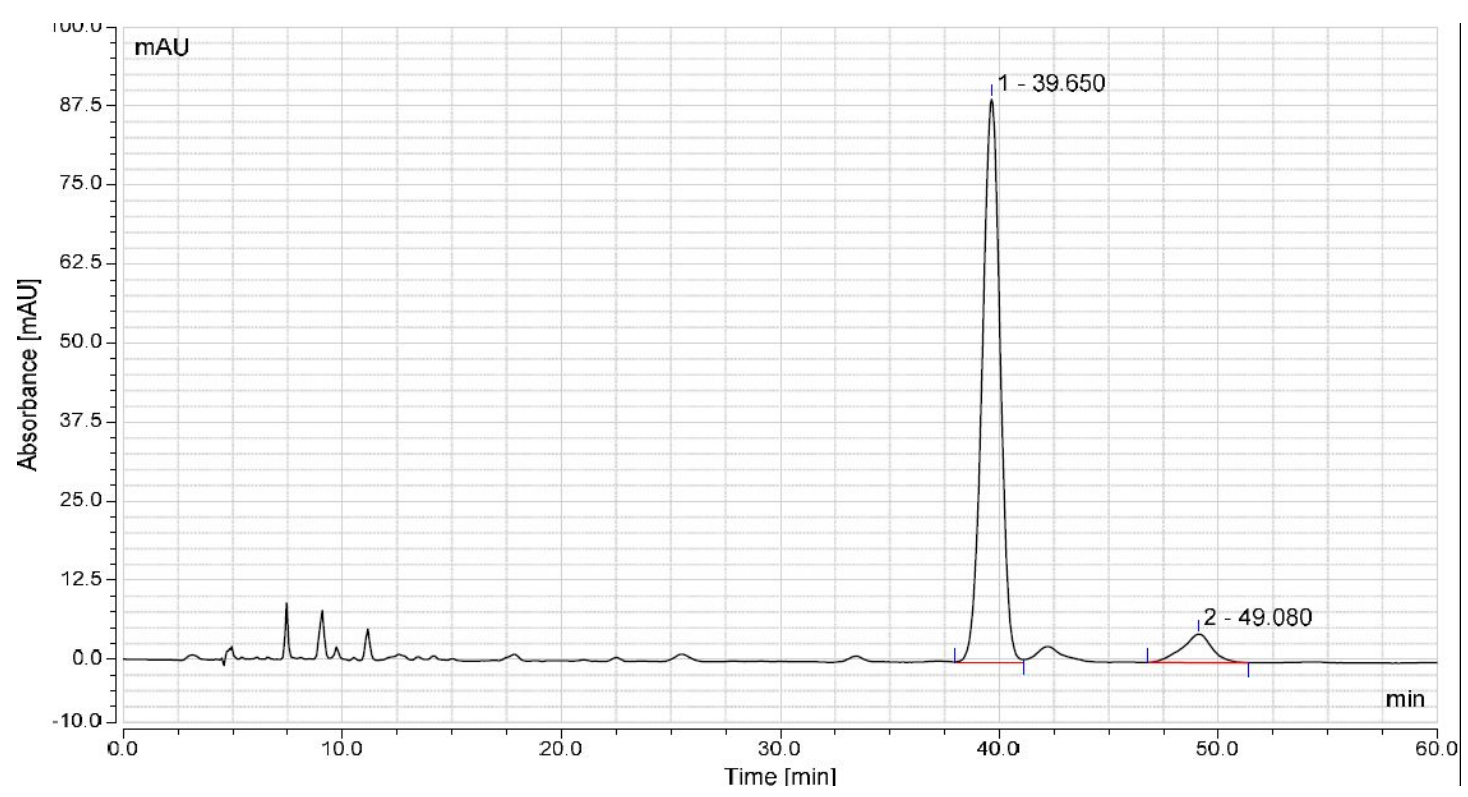

\section{Integration Results}

\begin{tabular}{l|l|c|c|c|c}
\hline No. & $\begin{array}{l}\text { Retention Time } \\
\text { min }\end{array}$ & $\begin{array}{c}\text { Retention Time } \\
\text { min }\end{array}$ & $\begin{array}{c}\text { Area } \\
\mathrm{AAU}^{*} \min \end{array}$ & $\begin{array}{c}\text { Height } \\
\mathrm{mAU}\end{array}$ & $\begin{array}{c}\text { Relative Area } \\
\%\end{array}$ \\
\hline 1 & 40 & 39.650 & 84.386 & 89.060 & 92.15 \\
2 & 49 & 49.080 & 7.190 & 4.512 & 7.85 \\
\hline
\end{tabular}


triisopropyl (((1R,4S)-3-phenylbicyclo[2.2.1] heptan-2-yl)ethynyl)silane (d42) d42 - ${ }^{1}$ H NMR

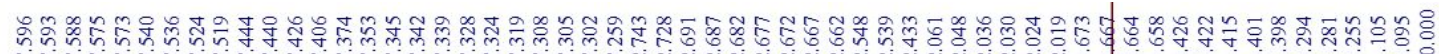

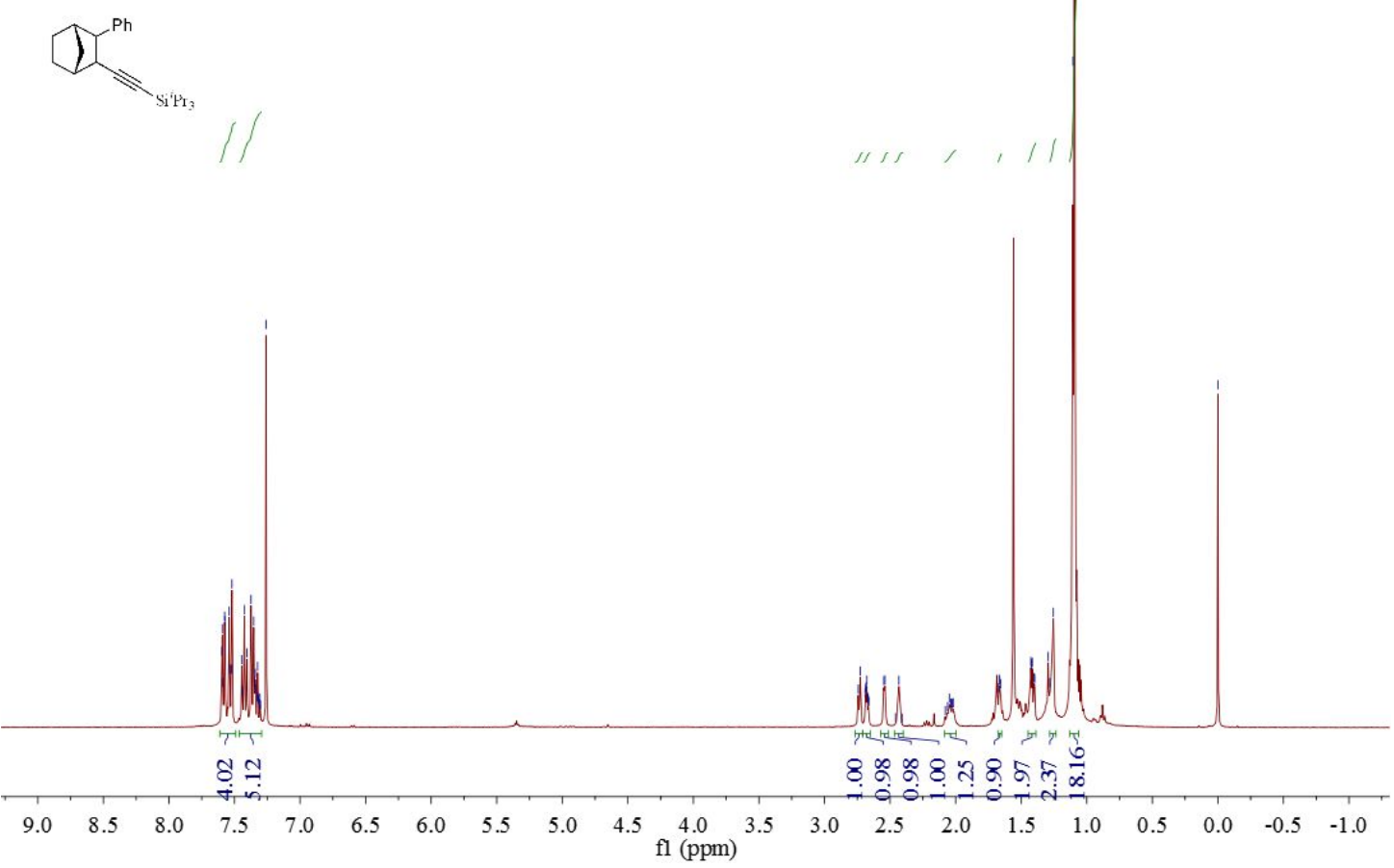

d42 - ${ }^{13}$ C NMR

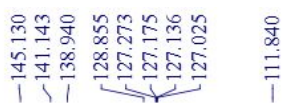

$\pi^{\mathrm{Ph}}$

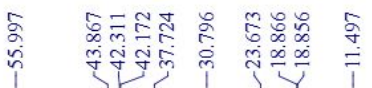

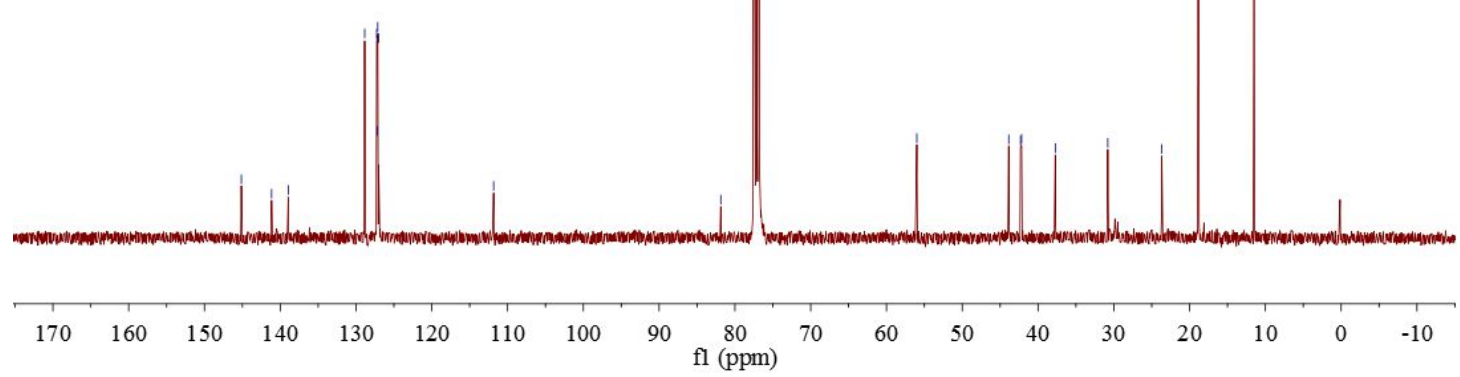


d42-HPLC

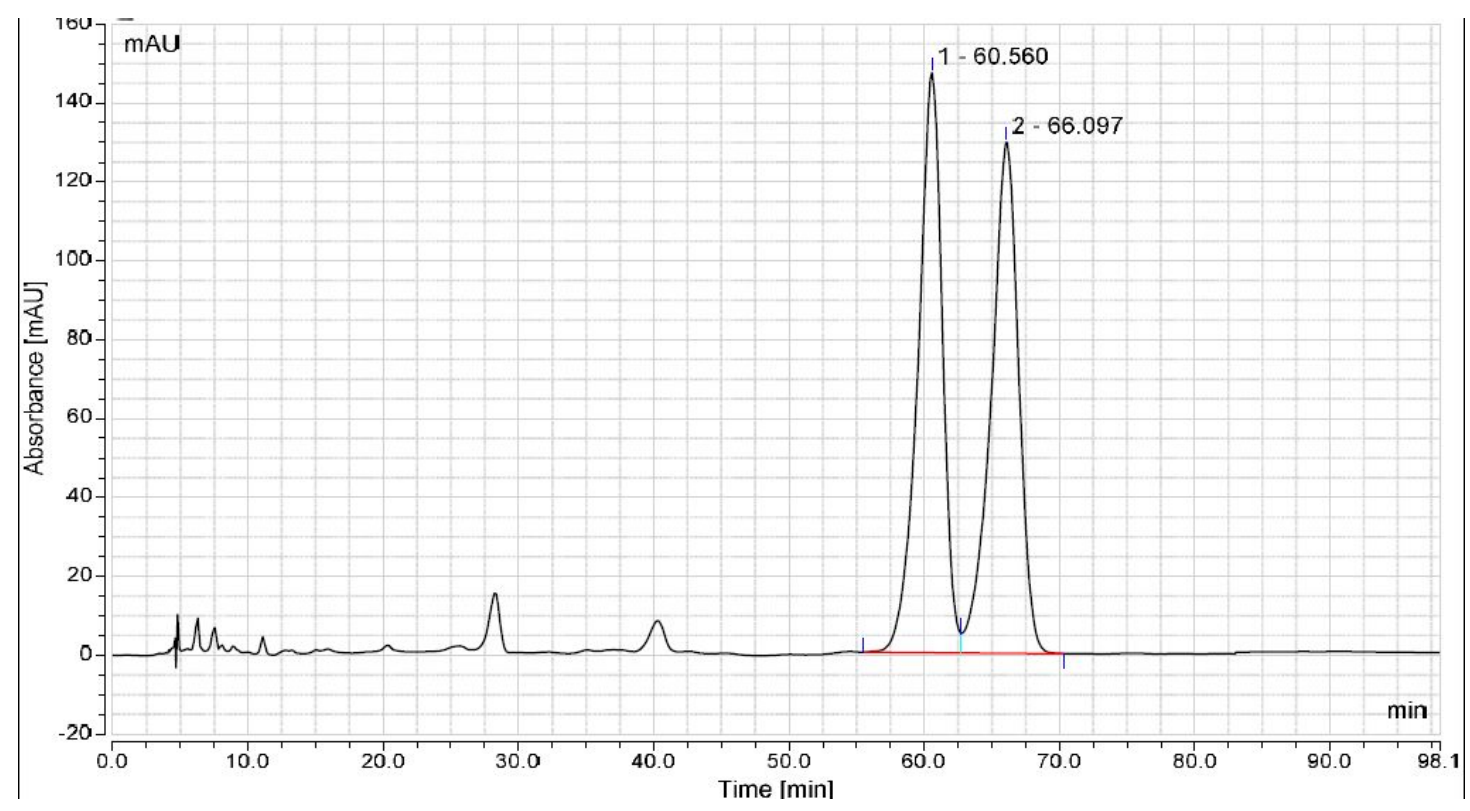

\section{Integration Results}

\begin{tabular}{|l|c|c|c|c|}
\hline No. & $\begin{array}{c}\text { Retention Time } \\
\min \end{array}$ & $\begin{array}{c}\text { Area } \\
\mathrm{mAU}^{*} \min \end{array}$ & $\begin{array}{c}\text { Height } \\
\mathrm{mAU}\end{array}$ & $\begin{array}{c}\text { Relative Area } \\
\%\end{array}$ \\
\hline 1 & 60.560 & 307.9201 & 147.0452 & 50.168 \\
2 & 66.097 & 305.8553 & 129.5594 & 49.832 \\
\hline Total: & $\mathbf{6 1 3 . 7 7 5}$ & $\mathbf{1 4 0 1 . 9 9 8}$ & $\mathbf{1 0 0 . 0 0 0}$ \\
\hline
\end{tabular}

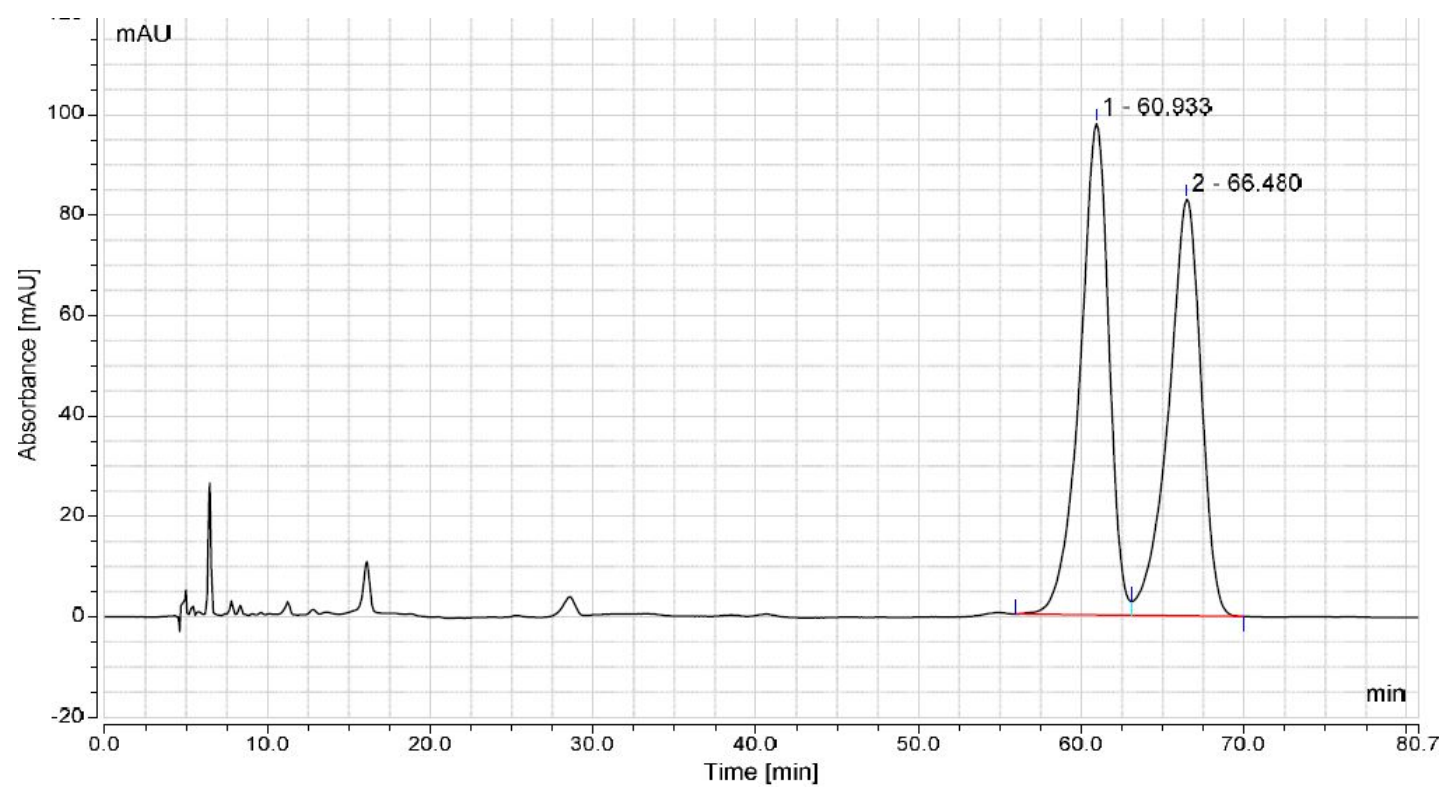

\begin{tabular}{|c|c|c|c|c|}
\hline \multicolumn{5}{|c|}{ Integration Results } \\
\hline No. & $\begin{array}{c}\text { Retention Time } \\
\text { min }\end{array}$ & $\begin{array}{c}\text { Area } \\
\mathrm{mAU* \operatorname {min }}\end{array}$ & $\begin{array}{l}\text { Height } \\
\text { mAU }\end{array}$ & $\begin{array}{c}\text { Relative Area } \\
\% \\
\end{array}$ \\
\hline $\begin{array}{l}1 \\
2\end{array}$ & $\begin{array}{l}60.933 \\
66.480\end{array}$ & $\begin{array}{l}202.0621 \\
192.3164\end{array}$ & $\begin{array}{l}97.8997 \\
83.0051\end{array}$ & $\begin{array}{l}51.236 \\
48.764\end{array}$ \\
\hline \multicolumn{2}{|c|}{ Total: } & 394.378 & 1401.998 & 100.000 \\
\hline
\end{tabular}


(R)-trimethyl (3-phenylhept-1-yn-1-yl) silane (d43) d43 - ${ }^{1}$ H NMR

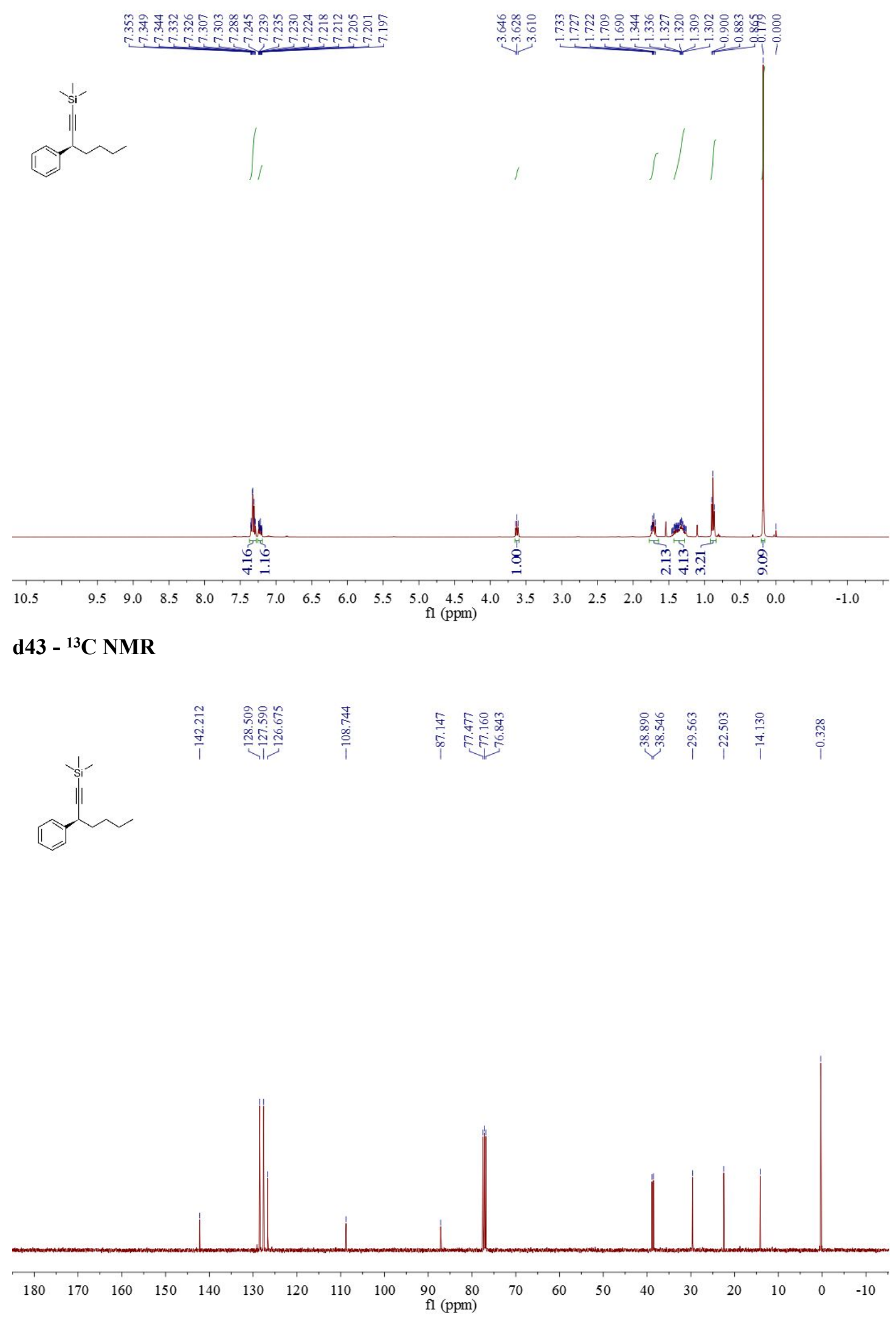


d43 - HPLC

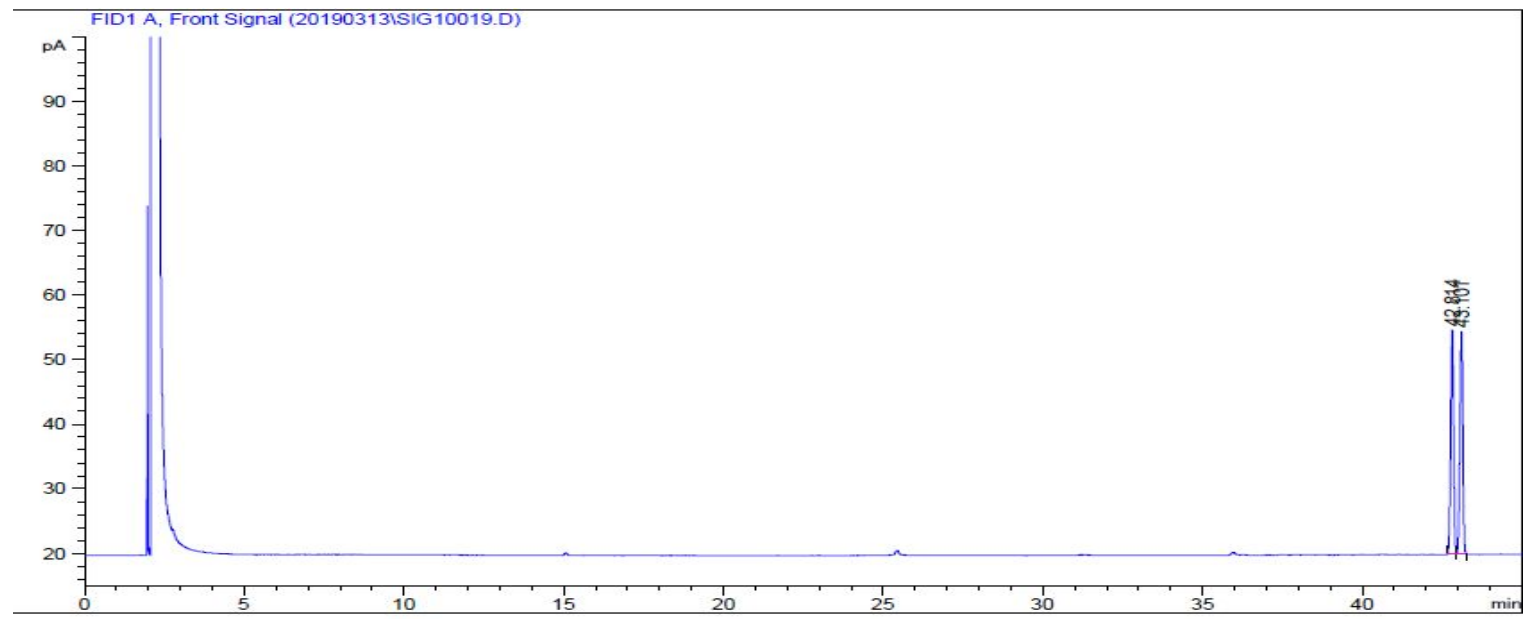

\begin{tabular}{|c|c|c|c|c|c|c|}
\hline $\begin{array}{l}\text { eak } \\
\text { \# }\end{array}$ & $\begin{array}{c}\text { RetTime } \\
\text { [min] }\end{array}$ & Tyре & $\begin{array}{l}\text { Width } \\
\text { [min] }\end{array}$ & $\begin{array}{r}\text { Area } \\
{\left[\mathrm{pA}^{*} \mathrm{~s}\right]}\end{array}$ & $\begin{array}{l}\text { Height } \\
{[\mathrm{pA}]}\end{array}$ & $\begin{array}{c}\text { Area } \\
\%\end{array}$ \\
\hline & & & & & & \\
\hline 1 & 42 & $3 \mathrm{~V}$ & 0.1 & 223 & 34 & 50 \\
\hline 2 & 43.101 & B & $0 . \epsilon$ & 222.61255 & 34.51304 & 49.86084 \\
\hline
\end{tabular}

Totals :

$446.46771 \quad 69.17459$

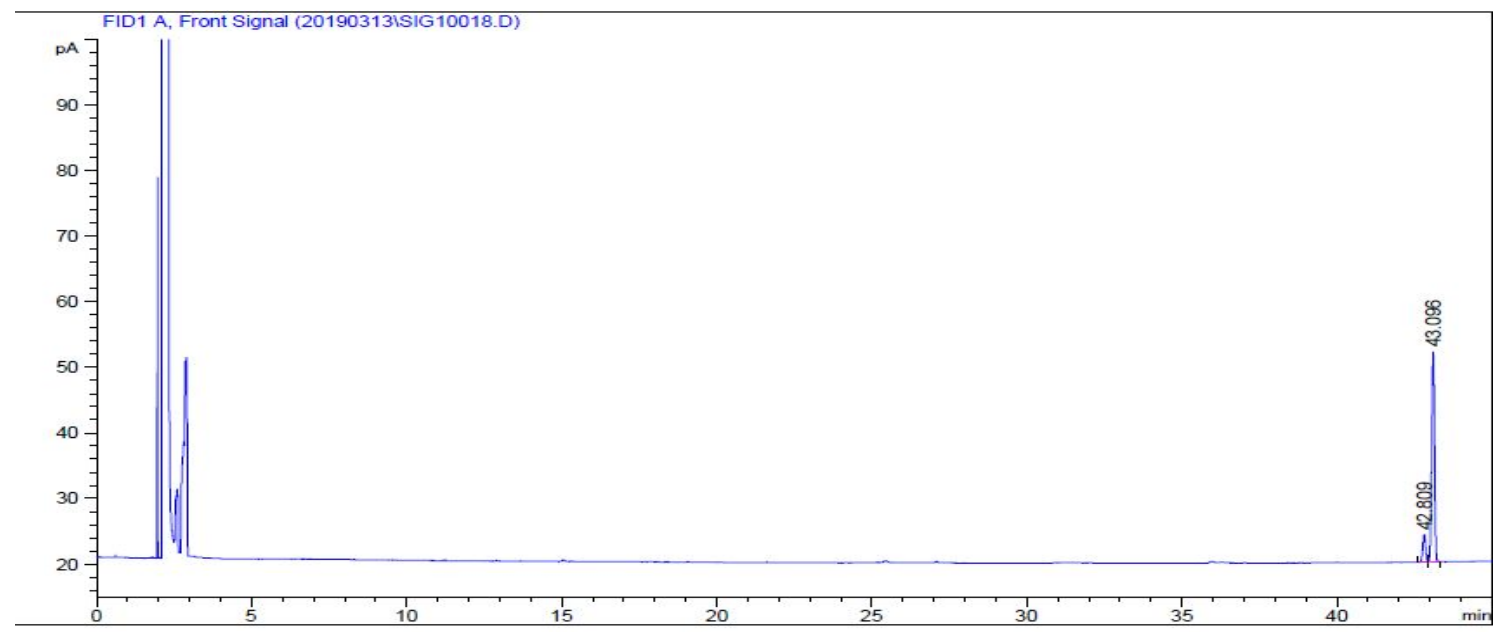

\begin{tabular}{|c|c|c|c|c|c|c|}
\hline $\begin{array}{c}\text { eak } \\
\#\end{array}$ & $\begin{array}{c}\text { RetTime } \\
\text { [min] }\end{array}$ & Type & $\begin{array}{l}\text { Width } \\
\text { [min] }\end{array}$ & $\begin{array}{r}\text { Area } \\
{\left[\mathrm{pA}^{*} \mathrm{~s}\right]}\end{array}$ & $\begin{array}{l}\text { Height } \\
{[\mathrm{pA}]}\end{array}$ & $\begin{array}{c}\text { Area } \\
\%\end{array}$ \\
\hline & & & & - & - & \\
\hline 1 & 42 & $F R$ & 0 . & 338 & 4.2 & 11. \\
\hline 2 & 43 & M & 58 & 205.57477 & 32.07396 & 88 \\
\hline
\end{tabular}

Totals :

$232.39814 \quad 36.28028$ 
(R)-4-(6, 6, 6-trifluorohex-1-yn-3-yl)-1, 1'-biphenyl (e1)

\section{e1 - ${ }^{1}$ H NMR}

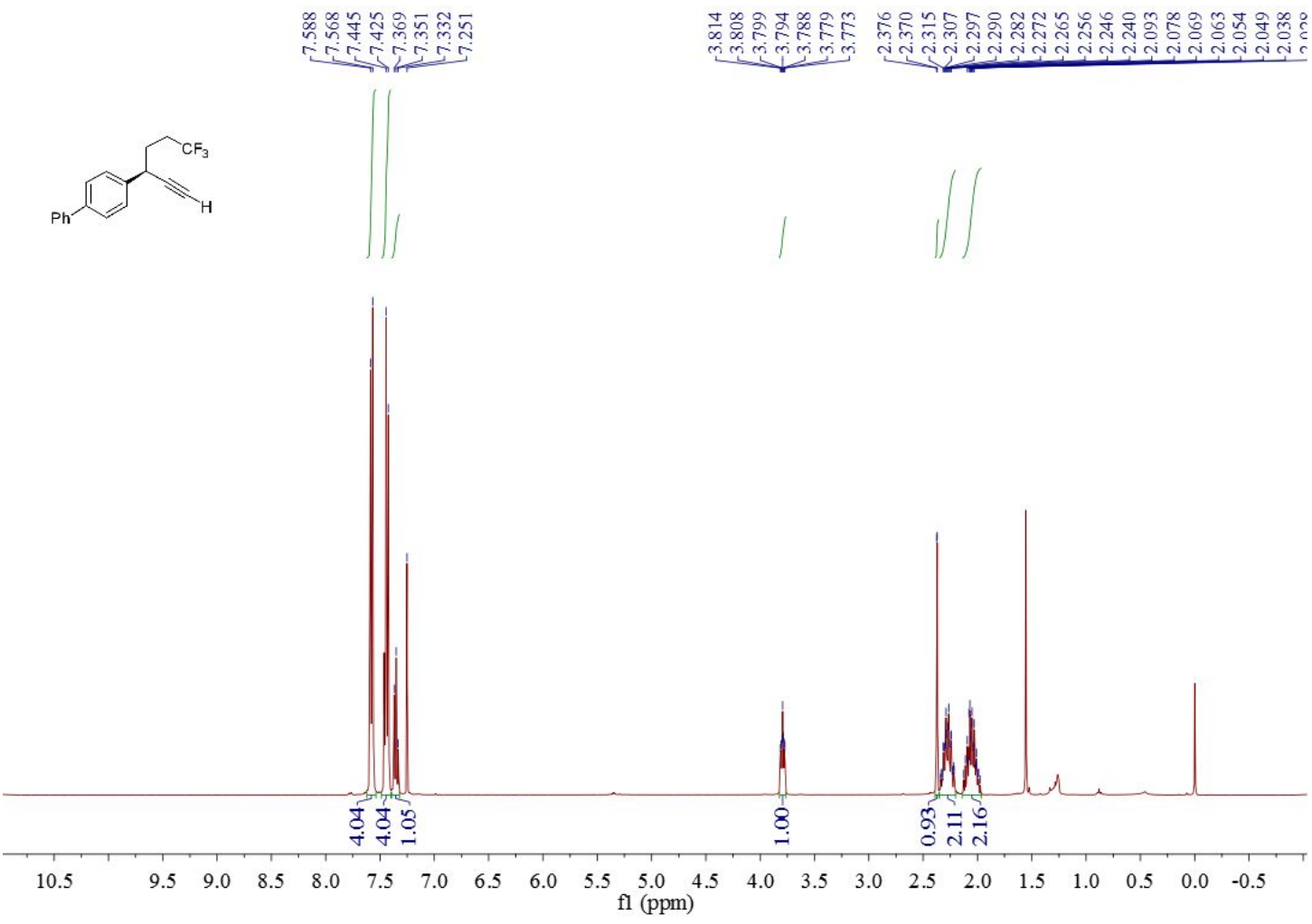

e1 - ${ }^{13}$ C NMR

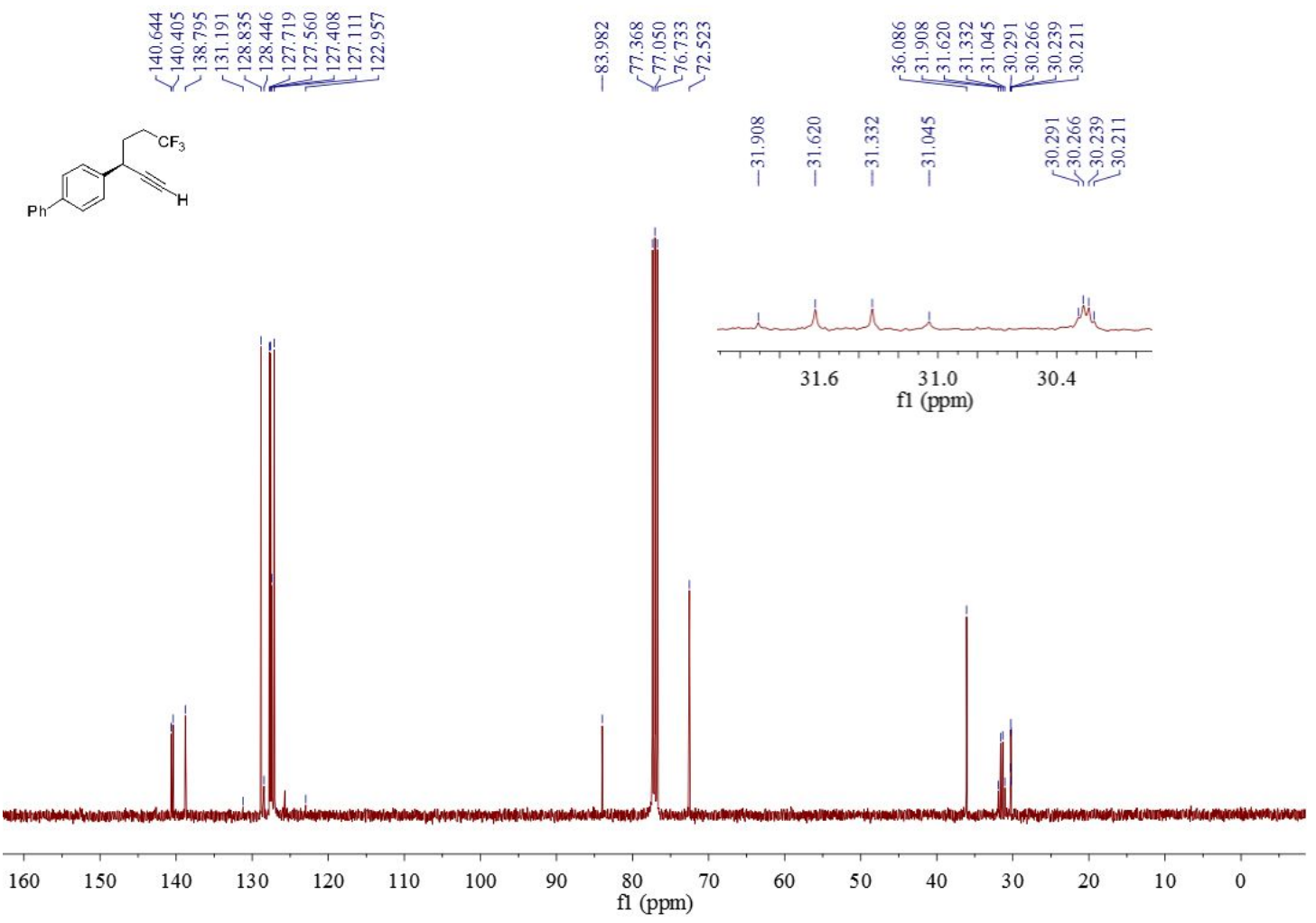




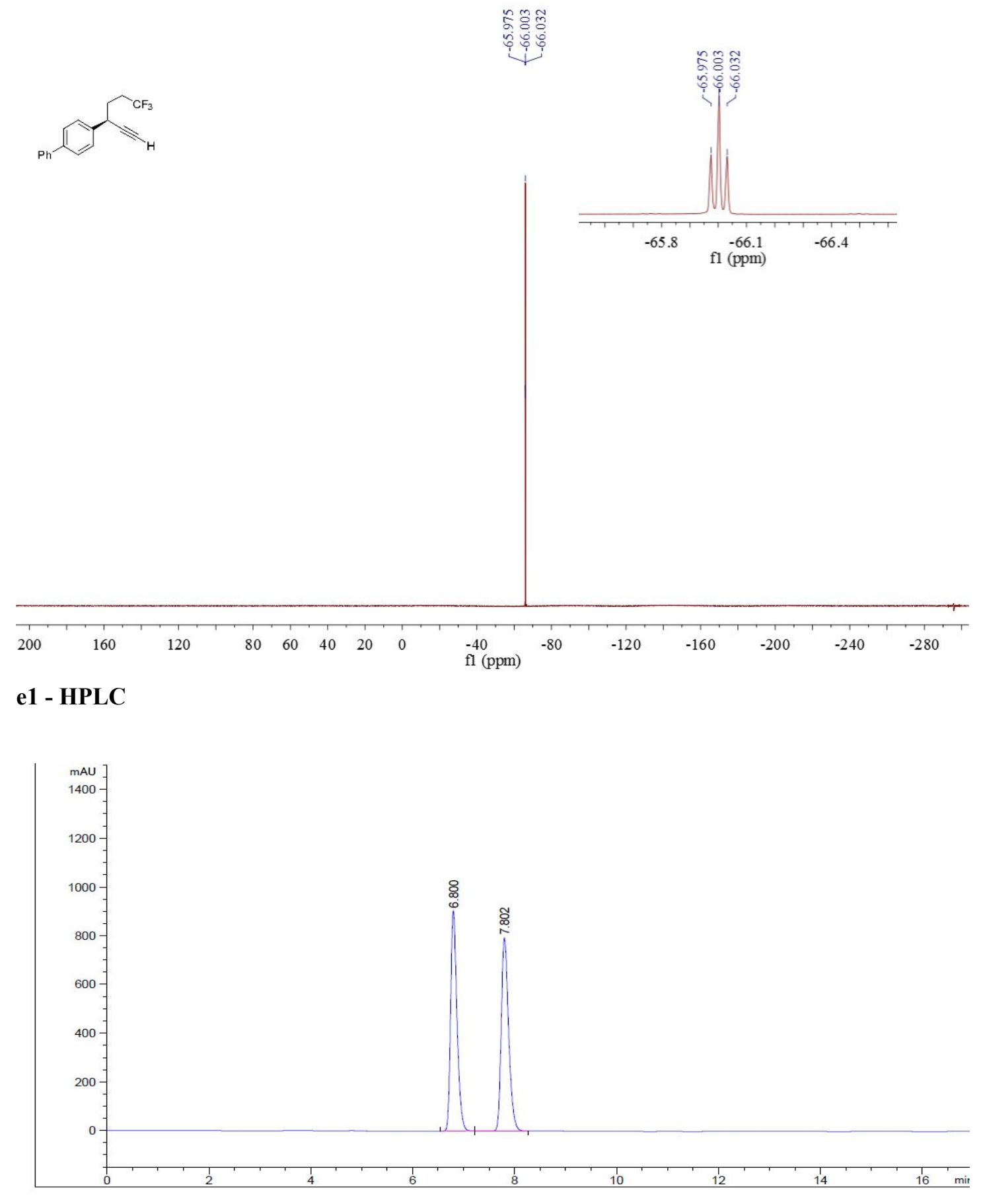

\begin{tabular}{cccccc}
$\begin{array}{c}\text { Peak RetTime Type } \\
\text { Width } \\
\text { [min] }\end{array}$ & $\begin{array}{c}\text { Area } \\
{[\mathrm{min}]}\end{array}\left[\begin{array}{c}\text { HAU*s }] \\
{[\mathrm{mAU}]}\end{array}\right.$ & $\begin{array}{c}\text { Area } \\
\%\end{array}$ \\
\hline 1 & 6.800 BB & 0.1329 & 7892.41113 & 903.48364 & 49.8936 \\
2 & 7.802 VB R & 0.1538 & 7926.05713 & 791.96484 & 50.1064
\end{tabular}

Totals : $\quad 1.58185 \mathrm{e} 4 \quad 1695.44849$ 


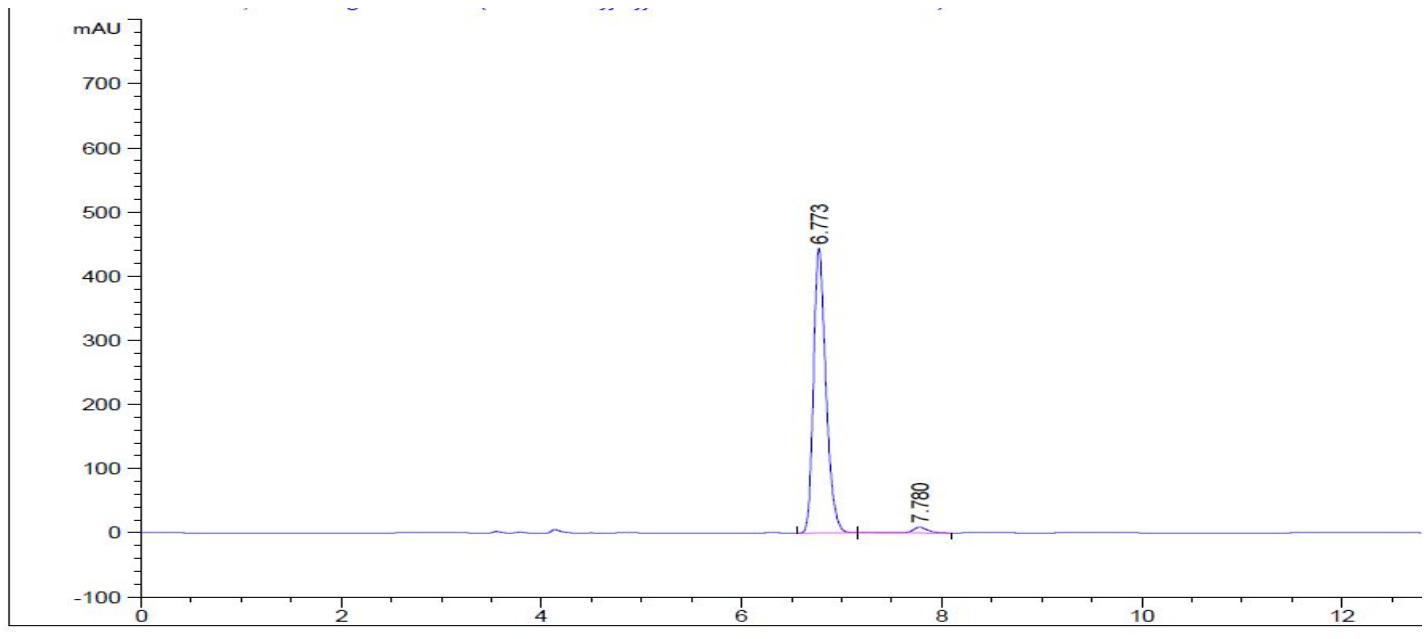

\begin{tabular}{|c|c|c|c|c|c|c|}
\hline $\begin{array}{c}\text { Peak } \\
\quad \#\end{array}$ & $\begin{array}{c}\text { RetTime } \\
\text { [min] }\end{array}$ & Type & $\begin{array}{l}\text { Width } \\
\text { [min] }\end{array}$ & $\begin{array}{c}\text { Area } \\
{\left[\mathrm{mAU}^{*} \mathrm{~s}\right]}\end{array}$ & $\begin{array}{l}\text { Height } \\
{[\mathrm{mAU}]}\end{array}$ & $\begin{array}{c}\text { Area } \\
\%\end{array}$ \\
\hline 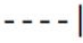 & - & & & |-...- & -- & --- \\
\hline 1 & 6.773 & BB & 0.1308 & 3833.71533 & 443.91144 & 97.6625 \\
\hline 2 & 7.780 & VB $R$ & 0.1553 & 91.75640 & 8.82810 & 2.3375 \\
\hline & : & & & 3925.47173 & 452 & \\
\hline
\end{tabular}

4-(6, 6, 6-trifluorohexa-1, 2-dien-3-yl)-1, 1'-biphenyl (f1)

f1 - ${ }^{1}$ H NMR

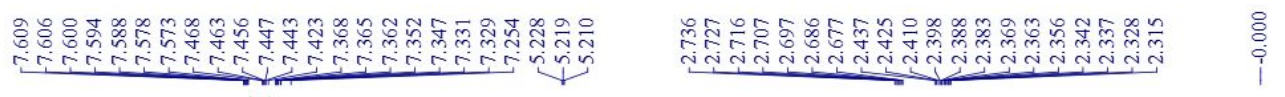
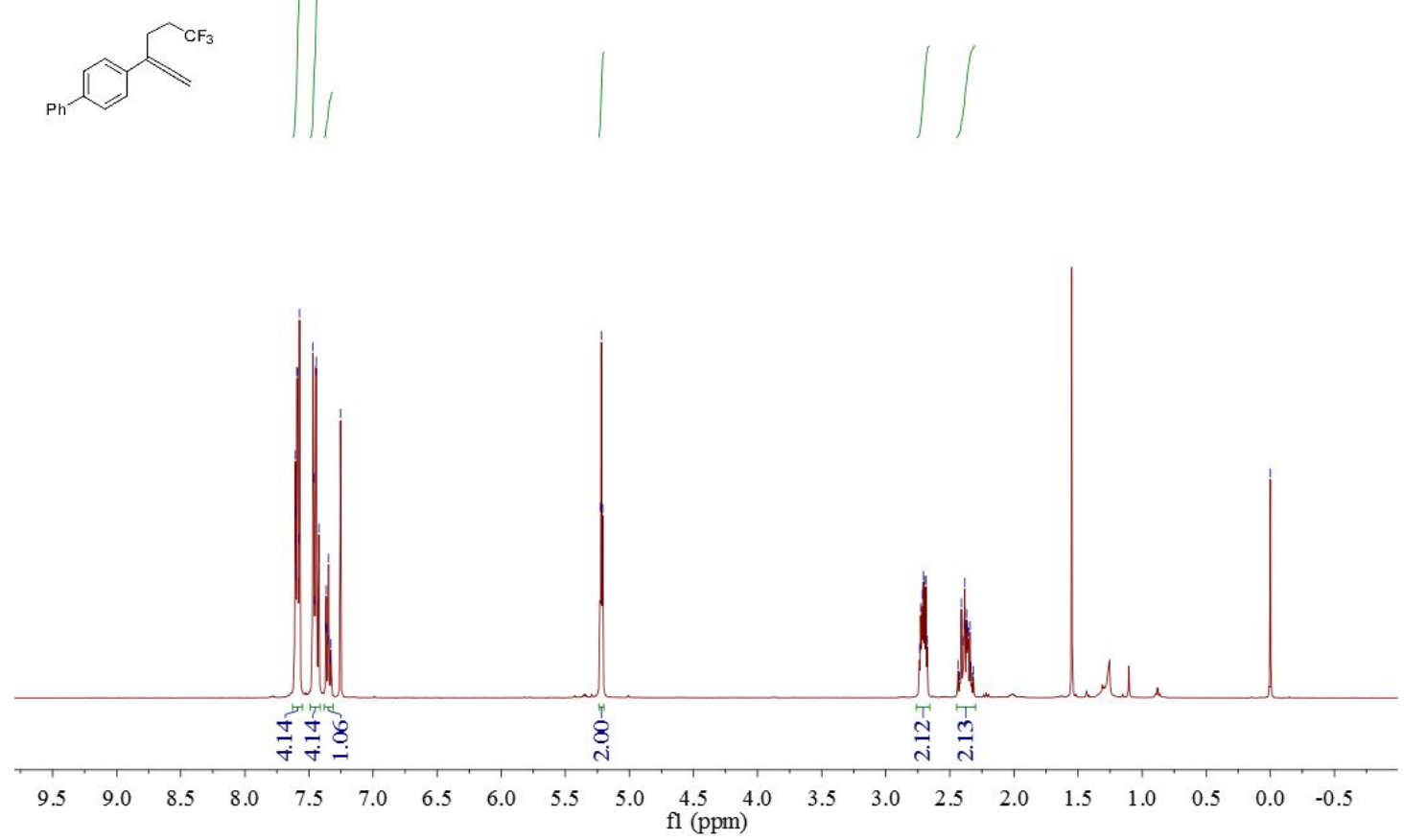
f1 $-{ }^{13}$ C NMR

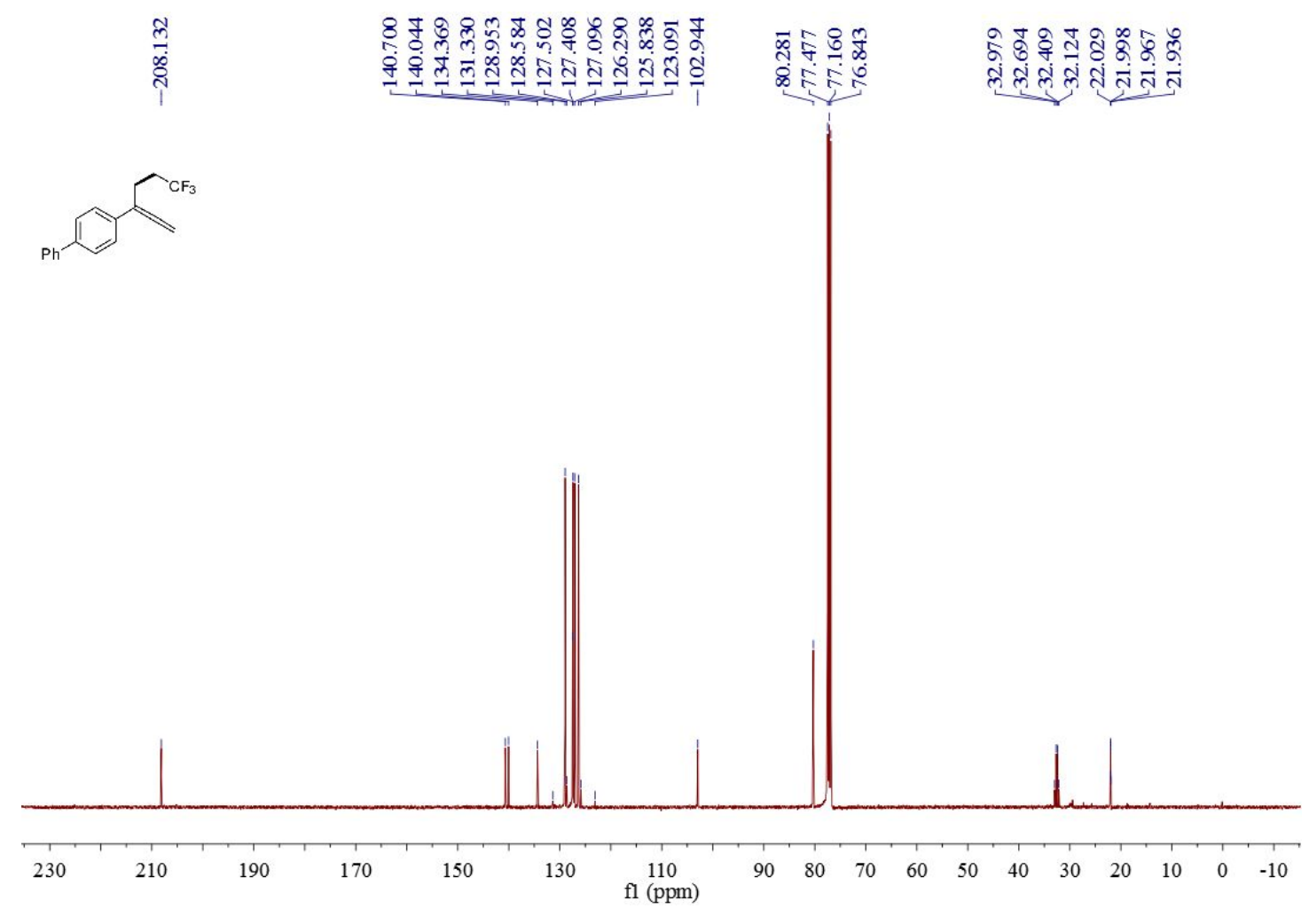

f1 $-{ }^{19}$ F NMR

일
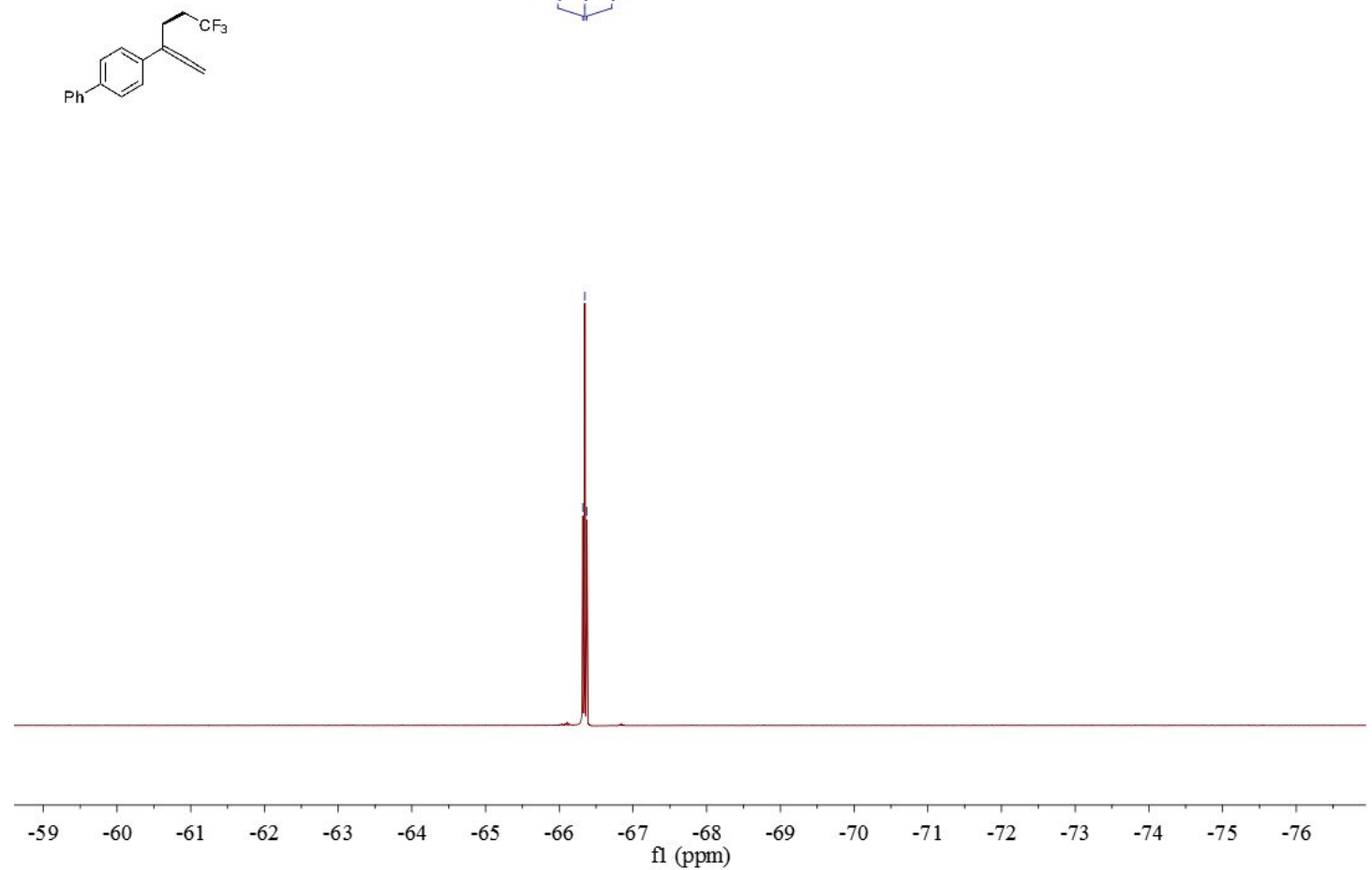
2, 2, 6, 6-tetramethyl-1-(2, 2, 2-trifluoroethoxy) piperidine

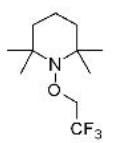

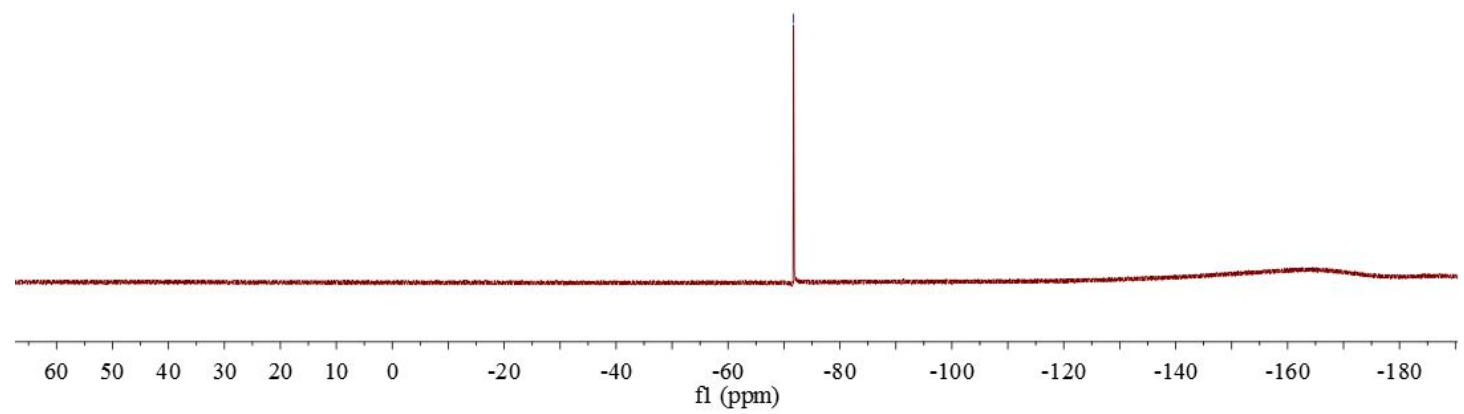

